Joachim Gassen

\title{
Datenbankgestützte Rechnungslegungspublizität
}

Ein Beitrag zur Evolution der Rechnungslegung 


\section{Joachim Gassen}

\section{Datenbankgestützte Rechnungslegungspublizität}

Rechnungslegung und Publizität stehen vor neuen Herausforderungen. Vor allem den Kapitalmarkt sollen sie mit entscheidungsrelevanten Informationen versorgen.

In dieser Arbeit wird untersucht, inwiefern die bestehenden Strukturen dieser Aufgabe gerecht werden. Basierend auf der Unmöglichkeit, eine perfekte Rechnungslegungspublizität theoretisch abzuleiten, werden grundlegende Änderungen in dem Prozeß der Datengewinnung und Präsentation vorgeschlagen, welche die Evolution der Rechnungslegung durch konkurrierende Konzepte erleichtern sollen.

Die Modifikationen führen zur Verlagerung weg von der monodimensionalen, zeitgebundenen Berichtspublizität hin zu einer mehrdimensionalen, zeitkontinuierlichen Datenbankpublizität. Hierfür wird eine exemplarische Client-Server-Umsetzung beschrieben.

Joachim Gassen, geboren 1971 in Göttingen, studierte von 1991 bis 1995 Volkswirtschaftslehre in Münster. Nach dem Abschluß als Diplom-Volkswirt arbeitete er bis zur Promotion 2000 als wissenschaftlicher Mitarbeiter am Lehrstuhl für Internationale Unternehmensrechnung von Herrn Prof. Dr. Pellens erst an der Universität Münster, dann ab 1997 an der Universität Bochum. Seit der Dissertation ist er als wissenschaftlicher Assistent am gleichen Lehrstuhl tätig. 
Datenbankgestützte Rechnungslegungspublizităt 


\section{BOCHUMER BEITRÄGE ZUR UNTERNEHMUNGSFÜHRUNG UND UNTERNEHMENSFORSCHUNG}

Herausgegeben von Prof. Dr. Dr. h.c. mult. Walther Busse von Colbe, Prof. Dr. Dr. h.c. Werner H. Engelhardt, Prof. Dr. Roland Gabriel, Prof. Dr. Arno Jaeger, Prof. Dr. Gert Laßmann, Prof. Dr. Wolfgang Maßberg, Prof. Dr. Bemhard Pellens, Prof. Dr. Marion Steven, Prof. Dr. Rolf Wartmann, Prof. Dr. Brigitte Werners, Prof. Dr. Daniel Zimmer

\section{Band 59}

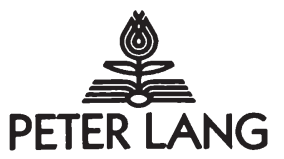

Frankfurt am Main - Berlin - Bern - Bruxelles - New York · Oxford - Wien 
Joachim Gassen

\section{Datenbankgestützte Rechnungslegungspublizität}

Ein Beitrag zur Evolution der Rechnungslegung

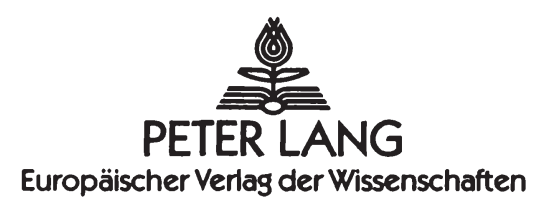


Die Deutsche Bibliothek - CIP-Einheitsaufnahme

Gassen, Joachim:

Datenbankgestützte Rechnungslegungspublizität : ein Beitrag zur Evolution der Rechnungslegung / Joachim Gassen. - Frankfurt am Main ; Berlin ; Bern ; Bruxelles ; New York ; Oxford ; Wien : Lang, 2000

(Bochumer Beiträge zur Unternehmungsführung und

Unternehmensforschung ; Bd. 59)

Open Access: The online version of this publication is published on www.peterlang.com and www.econstor.eu under the international Creative Commons License CC-BY 4.0. Learn more on how you can use and share this work: http://creativecommons.org/licenses/by/4.0.

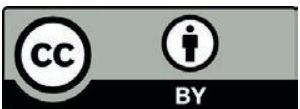

This book is available Open Access thanks to the kind support of ZBW Leibniz-Informationszentrum Wirtschaft.

Zugl.: Bochum, Univ., Diss., 2000

ISBN 3-631-36727-9

Gedruckt auf alterungsbeständigem, säurefreiem Papier.

D 294

ISSN 0175-7105

ISBN 3-631-36727-9

ISBN 978-3-631-75496-2 (eBook)

(C) Peter Lang $\mathrm{GmbH}$

Europäischer Verlag der Wissenschaften

Frankfurt am Main 2000

Alle Rechte vorbehalten.

Das Werk einschließlich aller seiner Teile ist urheberrechtlich geschützt. Jede Verwertung außerhalb der engen Grenzen des

Urheberrechtsgesetzes ist ohne Zustimmung des Verlages unzulässig und strafbar. Das gilt insbesondere für

Vervielfältigungen, Übersetzungen, Mikroverfilmungen und die Einspeicherung und Verarbeitung in elektronischen Systemen.

Printed in Germany 123467 


\section{Geleitwort}

Rechnungslegung und Unternehmenspublizität sind seit Mitte der 90er Jahren erheblichen Veränderungen ausgesetzt. Dachte man gegen Ende der 80er Jahre noch, mit der Transformation der EG-Bilanzrichtlinien in nationales Recht einen großen Meilenstein erreicht zu haben, stehen Forschung und Praxis 10 Jahre später vor vollkommen neuen Herausforderungen: Im Zuge der Internationalisierung der Produkt-, Arbeits- und vor allem der Kapitalmärkte wurde die deutsche Rechnungslegung aus ihrem beschaulichen Nischendasein förmlich herausgerissen. Plötzlich werden neue Anforderungen an sie gestellt: International akzeptiert, true und fair und -am wichtigsten- kapitalmarktorientiert solle sie sein. Schließlich verlangen Millionen neuer Teilnehmer an den boomenden nationalen und internationalen Aktienmärkten nach Hilfe bei Ihren Investitionsentscheidungen. Wie das in einer meinungspluralistischen Welt guter Brauch ist, entstand sofort eine nicht enden wollende Diskussion um die richtige oder falsche, gute oder schlechte Rechnungslegung. In Ermangelung schlagkräftiger ökonomischer Argumente ist die Debatte inzwischen überwiegend von politischem Interesse.

Was machen derweil die Rechnungslegungsadressaten? Stürzen sie sich begeistert auf die schöne neue Rechnungslegungswelt? Die Antwort fällt ernüchternd aus. Der bescheidene Zusammenhang zwischen Aktienrenditen und Bilanzdaten scheint wenig dafür zu sprechen, daß sich die internationale Entwicklung der Rechnungslegung auf einem guten Weg befindet. Das verwundert wenig, wenn der radikale Wandel berücksichtigt wird, der die Weltwirtschaft in den letzten Jahren von der Dienstleistungs- zur Informationsgesellschaft umstrukturiert. Während z.B. an den Aktienmärkten die Unternehmenspreise auf Basis von künftig erwarteten Patenten gebildet werden, wird im Umfeld der Rechnungsleger noch darüber gestritten, ob bereits bestehende selbsterstellte Patente aktiviert werden dürfen.

Warum ist die Rechnungslegung trotz vielfältiger Bemühungen derart weit von der Erfüllung der Informationsbedürfnisse der Adressaten entfernt, und wie kann sie auf Kurs gebracht werden? Auf diese Fragen präsentiert Joachim Gassen mögliche Antworten. Er konstatiert einen durch Überregulierung und vertikale Integration induzierten Mangel an evolutionärem Wandel in den Bereichen der Rechnungslegung und Publizität. Da er keine Möglichkeit sieht, auf normativen Weg die ,ideale Rechnungslegungspublizität“ zu entwerfen, schlägt er einen interessanten Ansatz vor: Nicht die Abbildungsregeln der Rechnungslegung und Publizität selber sollen verändert werden, sondem die Art, wie Rechnungslegungsdaten gewonnen und vor allem publiziert werden. Durch den Einsatz von standardisierten aber dennoch dynamischen Datenbankmodellen, die zur Generierung und Publizität von multidimensionalen Rechnungslegungsdaten dienen, erhofft er sich einerseits eine Belebung des Wettbewerbs um alternative Wertansätze und anderer- 
seits eine Stärkung der Evolutionsfähigkeit des nachgelagerten Marktes für Unternehmensanalyse. Diesem kommt die wichtige Aufgabe zu, den Adressaten bei der Entscheidungsfindung durch Aufbereitung der verfügbaren Information zu assistieren.

Herr Gassen kombiniert in seiner Arbeit sehr eindrucksvoll Ansätze der Volks- und Betriebswirtschaftslehre sowie der Wirtschaftsinformatik. Er fordert nicht nur eine prozessurale Neukonzeption der Rechnungslegungspublizität, er belegt auch sogleich im Rahmen einer Beispielimplementierung die grundsätzliche technische Umsetzbarkeit seiner Konzeption. Dadurch stellt die Arbeit eine Grundlage für vielfältige interessante Weiterentwicklungen im gesamten Bereich der elektronischen Untemehmenspublizität dar.

Es bleibt abzuwarten, inwieweit die Ansätze von Herrn Gassen in die betriebswirtschaftliche Praxis künftig umgesetzt werden. In den letzten Jahren hat sich gezeigt, wie grundlegend die neue Informationstechnologie unser berufliches und privates Umfeld verändert hat. Die sich immer deutlicher abzeichnenden Änderungen dürften vor diesem Hintergrund nur den Anfang einer weitreichenden Elektronisierung der Unternehmenspublizität darstellen. Von daher erscheint ein vorsichtiger Optimismus hinsichtlich der praktischen Umsetzung angebracht.

Bernhard Pellens 


\section{Vorwort}

Die vorliegende Arbeit wurde im Februar 2000 von der Fakultät für Wirtschaftswissenschaft der Ruhr-Universität Bochum als Dissertation angenommen. Sie entstand während meiner Tätigkeit als wissenschaftlicher Mitarbeiter am Lehrstuhl für Internationale Unternehmensrechnung zuerst an der Westfälischen Wilhelms-Universität Münster und dann ab Herbst 1997 an der Ruhr-Universität Bochum. In diesen gut vier Jahren haben sich mein Doktorvater Herr Prof. Dr. Bernhard Pellens und das gesamte Team des Lehrstuhls viel Mühe gegeben, aus dem Volkswirten mit Hang zur Informatik einen waschechten Rechnungsleger zu machen. Es ist ihnen wohl nicht gelungen.

Zur Schadensbegrenzung ermunterte Herr Prof. Dr. Pellens mich, eine Doktorarbeit etwas abseits der klassischen Bilanzierungspfade anzufertigen. Ihm gebührt Dank dafür, an seinem Lehrstuhl einerseits den notwendigen kreativen Freiraum zu schaffen und andererseits stets als kritischer Diskussionspartner für Gedanken ,in allen Gärstufen“ zur Verfügung zu stehen. Vor allem von diesen lebhaften und kurzweiligen Diskussionen habe ich wissenschaftlich und persönlich sehr profitiert.

Im Rahmen des Dissertationsverfahren erklärte sich Herr Prof. Dr. Roland Gabriel freundlicherweise zur Übernahme des Zweitgutachtens bereit. Ihm danke ich für seine Tips und Hinweise insbesondere zum informatischen Teil dieser Arbeit.

Auch meine Kolleginnen und Kollegen haben großen Anteil am Entstehen dieser Arbeit. $\mathrm{Da}$ ist zunächst die Lehrstuhlbesatzung zu nennen, die -erfrischend heterogen- dafür gesorgt hat, daß es nie langweilig wurde. Besonders hervorheben möchte ich hierbei meinen Freund und Kollegen Rolf Uwe Fülbier, der mir seit meinen Anfangstagen am Lehrstuhl stets mit Rat und Tat beiseite stand und einige meiner abstrusesten Thesen ertragen und sezieren mußte. Er hat diese Arbeit in der Entstehung begleitet und sorgfältig und kritisch gelesen. Seine Anmerkungen waren sehr hilfreich und seine freundschaftlich moralische Unterstützung hat mir ungemein geholfen. Mit Silke Sürken habe ich vor allem in unseren Bochumer Anfangstagen eine tolle gemeinsame Zeit verbracht. Ihre positive Art war sehr motivierend und hat dazu beigetragen, daß ich mich nach dem Lehrstuhlaufbau in Bochum direkt an die Dissertation gewagt habe. Weiterhin möchte ich Nils Crasselt und Ralf Schremper danken, die Teile meiner Arbeit kritisch gelesen haben. Neben meinen Kollegen haben auch die studentischen Hilfskräfte des Lehrstuhls, insbesondere Silvia Draganinska, Andree Elsner und Marc Richard beim Entstehen des Werkes mitgeholfen. Außerhalb des Lehrstuhls haben sich Jochen Müller und Eckehard Schulz durch Abschnitte dieser Arbeit gekämpft. Mit Joachim Schelp konnte ich Teile meiner Datenbankkonzeption besprechen. Ihnen allen danke ich sehr. 
Ich hatte das Glück, erste Ansätze dieser Arbeit 1998 im Rahmen des C\&L Doctoral Colloquiums der European Accounting Association präsentieren zu dürfen. Die Anmerkungen und Hinweise der dort anwesenden Hochschullehrer und anderen Teilnehmer haben der Arbeit wesentlich auf die Sprünge geholfen und die Entstehung maßgeblich beeinflußt. Dem Direktorium des Instituts für Unternehmungsführung und Unternehmensforschung und dem Peter Lang Verlag bin ich für die Aufnahme meiner Arbeit in diese Schriftenreihe zu Dank verpflichtet.

$\mathrm{Da}$ ich die gesamte Zeit der Promotion nicht nur beruflich sondern vor allem auch privat in bester Erinnerung behalte, verdanke ich meiner Familie und meinen Freunden: Meine Eltern haben mich finanziell und vor allem emotional stets unterstützt und mir damit meine Ausbildung ermöglicht. Meine Frau Birgit zeigt mir jeden Tag aufs neue, was wirklich zählt, hat mir in der „heißen“ Phase der Dissertation den Rücken freigehalten und quasi nebenbei die Arbeit auch noch Korrektur gelesen, genauso wie Ruth $\mathrm{Pa}$ genkemper und Gerrit Lemkau, denen ich ganz herzlich dafür danke. Unser Sohn Richard hat den Endzeitpunkt dieser Arbeit wesentlich mitbestimmt, deswegen gebührt Ihm hier auch der letzte Dank.

Bochum, im April 2000

Joachim Gassen 


\section{Inhaltsverzeichnis}

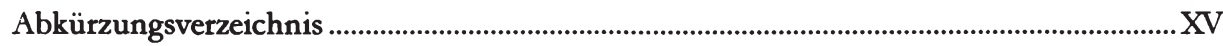

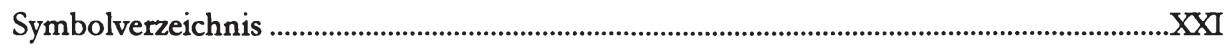

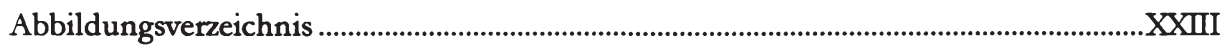

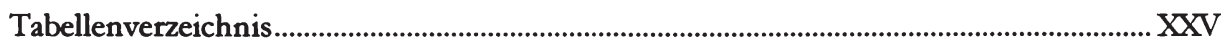

\section{Kapitel I}

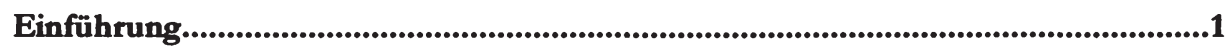

1 Problemstellung und Ziel der Untersuchung ............................................................................

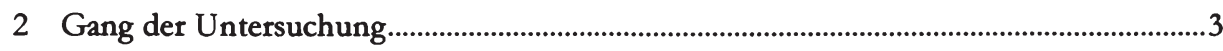

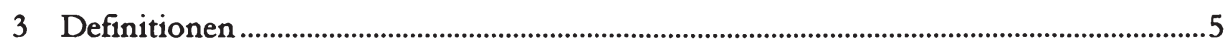

\section{Kapitel II}

Theorie der Rechnungslegung als Meßrahmen für eine normative Analyse............... 9

1 Ökonomische Begründung für Rechnungslegungspublizität ...........................................9

1.1 Zielsetzung der Rechnungslegungspublizität ........................................................9

1.2 Informationsökonomisch geprägte Ansätze ..........................................................12

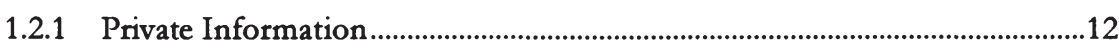

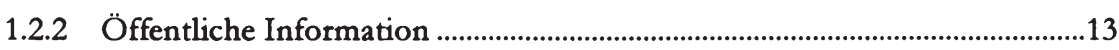

1.3 Institutionstheoretisch geprägte Ansätze.................................................................19

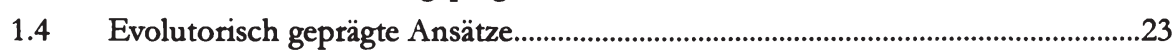

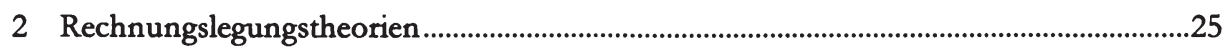

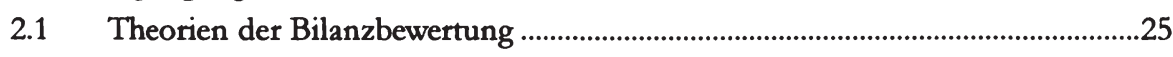

2.2 Entscheidungsorientierte Theorien.....................................................................

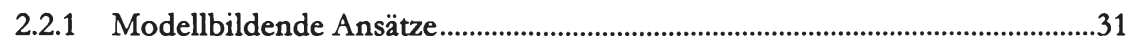

2.2.1.1 A Statement of Basic Accounting Theory ................................................31

2.2.1.2 Statement on Accounting Theory and Theory Acceptance..................35

2.2.2 Empirische Forschungen zur Entscheidungsrelevanz .......................................37

2.2.2.1 Untersuchungen auf Individualebene......................................................37

2.2.2.2 Untersuchungen auf Marktebene............................................................38

2.2.2.3 Test der Eignung von Rechnungslegungsdaten zu

Prognosezwecken ..........................................................................................39

IX 
3 Theoretische Untersuchungen als normative Entscheidungshilfe? ....................................42

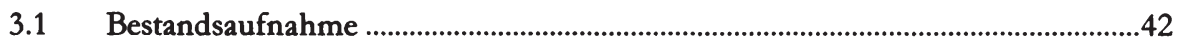

3.2 Skizzierung eines normativen Leitsatzes der Rechnungslegungspublizitätsregulierung ...........................................................44

3.3 Transaktionskostentheoretische Spezifizierung des Leitsatzes...............................47

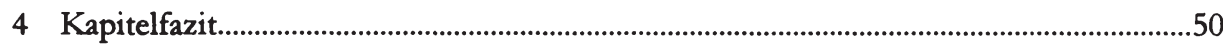

\section{Kapitel III}

Ausgestaltung der Rechnungslegungspublizität im internationalen Vergleich .

1 Historische Entwicklung der Publizitätsregulierung............................................................51

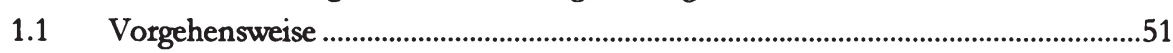

1.2 Merkantilismus ................................................................................................52

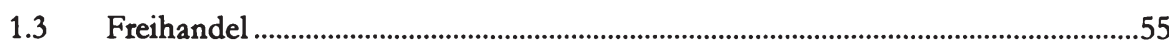

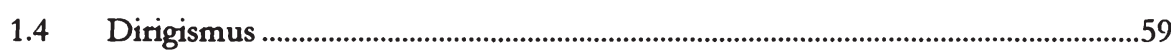

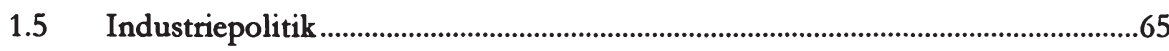

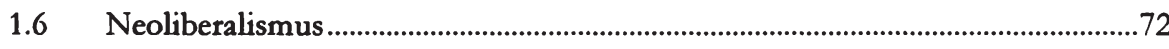

2 Positive Analyse der Rechnungslegungspublizitätsregulierung ...........................................78

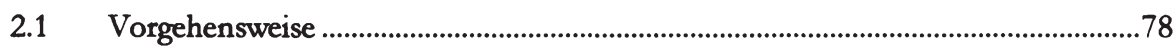

$2.2 \quad$ Außerökonomische Motive.....................................................................................78

2.3 Positive Erklärungsansätze ...................................................................................79

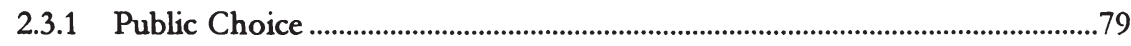

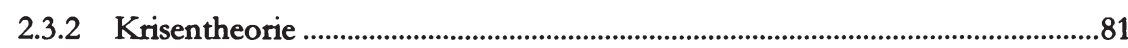

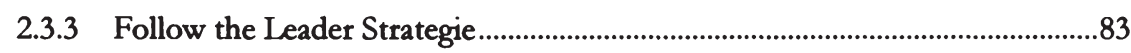

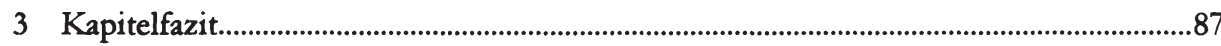

\section{Kapitel IV}

Eignung der bestehenden Publizitätssysteme zur Generierung von Marktprozessen.87

1 Charakterisierung der relevanten Rechnungslegungsmärkte.............................................87

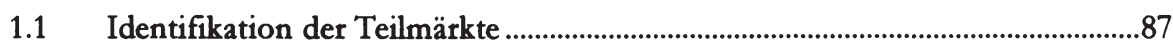

1.2 Markt für Rechnungslegungssysteme.....................................................................89

1.3 Markt für die Erstellung von Rechnungslegungsdaten ...........................................91

1.4 Markt für die Distribution von Rechnungslegungsdaten......................................93

1.5 Markt für die Analyse von Rechnungslegungsdaten ..............................................94 
2 Einfluß des deutschen Rechnungslegungspublizitätssystems auf die identifizierten

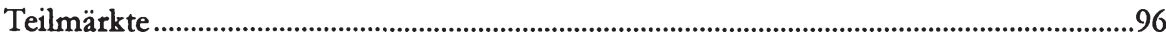

2.1 Markt für Rechnungslegungssysteme ..............................................................96

2.2 Publizitätsprozeß ..............................................................................................98

3 Einfluß des US-amerikanischen Rechnungslegungspublizitätssystems auf die identifizierten Teilmärkte ............................................................................................... 102

3.1 Markt für Rechnungslegungssysteme........................................................................ 102

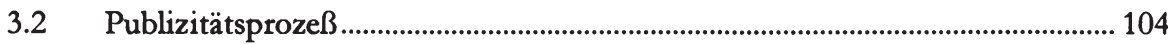

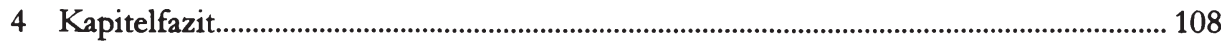

\section{Kapitel V}

Entwurf eines datenbankbasierten Publizitätssystems..................................................111

1 Theoretische Vorläufer ...................................................................................................... 111

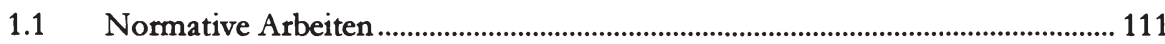

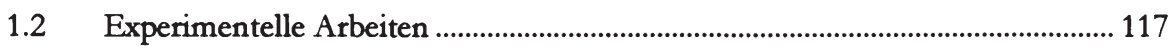

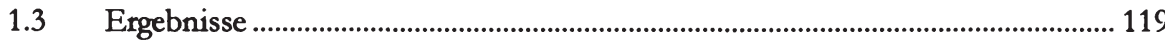

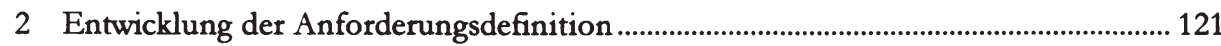

2.1 Datenhaltung im Unternehmensverbund ......................................................... 121

2.2 Öffentliches Informationssystem vs. internes Informationssystem................... 123

2.3 Eingliederung des öffentlichen Informationssystems in den Verbund der Unternehmensdaten........................................................................................................ 124

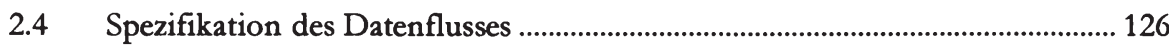

2.5 Zusammenfassung der Anforderungsdefinition ............................................... 128

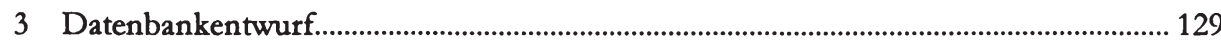

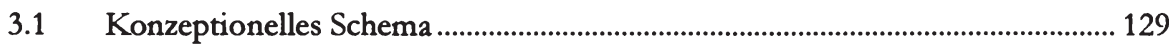

3.1.1 Starmodel als Konzeptionsgrundlage ............................................................ 129

3.1.2 Definition der Fakten ....................................................................................... 132

3.1.3 Definition der Dimensionen........................................................................... 134

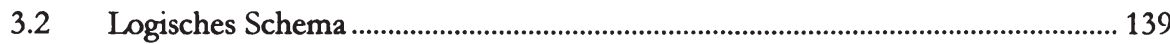

4 Implementierung eines Public-Accounting-Information-System-Server........................ 141

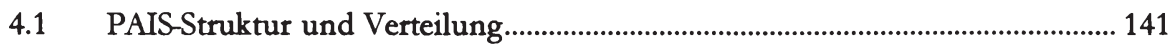

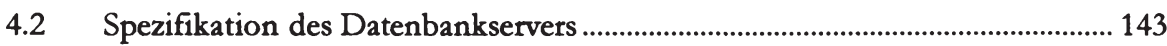

4.3 Spezifikation des Applikationsservers................................................................. 144

4.4 Generierung der PAIS-Datenbasis ...................................................................... 145

4.4.1 Schnittstelle zu operationalen Unternehmensdaten...................................... 145 
4.4.2 Erfassung von Ansatz- und Bewertungswahlrechten .................................... 148

4.4.3 Erstellung des PAIS-Datenmodells 1. Stufe ................................................... 151

4.4.4 Erstellung des PAIS-Datenmodells 2. Stufe ................................................... 152

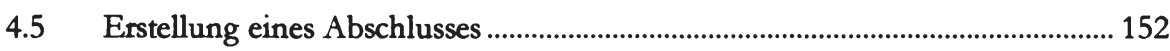

4.5.1 Charakterisierende Daten für den Abschluß …............................................ 152

4.5.2 Ermittlung der Postenbeträge .......................................................................... 153

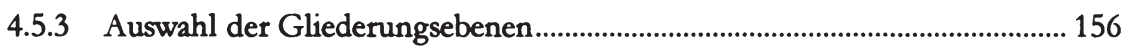

4.6 Abschlußanforderung durch den Client ................................................................. 157

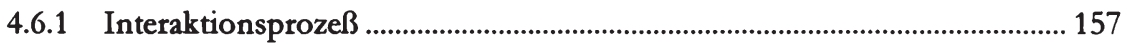

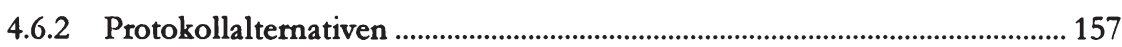

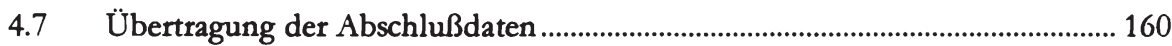

4.7.1 Mögliche Untergliederungen der Posten ................................................... 160

4.7.2 Übertragungsalternativen ......................................................................... 162

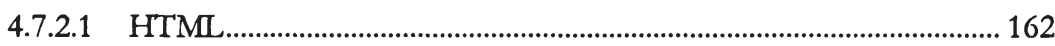

4.7.2.2 Extensible Markup Language (XML) ............................................... 164

4.7.2.3 Datenbankzugriff über ODBC/SQL................................................... 169

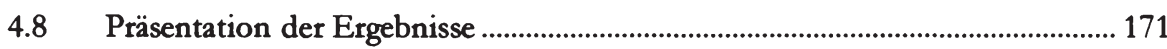

4.8.1 Rückgriff auf zentrale Präsentationsschemata ............................................... 171

4.8.2 Lokale Aufbereitungsmöglichkeiten der Daten ............................................. 173

5 Zusammenfassung und mögliche Erweiterungen ............................................................... 173

\section{Kapitel VI}

Marktwirtschaftliche Auswirkungen einer datenbankgestützten

Unternehmenspublizität ........................................................................................................177

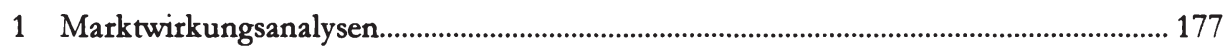

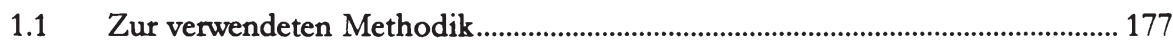

1.2 Zur Auswahl der betrachteten Märkte ............................................................. 178

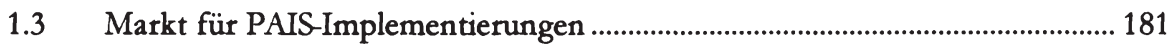

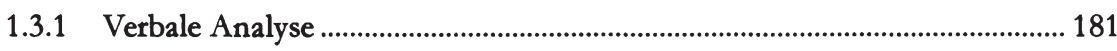

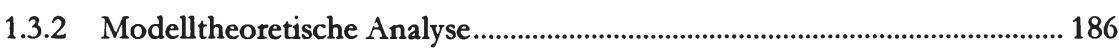

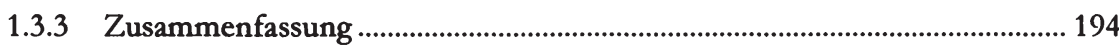

1.4 Markt für Daten- und Methodenprüfungen ....................................................... 194

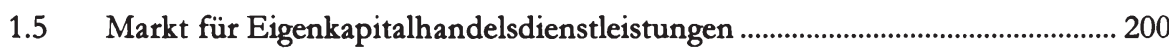

1.6 Markt für Rechnungslegungsregeln...................................................................... 202

1.7 Markt für externe Unternehmensanalyse.......................................................... 205

2 Auswirkungen auf die gesetzlichen Rahmenbedingungen............................................... 208 
Kapitel VII Zusammenfassung...................................................................... 213

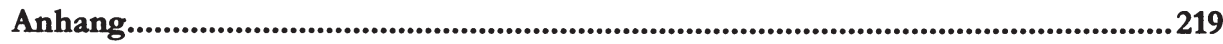

Literaturverzeichnis........................................................................................225

Verzeichnis der Rechtsquellen, Rechnungslegungspublizitätsnormen, Urteile und technischen Normen.

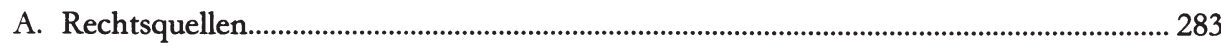

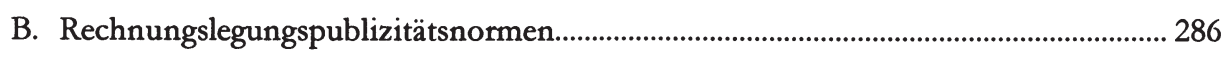

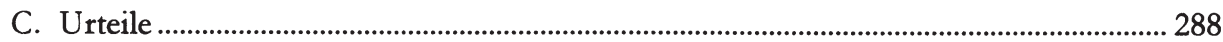

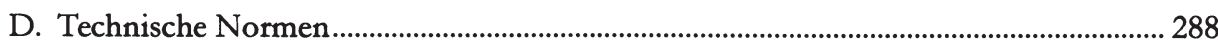


Joachim Gassen - 978-3-631-75496-2

Downloaded from PubFactory at 01/11/2019 04:12:56AM

via free access 


\section{Abkürzungsverzeichnis}

a.F.

AAA

AAER

ABl. EG

ABR

Abs.

AccRev.

ADHGB

AER

AG

AICPA

AIMR

ALR

APB

ASOBAT

ASR

BAWe

BB

BBK

$\mathrm{BFH}$

BFuP

BGBl.

BiRiLiG

BLOB

BMF

BMJ

BörsG

BörsZulVO

BR-Drucks.

BT-Drucks.

Bus. Info. Rev. alte Fassung

American Accounting Association

Accounting and Auditing Enforcement Release

Amtsblatt der Europäischen Gemeinschaften

Accounting and Business Research

Absatz

The Accounting Review

Allgemeines Deutsches Handelsgesetzbuch

American Economic Review

Aktiengesellschaft; Die Aktiengesellschaft (Zeitschrift)

American Institute of Certified Public Accountants

Association for Investment Management and Research

Allgemeines Landrecht für die preußischen Staaten

Accounting Principles Board

A Statement of Basic Accounting Theory

Accounting Series Release

Bundesaufsichtsamt für Wertpapierhandel

Betriebs-Berater (Zeitschrift)

Betrieb und Rechnungswesen: Buchführung, Bilanz, Kostenrechnung (Zeitschrift)

Bundesfinanzhof

Betriebswirtschaftliche Forschung und Praxis (Zeitschrift)

Bundesgesetzblatt

Bilanzrichtlinien-Gesetz

Binary Large Object

Bundesministerium der Finanzen

Bundesministerium der Justiz

Börsengesetz

Börsenzulassungs-Verordnung

Bundesrats-Drucksache

Bundestags-Drucksache

Business Information Review 
c.p.

Cal.Gen.Corp.L.

CAP

CAR

CGI

Const.

CR

DB

DBMS

DBW

Del.Gen.Corp.L.

DIN

DRSC

DStR

DTD

DVFA

DVFA/SG

e.V.

EAA

EAR

EDGAR

ERM

et al.

EU

EWG

F\&E

f.

FASB

FB

FRR

FTC

GAAP

gem. ceteris paribus

California General Corporation Law

Committee on Accounting Procedure

Contemporary Accounting Research (Zeitschrift)

Common Gateway Interface

Constitution of the United States

Computer und Recht (Zeitschrift)

Der Betrieb (Zeitschrift)

Datenbankmanagementsystem

Die Betriebswirtschaft (Zeitschrift)

Delaware General Corporation Law

Deutsches Institut für Normung e.V.

Deutsches Rechnungslegungs Standards Committee

Deutsches Steuerrecht (Zeitschrift)

Document Type Definition

Deutsche Vereinigung für Finanzanalyse und Anlageberatung e.V.

Deutsche Vereinigung für Finanzanalyse und Anlageberatung e.V./Schmalenbach-Gesellschaft - Deutsche Gesellschaft für Betriebswirtschaft e.V.

eingetragener Verein

European Accounting Association

European Accounting Review (Zeitschrift)

Electronic Data Gathering, Analysis, and Retrieval

Entitäten-Relationen-Modell

et alii

Europäische Union

Europäische Wirtschaftsgemeinschaft

Forschung und Entwicklung

und folgende

Financial Accounting Standards Board

Finanz Betrieb (Zeitschrift)

Financial Reporting Release

Federal Trade Commission

Generally Accepted Accounting Principles

gemäß 
ggf.

$\mathrm{GmbH}$

GNU

GPL

GS.

$\mathrm{GuV}$

HB

HFA

HGB

Hrsg.

hrsg.

HTML

HTTP

HWF

HWR

i.d.F.

i.V.m:

IAS

IASC

IDW

IETF

IKR

Inc.

insb.

ISO

JAE

JAR

JbeNatStat

Jg.

JITE

JoA

JoF

KapAEG

KonTraG

$\operatorname{krp}$ gegebenenfalls

Gesellschaft mit beschränkter Haftung

GNU is not Unix

General Public License

Preußische Gesetzessammlung

Gewinn- und Verlustrechnung

Handelsblatt

Hauptfachausschuß

Handelsgesetzbuch

Herausgeber

herausgegeben

Hypertext Markup Language

Hypertext Transfer Protocol

Handwörterbuch des Bank- und Finanzwesens

Handwörterbuch des Rechnungswesens

in der Fassung

in Verbindung mit

International Accounting Standard

International Accounting Standards Committee

Institut der Wirtschaftsprüfer

Internet Engineering Task Force

Industrieller Kontenrahmen

Incorporated

insbesondere

International Organization for Standardization

Journal of Accounting and Economics

Journal of Accounting Research

Jahrbücher für Nationalökonomie und Statistik

Jahrgang

Journal for Institutional and Theoretical Economics

Journal of Accountancy

Journal of Finance

Kapitalaufnahmeerleichterungsgesetz

Gesetz zur Kontrolle und Transparenz im Unternehmensbereich

Kostenrechnungspraxis 
M.B.C.A.

m.w.N.

MOG

MSS

N.Y.Bus.Corp.L.

NJW

Nr.

o. Jg.

o.a.

ODBC

OLAP

OSI

PAIS

Par.

PDF

PublG

R.M.B.C.A.

REA

RFC

RGBl.

RIW

ROHG

$\mathrm{S}$.

SA

SARB

SEA

SEC

sec.

SFAC

SFAS

SGML

SMAX

SoP

Sp.

SQL
Model Business Corporation Act

mit weiteren Nachweisen

Mindestoptimale Betriebsgröße

Management Support System

New York Business Corporation Law

Neue Juristische Wochenschrift

Nummer

ohne Jahrgang

oben angegeben(e)

Open Database Connectivity

Online Analytical Processing

Open System Interconnection

Public Accounting Information System

Paragraph

Portable Document Format

Publizitätsgesetz

Revised Model Business Corporation Act

Ressource-Event-Agent

Request for Comment

Reichsgesetzblatt

Recht der Internationalen Wirtschaft (Zeitschrift)

Reichsoberhandelsgericht

Seite

Securities Act

Status of Accounting Research Bulletins

Securities Exchange Act

Securities and Exchange Commission

section

Statement of Financial Accounting Concepts

Statement of Financial Accounting Standards

Standard Generalized Markup Language

Small Cap Exchange

Statement of Position

Spalte

Structured Query Language 
StuB

StückAG

StuW

TCP/IP

Tz.

Unif.S.A.

US

usw.

VerkProspG

VerkProspVO

Vgl.

Vol.

W3C

WiSt

WISU

WM

WPg

WpHG

WPK

WWW

XFRML

XML

z.B.

$\mathrm{ZB}$

$\mathrm{ZBB}$

$\mathrm{ZfB}$

$\mathrm{ZfbF}$

$\mathrm{ZfhF}$

ZGR

ZHR
Steuern und Bilanzen (Zeitschrift)

Stückaktiengesetz

Steuer und Wirtschaft

Transmission Control Protocol/Internet Protocol

Textziffer

Unified Securities Act

United States (of America)

und so weiter

Verkaufsprospektgesetz

Verkaufsprospekt-Verordnung

Vergleiche

Volume

The World Wide Web Consortium

Wirtschaftswissenschaftliches Studium (Zeitschrift)

Das Wirtschaftsstudium (Zeitschrift)

Wertpapier-Mitteilungen (Zeitschrift)

Die Wirtschaftsprüfung (Zeitschrift)

Wertpapierhandelsgesetz

Wirtschaftsprüferkammer

World Wide Web

XML-based Financial Reporting Markup Language

Extensible Markup Language

zum Beispiel

Zahlungsbereitschaft

Zeitschrift für Bankrecht und Bankwirtschaft

Zeitschrift für Betriebswirtschaft

Zeitschrift für betriebswirtschaftliche Forschung

Zeitschrift für handelswissenschaftliche Forschung

Zeitschrift für Unternehmens- und Gesellschaftsrecht

Zeitschrift für das gesamte Handels- und Wirtschaftsrecht 
Joachim Gassen - 978-3-631-75496-2

Downloaded from PubFactory at 01/11/2019 04:12:56AM

via free access 


\section{Symbolverzeichnis}

\begin{tabular}{|c|c|}
\hline$\vec{b}$ & Bewertungsvektor \\
\hline$\gamma$ & $\begin{array}{l}\text { Elastizität der Ausbringungsmenge hinsichtlich der } \\
\text { Nachfrage }\end{array}$ \\
\hline$\delta$ & $\begin{array}{l}\text { Elastizität der Produktionskosten hinsichtlich der Aus- } \\
\text { bringungsmenge }\end{array}$ \\
\hline$f$ & multiplikativer Kostenfaktor \\
\hline$i$ & Zählvariable \\
\hline$k$ & Konto \\
\hline$K$ & Kosten \\
\hline$m$ & Produktionsmenge \\
\hline$n$ & Anzahl der jeweiligen Vektorelemente \\
\hline$\rho$ & $\begin{array}{l}\text { Elastizität der Produktionskosten hinsichtlich der Nach- } \\
\text { frage }\end{array}$ \\
\hline$\vec{r}$ & realwirtschaftlicher Phänomenvektor \\
\hline$t$ & Zeitpunkt \\
\hline$w$ & Monetärer Buchungsbetrag \\
\hline$x$ & Nachgefragte Menge \\
\hline
\end{tabular}


Joachim Gassen - 978-3-631-75496-2

Downloaded from PubFactory at 01/11/2019 04:12:56AM

via free access 


\section{Abbildungsverzeichnis}

Abb. 1: Vertragsformen bei Transaktionen................................................................................4

Abb. 2: Rechnungslegungspublizitätsprozeß...............................................................................49

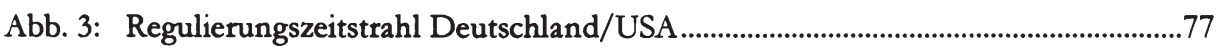

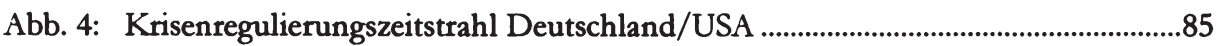

Abb. 5: Marktsegmentierung im Rechnungslegungsprozeß....................................................88

Abb. 6: Marktsegmentierung in der Abschlußerstellung ......................................................92

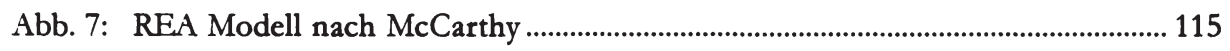

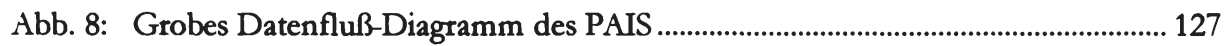

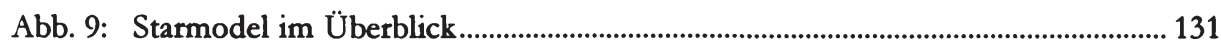

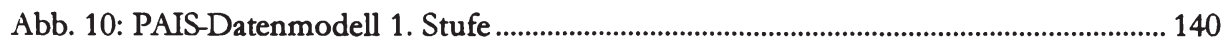

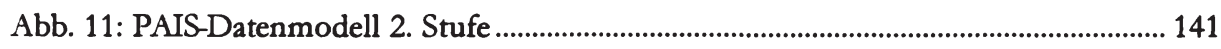

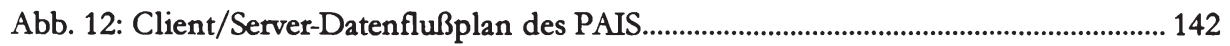

Abb. 13: Screenshot Relation Buchungen.................................................................................. 146

Abb. 14: Screenshot Herstellungskostenbuchungen ................................................................ 149

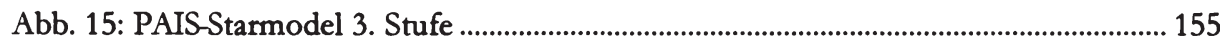

Abb. 16: HTML basierte PAIS-Benutzerschnittstelle ....................................................... 159

Abb. 17: Exemplarischer Datenwürfel des Forderungspostens............................................ 161

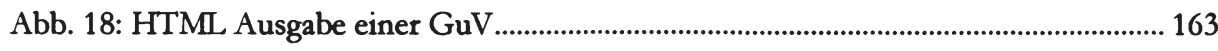

Abb. 19: Extensive Darstellung des Veröffentlichungsspiels.................................................. 187

Abb. 20: Rechnungslegungspublizitätsprozeß mit PAIS........................................................... 217 
Joachim Gassen - 978-3-631-75496-2

Downloaded from PubFactory at 01/11/2019 04:12:56AM

via free access 


\section{Tabellenverzeichnis}

Tab. 1: Anforderungen an die Konzeption des Publizitätssystems.................................. 128

Tab. 2: Beispielgeschäftsvorfälle Anlagenbuchungen.......................................................... 146

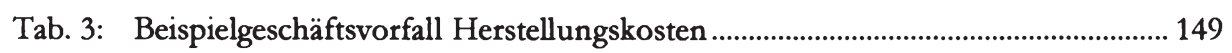


Joachim Gassen - 978-3-631-75496-2

Downloaded from PubFactory at 01/11/2019 04:12:56AM

via free access 


\title{
Kapitel I
}

\section{Einführung}

\begin{abstract}
"Wir müssen uns aber stets bewußt bleiben, daß die Bilanz ein in tausend Gestalten erscheinendes Gebilde des Lebens, des sich ständig in Form und Inhalt wandelnden Wirtschaftslebens ist. Wie alle Dinge der Wirklichkeit ist auch sie zu mannigfaltig und unerschöpflich, um unter e i n e $m$ Gesichtspunlet erfaßt werden zu können."
\end{abstract} Karl Käfer, Die Bilanz als Zukunftsrechnung, Zürich 1962, S.6.

\section{Problemstellung und Ziel der Untersuchung}

Rechnungslegung ist einer der zentralen Pfeiler der betriebswirtschaftlichen Lehre und Forschung. Um so verwunderlicher erscheint es, daß in der Literatur keine einheitliche Meinung erkennbar ist, was Rechnungslegung leisten sollte, leisten könnte und letztendlich leistet. Während Vertreter einer Extremposition den Sinn der Rechnungslegung lediglich in der Lösung von Verteilungsproblemen wie z.B. in der Festsetzung von Dividendenzahlungen und als Steuerbemessungsgrundlage sehen, gehen die Wissenschaftler der anderen Extremposition davon aus, daß die Rechnungslegung ein zentrales Informationsinstrument auf den unterschiedlichsten Märkten darstellt. ${ }^{1}$

Diese unterschiedlichen Sichtweisen resultieren auch aus der historischen und juristischen Entwicklung, die dem Instrument Rechnungslegung beschieden war. ${ }^{2}$ So ist die kontinentaleuropäische Auffassung der Rechnungslegung seit ihrem Anbeginn von deren Verteilungsaufgabe geprägt. Im Gegensatz dazu ist die verteilungsorientierte Rechnungslegung im angloamerikanischen Rechtsraum aus betriebswirtschaftlicher Sicht von eher untergeordneter Bedeutung. So erklärt sich, daß europäische Staaten schon seit Jahrhunderten regulatorisches Interesse für die Rechnungslegungspublizität aufbringen, während erst durch die zentrale Bedeutung der organisierten Kapitalmärkte öffentliche Rechnungsle-

1 Vgl. beispielhaft zur Subsumierung der gesamten Rechnungslegung unter dem 'Zweck der Information Pellens (1999), S. 10 f.; zu einer klaren Zweckdifferenzierung Schildbach (1998); die Informationsfunktion der Rechnungslegung kategorisch ablehnend Schneider (1997), S. 200-232.

2 Vgl. zu der Wirkungsbeziehung zwischen Umfeld und Rechnungslegungssystem Gray (1988); Bökkem/d'Arcy (1999); Pellens (1999), S. 22-29. 
gung als Instrument für den US-amerikanischen Gesetzgeber interessant wurde. Als Reaktion auf den schwarzen Donnerstag am 24. Oktober 1929 wurde der Rechnungslegung die wichtige Rolle zuerkannt, bei der Identifikation der „faulen Eier“ an der Börse hilfreich zu sein, indem die Anleger mit Unternehmensinformationen versorgt wurden.

So entwickelten sich die deutsche Rechnungslegungspublizität als ein typisch kontinentaleuropäisch geprägtes System und die US-amerikanische Rechnungslegungspublizität gemäß ihrer divergierenden Ziele im Zeitablauf in unterschiedliche Richtungen. Doch die Zeiten haben sich geändert. Im Zuge der international globalisierten Wettbewerbswirtschaft können auch die nationalen Regulierungen nicht mehr vollkommen unabhängig voneinander existieren. Erste Fallstudien zum Phänomen der Konkurrenz nationaler Regulierungsansätze ließen sich bereits im Zuge der Rechtsharmonisierung innerhalb der EU anfertigen. Spätestens mit dem erwachten Interesse deutscher Konzerne am US amerikanischen Kapitalmarkt jedoch wurden die unterschiedlichen Unternehmenspublizitätskonzepte frappant.

Daraus entwickelte sich eine Art Wettbewerb der Konzeptionen, aus dem der US amerikanische Ansatz der informationsorientierten Rechnungslegung zumindest in der ersten Runde als Sieger hervorgegangen ist. Natürlich liegt dieser Sieg nicht allein an der vermeintlichen Superiorität des Rechnungslegungssystems, sondern, wie bei Siegen üblich, an der überzeugenderen Gesamtleistung des Komplettpakets, zu dem der gesamte Bereich des Kapitalmarkts und dessen Regulierung, aber auch der politische Prozeß und die gezielte Abschottung des amerikanischen Kapitalmarkts gegen Unternehmen mit einer abweichenden Unternehmenspublizität gehören. Nichtsdestotrotz sehen sich nunmehr auch deutsche Konzerne mit Interesse am organisierten Kapitalmarkt gezwungen, informationsorientierte Rechnungslegungspublizität zu betreiben.

Doch was ist denn nun diese informationsorientierte Rechnungslegung? Wie kann ein Unternehmen oder ein Regulierer sicherstellen, daß das Ergebnis der Rechnungslegung von den Rechnungslegungsadressaten als Information geschätzt wird? Eine bloße Übernahme von US-amerikanischen Konzeptionen dürfte wohl zu kurz greifen. Wenn die deutsche Rechnungslegungspublizität grundlegend neue Ansprüche zu erfüllen hat, dann muß auch die grundlegende Konzeption zur Diskussion stehen. Diese Diskussion in Verbindung mit einem konstruktiven Modifikationsentwurf ist der Inhalt dieser Arbeit. Hierbei soll der Begriff informationsorientierte Rechnungslegung implizieren, daß an deren Ergebnisse keine unmittelbaren Rechtsfolgen im Sinne von Verteilungswirkungen gebunden sind. Diese der Rechnungslegung zugeordneten Verteilungswirkungen führen realiter zu Zielkonflikten und machen die Entwicklung einer konsequent informationsorientierten Rechnungslegung unmöglich. 
Zur Bewertung unterschiedlicher Rechnungslegungspublizitätssysteme wird ein Leitfaden, eine normative Konzeption benötigt. Dieser kann, wie noch aufzuzeigen sein wird, kein Patentrezept zur Weiterentwicklung der Rechnungslegungspublizität darstellen. Letztendlich bleibt die Besinnung auf das beste Informationssystem, das Ökonomen kennen: den Markt. Ziel dieser Untersuchung ist also festzustellen, inwiefern marktwirtschaftliche Prozesse im Bereich der Rechnungslegungspublizität bereits greifen und wie sie gegebenenfalls durch Modifikationen des Rechnungslegungspublizitätssystems gestärkt werden können. Dabei wird insbesondere auf die Neuerungen der elektronischen Datenverarbeitung einzugehen sein, die in den vergangenen Jahren die Welt der Informationsvermittlung außerhalb der traditionellen Rechnungslegung elementar verändert haben. ${ }^{3}$ Der für dieses Ziel gewählte Untersuchungsaufbau wird im folgenden Abschnitt erläutert.

\section{Gang der Untersuchung}

Im folgenden zweiten Kapitel wird ein Leitsatz zur Weiterentwicklung der Rechnungslegungspublizität entwickelt. Dazu werden zunächst durch den Rückgriff auf unterschiedliche Theorien Anhaltspunkte dafür generiert, warum es Rechnungslegungspublizität überhaupt gibt. Als Ergebnis wird ein Metazweck der Rechnungslegungspublizität skizziert, dem letztendlich die in der Einleitung genannten Motive der Rechnungslegung untergeordnet werden können. Anschließend werden die bestehenden theoretischen Überlegungen zur normativen Theorie der Rechnungslegung dargestellt und ihre Eignung zur Entwicklung eines normativen Leitbilds kritisch beurteilt. Im Anschluß daran wird versucht, durch Rückgriff auf die institutionell geprägte Vertragstheorie und die evolutionäre Ökonomie den erforderlichen Leitsatz zu spezifizieren.

Im dritten Kapitel wird unter Bezugnahme auf die historische Entwicklung die momentan praktizierte Rechnungslegungspublizitätspraxis in den USA und Deutschland beschrieben und einer positiven Analyse unterzogen. Die Wahl des nationalen Vergleichssystems fiel auf die USA, da das dortige Rechnungslegungspublizitätssystem wohl als international führend anzusehen ist, mit großem Aufwand kontinuierlich weiterentwickelt wird und ihm nachgesagt wird, besonders informationsorientiert $\mathrm{zu}$ sein. Die positive Analyse untersucht einerseits, ob unterschiedliche normative wirtschaftspolitische Leitbilder für die regulierenden Eingriffe in das Wirtschaftsgeschehen verantwortlich sein könn-

3 Hiermit soll nicht der Eindruck enweckt werden, daß die Veränderungen der Informationstechnologie spurlos an der Rechnungslegung und Publizität vorbei gegangen seien. Während im Erstellungsbereich die elektronische Datenverarbeitung das Berufsfeld des Rechnungslegers in den letzten Jahrzehnten bereits nachhaltig verändert hat, ist in den letzten Jahren auch die Unternehmenspublizität zunehmend im Umbruch begriffen. Vgl. als Überblick Lymer et al. (1999) sowie die Special Section "I The Internet and corporate reporting in Europe" der zweiten Ausgabe des 8. Volumes der European Accounting Review. 
ten und ob sich andererseits das Verhalten der Regulierer aus Sicht der Public Choice Theory erklären läßt.

Das vierte Kapitel vergleicht die Rechnungslegungspublizitätspraxis mit dem im zweiten Kapitel entwickelten Leitsatz. Nach der Identifizierung von relevanten Teilmärkten werden diese auf die Art und den Umfang der möglichen Beeinflussung durch die Rechnungslegungspublizität hin untersucht. Somit wird festgestellt, ob und wie die bestehenden Konzepte die Evolutionsfähigkeit des Wettbewerbs hinsichtlich der unterschiedlichen Märkte fördern und ob sie evolutorische Prozesse zur eigenen Weiterentwicklung selber nutzen.

An diesem Punkt der Arbeit soll deutlich werden, warum der Versuch einer Weiterentwicklung der Rechnungslegungspublizität im Rahmen dieser Arbeit angestrebt wird. Im anschließenden fünften Kapitel sollen die herausgearbeiteten prozeßbedingten Schwachpunkte der traditionellen Publizitätssysteme durch den Entwurf eines datenbankbasierten Rechnungslegungspublizitätssystems zumindest teilweise geheilt werden. Hierfür werden zunächst bereits in der Literatur bestehende Überlegungen dargestellt und analysiert, um im Anschluß daran ein multidimensionales Datenmodell für eine weitgehend dissagreggierte Rechnungslegungspublizität zu entwickeln. ${ }^{4}$ Für die Generierung der entsprechenden Daten und für das Retrieval der aus diesen Daten zu generierenden Abschlüsse wird eine beispielhafte Implementierung entwickelt.

Um seine evolutionsfördernde Wirkung zu entfalten, ist es von großer Bedeutung, daß das neu entwickelte Publizitätskonzept auch vom Markt akzeptiert wird und dementsprechende innovatorische Prozesse auslösen kann. Im sechsten Kapitel wird deswegen mittels unterschiedlicher Ansätze untersucht, wie ein solches Publizitätssystem auf eine von unvollkommenen Märkten geprägte Umgebung wirkt und wie vor- und nachgelagerte Märkte auf eine dergestalt modifizierte Möglichkeit zur Unternehmenspublizität reagieren könnten. Ferner wird untersucht, ob von einem zunächst als freiwillig einzustufenden Publizitätssystem auch Einflüsse auf die nationalen Regulierungskonzeptionen ausgehen könnten.

Eine Zusammenfassung der Ergebnisse im siebten Kapitel rundet die Arbeit ab.

4 Schon hier soll darauf hingewiesen werden, daß das Adjektiv „multidimensional“ hier nicht im Sinne der dreifachen Buchhaltung von Ijiri zu verstehen ist. Vgl. zu diesem Ansatz Ijiri $(1986 ; 1989)$, ausführend Altenburger (1996) und kommentierend Fraser (1993). 
Um die folgenden Ausführungen auf eine einheitliche Basis zu stellen, sind zunächst einige Begriffe zu definieren.

Unter einem Gut wird im folgenden ein materieller oder immaterieller Gegenstand verstanden, an den Verfügungsrechte gebunden sind. Als ein freies Gut gilt ein Gut, das nicht knapp ist. Solche freien Güter werden von den Wirtschaftssubjekten bis zum Sättigungsgrad konsumiert und sind deswegen nicht Teil der ökonomischen Analyse. Die an das Gut gebunden Verfügungsrechte umfassen das Recht des Gebrauchs und der Änderung, das Recht, über den Nutzenzufluß aus dem Gebrauch zu verfügen und das Recht, diese Rechte zu veräußern. ${ }^{5}$

Eine Institution ist ein System formeller und informeller Regeln einschließlich von Verfahren zu deren Durchsetzung. ${ }^{6}$ Organisationen sind die Kombination von Institutionen und den Wirtschaftssubjekten, die sich innerhalb dieser Institutionen bewegen. ${ }^{7}$

Ein Unternehmen ist eine im Regelfall formale Organisation, die als Aufgabe die Verringerung der Einkommensunsicherheiten ihrer Mitglieder hat. ${ }^{8}$ Im Gegensatz dazu ist ein Markt eine im Regelfall informell entstandene Institution, die den Tausch von Verfügungsrechten zwischen Anbietern und Nachfragern dezentral regelt. ${ }^{9}$ Aus den Definitionen von Markt und Unternehmen wird deutlich, daß nicht in jedem Fall eine klare definitorische Trennung möglich ist. Speziell die Dimensionsausprägungen der Transaktion zwischen Anbieter und Nachfrager ${ }^{10}$ können als genauere Differenzierung zwischen Markt und Unternehmen herangezogen werden. ${ }^{11}$ Im Verlauf der Arbeit wird im Bedarfsfall eine weitere Spezialisierung der Unterteilung zwischen Markt und Unternehmen vorgenommen.

5 Vgl. zu dieser Gutdefinition und zu der Systematisierung der Verfügungsrechte Eggertsson (1990), S. 34 f.; North (1990), S. 3 f.; Richter/Furubotn (1996), S. 82 f; Erlei (1998), S. 29; grundsätzlich und umfassend zum eigentlichen Wirtschaftssubjekt Kirchgässner (1991).

6 Vgl. Schmoller (1900), S. 61; eine detailliertere Definition bietet Ostrom (1985) und zusammenfassend Ostrom (1990), S. 51.

7 Vgl. North (1990), S. 4 f., der klar zwischen Institutionen und Organisationen trennt.

8 Vgl. Richter/Furubotn (1996), S. 8; Schneider (1995), S. 1-5; Erlei (1998) unterscheidet zwischen organischen Unternehmen, die er als flexibel, dezentral und anreizintensiv bezeichnet und mechanistischen Firmen, die er als relativ starr, zentralisiert und weniger anreizintensiv beschreibt.

9 Vgl. Richter/Furubotn (1996), S. 8. In der neoklassischen Mikroökonomie wird der Markt häufig mechanistisch definiert, also als Mechanismus, der die dezentralen Witschaftspläne der Wirtschaftssubjekte über Preise koordiniert. Vgl. Schumann (1992), S. 9-11.

10 Vgl. hierzu Williamson (1985), S. 68-84.

1 Vgl. hierzu als Anwendungsbeispiel für die Frage der Abgrenzung von rechnungslegenden Einheiten Sürken (1999). 
Information wird in dieser Arbeit über den Begriff „entscheidungsrelevant“ definiert. Information ist somit die entscheidungsrelevante Teilmenge der Umweltdaten. Diejenigen Umweltdaten, die keine Information darstellen, werden auch als „Echo“ bezeichnet. ${ }^{12}$ Diese Definition kann als Teilmenge des Informationsbegriffs nach Wittmann aufgefaßt werden, der Information als „Zweckorientiertes Wissen“ definiert, ${ }^{13}$ wenn der Prozeß der Entscheidung(sverbesserung) als Zweck aufgefaßt wird. ${ }^{14}$ In diesem Zusammenhang ist auch auf die Trennung zwischen Daten und Informationen hinzuweisen. Je nachdem, ob Daten notwendigerweise zur elektronischen Weiterverarbeitung geeignet sein müssen oder nicht, bilden Daten und Informationen eine Schnittmenge, oder Informationen sind die entscheidungsrelevante Teilmenge der Daten. ${ }^{15}$ Im Rahmen dieser Arbeit soll für Daten nicht per se die Möglichkeit zur elektronischen Weiterverarbeitbarkeit gefordert werden. Wenn also von einem Rechnungslegungspublizitätssystem die Rede ist, so ist damit ein System gemeint, daß einerseits ökonomische Sachverhalte innerhalb eines Unternehmens in quantitativen Daten abbildet (das Rechnungslegungssystem) und diese Daten einer grundsätzlich unbegrenzten Öffentlichkeit verfügbar macht (das Publizitätssystem i. e. S.) ${ }^{16}$.

Als letzter Begriff soll noch das einen Prozeß beschreibende Adjektiv evolutionär im ökonomischen Kontext definiert werden. Ein evolutionärer Prozeß kann durch Anleihen an die biologische Terminologie in die repetierenden Phasen Selektion, Mutation und Reproduktion geteilt werden. ${ }^{17}$ Übertragen auf den ökonomischen Koordinationsprozeß ist das zentrale Instrument zur Selektion der Marktmechanismus, der unterschiedliche Handlungskonzepte vergleicht und bewertet. Die am Markt superioren Verhaltensweisen werden von den Wirtschaftssubjekten reproduziert, durch zufällige Prozesse mutiert und aufgrund des Strebens nach Verbesserung selektiert. Die Gesamtheit dieser Schritte stellt

12 Vgl. Schildbach (1986), S. 6.

13 Vgl. Wittmann (1959), S. $14 \mathrm{f}$.

14 Vgl. zu gängigen Informationsdefinitionen Bode (1997); Wessling (1991), S. 11-30 und zu deren Problemen im Überblick Schneider (1997a), S. 71-82.

15 Das definitorische Merkmal der elektronischen Verarbeitbarkeit für Daten findet sich in der wirtschaftsinformatischen Literatur, die Gesamtheit von Daten und Informationen als Wissen bezeichnet. Diesem Sprachgebrauch soll hier nicht gefolgt werden, da Wissen aufgrund der umgangssprachlichen Bedeutung eng mit einem jeweils wissenden Subjekt verbunden erscheint. Vgl. zu gängigen wirtschaftsinformatisch geprägten Begriffsdefinitionen rund um den Terminus Information Müller (1999), S. 5-8 m.w.N.

16 Vgl. zu weiteren Publizitätsdefinitionen Kronstein/Claussen (1960), S. 9, Schredelseker/Kopetsch/Maybüchen (1986), S. 38-39, Pellens (1995), Sp. 1589-1590. Schneider (1997), S. 29 trennt die Rechnungslegung in einen internen Bereich, der nicht uneingeschränkt recherchierbar ist, und in einen öffentlich verfügbaren externen Bereich. Ähnlich geht auch Coenenberg (1998), S. 129 vor, der von öffentlicher Rechnungslegung spricht. Diese beiden Definitionen entsprechen im Groben der oben gegebenen; eine eher semantisch geprägte Definition unternimmt Fülbier (1998), S. 20 f.

17 Diese Unterteilung wird hier lediglich zur Verbesserung der Verständlichkeit vollzogen. Vgl. zur Kritik an der unreflektierten Übertragung biologischer Begriffswelten auf ökonomische Forschungsprogramme Nowak (1999), S. 208-213. 
einen evolutionären ökonomischen Prozeß dar. Das Ergebnis eines solchen Prozesses ist das Ergebnis menschlichen Handels, jedoch nicht das Ergebnis menschlichen Entwurfs. ${ }^{18}$

Im folgenden sollen weiterhin das Rechnungslegungs- und das Publizitätssystem zusammenfassend als Rechnungslegungspublizitätssystem bezeichnet werden. $\mathrm{Da}$ im Rahmen dieser Arbeit nur die externe Rechnungslegung betrachtet wird und diese Rechnungslegung nur durch die anschließende Publizität ihren Sinn erhält, sei diese etwas ungewohnte Nomenklatur gestattet, um sprachlich unschöne Formulierungen zu vermeiden. ${ }^{19}$

18 Vgl. zu weiteren Definitionen im Bereich der evolutionären Ökonomik Schneider (1995), S. 102-195; Kunz (1985), S. 1-4; detailliert zur Entwicklung Nowak (1999), S. 191-237.

19 Hier besteht eine gewisse Analogie zum US-amerikanischen Sprachraum, wo der Begriff financial reporting als Oberbegriff für financial accounting und reporting benutzt wird. Vgl. SFAC No. 1, Par. 5-8; Carmichael/Lilien/Mellman (1999), chapter 2, S. 2. 
Joachim Gassen - 978-3-631-75496-2

Downloaded from PubFactory at 01/11/2019 04:12:56AM

via free access 


\title{
Kapitel II
}

\section{Theorie der Rechnungslegung als Meßrahmen}

\author{
für eine normative Analyse
}

Eine Arbeit, deren Ziel auch in der Weiterentwicklung der Rechnungslegungspublizität besteht, trägt einen nicht zu leugnenden normativen Charakter. Wenn es um die Frage nach „richtig oder falsch“ geht, tut sich die Ökonomie als primär beobachtende Wissenschaft naturgemäß schwer. Nichtsdestotrotz sollen in diesem Kapitel Ansatzpunkte für eine aus ökonomischer Sicht wünschenswerte Weiterentwicklung der Rechnungslegungspublizität gesucht werden. Dafür wird wie folgt vorgegangen: Zunächst wird eine ökonomische Begründung für die Existenz von Rechnungslegungspublizität und deren Regulierung skizziert. $\mathrm{Da}$ die Regulierung der Rechnungslegungspublizität national und international ein empirisch zu beobachtender Fakt ist, soll das „ob“ der Regulierung hier nicht weiter diskutiert werden. Die Regulierer werden für das „wie“ der Regulierung eine Hilfestellung auch und gerade von Wissenschaftlern nachfragen. ${ }^{1}$ Deswegen werden daraufhin die unterschiedlichen Ansätze der normativen Rechnungslegungstheorie auf ihre Plausibilität und Funktionstüchtigkeit zur Legitimierung für die Rechnungslegungsregulierung hin untersucht. Des weiteren wird hinterfragt, inwiefern sich aus positiven Wirkungsanalysen der Rechnungslegungspublizität normative Handlungsempfehlungen ableiten lassen. Am Ende dieses Kapitels wird versucht, basierend auf den Vorüberlegungen eine normative Leitlinie für die Entwicklung der Rechnungslegungspublizität zu generieren.

\section{1 Ökonomische Begründung für Rechnungslegungspublizität}

\subsection{Zielsetzung der Rechnungslegungspublizität}

Wie bereits in den einleitenden Definitionen festgestellt, können Unternehmen als auBerhalb des Marktes institutionalisierte Verbindungen von Wirtschaftssubjekten zur Reduktion von Einkommensunsicherheiten analysiert werden. ${ }^{2}$ Aus dieser Definition wird

1 Vgl. zu dem Themenkomplex der Regulierungsbegründung und -ausgestaltung beispielhaft Streim (1985); Schildbach (1986), S. 82-98; Busse von Colbe (1987), S. 117-119; Hax (1988); Feldhoff (1992; 1994); Walz (1993); Breidenbach (1997) und Pellens/Gassen (1998). Für die US-amerikanische Forschung vgl. Horngren (1972; 1985) und Zeff $(1978 ; 1984)$.

2 Vgl. Schneider (1995), S. 30-41. 
bereits deutlich, daß eine klare Trennung zwischen Markt und Unternehmen von der jeweiligen Sichtweise des Betrachters abhängt. Im Rahmen dieser Arbeit wird die Trennung zwischen Markt und Unternehmen nicht als Fakt, sondern vielmehr als Analysehilfsmittel gesehen. Dadurch ergibt sich die Möglichkeit, ggf. eine andere MarktUnternehmensgrenze zu definieren, wenn es der jeweiligen partialanalytischen Sicht dienlich erscheint. Einkommensunsicherheiten bestehen für die am Unternehmen beteiligten Wirtschaftssubjekte hinsichtlich der zukünftigen Höhe und zeitlichen Verteilung des Einkommensstroms. Somit besteht für sie ein Anreiz, diese Unsicherheit durch den Abschluß von Verträgen zu reduzieren. Diejenige Person, die die Stillhalterposition bei solchen Verträgen übernimmt, wird als Unternehmer bezeichnet. ${ }^{3}$

Können die Unternehmensbeteiligten ihre Vertragsansprüche in einer sicheren Umwelt durchsetzen, so haben sie kein besonderes Bedürfnis an Informationen über die wirtschaftliche Lage des Unternehmens. Da jedoch Verträge in der unsicheren Realität unvollständig, zeitlich begrenzt und nicht immer mit verhältnismäßigen Mitteln durchsetzbar sind, haben alle Unternehmensbeteiligten ein Interesse an der wirtschaftlichen Entwicklung des Unternehmens, um ihre residuale Einkommensunsicherheit bewerten zu können und ggf. auf Änderungen zu reagieren.

Besitzt der Unternehmer totale Transparenz über die Tätigkeiten seines Unternehmens, so ist für ihn die Rechnungslegung bedeutungslos. Wird jedoch wie hier ein Unternehmen als ein institutionalisiertes Vertragsgeflecht aufgefaßt, ${ }^{4}$ so folgt daraus, daß auch der Unternehmer bei unvollständigen Verträgen ${ }^{5}$ und begrenzter Rationalität ${ }^{6}$ keine totale Transparenz über sein Unternehmen besitzt. Somit wird eine von Dritten erstellte Rechnungslegung für ihn zur Informationsquelle. Setzt der Unternehmer einen oder mehrere Agen-

3 Vgl. Schneider (1995), S. 32; allgemein zur Anwendung von mikroökonomischen Methoden und Konzepten in der betriebswirtschaftlichen Forschung Neus (1998); Wagenhofer/Ewert (1997); Gümbel (1996).

4 Vgl. zu dieser Unternehmenssichtweise grundlegend Commons (1934); Coase (1937); Alchian/Demsetz. (1972); Jensen/Meckling (1976); Grossman/Hart (1986); Hart/Moore (1990). Einen Überblick über die institutional geprägte Unternehmenstheorie bieten Williamson (1981); Holmstrom/Tirole (1989); Erlei/Leschke/Sauerland (1999), S. 175-228; zu institutionellen Vorläufern vgl. Richter/Furubotn (1996), S. 441-443; kritsch Schneider (1996), insb. S. 479-483.

5 Vgl. zu den hier verwendeten Vertragsformen Williamson (1985), S. 68-80; Macneil (1974).

6 Vgl. Williamson (1985), S. 45-46; Simon (1957) und Simon (1976), S. xxvi-xxxi, sowie S. $61-78$ und einführend zu ökonomischen Verhaltensmodellen Karpe (1999). 
ten (Manager) als stellvertretende Leiter des Unternehmens ein, so steigt das Informationsbedürfnis durch die etablierte Principle-Agent-Beziehung noch weiter an. ${ }^{7}$

Somit haben alle Unternehmensbeteiligten im wesentlichen aufgrund von asymmetrischer Informationsverteilung, unvollständiger Verträge und beschränkter Rationalität in Verbindung mit dem Opportunismus der Akteure einen Bedarf an Informationen über die zukünftige Situation des Unternehmens. Ferner besteht eine Nachfrage nach Informationen, die als Argumentationshilfe für die aktuellen Verteilungsprobleme innerhalb des Unternehmens dienen können. Somit kommen einer externen Rechnungslegung, die sich also neben dem Management auch an weitere, unternehmensexterne Adressaten wendet, zwei zentrale Aufgaben zu: Erstens soll über die abgelaufene Periode Bericht erstattet werden und zweitens soll die Unsicherheit über die Zukunft des Untemehmens reduziert werden. ${ }^{8}$ Diese beiden Zwecke sind nicht überschneidungsfrei. So werden die Publizitätsadressaten die berichtete vergangene Leistung des Managements und des Unternehmens in Ermangelung anderer Möglichkeiten regelmäßig in die Zukunft extrapolieren. Darüber hinaus enthalten die vorgenommenen Bewertungen in der Regel Informationen über den zukünftigen ökonomischen Nutzen der Vermögensgegenstände und Schulden.

Insofern ist die eigentliche Zielsetzung der Unternehmenspublizität leicht definiert: Es geht um den Abbau von asymmetrischer Information. ${ }^{9}$ Dies soll jedoch nicht durch die selektive Versorgung von Vertragspartnern mit privater Information, sondern durch die Verfügbarmachung von öffentlicher Information im Rahmen des Publizitätsprozesses gewährleistet werden. Während private Information nur einem begrenzten Personenkreis zur Verfügung steht, ist öffentliche Information in ihrem Adressatenkreis unbeschränkt, was nicht heißt, daß öffentlich verfügbare Information tatsächlich der gesamten Welt bekannt ist, sondern lediglich, daß sie aus Sicht des Informationsemittenten der ganzen Welt bekannt werden könnte. ${ }^{10} \mathrm{Um}$ diese Tätigkeit ökonomisch zu begründen, müssen also zwei Fragen untersucht werden: Besteht eine Nachfrage nach privater Information

7 Vgl. zu der Eigentümer-Manager-Beziehung bereits Smith (1776), S. 800; zu der ökonomischen Formulierung der daraus resultierenden Agencyproblematik grundlegend Jensen/Meckling (1976); sowie Holmstrom/Tirole (1989), S. 86-106. Es läßt sich empirisch zcigen, daß Unternehmen, deren Eigentum zu einem signifikanten 'Teil im Managerbesitz ist, ein Ergebnis veröffentlichen, das besser den Aktienkurs erklärt, als das Ergebnis von Unternehmen mit strikter Eigentümer-Manager-Trennung, vgl. Warfield/Wild/Wild (1995).

8 Diese Zweiteilung des Rechnungslegungszwecks findet sich sowohl in der deutschen als auch in der angloamerikanischen Bilanzierungsforschung. Vgl. AAA (1966), S. 12-36; Coenenberg (1998), S. 127.

9 Vgl. Pellens (1999), S. 10 f.; mit einer kritischen Diskussion Schneider (1998), insb. S. 1473.

10 Vgl. zu der Unterscheidung zwischen privater und öffentlicher Information u.a. Schneider (1997a); S. 77 f. 
und besteht ein Anreiz für Besitzer von privater Information, ihre Information durch Unternehmenspublizität öffentlich verfügbar zu machen? ${ }^{11}$

\subsection{Informationsökonomisch geprägte Ansätze}

\subsubsection{Private Information}

Die Informationsökonomie befaßt sich mit dem Angebot von und der Nachfrage nach Informationen. ${ }^{12}$ Die Nachfrage nach Informationen läßt sich mikroökonomisch in Abhängigkeit von dem Entscheidungsfeld eines Individuums modellieren. ${ }^{13}$ Dieses Individuum sieht sich gezwungen, eine Handlungsalternative auszuwählen, ohne Gewißheit über die ihn tangierende ökonomische Konsequenz seines Handelns zu besitzen. Diese hängt annahmegemäß von der ex ante nicht zu beobachtenden Ausprägung einer Umweltvariable, dem Umweltzustand, ab. Ist die Ausprägung dieser Variable dem Individuum bekannt, so kann dieses daraus mit Sicherheit auf den ökonomischen Nutzen seiner gewählten Handlungsalternative schließen. Ausgehend von dieser modellhaften Wiedergabe einer Entscheidungssituation ${ }^{14}$ kann Information auf unterschiedliche Art und Weise wirken: Einerseits kann Information dazu beitragen, die Menge von möglichen Umweltzuständen zu verändern, sie kann dazu führen, daß das entscheidende Wirtschaftssubjekt den möglichen Umweltzuständen Eintrittswahrscheinlichkeiten zuordnen, oder bestehende Eintrittswahrscheinlichkeiten modifizieren kann. Andererseits kann Information auch dazu führen, daß die Menge der Handlungsalternativen modifiziert wird. Wenn das entscheidende Individuum Wahrscheinlichkeiten für das Eintreten unterschiedlicher Umweltsituationen aus einer geschlossenen Menge von Umweltsituationen ${ }^{15}$ angeben kann, wird von einer „Risikosituation“ gesprochen, ist es dazu nicht in der Lage, so liegt eine „Ungewißheitssituation“ vor.

Wenn Rechnungslegungsinformationen verfügbar sind, besteht die Möglichkeit, die Entscheidungssituation durch diese zusätzlichen Information zu verbessern. So kann eine

1 Vgl. zur empirischen Relevanz von privater und öffentlicher Information als parallel angewendete Instrumente der Rechnungslegung beispielhaft Holland (1998).

12 Vgl. umfassend zur Informationsökonomie Lawrence (1999); zur Bedeutung der Informationsökonomie für den Bereich des Management Accountings Demski/Kreps (1982).

13 Vgl. überblicksmäßig zur Verwendung der Informationsökonomie im Bereich der Rechnungslegungstheorie Verrecchia (1982), S. 3-18; Walker (1988).

14 Vgl. zur Entscheidungstheorie Bamberg/Coenenberg (1996), insb. S. 56-153, sowie Sieben/Schildbach (1994), insb. S. 46-85.

15 Die Annahme einer geschlossenen Menge von zukünftigen Umweltzuständen ist aus streng ökonomischer Sicht nicht zu rechtfertigen, sondern stellt vielmehr eine Vereinfachung zur Ermöglichung einer quantitativen Analyse dar. Vgl. Adam (1996), S. 224224, der für diesen allgemeineren Fall auch von einer Entscheidung unter Unsicherheit mit zeitlich offenem Entscheidungsfeld spricht. 
Ungewißheitssituation in eine Entscheidung unter Risiko überführt werden und ein Entscheidungsfeld, für das bereits eine ungenaue a-priori Wahrscheinlichkeitsverteilung für die möglichen Umweltzustände gebildet wurde, kann durch zusätzliche Information und eine daraus gebildete a-posteriori Wahrscheinlichkeit verengt werden. Diese Schärfung des Entscheidungsfeldes kann durch die Ermittlung der Differenz der jeweils erwarteten Schäden aus der Entscheidung bewertet werden. Durch eine Aggregation dieser individuellen Zahlungsbereitschaften kann eine Nachfragefunktion nach Rechnungslegungsinformationen entwickelt werden. ${ }^{16}$ Somit läßt sich die Nachfrage eines bestimmten Adressaten nach Rechnungslegungsinformationen modelltheoretisch analysieren. ${ }^{17}$

Aus individueller Sicht wird ein Wirtschaftssubjekt also zusätzliche Informationen immer dann nachfragen, wenn es durch diese eine Verbesserung seines Entscheidungsfeldes erwartet und die Kosten der zusätzlichen Information durch diesen Vorteil überkompensiert werden. Falls die Informationen dem Adressaten kostenlos zur Verfügung stehen, gilt das Blackwell Theorem: Der Adressat wird die zusätzlichen Informationen nachfragen. ${ }^{18}$ Somit hat Information aus individueller Sicht immer einen positiven Bruttonutzen, der ggf. jedoch von den Kosten der Informationsbeschaffung überkompensiert werden kann. Diese Aussage gilt vor dem Hintergrund des hier verwendeten Theorierahmens. Fraglich ist, wie sich diese partialanalytische Sichtweise verändert, wenn die über Märkte transferierten Wechselwirkungen individuellen Verhaltens berücksichtigt werden.

\subsection{2 Öffentliche Information}

Wird statt lediglich privater Information nun die Wirkung von öffentlich verfügbarer Information analysiert, so stellt sich zunächst die Frage, ob sich die Aussage, Information habe immer einen positiven Bruttonutzen, auch unter Berücksichtigung der intersubjektiven Wechselwirkungen halten läßt. ${ }^{19}$ So läßt sich zeigen, daß unter bestimmten Bedingungen Wirtschaftssubjekte die Veröffentlichung von Information in einem reinen Gütertauschmodell ablehnen, ja sogar bereit sind, dafür zu zahlen, daß diese Informationen

16 Dieses Verfahren entspricht der Bayes-Regel, vgl. grundlegend Bayes (1763); einführend Sicben/Schildbach (1994), S. 50-53; Hopf (1983), S. 102-107; in der Anwendung auf Rechnungslegung Feldhoff (1992), S. 84-86; kritisch anmerken läßt sich, daß je nach Entscheidungssituation mehrere alternative rationale Entscheidungen möglich sind und so eine Entscheidung anhand der Bayes-Regel nicht möglich ist. Vgl. hierzu beispielhaft Heap/Varoufakis (1991).

17 Vgl. grundlegend Feltham (1968); Butterworth (1972); Marshall (1972); zur Analyse des Umsetzungsprozesses Uecker (1978).

18 Vgl. erläuternd und diskutierend zum Blackwelltheorem Ballwieser (1985).

19 Vgl. einführend Elliot/Jacobson (1994), die mit einem eher deskriptiven Ansatz versuchen, die gesamtwirtschaftlichen Effekte von öffentlichen Unternehmensinformationen zu beschreiben. 
nicht beschafft werden. ${ }^{20}$ Dies gilt auch für private Informationen, wenn die Marktpartner in der Lage sind, vom Marktverhalten des Informierten auf seinen Informationsstand zu schließen. Hakansson/Kunkel/Oblson untersuchten diese Bedingungen und zeigen, daß für die Ablehnung des gesamtwirtschaftlichen Werts öffentlich verfügbarer Information sehr restriktive Annahmen nötig sind. Diese betreffen vor allem die Allokationseffizienz der zugrundeliegenden Märkte, die Verteilung der subjektiven Erwartungen vor der Information und die subjektive Auswertung der Information. Sie kommen zu dem Ergebnis, daß wegen dieser engen Annahmen nicht grundsätzlich angenommen werden kann, $\mathrm{da} ß$ öffentlich verfügbare Information keinen sozialen Wert habe. Ebenso läßt sich allerdings nicht argumentieren, daß öffentlich verfügbare Information in jedem Fall die gesellschaftliche Wohlfahrt positiv beeinflußt. ${ }^{21}$ Des weiteren ist zu berücksichtigen, daß aus modelltheoretischer Sicht öffentlich verfügbare Information auch die Nachfrage nach privater Information dergestalt beeinflussen kann, daß die private Information von weniger Wirtschaftssubjekten nachgefragt wird und sich somit das Informationsgefälle zwischen informierten und uninformierten Marktteilnehmern noch weiter verstärkt. ${ }^{22}$

Gerade weil sich ein gesamtwirtschaftlicher Vorteil einer Unternehmenspublizität selbst unter Nichtberücksichtigung von Kosten der Informationsproduktion nicht zweifelsfrei modelltheoretisch ableiten läßt, ${ }^{23}$ ist von großer Bedeutung, ob der marktwirtschaftliche Steuermechanismus in der Lage ist, das Koordinationsproblem der öffentlichen Information von sich aus zu lösen. Wäre dies der Fall, so wäre jegliche Regulierung von Rechnungslegung und Publizität nutzlos und kontraproduktiv. Demzufolge gäbe es keine Notwendigkeit für normative Rechnungslegungstheorie mehr, der Markt könnte selber entscheiden, was für ihn gut ist, das Propagieren unterschiedlicher vermeintlich superiorer Rechnungslegungsstandards käme einer Anmaßung von Wissen ${ }^{24}$ gleich. $^{25}$

20. Vgl. Hirshleifer (1971); Fama/Laffer (1971); Marschall (1974); als Überblick wiederum Verrecchia (1982); Ewert (1989).

21 Vgl. Hakansson/Kunkel/Ohlson (1982); Ohlson/Buckman (1980; 1981); ähnlich relativierend Trueman (1983); mit einem anschaulichem Zahlenbeispiel Feldhoff (1992), S. 87-102. Andere Untersuchungen beleuchten auch Alternativen zur Veröffentlichung der privaten Information, wie z.B. das Auflegen von Fonds. Vgl. Admati/Pfleiderer (1988).

22 Vgl. Lundholm (1991); des weiteren kann öffentliche Information auch die Ausbringung von öffentlichen Gütem aus Wohlfahrtssicht negativ beeinflussen, vgl. Teoh (1997).

23 Als nicht erfolgreich muß letztlich auch der Versuch von Lev (1988) bewertet werden, der argumentiert, daß eine Behebung von Informationsasymmetrien der Chancengleichheit zuträglich und somit sozial sinnvoll sei. Diese Argumentation erscheint jedoch aus Sicht der starken evolutionären Bedeutung von selbstgeschaffenen Informationsvorsprüngen recht zweifelhaft. Vgl. auch Hayek (1960), S. 85-90.

24 Vgl. Hayek (1989).

25 Vgl. mit ähnlichen Ausführungen AAA (1977), S. 23; Belkaoui (1992), S. 85; Wolk/Francis/Tearny (1991), S. 77-89; Pellens/Gassen (1998). 
Um den Markt für Unternehmensinformationen auf Marktversagen zu testen, ${ }^{26}$ ist zunächst das Gut Information und dessen gesellschaftlicher Wert genauer zu untersuchen. Oben wurde schon gezeigt, warum Wirtschaftssubjekte private Information nachfragen. Diese Erkenntnisse lassen sich jedoch nicht ohne weiteres auf öffentliche Information übertragen. Dies gilt insbesondere auch wegen des grundsätzlichen Modellrahmens, der keine Produktions- und Kapazitätseffekte abbilden kann. ${ }^{27}$

Auch wenn davon ausgegangen wird, daß öffentlich verfügbare Informationen in der Regel einen gesellschaftlichen Nutzen induzieren, ist damit keineswegs automatisch eine Regulierungsnotwendigkeit gegeben. Untersuchungen zeigen in den USA, daß auch vor dem Securities Act (SA) und Securities Exchange Act (SEA) bereits Rechnungslegungsdaten von den börsennotierten Unternehmen publiziert wurden, und daß sich die Publizitätsqualität durch die Etablierung der Securities and Exchange Commission (SEC) nicht wesentlich besserte. ${ }^{28}$ Somit bestand auch vor dem Eingreifen der SEC ein Markt für öffentliche Rechnungslegungsinformation. Die Regulierung der Unternehmenspublizität könnte durch die Vermutung von Marktversagen gerechtfertigt werden, wenn dieser Markt für nicht funktionsfähig gehalten wird. Die Unterstellung, daß Märkte versagen können, impliziert ein normatives Leitbild, wie Märkte sich zu verhalten haben. Daß dies hochgradig problematisch ist, soll hier mit einem Verweis auf von Hayek ${ }^{29}$ nicht weiter thematisiert werden. Marktversagen wird häufig vermutet, wenn das zu handelnde Gut Charakterzüge eines Kollektivguts aufweist, wenn die langfristigen Durchschnittskosten produktionsbedingt über die Gesamtnachfrage hinweg fallend verlaufen, oder wenn externe Effekte bedingen, daß die gesellschaftliche Zahlungsbereitschaft von der Summe der individuellen Zahlungsbereitschaften abweicht. ${ }^{30}$

Kollektivgüter sind gekennzeichnet durch eine fehlende Rivalität im Konsum und durch die Nichtausschließbarkeit von Nichtzahlern. Auch wenn nur eine der beiden Eigenschaften auf Rechnungslegungsinformationen zutrifft, kann die marktwirtschaftliche Bereitstellung gestört sein. Rechnungslegungsinformationen wird gelegentlich eine fehlende

26 Vgl. grundsätzlich zu dem Begriff Marktversagen Bator (1958); erläuternd und diskutierend Fritsch/Wein/Ewers (1999), S. 91-346; Musgrave/Musgrave/Kullmer (1994), S. 68-72; allgemein zu Informationen und Wissen bereits Arrow (1962).

27 Vgl. Hakansson/Kunkel/Ohlson (1982), S. 1180-1181.

28 Vgl. Stigler (1975), S. 78-100; Benston (1969; 1973); relativierend Ingrim/Chewing (1983). Ähnliches gilt wohl auch für die unterschiedlichen amerikansischen Standard-Setter, vgl. Ely/Waymire (1999).

29 Vgl. sehr eindrucksvoll Hayek (1989), indem er den Versuch der Wirtschaftswissenschaft kritisiert, das Verhalten von Märkten zu prognostizieren und daraus folgend Marktfehlverhalten bei Abweichungen von der Prognose zu diagnostizieren.

30 Vgl. zu extemen Effekten einführend Wiese (1998). 
Rivalität im Konsum unterstellt. ${ }^{31}$ Während dies für öffentlich verfügbare Daten der Rechnungslegungspublizität quasi definitorisch zutrifft, beruht diese Annahme bei der Möglichkeit von privaten Informationen auf einer sehr engen Definition des Konsumprozesses der Information. Es läßt sich argumentieren, daß keine Rivalität im Konsum vorliegt, da der Erwerb der Information durch einen Nachfrager keine Neuproduktion der Information seitens der Anbieter notwendig macht. Andererseits tangiert die Konsumtion der Information über die daraus resultierenden Markttransaktionen sehr wohl die Nutzenniveaus anderer Wirtschaftssubjekte, die diese Information bislang noch nicht hatten. Ähnlich läßt sich allerdings auch für andere typische Kollektivgüter argumentieren: Rechtsschutz verbessert die Nutzensituation der Individuen, die Rechtsschutz genießen, zuungunsten derer, die keinerlei Absicherung vor Regelbruch erfahren. Es ist somit theoretisch zumindest vorstellbar, daß die Nutzenposition eines Rechtsschutz genießenden Individuums durch die Gewährung von Rechtsschutz an andere Individuen negativ beeinflußt wird. Der Widerstand der Sklavenhalter gegen die Auflösung der Sklaverei möge als empirischer Beleg für diese Nutzenbeeinflussung gelten. Somit wird hier folgender Standpunkt vertreten: Zwar herrscht aus streng ökonomischer Sicht keine Rivalität im Konsum, da jedoch der Nutzen der Informationskonsumtion erst im weiteren Marktprozeß entsteht und dieser Nutzen maßgeblich vom Informationsstand anderer Wirtschaftssubjekte abhängig ist, wird davon ausgegangen, daß eine Art „derivative“ Rivalität im Konsum von Information vorherrscht.

Die Möglichkeit des Ausschlusses von Nichtzahlern ist hierfür von großer Bedeutung. Ist diese gegeben, so ist eine Bereitstellung von privaten Unternehmensinformationen möglich und dürfte sich aufgrund der oben erfolgten theoretischen Überlegungen auch einstellen. Empirische Indizien wie private Auskunftsbüros etc. sprechen für das Vorhandensein einer solchen Nachfrage. Da der Nutzen, den ein Individuum aus einer bestimmten Information ziehen kann, zwischen unterschiedlichen Individuen jedoch stark differiert, ist die Exkludierung von Nichtzahlern ständig in Gefahr. Zum Beispiel: Manager besitzen private Informationen, die sie aufgrund von bestehenden Insidergesetzen nicht direkt nutzbringend am Markt einsetzen können. Dazu ist jedoch ein potentieller Eigentümer in der Lage. Im Rahmen einer verdeckten Preisverhandlung wird zwischen Management und potentiellem Eigentümer ein Kaufpreis für die private Information ermittelt, indem das Management mehr oder weniger verläßliche Signale über den Nutzen der privaten Information für den Adressaten emittiert. Nachdem die private Information an einen Marktteilnehmer veräußert wurde, besteht für das Management immer noch ein Anreiz, die private Information nach erfolgter Transaktion auch an andere Wirtschaftssubjekte zu

31 Vgl. Gonedes/Dopuch (1974), S. 65; diesen Ansatz kritisierend und ausführend Leftwich (1980); Feldhoff (1992), S. 103-108; zusammenfassend Watts/Zimmerman (1986), S. 162-169; Walker (1988); Feldhoff (1994). 
veräußern. Dieser Anreiz besteht, solange das Nutzenpotential der Information für Teilnehmer am Markt für Eigenkapitaltitel noch nicht vollkommen verzehrt wurde. Somit kann der Nutzen des ersten Investoren schleichend aufgezehrt werden. Ferner besteht unter Umständen ein Anreiz für Besitzer privater Information, die selber aus dieser Information direkt keinen Nutzen ziehen können und die die Verhandlungskosten einer privaten Informationsüberlassung scheuen, die Information öffentlich verfügbar zu machen um so ihre Bekanntheit zu steigern, wenn sie aus Prominenz Nutzen erzielen.

Die Gutcharakterisierung und hier vor allem die Grenzkosten der Informationsproduktion sprechen dafür, daß Information Charakterzüge eines Kollektivguts aufweist. Andererseits sprechen die hohen Nutzeninterdependenzen der Informationsnachfrager für einen privaten Charakter des Gutes Information. Daraus folgt, daß eine Bereitstellung nicht notwendigerweise vollkommen losgelöst vom Markt geschehen muß. Je nach Ausprägung des Kollektivguts können auch Bereitstellungsformen über Clubs, Verbände und durch private Anbieter möglich sein. ${ }^{32}$

Die Frage nach dem möglichen Bereitsteller von Rechnungslegungsinformationen ist somit nicht durch den Gutscharakter determiniert. Die Produktion von Rechnungslegungsinformationen bedingt das Erwerben von unternehmenspezifischem Know-How. Während dies im Rahmen der Managementtätigkeit quasi als Kuppelprodukt anfällt, ${ }^{33}$ ist der Erwerb dieser produktionsnotwendigen Kenntnisse für unternehmensexterne Anbieter in der Regel mit prohibitv hohen Fixkosten verbunden. Deswegen besitzt das anbietende Unternehmen quasi ein natürliches Monopol auf seine Rechnungslegungsinformationen. ${ }^{34}$ Ein natürliches Monopol allein macht jedoch noch kein Marktversagen aus. Hier ist es von entscheidender Bedeutung, ob die Nachfrager des Gutes über ausreichende Marktmacht verfügen, um ein Setzen von Monopolpreisen durch den natürlichen Monopolisten $\mathrm{zu}$ verhindern. ${ }^{35}$ Die meisten Vertragspartner eines rechnungslegenden und publizierenden Unternehmens sind gleichzeitig Adressaten der Rechnungslegungsdaten. Wenn diese Adressaten den Eindruck haben, daß das Angebot des Untemehmens nicht befriedigend ist, dürften sie ihre durch die Vertragssituation gegebene Marktmacht ausnutzen um satisfiszierende Informationen $\mathrm{zu}$ erhalten. In diesem Fall wäre also trotz natürlicher Monopolstellung des Anbieters ein regulierendes Eingreifen nicht angeraten.

32 Vgl. zur Clubtheorie grundlegend Buchanan (1965) und einführend Breuer (1998). Zu einer Implementierung in ein standardisiertes Vorgehensschema zur Bereitstellung von Kollektivgütern Grossekettler (1995), S. 496-526; zur Anwendung im Bereich der Publizitätsregulierung Bird/Locke (1981) und Pellens/Gassen (1998).

33. Vgl. ähnlich auch die Modellannahmen in Hakansson (1977).

34 Vgl. auch Wolk/Francis/Tearny (1991), $81 \mathrm{f}$.

35 Vgl. zu den marktwirtschaftlichen Problemen von natürlichen Monopolen Scherer/Ross (1990), S. 21-55; Fritsch/Wein/Ewers (1999); S. 178-262 
Der öffentlich verfügbaren Rechnungslegung wird auch die Emission externer Effekte zugeschrieben. ${ }^{36}$ Unter externen Effekten werden teilweise auch pekuniäre externe Effekte verstanden, also Nutzenwirkung einer Person, die durch den Marktmechanismus übertragen werden und letztendlich durch die wirtschaftliche Disposition eines anderen Wirtschaftssubjekt induziert wurden. Rechnungslegungsinformationen eines am Markt erfolgreichen Unternehmens können pekuniäre externe Effekte emittieren, die auf andere weniger erfolgreiche Unternehmen ausstrahlen, die es dadurch an ihren Vertragsmärkten schwieriger haben. Solche pekuniären extemen Effekte sind nicht schädlich, im Gegenteil: Sie sind maßgeblich für das Funktionieren einer Marktwirtschaft. Technologische externe Effekte hingegen entstehen, wenn durch die Dispositionen eines Wirtschaftssubjektes neben dem eigentlich geplanten Gut mindestens ein Kuppelprodukt entsteht, dem sich die anderen Wirtschaftssubjekte nicht entziehen können. Ein solcher technologischer externer Effekt wird jedoch durch das Anbieten von Rechnungslegungsnormen nicht induziert. Der in diesem Zusammenhang hin und wieder referenzierte „öffentliche Nutzen“ der Rechnungslegung, der das „Informationsinteresse der Allgemeinheit“ befriedigen soll, ist kein positiver technologischer externer Effekt der publizierten Rechnungslegung, sondern vielmehr eine ungenaue Definition des Nachfragerkeises. ${ }^{37}$ Die interessierte Öffentlichkeit wird nicht mit einem unvermeidbaren Kuppelprodukt konfrontiert, sondern fragt gerade das originäre Gut, die Rechnungslegungsinformationen nach.

Rechnungslegung läßt sich abweichend von der bisherigen Betrachtungsweise auch nicht selber als ökonomisches Gut, sondern als Informationssignal interpretieren, welches Marktversagen auf anderen Märkten, hauptsächlich auf dem Kapitalmarkt, vermeiden soll. Findet keine Veröffentlichung von Rechnungslegungsdaten statt, so droht nach Akerlof ein Adverse Selection Proze $\beta^{38}$, der dazu führen kann, daß der Kapitalmarkt funktionsuntüchtig wird. ${ }^{39}$

Nach der Theorie des Informationsgleichgewichtes auf Märkten mit asymmetrischer Informationsverteilung werden Anbieter von Gütern, über deren Qualität sich die Nachfrager kein Bild machen können, von sich aus zur Veröffentlichung von Signalen tendieren, um ihre Produkte besser absetzen zu können. So bildet sich ein Informationsgleichgewicht, bei dem auch die weniger gut informierten Marktteilnehmer in der Lage sind,

36 Vgl. Wolk/Francis/Tearny (1991), S. 83.

37 Vgl. zu einer detaillierten Analyse des Adressatenkreises des Rechnungswesens Schildbach/Feldhoff (1993).

38 Vgl. Akerlof (1970).

39 Vgl. zu dieser Argumentationskette Feldhoff (1992), S. 119-158, zu einem spieltheoretischen Ansatz Feldhoff (1992a). 
die Qualität der angebotenen Produkte zu bewerten. ${ }^{40}$ Riley zeigt jedoch, daß sich dieses Informationsgleichgewicht bei kontinuierlichen Qualitätsunterschieden der am Markt angebotenen Produkte nicht notwendigerweise einstellt. ${ }^{41}$

Festzuhalten bleibt, daß aus informationsökonomischer Sichtweise die Notwendigkeit einer Regulierung der Rechnungslegungspublizität nicht zweifelsfrei gerechtfertigt werden kann. ${ }^{+2}$ Deswegen soll im folgenden das Phänomen Rechnungslegungspublizität auch noch aus dem Blickwinkel der institutionentheoretischen Vertragstheorie gesehen werden.

\subsection{Institutionstheoretisch geprägte Ansätze}

Aus Sicht der transaktionskostenbasierten Vertragstheorie ist das ökonomische Koordinationsproblem im wesentlichen ein Problem der Vertragsgestaltung. ${ }^{43} \mathrm{Da}$ sich die Neue Institutionenökonomik in ihrem Zweig der Transaktionskostenanalyse ${ }^{44}$ deutlich von den rigiden Modellannahmen der Neoklassik differenziert, ist ein Abschluß von vollständigen Verträgen, die sämtliche Eventualitäten koordinieren, in der Regel nicht mehr möglich, geschweige denn ökonomisch sinnvoll.

Durch den von Herbert $A$. Simon eingeführten neuen Fokus der sozialwissenschaftlichen Forschung auf das von Restriktionen geprägte Verhalten der Akteure, der unter der Bezeichnung „cognitive turn“ in die Literatur eingegangen ist, ${ }^{45}$ wurde deutlich, daß Institutionen nicht kostenfrei funktionieren können und somit als Teil des ökonomischen Systems explizit berücksichtigt werden müssen. Wegen begrenzter Rationalität wird es für Wirtschaftssubjekte möglich, sich den rigiden Verhaltensvorschriften von Institutionen $\mathrm{zu}$ widersetzen und durch opportunistisches Verhalten auszubrechen. Somit wird das Verhandeln von speziellen, neugeschaffenen Vertragsinstitutionen zu einer sinnvollen Tätigkeit im ökonomischen Alltag. Diese Institutionen treten neben die bereits bestehenden Institutionen, welche von einer staatlichen Stelle zentralistisch erstellt und den Wirt-

4) Vgl. Riley (1979); allgemein zu adverse selection und signalling Kreps (1990), S. 625-652; zum Anfang der Theorie des Informationsgleichgewichtes vgl. Spence (1974); Rothschild/Stiglitz (1976) und Wilson (1977). $\mathrm{Zu}$ einer Übertragung dieser Theorie auf die Rechnungslegung vgl. beispielhaft Dye (1983) und HartmannWendels (1994).

+1 Vgl. Riley (1979), S. $352 \mathrm{f}$.

t2 So auch Gonedes (1975), insb. S. 859-861, der anhand eines spieltheoretischen einperiodigen Modells einen Mechanismus aufzeigt, der an Kapitalmärkten zur Informationsproduktion führt und somit keine Notwendigkeit für Publizitätsnormen anerkennt.

43 Vgl. als grundlegenden Einstieg zur Transaktionskostentheorie Williamson (1979).

4 Vgl. zur Struktur der Neuen Institutionenökonomik Erlei/leschke/Sauerland (1999), S. 1-56, insb. S. 42-45; zum Begriff auch Coase (1984).

45 Vgl. als wohl zentrales Werk Simon (1957); vgl. auch Lindenberg (1998). 
schaftssubjekten oktroyiert wurden. ${ }^{46}$ In Zusammenarbeit mit den ebenfalls verhandelten Durchsetzungsmechanismen regeln sie die Transaktionen zwischen den ex ante unabhängigen Wirtschaftssubjekten.

Dieser Vertragsprozeß läßt sich zu Analysezwecken in unterschiedliche Phasen unterteilen. ${ }^{47} \mathrm{Er}$ beginnt mit der Vertragsanbahnung. Um einen möglichen Vertragspartner zu eruieren, sind Such- und Informationstätigkeiten vorzunehmen. Aus Sicht eines Käufers ist eine zentrale Aufgabe die Suche nach dem günstigsten Preis eines bestimmten Gutes ${ }^{\text {}}$, sowie bei Produktdifferenzierung, die Suche nach einem Produkt mit den gewünschten Eigenschaften. ${ }^{49}$ Speziell für diese Suchaufgabe spielt die Erfahrung des Suchenden eine große Rolle, da die Qualität des zu bewertenden Produkts nicht kostenlos in Erfahrung zu bringen ist. Der Anbieter der Produkte hat einige Möglichkeiten, die Feststellung der Qualität seines Produktes zu steuern. So kann er Werbung betreiben, Produktgarantien aussprechen, einen Markennamen aufbauen und eben auch wichtige Informationen im Rahmen der Rechnungslegungspublizität veröffentlichen. All diese Tätigkeiten führen zu Institutionen, die den Vertragsabschluß erleichtern sollen. In gewissen Fällen, in denen die Suche von möglichen Vertragspartnem mit erheblichen Problemen verbunden ist, kann auch die Integration der Transaktion in einen hierachischen Unternehmensverbund transaktionskostenmindernd sein. Für die Unternehmensleitungs-Eigentümerbeziehung ist dies in der eigentümergeleiteten Unternehmung der Fall. Grundsätzlich kann die Integration sowohl vertikal, horizontal als auch diagonal erfolgen. Falls eine direkte Integration zu starr erscheint, ist auch die Bildung impliziter Vereinbarungen oder strategischer Allianzen möglich. ${ }^{50}$

Wenn der gewünschte Vertragspartner für die Transaktion gefunden wurde, schließt sich als nächste Phase der Vertragsabschluß an. Bei marktnahen Transaktionen ist einer der zentralen Vertragsparameter der Preis. Die beobachtbaren Preisstarrheiten ${ }^{51}$ sprechen dafür, daß das reine Preisbildungsmodell der Neoklassik mit sich kontinuierlich an die Entwicklung von Angebot und Nachfrage anpassenden Preisen in der Realität nur selten Verwendung findet. Administrierte Preise lassen sich erklären, wenn sich durch Konstanz

* Vgl. zu der Differenzierung von spontan entstandenen und zentralistischen Institutionen Williamson (1973); Hayek (1973), S. 20-21.

47 Vgl. Richter/Furubotn (1996), S. 51-53 und speziell zu den Transaktionskosten auf Märkten S. 318-340.

48 Vgl. dazu grundlegend aus Sicht der neoklassischen Informationsökonomik Stigler (1961); zur kritischen Reflexion Kunz (1985), S. 35-41.

49 Vgl. Nelson (1970) und Akerlof (1970).

so Vgl. zu den unterschiedlichen Institutionen für den marktwirtschaftlichen Suchprozeß Barzel (1982); allgemein zu Institutionen zwischen dem neoklassischen Punktmarkt und dem hierachischen Untemehmen Richter/Furubotn (1996), S. 317-326.

51 Vgl. z.B. Kaufer (1980), S. 578-590. 
der Preise die Suchphase vor Vertragsabschluß verkürzen läßt. Ebenso reduzieren feste Listenpreise die Verhandlungsdauer zwischen den Transaktionspartnern, somit in der Regel auch die daraus resultierenden Transaktionskosten. ${ }^{52}$

Nach dem eigentlichen Vertragsabschluß ist die Transaktion aus Sicht der Neuen Institutionenökonomik noch nicht beendet. So muß ex post noch die Erfüllung des Vertrages, die Kontrolle und ggf. auch die Durchsetzung eventuell verbliebener Ansprüche geregelt werden. Auch für diese Vertragsphase kann die Rechnungslegungspublizität eine sinnvolle Institution darstellen. ${ }^{53}$ Die Vertragspartner befinden sich nach Abschluß des Vertrages in einer Art bilateralem Monopol. Dadurch steigen die potentiellen Erträge opportunistischen Verhaltens. ${ }^{54}$ Ein generelles Abwickeln der Probleme nach Vertragsabschluß über die zentrale Institution des Rechtssystems, wie von der neoklassischen Theorie kostenfrei unterstellt, ist wegen der Verhaltensannahmen nicht zweckmäßig. Je nach den Determinanten der zu koordinierenden Transaktion sind unterschiedliche Institutionen zur Koordination der Vertragserfüllung sinnvoll. So ist die Häufigkeit der Transaktion von Bedeutung. Handelt es sich um wiederkehrende Geschäfte, so besteht für beide Seiten ein großer Anreiz, auf opportunistisches Verhalten zu verzichten, solange der Grad der Spezifität vergleichsweise gering ist. In diese Richtung kann auch ein über dem Konkurrenzpreis liegender Vertragspreis wirken, da er das Bestreben des Anbieters, weiter als zukünftiger Vertragspartner zu fungieren, noch verstärkt. Besitzt die betrachtete Transaktion jedoch eine hohe Spezifität, so wird der Anreiz zur Integration der Transaktion in eine hierachische Unternehmensstruktur höher. Bei seltenen, sich nicht regelmäßig wiederholenden Vertragsabschlüssen, ist unter Umständen die Einschaltung eines Dritten denkbar, der die ex post Abwicklung des Vertrages überwacht. Dies umfaßt auch eine mögliche Kontrolle der gelieferten Gutsqualität. In bestimmten Fällen, in denen die Vertragserfüllung über eine lange Zeitdauer geplant ist und mit großer Unsicherheit belastet ist, kann eine Regulierung durch den Staat eine institutionelle Alternative zur vertikalen Integration werden, um opportunistisches Verhalten zu verhindern. ${ }^{55}$ Somit wird deutlich, daß die nach Vertragsabschluß entstehenden Transaktionsprobleme einen großen Einfluß auf das Entstehen von Unternehmen einerseits und Märkten anderseits haben.

52 Vgl. Alchian/Woodward (1987).

53 Inwiefern der Charakter der Rechnungslegungspublizität sich nach Vertragsabschluß wandelt, hängt u.a wohl davon ab, inwieweit die Investoren spezifische Investitionen zum Vertragsabschluß getätigt haben und ob ihre Vertragsrechte auf Sekundärmärkten zu geringen Transaktionskosten handelbar sind.

54 Vgl. Williamson (1985), S. 61-63; der dieses Phänomen als „fundamental transformation“ bezeichnet.

55 Vgl. hierzu die Diskussion von Demsetz (1968); Williamson (1976) und Goldberg (1976). Während Demsetz die Regulierung von natürlichen Monopolen grundsätzlich ablehnt, sehen Williamson und Goldberg unter bestimmten Bedingungen eine Regulierung aufgrund der hohen Gefahr opportunistischen Verhaltens als sinnvoll an, zumal eventuelle Nachverhandlungen mit dem Monopolanbieter wohl kaum unter Marktbedingungen stattfinden können. 
Nach Williamson wird der Charakter der vertraglichen Koordination also im wesentlichen von drei Determinanten bestimmt: Von dem Grad der Unsicherheit, der Spezifität der eingesetzten Faktoren und der Häufigkeit, mit der eine bestimmte Transaktion durchgeführt wird. ${ }^{56}$ Gerade die Unsicherheit kann durch die verfügbare Menge an Information reduziert werden, so daß die mit der Transaktion verbundenen Kosten sinken und eine näher am Ideal des klassischen Vertrags orientierte Koordinationsform zustande kommen kann. Diese Argumentation spricht für den Austausch von Information zwischen Vertragspartnern und kann somit Anhaltspunkte für die Erklärung von privatem Informationsangebot und privater Informationsnachfrage liefern. Fraglich ist jedoch, ob dadurch auch ein Anreiz für die Bereitstellung von öffentlicher Information und somit für Unternehmenspublizität geliefert werden kann. Wenn das Management als potentielle Vertragsseite den Vertragspartnern bereits vor Vertragsabschluß die Bereitschaft zur Reduktion der durch die Informationsasymmetrie begründeten Unsicherheit kommunizieren will, dann kann die glaubhafte Versicherung, ein Publizitätssystem einzusetzen, zuträglich sein. Eine solche glaubhafte Versicherung kann z.B. ein öffentliches Versprechen verbunden mit der Reputation des Managements als Geisel sein. Falls dem Management keine Methoden zur Verfügung stehen, die kontinuierliche Publikation von Unternehmensdaten auch für die Zukunft glaubhaft zu versichem, könnte das Management vom Regulierer eine gesetzliche Absicherung der Selbstverpflichtung nachfragen. ${ }^{57}$ Geht die Initiative für die Produktion und Veröffentlichung von Rechnungslegungsdaten nicht von dem Management, sondern von den aktuellen Eigentümern aus, so obliegt diesen die Aufgabe, sich gegen das opportunistische Verhalten des Managements abzusichern. Dies kann einerseits über die Manifestation entsprechender Vorschriften in der Satzung, oder andererseits durch die Etablierung von gesetzlichen Normen geschehen, die das Management zur Einhaltung bestimmter Publizitätsregeln verpflichten. Letztere Variante ist vor allem von Bedeutung, da neben den bestehenden Eigentümern auch die künftigen oder potentiellen Eigentümer in den Nutzen der Rechnungslegungspublizität kommen sollen.

Aus vertragstheoretischer Sicht kann eine gesetzlich erzwungene Rechnungslegungspublizität also vorrangig aus Interesse der potentiellen Vertragspartner von Bedeutung sein. Die bestehenden Vertragspartner könnten jedoch aus derivatem Interesse eine Publizität nachfragen, wenn sie durch diese eine Verbesserung ihrer Vermögensposition erwarten. So könnten bestehende Aktionäre eine Unternehmenspublizität einfordern, wenn sie dadurch eine Reduktion der Spezifität ihres Eigenkapitalanteils erwarten und deswegen eine transaktionskostenärmere Veräußerung realisieren könnten. Des weiteren kann eine starre Verteilungsregel die Ermittlung von bestimmten Rechnungslegungsdaten notwendig

\footnotetext{
56 Vgl. Williamson (1985), S. 68-84.

57 Vgl. ähnlich Walker (1988).
} 
machen. Diese Verteilungsregel kann, muß aber nicht gesetzlich reguliert sein. Wie in der Problemstellung dieser Arbeit bereits erwähnt, führt eine solche rechnungslegungsabhängige Verteilungsregel zu einem gewissen Trade-Off der Informationswirkung des Abschlusses für einen ex ante unbekannten Adressaten, so daß ein gewisser Widerstand gegen die Implementierung solcher Verteilungsregeln zu erwarten ist.

Aus institutionstheoretischer Sicht läßt sich die Existenz von Rechnungslegungspublizität und, mit Abstrichen, auch deren Regulierung erklären. ${ }^{58}$ Eine normative Rechtfertigung der Pflicht zur Publizität erscheint jedoch nicht möglich. Im folgenden soll als letzter theoretischer Ansatz die evolutionäre Ökonomik behandelt werden, die im Gegensatz zu den bisher verwendeten Modellen, eher den Prozeß der Koordination und Institutionenentwicklung in den Vordergrund der Betrachtung stellt.

\subsection{Evolutorisch geprägte Ansätze}

Aus evolutorischer Sicht befindet sich das Wirtschaftssystem in einem Zustand, der von den Wirtschaftssubjekten aufgrund von konstitutioneller Unwissenheit als Unordnung interpretiert wird. Um in diesem subjektiven Chaos zu überleben, suchen die Wirtschaftssubjekte nach strukturierenden Prinzipien. Somit bilden sie Institutionen und Organisationen. Ein zentrales Gemenge von Institutionen zur Strukturierung des Wirtschaftssystems ist das Marktsystem. Je mehr spontane Regeln innerhalb des Institutionengemenges Markt von den Wirtschaftssubjekten geschaffen werden, desto mehr wird die Komplexität des Wirtschaftssystems in das Marktsystem übertragen. ${ }^{59}$ Somit reduziert die Nutzung des Marktes für die Wirtschaftssubjekte die Komplexität des wirtschaftlichen Umfeldes, der Markt funktioniert, wie von Hayek das ausdrückt, als ein Such- und Entdeckungsverfahren. ${ }^{60}$ Die Marktteilnehmer erfahren durch den Marktprozeß, wie sich der Wert ihrer Handlungsalternativen im Zeitablauf verändert. Dadurch werden sie in die Lage versetzt, darauf $\mathrm{zu}$ reagieren und ggf. sich durch einen anderen Wirtschaftsplan neue Handlungsalternativen zu generieren. $\mathrm{Da}$ der Marktmechanismus als Kommunikationsinstrument gesehen wird, bedeutet nicht, daß keine anderen Institutionen zum Austausch von Informationen vorstellbar sind. Rechnungslegungspublizität könnte aus evolutorischer Sicht als spontane Institution zur Reduktion der Einkommensunsicherheit interpretiert werden, solange sie auf freiwilliger Basis erfolgt.

\footnotetext{
58 Vgl. dazu auch King/Waymire (1993); Picot/Dietl (1994); allgemein zur Wechselwirkung von Unternehmensorganisation und korrespondierendem Unternehmensrecht Williamson (1991).

59 Vgl. Kunz (1985), S. 1.

(1) Vgl. Hayek (1945), insb. S. 26.
} 
$\mathrm{Da}$ die Funktionsweise des Marktes nicht modelliert werden kann, gibt es keine Möglichkeit, Marktprozesse oder auch die Auswirkungen von anderen Institutionen hinsichtlich ihrer Effizienz zu beurteilen. ${ }^{61}$ Somit kann die Forderung der evolutorischen Ökonomik nur lauten, die Funktion des Marktes als Entdeckungsverfahren zu unterstützen. Für diese Funktion sind bestimmte Rahmenbedingungen von Bedeutung. Damit spontane Institutionen entstehen können, ist die Existenz von Verfügungsrechten unerlässlich. ${ }^{62}$ Von Hayek betont ferner die Bedeutung von allgemeinen abstrakten Regeln (laws) und grenzt diese von speziellen Regeln (commandos) $a b .{ }^{63}$ Während allgemeine Regeln ursprünglich spontane Institutionen sind und durch zunehmende Akzeptanz zu Regeln abstrahiert werden, sind spezielle Regeln immer das Ergebnis von konstruktivistischinterventionistischen Eingriffen einer hoheitlichen Instanz und als solche ein Fremdkörper im Marktprozeß. Während das grundsätzliche Konzept der Rechnungslegungspublizität als bedingt spontane Institution angesehen werden $\mathbf{k a n n}^{64}$, muß die Verpflichtung zur Unternehmenspublizität wohl als eine spezielle Regel eingestuft werden. Allgemeine Regeln schaffen einheitliche Bedingungen für alle Marktteilnehmer und entsprechen so von Hayek's Grundsatz der Gleichheit vor dem Gesetz. ${ }^{65}$

Somit bietet die evolutorische Ökonomik Anhaltspunkte, interventionistisches Handeln des Staates zu erkennen. Unter Umständen kann die Beobachtung von Marktprozessen Anhaltspunkte für fehlende Evolution bieten. Ein Markt, der über längeren Zeitraum keine prozessuralen Änderungen erkennen läßt, wird in diesem Zusammenhang als überprüfungswürdig angesehen. ${ }^{66}$ Dies mag aufgrund der vorherrschenden Regulierungen auch zumindest zeitweilig für den deutschen Markt für Rechnungslegungspublizitätsinformationen gelten, da dieser, abgesehen von den Gesetzesänderungen mit dem AktG 1965 und HGB 1985 bis in die neunziger Jahre von relativ wenig Modifikationen betroffen war.

Es läßt sich festhalten, daß auch aus evolutorischer Sicht mögliche Begründungen für das Phänomen Rechnungslegungspublizität ableitbar sind. ${ }^{67}$ Eine normative Begründung für

61 Vgl. Kunz (1985), S. 3.

62 Vgl. zu den Rahmenbedingungen einer Wirtschaftsordnung bereits Eucken (1965), S. 241 f., der die Enthaltung des Staates von Eingriffen in den Marktprozeß fordert jedoch gewisse Interventionen zur Vermeidung von monopolistischen Marktformen für notwendig hält und sich insofern klar von der evolutorischen Ökonomie distanziert.

${ }^{63}$ Vgl. Hayek (1960), S. 148-151; vgl. dazu auch Kunz (1985), S. 156 f.

64 Vgl. Schneider (1997), S. 27.

65 Vgl. Hayek (1960), S. 85-88.

66 Vgl. Wegner (1996), S. 75-79; vgl. übertragend auf den Bereich der Rechnungslegung bereits Ordelheide (1995).

67 Vgl. wiederum Ordelheide (1995); Schneider (1997), S. 27. 
dessen Regulierung läßt sich jedoch nicht entwickeln. Da eben diese Regulierung empirisch jedoch als Ergebnis des politischen Prozesses international evident ist, besteht Bedarf an normativen Handlungsempfehlungen, wie denn die bestehende Rechnungslegungspublizität weiterzuentwickeln sei.

Um jedoch zu der deswegen notwendigen operationalen Bewertungsskala für unterschiedliche Rechnungslegungspublizitätssysteme zu gelangen, müssen einige Annahmen über Wirkungszusammenhänge getroffen werden. Der skizzierte Metazweck der Rechnungslegungspublizität ist hierfür zu wenig operational. Wie können die Adressaten adäquat über die abgelaufene Periode informiert werden? Welche Größe ist für die Regelung ihrer Verteilungsprobleme ideal? Welche beobachtbaren Merkmalsausprägungen besitzen eine Proxyfunktion für zukünftige Ereignisse? Aus der oben gegebenen funktionalen Publizitätsbeschreibung ergeben sich zahlreiche Anwendungsprobleme. Die in der Wirtschaftsgeschichte entstandenen Publizitätssysteme sind intendiert rational geschaffene Konstrukte, die einer bestimmten Zielvorstellung entsprechen sollen. Hierbei handelt es sich um die gesuchten operationalen Ausgestaltungen des Metazwecks der Rechnungslegungspublizität. Im folgenden Abschnitt werden zunächst die unterschiedlichen in der Literatur entwickelten Zielvorstellungen dargestellt. Um diese Zielvorstellungen zu erhalten, wird ein Überblick über die divergierenden Richtungen der Bilanztheorie vorgenommen. Aus diesen lassen sich die jeweilig korrespondierenden Ziele der Rechnungslegung ableiten. Im Anschluß daran wird untersucht, inwieweit diese Ziele eine ökonomisch plausibel erscheinende Operationalisierung darstellen. Abschließend und darauf aufbauend soll dann der für diese Arbeit verwendete normative Rahmen der Rechnungslegungspublizitätsentwicklung abgesteckt werden.

\section{Rechnungslegungstheorien}

\subsection{Theorien der Bilanzbewertung}

Konstituierendes Merkmal der Rechnungslegungspublizität ist die Abbildung von realökonomischen Sachverhalten in monetär quantitativen Daten für einen ex ante unbestimmten Adressatenkreis. Um dies zu erreichen, müssen die Sachverhalte bewertet werden. Erst durch Bewertung entstehen letztlich die Rechnungslegungsgrößen Aufwand und Ertrag, Vermögensgegenstand und Verbindlichkeit. Sowohl in Deutschland als auch in den USA prägt die Idee der Verteilung der Ein- und Auszahlungen auf die unterschiedlichen Perioden die frühen Theorien der Rechnungslegung. ${ }^{68}$ Die Periodisierung unter-

68 Vgl. Rückle (1993), Sp. 251, Beaver (1989), S.3; überblicksmäßig zur US-amerikanischen Bilanztheorie auch Ballwieser (1993); Haller (1994), S. 79-195. 
scheidet die Bilanz und Gewinn- und Verlustrechnung von der Einzahlungs- und Auszahlungsrechnung. ${ }^{69}$ Durch die Periodenabgrenzung soll es den Rechnungslegungsadressaten besser möglich sein, den Nutzen ihres vertraglichen Engagements zu beurteilen, als dies lediglich auf Basis einer Finanzierungsrechnung möglich ist. ${ }^{70}$ Somit wird die Frage der Bilanzierung als eine Frage der korrekten Periodisierung von Ein- und Auszahlungen gesehen.

Die Ansicht, daß nur durch eine Periodisierung das Vermögen und der Erfolg eines Unternehmens zuverlässig gemessen werden können, ist keinesfalls unumstritten. So argumentiert Ijiri, daß Cash Flow Accouting dem Accrual Concept überlegen sei, da die Größe „Zahlungsüberschuß“ vom Adressaten weniger leicht mit der eigentlichen Zielgröße „zukünftiges ökonomisches Einkommen“ verwechselt würde als eine Erfolgsgröße. Zudem besäße der zukünftige Cash Flow als Prognoseziel einen direkten logischen Bezug zu vergangenen Cash Flows. ${ }^{71}$ Ebenso wird der zusätzliche Aufwand und die mangelnde Willkürfreiheit kritisiert, die eine Periodisierung der Ein- und Auszahlungen mit sich bringt. Auch wenn wohl nach herrschender Meinung die Vorteile einer Periodisierung die Nachteile überkompensieren, so ist die internationale Etablierung einer Kapitalflußrechnung, welche die anderen Rechenwerke des Jahresabschlusses flankiert, als folgerichtig zu begrüßen. $^{72}$

Erst die Einführung des Periodisierungskonzeptes ermöglichte die Ermittlung eines über die reine Liquidität hinausgehenden Wertes des Vermögens des gesamten Unternehmens ohne auf eine komplette Neubewertung im Rahmen einer Inventur zurückzugreifen. Im deutschsprachigen Raum ist mit dem Bilanzierungsziel „Vermögenswertermittlung“ immer noch die statische Bilanzauffassung verbunden. So findet sich in dem Urteil des preußischen Reichsoberhandelgerichtes (ROHG) von 1873 eine die statische Auffassung kennzeichnende Definition des Bilanzzwecks: „Die kaufmännische Bilanz hat den Zweck, die Uebersicht und Feststellung des Vermögensbestandes in einem bestimmten Zeitpunkte und damit zugleich, vermittelst der Vergleichung der für verschiedene Zeiten aufgenommenen Bilanzen, auch des Resultates der Geschäftsfübrung wäbrend der dazwischen liegenden Perioden zu bewirken. "a3. Hier wird bereits deutlich, daß der Periodenerfolg sich aus statischer Sicht als Vergleich des Vermögens zwischen zwei Bilanzzeitpunkten ergibt. Auch Simon betont die duale Zielsetzung der statischen Bilanzauffassung, indem er aufzeigt, daß nur durch eine kor-

69 Vgl. Chmielewicz (1993), Sp. $1514 \mathrm{f}$.

70 Vgl. Paton/Littleton (1940).

71 Vgl. Beaver (1989), S. 7; Ijiri (1978).

72 Vgl. Chmielewicz (1993), Sp. 1523 f.; zu der Forderung nach der Veröffentlichung einer zusätzlichen Finanzierungsrechnung im deutschsprachigen Raum Busse von Colbe (1966) und Moxter (1966).

73 ROHG (1873), S. 17, die Hervorhebungen des Originals wurden hier nicht wiedergegeben. 
rekte Vermögensermittlung die Feststellung des Gewinns möglich sei. Die Bedeutung der Gewinnermittlung sieht er vor allem bei Gesellschafterbeziehungen der rechnungslegenden Unternehmen. ${ }^{74}$ Diese Sichtweise: „Gewinnermittlung durch Vermögensvergleich“ findet sich auch in den Anfängen der US-amerikanischen Bilanztheorie. ${ }^{75}$

Neben diesem eher juristisch geprägten Konzept der Vermögensermittlung, das speziell für die Anbindung spezieller Rechtssätze konzipiert wurde, besitzt die Idee der Vermögensermittlung auch eine ökonomische Bedeutung. Aus betriebswirtschaftlicher Sicht ist nicht nur die absolute Höhe des Vermögens interessant, sondern auch seine Aufgliederung in einzelne Komponenten. Diese Sichtweise führt nicht nur zu anderen Ansatzsondern auch zu anderen Bewertungsempfehlungen als das Konzept Simons. So fordert Osbabr, daß für jede Bewertungsentscheidung lediglich die Auffassung des bilanzierenden Unternehmens entscheidend sei, gegebenenfalls seien die angegebenen Werte zu erläutern. ${ }^{76}$ Des weiteren seien sämtliche Wertansätze in der Bilanz entsprechend ihrer zukünftigen Wirkung vor allem auf die Liquidität hin auszuweisen und zu präsentieren. ${ }^{77}$ Letztendliches Ziel der vorgeschlagenen Bilanzierungssysteme war jedoch vorrangig die Ermittlung eines aus dem Vermögensvergleich ermittelten Reingewinns, der dann zur Lösung der das Untemehmen betreffenden Verteilungsprobleme verwendet werden konnte. Der betriebswirtschaftliche Informationscharakter für kommende Perioden ${ }^{78}$ war von geringer Bedeutung. Da neben den privatrechtlichen Verteilungsproblemen an die Bilanzergebnisse auch spezielle Rechtssätze gebunden wurden, wurde die Publizität in Deutschland auch eine juristische Notwendigkeit ${ }^{79}$, um den Adressaten den Zugriff auf die Daten zu ermöglichen. Im Gegensatz dazu forderten Paton/Littleton in den USA, daß die informationsorientierte Publizität streng von juristischen Fragestellungen zu trennen sei: "It is the task of law to measure the impairment of rights and to see that the status of the party damaged is restored; it is the task of accounting to make the most truthful and significant measurements possible of the continuos flow of business activity ${ }^{180}$ Gemeinsam ist beiden jedoch das Streben nach dem vom jeweiligen Zweck der Rechnungslegung determinierten „richtigen“ Vermögens- und

74 Vgl. Simon (1899), S. 5.

75 Vgl. Canning (1929), insb. S. 47-58; Paton/Littleton (1940), S. 9, die ebenso wie die deutschen Statiker die wahre Gewinnermittlung jedoch nur durch die Totalbilanz für möglich halten, Vgl. ebenda S. 10.

76 Vgl. Osbahr (1923), S. 33-34; diametral anderer Ansicht Fischer (1909).

7 Vgl. Osbahr (1923), S. 79 f.

78 Vgl. Osbahr (1923), S. 122.

79 Vgl. Schmalenbach (1962), S. 18-19, der behauptet, daß der aus dem Ordonnance de Commerce hervorgegangene napoleonische Code de commerce in der Folgezeit unreflektiert in die kontinentaleuropäischen Handelsgesetzbücher übernommen wurde und somit sich die Auffassung durchsetzte, "daß ein ordentliches Handelsgesetzbuch auch Bestimmungen über die Bilañ enthalten müsse", Schmalenbach (1962), S. 19; ähnlich auch Simon (1899), S. 34, Fn. 20.

8) Paton/Littleton (1940), S. 11. 
Gewinnausweis. ${ }^{81}$ Auf dieser Suche nach dem richtigen Periodenerfolg befand sich auch Schmalenbach mit der Entwicklung der dynamischen Bilanzauffassung. ${ }^{82}$ Er sieht den Bilanzzweck primär in der Prognose drohender Konkurse und Liquiditätsprobleme (Zahlungsstockungen), insofern entspricht seine Zielsetzung einer Teilmenge des oben beschriebenen Metazwecks der Rechnungslegungspublizität. ${ }^{83}$ Für Aktiengesellschaften spricht zwar Schmalenbach einem Großteil der Aktionäre das Interesse an publizierten Bilanzen ab, da sie als Spekulanten lediglich am Kursverlauf der Aktien interessiert seien, ${ }^{84}$ gleichzeitig erkennt er jedoch die Bedeutung einer regelmäßigen Publizität für die Allokationseffizienz von Aktienmärkten und beklagt, daß der Gesetzgeber bei dem Entwurf des Aktiengesetzes von $1884 \mathrm{zu}$ viel juristisch und zuwenig ökonomisch gehandelt habe. ${ }^{85}$ Aus diesen Überlegungen folgert er, daß der eigentliche Zweck der Bilanz die Ermittlung des „richtigen Erfolges“ sei. Mit diesem Ergebnis stimmt er mit der angloamerikanischen Bilanzforschung bis etwa zu den 60er Jahren dieses Jahrhunderts überein. $^{86}$

Die gesamte hier vorgestellte Bilanzierungsforschung ist also auf der Suche nach dem „richtigen“ Wertansatz. Auch wenn den Autoren sehr wohl bekannt ist, daß es den ökonomischen, objektiven Wert eines Gutes nicht gibt, ${ }^{87}$ so suchen sie trotzdem nach einer absoluten Lösung des Bewertungsproblems, da vor allem die Gewinnermittlung als zentrale Grundlage für die Lösung von Verteilungsproblemen gesehen wird. Deswegen wurde eine sehr detaillierte normative Wertlehre entwickelt, die in ihren Grundlagen das Kernproblem der Rechnungslegungspublizität, die Frage der Bewertung, bis heute wesentlich prägt. Zur Verdeutlichung der aus diesen Anstrengungen resultierenden Ergebnisse wird im folgenden beispielhaft auf die Ansätze Osbahrs eingegangen. ${ }^{88} \mathrm{Er}$ unterscheidet gemäß

81 Vgl. Osbahr (1923), S. 123. Dies scheint jedoch für die Entwickler des allgemeinen deutschen Handelsgesetzbuches von 1862 nur eingeschränkt zu gelten. Schmalenbach (1962), S. 23 wertet: „Man bat den Eindruck, daß niemand in der Komission genau wußte, welche Zwecke mit den Bilanzuorschriften erfüllt werden sollten.".

82 Vgl. Schmalenbach (1919; 1962) und Münstermann (1966).

83 Vgl. Schmalenbach (1962), S. 25-26.

84 Vgl. Schmalenbach (1962), S. 30.

85 Vgl. Schmalenbach (1962), S. 33.

86 Vgl. dazu die aufzählende Analyse in AAA (1977), S. 5-10.

87 Vgl. dazu schon die Ausführungen von Platon (399 v. Chr. - 347 v.Chr), 304b, der auf den vermeintlichen Widerspruch in der Bewertung von Wasser hinweist, daß zwar unersetzlich doch trotzdem quasi kostenfrei sei und feststellt, daß nur Knappheit ein Gut wertvoll mache. Diese Ausführungen wurden von Smith (1776), 31-32 aufgegriffen, der als Gegenbeispiel Diamanten anführt und zwischen dem ,"alue in use "und dem "value in exchange " unterscheidet. Vgl. als weiteren Vordenker Ricardo (1817), S. 17-44.

88 Vgl. zu den nun folgenden Wertansätzen Osbahr (1923), S. 16-25; zu anderen deutschen Bewertungskonzepten vgl. Simon (1899), insb. S. 290-325 zusammenfassend Moxter (1984), S. 15-22; zu den dynamischen Bewertungskonzepten vgl. Schmalenbach (1962), S. 77-172 und zusammenfassend Moxter (1984), S. 38-47. 
seiner tendenziell betriebswirtschaftlich geprägten Sichtweise zwischen unterschiedlichen Herkunfts- und Verwendungsalternativen der zu bewertenden Vermögensgegenstände.

Der Veräußerungswert ${ }^{\beta 9}$ entspricht dem Wert bei Verkauf des Gutes an Unternehmensdritte. Hierbei unterscheidet Osbabr zwischen einem objektiven Veräußerungswert, der auf einem funktionstüchtigen Markt ohne Marktmacht zustande kommt und dem subjektiven Veräußerungswert. Der objektive Veräußerungswert entspricht im wesentlichen der angloamerikanischen Definition des fair values ${ }^{20}$. Der subjektive ${ }^{91}$ Ansatz hingegen, den Osbahr auch als Sonder-Veräußerungswert oder Sonderverkaufswert bezeichnet, berücksichtigt die bei der spezifischen fiktiven Transaktion vorliegenden Gegebenheiten. Je nach der Sichtweise wird zwischen einem persönlichen Sonderverkaufswert aus Sicht des Bilanzierenden und einem geschäftlichen Sonderverkaufswert aus Sicht des bilanzierenden Unternehmens unterschieden.

Als weiterer Wertansatz wird der Gebrauchswert angeführt. Osbabr betont hier die Unterscheidung zwischen der bilanziellen und der volkswirtschaftlichen Wertauffassung. Er stellt klar, daß es nicht im Rahmen der Bilanz möglich sei, den objektiven Gebrauchswert im ökonomischen Sinn zu ermitteln. Vielmehr ginge es bei der Differenzierung zwischen Veräußerungs- und Gebrauchswert um die Rolle, welche die jeweiligen Vermögensgegenstände im Unternehmensverbund spielten. Ein Vermögensgegenstand, der nicht nur vorübergehend, sondern vielmehr dauerhaft zur Produktion zur Verfügung steht, ist nach Osbabr mit dem Gebrauchswert zu bewerten. Diesen sieht er in zwei Ausprägungen analog zum Veräußerungswert: Der persönliche Gebrauchswert wird aus Sicht des bilanzerstellenden Unternehmers gesehen, der geschäftliche aus Sicht des gesamten Unternehmens. Als bessere Bezeichnung für den Gebrauchswert schlägt Osbahr den Begriff „Betriebswert“ vor. Als Ermittlungsgrundlage dienen die Selbstkosten, so daß nur noch eventuelle Wertkorrekturen nach unten oder oben als subjektive Bewertungskomponente vorzunehmen sind. Insofern entspricht dieser Wertansatz in Grundzügen dem Konzept der fortgeführten Anschaffungskosten.

89 Simon spricht hier von Verkehrswert, vgl. Simon (1899), S. 294.

9) Vgl. SFAS No. 107, Par. 5,6,11, Appendix A; SFAS No. 122, Par. 3(f) und IAS 32, Tz. 5; zu Problemen bei der Ermittlung eines zukunftsorientierten Fair Values AAA's Financial Accounting Standards Committee (1998).

91 Vgl. Simon (1899), S. 295, der die Begriffspaare subjektiv/objektiv und absolut/relativ in Verbindung mit Wertbegriffen wegen des definitionsinhärenten Widerspruchs derselben ablehnt. Vgl. jedoch Osbahr (1923), S. 14, der argumentiert, daß „objektiv“ in Verbindung mit bilanziellen Wertbegriffen einen Ansatz beschreibt, "den ein Gegenstand in der Beurteilung einer Vielheit von werturteilenden Mensiben besityt" (ebendort). Osbahr ist sich anscheinend über die Schwammigkeit dieser Definition im klaren, er befürwortet auch wie Simon das Begriffpaar allgemein/besonders, ohne es jedoch im folgenden zu benutzen. 
Auch der Selbstkosten wert kann aus objektiver oder subjektiver Sicht ermittelt werden. Die Selbstkosten aus objektiver Sicht resultieren entweder aus den Marktpreisen der zur Produktion aufgewendeten Güter zum jeweiligen Erwerbszeitpunkt (herstellungsorientierter Selbstkostenwert) oder zum Bewertungszeitpunkt (wiederbeschaffungsorientierter Selbstkostenwert). Osbabr sieht weiterhin die Möglichkeit eines subjektiven Selbstkostenwertes, wenn nicht die objektiven Verkehrswerte, sondern jeweils die subjektiven Verkehrswerte zur Bewertung herangezogen werden. Die objektiven Selbstkosten zum herstellungsorientierten Wert entsprechen dem Anschaffungskostenprinzip.

Als letzte Bewertungsgruppe führt Osbahr den Ertragswert an. Zu dessen Emittlung werden die in der Vergangenheit realisierten Erträge, die dem zu bewertenden Gut beizulegen sind, unter Berücksichtigung besonderer Bedingungen in die Zukunft extrapoliert und mit Hilfe eines "landesüblichen Zinsfußes ${ }^{102}$ abgezinst. Osbahr weist auf die Probleme hin, mit Hilfe des Ertragswertverfahrens einzelne Vermögensgegenstände bewerten zu wollen. Er sieht die Aufgabe der Bewertungsmethode eher im Bereich der Gesamtunternehmensbewertung und betont die besondere Bedeutung des Ertragswerts als volkswirtschaftlich vertretbarer Wertansatz.

Es wird deutlich, daß die meßorientierten Bilanzauffassungen eine Vielzahl von unterschiedlichen Bewertungskonzeptionen ermittelt haben, die sich auch heute noch in den Diskussionen zu aktuellen Normungsfragen widerspiegeln. Bei der Frage, welche Bewertungsmethode aus normativer Sicht zu befürworten ist, sind die frühen Werke wenig informativ, da ihre Empfehlung sich weniger auf wissenschaftstheoretisch saubere Modelle, als auf mehr oder weniger einleuchtende Meinungen berufen. So gelingt es den pragmatischen bewertungsorientierten Ansätzen nicht, eine normative Theorie der Bilanzierung zu entwickeln ${ }^{93}$, da sie keine entsprechende Analyse der letztendlich die Rechnungslegung induzierenden Entscheidungsprobleme vomehmen. Daß die Rechnungslegungspublizität auch für Entscheidungen eine wichtige Rolle spielte, war jedoch auch den Vertretern der bewertungsorientierten pragmatischen Richtung klar. So stellt Osbahr fest, daß der Zweck von wirtschaftsgeschichtlichen Aufzeichnungen im Erkenntnisgewinn für zukünftiges Handeln bestehe. ${ }^{4}$ Paton/Littleton erkannten die Befriedigung des Informationsinteresses der Adressaten als eine der zentralen Aufgaben des Rechnungswesens an. ${ }^{95}$ In den 50er Jahren wurden vorrangig im Bereich der Theorie des internen Rechnungswesens erste Stimmen laut, die eine stärkere Ausrichtung der Rechnungswesenskonzeption

22 Osbahr (1923), S. 23.

93 Vgl. Caplan (1972), S. 438-440, der diese Forschungsansätze als „armchair theorizing “bezeichnet.

94 Vgl. Osbahr (1923), S. 122.

95 Vgl. Paton/Littleton (1940), S. 1; zu weiteren frühen Quellen, welche die Bedeutung der Adressateninteressen für das Rechnungswesen hervorheben vgl. AAA (1977), S. $10 \mathrm{f}$. 
an den Informationsinteressen der Adressaten forderten. ${ }^{6}$ Dies führte in den USA in den 60er Jahren zu dem Versuch, die bilanztheoretische Forschung in eine neue Richtung zu bewegen. ${ }^{77}$

\subsection{Entscheidungsorientierte Theorien}

\subsubsection{Modellbildende Ansätze}

\subsubsection{A Statement of Basic Accounting Theory}

Mit Veröffentlichung der Ergebnisse eines Komitees der American Accounting Association (AAA) 1966, A Statement of Basic Accounting Theory ${ }^{\circ 8}$ (ASOBAT) begann die konsequente Ausrichtung der Rechnungswesenforschung am Informationsnutzen der Adressaten des Rechnungswesens. Das ASOBAT ist als theoretisches Rahmenkonstrukt sowohl für die (externe) Rechnungslegung als auch für das interne Rechnungswesen konzipiert. Als Zielsetzung des Rechnungswesens wird die Versorgung der Adressaten mit Informationen für folgende Zwecke definiert:

1.) Entscheidungen zur Ressourcenallokation, inklusive zur Bestimmung von Entscheidungsräumen und möglichen Zielsetzungen.

2.) Interne Unternehmenssteuerung.

3.) Rechenschaft über verwendete Ressourcen.

4.) Erleichterung der sozialen Kontrolle.?

Diese Informationsziele sollen durch die Verfügbarmachung von Daten, die sowohl historisch als auch zukunftsorientiert sein können, realisiert werden. Das Komitee betont die Bedeutung der Zukunftsrelevanz und stellt klar, daß die Adressaten auch vergangene Daten auf ihren Informationsgehalt zur Prognose zukünftiger Ereignisse hin untersuchen. Somit sei eine strenge Fokussierung des Bilanzierungsprozesses auf transaktionenbasierte, also auf die physischen Aktionen im Unternehmensverbund charakterisierenden Daten nicht zweckmäßig. ${ }^{100} \mathrm{Um}$ die für relevant erachteten Daten den Adressaten zu kommunizieren, ist auch ein Bewertungsprozeß notwendig. Das Komitee sah seine

\footnotetext{
Vgl. als Beispiel Chambers (1955).

Vgl. hierzu auch Hakansson (1969); Dyckman/Zeff (1984), S. 226-233.

Vgl. AAA (1966).

? Vgl. AAA (1966), S. 4 und erläuternd Wolk/Francis/Tearny (1991), S. 148-150.

100) Vgl. AAA (1966), S. 5-6.
} 
Hauptaufgabe darin, Standards zu entwickeln, die bei Beachtung eine zielkonforme Entwicklung von Rechnungslegungsvorschriften garantieren. Als Ergebnis präsentieren sie vier Eckpfeiler:

Relevance bedeutet, daß die zu berichtende Information relevant für die o,a. vier Kategorien ist. Das Komitee macht deutlich, daß unterschiedliche Wertansätze gleichzeitig ggf. für unterschiedliche Adressaten relevant sein können. Verifiability steht für eine intersubjektive Plausibiltät des Wertansatzes. Unterschiedliche Wirtschaftssubjekte sollen bei gleichem Informationsstand zu ähnlichen Wertansätzen gelangen. Dieser Grundsatz soll vor allem gewährleisten, daß Wirtschaftssubjekte, die keinen direkten Zugang zur originären Datenbasis, bzw. keine Kompetenz zur Auswertung besitzen, nicht von den Erstellem der Rechnungslegungsinformation hintergangen werden können. Freedom from bias soll das Verwenden einseitiger Bewertungsmethoden verhindern, die bestimmte Adressatengruppen gegenüber anderen bevorteilen würden. Das Komitee geht davon aus, daß bei dem unbegrenzten Adressatenkreis der externen Rechnungslegung eine Bevorteilung einer Gruppe nie positiv sein kann. Im internen Rechnungswesen hält sie jedoch eine Tolerierung von einseitig ermittelten Daten für zulässig, solange die Adressatengruppe abschließend charakterisiert werden kann. Quantifíability bedeutet schließlich die Möglichkeit zur Quantifizierung der präsentierten Informationen. Hierbei beschränkt sich das Komitee nicht auf monetäre Bewertungen auf Basis von historischen oder Marktwerten, sondern hält grundsätzlich auch andere Bewertungsgrundlagen für möglich. So wird auch speziell auf Bandbreiten oder geschätzte Werte hingewiesen. ${ }^{101}$

Die entsprechend dieser Standards ermittelte Information erreicht die Adressaten über einen speziellen Kommunikationskanal. Dieser ist für den Grad der Zielerreichung nach Ansicht des Komitees von großer Bedeutung. Deswegen definieren sie ein Anforderungsprofil, um ein Funktionieren dieses Kanals zu garantieren. Die Berichterstattung soll auf den jeweiligen Zweck zugeschnitten sein. Diese Forderung ist vor allem bei speziellen Abschlüssen für eingeschränkte Adressatengruppen relevant. So erwähnt das Komitee die Notwendigkeit, Steuerabschlüsse speziell auf die Bedürfnisse der Steuerbehörden hin zuzuschneiden. Es wird betont, daß der Adressatenkreis und der Publizitätszweck neben dem Kommunikationskanal auch die anzuwendenden Bewertungsmethoden und die weiteren Erstellungsmodalitäten beeinflussen. Wenn in einem Abschluß für unterschiedliche Adressatengruppen spezielle Informationen gegeben werden, so sind diese besonders zu kennzeichnen. Die Präsentation der Daten soll eine Interpretation der unterliegenden ökonomischen Ereignisse ermöglichen, deswegen ist eine zu starke Aggregation zu vermeiden. Generell ist bei der Präsentation der Rechnungslegungsdaten immer darauf zu 
achten, daß keine wichtigen Zusammenhänge verschüttet werden. Darüber hinaus sind relevante Umweltinformationen anzugeben, die für das Verständnis der präsentierten Daten sinnvoll sind. Dies kann auch Begründungen der Bewertungswahl beinhalten, speziell dann, wenn von den allgemein akzeptierten Bewertungsnormen abgewichen wird. Innerhalb eines einzelnen Abschlusses sollen möglichst einheitliche Bewertungsmethoden vorgenommen werden, um die Relation der einzelnen Posten zueinander nicht zu verzerren. Über die Anforderungen an einen einzelnen Abschluß formuliert das Komitee auch noch Forderungen an die Vergleichbarkeit der Abschlüsse. Diese sollen sowohl temporal als auch zwischen unterschiedlichen Unternehmen vergleichbar gehalten werden. ${ }^{102}$

Im nächsten Abschnitt des Statements konzentriert sich das Komitee besonders auf die externen Adressaten des Rechnungswesens. Es identifiziert unterschiedliche Adressatengruppen, wie Aktionäre, Gläubiger, Kunden, Steuerbehörden, Arbeitnehmer, Regulierungsinstitutionen und Intermediäre der genannten Gruppen. Obwohl diese Gruppen unterschiedliche Interessen haben und dementsprechend zumindest teilweise unterschiedliche Berichtssysteme nachfragen, geht das Komitee von der Möglichkeit aus, konsensfähige Bewertungsprinzipien und Berichtsnormen zu generieren, die einem Großteil der externen Adressaten gerecht werden. ${ }^{103}$

Um dies zu erreichen, versucht das Komitee zunächst, das zentrale Anwendungsgebiet der Rechnungslegungsdaten für externe Adressaten zu bestimmen. Nach Ansicht der Verfasser ist dies die Vorhersage von zukünftigen ökonomischen Ereignissen. Als Prognoseziele werden der zukünftige Periodenüberschuß, die zukünftige Liquidität, die künftige Effektivität des Managements und, von der Prognose eher losgelöst, die Überwachungsmöglichkeit des Managements durch die extemen Adressaten gesehen. Die künftige Effektivität der Unternehmensleitung wirkt sich natürlich auf die anderen zu prognostizierenden Größen aus und ist als solche immer relevant. Die Vertreterfunktion des Managements (stewardship) kann ohne Rechnungslegungsdaten nicht von den unternehmensexternen Adressaten beobachtet werden, deswegen liegt hier neben der Prognose eine weitere wesentliche Aufgabe der Rechnungslegung. ${ }^{104}$ Die Anwendungsgebiete sind indes nicht überschneidungsfrei. Da die Adressaten unsicher über die Entwicklungen der Zukunft sind, werden sie regelmäßig das Verhalten des Managements in der Vergangenheit als Proxy für dessen zukünftigen Erfolg verwenden.

Aufgrund der unterschiedlichen Adressatengruppen und der ebenfalls differierenden Zielsetzungen sieht das Komitee die Ermittlung des einen „richtigen“ Wertansatzes als

\footnotetext{
102 Vgl. AAA (1966), S. 13-18.

103 Vgl. AAA (1966), S. 19-23.

104 Vgl. AAA (1966), S. 23-27.
} 
nicht möglich an. ${ }^{105}$ Speziell die gleichzeitige Veröffentlichung von historischen Kosten und Marktwerten wird jedoch für tendenziell geeignet gehalten, um die ex ante unbekannten Informationsbedürfnisse der Adressaten zu befriedigen. In diesem Zusammenhang diskutieren die Verfasser den grundsätzlichen trade-off zwischen relevance und verifiability. Aus ihren Ausführungen entsteht der Eindruck, als sei ein gewisses Mindestmaß an verifiability ein notwendiges aber nicht hinreichendes Kriterium, um einen Bewertungsmaßstab für geeignet zu erklären. Somit können hochgradig relevante Bewertungsmaßstäbe, wie zum Beispiel auf Insiderbasis prognostizierte zukünftige Erlöse grundsätzlich nicht als zulässiger Bewertungsmaßstab angesehen werden, da ihnen der notwendige Grad an verifiability abgesprochen werden muß. Das Komitee weist auch darauf hin, daß Bewertungsverfahren, die auf historischen Kosten beruhen, häufig Verzerrungen in Richtung auf einen niedrigen Gewinnausweis beinhalten. Dieser bias kann durch eine Verwendung von Marktwerten vermieden werden. Außerdem wird hinsichtlich der Quantifizierung auf die Möglichkeit der Angabe von Bandbreiten anstatt von Einzelwerten hingewiesen. ${ }^{106}$

Zusätzlich zu diesen eher allgemeinen Überlegungen entwickelt das Komitee Vorstellungen zu speziellen Rechnungslegungsbestandteilen: So fordert es eine Erfassung von schwebenden Verträgen in der Bilanz, wenn die Verträge hinreichend lang laufen und die Informationen relevant sind. Die Bewertung von Unternehmenszusammenschlüssen nach der Pooling of Interest Methode wird von den Verfassern abgelehnt, da die dann als Bewertungsgrundlage geltenden Buchwerte der alten Unternehmen keinerlei relevance besitzen. Statt dessen ist, so weit möglich, die Purchase Methode anzuwenden. Neben Marktwerten sind auch für eine Prognose relevante Umwelteinflüsse anzugeben, ferner auch geschätzte Marktwerte für neu entdeckte Rohstoffquellen. Bei der Bewertung zu Marktwerten sind grundsätzlich auch latente Steuern anzusetzen. Wenn Vermögensgegenstände abgeschrieben werden, hat das Unternehmen sich stärker als bislang an den korrespondierenden Erträgen auszurichten. Falls ein Vermögensgegenstand keinen zukünftigen Rückfluß mehr erwarten läßt, soll er aus der Bilanz erfolgswirksam ausscheiden. Immaterielle Vermögensgegenstände, die zukünftigen Nutzen versprechen und den Ansatzkriterien entsprechen, sind in der Bilanz zu erfassen. ${ }^{107}$

Die besondere Bedeutung des ASOBAT liegt weniger in der expliziten Formulierung von einzelnen Rechnungslegungsempfehlungen, sondern vielmehr in der Neuausrichtung der Rechnungslegungsforschung. Die grundsätzliche Ausrichtung der Rechnungslegungstheo-

\footnotetext{
105 Diese amerikanische Wertdiskussion wurde auch im deutschen Sprachraum reflektiert und weiterentwickelt. Vgl. beispielhaft und m.w.N. Coenenberg (1968) und Moxter (1966).

106 Vgl. AAA (1966), S. 27-30.

107 Vgl. AAA (1966), S. 32-36.
} 
rie an den Entscheidungsproblemen der Adressaten öffnete der Forschung eine methodische Grundlage, auf die in der Zukunft aufgebaut werden sollte. ${ }^{108}$

\subsubsection{Statement on Accounting Theory and Theory Acceptance}

Im Anschluß an die Veröffentlichung des ASOBAT versuchte sich zunächst das Accounting Principles Board (APB) an der Umsetzung der neuen Denkweise in einem Grundsatzstandard. Zu der Zeit befand sich das APB sehr stark in der öffentlichen Diskussion und so bestand die Hoffnung, mit dem neuen Standard das bisherige rule-making auf ein stabiles theoretisches Fundament zu stellen. Jedoch wurde im wesentlichen in dem veröffentlichten APB Statement No. 4 nur bisherige Praxis mit den Ergebnissen von ASOBAT vermischt, so daß eine wirkliche theoretische Neukonzeption nicht stattfand. ${ }^{109}$ Das vom AICPA eingesetzte Trueblood Committee hatte die Aufgabe, basierend auf APB Statement No. 4, die Ziele der Rechnungslegung umfassend zu definieren. ${ }^{110}$ Es entwickelte einen Zielkatalog mit 12 Punkten, die das Zielproblem der Rechnungslegung aus Adressatensicht recht umfassend schildem. Leider ist aus dem Zielsystem keine operativ normative Erstellungempfehlung für Rechtsnormen ableitbar ${ }^{111}$.

Die angloamerikanische bilanztheoretische Forschung stand somit Anfang der 70er Jahre vor vielen ungelösten Problemen. ${ }^{112}$ Trotz zahlreicher Versuche war es nicht gelungen, das Rechnungswesen auf eine theoretische Ebene zu stellen, welche die Deduktion von normativ gewünschten Rechnungslegungsstandards erlaubte. So beauftragte das Executive Committee der AAA eine Gruppe von anerkannten Forschern im Bereich des Rechnungswesens, einen Bericht über den momentanen Stand der Rechnungswesenstheorie und über deren Akzeptanz zu erstellen: das "Statement on Accounting Theory and Theory Acceptance"(SATTA).

Dieses aus 9 Hochschullehrem bestehende Komitee gliedert zunächst die bisherige Literatur zur Accounting Theory und identifiziert dabei zwei Strömungsrichtungen des Decision-Usefulness-Approach: Die Decision-Model-Orientation und die Decision-MakerOrientation. ${ }^{113}$ Die Decision-Model-Orientation fußt auf den Entwicklungen des ASOBAT

\footnotetext{
108 Vgl. als Ansatz Crandall (1969), der sich explizit auf ASOBAT bezicht, jedoch bemängelt, daß die Informationsökonomie nicht in der Lage ist, operationale Handlungsanweisungen für die Entwicklung von Rechnungslegungsregeln zu liefern.

109 Vgl. APB Statement No. 4 und Wolk/Francis/Tearny (1991), S. 153-155.

110 Vgl. zu den Ergebnissen dieses Committees den Supplementband zum Volume 12 des Journal of Accounting Research, insb. Sorter/Gans (1974).

111 Vgl. Miller (1974) und Cyert/Ijiri (1974).

112 Vgl. Wheeler (1970); Hakansson (1969) und Dyckman/'Zeff (1984), S. 226-233.

113 Vgl. AAA (1977), S. 10.
} 
und versucht, Modelle über das informationsabhängige Entscheiden der Rechnungslegungsadressaten zu generieren, um mit deren Hilfe die unterschiedlichen Rechnungslegungskonzepte nach dem Adressatennutzen zu beurteilen. Diese Entscheidungsmodelle hängen entweder vom beobachteten Ertragswert des Engagements oder von einer getrennten Rendite/Risiko Betrachtung ab. Dies führte häufig zu einer Betonung von Cash-FlowWerten im Bereich der Rechnungslegung ${ }^{114}$. Chambers lehnt die Orientierung der Entscheidung an geschätzten zukünftigen Zahlungsströmen grundsätzlich ab und hält eine Orientierung an aktuell realisierbaren Veräußerungserlösen für angeraten, da nur diese relevant und objektivierbar seien. ${ }^{115}$ Die Decision-Maker-Orientation versucht im Gegensatz, das Entscheidungsverhalten der Rechnungslegungsadressaten empirisch zu ergründen. Dies kann entweder auf Individual- oder Marktebene geschehen. ${ }^{116}$

Das Komitee trennt sich von den vier Anforderungskategorien an zu berichtende Sachverhalte, wie sie von der ASOBAT-Studie dargestellt wurden. Sie identifizieren nur die zwei Charakteristika Relevance und Reliability, wobei letzteres die ASOBAT Anforderungen Freedom of bias, verifiability und quantification im wesentlichen umfaßt. Es wird explizit klargestellt, daß sowohl ein gewisses Minimum an Reliability als auch ausreichende Relevance jeweils für sich gesehen ein notwendiges, aber nicht hinreichendes Kriterium darstellen. Im Gegensatz zu ASOBAT betonen die Verfasser zusätzlich die Bedeutung der Zeitnähe. Außerdem wird die explizite Abwägung von Informationskosten und -nutzen gefordert. $^{117}$

Die aus dem Grundsatz der Decision Usefulness abgeleiteten Leitsätze zur Erstellung von Rechnungslegungs- und Publizitätsregeln bleiben so allgemein, daß eine Operationalisierung nur sehr beschränkt möglich erscheint. Wann ist ein Wertansatz relevant? Es ist wohl davon auszugehen, daß Relevanz keine binäre Eigenschaft ist. Welcher Wertansatz ist also „am relevantesten“? Diese Fragen sind schon für einen Adressaten schwer zu beantworten, wenn Rechnungslegungsdaten publiziert werden, werden sie wohl unlösbar. Auch die Verläßlichkeit von Informationen ist kein intersubjektiv zu beobachtender Fakt. Demzufolge erstaunt es nicht, daß die verwendeten Modelle zur Ermittlung der Zieleignung von Rechnungslegungsdaten sehr allgemein und oberflächlich bleiben. Für den weiteren Erkenntnisfortschritt war die genauere Analyse der Rezeption von Rechnungslegungsdaten und deren Wirkungsmechanismen im Entscheidungsprozeß unerlässlich. Dies

\footnotetext{
114 Vgl. als Beispiel Revsine (1973), insb. S. 86-136; zu weiteren Vertretern vgl. AAA (1977), S. 13 f.

115 Vgl. Chambers (1966), insb. S. 220-266.

116 Vgl. AAA (1977), S. 17-21; zu den Ergebnissen der empirischen Rechnungslegungsforschung vgl. auch die Ausführungen des folgenden Unterkapitels.

117 Vgl. AAA (1977), S. 16 f.
} 
bewirkte die verstärkte Hinwendung der Forscher zur empirischen Analyse des Untersuchungsobjektes. ${ }^{118}$ Diese Untersuchungen werden im folgenden vorgestellt.

\subsubsection{Empirische Forschungen zur Entscheidungsrelevanz}

\subsubsection{Untersuchungen auf Individualebene}

Nicht zuletzt durch die unbefriedigenden Ergebnisse der modellorientierten normativen Theoriebildung veranlaßt, fand in den 70er Jahren eine Besinnung auf die Verwendung von empirisch fundierten Methoden als Grundlage zur induktiven Theoriebildung statt. So entstand das Behavioral Accounting Research, dessen Ziel es ist, mit sozialwissenschaftlichen Methoden zu untersuchen, wie Wirtschaftssubjekte Rechnungsweseninformationen aufnehmen, auswerten und zur Entscheidungsfindung nutzen. Auf der Basis dieser Ergebnisse sollen Wirkungsmodelle entwickelt werden, die es dann auch ermöglichen, normative Leitlinien für die Entwicklung von Standards zu formulieren. ${ }^{119}$

Die unterschiedlichen Ausprägungen des Forschungsgebiets lassen sich nach den Fragestellungen differenzieren: ${ }^{120}$ Ein erstes Untersuchungsgebiet besteht in der Beobachtung der von den Adressaten vorgenommenen Analysetätigkeiten. Sind diese weitgehend bekannt, so können daraus Anhaltspunkte für Publizitätsvorschriften gewonnen werden, die den Adressatenbedürfnissen entsprechen. ${ }^{121}$ Ein anderes Verfahren besteht in Expertenbefragungen, bei denen ausgewählte Adressaten unterschiedliche Publizitätsbestandteile bzw. Bewertungskonzepte hinsichtlich ihrer Informationsrelevanz beurteilen. ${ }^{122}$ Dies muß nicht nur als Befragung sondem kann auch als Laborexperiment geschehen. ${ }^{123}$ Schließlich werden auch Adressaten direkt nach Ihren Präferenzen hinsichtlich unterschiedlicher Bewertungsalternativen oder nach der subjektiven Wichtigkeit bestimmter Unternehmensdaten befragt. ${ }^{124}$ Auch wenn die Untersuchungen auf Individualebene interessante neue Erkenntnisse über die Informationsinteressen der Rechnungslegungsadressaten boten, so scheitert auch die Umsetzung ihrer Ergebnisse in normative Theorien zur Wei-

118 Vgl. zu diesem Prozeß auch Dyckman/Zeff (1984); für einen Überblick über deutsche Ansätze vgl. auch Ballwieser (1996).

119 Vgl. als einen der Auslöser Devine (1960).

120) Vgl. zu der hier vorgenommen Unterteilung AAA (1977), S. 18-19, Dyckman/Gibbins/Swieringa (1978).

121 Vgl. als frühen Vertreter dieser Richtung Horngren (1955); jüngst Hunton/McEwan (1997) und McEwan/Hunton (1999).

122 Vgl. als Beispiel Baker/Haslem (1973) und Abdel-Kahalik (1973).

${ }^{123}$ Für die Durchführung als Laborexperiment vgl. Falk/Ophir (1973); grundsätzlich zu unterschiedlichen Untersuchungstypen im Bereich der behavioral accounting research auch Rhode (1972) und Dyckman/Gibbins/Swieringa (1978); zu den durchgeführten Laborexperimenten in den 70er Jahren Swieringa/Weick (1982).

124 So z.B. bei Copeland/Francia/Strawser (1973). 
terentwicklung der Rechnungslegung letztendlich an der Unmöglichkeit, von den Einzelbeobachtungen zu einer nicht ohne weiteres falsifizierbaren Theorie für die Adressatengesamtheit zu gelangen. ${ }^{125}$ Dieses Problem läßt sich mit auf Marktebene ansetzenden empirischen Untersuchungen umschiffen. ${ }^{126}$

\subsubsection{Untersuchungen auf Marktebene}

Ergebnisse der finanzierungstheoretischen Forschung über die Effizienz der Kapitalmärkte ermöglichten eine Neuorientierung der empirisch geprägten Rechnungslegungsforschung. Fama charakterisiert drei Formen der Kapitalmarkteffizienz. In der schwachen Form werden sämtliche historischen Preise der Kapitalmarkttitel von den Marktteilnehmern in der Kursbildung verarbeitet. Preise auf einem Markt mit halbstrenger Effizienz reflektieren alle öffentlich verfügbaren Informationen und ein streng effizienter Kapitalmarkt beinhaltet sämtliche Informationen, also auch jene, die nur privat verfügbar sind. ${ }^{127}$ Die Annahme, daß der US-amerikanische Kapitalmarkt halbstreng ist, versuchen Fama et al. durch eine Untersuchung von Aktienkursreaktionen auf die Ankündigung von Aktiensplitts zu belegen. ${ }^{128}$ Die These der halbstrengen Kapitalmarkteffizienz ist logischerweise nicht verifizierbar, sondern lediglich faslifizierbar, indem eine öffentlich verfügbare Information aufgezeigt wird, die nicht durch den Markt abgebildet wird. Der Begriff „Information“ ist jedoch nicht zweifelsfrei bestimmbar. Im Rahmen der Kapitalmarktforschung wird der Informationsbegriff im Umkehrschluß durch eine Kapitalmarktreaktion gemessen. So ergibt sich die Definition: Eine Information liegt vor, wenn der Kapitalmarkt statistisch abgesichert mit einer abnormalen Rendite reagiert. So gesehen verkommt die These der halbstrengen Kapitalmarkteffizienz zur Tautologie. ${ }^{129}$

Da zahlreiche Arbeiten eine halbstrenge Kapitalmarkteffizienz zumindest des USamerikanischen Kapitalmarkts vermuten ließen und lassen, ${ }^{130}$ kann das $\mathrm{Ma}$ der abnormalen Rendite als Anzeichen für den Nutzen einer Rechnungslegungsinformation verstanden werden. Eine abnormale Rendite ist die Differenz zwischen der beobachteten

125 Vgl. zur wissenschaftstheoretisch fundierten Modellbildung grundlegend Popper (1989).

120 Vgl. zu dieser Entwicklung auch Gonedes (1972), Gonedes/Dopuch (1974), S. 50.

127 Vgl. Fama (1970), S. 388 und erweiternd weiterhin Fama (1991).

128 Vgl. Fama et al. (1969); kritisch zur Vorgehensweise Schildbach (1986), S. 37 f.

129 Vgl. ähnlich Barker (1998), S. 3; Merton (1987); weiterhin modelltheoretisch kritisch Grossman/Stiglitz (1980); zur These der Kapitalmarkteffizienz und zu sie betreffenden Irtümem und Mißverständnissen Schmidt (1976), S. 375-417; Schneider (1993), S. 1429; Schildbach (1986), S. 33-45. Ein Anatz zur modelltheoretischen Erklärung der Informationseffizienz des Kapitalmarkts liefern Gonedes/Dopuch (1974); Gonedes (1975), der auch auf Basis dieses Modells keine Begründung für Rechnungslegungsnormen sieht.

130 Vgl. als Überblick Fama (1991). Vgl. zur Kapitalmarkteffizienz auf dem deutschen Markt Schmidt (1982); Möller (1985). 
Rendite eines Kapitalmarkttitels und der durch ein allgemeines Marktmodell prognostizierten Rendite. ${ }^{131}$ Wenn solche abnormalen Renditen bei Unternehmen zeitgleich zu der Veröffentlichung bestimmter Rechnungslegungsinformationen entstehen, kann statistisch getestet werden, mit welcher Fehlerwahrscheinlichkeit die abnormale Rendite durch die Rechnungslegungsinformation induziert wurde. Dazu muß jedoch die jeweilige Tendenz der abnormalen Rendite prognostizierbar sein. Somit kommt auch die empirische Kapitalmarktforschung nicht ohne Annahmen hinsichtlich des Verhaltensmodells der Marktteilnehmer aus. ${ }^{132}$ Gonedes/Dopuch zeigen weiterhin, daß eine abnormale Rendite auf einem halbstrengen Kapitalmarkt nicht als Beweis dafür gelten kann, daß die sie induzierende Rechnungslegungsinformation aus gesamtwirtschaftlicher Sicht gewünscht ist, ${ }^{133}$ sondem lediglich, daß einer Gruppe von Marktteilnehmem Nutzen stiftet. Dies resultiert aus der Annahme, daß die Produktion der Information nicht kostenfrei ist und daß Freerider nicht vom Konsum der Information ausgeschlossen werden können. ${ }^{134}$

Somit eignet sich die kapitalmarktorientierte Rechnungslegungsforschung zwar zum Testen, ob Rechnungslegungsinformationen Entscheidungsrelevanz besitzen, sie ist jedoch kein Mittel, um zweifelsfrei superiore Rechnungslegungsnormen und vor allem Publizitätsnormen zu identifizieren. Dies gilt um so mehr, da es nicht ohne weiteres (also ohne Regulierungsumgestaltung zur Einräumung von Wahlrechten etc.) möglich ist, alternative Rechnungslegungsnormen hinsichtlich des Grades ihrer Entscheidungsrelevanz zu klassifizieren.

\subsubsection{Test der Eignung von Rechnungslegungsdaten zu Prognosezwecken}

Neben den Untersuchungen, ob Rechnungslegungsdaten direkt Marktreaktionen induzieren und der individuellen Befragung von Rechnungslegungsadressaten kann auch getestet werden, inwieweit sich Daten der Rechnungslegung zur Abschätzung künftiger Sachverhalte, wie Aktienkursentwicklungen, Dividendenzahlungen und Insolvenzen, eignen. Außerdem können auch zukünftige Jahresabschlußdaten, wie der künftige Periodenerfolg prognostiziert werden. Da die Vertreter der modellbildenden entscheidungsorientierten Bilanztheorie übereinstimmend der Ansicht waren, daß das vorrangige Entscheidungs-

131 Vgl. Ball/Brown (1968), S. 160-164.

132 Vgl. zu weiteren Kapitalmarktstudien Gonedes (1973); Gonedes/Dopuch (1974), S. 80-103; die Übersicht von Perlitz (1973), S. 18-45; Kaplan (1978); Lev/Ohlson (1982), insb. S. 258-266; Haller (1994), S. 177-186; White/Sondhi/Fried (1997), S. 215-251. Zu Kapitalmarktstudien für den deutschen Markt Kleine-Doepke (1981); Brandi (1977); Berndsen (1978; 1979); Coenenberg et al. (1978); Möller (1986), S. 1; Coenenberg (1997), 790 f.; Coenenberg/Federspieler (1999); Pellens/Tomaszewski (1999); Möller/Keller (1999). Allgemein zur Kurswirkungen von Ereignissen am deutschen Aktienmarkt umfassend Röder (1999); zu Problemen bei der Ereignisidentifikation Bhattacharya et al. (2000).

133 Vgl. Gonedes/Dopuch (1974) und mit ähnlichem Ergebnis Beaver/Demski (1974), S. $175 \mathrm{f}$

134 Vgl. Gonedes/Dopuch (1974), S. 78 f.; zusammenfassend und vertiefend Lev/Ohlson (1982), S. 292-294. 
problem der Publizitätsadressaten in der Prognose zukünftiger Ereignisse liegt ${ }^{135}$, ist davon auszugehen, daß ein Rechnungslegungssystem mit hoher Prognosetauglichkeit von ihnen gegenüber einem mit geringer Prognosetauglichkeit bevorzugt werden dürfte. ${ }^{136}$ Insofern stellt dieser Ansatz die Verbindung von individueller und marktweiter Analyse dar: Auf der Hypothese der Relevanz von Marktdaten für individuelle Entscheidungen fußend, wird untersucht, ob Rechnungslegungsdaten in der Lage sind, diese Informationen zu geben.

In Abhängigkeit von unterschiedlichen Adressatentypen sind spezielle Prognoseverfahren geeignet. Für die Prognosebedürfnisse von Fremdkapitalgebern, deren Hauptrisiko in der Illiquidität des Schuldnerunternehmens besteht, zeigte Beaver, daß sich durch die Diskriminanzanalyse auf Basis von Jahresabschlußdaten signifikante Prognoseverbesserungen erzielen lassen. ${ }^{137}$ Das Entscheidungsproblem von Fremdkapitalgebern ist also im wesentlichen von einer Binärvariablen bestimmt, wenn von der Möglichkeit einer von null differierenden Konkursquote abgesehen wird. Durch diese besondere Entscheidungssituation wird die Möglichkeit der Prognose wesentlich erleichtert. ${ }^{138}$

Aus Sicht der Eigenkapitalgeber versuchen Beaver/Dukes aus drei unterschiedlichen Gewinnkonzeptionen diejenige zu ermitteln, die den größten Erklärungsgehalt für Aktienkurse enthält. ${ }^{139}$ Dafür bildeten sie Abnormal Price Indices für zwei unterschiedliche Portfolios. Ein Portfolio bestand aus Unternehmen, deren veröffentlichtes Ergebnis höher war als das prognostizierte, das andere Portfolio bestand aus Unternehmen mit einem der jeweiligen Konzeption entsprechenden Ergebnis unter dem Prognosewert. Die beiden Portfolios wurden dann eine gewisse Zeit gehalten. Beaver/Dukes ermittelten, daß eine Gewinnkonzeption mit Periodenabgrenzung einer reinen Cash-Flow-Konzeption überlegen sei, da sich auf Basis dieser Ergebnisdefinition höhere abnormale Renditen erwirtschaften ließen. ${ }^{140}$ Problematisch an diesem Vorgehen ist einerseits die Prognose des Ver-

135 Vgl. AAA (1966), S. 19.

136 Vgl. Beaver/Kennely/Voss (1968); Belkaoui (1992), S. $138 \mathrm{f}$.

137 Vgl. Beaver (1966); Altman (1968); einführend zur Methodik Werkheim (1998). Barth/Beaver/Landsman (1998) untersuchen weiterhin, wie der Aussagegehalt vom Buchwert des Eigenkapitals und der Gewinngröße für den Marktwert des Eigenkapitals sich bei Verschlechterung der finanziellen Situation verändern. Vgl. zu unterschiedlichen Methoden der empirischen Insolvenzforschung und zur kritischen Diskussion ihrer Ergebnisse Laitinen/Kankaanpää (1999).

138 Vgl. zur empirisch basierten Insolvenzprognose auf Basis von Jahresabschlußdaten in Deutschland grundlegend Perlitz (1973); Beermann (1976), insb. S. 29-126; Gebhardt (1980), insb. S. 48-97; sowie in jüngerer Zeit Krause (1993); Baetge et al. (1994); Dittmar/Hilbert (1998); Anders/Szczesny (1998) und unter Einbeziehung von qualitativen Informationen Blochwitz/Eigermann (2000). Grundsätzlich zu der Verwendung von neuronalen Netzen in der Rechnungslegungsforschung Trigueiros/Taffler (1996); zum generellen Erklärungsgehalt von Bilanzkennzahlen für das Unternehmensrisiko Templin (1998) und Billings (1999).

140 Relativierend zu diesem Ergebnis z.B. Pfeiffer et al. (1998). 
gleichsgewinns für die Bildung der Portfolios und andererseits der nur sehr schwache Prognosecharakter, der durch das Halten der Portfolios zustande kommt. In einer weiteren Arbeit versuchen Brooks/Buckmaster/Dascher auf Basis linearer Regression die Prognose von Aktienkursentwicklungen durch die Verwendung von unterschiedlichen Gewinngrößen. Sie stellen keine signifikanten Unterschiede zwischen den verwendeten Gewinnkonzeptionen fest. ${ }^{141}$ Dechow hingegen bestätigt die Hypothese, daß der Jahresüberschuß besser als der Cash Flow der gewöhnlichen Geschäftstätigkeit geeignet ist, die Aktienrendite zu erklären. ${ }^{142}$ Dieses Ergebnis scheint sich durch einige ähnliche Untersuchungen für unterschiedliche Rechnungslegungssysteme zu bestätigen. ${ }^{143}$ Im Laufe der weiteren Forschungsentwicklungen wurden zahlreiche Modelle mit dem Ziel entwickelt, den Erklärungsgehalt von Rechnungslegungsdaten für Aktienkurse und Aktienkursentwicklungen festzustellen. ${ }^{144}$ Dabei wurden auch andere erklärende Variablen als Ergebnis- und Eigenkapitalgrößen eingesetzt ${ }^{145}$ und spezielle Rechnungslegungsdaten in ihrer Wirkung gesondert betrachtet. ${ }^{140}$

Auch wenn ein signifikanter Zusammenhang zwischen Rechnungslegungsdaten und Aktienkursen bzw. Renditen wohl als hinreichend gesichert gelten kann, sind die Versuche, aus Rechnungslegungsinformationen Aktienkurse zu prognostizieren, im großen und ganzen als gescheitert zu beurteilten. Da künftige Aktienkurse eine Hauptdeterminante im Entscheidungsfeld der aktuellen und potentiellen Aktionäre, einer der Adressatengruppen der Unternehmenspublizität, darstellen, wirft dies ein eher negatives Licht auf

$1+1$ Vgl. Brooks/Buckmaster/Dascher (1975).

142 Dies gilt vor allem, wenn der Berichtszeitraum kurz, das unternehmenstypische Produktionsintervall lang und der Liquiditäts- und Finanzbedarf des Unternehmens stark volatil ist. Vgl. Dechow (1994).

143 Vgl. beispielhaft Ou/Penman (1989); Holthausen/Larcker (1992); Harris/Lang/Möller (1994); Cheng/Liu/Schaefer (1996); Sloan (1996); als Überblick über weitere Studien White/Sondhi/Fried (1997), S. 42 f.; Charitou (1997), insb. S. 631 f.; sowie in jüngster Zeit Charitou/Panagiotides (1999), mit einer Übersicht über frühere Studien auf S. 287-291 und Dechow/Kothari/Watts (1998).

144 Vgl. für deutsche Unternehmen Pellens (1989); zur Hypothesenbildung die unterschiedlichen auf Edwards/Bell (1961) zurückgehenden Bewertungsmodelle von Ohlson (1995); Feltham/Ohlson (1995, 1999); Ohlson/Zhang (1998); Barth/Beaver/Landsman (1998); Collins/Pincus/Xie (1999); Morel (1999), im Überblick auch White/Sondhi/Fried (1997), S. 249 f.; Kothari/Zimmerman (1995) und für frühere Ansätze Atiase/Tse (1986).

145 Vgl. z.B. Ou (1990); Ohlson/Penman (1992); Lev/Thiagarajan (1993); Lamont (1998); zum Vergleich von financial und nonfinancial Information für die Branche der Telekommunikation Amir/Lev (1996); Shevlin (1996); für Pensionsverbindlichkeiten Davis-Friday et al. (1999); zur Publikation der Kundenbindung Ittner/Larcker (1998); zur Beziehung von qualitativen und quantitativen Informationen in der Luftfahrtindustrie Behn/Riley (1999) und zu den unterschiedlichen Komponenten des Comprehensive Income Concepts des FASB Dhaliwal/Subramanyam/Trezevant (1999).

146 Vgl. beispielhaft jüngst für neubewertete Vermögensgegenstände Barth/Clinch (1998); speziell auch für immaterielle Aboody/Lev (1998); für die Wirkung von Goodwillbilanzierung in Deutschland Krämling (1998), insb. S. 153-246; für den Fair-Value-Wertansatz bei Finanzinstrumenten Simko (1999) und zum Vergleich von IAS und USGAAP auf der Basis von Überleitungsrechnungen Harris/Muller (1999). 
die Entscheidungsrelevanz der bisherigen Jahresabschlußgestaltung. ${ }^{147}$ Dies gilt um so mehr, als daß sich zeigen läßt, daß andere Daten, die nicht der Rechnungslegung entstammen, eine wesentliche Verbesserung der Aktienkursprognose ermöglichen. ${ }^{1+8}$

\section{Theoretische Untersuchungen als normative Entscheidungshilfe?}

\subsection{Bestandsaufnahme}

Die in den Vorabschnitten vorgestellten Untersuchungen versuchen aus unterschiedlicher Motivation und mit unterschiedlichen Methoden, sich dem praktischen Phänomen Rechnungslegungspublizität theoretisch zu nähern. Dies geschieht zunächst auf einer positiven Basis, also mit der Fragestellung: „Warum wird so bilanziert, wie bilanziert wird?" ${ }^{149}$ Ziel einer solchen Analyse ist unter anderem die Ermittlung von Theorien, die das Verhalten von an dem Rechnungslegungsprozeß beteiligten Wirtschaftssubjekten vorhersagen. Die normative Fragestellung „Wie sollte Rechnung gelegt werden?“ ist bei funktionierenden Marktmechanismen nach Auffassung der ökonomischen Theorie einzig und allein vom Markt zu beantworten. ${ }^{150}$ Insofern ist es nicht verwunderlich, daß die bilanztheoretischen Konzeptionen nicht in der Lage sind, zufriedenstellende normative Entscheidungshilfen zu präsentieren. So sind die frühen Konzeptionen zur Bewertung in wesentlichen Belangen als unwissenschaftlich zu beurteilen, da sie aus der Praxis abgeleitete Bewertungsverfahren im wesentlichen willkürlich als geeignet oder weniger geeignet beurteilen. Schon der Versuch, einen für alle Adressaten korrekten Wertansatz zu ermitteln, ist aus rein logischen Überlegungen zum Scheitem verurteilt. ${ }^{151} \mathrm{Da}$ es keinen objek-

147 Vgl. mit ähnlichen Ergebnissen Pohlmann (1981), S. 185 f. und Lev/Zarowin (1999), die weiterhin auf der Basis von Rechnungslegungsdaten-Kursregressionen behaupten, daß der Informationsgehalt der US amerikanischen Rechnungslegung in den letzten $20 \mathrm{Jahren}$ tendenziell abgenommen habe.

148 Vgl. z.B. Deng/Lev/Narin (1999), die feststellen, daß patententbezogene Informationen signifikant und mit $\mathrm{R}^{2}$-Werten im Bereich von 0,3-0,4 mit künftigen Aktienkursen korrelieren.

149 Auf diese Fragestellung wurde im bilanztheoretischen Abriß nicht eingegangen, da sie für die Entwicklung eines normativen Bewertungssystems nur tangential relevant ist. Vgl. zur positiven Accounting Theory grundlegend Watts/Zimmerman (1986; 1990).

150 Vgl. bereits Smith (1776), S. 15, der den Marktmechanismus und Eigeninteresse als das Verfahren zur Lösung von Bereitstellungsproblemen erkennt und eindrucksvoll Hayek (1989), der davor warnt, die Ergebnisse von Märkten aufgrund normativer Überlegungen verbessem zu wollen.

151 Dies zeigt die Diskussion um die objektive und subjektive Wertlehre in der Nationalökonomie des 18. und 19. Jahrhunderts. Vgl. beispielhaft Smith (1776), S. 31-32 und Ricardo (1817), S. 17-44. Als Ergebnis läßt sich festhalten, daß es keinen objektiven Wertansatz für Güter geben kann, da jedes Wirtschaftssubjekt die subjektiv empfundene Knappheit von Gütern anders bewertet und somit zu einem individuellen Wertansatz für das betrachtete Gut gelangt. 
tiven Unternehmenswert geben kann, kann auch kein objektiv korrekter Periodenerfolg ermittelt werden. ${ }^{152}$

Während sich ein Teil der bilanztheoretischen Forschung aufgrund der an den Periodenerfolg gebundenen Rechtsfolgen noch auf der Suche nach dem "wahren Periodenerfolg“ befindet, ${ }^{153}$ wechselte der Schwerpunkt vor allem der internationalen Forschung von der Erstellungsseite auf die Analyse der Anteilseignerinformationsinteressen. Dieser Ansatz ermöglichte das Verwenden empirischer Forschungsmethoden und kann wohl als eigentlicher Startpunkt der wissenschaftlich fundierten Rechnungslegungsforschung angesehen werden. Die Möglichkeit, Informationsinteressen einzelner Rechnungslegungsadressaten durch Befragung zu ermitteln, ist sicherlich hilfreich, doch kann daraus keine allgemeine Präferenz für bestimmte Verfahren abgeleitet werden. Dies folgt letztlich aus der Unmöglichkeit des interpersonellen Nutzenvergleichs. Eine Rechnungslegungsänderung ist also nur dann normativ zu fordern, wenn niemand durch sie einen Nutzenverlust erleiden würde. Dies bedingt jedoch, daß sämtliche Informationen vor Rechnungslegungsänderung auch nach Rechnungslegungsänderung vorhanden sind, und daß die Erstellung und Auswertung der Rechnungslegungsdaten kostenfrei möglich ist. ${ }^{154}$ Somit kann durch die Befragung von Adressaten nicht die „eine richtige Rechnungslegung“ ermittelt werden. Die empirische Kapitalmarktforschung ist hier im Vorteil, da sie nicht einzelne Präferenzen direkt, sondern über den Kapitalmarkt abgebildet aggregiert erfaßt. Sie beruft sich also konform zur ökonomischen Theorie auf die Bedeutung der Märkte zur Evaluation wirtschaftlichen Handelns. ${ }^{155}$ Mit ihrer Hilfe können bestehende Rechnungslegungsverfahren unter Berücksichtigung von bestimmten Preisbildungsmodellen auf ihre Marktwirkung hin überprüft werden, noch nicht angewendete Bilanzierungs- bzw. Publizitätsmethoden können jedoch naturgemäß nicht auf ihre Wirkung hin analysiert werden. Außerdem besteht auch bei kapitalmarkttechnisch entscheidungsrelevanten Informatio-

152 So bereits Rieger (1928), S. 203-210, der somit die Totalbilanz als einzig verläßliche ansieht. Vgl. zu der Wertdiskussion im Rahmen der Untemehmensbewertung beispielhaft Ballwieser (1995) sowie Bökking/Nowak (2000) und aus eher juristisch geprägter Sichtweise Ludewig/Ludewig-Husheer (1999) sowie Großfeld (2000). In jüngerer Zeit wird die Unternehmensbewertung eher zur Entwicklung subjektiver Wertansätze unter Verwendung optionspreistheoretischer Methoden weiterentwickelt, vgl. jüngst beispielhaft Tomaszewski (1999) oder aus prozessuraler Sicht analysiert, vgl. beispielhaft Rockholtz (1999).

153 Als Beispiel dafür mag die nicht verstummende Diskussion gelten, ob das deutsche Rechnungslegungssystem besser, schlechter, oder vergleichbar mit seinen internationalen Konkurrenten sei. Vgl. zusammenfassend Schildbach (1999a).

154 Vgl. als Beweis für diese Behauptung selbst für den Einpersonenfall Demski (1973); ein Versuch der gegenteiligen Behauptung unternimmt Cushing (1977), dieser geht jedoch von homogenen Bilanzadressaten aus. Vgl. erläuternd Ballwieser (1982). Letztlich geht die Argumentation von Demski auf das bekannte ArrowParadoxon zurück, vgl. dazu crläuternd Lenk/Teichmann (1999) und diskutierend Gaertner (1998).

155 Genau diese Marktorientierung wird der Forschungsrichtung jedoch fälschlicherweise auch vorgeworfen. So z.B. Thomas (1974), insb. S. 86-93, der damit ein gutes Beispiel für „Anmaßung von Wissen“ im Sinne von Hayek (1989) bietet. 
nen weiterhin das Problem des nicht sicheren gesamtwirtschaftlichen Nutzens von öffentlich verfügbarer Information. Des weiteren reduzieren die notwendigen Annahmen hinsichtlich der Renditenverteilung und des Marktmodells den Aussagegehalt der auf der halbstrengen Informationseffizienz fußenden Untersuchungen die Aussagen nicht unwesentlich. ${ }^{156}$ Deswegen ist auch die empirische Kapitalmarktforschung nicht in der Lage, als Meßlatte für neue Publizitätssysteme vor deren Implementierung zu dienen. ${ }^{157}$

Die Gesamtbetrachtung der bisherigen Forschungsergebnisse macht das Problem der normativen Bilanztheorie deutlich: Es gibt kein theoretisch valides Verfahren, welches in der Lage ist, das Entdeckungsverfahren des Marktes zu ersetzen, oder auch nur abzubilden. ${ }^{158}$ Also kann es eine befriedigende normative Theorie der Rechnungslegung im engen Sinn nicht geben. Jeglicher Versuch, eine solche Theorie zu formulieren kann wohl eher als das Votieren für bestimmte Rechnungslegungsvarianten interpretiert werden. ${ }^{159}$ Somit werden normative Veröffentlichungen zu Zeichen von einer Nachfrage nach bestimmten Änderungen des bestehenden Rechnungslegungssystems. Da die Regulierung der Rechnungslegung und Publizität, sei sie nun informationsökonomisch begründbar oder nicht, eine Lösung der normativen Probleme über den Markt unmöglich macht, stehen die Regulierer, die Ersteller, die Prüfer und die Adressaten von Rechnungslegungsinformationen vor einem Dilemma: Sie bräuchten ein Instrument, welches ihnen die Möglichkeit bietet, zwischen unterschiedlichen Methoden und sonstigen Ausprägungsmerkmalen der Rechnungslegung auszuwählen. Dieses kann jedoch ohne den Markt nicht generiert werden.

\subsection{Skizzierung eines normativen Leitsatzes der Rechnungslegungspublizitätsre- gulierung}

Als Ergebnis der theoretischen Betrachtung kann also festgehalten werden, daß der Regulierer der Rechnungslegung und Publizität kein „normatives Patentrezept“ der Forschung erwarten kann, welches ihm die richtige Regulierung der Rechnungslegung empfiehlt. Die einzige Empfehlung an ihn kann nur lauten: So viel marktwirtschaftliche Koordination wie möglich. Eine vollständige Deregulierung der Rechnungslegung und Publizität wäre zwar theoretisch denkbar, erscheint jedoch praktisch nicht gangbar. In der positiven

Vgl. Schneider (1997), S. 366-376 und Schildbach (1986), S. 33-43.

157 Nichtsdestotrotz wird sie von Regulierem in Ermangelung besserer Alternativen als Entscheidungsgrundlage genutzt. Vgl. als Beispiel für die SEC Pownall/Schipper (1999).

158 Vgl. mit ähnlichem Ergebnis bereits Ballwieser (1982).

159 Vgl. hierzu Watts/Zimmerman (1986), S. 338-436; die normativ geprägte Rechnungslegungsforschung als Versuch werten, bestimmte von der Praxis nachgefragte Rechnungslegungsmethoden via Argumentation zu akzeptierten Verfahren zu machen; ähnlich auch Ballwieser (1997), insb. S. 391. 
Theorie der Regulierung wird Regulierung als Gut verstanden, welches von privaten Wirtschaftssubjekten nachgefragt und daraufhin vom Staat, bzw. ihm untergeordneten Instanzen mit delegiertem Machtmonopol, angeboten wird. Dieser Nachfrage nach Regulierung kann auch eine Nachfrage nach Deregulierung entgegen gestellt werden, allerdings ist dabei zu beachten, daß häufig Regulierungen im Rahmen des rent seekings von relativ gut organisierten kleinen Gruppen nachgefragt werden, die einen großen Nutzen davon haben. Die korrespondierende Nutzeneinbusse der anderen Wirtschaftssubjekte verteilt sich auf eine große, schlecht organisierte Gruppe, im Extremfall auf die allgemeine Öffentlichkeit. Somit kann erwartet werden, daß in diesem Fall die Nachfrage nach Deregulierung geringer ausfallen wird, als die nach Regulierung. ${ }^{160}$ Im speziellen Fall der Regulierung der Rechnungslegung ist jedoch die unterschiedliche Marktmacht der Regulierungsnachfrager und der von der Regulierung negativ betroffenen Wirtschaftssubjekte nicht sonderlich stark ausgeprägt, es kann sogar nicht zweifelsfrei interpretiert werden, welche der von der Regulierung tangierten Parteien zu welcher Gruppe gehört. Es ist jedenfalls nicht zu beobachten, daß eine komplette Deregulierung von den beteiligten Wirtschaftssubjekten vehement nachgefragt wird. ${ }^{161}$ Daraus wird hier gefolgert, daß eine grundsätzliche Deregulierung der Rechnungslegung hier nicht zur Disposition steht.

Es geht also um eine möglichst effiziente Ausgestaltung der Regulierung von Rechnungslegung und Publizität. ${ }^{162}$ Dies kann nicht geschehen, indem Marktergebnisse vorweggenommen werden, sondern vielmehr durch die Etablierung möglichst vieler Marktprozesse im Rechnungslegungs- und Publizitätsprozeß. Eine - zugegeben stark operationalisierungsbedürftige - normative These zur Regulierung der Rechnungslegung und Publizität lautet also:

„Um eine marktwirtschaftlich wünschenswerte Rechnungslegungspublizität zu er-
balten, gestalte die Regulierung der Rechnungslegung und Publizität so, daß, wo
immer möglich, partielle Marktprozesse etabliert werden, in denen Nachfrager von

160 Vgl. zur positiven Theorie der Regulierung grundlegend Stigler (1971; 1975); Posner (1974) und Peltzman (1976); einführend Fülbier (1999); zusammenfassend und diskutierend Priest (1993).

101 Zwar kann das von deutschen Konzemen nachgefragte und auch durchgesetzte KapAEG als eine Nachfrage nach Lockerung der Rechnungslegungsnormen interpretiert werden, eine Nachfrage nach genereller Liberalisierung der Rechnungslegung und Publizität kann daraus jedoch nicht geschlossen werden; vgl. auch Pellens (1999), S. $529 \mathrm{f}$.

162 Dieser Weg der Abkehr von der normativen Fragestellung der Auswahl aus unterschiedlichen Rechnungslegungsalternativen, hin zu einer regulierungsprozeßorientierten Sichtweise, die versucht, den Regulierungsprozeß so zu gestalten, daß möglichst effiziente Ergebnisse entstehen, wird momentan von einem Großteil der Rechnungslegungsliteratur und Praxis gewählt. Vgl. als modelltheoretischen Vorläufer Demski (1974); für Deutschland Pellens/Gassen (1998); Fülbier/Gassen (1999) und für die US-amerikanische Literatur die Diskussion in Accounting Horizons, Vol. 12, z.B. Jonas/Young (1998); Levitt (1998); Kaplan/Fender (1998); Knutson/Napolitano (1998); Reither (1998); Rogero (1998) und Smith (1998). 
Unternehmensinformationen und Anbieter derselben obne Intervention des Regulie-

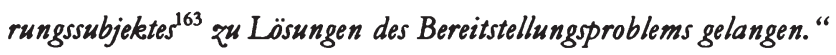

Diese Wirkungsthese fußt auf dem definitorischen Zusammenhang, daß ein effizienter Markt zu effizienten Allokationswirkungen führt. Fraglich ist, welche strukturellen Maßnahmen das Regulierungssubjekt ergreifen kann, um effiziente Marktprozesse zu ermöglichen und wie diese zu identifizieren sind. Die Effizienz eines Marktes wird teilweise sehr unterschiedlich definiert. ${ }^{164}$ Hier soll nicht auf eine bestimmte Effizienzdefinition zurückgegriffen werden. Vielmehr wird der allgemeinere Term „funktionsfähig“ in einer evolutorischen Sichtweise verwendet. Ein Markt soll als funktionsfähig angesehen werden, wenn er ohne ordnungspolitische Intervention Transaktionen realisiert und wenn im Zeitablauf Änderungen der gehandelten Verfügungsrechtsbündel, der Marktteilnehmer oder der Transaktionskonditionen zu beobachten sind. Um mögliche Märkte zu erkennen und zur Untersuchung des Problems, welche intendierten und systembedingten Markteintrittsbarrieren oder sonstige Hemmnisse vorliegen, soll die Transaktionskostentheorie verwendet werden. ${ }^{165}$ Sie bietet ein Instrumentarium, um Schnittstellen zu erkennen, an denen Wirtschaftssubjekte Verfügungsrechte transferieren (können) und an denen somit marktwirtschaftliche Koordination entstehen kann. Im folgenden wird ein operationales Verfahren vorgestellt, wie der Rechnungslegungs- und Publizitätsprozeß nach solchen technologisch separierbaren Schnittstellen durchsucht werden kann, die an ihrem Übergang die Implementierung von Marktprozessen möglich machen könnten. Je nach Konzeption der Regulierung lassen sich an diesen Schnittstellen dann unterschiedliche Rahmenbedingungen hinsichtlich Häufigkeit der Transaktion, Spezifität der Transaktion und deren Sicherheit identifizieren. Diese ermöglichen in ihrer Kombination dann eher marktnahe oder marktferne Kontraktlösungen. ${ }^{166}$ Aufgabe des Regulierungssubjekts ist es dann, an den identifizierten Schnittstellen Rahmenbedingungen zu schaffen, die eher marktnahe Kontrakte ermöglichen.

163 Vgl. zur Definition von Regulierungssubjekt, Regulierungsobjekt und Instrument der Regulierung Fülbier (1998), S. 11-18.

164 Eine der klassischen Definitionen ist die der vollständigen Konkurrenz, vgl. als Überblick Schumann (1992), S. 211-213. Deren Annahme der vollständigen Markttransparenz macht das Modell jedoch als effizienten Referenzmarkt nicht operationalisierbar.

165 Die Transaktionskostentheorie geht im wesentlichen auf zwei Autoren zurück. Coase (1937) zeigt die Bedeutung von Transaktionskosten für das Entstehen von Vertragslösungen. Williamson (1985) greift die Gedanken von Coase auf und entwickelte so eine auf Transaktionskosten basierte Vertragstheorie.

166 Vgl. zu diesem Vorgehen und zur Systematik der Transaktionsrahmenbedingungen Williamson (1985), S. 68-84. 


\subsection{Transaktionskostentheoretische Spezifizierung des Leitsatzes}

Wie der vorhergehende Abschnitt gezeigt hat, ist der hier gebildete normative Leitsatz der Rechnungslegung und Publizität weniger am Ergebnis, als vielmehr am Prozeß ausgerichtet. Deswegen wird nun der Rechnungslegungs- und Publizitätsprozeß vorgestellt. Dabei werden die technologisch separierbaren Schnittstellen innerhalb des Prozesses möglichst differenziert identifiziert. Wenn die Unternehmensinformationen über diese Schnittstellen transformiert werden, entstehen Transaktionskosten. ${ }^{167}$ Diese Transaktionskosten beeinflussen die Wahl der Vertragskonzeption, die den Austausch des Gutes über die Schnittstelle koordiniert. Je nach Ausprägung der oben erwähnten Rahmenbedingungen erscheinen unterschiedliche Vertragslösungen vorteilhaft, wie in Abb. 1 ersichtlich. $\mathrm{Zu}-$ sätzlich zu den dort skizzierten Eigenschaften einer Transaktion ist jeweils noch der Grad der Unsicherheit zu berücksichtigen.

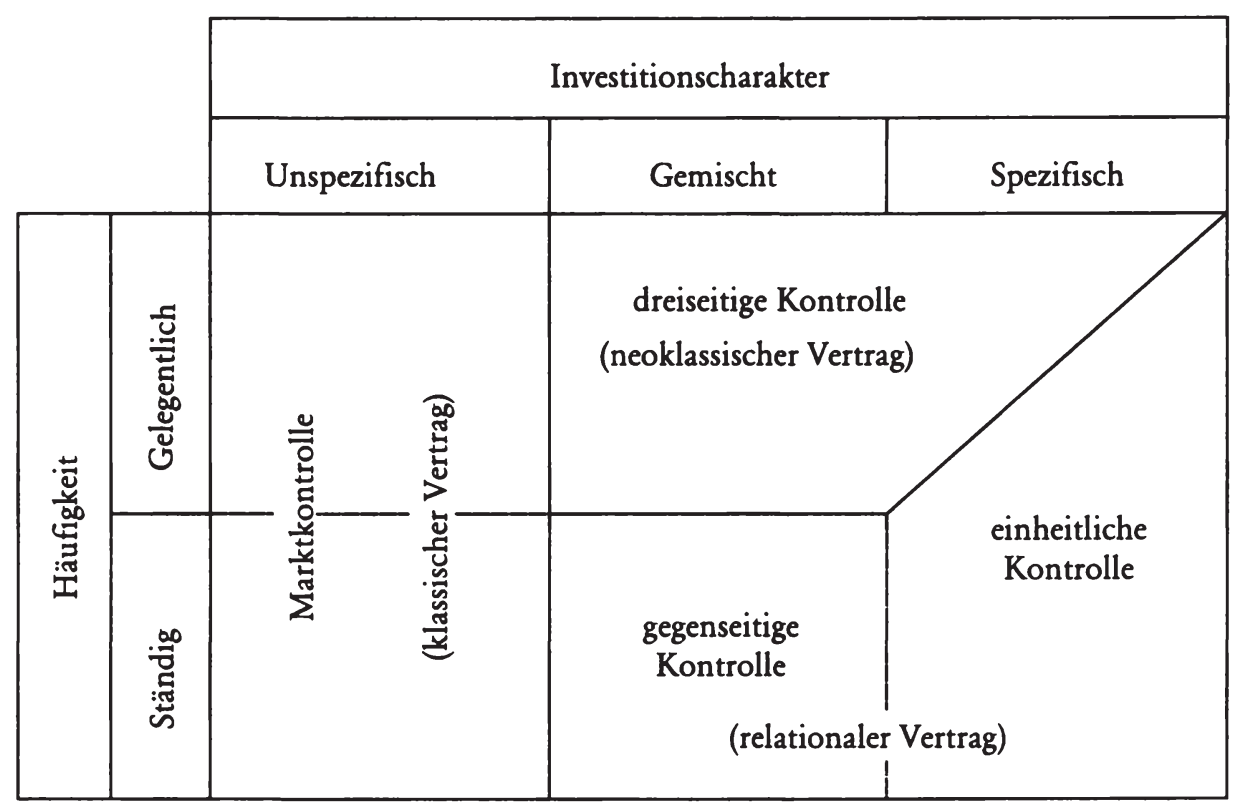

Abb. 1: Vertragsformen bei Transaktionen

Quelle: Übersetzt nach Williamson (1985), S. 79.

Ein im Sinne der oben skizzierten Funktionsfähigkeit gewünschtes Ergebnis ist wohl am ehesten bei vorliegender Marktkontrolle zu erwarten, also wenn die Investitionen, die für die Transferierung über die Schnittstelle benötigt werden, unspezifisch sind. Im Extremfall der einheitlichen Kontrolle ist der Transfer komplett in eine Institution verlegt. Das

167 Vgl. Williamson (1985), S. 1. 
daraus resultierende Ergebnis kann nur durch Zufall mit dem eines funktionsfähigen Marktes übereinstimmen. An den Schnittstellen, an denen ex ante unabhängige Wirtschaftssubjekte Verträge schließen, können Regulierungen etabliert werden, welche die Rahmenbedingungen der Transaktionen verändern. Aus Sicht der Vertragstheorie sind die Rahmenbedingungen so zu verändern, daß die Wirtschaftssubjekte eher in der Lage sind, marktnahe Vertragskonstellationen zu wählen. Hierbei ist jedoch zu berücksichtigen, daß durch die Regulierung den Wirtschaftssubjekten ein Teil ihrer ökonomischen Dispositionsfreiheit geraubt wird. So erscheint es wenig sinnvoll, Jahresabschlußerstellern eine rigorose Feingliederung des Lageberichts vorzuschreiben, um den Grad der spezifischen Investition zu verringem, den die Adressaten für das Verständnis desselben aufbringen müssen. In diesem Fall dürfte die ökonomische Nutzeinbuße durch den starren Rahmen den Vorteil der leichteren Verständlichkeit wohl überkompensieren.

In Abb. 2 wird die Unternehmens-, Adressaten- und Marktsphäre des Rechnungslegungspublizitätsprozesses dargestellt. Die hier identifizierten Prozeßschritte sind durch Schnittstellen miteinander verbunden. An diesen Schnittstellen wird eine Tätigkeit vorgenommen, um das Gut Unternehmensinformation für den nächsten Prozeßschritt vorzubereiten. So werden die Transaktionsdaten an einer Schnittstelle durch Aggregation für den nächsten Arbeitsschritt zusammengefaßt. Für jede Schnittstelle lassen sich die entsprechenden Rahmenbedingungen der Transaktion angeben. So läßt sich für ein bestehendes Rechnungslegungs- und Publizitätssystem angeben, inwieweit es an den identifizierten Schnittstellen marktnahe Transaktionen zuläßt. Die erste Schnittstelle zwischen der Umwelt (also der Gesamtheit der in diesem Schaubild exogen erfaßter Wirtschaftssubjekte) und der Transaktionsdatenbank beinhaltet die Aufgabe, die für die berichtende Einheit relevanten Informationen zu identifizieren. Unter einer Transaktionsdatenbank wird in diesem Zusammenhang die originäre mengenmäßige Datenerfassung des Unternehmens verstanden. $\mathrm{Da}$ hierfür detaillierte Kenntnisse über die Unternehmensinterna notwendig sind, muß die ausführende Institution hochgradig spezifische Investitionen tätigen, um diese Aufgabe erfüllen zu können. Auch wenn die Tätigkeit häufig wiederkehrend ist, so ist die für diese Transaktion vorherrschende Kontraktform entsprechend Abb. 1 die der einheitlichen Kontrolle. Ähnliche Betrachtungen lassen sich für alle Prozeßschritte der einzelnen Rechnungslegungspublizitätssysteme anstellen. 


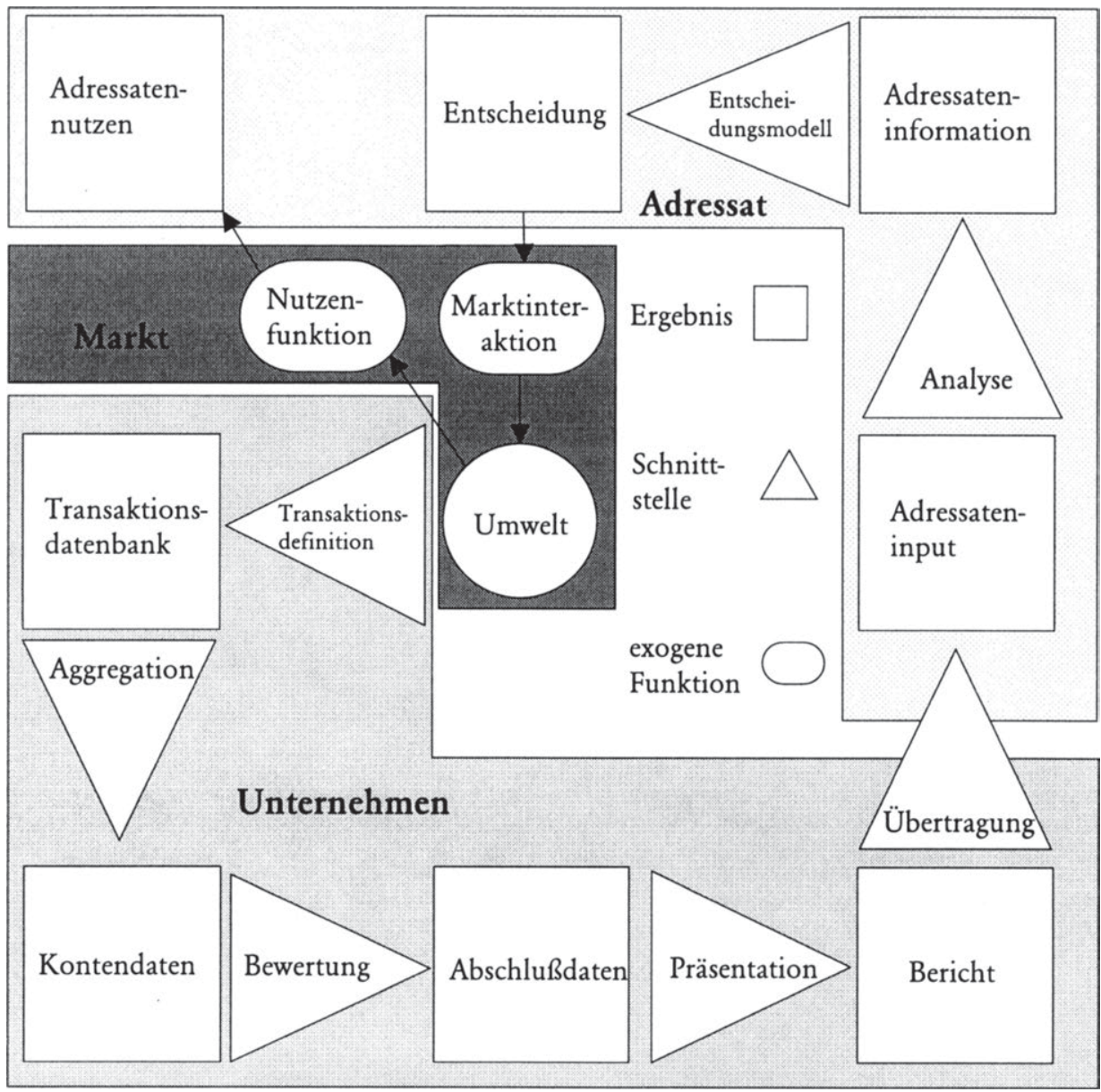

Abb. 2: Rechnungslegungspublizitätsprozeß

Quelle: In Anlehnung an Crandall (1969), S. 459. ${ }^{168}$

Mittels dieser Betrachtungsweise werden unterschiedliche Rechnungslegungspublizitätssysteme hinsichtlich ihrer Verwendung von Marktmechanismen vergleichbar und anhand des oben formulierten normativen Leitsatzes auch bewertbar. Der Leitsatz läßt sich dann transaktionstheoretisch weiter operationalisiert wie folgt formulieren:

„Ein Rechnungslegungs- und Publizitätssystem ist um so eher marktwirtschaftlich wünschenswert, desto eher dem klassischen Ideal nahe Vertragslösungen ${ }^{169}$ an den

168 Ähnliche Abbildungen finden sich auch bei Gebhardt (1980), S. 24 und Pellens (1989), S. 57.

169 Vgl. zu den unterschiedlichen Vertragsdefinitionen Macneil (1978), zu der klassischen insbesondere S. 856865. 
im Rechnungslegungs- und Publizitätsprozeß identifizierten technologisch separierbaren Schnittstellen unter Berücksichtigung der korrespondierenden Organisationskosten realisiert werden können."

\section{Kapitelfazit}

Das Ziel dieses Kapitels war die Ermittlung eines Beurteilungsrahmens für die Qualität unterschiedlicher Rechnungslegungspublizitätssysteme. Als Ergebnis bleibt festzuhalten, $\mathrm{da}$ es nicht möglich erscheint, das Ergebnis eines Publizitätsprozesses, also die eigentliche Unternehmensinformation, zweifelsfrei zu bewerten, um so zu beurteilen, welches Berichtssystem für die Informationsbedürfnisse eines ex ante unbekannten Adressaten am geeignetsten erscheint. Nichtsdestotrotz besteht die faktische Notwendigkeit für die Regulierer der Rechnungslegung und Publizität, aus bestehenden Alternativen für unterschiedliche Fragestellungen auszuwählen. Deswegen wird hier ein andersartiger Weg für die Bewertung von unterschiedlichen Berichtssystemen vorgeschlagen, der nicht an den Ergebnissen, also an den letztendlich regulierten Verfahren ansetzt, sondern vielmehr an den Methoden, wie diese Verfahren generiert werden.

Zentraler Gedanke ist dabei die alte Erkenntnis, daß in einer Marktwirtschaft der Markt als superiores Koordinationsinstrument wenn immer möglich eingesetzt werden soll. Dies erfordert teilweise die Änderung bestehender Infrastrukturen, um Transaktionen, die bislang nicht von Marktprozessen koordiniert werden, an diese heranzuführen. Eine wünschenswerte Regulierung der Rechnungslegung und Publizität läßt, wenn immer möglich, die Probleme der Unternehmensberichterstattung von den letztendlich Betroffenen über die simplen Gesetze von Angebot und Nachfrage lösen.

Im weiteren ist also zu untersuchen, inwieweit es unterschiedliche Grade der Marktinvolvierung bei bestehenden Publizitäts- und Rechnungslegungssystemen gibt. Dies soll in den nun folgenden Kapiteln III und IV geschehen. Im Anschluß daran wird der Versuch unternommen, ein alternatives System zu skizzieren, das durch Ausnutzung neuer Informationstechnologien in der Lage ist, zusätzliche Marktmechanismen zu implementieren. 


\section{Kapitel III}

\section{Ausgestaltung der Rechnungslegungspublizität}

\section{im internationalen Vergleich}

In diesem Kapitel wird das US-amerikanische und das deutsche Rechnungslegungspublizitätssystem vorgestellt und aus positiver Sicht analysiert, indem zunächst die historische Entwicklung der Regulierungen aufgezeigt wird. Dazu werden einerseits die gesellschaftsrechtlichen Vorschriften analysiert mit einer schwerpunktmäßigen Betrachtung der Rechtsform der Aktiengesellschaft, respektive der Corporation. Andererseits wird auf die kapitalmarktrechtlichen Vorschriften eingegangen, wobei zu betonen ist, daß diese strikte Trennung nicht immer durchzuhalten ist.' ${ }^{1}$ Um erste Anhaltspunkte für die Gründe der jeweiligen Ausgestaltung zu erhalten, werden diese Ausführungen anhand der historisch vorherrschenden wirtschaftspolitischen Leitbilder strukturiert. Anschließend werden die resultierenden Normen mittels der positiven Regulierungstheorie analysiert, um die beobachteten Systeme ökonomisch erklären zu können. Ziel des Kapitels ist es, ungeachtet der im zweiten Kapitels entworfenen Konzeption der Rechnungslegungspublizität, Anhaltspunkte für die positive Erklärung der realiter herrschenden Normen zu geben.

\section{Historische Entwicklung der Publizitätsregulierung}

\subsection{Vorgehensweise}

Zur Strukturierung der historischen Entwicklung wird auf die von der wirtschaftsgeschichtlichen Forschung identifizierten wirtschaftspolitischen Leitbilder zurückgegriffen, die sich ebenso wie die zu beschreibenden Regulierungen im Zeitablauf entwickelt haben. Diese Leitbilder basieren in wesentlichen Bestandteilen auf Entwicklungen der mikroökonomischen Theorie. Zwar wird in dieser Arbeit nicht die Ansicht vertreten, daß sich politische Entscheider aus altruistischen Motiven gemäß einer normativen Theorie verhalten, andererseits soll nicht ausgeschlossen werden, daß der politische Druck zumindest partiell so gut funktioniert, daß Politiker die beste Lösung darin sehen, sich gemäß der von der Forschung gerade favorisierten normativen Leitbilder zu verhalten, um so

1 Vgl. Fülbier (1998), S. 33-37 und allgemein zur zunehmenden Verschmelzung von unterschiedlichen Rechtsgebieten auch über nationale Grenzen hinweg Großfeld (1997). 
ihre Handlungen intersubjektiv legitimieren zu können. Andererseits lassen sich auch normative Leitbilder positiv als von der Wirtschaftspolitik nachgefragte Rechtfertigungsschemata interpretieren. Auch aus dieser Sichtweise macht eine Referenzierung von wirtschaftspolitischen Leitbildem in der politischen Analyse Sinn.

Die hier gewählte Einteilung der wirtschaftspolitischen Epochen ist nicht frei von einer gewissen Willkür. Auch die Zuordnung von Zeitphasen zu diesen Epochen divergiert zwischen den unterschiedlichen nationalen Umfeldern. Nichtsdestotrotz wurde dieser vereinfachende Gliederungsansatz gewählt, da er die Argumentationsrichtung der Ausführungen am besten strukturiert.

\subsection{Merkantilismus}

Das erste eventuell für die Entwicklung der Rechnungslegungspublizität relevante wirtschaftspolitisches Leitbild ${ }^{2}$ ist das Konzept des Merkantilismusses, welches zur Zeit der Entwicklung des Ordonnance de Commerce in Frankreich vorherrschend war. ${ }^{3}$ Nach der schriftlichen Fixierung von Buchführungsregeln in den italienischen Stadtstaaten übernahm die französische Rechtsetzung diese Vorschriften konzeptionell in den Ordonnance de Commerce von $1673^{4}$, der bereits in Titel III, Artikel VIII eine Inventarpflicht enthält, deren Veröffentlichung jedoch durch Artikel IX deutlich eingeschränkt wird. Die französische Weiterentwicklung, der Code de Commerce von 1807, fungierte als eines der bedeutenden Vorbilder für das deutsche Gesellschaftsrecht des 19. Jahrhunderts. ${ }^{5}$ Der Merkantilismus ist von einer protektionistischen und dirigistischen Grundtendenz geprägt. Dies wird auch in den Vorschriften zur Rechnungslegung deutlich. Das Hauptmotiv für die Institutionalisierung dieser Vorschriften lag in zahlreichen Konkursen ${ }^{6}$, deren Zustandekommen teilweise auf dolose Handlungen der Eigentümer zurückgeführt waren und die nicht im Sinne der zentralistischen Leitungsinstanz Frankreichs waren. Somit kann argumentiert werden, daß die Vorschriften zur Rechnungslegungspublizität im Rahmen des normativen Trends der Zeit lagen.

2 Die hier verwendeten Leitbilder werden auch häufig als wettbewerbspolitische Leitbilder bezeichnet. Um nicht schon a priori nicht markwirtschaftliche Konzeptionen wie das Konzept der sozialistischen Planwirtschaft auszuschließen, wird hier der etwas weitere Begriff des wirtschaftspolitischen Leitbildes gebraucht.

3 Vgl. einführend zum Prinzip des Kameralismusses Schmidt (1994), insb. S. 38-48.

4 Vgl. zu der italienischen Buchhaltungstechnik Penndorf (1930); zur Entstehungsgeschichte der Buchhaltung, Rechnungslegung und Publizität in Deutschland Penndorf (1913); Vehn (1929); Schön (1997) überblicksweise Schneider (1993a). Speziell zum Ordonnance de Commerce sind die Ausführungen von Savary (1675) grundlegend.

5 Vgl. Barth (1953), S. 65-71.

6 Vgl. Schmalenbach (1962), S. 18. 
Der Ursprung der deutschen gesellschaftsrechtlichen Rechnungslegung liegt im preußischen Allgemeinen Landrecht (ALR) von 1794, welches seinerseits wiederum vom Ordonnance de Commerce geprägt war. $\iint$ 642-646 ALR beinhalteten Vorschriften für die Rechnungslegung von Handelsgesellschaften. Ein wesentlicher Bestandteil war die Festsetzung der Bewertungshöchstgrenze für Rohstoffe und Handelswaren. Diese waren zum niedrigeren Wert der beiden Alternativen Anschaffungskosten und erzielbaren Veräußerungswert anzusetzen. Verloren Waren durch Lagerung an Wert, so war dieser Verlust durch Abschreibungen zu erfassen. Auch der Wertverlust bei zweifelhaften und uneinbringlichen Forderungen mußte in der Rechnungslegung wiedergegeben werden. Diese Bewertungsvorschriften waren jedoch nur anzuwenden, wenn im Gesellschaftervertrag keine bestimmten Anweisungen zur Rechnungslegung enthalten waren. Es ging den Verfassern des ALR also weniger um eine einheitliche Gestaltung der Gesellschafterpublizität von Handelsgesellschaften, sondern vielmehr um die Vermeidung von Streitfällen, falls im Gesellschaftervertrag keine Vorschriften für die Bilanzerstellung enthalten waren. Somit können diese Vorschriften nicht so eindeutig einem merkantilistischen wirtschaftspolitischen Konzept zugeordnet werden, wie die französischen Vorbilder.

Die US-amerikanischen Normen zur Rechnungslegungspublizität entwickelten sich in einem vom kontinentaleuropäischen Rechtssystem ${ }^{7}$ fundamental unterschiedlichen Umfeld. Die Wurzeln des US-amerikanischen Rechtssystems liegen in England. Obwohl England über 500 Jahre von einer römischen Besatzermacht kontrolliert wurde, hat das römische Rechtssystem keinen Einzug gehalten. Nach der römischen Epoche war das Rechtssystem angelsächsisch geprägt. Durch die Eroberung Englands durch die Normannen 1066 wurde dieses Stammesrecht von dem normannischen Recht verdrängt. Aus diesem entwickelte sich das Common Law System, welches England im Zuge seiner imperialistischen Aktivitäten in der Neuzeit in die halbe Welt exportierte. ${ }^{8}$

Auch die amerikanischen Einwanderer nahmen im wesentlichen ihr heimatliches Rechtssystem mit in das neue Land. Von einigen Ausnahmen abgesehen', führte dies in den Großteil der Bundesstaaten zur Übernahme des britischen Common Law Systems. Die Entscheidungsfindung bei Konfliktfällen obliegt dem jeweiligen Richter, der bei seiner Urteilsfindung auf vergleichbare Gerichtsentscheidungen zurückzugreifen hat. Von diesen kann er nur abweichen, wenn deren Anwendung durch Änderung der Rahmenbedingungen des neuen Falls nicht akzeptabel erscheint. Somit wird nicht ausgehend von grund-

7 Vgl. zum gemeinsamen Ursprung des europäischen Rechtssystems Coing (1968).

8 Vgl. zur Rechtsgeschichte Englands und zur Entwicklung des common law sytems David/Grasmann (1988), Tz. 284-298.

? So besitzen Louisiana und die ehemaligen spanischen Kolonien ein eher kontinental europäisch geprägtes Rechtssystem, vgl. Haller (1994), S. 13. 
sätzlichen Gesetzen deduktiv entschieden, vielmehr wird aus Einzelfallregeln induktiv eine Entscheidung gesucht. Diese Vorgehensweise behindert das Oktroyieren von wirtschaftspolitischen Konzepten durch die Legislative auf die Judikative. ${ }^{10}$

Zusätzlich zu der Common Law-Konzeption führt auch die Organisation der USA als Bund unabhängiger Einzelstaaten dazu, daß in der US-amerikanischen Rechtsgeschichte der Rechnungslegungspublizität staatliche Einflußnahme im 18. und 19. Jahrhundert nur schwer auszumachen ist. Lediglich die durch die Constitution an den Staat delegierten Befugnisse, wie z.B. das Steuer- und Währungswesen sowie der innerstaatliche Handel werden durch bundesrechtliche Institutionen geregelt. ${ }^{11} \mathrm{Da}$ somit der judikative Zuständigkeitsbereich des Bundes eher gering ist, besitzen die Einzelfallregelungen auf nationalstaatlichem Niveau eher geringe Relevanz. Hier sind vor allem die vom Congress erlassenen Gesetze Entscheidungsgrundlage der Bundesgerichte. Die jeweiligen Bundesstaaten hingegen haben ihr eigenes individuelles System von Einzelentscheidungen (Case Law System). Für den Fall, daß bei einem zu entscheidenden Fall kein Präzedenzfall auffindbar ist, ist es gängige Praxis, auf Urteile anderer Bundesstaaten zurückzugreifen. Somit entwickeln sich die einzelnen Case Law Systeme zwar grundsätzlich selbständig, jedoch nicht vollkommen unabhängig voneinander. Im Laufe der Zeit wurden von den USamerikanischen Einzelstaaten jedoch zusätzlich zu den zahlreichen Einzelfallentscheidungen von der Legislative zahlreiche allgemeine Gesetze erlassen, so daß heutzutage das US-amerikanische Rechtssystem eher eine Zwitterposition zwischen der reinen Common Law Philosophie englischer Prägung und dem kontinental europäisch geprägten Civil Law System einnimmt.

Festzuhalten bleibt also, daß im kontinentaleuropäischen Bereich schon im 18. und 19. Jahrhundert der staatliche Einfluß auf das Gesellschaftsrecht und damit auch auf die Rechnungslegungspublizität deutlich erkennbar ist. Entsprechend des damaligen wirtschaftspolitischen Leitbilds des Merkantilismus sind die Vorschriften von dem Geist geprägt, das untemehmerische Handeln von staatlicher Seite zu kontrollieren und unerwünschtes Verhalten zu vermeiden. Im Gegensatz dazu ist das US-amerikanische Gesellschaftsrecht subsidiär ausgestaltet und läßt zumindest historisch keine Einflüsse eines frühen wirtschaftspolitsch motivierten staatlichen Einflusses erkennen.

10 La Porta et al. (1998) sehen die unterschiedlichen Rechtssysteme als zentralen Grund für die unterschiedliche Ausgestaltung des Kapitalmarktrechts und argumentieren, daß kontinentaleuropäische Rechtssysteme generell weniger zum Individualschutz neigen.

1 Der Rest der Gesetzgebungskompetenz steht somit den Bundesstaaten zu. Vgl. zur Gesetzgebungskompetenz des Congress Const. Art. I, sec. 8. 


\subsection{Freihandel}

Die im 18. und 19. Jahrhundert entwickelte klassische Nationalökonomie bildet die Grundlage der modernen Ökonomie. ${ }^{12}$ Es ist ihr Verdienst, den positiven Einfluß des Wettbewerbs auf den gesamtwirtschaftlichen Wohlstand erkannt und formuliert zu haben. ${ }^{13}$ Somit ist zentrale Forderung des wirtschaftlichen Leitbilds der Klassik, der invisible hand $^{14}$ wo immer möglich, den nötigen Spielraum zur Entfaltung zu gewähren. Eine der konstituierenden Forderungen klassischer Wirtschaftspolitik war das Fördern des nationalen und internationalen Freihandels und in diesem Zug der Abbau von realen und indirekten Handelsbarrieren.

Die Rolle von Information und Wissen besteht in der klassischen Sichtweise hauptsächlich darin, die Wirtschaftssubjekte in die Lage zu versetzen, eigenverantwortlich ihr wirtschaftliches Geschick durch die Teilnahme an Märkten lenken zu können. Smith sieht Bildung so als ein meritorisches Gut an, welches den unterpriviligierten Bevölkerungsschichten zur Verfügung gestellt werden sollte, damit sie in der Lage sind, aktiv am Wirtschaftsgeschehen teilzunehmen. ${ }^{15}$ Generell wird die Information der Konsumenten über die Qualität der Produkte als staatliche Aufgabe gesehen, wenn die Auswahlentscheidung der Konsumenten aufgrund von Informationsmängeln verzerrt sind. ${ }^{16}$ Es liegt nahe, daß gerade für abstrakte Güter, wie Ansprüche auf Realvermögen aus Gesellschafterverhältnissen, eine recht hohe Unsicherheit der Marktteilnehmer unterstellt werden kann. Aus dieser Sicht läßt sich wohl im gewissen Rahmen eine Pflicht zur Rechnungslegungspublizität aus Sicht der klassischen Wettbewerbskonzeption rechtfertigen, solange sie zur Reduktion der Informationsasymmetrie auf Märkten beiträgt.

Diese Denkweise findet sich vor allem in den gesellschaftsrechtlichen Grundlagen des britischen und US-amerikanischen Rechts wieder. Die vorherrschende Gesellschaftsform der Vereinigten Staaten ist die Rechtsform der corporation. Sie ist in etwa mit der deutschen Aktiengesellschaft vergleichbar. ${ }^{17} \mathrm{Da}$ das Gesellschaftsrecht nicht zum Kompetenzbereich des Bundes gehört, gibt es in den Einzelstaaten unterschiedliche Rechtsausprägungen. Das jeweils anzuwendende Gesellschaftsrecht ergibt sich nicht aus dem aktuellen Firmensitz, sondern vielmehr aus dem Ort der Gründung. ${ }^{18}$ Somit ist es für Unterneh-

\footnotetext{
12 Vgl. als zwei der Hauptvertreter Smith (1776) und Ricardo (1817), einen guten Überblick gibt Schefold/Carstensen (1994).

13 Vgl. z.B. Smith (1776), S. 15.

14 Vgl. Smith (1776), S. 485.

15 Vgl. Smith (1776), S. 842.

16 Vgl. Alsmöller (1982), S. 62 f.

17 Vgl. Merkt (1991), Tz.203-216.

18 Vgl. Merkt (1991), Tz. 147-153.
} 
mensgründer mit relativ geringen Kosten verbunden, sich dem ihren Ansprüchen am besten entsprechendem Gesellschaftsrecht zu unterwerfen. Dies führt zu einer Wettbewerbssituation der unterschiedlichen einzelstaatlichen Gesellschaftsrechtskonzeptionen, ${ }^{19}$ aus dem vor allem der Staat Delaware als Marktführer herausgegangen ist. ${ }^{20}$ Die Unterschiede in den einzelnen gesellschaftsrechtlichen Ausgestaltungen veranlaßten die American Bar Association 1950 dazu, mit dem Model Business Corporation Act (M.B.C.A.) eine Standardisierungsempfehlung für das Gesellschaftsrecht zu erarbeiten. Diese wurde 1984 in einer überarbeiteten Version als Revised Model Business Corporation Act (R.M.B.C.A.) veröffentlicht. Obwohl diese Empfehlungen nur partiell Eingang in die einzelstaatliche Gesetzgebung gefunden haben und somit weiterhin wesentliche Unterschiede im US-amerikanischen Gesellschaftsrecht verbleiben, orientieren sich die folgenden Ausführungen zur gesellschaftsrechtlich fundierten Publizität im wesentlichen an den Empfehlungen des R.M.B.C.A, da sie einen gewissen gemeinsamen Nenner der gesellschaftsrechtlichen Konzeptionen der USA darstellen.

Zentraler Bestandteil der gesellschaftsrechtlichen Publizitätsregulierung der USA ist eine allgemeine Auskunftspflicht der corporation gegenüber ihren Aktionären. Diese wird im Rahmen des Common Law sehr weitläufig ausgelegt. Nach stehender Rechtssprechung hat jeder Aktionär, der ein gerechtfertigtes Interesse vorweisen kann, Anrecht auf alle Informationen, die mit der Geschäftstätigkeit der Gesellschaft direkt zu tun haben. Als gerechtfertigtes Interesse gilt z.B. schon das Bedürfnis des Aktionärs, den momentanen Wert seines Anteils an der Gesellschaft zu ermitteln. Diese weitreichende Informationspflicht wird jedoch durch die Unternehmenspraxis, den Aktionären ein derartiges Recht regelmäßig zu verwehren, stark eingeschränkt. ${ }^{21}$ In den Gesetzesvorschriften finden sich Hinweise darauf, welche Daten von den Unternehmen auf jeden Fall vorgehalten werden müssen, und welche nur nach Aufforderung des Aktionärs vorzulegen sind. ${ }^{22}$

Das Einsichtsrecht des Aktionärs impliziert auch eine Berichtspflicht des Unternehmens. So sind Unternehmen verpflichtet, Aktionärsbücher zu führen und die Gesellschafterver-

19 Vgl. Brotte (1997), S. 112; Merkt (1991), Tz. 17-26, der diesen Sachverhalt nach einem Gerichtsurteil als „race for laxity“ bezeichnet und ihn somit aus Juristensicht in einem eher negativen Iicht darstellt. Vgl. aus ökonomischer Sicht zum Wettbewerb der Regulierer Sinn (1997) und für die ähnliche Situation in der EU Sicbert/Koop (1990); Hauser (1993); Streit/Mussler (1994). Jüngst zum Regulierungswettbewerb zwischen SFC und IASC Cox (1999).

20 Vgl. Hatzis-Schoch (1992); Merkt (1991), Tz. 23 und Dodd/Leftwich (1980), die durch eine Kapitalmarktstudie aufzeigen, daß nicht, wie von Juristen angenommen, die Aktionäre unter diesem Deregulierungswettlauf leiden, sondern im Gegenteil davon eher profitieren. Aus juristischer Sicht relativierend Kamar (1998).

21 Diese Situation findet sich ähnlich im britischen Gesellschaftsrecht. Pratt/Storrar (1997) zeigen Gründe auf, welche die faktische Einschränkung des Auskunftsrechts erklären.

22 Vgl. Merkt (1991), Tz. 585 f., mit Verweisen auf entsprechende Urteile; eine exemplarische Umsetzung ist z.B. $\ 220$ Del.Gen.Corp.L.; die generelle Empfehlung findet sich in $₫ 16$ R.M.B.C.A. 
sammlungen angemessen zu protokollieren. Außerdem ist ein gewisses Mindestmaß an Buchführung und Publizität einzuhalten. ${ }^{23}$ Diese Daten können auf Antrag von den Aktionären eingesehen werden, teilweise müssen sie ihnen auch auf regelmäßiger Basis zugesendet werden. ${ }^{24}$ Die US-amerikanischen gesellschaftsrechtlichen Grundlagen der Unternehmenspublizität sind von der grundsätzlichen Konzeption und deren Auslegung durch die Urteile des Case Law sehr weitgehend. Sie bieten dem Aktionär, der ein plausibles Interesse belegen kann, grundsätzlich die Möglichkeit, nahezu unbeschränkt wirtschaftliche Informationen vom Untemehmen einzufordern. Das Management des betroffenen Unternehmens verhält sich jedoch in der Regel sehr unkooperativ, so daß die Einforderung der Informationen für Unternehmensbeteiligte mit prohibitiv hohen Transaktionskosten verbunden ist. ${ }^{25}$ Nichtsdestotrotz gewährt das US-amerikanische Gesellschaftsrecht auch vor dem Hintergrund seiner Publizitätsforderungen den beteiligten Wirtschaftssubjekten eine recht große Vertragsfreiheit und scheint somit den Ansprüchen einer klassischen Wirtschaftspolitik weitgehend zu entsprechen.

Im krassen Gegenteil dazu steht das deutsche Handelsgesetz bis 1870, das die Gründung von Aktiengesellschaften von einer staatlichen Konzession abhängig macht ${ }^{26}$ und somit die Wettbewerbsfreiheit wesentlich einschränkt. Trotz vieler Vorstoße einzelner deutscher Staaten ${ }^{27}$ wurde bis in die zweite Hälfte des 19. Jahrhunderts kein allgemeines deutsches Handelsrecht kodifiziert. So entwickelten sich die gesellschaftsrechtlichen Vorschriften zur Rechnungslegungspublizität zunächst in speziellen Vorschriften zu einzelnen Gesellschaftsformen. Das preußische Gesetz über die Aktiengesellschaften vom 9.11.1843 verlangte in $\ 2 \mathrm{Nr} .4$ die Aufnahme von Bilanzierungsregeln in den Gesellschaftsvertrag, der aus dem Jahresabschluß resultierende Überschuß wurde in $\int 17$ als Ausschüttungsobergrenze festgesetzt und der Vorstand der Aktiengesellschaft wurde in $\$ 24$ verpflichtet, geschäftsjährlich Bilanz zu ziehen und diese der preußischen Regierung mitzuteilen. Das preußische Konzessionssystem für Aktiengesellschaften gab dem Staat nach dem preußischen Gesetz für Aktiengesellschaften von 1843 i.V.m. Nr. 16 des Aktienregulativs vom 29.3.1856 die Möglichkeit, auf die Methoden der Bilanzerstellung Einfluß zu nehmen, da der Gesellschaftsvertrag, welcher nach $\ 2 \mathrm{Nr}$. 4 die Bilanzierungsgrundsätze bestimmen mußte, bei Unternehmensgründung der Konzessionsstelle zur Genehmigung vorzulegen war. Des weiteren wurde mit Nr. 16 des Aktienregulativs eine Publizitätspflicht der erstellten Bilanz kodifiziert. Die gesamten deutschen Regelungen der ersten Hälfte des 19. Jahr-

\footnotetext{
23 Vgl. Merkt (1991), Tz. 590.

24 Vgl. z.B. $\oint 624$ N.Y.Bus.Corp.L; $\int \oint 1501,2200$ Cal.Gen.Corp.L.

25 Vgl. Merkt (1991), Tz. 586.

26 Das Beschränken des Marktzuganges wird von Smith im anderen Zusammenhang für corperations kritisiert, vgl. Smith (1776), S. $136 \mathrm{f}$.

27 Vgl. Barth (1953), S. 67 f.
} 
hunderts erscheinen somit nicht vordergründig von der klassischen Idee der Selbstregulierung geprägt.

Das Allgemeine Deutsche Handelsgesetzbuch (ADHGB) von 1861 löste das preußische Aktiengesetz von $1843 \mathrm{ab}$ und enthielt weitergehende Vorschriften zur Rechnungslegung in den $\iint 27-32 \mathrm{ADHGB}$. In diesen wurde unter anderem die Pflicht zur Rechnungslegung und gewisse Bewertungsregeln erfaßt. Bei den Lesungen des Entwurfs zum ADHGB gab es Differenzen hinsichtlich der Notwendigkeit der speziellen Vorschriften zur Rechnungslegung. So forderten die Vertreter aus Hamburg und aus Kurhessen, diese ersatzlos zu streichen. ${ }^{28}$ Dieser Vorschlag fand jedoch keine Mehrheit, so daß sich im ADHGB Vorschriften zur Bilanzerstellung wiederfanden. So waren die Vermögensgegenstände zu dem ihnen zum Abschlußzeitpunkt beizulegenden Wert in der Bilanz anzusetzen. ${ }^{29}$ Im Rahmen der Weiterentwicklung des Gesellschaftsrechts wurden in der Aktienrechtsnovelle vom 11.6.1870 die Aufhebung der Konzessionspflicht für Aktiengesellschaften beschlossen. Diese Gesetzesnovellen können als ein Versuch angesehen werden, den entstehenden deutschen Einheitsstaat mit einem Gesellschaftsrecht auszustatten, das den Freihandel als konstituierendes Wirtschaftsprinzip fördern sollte.

Vor diesem Hintergrund erstaunt es nicht, daß diese Gesetzesänderungen zu einem rapiden Wachstum der Zahl der Aktiengesellschaften führten, die Anzahl vervierfachte sich in vier Jahren. ${ }^{30} \mathrm{Da}$ gleichzeitig das Kapital der Aktiengesellschaften nur unwesentlich stieg und bei den neugegründeten Gesellschaften das Kapital sogar beträchtlich schrumpfte, wurde in der Öffentlichkeit von einer Krise der Aktiengesellschaften ausgegangen, die mit der Aktienrechtsnovelle von 1884 gesetzgeberisch eingedämmt werden sollte. ${ }^{31}$ So wurden weitergehende Vorschriften erlassen, die das Bilden von nicht überlebensfähigen Aktiengesellschaften erschweren sollten. Neben strengeren Bewertungsvorschriften, als zentraler Grundsatz wurde das Niederstwertprinzip kodifiziert ${ }^{32}$, und schärferen Strafbestimmungen $^{33}$ wurde die Verpflichtung zur Erstellung einer Gewinn- und Verlustrechnung ${ }^{34}$, sowie die Pflicht zur deren Veröffentlichung zusammen mit der Bilanz binnen 6 Monate nach

28 Vgl. Schmalenbach (1956), S. 23.

29 Vgl. ADHGB, Art. 31; massive Kritik an dieser auch in $\int 40$ HGB i.d.F. vom 10.5.1897 weiterlebenden Regel übt Fischer (1909), S. 83: „Die „objektiven“ und „wabren“ Werte einer selbständigen Bewertungsmethode existieren allein in der Vorstellung der Urbeber des Art. 31 A. D. HB. und des $\int 70 \mathrm{R}$. HB. Sie mögen durchaus entschuldbar geirrt haben, aber sie haben schwer geirrt.".

30 Vgl. Barth (1953), S. 75 f. Hierzu ist anzumerken, daß auch die französischen Reparationszahlungen im Zuge des deutsch-französichen Krieges für die Gründungswelle mitverantwortlich sein dürften.

31 Vgl. Barth (1953), S. 75; Schubert (1985); Hommelhoff (1985).

32 Aktienrechtsnovelle vom 18.7.1884, Artikel 185a, Ziffer 1.

33 Aktienrechtsnovelle vom 18.7.1884, Artikel 249.

34 Aktienrechtsnovelle vom 18.7.1884, Artikel 185a, Ziffer 6 .i.V.m Artikel 185c. und Artikel 239b. 
Abschlußerstellung kodifiziert ${ }^{35}$. Insofern ist im Bereich des deutschen Gesellschaftsrechts nur ein sehr kurzer und geringer Einfluß der Ideen der klassischen Ökonomie zu erkennen.

\subsection{Dirigismus}

Teilweise als Gegenentwurf zur klassischen Nationalökonomie, teilweise als Reaktion auf beobachtete wirtschaftliche Mißstände, bildeten sich im 19. und vor allem in der ersten Hälfte des 20. Jahrhunderts wirtschaftspolitische Konzepte heraus, die das grundsätzliche Prinzip der Selbstorganisierung der marktwirtschaftlichen Kräfte mehr oder weniger stark ablehnten. ${ }^{36}$ Diesen Ansätzen ist gemein, daß sie die Staatsaufgabe im Wirtschaftssystem im Umfang deutlich größer einschätzen, als die des „Nachtwächterstaats“ klassischer Prägung. Diese Ansätze, die neben diversen zentralistischen sozialistischen Konzepten auch marktwirtschaftliche Konzepte wie den Keynsianismus und den deutschen Kathedersozialismus umfassen, sollen hier unter dem vereinfachenden Sammelbegriff „Dirigismus" in ihrer Auswirkung auf die Rechnungslegung behandelt werden.

Genau in diese Epoche fiel 1897 die Umarbeitung des ADHGB zum Handelsgesetzbuch (HGB) im Rahmen der Neugestaltung des bürgerlichen Rechts. ${ }^{37}$ Dabei blieb sein wesentlicher Charakter erhalten. Neuerungen für die gesellschaftsrechtliche Publizität gab es durch den Entwurf eines Gesetzes für Aktiengesellschaften und Kommanditgesellschaften auf Aktien des Reichsjustizministeriums von 1930, der im Rahmen der Aktienrechtsnovelle vom 19.9.1931 und dem AktG 1937 fast vollständig in gültiges Recht transformiert wurde. ${ }^{38}$ Die Grundkonzeption des Gesetzesentwurfs war vom Gedanken des Unternehmens an sich geprägt, so heißt es in der Entwurfsbegründung, daß ,das Interesse des Einzelaktionärs einerseits und das Interesse der Gesellschaft und der Allgemeinbeit anderseits auszugleichen und qu versöhnen wäre ${ }^{(39}$. Daraus schließen die Gestalter des Entwurfs, daß das Unternehmensmanagement sein Verhalten lediglich am Unternehmen auszurichten habe und nicht an dem Gewinnstreben der Aktionäre. Diese Sichtweise, die ein gewisses diffuses Allgemeinwohl über das Interesse des einzelnen stellt, ist typisch für die dirigistische

35 Aktienrechtsnovelle vom 18.7.1884, Artikel 185c.

36 Für den deutschen Sprachraum ist hier vor allem die Bedeutung der jüngeren Historischen Schule um von Schmoller und der 1872 als Socialreformverein gegründete Verein für Socialpolitik zu nennen. Vgl. einführend Rieter (1994). Für die maßgebliche Rolle des Vereins für Socialpolitik in der Aktienrechtsreform 1884 Schubert (1985), S. 2-7; allgemein zu der Entwicklung der Aktionärsschutzvorschriften des deutschen Aktienrechts Pellens (1994), S. 18-46.

37 Vgl. allgemein zur Transformation des ADHGB in das HGB Schmidt (1997); Wöhe (1999), S. 66-68.

38 Vgl. Barth (1953), S. 84.

39 Erläuternde Bemerkungen des Reichsjustizminsiteriums zum Entwurf von 1931 in Hommelhoff/Schilling (Hrsg.) (1987), S. 922. 
Grundtendenz der damaligen Zeit. Den bevormundeten Aktionären soll im Gegenzug, nach nordamerikanischen und englischem Vorbild eine gesteigerte Offenlegung der Unternehmenstätigkeit im Rahmen des Geschäftsberichts garantiert werden, wobei jedoch eine begründete Auskunftsverweigerung durch den Vorstand möglich war. ${ }^{40}$ Die Möglichkeit zur Legung von stillen Reserven wurde als für das Unternehmen wichtiges Schutzinstrument weiterhin beibehalten. Die Funktion dieses Schutzinstrumentes versteht sich vor allem vor dem Hintergrund der aktienrechtlich kodifizierten Ausschüttungsregeln. Der Verbund dieser mit der Möglichkeit des Vorstands, den Jahresüberschuß durch das Legen bzw. Heben von stillen Reserven quasi beliebig zu manipulieren, sollte de facto dem Vorstand die Möglichkeit geben, die Dividenden im Sinne des „Unternehmens an sich" festzusetzen. ${ }^{41}$ Des weiteren wurde die generelle Prüfungspflicht für die Abschlüsse von Aktiengesellschaften eingefürt. Die Gesamtheit des Entwurfs kann als deutsche Reaktion auf die Zusammenbrüche der Weltwirtschaftskrise gedeutet werden ${ }^{42}$ und wurde in einem ersten Schritt in der Aktienrechtsnovelle 1931 umgesetzt. Diese führte u.a. Gliederungsvorschriften für Bilanz und Gewinn- und Verlustrechnung, sowie zahlreiche weitere Angabepflichten ein. Durch diese Novelle findet sich auch erstmals die Generalnorm der Rechnungslegung, daß der Jahresabschluß so aufzustellen sei, daß er einen möglichst sicheren Einblick in die Lage der Gesellschaft gewähre. ${ }^{43}$ Diese Norm war jedoch von untergeordneter Bedeutung und konnte sich gegen den Geist des HGB 1931, welches künstliche Unterbewertung explizit förderte, nicht durchsetzen. ${ }^{44}$ Das folgende separate Aktiengesetz (AktG) von 1937 brachte wenig Veränderungen für die Rechnungslegungspublizität. Nicht zuletzt deswegen war es in der Literatur recht umstritten. ${ }^{45}$

Neben den Änderungen des Gesellschaftsrechts führte die dirigistische Grundtendenz der Legislative auch zu einer Erhöhung der Regulierungsdichte im deutschen Kapitalmarktrecht. Die bereits erwähnte Gründungswelle durch die Aufgabe des Konzessionsrechts für Aktiengesellschaften im Rahmen der Aktienrechtsnovelle von 18.11.1870, in deren Zug auch viele Scheingründungen vorgenommen wurden, führte zu einer anschlieBenden Konkurswelle, verbunden mit erheblichen Verlusten von Kleinanlegern. Dies war einer der Gründe für das Entstehen des Börsengesetzes (BörsG) von 1897. Ein weiterer Grund war das Bestreben der Vertreter des Agrarsektors, den Terminhandel mit ihren

\footnotetext{
40 Vgl. Barth (1953), S. 85.

41 Vgl. Herbig/Geßler/Hefermehl (1937), insb. S. 188.

42 Vgl. Barth (1953), S. 84, der speziell auf den Fall der Frankfurter Allgemeinen Versicherungs-AG hinweist; Gessler (1965), S. 146.

43 Vgl. \260b Abs. 2 HGB 1931.

4 Vgl. Gessler (1965), S. 146 f.

45 Vgl. Gessler (1965), S. 151, Schmalenbach (1962), S. 41; einführend zum Inhalt des AktG 1937 Quassowski (1937).
} 
Produkten zu unterbinden, da sie glaubten, dieser sei ihrer Marktposition abträglich. Diese Gründe, zusammen mit einer dem Börsenhandel sehr kritisch gegenüberstehenden Legislative und Judikative führten zu einem Börsengesetz, das den Börsenhandel eher behinderte, als förderte. ${ }^{46}$ So enthielt das BörsG 1897 sehr unklare Vorschriften zur Börsenordnung, die nicht einmal regelte, wer diese Ordnung erlassen sollte und ob sie einen öffentlichen oder einen privaten Rechtscharakter hatte. Außer der Emissionspublizität im Rahmen eines Prospekts ${ }^{47}$ wurde von den Unternehmen keine über die gesellschaftsrechtlichen Publizitätspflichten hinausgehenden Informationen gefordert. Lediglich ausländische Unternehmen hatten nach $₫ 39$ (2) BörsG 1897 im fünfjährigen Abstand eine Bilanz zu veröffentlichen. Die Vorschriften für den Terminhandel verboten den Handel für bestimmte Produktbereiche, darunter auch Getreide und Mühlenprodukte. Außerdem verlangte das BörsG von allen Teilnehmem am Terminmarkt, daß sie sich in ein Börsenregister eintragen lassen, bevor sie am Terminmarkt teilnehmen können. Diese Vorschriften führten zu einem starkem Rückgang des Börsenhandels, insbesondere im Terminhandel, der quasi vollkommen zusammenbrach.

Der Gesetzgeber versuchte, diese Probleme 1908 durch eine Reform des BörsG in den Griff zu kriegen, da vor allem die große volkswirtschaftliche Bedeutung des Terminhandels erkannt worden war. Das Börsenregister entfiel, ebenso wie die unglückliche Termingeschäftsdefinition, welche durch spezielle Vertragsgestaltungen zu umgehen war. ${ }^{48}$ An den Publizitätspflichten wurde jedoch nichts geändert. In einer Börsengesetzreform von 1934 wurde die Ermittlung des amtlichen Kurses allein den staatlich anerkannten Börsenmaklern zugestanden, um die Kursfindung unparteiisch zu halten. Sämtliche Änderungen an dem BörsG vor dem zweiten Weltkrieg änderten einzelne Ausgestaltungsschwächen des BörsG, ohne jedoch die grundsätzlich börsenkritische Ausrichtung des Gesetzes zu korrigieren.

Im Gegensatz zu den deutschen Vorschriften sind in den USA dirigistische Tendenzen vornehmlich im Kapitalmarktrecht zu erkennen. Auch in den USA entstanden aufgrund von zahlreichen betrügerischen Handlungen an Kapitalmärkten in fast allen Bundesstaaten gesetzliche Regelungen zum Kapitalmarkthandel. ${ }^{49}$ Ziel dieser war nicht primär der Schutz von Anlegern durch mehr Publizität, da den Anlegern selbst bei ausreichendem Informationsstand die Fähigkeit abgesprochen wurde, betrügerische Handlungen zu er-

46 Vgl. Mues (1997), S. 15. Vgl. ausfühlich zur Geschichte des BörsG 1896 die Monographien Meier (1992) und Schulz (1994).

47 Vgl. § 38 BörsG 1897; vgl. zum Inhalt Weber (1897), S. 237.

48 Vgl. Mues (1997), S. 20-22.

$49 \mathrm{Zu}$ beachten ist allerdings, daß neben den einzelstaatlichen Vorschriften auch die Börsen selbstverwaltend tätig wurden. Vgl. Mahoney (1997), S. 1457-1464. 
kennen. Es ging also darum, diejenigen Kapitalnachfrager zu stoppen, die den Kapitalanbietern das „blaue vom Himmel“ versprechen, diese Versprechungen jedoch nicht einhalten. So erklärt sich auch die Bezeichnung „blue sky laws“, die sich für diese Gruppe von Einzelstaatengesetze eingebürgert hat. ${ }^{50}$ Zentraler Fokus der Regulierungen ist nicht der Sekundär-, sondern der Primärhandel. Es existiert ein Standardisierungsversuch zu den einzelstaatlichen Regelungen, der Uniform Securities Act (Unif.S.A.) von 1956, der jedoch nur von etwa 36 Bundesstaaten in lokales Recht übernommen wurde. ${ }^{51}$ Dieser beinhaltet vor allem drei unterschiedliche Ansatzpunkte für Vorschriften zum innerstaatlichen Kapitalmarkthandel. Zum einen wird der Aufsichtsbehörde die Möglichkeit eingeräumt, bei Verdacht auf betrügerische Handlung beim An- oder Verkauf von Wertpapieren geeignete Untersuchungen einzuleiten und ggf. Warnungen zu veröffentlichen. Ferner wird von allen Händlern, die an der Emission oder dem Verkauf von Wertpapieren beteiligt sind, eine Registrierung bei dem betreffenden Bundesstaat verlangt. Schließlich müssen alle $\mathrm{zu}$ handelnden Papiere registriert werden. ${ }^{52}$

Die an sich sehr weitreichenden einzelstaatlichen Regulierungsmöglichkeiten für den Kapitalmarkt haben trotz der erwähnten Offenlegungspflichten nicht primär die Reduktion der Informationsasymmetrie als Ziel. Vielmehr scheint die Regulierungsmotivation zu sein, „schlechten“ Untemehmen den Zugang zum Kapitalmarkt zu verwehren. Die Grenze zwischen guten und schlechten Unternehmen ist hierbei jedoch schwer zu ziehen. Es besteht die Gefahr, daß risikoreiche Unternehmen und Entrepreneure tendenziell eher vom Kapitalmarktzugang ausgeschlossen werden. Betrügerisches Verhalten kann durch die einzelstaatlichen Gesetze auch nicht ausgeschlossen werden. Dies und die zahlreichen Ausnahmebereiche der bestehenden Einzelstaatenregelungen ${ }^{53}$ haben neben der überragenden Bedeutung des Wertpapierhandels auf Bundesebene dazu geführt, daß die bundesstaatlichen Regelungen, welche im folgenden vorgestellt werden, die einzelstaatlichen Vorschriften in ihrer Bedeutung bei weitem übertreffen.

Als Hauptgrund für die US-amerikanische Kodifizierung des Kapitalmarktrechts auf Bundesebene wird der Börsencrash Ende der 20er Jahre dieses Jahrhunderts gesehen. Dessen Eintreten erschütterte das Vertrauen in die Koordinationseffizienz der Eigenkapitalmärkte zutiefst. Als möglicher Grund wurde die aufgrund mangelnder Information beschränkte Möglichkeit der Marktteilnehmer eruiert, sich ein Bild über die Qualität der

\footnotetext{
so Vgl. Brotte (1997), S. 116.

51 Vgl. Merkt (1991), Tz. 450; der auch darauf verweist, daß der Revised Unif.S.A. von 1985 erst von wenigen Staten übernommen wurde.

52 Vgl. Merkt (1991), Tz. 451.

53 Vgl. Brotte (1997), S. 120.
} 
gehandelten Wertpapiere zu machen. ${ }^{54}$ Um dies in Zukunft sicherzustellen und um den Marktzugang für unseriöse und betrügerische Risikokapitalnachfrager einzudämmen, wurde die Securities and Exchange Commission (SEC) 1934 gegründet. ${ }^{55}$ Ihre Aufgaben wurden durch den Securities Act (SA) von 1933 und den Securities Exchange Act (SEA) von 1934 geregelt. ${ }^{56} \mathrm{Zu}$ dieser Zeit versuchte der neu ins Amt gewählte Präsident Roosevelt, durch die „New Deal“-Gesetzgebung die Vereinigten Staaten nach durchlittener Weltwirtschaftskrise ökonomisch wieder in Schwung zu bringen. Die regulatorischen Eingriffe in den Wertpapiermarkt waren ein Teil dieses Versuchs.

Der SA koordiniert den interstaatlichen Primärmarkt. Sämtliche Wertpapieremittenten ${ }^{57}$ haben vor der Zulassung zum Handel der SEC Informationen in einem sogenannten registration statement zukommen zu lassen. Dieses enthält unter anderem zahlreiche Informationen zu sämtlichen an der Emission beteiligten Personen, zu dem Bereich der Geschäftstätigkeit, zu der Höhe des durch die Emission zu erzielenden Eigenkapitals, zu dem geplanten Ausgabekurs der Aktien, zu den geschätzten Kosten der Emission, sowie ein Jahresabschluß, der höchstens 90 Tage alt sein darf. ${ }^{58}$ Nach sec. 11 SA haften Emittent und Emissionsbank gesamtschuldnerisch für eventuelle Fehlinformationen. Die Registrierung bei der SEC erfolgt automatisch 20 Tage nach dem Einreichen der geforderten Unterlagen bei der SEC, solange diese keinen Einspruch wegen Unvollständigkeit oder sonstiger Mängel der Unterlagen und somit die Registrierung aufschiebt. ${ }^{59}$

Der SEA hingegen regelt den Sekundärhandel mit bereits entsprechend dem SA registrierten Wertpapieren, die an den nationalen Börsen der USA gehandelt werden. ${ }^{60}$ Zentraler Bestandteil ist die Verpflichtung der gelisteten Unternehmen zur regelmäßigen und fallweisen Unternehmenspublizität. Die regelmäßige Publizität umfaßt den Jahresabschluß auf Formular 10-K (für ausländische Unternehmen Formular 20-F) und die Quartalsberichterstattung, die dem Formular 10-Q entsprechend durchgeführt werden soll. Die

54 Vgl. Skousen (1991), S. 47; Mahoney (1997), S. 14641475.

55 Vgl. einführend zur SEC Fitzgerald/Carnall (1999); Skousen (1991).

56 Neben diesen beiden Gesetzen, die den Kem der US-amerikanischen Kapitalmarktpublizität ausmachen, gibt es noch einige weitere, die Spezialbereiche regeln. Vgl. zur Übersicht Brotte (1997), S. $121 \mathrm{f}$.

57 Davon ausgeschlossen sind nur nicht-öffentliche Emissionen, Fremdkapitaltitel mit weniger als neun Monaten Laufzeit und Wertpapiere spezieller Institutionen, wie z.B. der USA und untergeordneten Gebietskörperschaften, der Bundesbank oder ihrer Ableger und gemeinnützigen Organisationen, vgl. sec. 3 und $4 \mathrm{SA}$.

58 Vgl. sec. 7 SA und Schedule A SA sowie die detailliertere Regelung in Reg. S-K, $\$ 222.501-512$ und die entsprechenden S und F Forms der SEC. Seit 1982 besteht das Integrated Disclosure System der SEC, welches Primär- und Sekundärpublizität in weiten Teilen auf eine einheitliche Basis stellt und vor allem den Verweis auf der SEC bereits vorliegende Unterlagen ermöglicht, vgl. hierzu Brotte (1997), S. 133-136.

59 Vgl. sec. 8 und 8A SA.

wo Seit dem Securities Act Amendment von 1964 wird auch der over-the-counter Handel mit reguliert. 
fallweise Ad-hoc-Publizität ist vom Eintreten bestimmter Sachverhalte abhängig, ihr Berichtsumfang wird durch Formular $8-\mathrm{K}$ geregelt. ${ }^{61}$ Diese Berichterstattungspflicht gilt jedoch nicht gegenüber den Aktionären der berichtenden Gesellschaften, eine solche Pflicht müßte gesellschaftsrechtlich fundiert werden und fält somit nicht in den Zuständigkeitsbereich des Bundes. Vielmehr handelt es sich bei den o.a. Berichtspflichten um eine Berichterstattung gegenüber der SEC. Die SEC wiederum macht die bei ihr eingereichten Berichte der breiten Öffentlichkeit zugänglich. Während dies in der Vergangenheit primär durch Auslegung in den Büros der SEC geschah und ggf. die Berichte über Mikrofiche und Fotokopien bezogen werden konnten, setzt sich seit Beginn der 80er Jahre die elektronische Erfassung der Daten im Electronic Data Gathering, Analysis, and Retrieval (EDGAR) System der SEC durch. In der ersten Hälfte der 80er Jahre wurde dieses System von der SEC entwickelt, um die ständig wachsende Flut von Filings zu bewältigen. ${ }^{62}$ Als ,angenehmer Nebeneffekt“ sind die nunmehr elektronisch archivierten Daten weltweit problemlos abrufbar. ${ }^{63}$

Als weiterer Bestandteil der von der SEC geforderten Unternehmenspublizität ist die Gruppe der Proxy-Statements den Aktionären direkt zuzuleiten. Unternehmensleitungen, die von ihren Aktionären die Stimmrechtsvollmacht (proxy) erhalten wollen, sind nach Regulation 14A, Rule 14a-3(a) i.V.m. sec. 14 SEA verpflichtet, diese Proxy-Statements zu erstellen. Der Inhalt der Proxy-Statements entspricht im wesentlichen dem Jahresabschluß des Unternehmens und einer Tagesordnung der Hauptversammlung. ${ }^{64}$

Die Befugnis des SEA, explizite Rechnungslegungsregeln zu erlassen, hat die SEC jedoch aus ihren Bereich in den privaten Sektor an das Financial Accounting Standards Board (FASB) zurück delegiert, so daß die einzelnen Rechnungslegungsstandards nicht wie in

${ }^{61}$ Vgl. zur Form 8-K Berichtspraxis und zur Kapitalmarktrelevanz der Ad-hoc-Informationen Carter/Soo (1999).

62 So stieg die Zahl der Registrierungen von ca. 30.0001978 auf über 40.000 1983. Vgl. Skousen (1991), S. 42; dies führte u.a. dazu, daß nur noch ein Teil der Berichte von der SEC wirklich geprüft werden konnten. So konnten 1985 nur $22 \%$ aller eingereichten 10-K Forms untersucht werden, vgl. Horton (1993), S. 27.

63 Vgl. Pagell (1995); es gibt mittlerweile zahlreiche Dienstleister, welche die EDGAR-Daten über das Internet anbieten, das Intemetangebot der SEC wird mit 24 Stunden Verzögerung eingespeist. Vgl. auch Lymer et al. (1999), S. 57.

64 Die Autorität, die eigentlich das Gesellschaftsrecht betreffende Proxyregulierung vorzunehmen, leitet die SEC aus der Tatsache ab, daß Proxymitteilungen über die Bundespost versendet werden, vgl. Merkt (1991), Tz. 635, der die Proxyregeln als ein „Haupteinfalltor für die bundesrechtliche Regulierung des corporation law" (ebendort) bezeichnet. 
Deutschland vor dem DRSC durch gesetzliche Vorschriften entstehen, sondern vielmehr auf privatwirtschaftlicher Basis generiert werden. ${ }^{65}$

Durch den SA und SEA erhöhte der US-amerikanische Gesetzgeber die Regulierungsdichte im Kapitalmarktrecht beträchtlich. Das mangelnde Vertrauen in die Selbstorganisierungsfähigkeit der Kapitalmärkte führte letztlich zu dem Entstehen eines der komplexesten Kapitalmarktsysteme weltweit.

\subsection{Industriepolitik}

Während die klassische Theorie von einer grundsätzlichen Superiorität der marktwirtschaftlichen Koordination ausgeht und erwartet, daß sich auch ohne regulatorisches Zutun funktionsfähige Märkte bilden, ist das neoklassisch fundierte wirtschaftspolitische Leitbild der traditionellen Industrieökonomik hier etwas pessimistischer, jedoch ohne grundsätzlich staatliche Wirtschaftslenkung im Sinne der dirigistischen Ansätze vorbehaltlos zu legitimieren. Nach ihr gilt eine kausale Relation MarktstrukturMarktverhalten - Marktergebnis. Somit determiniert die Marktstruktur letztendlich das Engebnis des wirtschaftlichen Koordinationsprozesses. ${ }^{\text {ob }}$ Ein funktionsfähiger Markt bedarf dann grundsätzlich keiner Regulierung. Als funktionsfähig wird ein Markt angesehen, wenn seine Struktur vollkommen ist und auf ihm vollständige Konkurrenz herrscht. ${ }^{67}$ Falls dies auf allen Märkten der Fall ist, dann gilt das totale Konkurrenzgleichgewicht nach Walras, welches gesamtwirtschaftlich pareto-optimale Engebnisse als Modellergebnisse erwarten läßt. Sobald jedoch einer der beobachteten Märkte von diesen Modellbedingungen abweicht, gilt diese strenge Kausalität nicht mehr. Aus diesen theoretischen Überlegungen ergibt sich für die Wirtschaftspolitik zunächst die Folgerung, daß alle realiter beobachteten Märkte, die nicht die für die vollkommene Konkurrenz adäquate Marktstruktur eines Polypols aufzeigen, nicht notwendigerweise für die gesamtwirtschaftliche Wohlfahrt optimal sind. Speziell das Vorliegen von Monopolen wurde als für die Gesamtwirtschaft negativ interpretiert. Weitere Gründe für das

65 Das FASB besitzt den sog. Substantial Authorative Support der SEC nach ASR No. 4, 1938, ASR No. 155, FRR 1; vgl. zu dieser Kompetenzdelegation auch Merkt (1991), Tz. 202; Carmichael/Lilien/Mellman (1999), chapter 2, S. 18 f. Vgl. zu den Vorgängerinstitutionen Horngren (1972); Zeff (1972; 1984); Haller (1994), S. 37-40 und S. 52; Miller/Redding/Bahnson (1998), S. 55-58.

66 Vgl. grundlegend Mason (1939; 1949); Bain (1971); zusammenfassend zur wirtschaftspolitischen Sichtweise des Terminusses Industriepolitik Oberender/Daumann (1995).

67 Vollständige Konkurrenz liegt nach Schumann (1992), S. 211 f. vor, wenn der Markt vollkommen ist und wenn es am Markt sehr viele Anbieter und Nachfrager gibt (atomistische Angebots- bzw. Nachfragestruktur). Auf einem vollkommenen Markt gibt es 1.) keine Präferenzen persönlicher, räumlicher oder sachlicher Art und 2.) vollständige Markttransparenz vor allem im Sinne von vollständiger Preisinformation. Vgl. weiterhin zu den Begriffen der vollkommenen Märkte und der vollständigen Konkurrenz Wied-Nebbeling (1997), S. 3-5 und für den angloamerikanischen Sprachraum und den analogen Begriff perfect competition Kreps (1990), S. 264 f. 
Abweichen von der gewünschten Marktstruktur liegen in dem Vorliegen von externen Effekten und asymmetrischer Informationsverteilung. All diese Phänomene beeinflussen die Marktstruktur und werden in der neoklassischen Theorie mit dem Begriff „Marktversagen" tituliert.

Basierend auf dem Konzept, daß die Marktstruktur letztendlich das Marktergebnis determiniert, wurden in Deutschland und den USA detaillierte Konzepte für „die richtige Marktstruktur“ entwickelt, die hier unter dem Begriff Industriepolitik zusammengefaßt werden. So sieht Kantzenbach die korrekte Marktstruktur im weiten Oligopol mit mäßiger Markttransparenz beheimatet ${ }^{68}$, während die Harvard-Richtung der US-amerikanischen Wettbewerbstheorie das Konzept der workable competition kreierte, welches mehr Wert darauf legt, daß Marktzutrittsschranken einen möglichen Wettbewerb nicht verhindern. ${ }^{69}$

Der Versuch, durch regulatorischen Eingriff bestehende Marktstrukturen zu verändern, findet sich vor allem im jüngeren deutschen Kapitalmarktrecht. So wurden die Finanzmarktförderungsgesetze mit dem erklärten Ziel lanciert, die Anzahl der Kapitalmarktteilnehmer zu erhöhen. Ähnliche Motive lassen sich auch beim SA und SEA finden. Grundsätzlich kann die gesamte Unternehmenspublizität als Versuch gedeutet werden, den Grad der asymmetrischen Information zwischen den Marktteilnehmern zu reduzieren, da asymmetrische Information über adverse selection Mechanismen zu Marktversagen im neoklassischen Sinn führen kann. ${ }^{70}$ Des weiteren wird argumentiert, daß Unternehmensinformationen wegen der schlechten Exkludierbarkeit der Nutzer und der nur indirekten Rivalität im Konsum den Charakter eines öffentlichen Gutes besitzen. ${ }^{71}$

Nach dem zweiten Weltkrieg und nach Vorschriften der Siegermächte zur Unternehmenspublizität wurde das AktG von 1965 entworfen. ${ }^{72}$ War bislang die gesellschaftliche Publizität vorrangig von Gläubigerinteressen geprägt, so bahnte sich durch das AktG 1965 eine stärkere Einbeziehung der Aktionärsinteressen an. ${ }^{73}$ Insofern kann das AktG 1965 als ein Versuch gewertet werden, durch mehr Information die Struktur des Kapitalmarktes zu modifizieren. So finden sich im AktG 1965 nicht nur Wertobergrenzen für Vermögensge-

68 Vgl. Kantzenbach (1967); kritisch dazu Hoppmann (1966).

69 Vgl. grundlegend Baumol/Panzar/Willig (1988); zusammenfassend auch Kaufer (1980).

70 Vg. grundlegend zu dieser Argumentation Akerlof (1970).

71 Vgl. z.B. May/Sundem (1976), S. 749; Pellens/Gassen (1998); zur Einführung von öffentlichen Gütern in die Neoklassik Samuelson (1954).

72 Dieses AktG 1965 wurde faktisch in zwei Stufen entwickelt: In der sog. kleinen Aktienrechtsform, dem Gesetz über die Kapitalerhöhung aus Gesellschaftermitteln und über die Gewinn- und Verlustrechnung und in dem eigentlichen neuen AktG, vgl. Gessler (1965), S. $151 \mathrm{f}$.

73 Diese Kapitalmarktorientierung war schon mit der kleinen Aktienrechtsreform von 1959 eingeleitet worden, vgl. Goerdeler (1992). 
genstände, sondern ebenfalls Wertuntergrenzen, die das Bilden stiller Reserven erschweren sollen. ${ }^{74}$ Des weiteren werden Obergesellschaften mit Untergesellschaften im Inland dazu verpflichtet, einen Konzemabschluß zu veröffentlichen. Neben den Vorschriften zu dem Umfang und der Ausgestaltung des zu erstellenden Jahresabschlusses, wurden auch Auskunftsrechte des Aktionärs kodifiziert, welche er notfalls auch vor Gericht erstreiten kann. ${ }^{75}$ Somit wandelte sich die gesellschaftsrechtliche Publizität mit dem AktG 1965 von einem rein an Gläubigerinteressen ausgerichteten Instrument, dessen hauptsächliche Aufgabe seit dem ALR von 1794 die Einkommensbemessung war, zumindest ansatzweise zu einem Informationsinstrument für die Teilnehmer am anonymen Kapitalmarkt. Das AktG 1965 war bis in die 80er Jahre hinein der regulatorische Kem für die deutsche Rechnungslegungspublizität. ${ }^{76}$

Trotz der teilweise gemeinsamen Rechtsentwicklung haben sich die nationalen Gesellschaftsrechtskonzeptionen Europas unterschiedlich entwickelt. Diese sollten im Rahmen der durch den EG-Vertrag konstituierten Gründung der Europäischen Wirtschaftsgemeinschaft aneinander angeglichen werden, um den europäischen Binnenmarkt zu stärken und dadurch die bestehende Marktstruktur zu verändem. Somit wurden auch Richtlinien zur europaweiten Harmonisierung des Bilanzrechts und der gesellschaftsrechtlichen fundierten Rechnungslegungspublizität erlassen. Die erste Richtlinie (68/151/EWG) fordert im wesentlichen die Veröffentlichung von einer Bilanz und Gewinn- und Verlustrechnung aller Kapitalgesellschaften der Mitgliedstaaten. Detaillierte Vorschriften zur Ausgestaltung der Rechenwerke enthält sie nicht. Diese wurden erst in der vierten EG-Richtlinie (78/660/EWG) für den Einzelabschluß implementiert. Hier wurde festgesetzt, daß ein zu veröffentlichender Jahresabschluß aus Bilanz, Gewinn- und Verlustrechnung sowie Anhang besteht. Des weiteren ist auch ein Lagebericht zu veröffentlichten. Die vierte Richtlinie sieht weiterhin größenabhängige Erleichterungen für kleine Kapitalgesellschaften vor.

Die siebente EG-Richtlinie (83/349/EWG) verpflichtet Unternehmen zur Erstellung und Veröffentlichung eines Konzernabschlusses und eines Konzemlageberichts, wenn sie Mutterunternehmen eines Konzerns sind, in denen ein oder mehrere Kapitalgesellschaften i.S.d. Art. 4 Abs. 1 Siebente EG-Richtlinie enthalten ist. ${ }^{77}$ Die Richtlinie gilt grundsätzlich unabhängig von der Rechtsform des Mutterunternehmens, im Rahmen der ein-

74 Vgl. als Beispiel of 153-155 AktG 1965.

75 Vgl. If 131-132 AktG 1965.

76 Vgl. zu empirischen Untersuchungen über die Publizitätspraxis deutscher Untemehmen vor dem BiRiLiG Wysocki (1976); Berndsen (1978); zu der Wirkung dieser Publizität Coenenberg/Möller (1979); Gebhardt (1980) und Pellens (1989).

$\pi$ Vgl. zu der 4. und 7. EG-Richtlinie Schwark (1978); mit der damaligen Situation in den USA vergleichend Hopt (1980). 
zelstantlichen Umsetzung in geltendes Recht wird jedoch die Möglichkeit für die Beschränkung der verpflichteten Rechtsform auf Kapitalgesellschaften nach Art. 4 Abs. 2 Siebente EG-Richtlinie gegeben. Ebenso können Konzerne, die den Größenkriterien der Vierten EG-Richtlinie entsprechend als klein oder mittelgroß zu charakterisieren sind, von der Publizitätspflicht nach der Siebenten EG-Richtlinie entbunden werden, wenn die Mitgliedstaaten dies bei der Transformation der Richtlinien in nationales Recht dementsprechend kodifizieren. ${ }^{78}$

Die Umsetzung der EG-Richtlinien in deutsches Recht geschah im Rahmen des Bilanzrichtlinien-Gesetzes (BiRiLiG) vom 19. Dezember 1985. ${ }^{79}$ Diese Kodifizierung vergrößerte vor allem für $\mathrm{GmbH}$ die Publizitätspflichten beachtlich. Der Gesetzgeber gab alle größenabhängigen Befreiungsmöglichkeiten an die Untemehmen weiter. Das durch das BiRiLiG neu in das HGB eingefügte 3. Buch ( $\$$ 238-339 HGB) regelt recht detailliert die Rechnungslegungvorschriften. Die Publizität der Jahresabschlußdaten wird in $\int \$$ 325-329 HGB geregelt. Für den Jahresabschluß werden Ansatz-, Bewertungs- und Ausweisregeln sowie Angabepflichten für den Anhang formuliert. Ferner werden Inhalte des den Jahresabschluß ergänzenden Lageberichts festgeschrieben. Grundsätzlich hängt der Detailierungsgrad der geforderten Angaben sowie die Publizitätsmethode von der Rechtsform und der Größe des jeweiligen Unternehmens ab. Hinsichtlich der Größe wird zwischen drei Kategorien und hinsichtlich der Rechtsform wird zwischen Personen-, Kapitalgesellschaften und Genossenschaften unterschieden. Zusätzlich zu den Kriterien des HGB fordert das PublG gem. IS 1 und 9 die Veröffentlichung von Rechnungslegungsdaten auch unabhängig von der Rechtsform für Unternehmen, die bestimmte Größenkriterien überschreiten. Diese haben dann die HGB Vorschriften für Kapitalgesellschaften anzuwenden. Analoges gilt für den Konzernabschluß nach \$\$ 11-15 PublG.

Neben der Vierten und Siebenten EG-Richtlinie wurde durch das BiRiLiG auch die Achte EG-Richtlinie in deutsches Recht transformiert. Diese regelt die Prüfung von zu veröffentlichenden Einzel- und Konzernabschlüssen und die Zulassung der mit der Prüfung beauftragten Personen.

Mit der Umsetzung der EG-Richtlinien in deutsches Gesellschaftsrecht nahm der Grad der Rechnungslegungs- und Publizitätsregulierung beträchtlich zu, ohne daß das eigentliche Hauptziel der europaweiten Harmonisierung der gesellschaftsrechtlich fundierten Unternehmenspublizität und die damit verbundene Stärkung des europäischen Binnen-

n Vgl. umfassend zur Konzemrechnungslegungspflicht nach HGB Busse von Colbe/Ordelheide (1993), S. 4762.

7. Vgd zum BiRiliG Biener/Berneke (1986); Biener (1989). Eine positive Analyse der lobbyistischen Einflußnahme im Rahmen des Gesetzgebungaprozeß unternimmt Ordelheide (1997; 1998a); und empirisch detaillierter Mcleag/Ordelheide/Young (1999). 
marktes erreicht wurde. ${ }^{80}$ Mitverantwortlich dafür waren die zahlreichen Wahlrechte und Umsetzungsspielräume der EG-Richtlinien, welche es den Mitgliedsstaaten ermöglichten, ihre nationalen Bedürfnisse umzusetzen. ${ }^{81}$

Auch im Bereich des Kapitalmarktrechts waren es neben der Novelle des BörsG von 1975 vor allem die EG-Richtlinien zur Harmonisierung der europäischen Kapitalmärkte, die das Kapitalmarktrecht der Bundesrepublik nachhaltig veränderten. ${ }^{82}$ Die Zulassungsrichtlinie regelt die Bedingungen, die von Wertpapieremittenten erfüllt werden müssen, welche die amtliche Notierung an einer europäischen Börse anstreben. Die Prospektrichtlinie wurde von der Kommission mehrfach überarbeitet und fordert, nach dem Beitritt Großbritanniens zur EG von der angloamerikanischen Publizitätsphilosophie geprägt, ${ }^{83}$ zahlreiche Informationen über das emittierende Unternehmen. Dies gilt ebenso für die Zwischenberichtsrichtlinie, die eine regelmäßige Publizität der börsennotierten Unternehmen verlangt. Die Umsetzung der EG-Richtlinien erfolgte zum Teil im BörsG, zum Teil in der Börsenzulassungsverordnung (BörsZulVO). So ist $₫ 38$ (1) Nr. 2 BörsG die Rechtsgrundlage für den Zulassungsprospekt, die eigentliche Ausgestaltung wird allerdings in den

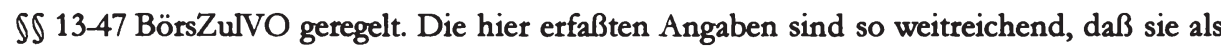
mit denen der US-amerikanischen Börsenaufsichtsbehörde SEC als vergleichbar angesehen werden. ${ }^{84}$ Die regelmäßige Publizität umfaßt neben der Übernahme der gesellschaftsrechtlich fundierten jährlichen Publizität ${ }^{85}$ vor allem die Verpflichtung der im amtlichen Markt notierten Unternehmen, nach $₫ 44 \mathrm{~b}$ BörsG auf halbjährlicher Basis einen Zwischenbericht $\mathrm{zu}$ veröffentlichen. Auch hier wird der eigentliche Inhalt des Zwischenberichts von $\iint 53-62$ BörsZuIVO geregelt. Der Zwischenbericht soll den Kapitalmarktteilnehmern ermöglichen, die Geschäftstätigkeit des Unternehmens in den letzten sechs Monaten zu beurteilen. Dazu müssen mindestens die Umsatzerlöse und das Ergebnis vor und nach Steuem angegeben werden. Erstellt der Emittent einen Konzernabschluß, so kann er wählen, ob er sich auf diesen oder auf den Einzelabschluß bezieht, hierbei hat die Zulassungsstelle jedoch die Möglichkeit, ihrer Meinung nach durch die Ausübung dieses

80 Vgl. allgemein zum Stand der Publizitäts- und sonstigen Informationsvorschriften des Gesellschaftsrechts Wilde (1998); zu den durch das BiRiLiG eingeführten Wahlrechten hinsichtlich der GuV-Struktur Baetge/Fischer (1987; 1988).

81 Vgl. zum Harmonisierungsgrad der europäischen Rechnungslegung vor und nach den EG-Richtlinien Tas (1992); Krisement (1994), insb. S. 149-189; allgemein zu den Auswirkungen der EU auf den Bereich der Rechnungslegung und Prüfung Hegarty (1997) und zu den erwartenen Entwicklungen im europäischen Untemehmensrecht Lutter (2000).

82 Diese sind im einzelnen die Zulassungsrichtlinie (79/279/EWG) vom 5. März 1979, die Prospektrichtlinie (80/390/EWG) vom 17. März 1980 und die Zwischenberichtsrichtlinie (82/121/EWG) vom 15. Februar 1982.

83 Vgl. Schwark (1987), S. 2042.

84 Vgl. Schwark (1987), S. 2044.

85 Vgl. $§ 44$ (1) Nr. 3 Abs. 2 BörsG i.V.m. $\ 65$ BörsZulVO. 
Wahlrechtes fehlende Informationen einzufordern. Eine Prüfung der veröffentlichten Rechnungslegungsdaten ist nicht erforderlich.

Eine weitere EG-Richtlinie, die Emissionsprospektrichtlinie (89/298/EWG), regelt die Erstellung des eigentlichen Verkaufsprospektes, der zum Zeitpunkt der Emission zu erstellen ist, also vor einem eventuellen Börsenlisting und somit insbesondere von Bedeutung ist, wenn Untemehmen keine Börsenzulassung beabsichtigen. Die Umsetzung der Richtlinie in deutsches Recht erfolgte in dem Verkaufsprospektgesetz (VerkProspG). Die eigentlichen Inhalte regelt die Verkaufsprospekt-Verordnung (VerkProspVO). Die geforderten Inhalte des Verkaufsprospektes hängen auch davon ab, ob ein Listing am amtlichen Handel geplant ist oder nicht und entsprechen im wesentlichen denen des Zulassungsprospektes respektive denen des Unternehmensberichtes. ${ }^{86}$

Mit den o.a. EG-Richtlinien war die Tätigkeit des europäischen Regulierers im Kapitalmarktbereich noch nicht beendet. Drei weitere EG-Richtlinien ${ }^{87}$ wurden durch das zweite Finanzmarktförderungsgesetz in deutsches Recht transformiert. Durch diese Umsetzung wurde das Wertpapierhandelsgesetz (WpHG) neu geschaffen. ${ }^{88}$ Eine zentrale Aufgabe dieses Gesetzes ist die Kontrolle von Unternehmensinsidern und deren Geschäften. Um diese Aufgabe erfüllen zu können, wurde als Element der neuen Börsenaufsicht das Bundesaufsichtsamt für Wertpapierhandel (BAWe) institutionalisiert. Außerdem wurde die vorher in $₫ 44$ a BörsG kodifizierte Vorschrift zur Ad-hoc-Publizität, welche rechtsunsicher ausgestaltet war und als de facto wirkungslos kritisiert wurde, durch den neu konzipierten $\int 15 \mathrm{WpHG}$ im Rahmen der Insiderregelungen ersetzt. ${ }^{89}$ Zusätzlich zu den Vorschriften zur Vermeidung des Mißbrauchs von Insiderwissen umfaßt das WpHG Vorschriften zur Offenlegung bei Veränderungen des Stimmrechtsverhältnisses ${ }^{90}$ und setzt Verhaltensregeln für Wertpapierdienstleister. ${ }^{91}$

Im Rahmen der Bestrebungen, das deutsche Kapitalmarktrecht weiterzuentwickeln, wurde das dritte Finanzmarktförderungsgesetz entwickelt, welches am 1.4.1998 als Gesetz zur weiteren Fortentwicklung des Finanzplatzes Deutschland größtenteils in Kraft tat. Die für die Publizität relevanten Neuerungen umfassen im wesentlichen die Prospekthaftung ${ }^{92}$,

86 Vgl. Assmann (1993), S. $554 \mathrm{f}$.

87 Dies waren die Transparenzrichtlinie (86/627/EWG) vom 12 Dezember 1988, die Insiderrichtlinie (89/592/EWG) vom 13. November 1989 und die Wertpapierdienstleistungsrichtlinie (93/22/EWG) vom 10. Mai 1993.

88 Vgl. grundlegend zum WpHG Hopt (1995); kritisch diskutierend Cahn (1998).

89 Vgl. Pellens (1991); Pellens/Fülbier (1994); Fülbier (1998), S. 37-40 und Fülbier (1999a).

9 Vgl. of 21-30 WpHG.

91 Vgl. \$f 31-37 WpHG.

92 Vgl. zur Prospekthaftung Groß (1999). 
die Liberalisierung der Regeln zum Unternehmensbericht, eine Verschärfung der Verkaufsprospektvorschriften und die Entbindung ausländischer Emittenten von der Vorabmeldepflicht gegenüber des BAWe im Rahmen der Ad hoc Publizität, wenn sie bereits gegenüber ihrer nationalen Börsenaufsichtsbehörde meldepflichtig sind. Im Rahmen der Prospekthaftung hat der Adressat nunmehr ein sechsmonatiges Recht auf Schadensersatz, auch wenn er die Wertpapiere zum Antragszeitpunkt nicht mehr hält. Die Erstellung eines Unternehmensberichts wird erleichtert, indem bei Neuemissionen von Unternehmen, die noch keine 18 Monate existieren, kein Jahresabschluß mehr verlangt wird, ein Unternehmensbericht für mehrere deutsche Börsen nur noch einmalig geprüft wird und ausländische Emittenten ihren Unternehmensbericht nicht mehr unbedingt in deutscher Sprache, sondern auch in anderen im grenzüberschreitenden Wertpapierverkehr üblichen Sprachen verfassen können. Die Bedeutung des Verkaufsprospekts wird gestärkt, indem das BAWe ermächtigt wird, die Verkaufsprospekte auf Vollständigkeit hin zu überprüfen und gegebenenfalls das öffentliche Angebot von Wertpapieren zu untersagen, für die kein hinreichender Verkaufsprospekt angeboten wurde. ${ }^{93}$

Die Gesamtheit der durch die diversen EG-Richtlinien institutionalisierten neuen Publizitätsvorschriften für börsennotierte Unternehmen werden teilweise als künstliche Eintrittsbarriere in den Kapitalmarkt kritisiert. ${ }^{94}$ Es wird also deutlich, daß die EU mit ihren Kapitalmarktrichtlinien die Marktstruktur des europäischen Kapitalmarkts beeinflußt hat.

Die US-amerikanische Wirtschaftspolitik der 60er und 70er Jahre war relativ stark von industriepolitischem Gedankengut geprägt. ${ }^{95}$ Dies zeigt sich unter anderem im Anti-Trust Law. ${ }^{9}$ Im Bereich der Rechnungslegungspublizität läßt sich ein gezielter Eingriff in Marktstrukturen und in das Marktverhalten nur sehr begrenzt erkennen. Zwar kann generell das US-amerikanische Kapitalmarktrecht als ein steuemder Eingriff in die Marktstruktur und in das Marktverhalten des Marktes für Realkapital analysiert werden, eine generelle industriepolitische Intention läßt sich jedoch in den US-amerikanischen Normen zu Rechnungslegungspublizität nicht ausmachen. Andererseits besitzt die Hauptaufgabe der SEC, die Funktionsfahigkeit und die internationale Superiorität des US-

93 Vgl. zu den Änderungen des dritten Finanzmarktförderungsgesetzes überblicksartig Kusserow/Hofmann (1998).

94 Vgl. Schwark (1987), S. 2042.

95 Dies gilt vor allem vor dem Hintergrund der traditionell niedrigen Regulierungsgrad des US amerikanischen Wirtschaftssystems. Vgl. zur Praxis der US-amerikanischen Industriepolitik Oberender/Daumann (1995), S. 32-37.

V Vgl. zur ökonomischen Fundierung des Anti-Trust Law Scherer/Ross (1990), S. 317-352; Posner (1992), S. 285-320. 
amerikanischen Kapitalmarkts jetzt und in Zukunft zu gewährleisten, einen offensichtlichen industriepolitischen Charakter.

\subsection{Neoliberalismus}

Quasi als Reaktion auf die stark an der Marktstruktur ausgerichteten Theorieansätze der Industriepolitik bildete sich eine Gegenbewegung heraus, welche die dirigistische Grundhaltung der Harvard School als Anmaßung von Wissen zurückweist. ${ }^{97}$ Märkte werden aus neoliberaler Sicht nicht strukturell, sondern als Prozeß analysiert. Ein unbeeinflußtes Marktergebnis wird definitorisch als gesamtwirtschaftlich optimal betrachtet, da es das Ergebnis eines Evolutionsprozesses darstellt, wobei jedoch ein langfristiger Betrachtungszeitraum zugrunde gelegt wird. Aus dieser Überlegung leitet sich die Forderung nach einer extremen Zurückhaltung des wirtschaftspolitischen Regulierers ab. Dieser soll lediglich die Verfügungsrechte so verteilen, daß Wettbewerb ermöglicht wird, und ansonsten durch gezielte Verbote Wettbewerbshemmnisse beseitigen. Regulierungen mit Gebotscharakter sind nach neoliberalem Denken grundsätzlich abzulehnen. Ferner sind bei jeder Regulierung deren Durchsetzungskosten und die Eigendynamik der regulierenden Institution explizit zu berücksichtigen. ${ }^{98}$

Die Gedankenansätze des Neoliberalismus wurden in der wissenschaftlichen Literatur zu Fragen der Rechnungslegungsregulierung häufig aufgegriffen, um die Regulierungstiefe von Publizität und Rechnungslegung anzuzweifeln. ${ }^{99}$ Eventuell kann die Institutionalisierung des deutschen privaten Standardsetters DRSC als der Versuch des Staates gewertet werden, sich aus der Regulierung der Rechnungslegungspublizität zurückzuziehen. ${ }^{100} \mathrm{Im}$ allgemeinen ist die neoliberale Gedankenrichtung bei der Modifikation von bestehenden Publizitätssystemen jedoch nicht als Argumentationsgrundlage verwendet worden. Im Gegenteil: Die Implementierung der Kapitalmarktpublizität der USA durch das SA und SEA läuft dem neoliberalen Konzept einer eingriffsschwach ausgestalteten Wirtschaftspolitik diametral entgegen. Als Auslöser der Gesetzgebung wurden wie o.a. betrügerische Handlungen am Wertpapiermarkt angeführt. Anstatt diese durch ein Verbot zu verhin-

97 Vgl. zum Begriff „Anmaßung von Wissen“ Hayek (1989).

98 Vgl. zu den Grundsätzen der neoliberalen Wettbewerbs- und Wirtschaftspolitk, die auch unter den Begriffen Chicago School und Economic Theory of Law bekannt geworden ist, grundlegend Hayek (1994); Schmidtchen (1983); Hoppmann (1966) im Gegenentwurf zu Kantzenbach; zusammenfassend und vertiefend Posner (1992); diskutierend Schmidtchen (1991) in Anwendung auf den Kapitalmarkt Schneider (1986).

99 So vgl. z.B. Gonedes/Dopuch/Penman (1976), Ordelheide (1995); zur Regulierung des Insidertatbestandes Schömer (1991); Schneider (1993); jüngst auch Fülbier (1998); aus juristischer Sicht zur Deregulierung des AktG Spindler (1998).

100 Vgl. Biener (1996); Pellens/Gassen (1998); dies ergibt sich jedoch nicht aus der offiziellen Begründung, die ein perfektes Beispiel einer „follow the leader" Strategie gibt. Vgl. BT-Drucks. 13/9909 und 13/10038. 
dern, wie auch von liberaler Seite gefordert wurde ${ }^{101}$, entschloß man sich zu einem allgemeinen Publizitätsgebot.

Nichtsdestotrotz unterliegen auch Regulierungen evolutionären Prozessen. So verwundert es angesichts der national selbst innerhalb der EU stark divergierenden Rechnungslegungspublizität nicht, daß im Rahmen der zunehmenden globalen Transparenz der Kapitalmärkte seit Beginn der 90er Jahre der Konkurrenzdruck auf das gesellschaftsrechtlich geprägte Publizitätssystem der Bundesrepublik zunimmt. Da deutsche Konzeme mit dem freiwilligen Befolgen anderer Publizitätssysteme im Rahmen der gesetzlich determinierten Möglichkeiten quasi eine Abstimmung mit den Füßen vomahmen ${ }^{102}$, sah sich der Gesetzgeber 1998 gezwungen, die gesellschaftsrechtlichen Publizitätsanforderungen für ausländische Publizitätssysteme durch das KapAEG zeitlich bis 2004 befristet für andere Normensysteme zu öffnen. Er band jedoch diese Öffnung an den Börsenzugang der berichtenden Konzeme, insofem besteht hier ein gewisser Nexus zwischen Gesellschafts- und Kapitalmarktrecht. ${ }^{103}$ Des weiteren wurden mit dem Gesetz zur Kontrolle und Transparenz im Unternehmensbereich (KonTraG) weitere Vorschriften zur Erweiterung und Modifikation der Rechnungslegungspublizität erlassen. ${ }^{104}$

Zentrales Ergebnis dieser Änderungen war neben der o.a. Möglichkeit für deutsche börsennotierte Mutterunternehmen, für einen befristeten Zeitraum ihren Konzernabschluß nicht nach handelsrechtlichen, sondern nach alternativen international anerkannten Vorschriften zu erstellen ${ }^{105}$ und der Kodifizierung einer Pflicht zur Veröffentlichung einer Kapitalflußrechnung sowie einer Segmentberichterstattung ${ }^{106}$ die Institutionalisierung eines privatrechtlichen deutschen Standardsetters ${ }^{107}$. Mit dieser strukturellen Änderung der Publizitätsregulierung ermöglicht der deutsche Gesetzgeber eine Weiterentwicklung

101 Vgl. Brotte (1997), S. 122; Skousen (1991), S. 6 f.; Benston (1969; 1973).

102 Vgl. zu den Bemühungen deutscher Unternehmen, internationale Publizitätsvorschriften zu befolgen Pellens (1999), S. 501-519. Dieser Trend ist allerdings vorrangig bei börsennotierten Unternehmen anzutreffen. Ihren Zielen gemäß besitzen kleinere und mittlere Unternehmen nur ein geringes Interesse an der internationalen Rechnungslegungspublizität. Vgl. hierzu Küffner/Hock (1998).

103 Vgl. dazu auch explizit Möllers (1999).

104 Vgl. zu den hier erwähnten Änderungen auch Pape (1997); Pellens/Bonse/Gassen (1998); Scheffler (1999a). Das Kon'TraG enthält auch zahlreiche Änderungen im Bereich der Jahresabschlußprüfung und sonstiger Bereiche der Unternehmenskontrolle. Vgl. hierzu Wengert/Wengert (1997); Hommelhoff/Mattheus (1998); Zimmer (1998a). Für die Auswirkungen auf die GmbH Remmer/Theile (1998). In jüngster Zeit zeichnet sich durch die Transformation der GmbH \& Co-Richtlinie (90/605/EWG) eine auch realiter wirksame Publizitätspflicht mittelständischer Unternehmen ab, vgl. Bihr (1999); Emst (1999); Marx/Scharenberg (1999). Hierbei handelt es sich wohl eher um ein Relikt industriepolitischen Gedankenguts aus den EGRichtlinien, vgl. Heni (1999), S. 914.

105 Vgl. \292a HGB. Vgl. zur Umsetzung von \292a HGB den ersten Standard des DRSC DRS 1.

106 Vgl. $\$ 297$ (1) HGB.

107 Vgl. $₫ 342 \mathrm{HGB}$. 
der deutschen gesellschaftsrechtlichen Publizität auf privater Basis. ${ }^{108}$ Durch geeignete Formulierung des $\$ 342$ (2) HGB gibt dieser jedoch die Regulierung nicht komplett aus der Hand, sondern hält sich durch die Möglichkeit der Nichtveröffentlichung ein Einspruchsrecht vor. ${ }^{109}$ Als privater Standardsetter fungiert das im März 1998 gegründete Deutsche Rechnungslegungs Standards Committee (DRSC), welches sich die Entwicklung international anerkannter Standards zur Rechnungslegungspublizität sowie die Vertretung deutscher Interessen bei internationalen Standardsettern zum Ziel gesetzt hat. ${ }^{110}$

Im Bereich des deutschen Kapitalmarktrechts kann die Schaffung unterschiedlicher Marktsegmente als Versuch interpretiert werden, mögliche Markteintrittsbarrieren abzubauen, indem die unterschiedlichen Segmente des Aktienhandels, nach der Börsenrechtsreform von 1986 der amtliche und der geregelte Markt, unterschiedlich streng reglementiert wurden. Während eine Zulassung zum amtlichen Handel eine Art Qualitätssiegel für die betreffenden Unternehmen bedeutet, können den geregelten Handel auch solche Unternehmen in Anspruch nehmen, welche die hohen Publizitätsanforderungen noch nicht erfüllen können. Anstatt eines Emissionsprospektes müssen diese einen Unternehmensbericht einreichen, der weniger detaillierte Informationen einfordert. Der genaue Inhalt wird von den jeweiligen Börsenordnungen bestimmt. ${ }^{111}$ Eine regelmäßige Berichtspublizität im Sinne des $\$ 44 b$ BörsG ist für Unternehmen im geregelten Markt nicht erforderlich, bei nicht ausreichenden Angaben kann der Zulassungsauschuß jedoch weitere Informationen einfordem.

Zusätzlich zu diesen beiden Segmenten gibt es noch den in großen Teilen privatrechtlich organisierten Freiverkehr, der von einem von dem Börsenträgerverein genehmigten Freiverkehrsausschuß organisiert wird. ${ }^{112}$ Die zur Zulassung zum Freiverkehr geforderte Publizität ist sehr gering: Das sogenannte Exposé des Emittenten umfaßt in der Regel die letzten Geschäftsberichte sowie einige wenige weitere Angaben. Eine regelmäßige Publizität wird von kapitalmarktrechtlicher Seite nicht gefordert. ${ }^{113} \mathrm{Ob}$ diese partielle Deregulierung für den geregelten Markt und dem Freiverkehr für börsennotierte Untemehmen von

108 Ähnliche Schritte sind auch in anderen Ländem mit kontinentaleuropäischem Gesellschaftsrecht zu beobachten. Vgl. beispielhaft für die Schweiz Koller (1999).

109 Vgl. auch aus juristischer Sicht kritisch zu den Änderungen der Normengenerierung durch das KonTraG Hommelhoff/Schwab (1998); relativierend Heintzen (1999); ebenso Beisse (1999).

110 Vgl. zur Gründung des DRSC beispielhaft Moxter (1998); Knorr (1998); Pellens/Bonse/Gassen (1998); Drescher (1998); Budde/Steuber (1998); Pellens/Gassen (1998); Schildbach (1999); Schwab (1999); Breidenbach (1999); Scheffler (1999; 1999a).

111 Vgl $\int 73$ (1) Nr. 2 BöroG.

112 Vgd. $\$ 78$ Börs.

113 Vg. Schwark (1987), S. 2046; seit der Ānderung des $\lceil 267$ (3) Satz 2 HGB durch das Kon'TraG gelten zudem die normalen Größenerleichterungen für die im Freiverkehr notierten Unternehmen. Vg. zu den Auswirkungen Ostrowski (1999). 
großer Bedeutung sind, darf jedoch vor dem Hintergrund der zahlreichen anderen die Segementwahl beinflussenden Entscheidungsfaktoren ${ }^{114}$ wohl bezweifelt werden.

Mit der Gründung des Neuen Marktes wurde der Frankfurter Wertpapierbörse ein viertes Segment hinzugefügt, das teikweise privatrechtlich organisiert ist. ${ }^{115}$ Die Zulassungsvoraussetzungen des Regelwerks entsprechen im wesentlichen denen des geregelten Marktes. Untemehmen, die sich am neuen Markt listen lassen wollen, müssen das Zulassungsverfahren für den geregelten Markt durchlaufen. ${ }^{16}$ Die im Emissionsprospekt erforderlichen Angaben sind im Regelwerk des neuen Marktes sehr detailliert aufgelistet. ${ }^{117}$ Sämtliche Daten der Rechnungslegung müssen entweder IAS oder US-GAAP konform sein, wobei eine Überleitungsrechnung anerkannt wird. ${ }^{118}$ Nach erfolgter Zulassung ist das am Neuen Markt gelistete Unternehmen verpflichtet, Quartalsberichte zu veröffentlichen, deren Rechnungslegungsdaten nach den gleichen Vorschriften wie die Jahresabschlüsse ermittelt werden müssen. Bei Muttergesellschaften sind auch Konzemabschlußdaten einzubeziehen. ${ }^{119}$ Der Jahresabschluß muß veröffentlicht werden, die Frist hierfür ist mit drei Monaten nach Periodenende wesentlich kürzer als die nach $\$ 325$ HGB vorgeschriebene Frist von neun Monaten. ${ }^{120}$ Eine weitere publizitätswirksame Vorschrift des Regelwerks ist die Verpflichtung des Unternehmens, mindestens einmal im Jahr eine Analystenveranstaltung durchzuführen, wobei jedoch der genaue Inhalt und der Ablauf dieser Analystenveranstaltung nicht im Regelwerk spezifiziert werden. ${ }^{121} \mathrm{Da}$ diese detaillierten Publizitätsvorschriften nicht über gesetzliche Regulierungen, sondern über einen privatrechtlichen Vertrag institutionalisiert werden, kommt dieser Ansatz einer neoliberalen wirtschaftspolitischen Konzeption entgegen.

Im Gegensatz zur Entwicklung des deutschen Gesellschafts- und Kapitalmarktrechts ist im US-amerikanischen Regulierungsumfeld nur sehr begrenzt eine Tendenz für einen Rückzug des Gesetzgebers zu exkennen. Im Gegenteil: Weiterhin nimmt die SEC ihren gesetzlichen Auftrag zur Marktkontrolle wahr und greift in Bereichen, wo sie Notwendig-

114 Hier sind vor allem die Image und Aktienmarketingaspekte einer Segmentwahl zu beachten. Vgl. Harrer/Heidemann (1998); zu Rechtsfragen im Rahmen eines Börsengangs allgemein Picot/Land (1999).

115 Vgl. zum Neuen Markt allgemein Förschle/Helmschrott (1998); Gerke (1999); Gerke/Bank/Seitz (1999); Benz/Kiwitz (1999), zu den teilnehmenden Untemehmen Maute (1999).

116 Vgl. Regelwerk Neuer Markt, Abschnitt 2, Gliederungspunkt 23.

117 Vgl. Regelwerk Neuer Markt, Abschnitt 2, Gliederungspunkt 4.

118 Ebenfalls kann die Deutsche Börse AG den Emittenten von dieser Auflage zeitlich befristet entbinden. Vg1. Regelwerk Neuer Markt, Abschnitt 2, Gliederungspunkt 4.1.9, insb. Gliederungspunkt 4.1.9 (3) 3. Satz. Vgl. zur Bilanzierungspraxis der Unternehmen am neuen Markt Pellens (1999), S. 9; Peemöller/Finsterer/Neubert (1999) und d'Arcy/Leuz (2000).

119 Vgl. Regelwerk Neuer Markt, Abschnitt 2, Gliederungspunkt 7.1.

120 Vgl. Regelwerk Neuer Markt, Abschnitt 2, Gliederungspunkt 7.2.2 (2).

121 Vgl. Regelwerk Neuer Markt, Abschnitt 2, Gliederungspunkt 7.2.11. 
keit für Regeln ausmacht und das FASB (noch) nicht tätig wurde, über Gebote regulierend in die Rechnungslegungspublizität ein. ${ }^{122}$ Lediglich im Rahmen der Reformierung des Kapitalmarktrechts durch den Securities Litigation Reform Act von 1995, der unter anderem die Klagemöglichkeiten der Publizitätsadressaten enger faßt und die Haftung der betroffenen Unternehmen vor allem im Bereich der Prognosepublizität reduziert, kann eine gewisse neoliberale Tendenz vermutet werden. ${ }^{123}$

122 So z.B. die Verlautbarungen der SEC zur Derivatepublizität, vgl. Securities Act Release Nr. $33-7386$ vom vom 31.1.1998; o.V. (1997); Laupenmühlen/Münz (1998) und zum Jahr-2000-Problem, vgl. Securities Act Release Nr. 33-7558 vom 29.7.1999.

123 Vgl. kurz zum Securities Litigation Reform Act Markus (1996); zur eingehenderen Analyse und zur positiven Interpretation Guntz (1995). 


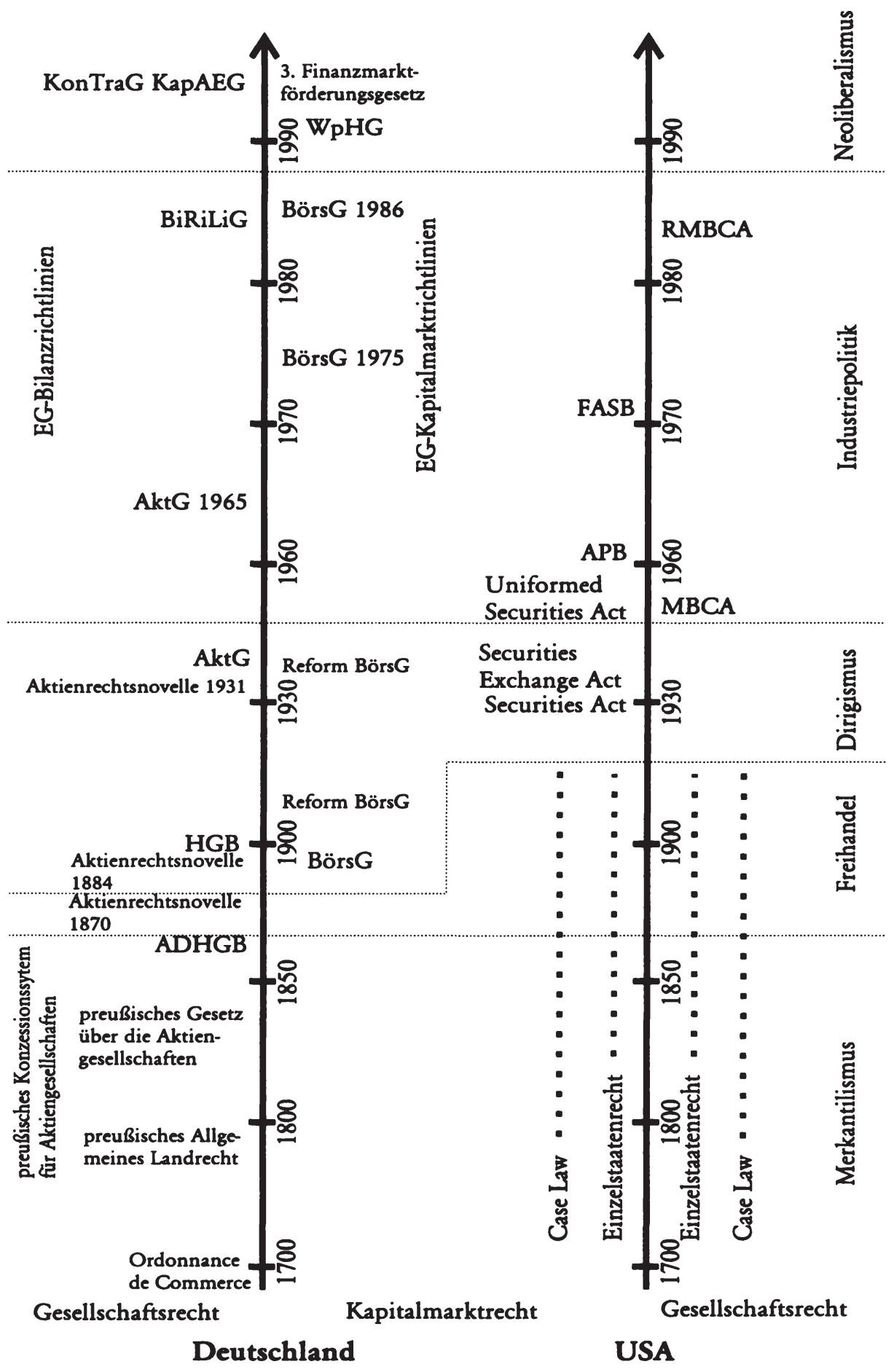

Abb. 3: Regulierungszeitstrahl Deutschland/USA 


\subsection{Vorgehensweise}

Nachdem im vorangegangenen Abschnitt die historische Entwicklung der Vorschriften zur Rechnungslegungspublizität dargestellt wurde, ist deutlich geworden, daß weder die deutschen noch die US-amerikanischen Regeln ein Publizitätssystem ,aus einem Guß" darstellen. Vielmehr bestehen beide aus einer Vielzahl von separaten Regulierungsansätzen, die den Markt für quantitative Unternehmensinformationen mit unterschiedlichen Zielsetzungen angehen. Diese lassen sich zumindest grob unterschiedlichen wirtschaftspolitischen Leitbildern unterordnen, wie hier in Abb. 3 geschehen. Es wird auch klar, daß eine alleinige Begründung der Regulierungen aus ökonomisch-normativen Ansätzen, egal welcher Epoche diese entstammen, keinesfalls möglich ist. Deswegen soll im folgenden versucht werden, Anhaltspunkte zu ermitteln, warum diese unterschiedlichen Publizitätssysteme von den jeweiligen Regulierungssubjekten den Marktteilnehmern oktroyiert wurden. Dafür wird zunächst kurz auf außerökonomische Begründungen für eine Regulierung der Rechnungslegungspublizität eingegangen. Anschließend wird die positiv orientierte Theorie der Regulierung verwendet, welche die politischen Entscheider wie die anderen Wirtschaftssubjekte auch dem Prinzip des methodologischen Individualismus entsprechend modelliert und somit den Regulierer nicht als exogene ausführende Instanz eines normativen Ergebnisses interpretiert, sondern ihn vielmehr modelltechnisch endogenisiert.

\subsection{Außerökonomische Motive}

Das Gerechtigkeitskriterium wird vor allem von juristischer Seite häufig für die Begründung von Gesetzesvorhaben genannt. ${ }^{124}$ Im Sinne des Rawlschen Schleiers der Ungewißheit ist eine Regel gerecht, wenn sie ex ante von allen potentiell betroffenen Wirtschaftssubjekten als so empfunden wird, ohne daß jene wissen, ob sie von dieser Regel positiv oder negativ betroffen sein werden. ${ }^{125}$ Die Forderung nach Veröffentlichung von Rechnungslegungsinformationen entspricht der Forderung nach einer Umverteilung eines knappen Gutes (der Information) von einer kleinen Gruppe (der Insider) auf die Allgemeinheit. Es ist keineswegs eindeutig, $\mathrm{da}$ eine solche distributive Maßnahme unter dem Schleier der

124 Vgl. statt vieler Belege SEA, Sec. 13 a, wo feststellt wird, daß eine Rechnungslegungspublizität notwendig sei "for the proper protection of investors and to insure fair dealing in the security" Die Tatsache, daß die hier vorgestellten Verteilungsargumente als nichtökonomisch bezeichnet werden, soll keine Wertung darstellen. Sie sind lediglich aufgrund ihres Charakters nicht durch ökonomisch basierte Modellbildung begründbar und entziehen sich so der ökonomischen Analyse. Nichtsdestotrotz werden sie häufig in Regulierungsbegründungen genannt und kommen somit als möglicher positiver Erklärungsansatz für das Verhalten des Regulierers in Frage.

125 Vgl. Rawls (1990), insb. S. 118-192 
Ungewißheit von allen Wirtschaftssubjekten befürwortet würde. ${ }^{126}$ Sowohl die Grundlage des US-amerikanischen Kapitalmarktrechts als auch des deutschen Gesellschaftsrechts zumindest in jüngerer Zeit besteht jedoch in der Forderung, die Marktteilnehmer bzw. Gesellschafter so mit Informationen zu versorgen, daß sie den gleichen Informationsstand haben. Diese Gleichverteilung von Informationen reduziert die Motivation der Wirtschaftssubjekte, sich diese auf eigene Rechnung zu beschaffen und benachteiligt dadurch diejenigen, die bei der Beschaffung von Information einen Konkurrenzvorteil besitzen. Die Entscheidung, inwiefern diese ökonomische Nutzeinbuße durch ein nicht ökonomisches Argument wie soziale Gerechtigkeit (über)kompensiert werden kann, kann letztlich nur durch den politischen Prozeß getroffen werden.

Festzuhalten bleibt, daß außerökonomische Umverteilungsargumente benutzt wurden, um die Regulierung der Rechnungslegungspublizität und hier vor allem die Implementierung einer generellen Publizitätspflicht zu rechtfertigen. Neben diesen kommen als ein weiterer Erklärungsansatz noch die bereits erwähnten positiven Erklärungsansätze zur Analyse in Frage.

\subsection{Positive Erklärungsansätze}

\subsubsection{Public Choice}

Kemidee der sogenannten Public Choice Theory ist die Übertragung des methodologischen Individualismus von der Ökonomie auf Fragestellungen der politischen, gesellschaftlichen Sphäre. ${ }^{127}$ Sie unterstellt der politischen Entscheidungsfindung nicht mehr ein übergeordnetes ideologisches Konzept, sondern geht vielmehr davon aus, daß der politische Entscheidungsprozeß letztlich aus der Summe der Individualentscheidungen resultiert. Auch Politiker werden somit zu eigennützig handelnden Individuen, die ihre Leistungen auf einem Markt für Politiker anbieten. Sie besitzen als Gestalter des Staates ein Monopol auf den Eingriff in die Verfügungstechtsverteilung, welches sie, bei Abwe-

126 Vgl. zu einer ökonomischen Betrachtung möglicher Gerechtigkeitskriterien auch Hayek (1960), S. 85-102; der belegt, daß ein Gleichverteilungspostulat letztlich die Freiheit als Abwesenheit von Zwang unmöglich macht und auch den Grundsatz der Gleichheit vor dem Gesetz verletzt.

127 Grundlegend zum Konzept der Public Choice Theory Buchanan/Tullock (1962), insb. S. 11-15; Truman (1971); als Übersichtsartikel Tollison (1982; 1988) sowie umfassend in der Anwendung auf juristische Fragen Posner (1992). Vgl. als wichtigen deutschen Vorläufer in den fünfziger Jahren Kaiser (1978); vgl. ferner Aranson (1998). Etwas weiter definiert Ballwieser (1996) die ökonomischen Analyse des Rechts und gibt einen Literaturüberblick über die deutsche Forschung zum Bereich Rechnungslegung. 
senheit klarer Spielregeln, zu ihrem eigenen Nutzen einsetzen werden. ${ }^{128}$ Somit sind sie Monopolist für die Regulierung der Rechnungslegungspublizität und werden von deren Nachfragern dementsprechend umworben.

Als potentielle Nachfrager einer Regulierung der Unternehmenspublizität kommen einerseits die von der Informationsasymmetrie betroffenen Vertragspartner des Unternehmensmanagements in Frage, andererseits jedoch auch die Manager selber, da diese durch einen regulierenden Eingriff in die Berichtserstattungspraxis einen ansonsten bestehenden Wettbewerb um die beste Unternehmenspublizität verhindern können. Ein spezieller Nachfragedruck der Rechnungslegungsadressaten läßt sich z.B. aus der Regierungserklärung zum KapAEG kaum ausmachen. Es wird nicht angeführt, daß die Kapitalmarktteilnehmer von den Unternehmen eine internationalen Regeln entsprechende Unternehmenspublizität verlangen, sondern vielmehr, daß der Aufwand einer doppelten Rechnungslegung deutschen Unternehmen nicht zuzumuten sei. ${ }^{129}$ Andererseits bezieht sich die praxisnahe Literatur zur Entwicklung der Rechnungslegungspublizität häufig auf einen Nachfragedruck nach internationalisierter Publizität, der von den Akteuren der internationalen Kapitalmärkte ausgehe. ${ }^{130}$ Im Gegensatz zu den eigentlichen Adressaten der Unternehmenspublizität bringen sich die rechnungslegenden Unternehmen häufig auch direkt als Nachfrager in den Regulierungsprozeß ein. So werden vor allem Regelungslükken in den speziellen Rechnungslegungsvorschriften kritisiert. ${ }^{131}$ Neben den Unternehmen treten in diesem Zusammenhang auch die Rechnungslegung prüfenden Instanzen auf, die ihrerseits detaillierte Vorschriften zur Rechnungslegung und Publizität anmahnen, gegebenenfalls aber auch auf eigenen Antrieb solche Normen anbieten. ${ }^{132}$

Weitere Nachfrager nach Regulierung sind die eigentlichen Ausgestalter der Regulierung selber. Unabhängig davon, ob die Ausgestaltung von öffentlicher Seite wie z.B. vom Bun-

128 In demokratischen Systemen wird dieses Problem durch die Einführung von Amtszeiten und Wiederwahlen abgeschwächt. Die Politiker erhalten also ein Monopol auf Zeit. Femer besteht für die Staatenbürger ggf. die Möglichkeit zum abwandern. Vgl. einführend Richter/Furubotn (1996), S. 453-476 und vertiefend Erlei/Leschke/Sauerland (1999), S. 307-515. Ferner wird das Verhalten der politischen Akteure auch durch die Beziehung von Parlament und Bürokratie beeinflußt. Vgl. für das Verhältnis SEC-Congress Weingast (1984) und für die Einflüsse der Constitution auf die Regulierung der Rechnungslegungspublizität Johnson (1981).

129 Vgl. BR-Drucks. 967/96.

130 Vgl. als wohl einer der ersten in Deutschland Liener (1992). Diese Sichtweise wird auch von Umfrageergebnissen gestützt. Vgl. Förschle/Glaum/Mandler (1995) und in signifikanterem Maße Förschle/Glaum/Mandler (1998).

131 Vgl. so die Anstrengungen der amerikanischen Industrie den SFAS No. 133 zu Finanzderivaten aufzuhalten, mit der Begründung, daß zahlreiche Unklarheiten und Probleme hinsichtlich seiner Anwendung beständen, vgl. SFAS No. 137, insb. Par. 5-11 sowie o.V. (1999); sowie den politischen Einfluß der Unternehmen in der Umsetzung der EG-Richtlinien in Deutschland. Vgl. McLeay/Ordelheide/Young (1999).

132 So. vgl. zum Beispiel die Veröffentlichungen des Hauptfachausschusses (HFA) der Wirtschaftsprüfer und die Statements of Position (SOP) des American Institute of Certified Public Accountants (AICPA). 
desministerium der Finanzen (BMF), vom Bundesministerium der Justiz (BMJ), vom Bundesfinanzhof $(\mathrm{BFH})$ oder von der SEC, oder von der privaten Seite, also z.B. von den Wirtschaftsprüferverbänden, von Börsen, vom DRSC, vom IASC oder vom FASB übernommen wird, in jedem Fall bewegt sich die dann ausführende Instanz außerhalb eines wettbewerbsintensiven Marktes, im Regelfall handelt es sich um eine budgetierte Institution. Die damit verbundene minimale Einkommensunsicherheit der in dieser Institution tätigen Wirtschaftssubjekte führt dazu, daß diese einer Deregulierung tendenziell ablehnend gegenüberstehen. ${ }^{133}$ Ein gutes Beispiel für dieses typische Bürokratieverhalten ist die EU-Kommission, die sich standhaft weigert, von der eher traditionell bestimmten europaweiten Regulierung der Rechnungslegungspublizität abzuweichen. ${ }^{134}$

Somit läßt sich durch die Theorie des public choice ansatzweise erklären, warum sich ein bestehendes Regulierungsgeflecht als sehr beständig erweist und weiter ausgebildet wird. ${ }^{135}$ Tendenziell unklar bleibt jedoch, warum ursächlich die Untemehmenspublizität überhaupt reguliert wurde. Eine gut formierte Nachfragerinstitution, die eine solche Regulierung hätte durchsetzen können, läßt sich historisch nicht eruieren. Deswegen soll im folgenden auf zwei spezielle Argumentationsketten zurückgegriffen werden, die auch auf dem methodologischen Individualismus der public choice theory basieren.

\subsubsection{Krisentheorie}

Als ein klassischer positiver Erklärungsansatz für das Phänomen der Regulierung versucht die Krisentheorie, regulatorische Maßnahmen als Reaktion der politischen Institutionen auf von der Öfentlichkeit wahrgenommene Mißstände zu interpretieren. Nachdem diese behoben sind, verbleibt die Regulierung in der Regel als bewährte Maßnahme im Rechtssystem. Selbst nicht erfolgreiche Regulierungen haben eine gewisse Beharrungstendenz, da einerseits eine Zurücknahme der bestehenden Vorschriften durch die amtierenden Politiker einem Fehlergeständnis gleichkommt, andererseits neue Politiker eher dazu tendieren, neue Regulierungen zu institutionalisieren als alte zurückzunehmen. ${ }^{136}$

133 Vgl. für eine institutionelle Analyse des FASB Fogarty (1992).

134 Vgl. dazu Hulle (1998).

135 Vgl. für weitere positive Analysen des Normensetzungsprozesses der Rechnungslegung beispielhaft Ordelheide (1997; 1998a); McLeay/Ordelheide/Young (1999). Den Versuch eines Modellentwurfs über die Motive der Einflußnahme unternimmt Hakansson (1981).

136 Vgl. grundlegend zur Krisentheorie Joskow (1974), insb. S. 297 f. der für regulierende Institutionen zwe Zustände unterscheidet: Ein gleichgewichtiger, der die Interessen des sozio-ökonomischen Umfeldes stets ausgleicht und ein innovativer, in dem die Institution mit Regulierungen auf Ungleichgewichte im Umfeld reagiert. 
Da Politiker in demokratischen Staatsformen letztendlich vom Votum der Wähler abhängig sind, müssen sie signifikante Nutzeinbußen befürchten, wenn sie keine Maßnahmen ergreifen, um ein von der Öffentlichkeit identifiziertes Problem zu bekämpfen. Somit läßt sich die Krisentheorie von zwei Seiten interpretieren. Einerseits können wirtschaftliche Ergebnisse mit extremen Verteilungswirkungen, die im gesellschaftlichen Konsens als Krise betrachtet werden, als Auslöser von regulatorischen Maßnahmen interpretiert werden, andererseits können Wirtschaftssubjekte, die Regulierungen vom Staat nachfragen, Meinungsintermediäre gezielt dazu bewegen, eine Krisenstimmung durch geeignete Informationssteuerung zu induzieren. Diese kann durch die Regulierungsnachfrager benutzt werden, um die gewünschten Gesetzesänderungen zu bewirken.

Das Entstehen der Rechnungslegungspublizitätsregulierung kann zumindest teilweise durch die Krisentheorie erklärt werden. So entstanden schon die Regeln des Ordonnance de Commerce als Reaktion auf die Verteilungseffekte der betrügerischen Gründungen und Bankrotte in Frankreich des 17. Jahrhunderts. ${ }^{137}$ Für die Implementierung des deutschen BörsG von 1897 versuchten die Agrarlobbyisten der Öffentlichkeit glaubhaft zu machen, daß insbesondere die Terminbörsen den gesamten Berufsstand der Landwirte existentiell bedrohten. Diese Bedrohung sollte sich auch auf die gesamte deutsche Wirtschaft auswirken und somit ein de facto Verbot des deutschen Terminhandels mit Agrarprodukten notwendig machen. ${ }^{138}$ Somit kann das BörsG 1897 eher als ein Produkt einer künstlich erzeugten Krisenstimmung gesehen werden.

Die Regulierung der Unternehmenspublizität am US-amerikanischen Kapitalmarkt entstand als direkte Reaktion auf die Weltwirtschaftskrise im Rahmen der New-DealGesetzgebung Roosevelts und wurde konzipiert, um künftige Krisen am Kapitalmarkt dieser Art zu verhindern. ${ }^{139}$ Auch wenn dieses Ziel nicht erreicht wurde, wie nachfolgende dramatische Kursverluste zeigen, ${ }^{140}$ so wurde die in den dreißiger Jahren eingeführte Kapitalmarktregulierung in der Öffentlichkeit wenig hinterfragt. ${ }^{141}$ Die gesamten New-DealVorschriften waren wohl weniger aus ihrer originären ordnungspolitischen Bestimmung heraus erfolgreich, sondem eher durch ihre Funktion als eine glaubhafte Zusicherung ${ }^{142}$ der US-amerikanischen Regierung, sich in Zukunft extrem für die Erholung der US amerikanischen Wirtschaft einzusetzen. Auch die einzelnen Erlasse der SEC lassen sich teilweise auf das Vorliegen bestimmter als Krisen interpretierter Situationen zurückfüh-

\footnotetext{
137 Vgl. zu dieser Kausalfolge Schmalenbach (1962), S. 18.

138 Vgl. Weber (1897), insb. S. 245.

139 Vgl. Skousen (1991), S. 1-7.

140 So verlor der Dow Jones Industrial Index im August 1987 alleine ca. 30 \%.

141 So jedenfalls Stigler (1964), S. 117.

142 Dieser Begriff ist hier im Sinne von Williamson (1985), S. 163-205 zu verstehen.
} 
ren. So sind die umfangreichen Vorschriften der SEC zur Unternehmenspublizität über Finanzderivate im wesentlichen wohl als eine Reaktion auf die derivatbedingten spektakulären Unternehmensschieflagen wie z.B. der Bearings Bank und der Metallgesellschaft zu verstehen, ähnliches gilt auch für das KonTraG.

Viele Regulierungsentscheidungen im Bereich der Rechnungslegungspublizität können durch zeitlich vorgelagerte Krisen erklärt werden. ${ }^{143}$ Völlig losgelöst von der Eignung der Maßnahmen, die sie induzierenden Probleme zu beseitigen, läßt sich also festhalten, daß die regulierenden Instanzen eher reaktiv als aktiv tätig geworden sind. Dies zeigt sich besonders deutlich im internationalen Zeitvergleich. Hier wird deutlich, daß Regulierungen eines Landes häufig mit zeitlicher Verzögerung von anderen Ländern übernommen wurden, teilweise sogar, obwohl die auslösende Krise im übernehmenden Land gar nicht, oder nur abgeschwächt zu beobachten war. Dieses Phänomen soll im folgenden unter dem Begriff „Follow the Leader Strategie“ etwas eingehender beschrieben werden.

\subsubsection{Follow the Leader Strategie}

Die aus der Managementlehre bekannte Strategie der Nachahmung von erfolgreichem Wirtschaftsverhalten ${ }^{144}$ läßt sich auch in den Bereich der public choice theory übertragen. Die Entscheidung, in welche Bereiche des wirtschaftlichen Lebens durch Regulierung wie eingegriffen werden soll, stellt für Politiker eine strategische Fragestellung dar, die sich durchaus mit der Markteinführung oder Weiterentwicklung eines bestimmten Produkts vergleichen läßt. Es ist für den Politiker rational, sich an Vorbildern zu orientieren. Er wird tendenziell nur solche Regulierungen institutionalisieren, deren Einführung anderen Politkern bereits Nutzen gestiftet hat. Dadurch läßt sich der Siegeszug bestimmter Regulierungskonzeptionen, wie der des Ordennance de Commerce durch das kontinentaleuropäische Handelsrecht oder der Delegation von Erstellungskompetenz an private Rechnungslegungsinstitutionen erklären. Besonders prägnant ausgeprägt ist dieses „Abkupfern" von nationalen Regulierungskonzeptionen auch in der gesamten Weiterentwicklung des deutschen Gesellschafts- und Kapitalmarktrechts zu beobachten. ${ }^{145}$ Durch dieses internationale Angleichen von Regulierungskonzeptionen reduzieren sich gleichzeitig die

143 Vgl. mit ähnlichem Engebnis für die deutsche Rechnungslegung Busse von Colbe (1996).

144 Vgl. zum strategischen Konzept „Follow the leader" Porter (1985), S. 181-191; zur Übertragung auf Fragen der politischen Sphäre sinngemäß Oberender/Cachej (1999), insb. S. 579 und explizit in einem anderen $\mathrm{Zu}$ sammenhang Shachar/Nalebuff (1999); allgemein zum Wettbewerb zwischen unterschiedlichen Regulierem Sauerland (2000).

145 So z.B. die Implementierung der nennwertlosen Aktie und die Ermöglichung des Rückkaufs eigener Aktien. Vgl. hierzu die Begründungen zum Stückaktiengesetz (StückAG) und zum KonTraG, BR-Drucks. 871/97 und 872/97; sowie Pellens/Bonse (1999); zu Stellungnahmen der Regulierer zu den Änderungen des Gesellschafts- und Kapitalmarktrechts vgl. Schmidt-Jortzig (1997) und Däubler-Gmelin (1999). 
Unterschiede zwischen den unterschiedlichen Staatssystemen. Da Unternehmen im Gegensatz zu individuellen Wirtschaftssubjekten in der Lage sind, eher unter Inkaufnahme akzeptabler Transaktionskosten eine Abstimmung mit den Füßen vorzunehmen und dadurch ein anderes Regulierungssystem wählen können, ist dieser Sachverhalt unter dem Titel Standortwettbewerb makroökonomisch von großer Bedeutung. Es ist somit für die Ausgewogenheit der Regulierung der Unternehmenspublizität wichtig, daß auch die Adressaten der Untemehmenspublizität die Möglichkeit besitzen, ihre vertraglichen Engagements international zu differenzieren, um einen fairen internationalen Wettbewerb der Regulierungssysteme zu ermöglichen, der nicht systematisch die Unternehmensseite bevorzugt.

Die Entwicklung der Untemehmenspublizität scheint also gegen Ende des 20. Jahrhunderts durch einen internationalen Regulierungswettbewerb gekennzeichnet $\mathrm{zu}$ sein, der letztendlich zu einer tendenziellen Angleichung der Systeme führt. Diese Beobachtung läßt darauf schließen, daß Regulierungen nicht unumstößlich sind und daß Marktmechanismen im internationalen Rahmen durchaus einen Einfluß auf deren Entstehung und Evolution haben. 


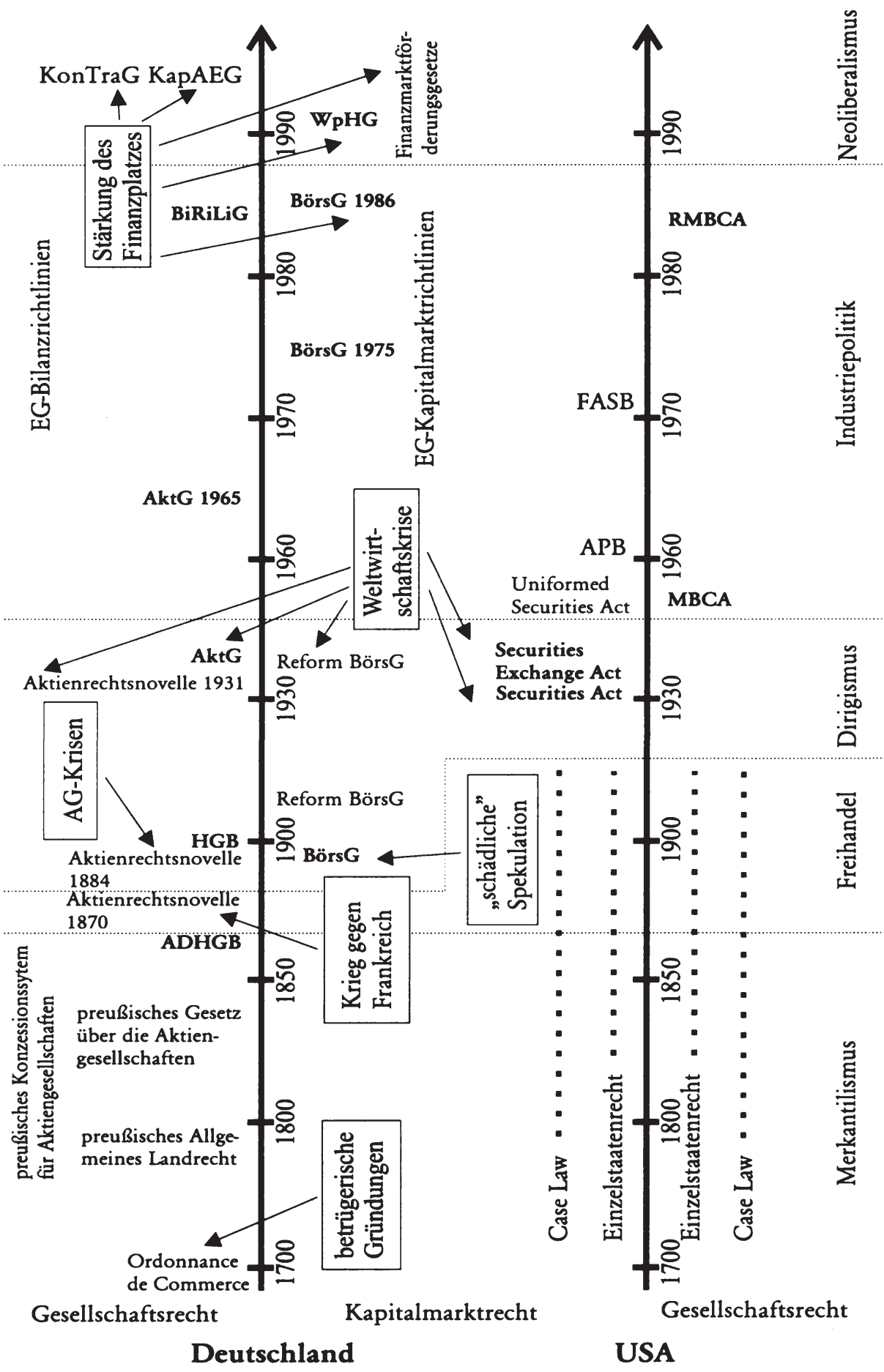

Abb. 4: Krisenregulierungszeitstrahl Deutschland/USA 


\section{Kapitelfazit}

Die bestehenden Rechnungslegungspublizitätssysteme sind historisch gewachsen. Sie entstammen nicht einer einheitlichen normativ basierten Argumentation, sondern sind vielmehr politisches Stückwerk als Reaktion auf als ungünstig eingeschätzte wirtschaftliche Situationen. Dieses Analyseergebnis wird auch durch Abb. 4 wiedergegeben. Auch wenn eine grundsätzliche Publizitätspflicht aus ökonomischer Sicht nicht vollkommen abwegig erscheint, so ist weder die deutsche noch die US-amerikanische Ausgestaltung im Detail normativ begründbar. ${ }^{146} \mathrm{Ihr}$ hauptsächlicher Vorteil liegt in dem Prozeß des historischen Wachstums, der die unterschiedlichen Publizitätssysteme kontinuierlich verändert. Speziell in den letzten Jahren kam der Wettbewerb unterschiedlicher nationaler Konzeptionen hinzu, dessen Resultat fast als Markt für Rechnungslegungspublizitätskonzeptionen bezeichnet werden kann. Auf diesem Markt scheint sich momentan der angloamerikanische Ansatz auf recht breiter Front durchzusetzen. Dies allein ist aus neoliberaler Sicht als Zeichen seiner Superiorität zu werten. Im folgenden Abschnitt soll nun untersucht werden, inwieweit die Ergebnisse der Regulierung die im zweiten Kapitel formulierten Ansprüche erfüllen. 


\section{Kapitel IV}

\section{Eignung der bestehenden Publizitätssysteme}

\section{zur Generierung von Marktprozessen}

Das im zweiten Kapitel formulierte Anspruchsprofil an eine Regulierung der Rechnungslegungspublizität, möglichst viele partielle Marktprozesse zu ermöglichen, ist, wie aus dem dritten Kapitel ersichtlich, bislang noch nicht explizit als Regulierungsbegründung angeführt worden. Nichtsdestotrotz können die durch den politischen Angebots- und Nachfrageprozeß entstandenen Regulierungssysteme sehr wohl diesem Anspruchsprofil genügen. Deswegen soll im nun folgenden Kapitel zunächst der in Kapitel II entwickelte Leitsatz aufgegriffen und anhand der technologisch separierbaren Schnittstellen des Rechnungslegungspublizitätsprozesses mögliche Teilmärkte identifiziert werden. ${ }^{1}$ Im Anschluß daran wird überprüft, inwieweit das deutsche und US-amerikanische Publizitätssystem in den einzelnen Stufen des Publizitätsprozesses jeweils das Entstehen von marktnahen Koordinationsmechanismen unterstützen.

\section{Charakterisierung der relevanten Rechnungslegungsmärkte}

\subsection{Identifikation der Teilmärkte}

Der Prozeß der Rechnungslegungspublizität setzt sich aus mehren Teilstufen zusammen, wie bereits im zweiten Kapitel angedeutet wurde. Hinzu kommt noch die Entwicklung der einzelnen Rechnungslegungsnormen, die durch die nationalen und internationalen Regulierungsgremien aus dem normalen Publizitätsprozeß weitgehend losgelöst wurde und hier somit auch zunächst getrennt betrachtet wird.

Der Prozeß der Rechnungslegungspublizität wird in Datenerstellung, -distribution und analyse differenziert. Diese Unterteilung mutet zwar angesichts der im zweiten Kapitel identifizierten technologisch separierbaren Schnittstellen etwas grob vereinfachend und arbiträr an, soll hier jedoch dennoch verwendet werden, da sie im wesentlichen den heute realiter beobachteten Teilmärkten entspricht.

1 Vgl. zu einer Analyse des Rechnungslegungsprozesses auch Gibbins/Richardson/Waterhouse (1990). 


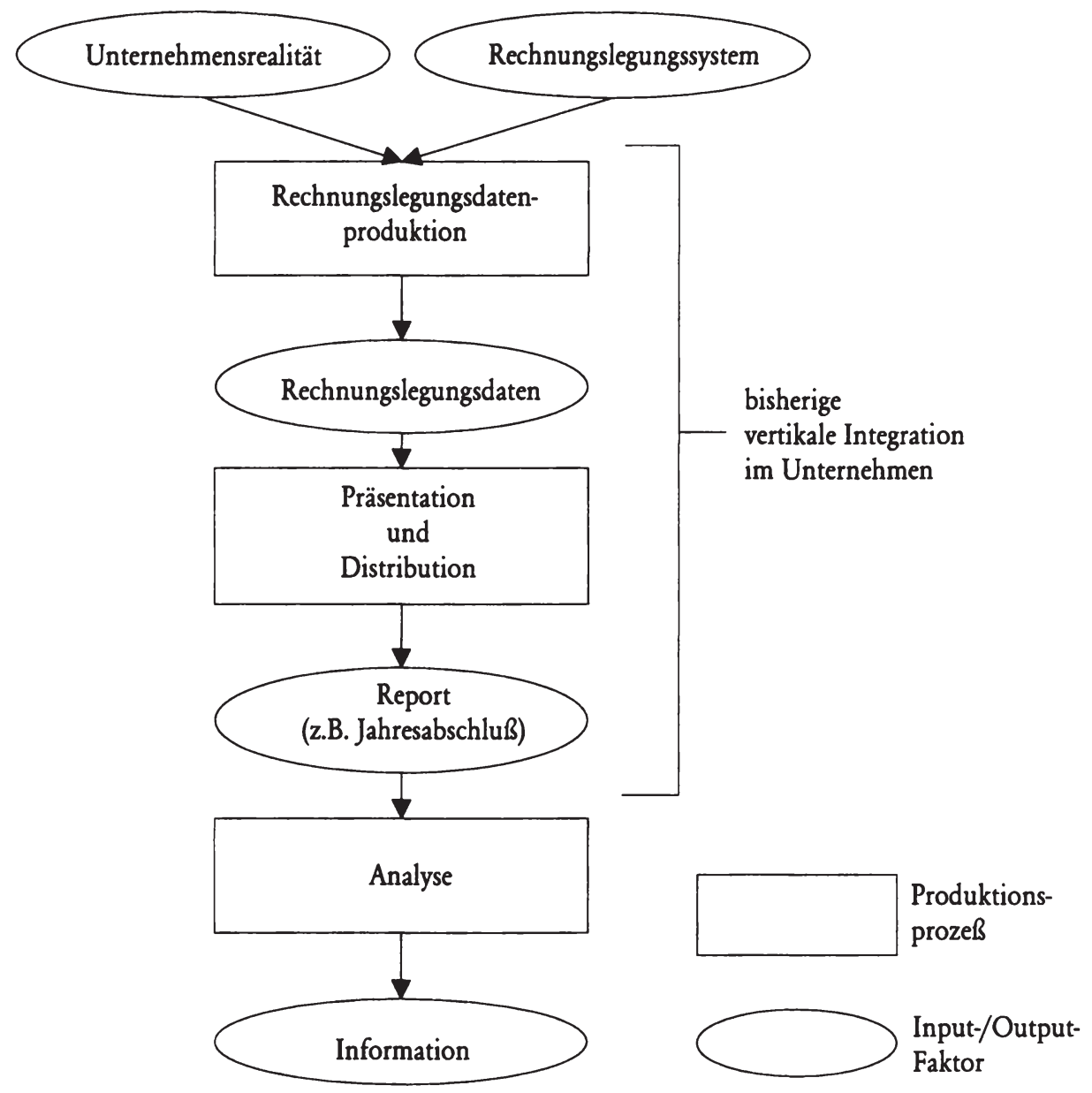

Abb. 5: Marktsegmentierung im Rechnungslegungsprozeß

Wie in Abb. 5 ersichtlich, ist der Großteil des Produktionsprozesses der Rechnungslegungspublizität bislang in den berichtenden Unternehmen angesiedelt. In den folgenden Abschnitten werden die einzelnen Produktionsstufen aus transaktionstheoretischer Sicht analysiert.

Dies soll nach folgendem standardisierten Schema geschehen: Zunächst wird das zu handelnde Gut, also das Ergebnis der jeweiligen Produktionsstufe und der korrespondierende Produktionsprozeß transaktionstheoretisch hinsichtlich der $\mathrm{zu}$ erwartenden Spezifität und Häufigkeit der Transaktion definiert, wobei auch mögliche Teilmärkte für separierbare Zwischenprodukte erwähnt werden. Im Anschluß daran werden potentielle Anbieter und Nachfrager charakterisiert. Abschließend werden mögliche Umwelteinflüsse auf den Marktprozeß dargestellt. 
Begonnen wird mit dem Markt für Rechnungslegungssysteme. Dieser ist zwar nicht direkt Bestandteil des Produktionsprozesses der Rechnungslegungspublizität, ist aber als Produzent einer der beiden Inputfaktoren für das Ergebnis der Rechnungslegungspublizität von zentraler Bedeutung.

\subsection{Markt für Rechnungslegungssysteme}

Unter einem Rechnungslegungssystem wird hier die Gesamtheit von Regeln, mit denen die Abbildung des ökonomischen Geschehens einer Unternehmung in quantitativen Datenstrukturen (den Rechnungslegungsdaten) koordiniert wird und die Regeln zu Aufbereitung der Daten für Veröffentlichungszwecke verstanden. So definiert, wird die Verwendung eines Rechnungslegungssystems unerlässlich, wenn eine Rechnungslegungsdaten enthaltende Unternehmenspublizität angestrebt wird. Ferner umfaßt ein Rechnungslegungssystem somit auch Teile von Regeln, die sonst eher dem Bereich der Publizität zugeordnet werden, insbesondere Ausweisfragen. Die Auswahl eines bestimmten Rechnungslegungssystems kann grundsätzlich unternehmensindividuell getroffen werden. Theoretisch kann es also so viele Rechnungslegungssysteme wie publizierende Unternehmen geben.

Zur Erläuterung der Marktstruktur ist zu untersuchen, wie ein so charakterisiertes Rechnungslegungssystem im wirtschaftlichen Prozeß bereitgestellt werden dürfte und welche Wettbewerbsprozesse entstehen. Das hier betrachtete Gut Rechnungslegungssystem ist hochgradig spezifisch, da es letztendlich den jeweiligen realwirtschaftlichen Besonderheiten eines jeden Unternehmens Folge zu leisten hat. Die eigentliche Transaktion, der Erwerb eines solchen Systems durch das rechnungslegende Unternehmen ist eine einmalige Transaktion, wenn keine im Zeitablauf erforderlich werdenden Anpassungsmaßnahmen berücksichtigt werden. Da diese jedoch bei sich ständig dem Umfeld anpassenden Unternehmen eher die Regel als die Ausnahme darstellen dürften, kann bei der Bereitstellung eines Rechnungslegungssystems eher von einer fortwährenden, dauerhaften Transaktion zwischen Anbieter und Nachfrager ausgegangen werden, deren Intensität und Komplexität zudem mit einer nicht unerheblichen Unsicherheit behaftet sind. Aus den Eigenschaften der hier beobachteten Transaktion kann in einem ersten Schritt geschlossen werden, $\mathrm{da} ß$ eine Marktkooperation im reinen Sinne wohl recht unwahrscheinlich ist. Statt dessen erscheint denkbar, daß das Angebot von Rechnungslegungssystemen in einer vertraglichen Konzeption realisiert wird, die der theoretischen Konzeption eines relationalen Vertrages mit vereinheitlichter Kontrolle ähneln könnte. ${ }^{2}$

2 Vgl. zur Theorie der relationalen Verträge Macneil (1978), S. 886-900. 
Die Bestimmung der potentiellen Vertragspartner für die Transaktion des Gutes Rechnungslegungssystem fällt für die Nachfragerseite leicht: Die Rechnungslegungsersteller eines jeden publizierenden Untemehmens werden, wie o.a., quasi definitorisch zu einem direkten Nachfrager, wenn sie die Produktion der Rechnungslegungsdaten selber übernehmen, ansonsten stellen sie indirekte Nachfrager dar. Tendenziell könnte dies wegen der transaktionskostengünstigen Lösung der vertikalen Integration dazu führen, daß jedes Management sein angewendetes Rechnungslegungssystem selber erstellt.

Diese Argumentation berücksichtigt jedoch noch nicht die Grenzkosten der Produktion eines Rechnungslegungssystems für einen neuen Anbieter und vor allem die ökonomischen Vorteile eines unternehmensübergreifend standardisierten Rechnungslegungssystems. Es kann davon ausgegangen werden, daß die Grenzkosten für die Versorgung eines weiteren Rechnungslegungssystemnachfragers über große Bereiche der relevanten Ausbringungsmenge eines normalen Anbieters monoton fallend sind, so daß für jeden Anbieter ein Anreiz besteht, möglichst viele Nachfrager mit einem Rechnungslegungssystem zu versorgen. Gleichzeitig kann davon ausgegangen werden, daß die Nutzenfunktion der Nachfrager eines Rechnungslegungssystems über gewisse Bereiche negativ korreliert mit den Interpretations- und Auswertungskosten der Rechnungslegungsadressaten. Diese Kosten korrelieren jedoch aufgrund von Netzwerkexternalitäten negativ mit dem Standardisierungsgrad der angebotenen Rechnungslegungssysteme, wenn davon ausgegangen wird, daß Rechnungslegungsadressaten in ihrer Gesamtheit Informationen von unterschiedlichen Unternehmen nachfragen und daß die spezifischen Investitionen, die geleistet werden müssen, um das Know-How für ein spezifisches Rechnungslegungssystem zu erwerben, größer als Null sind.

Somit weist der Markt für Rechnungslegungssysteme eine gewisse Tendenz zum Verdrängungswettbewerb mit einer Möglichkeit des natürlichen Monopols auf. Es läßt sich auch argumentieren, daß ein Rechnungslegungssystem Charakterzüge eines öffentlichen Gutes führt. ${ }^{3}$ Beides könnte aus marktstrukturtheoretischer Sicht der Wettbewerbsintensität abträglich sein: Ersteres, da ein möglicher Monopolist in der Lage wäre, seine Marktmachtstellung zur Rentenerzielung zu nutzen, letzteres, da die Summe der individuellen Nachfrager nach einem öffentlichen Gut geringer ist als die gesamtwirtschaftliche Nachfrage und somit eine tendenzielle Unterversorgung droht. Auf die letzte Fragestellung antwortet der Regulierer mit einer Verpflichtung zur Publizität, die letztendlich die Nachfrage nach einem Rechnungslegungssystem zwangsweise induziert, auf das erste Problem, indem er sich entweder als Anbieter in die Monopolposition begibt und somit

Vgl. z.B. Hax (1988); Pellens/Gassen (1998) und Pellens (1999), S. 549-553. 
die Produktion von Rechnungslegungssystemen verstaatlicht oder indem er einen bestimmten Anbieter auswählt und sich eine Vertragsabbruchoption offenhält.

Eine mögliche Marktaufsplittung hängt stark von den gewünschten Berichtsinhalten des Managements ab, so ist z.B. durchaus denkbar, daß der hier betrachtete Markt wiederum in Teilmärkte für ein System zu Erstellung der Berichtselemente Gewinn- und Verlustrechnung und Bilanz, sowie in ein Teilsystem der Kapitalflußrechnung, in ein Teilsystem der Segmentberichterstattung usw. zerfällt. ${ }^{4}$ Andererseits erscheint es auch denkbar, daß Unternehmenstypen, die z.B. hinsichtlich der Branchenzugehörigkeit oder der Finanzierungsmethoden unterschiedliche Merkmalsausprägungen besitzen, sich jeweils zu Nachfragergruppen von für sie adäquaten Rechnungslegungssystemen zusammenschließen. Für jeden Teilmarkt für sich gelten dann tendenziell wieder die gleichen Strukturüberlegungen.

\subsection{Markt für die Erstellung von Rechnungslegungsdaten}

Durch die Anwendung des Rechnungslegungssystems auf die ökonomische Realität des berichtenden Unternehmens entsteht ein quantitatives Abbild, die Gesamtheit der Rechnungslegungsdaten. Grundsätzlich ist vorstellbar, daß diese Produktion von Rechnungslegungsdaten über die Gesetze von Angebot und Nachfrage koordiniert werden. Bei dieser Tätigkeit hat der Ersteller der Daten, also der Anwender des Rechnungslegungssystems einen Vektor von Einflußmöglichkeiten, z.B. über Bilanzansatz-, Berwertungs- und Ausweiswahlrechte, dessen Dimensionalität vom Rechnungslegungssystem abhängt. Somit besteht auf den Markt für die Erstellung von Rechnungslegungsdaten die Möglichkeit einer heterogenen Konkurrenzsituation, da die Ersteller durch Setzen des Einflußvektors individuell geprägte Rechnungslegungsdaten anbieten können.

In seiner Gesamtheit ist die Produktion von Rechnungslegungsdaten wohl als sehr spezifisch anzusehen, da die Technik der Rechnungslegung sehr stark von individuellen Details der Unternehmung abhängt. Als Inputfaktor für ein periodisches Informationsmedium ist die Datenerstellung eine wiederkehrende Transaktion. Auf Basis dieser Überlegungen erscheint eine Lösung des Vertragsproblems sowohl durch vertikale Integration als auch durch Koordination mittels eines relationalen Vertrags denkbar. Hierbei ist jedoch zu beachten, daß die Spezifität der Rechnungslegung in den Phasen des Produktionsprozesses tendenziell abnimmt: So ist die Basis der Rechnungslegung, die transak-

4 Ähnliche Phänomene sind in Deutschland realiter zu beobachten gewesen, vgl. etwa die HFAVerlautbarungen zur Kapitalflußrechung, vgl. HFA 1/1978 und, in Zusammenarbeit mit dem Arbeitskreis Finanzierungsrechnung der Schmalenbach-Gesellschaft/Deutsche Gesellschaft für Betriebswirtschaft e.V., HFA 1/1995 sowie die DVFA/SG Stellungnahme zur Ermittlung einer speziellen Ergebnisgröße, dem DFVA-Ergebnis, vgl. Busse von Colbe et al. (Hrsg.) (2000). 
tionsorientierte Finanzbuchhaltung, wesentlich enger an die Besonderheiten des Unternehmens geknüpft als die Verwendung spezieller Bewertungsmethoden für einzelne Bestandskonten im Rahmen des Jahresabschlusses, da hier bereits die Rechnungslegungsdaten in einem standardisierten Kontenrahmen vorliegen. Bei Aufspaltung des hier betrachteten Marktes in mehrere produktionstechnisch hintereinander geschaltete Teilmärkte, wie in Abb. 6 geschehen, ist somit ein schrittweiser Übergang von der einheitlichen Kontrolle bis zum klassischen Vertrag als Koordinationsform denkbar.

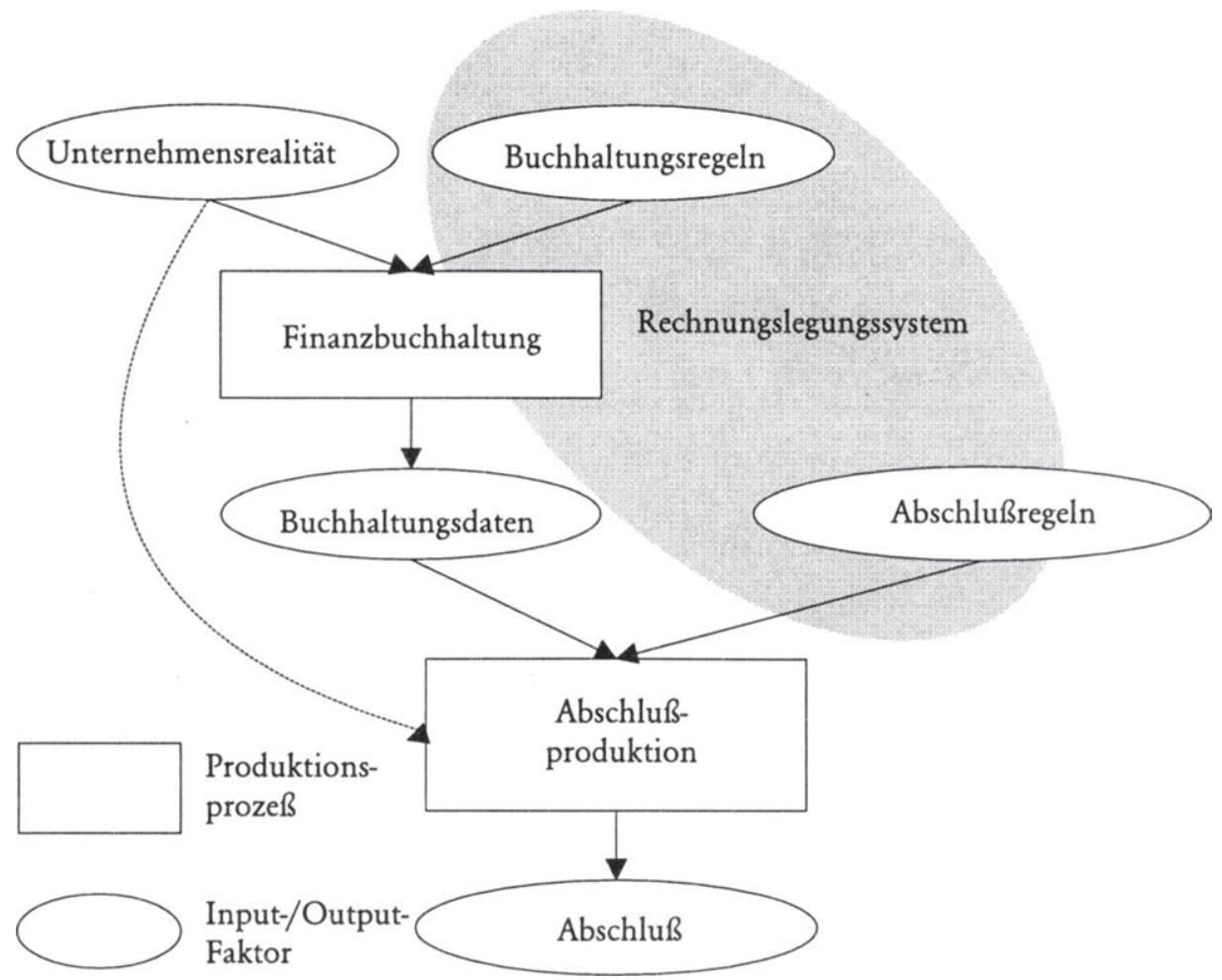

Abb. 6: Marktsegmentierung in der Abschlußerstellung

Ein wichtiger Einflußfaktor, der sich sowohl auf die Marktstruktur als auch auf die Wettbewerbsintensität auswirken kann, liegt in der Informationsasymmetrie zwischen Management und Unternehmensumwelt über die wirkliche Lage des Unternehmens. Hat das Management ein Interesse, diese Informationsasymmetrie zu erhalten, bzw. auszubauen, dann wird eine vertikale Integration der Rechnungslegungsdatenerstellung unumgänglich. Speziell in diesem Fall ist die mögliche Aufgliederung des Marktes von großer Bedeutung. Dadurch entsteht die Möglichkeit, die Bereiche der Rechnungslegung, deren Bearbeitung ein hohes $\mathrm{Maß}$ an Insiderwissen voraussetzt, im berichtenden Unternehmen 
selbst anzugliedern und die anderen, weniger spezifischen Teilbereiche marktnäheren Koordinationsformen zu überlassen. Für diese Teile der Rechnungslegung ermöglichen dann relativ groß dimensionierte Einflußvektoren eine Vielfalt von unterschiedlichen Datenangeboten, während im ersten, vertikal integrierten Bereich der Rechnungslegungsdatenerstellung Einflußvektoren lediglich die Möglichkeit zu verborgenen Handlungen des Managements erweitem und keine direkte Wettbewerbswirkung besitzen. ${ }^{5}$

Wie aus den obigen Ausführungen deutlich wird, hängt die Wettbewerbsintensität des Marktes für Erstellung von Rechnungslegungsdaten nicht unwesentlich von der jeweiligen Ausgestaltung des Rechnungslegungssystems ab. Wenn dieses einerseits die Möglichkeit der Separation des Rechnungslegungsprozesses in mehrere Stufen bietet und anderseits bei den weniger spezifischen Produktionsphasen den Datenerstellern einen relativ großen Einflußvektor bietet, wird zumindest in diesen Bereichen eine marktnahe vertragliche Koordination denkbar. Andererseits beeinflußt auch der Nachfragerkreis die Wettbewerbsintensität. Wenn das Management die Präsentation und Distribution der Rechnungslegungsdaten selber übernimmt, ist es der direkte Nachfrager der Rechnungslegungsdaten. In diesem Fall erscheint es plausibel, daß es auch die Produktion der Daten in Eigenregie vornimmt.

\subsection{Markt für die Distribution von Rechnungslegungsdaten}

Die produzierten Daten müssen noch geeignet präsentiert vom Produzenten zum letztendlichen Konsumenten, also zum Publizitätsadressaten gelangen. Zu diesem Zweck muß einerseits ein adäquates Medium ausgewählt werden, andererseits müssen die Zeitpunkte, bzw. Zeiträume des Angebots festgelegt werden. In ihrer Gesamtheit soll diese Fragestellung hier als Distributionskonzept bezeichnet werden. Nachgefragt wird ein solches Distributionskonzept naturgemäß vom Produzenten der Rechnungslegungsdaten oder von den rechnungslegenden Untemehmen. Die eigentliche Produktion und Realisation eines solchen Konzepts ist relativ unspezifisch und vergleichbar mit anderen Kommunikationskonzepten, wie Wirtschaftsagenturen, wirtschaftlichen Datenbanken und Investmentberatungsgesellschaften. Das Konzept wird zwar im wesentlichen einmalig entwickelt, ist aber ständig geänderten Rahmenbedingungen anzupassen. Die aus dem Konzept resultierende Publizität ist fortlaufend. Somit scheint eine Realisierung der Distribution von Rechnungslegungsdaten über marktnahe Koordinationsformen durchaus denkbar.

5 Diese Überlegungen finden sich analog in der Diskussion, ob Wahlrechte in einem Rechnungslegungssystem zuträglich oder abträglich für die Informationsfunktion sind. Vgl. zur "Discretion vs. Uniformity Debate" Dye/Verrecchia (1995); Wolk/Francis/Tearny (1991), S. 226-240. 
Für die Wettbewerbssituation auf diesem Markt sind die Nachfragerstruktur und die Grenzkosten der Nutzung eines weiteren Distributionskonzeptes entscheidend. Da speziell für elektronische Kommunikationskanäle die Grenzkosten der Informationsübertragung vergleichsweise gering sind und vor dem oben angeführten Hintergrund, daß in der bestehenden Literatur eine Vielzahl von Publizitätsadressaten ausgemacht werden ${ }^{6}$ und der Begriff der Publizität die allgemeine Öffentlichkeit umfaßt, kann angenommen werden, daß die Menge der unterschiedlichen Distributionskanäle in etwa der normalen Menge an allgemein zugänglichen Informationsmedien entspricht. Falls die Menge der vorhandenen Alternativen deutlich davon abweicht, kann dies wohl nur auf eine künstliche Eindämmung der Wettbewerbsintensität hindeuten. Ähnliches gilt, jedoch deutlich abgeschwächt, für die Zeitpunkte und -räume der Informationsdistribution. Hierbei ist jedoch zu bedenken, daß die Umstellung von einem zeitdiskreten zu einem zeitkontinuierlichen Distributionskonzept mit erheblichen Fixkosten verbunden ist. Ebenso ist in einem zeitdiskreten Distributionskonzept der Akt der Abschlußerstellung zu einem bestimmten Zeitpunkt ein wichtiger Kostentreiber für das publizierende Unternehmen. Somit ist zu erwarten, daß die Distributoren von Unternehmenspublizität wesentlich unelastischer hinsichtlich der Nachfrage nach zeitlicher Produktvariation reagieren als hinsichtlich Nachfrage nach Variation des Distributionskanals. ${ }^{7}$

Ein gewisser Grad der Standardisierung kann auch hinsichtlich der Distribution zur Realisation von Netztwerkexternalitäten führen. Standardisierte Daten lassen sich vom Adressaten leichter verarbeiten als solche, für die er unterschiedliche Distributionskanäle in Anspruch nehmen muß. Dies gilt insbesondere, wenn die Daten in elektronisch aggregierter Form vorliegen. Somit ist bei normaler Marktentwicklung zu erwarten, daß Distributionsanbieter entsprechend standardisierte Produkte anbieten werden.

\subsection{Markt für die Analyse von Rechnungslegungsdaten}

Die publizierten Daten der Rechnungslegung stellen per se kein klares Entscheidungssignal dar, da bei Ihrer Produktion die individuellen Maßstäbe des jeweiligen Rechnungslegungsadressaten nicht berücksichtigt werden können. Somit entsteht auf Adressatenseite ein Bedarf nach analytischer Aufbereitung der quantitativen Informationen unter Berück-

6 Vgl. hierzu die Abwägung der unterschiedlichen Adressatengruppen bei Kropff (1993), Sp. 1674; Schildbach/Feldhoff (1993).

7 Hierbei ist jedoch auf bereits referenzierte Vergleichsmärkte zu verweisen. So bieten zahlreiche Wirtschaftsdatenbanken, deren Angebot früher medienbedingt zeitdiskret war, mittlerweile ihre Dienstleistung zeitkontinuierlich an, vgl. dazu z.B. die Mittelstandsdatenbank des Hoppenstedt Verlages oder das Online Angebot von Investmentfirmen wie Bloomberg. 
sichtigung der Verwendung unterschiedlicher Rechnungslegungssysteme. ${ }^{8} \mathrm{Da}$ Rechnungslegungspublizität in der Regel mit einer periodischen Regelmäßigkeit erfolgt, ist die Tätigkeit der Analyse ebenfalls wiederkehrend. Die Spezifität hängt sehr wesentlich vom Standardisierungsgrad der verwendeten Rechnungslegungssysteme und dem jeweiligem Einflußvektor des Erstellers ab. Wenn viele Unternehmen vergleichbare Rechnungslegungssysteme mit relativ geringen Einflußvektoren verwenden, ist die Spezifität der einzelnen Analysetätigkeit verhältnismäßig gering. In diesem Fall erscheint eine marktnahe Koordinationsform denkbar. Je spezieller und unterschiedlicher jedoch die einzelnen Rechnungslegungssysteme und je größer die Einflußvektoren werden, desto mehr spezifische Investitionen sind seitens der Analyseanbieter vonnöten, um aus den publizierten Daten Entscheidungsrichtlinien für die nachfragenden Bilanzadressaten zu gewinnen. In diesem Fall werden aus transaktionskostentheoretischer Sicht eher Koordinationsformen zu erwarten sein, die stärker vom klassischen Vertragsschema abweichen.

Die realiter beobachtbare Marktsituation macht deutlich, daß eine vertikale Integration der Analyse von Rechnungslegungsdaten in die Nachfragergruppen zwar partiell erfolgt, wie z.B. bei institutionellen Nachfragern von Rechnungslegungsinformationen wie Banken als Fremdkapitalgeber, andererseits wird aber auch deutlich, daß es Marktteilnehmer gibt, die sich auf die Prozeßphase der Analyse einseitig spezialisiert haben und somit den Beweis erbringen, daß marktwirtschaftlicher Wettbewerb im Rechnungslegungspublizitätsprozeß durchaus denkbar ist. Ferner können Finanzanalysten als Anbieter von Rechnungslegungsdatenanalyse in ihrer Rolle als Informationsintermediäre helfen, die Auswirkungen der Informationsasymmetrie zwischen Management und Rechnungslegungsadressaten zu mindern. Dazu müssen sie durch glaubhafte Versicherungen sowohl das Management als auch den Adressaten davon überzeugen, daß sie ihr Insiderwissen nicht zum eigenen Vorteil nutzen. Dazu sind sie in dieser Dreiecksrelation eher als das Management in der Lage, da die spezifische Investition in ihren Geschäftsbetrieb viel besser als Geisel geeignet ist, als der Arbeitsvertrag oder die Reputation eines Managers.

Aus diesen Überlegungen wird deutlich, daß der Markt für Rechnungslegungsdatenanalyse für die Funktionserfüllung der rechnungslegungsorientierten Unternehmenspublizität von großer Bedeutung ist. Gerade der Wechsel des Anbieterkreises von den Managements der Unternehmen hin zu extem angesiedelten Finanzanalysten hat einen positiven Einfluß auf die Produktqualität und Nachfrage.

8 Vgl. zur Fragestellung der Bedeutung unterschiedlicher Rechnungslegungssysteme für die Probleme der Jahresabschlußanalyse Ordelheide (1998). 


\section{Einfluß des deutschen Rechnungslegungspublizitätssystems auf die identifizier- ten Teilmärkte}

\subsection{Markt für Rechnungslegungssysteme}

Das in Kapitel III skizzierte deutsche Regulierungssystem beeinflußt erwartungsgemäß die oben identifizierten Teilmärkte des Publizitätsprozesses nicht unwesentlich. Die eigentliche Normenentwicklung, die auf dem Markt für Rechnungslegungssysteme stattfindet, ist spätestens seit dem ADHGB von 1861 in öffentlicher Hand und somit in einer künstlichen Monopolsituation. Zuvor ließ das ALR den Gesellschaftern noch die Möglichkeit, die subsidär ausgestalteten Vorschriften der $\int \$$ 644-646 ALR durch eine andere Regelung im Gesellschaftervertrag außer Kraft zu setzen. Die letztendliche Produktion des Rechnungslegungssystems wurde im Zeitablauf von unterschiedlichen Organen des Staates unternommen. So war vor der Siebenten EG-Richtlinie vor allem das deutsche Justizministerium mit seinen Entwürfen, z.B. des AktG 1965 normbildend tätig.' Aufgrund des durch die Maßgeblichkeit und Umkehrmaßgeblichkeit induzierten engen Zusammenhangs des handels- und steuerrechtlichen Rechnungslegungssystems waren sowohl die Erlasse des Bundesministeriums der Finanzen als auch die Rechtsprechung des Bundesfinanzhofes de facto von großer Bedeutung für die Rechnungslegungsnormengenerierung. Im Rahmen der Entwicklung der EG-Richtlinien hat der deutsche Gesetzgeber den Großteil seiner Normensetzungskompetenz im Bereich der Rechnungslegung und Publizität an die europäische Kommission delegiert. Diese konzipierte, nach umfangreichen Diskussionen unter den Mitgliederstaaten, mit der vierten, siebenten und achten EGRichtlinie ein Normengebäude zur Rechnungslegung, welches die wesentlichen Eckpfeiler einer rechnungslegungsorientierten Unternehmenspublizität enthält. Durch die Transformation der EG-Richtlinien in nationales Recht mit dem BiRiLiG und dem daraus resultierenden HGB 1985 wurde das Angebot des deutschen Souveräns auf einem neuen Stand fixiert. Verbliebene Lücken schloß wie erwähnt die fiskalische Gesetzgebung sowie Rechtsprechung und Bereiche mit Auslegungsspielraum boten einen Tätigkeitsbereich für Wirtschaftsprüfer, Praktiker und die Vertreter von Lehre und Forschung. Der hier entstandene Markt für Normenauslegung kann als ein Teilmarkt für Rechnungslegungssysteme angesehen werden. Sein Entlohnungsmechanismus ist zwar im wesentlichen nicht monetär, scheint jedoch, von der Fülle der kommentierenden Literatur her geschlossen, durchaus funktionsfähig zu sein. ${ }^{10}$

9 Vgl. zu den Regulierungssubjekten in der deutschen Bilanzrechtsgeschichte Penndorf (1913); Vehn (1929); Barth (1953), S. 65-113.

10 Vgl. zu der marktwirtschaftlichen Interpretation der rechnungslegungsorientierten Forschung bereits Watts/Zimmermann (1979). 
Das deutsche Rechnungslegungssystem wird also von einer Vielzahl von Subanbietern unter einheitlicher Kontrolle des Staates angeboten. Da bislang die Anwendung dieses Konglomerats an Regeln durch gesetzliche Vorschriften den berichtenden Unternehmen oktroyiert wurde, konnte kein Wettbewerb auf dem Markt für Rechnungslegungssysteme stattfinden. Zwar kann auch der politische Willensbildungsprozeß mit seinen zahlreichen Teilnehmem in Sinne der public choice theory als Wettbewerbsprozeß angesehen werden, durch seine hohen Transaktionskosten, die wiederum zu Koordinationsmängeln führen, ist er jedoch im Regelfall als inferiorer Mechanismus anzusehen.

Durch die vom KapAEG und KonTraG eingeführten Änderungen des HGB verändert sich die Regulierungssituation auf dem deutschen Markt für Rechnungslegungssysteme deutlich. Einerseits wurde zumindest den börsennotierten Unternehmen erlaubt, für ihre Konzernabschlußpublizität andere, international anerkannte Rechnungslegungssysteme zu verwenden. Andererseits wurde mit $\ 342$ HGB die Möglichkeit für eine private Organisation geschaffen, sich an der Weiterentwicklung des deutschen Rechnungslegungssystems als Anbieter maßgeblich zu beteiligen. Dem mittlerweile gegründeten DRSC wird jedoch vom Gesetzgeber nicht die komplette Normensetzungskompetenz zugestanden, da er sich durch $\int 342$ (2) HGB über das Bundesministerium der Justiz die Möglichkeit offenhält, ihm nicht genehme Normen nicht zu veröffentlichen und ihnen somit den für die Relevanz maßgeblichen Vermutungscharakter nicht zu verleihen.

Die Möglichkeit für börsennotierte Unternehmen, alternative, intemational anerkannte Rechnungslegungsnormen zu befolgen, ist nach Artikel 5 KapAEG zeitlich bis zum 31.12.2004 befristet. Somit ist auch das Zeitfenster des Wettbewerbs auf dem Markt für Rechnungslegungsnormen begrenzt. Die zeitliche Beschränkung der Gültigkeit des $\int 292$ a HGB wurde im Gesetzesentwurf integriert, um dem deutschen Rechnungslegungssystem eine Übergangsphase einzuräumen, in dem es an die internationale Konkurrenz quantitativ und qualitativ herangeführt werden soll. $\mathrm{Daß}$ letztendlich wieder ein deutsches Rechnungslegungssystem den Markt monopolistisch beherrschen soll, steht beim Regulierer anscheinend außer Frage. ${ }^{11}$ Als Argumentation für ein solches Vorgehen wird vor allem von juristischer Seite ins Feld geführt, daß eine Einbettung der Rechnungslegungsvorschriften in das deutsche Rechtssystem, vor allem aufgrund der vielen Rechtsfolgen, unablässig ist. ${ }^{12}$ Die Existenz der in Verfügungsrechte eingreifenden Vorschriften, die an das Rechnungslegungssystem gebunden sind, ist ein Indiz für die lange Tradition der deutschen Rechnungslegungsregulierung und für die Eigenschaft institutionalisierter Vorschriften, weitere Regulierungen zu induzieren. Diese Rechtsfolgen, z.B. die Vorschrift

11 Vgl. hierzu die Stellungnahme des Rechtsausschusses vom 11. Februar 1998, BT-Drucks. 13/9909.

12 Vgl. dazu Hommelhoff/Schwab (1998); befürwortet wird eine dynamische Verweislösung von Heintzen (1999). 
zur Dividendenhöhe in $\$ 58 \mathrm{AktG}$, erschweren in der Tat einen möglichen Wettbewerb für Rechnungslegungssysteme wesentlich.

Auch wenn die deutsche Gesetzgebung in jüngster Zeit dem Wettbewerb auf dem Markt für Rechnungslegungssysteme tendenziell den Rücken gestärkt hat, ist keine wirkliche Marktkoordination zu beobachten und bei grundsätzlicher Beibehaltung der Vorschriften auch in Zukunft nicht zu erwarten. Für diese These sind drei Gründe anzuführen: Erstens ist die Möglichkeit für Unternehmen, andere Rechnungslegungsnormen zu verwenden, zeitlich und vom Umfang her beschränkt, somit ist die gleichzeitige Präsenz mehrerer Anbieter, obwohl international z.B. mit der EU-Komission, dem IASC und dem FASB vorhanden, am deutschen Markt umfassend und auf Dauer nicht möglich. Zweitens führen die Rechtsfolgen vor allem des Einzelabschlusses dazu, daß jede Rechnungslegungsnormenänderung Verteilungswirkungen induziert, die nicht marktwirtschaftlichen Reaktionsmechanismen entsprechen. Drittens ist das auf Basis von $₫ 342$ HGB ins Leben gerufene DRSC so in staatliche Zwänge eingebettet, daß es vorerst keine wirkliche Neuund/oder Weiterentwicklung des bestehenden Rechnungslegungspublizitätssystems auf privatwirtschaftlicher Basis vomehmen kann. Eine mögliche Lösung bestünde in der strikten Trennung des informationsorientierten Konzemabschlusses und des zahlungsorientierten Einzelabschlusses, wobei das Rechnungslegungssystem für ersteren eher marktnah und das System für letzteren durch Regulierung festgesetzt werden kann. ${ }^{13}$ Die Regulierung des Einzelabschlusses wäre dann zu rechtfertigen, wenn politischer Konsens darin bestünde, daß eine „objektivierte“ Ausschüttungsregel benötigt wird. Obwohl durch die Gesetzesänderungen der letzten Zeit ein gewisser Trend in diese Richtung zu erkennen ist, bleibt die Umsetzung noch abzuwarten.

\subsection{Publizitätsprozeß}

Im folgenden sind die Auswirkungen des deutschen Rechtsrahmens auf die drei Produktionsphasen der Rechnungslegungsinformation gemäß Abb. 5 und Abb. 6 zu betrachten. Als erste Stufe wird die Produktion der eigentlichen Rechnungslegungsdaten analysiert. Der vom verwendeten Rechnungslegungssystem gesteuerte Prozeß der Datenerstellung ist hauptsächlich von drei Determinanten abhängig: Von der Möglichkeit, den Produktionsprozeß in technologisch separierbare Teilschritte zu trennen, von dem Interesse des berichtenden Managements an den resultierenden Rechnungslegungsdaten und von dem Einflußvektor, den das Rechnungslegungssystem den jeweiligen Erstellern bietet. Die erste Determinante ist im wesentlichen von der informationstechnischen Infrastruktur der 
berichtenden Unternehmen geprägt und damit von der Regulierung der Rechnungslegung weitgehend unabhängig.

Das Management zieht Nutzen aus der Informationsasymmetrie zu den Unternehmensbeteiligten. Dies resultiert aus versteckten Handlungen: Ein Management, das die Ertragslage quasi beliebig verzerren kann, hat fast unbegrenzte Chancen zur versteckten Konsumtion von Anteilseignervermögen. Andererseits haben die Rechtsfolgen aus Rechnungslegungsdaten den Charakter eines Prinzipal-Agenten-Vertrages: Eine Ausschüttungsregelung wie $₫ 58$ (2) AktG greift in die Verteilung des Gewinns zwischen Management und Eigentümer ein und beeinflußt somit auch die Möglichkeiten zu versteckten Handlungen durch das Management. ${ }^{14}$ Durch den ausgelösten faktischen Mittelabfluß wird der Verfügungsbereich der Manager reduziert. Da dieser Mittelabfluß besser zu steuern ist, wenn das Management die Rechnungslegungsdatenerstellung selber übernimmt, ist zu erwarten, daß Rechtsfolgen von Rechnungslegungsdaten tendenziell dazu führen, daß das Management eher das Bedürfnis haben wird, die Rechnungslegungsdaten selber zu produzieren. Das gilt unter anderem auch dann, wenn die Entlohnung des Managements direkt oder indirekt von Rechnungslegungsdaten abhängig gemacht wird.

Somit sind auch für den Erstellungsprozeß der Rechnungslegungsdaten die in der deutschen Rechtslandschaft fest an diese Daten gebundenen Rechtsfolgen als wettbewerbshinderlich anzusehen, da sie die Bedeutung der Informationsasymmetrie weiter verstärken und somit die Bereitschaft des Managements, Teile der Datenerstellung Dritten zu überlassen, noch weiter reduziert. In diesem Zusammenhang wird auch deutlich, daß es in der Regel von großer Bedeutung ist, wem das Verfügungsrecht zusteht, unter den Anbietern für die Erstellung der Rechnungslegungsdaten auszuwählen. Kann das Management frei entscheiden, wer die Rechnungslegungsdaten erstellt, fällt ihm die Ausnützung seiner asymmetrischen Information ungleich leichter, als wenn diese Entscheidung zum Beispiel vom Aufsichtsrat oder gar von der Hauptversammlung gefällt würde, vorausgesetzt, die Rechnungslegungssysteme unterscheiden sich hinsichtlich der Beseitigung der Informationsasymmetrie. Bislang ist davon auszugehen, daß gemäß $₫ 76$ (1) AktG der Vorstand im Rahmen seiner allgemeinen Geschäftsführungskomptenz auch das Recht und die Pflicht hat, den Ersteller der Rechnungslegungsdaten auszuwählen. Sollte durch Separation der technischen Schnittstellen zwischen unterschiedlichen Erstellern ein Wettbewerb möglich werden, so ist jedoch eine Regelung analog zu dem durch das KonTraG geänderten $\S 111$ (2) AktG denkbar, die den Aufsichtsrat mit der Emennung des Datenproduzenten

14 Vgl. z.B. zur grundsätzlichen Wirkung der Thesaurierungsregeln auf die Beziehung von Management und Kapitalgebern auch Pfaff (1989). Zu unterschiedlichen Ausgestaltungen von Ausschüttungsregeln im internationalen Vergleich Leuz/Deller/Stubenrath (1998). 
beauftragt und so verhindert, daß das Management, dessen Nutzen nicht unwesentlich von den Rechnungslegungsdaten abhängt, deren Erstellung beliebig steuern kann.

Haupthinderungsgrund für einen Wettbewerb unterschiedlicher Rechnungslegungsdaten ist jedoch die Umsetzung der vierten und siebenten EG-Richtlinie in das deutsche Gesellschaftsrecht und die enge Bindung der steuerlichen Rechnungslegung an die Daten des Handelsrechts. Durch die rechtliche Verbindlichkeit bestimmter Rechnungslegungsverfahren haben diejenigen Rechnungslegungsdatenersteller, deren Daten diesen Anforderungen genügen, einen deutlichen Marktvorteil. Berichtende Unternehmen sind zur Erstellung entsprechender Rechenwerke gesetzlich verpflichtet, alternative Rechnungslegungsdaten können nur additiv angeboten werden. Innerhalb der gesetzlich oktroyierten Rechnungslegung exisitiert trotz bestehender Wahlrechte nur ein vergleichsweise kleiner Entscheidungsvektor für den Rechnungslegungsdatenersteller. Somit ist kein wirklicher Wettbewerb zwischen unterschiedlichen Anbietern möglich, da die Aktionsparameter innerhalb des gesetzlichen Rahmens zu eng sind. Die vor diesem Hintergrund bestenfalls zu erwartende homogene Konkurrenz dürfte einem starken Wettbewerb nicht zuträglich sein, sondern eher zum Erliegen des Marktes führen.

Nach der Produktionsphase der Rechnungslegungsdatenerstellung wird als nächster Prozeßschritt und damit als nächster potentieller Teilmarkt die Phase der Präsentation- und Distribution betrachtet. Die Produktion des kompletten Publizitätspaketes „Geschäftsbericht" und die Distribution desselben ist in der Bundesrepublik relativ wenig reguliert. So ist zwar eine Mindestberichtsmenge des Anhangs und des Lageberichts in den $\iint 284$ 289 HGB vorgeschrieben und deren Publizität in den $\iint 325-329$ HGB sowie für bestimmte Untemehmen im PublG gefordert, das berichtende Unternehmen kann jedoch im Geschäftsbericht zusätzliche Angaben vornehmen, die medientechnische Gestaltung ist weitgehend freigestellt. Die Veröffentlichung des (Konzern)-Jahresabschlusses, des Lageberichts, ggf. des Berichts des Aufsichtsrats und der Angaben zu Gewinnverwendung muß entsprechend $₫ 325$ HGB von Kapitalgesellschaften beim jeweilig zuständigen Handelsregister erfolgen. Große Kapitalgesellschaften gem. \$267 (3) HGB müssen diese Rechenwerke zunächst im Bundesanzeiger veröffentlichen. ${ }^{15}$ Somit sind bestimmte Distributionskanäle gesetzlich vorgeschrieben, weitere können jedoch frei gewählt werden. Dabei kann auch von der Schriftform als Kommunikationsmittel und von der physikalischen Distribution als gedrucktes Schriftstück abgewichen werden. ${ }^{16}$ Eine Beobachtung der

15 Die Publizitätspflicht von Personengesellschaften ist größenabhängig im PublG geregelt. Gemäß $\uparrow 1$ PublG große Personengesellschaften müssen im wesentlichen die gleichen Anforderungen wie große Kapitalgesellschaften erfüllen.

16 Vgl. zu den rechtlichen Rahmenbedingungen einer elektronischen handelsrechtlichen Publizität Windbichler (1988). 
bereits erfolgten Diversifikation der Kommunikationsmethoden für die Distribution von Rechnungslegungsdaten zeigt recht deutlich, daß hier bereits über die Pflichtdistribution hinaus Anbieter tätig sind. ${ }^{17}$

Die Analyse der Rechnungslegungsdaten und der weiteren Publizitätsinformationen als letzte Produktionsphase wird nur in recht geringem Umfang von den publizierenden Unternehmen selber angeboten. ${ }^{18}$ Ein Grund hierfür könnte die nicht vorhandene Rechtssicherheit seitens des Managements sein, in welchem Umfang es für eigene Analyseergebnisse juristisch haftbar gemacht werden kann. Dies ist vor allem bei Aussagen mit Prognosecharakter von großer Bedeutung. ${ }^{19} \mathrm{Da}$ das Management am deutschen Markt für Unternehmensanalyse sich eher zurückhält, müssen andere Anbieter in die Bresche springen, wenn der Markt für Unternehmensanalyse funktionieren soll. Der Bereich der Jahresabschlußanalyse ist in Deutschland abgesehen von den normalen privatrechtlichen Regelungen nicht reguliert. ${ }^{20}$ Somit steht es jedem Wirtschaftssubjekt frei, am Markt seine Dienstleistung anzubieten. Wie bereits oben erwähnt wurde, befinden sich zahlreiche Anbieter am Markt, die, teilweise auf bestimmte adressatenorientierte Teilmärkte spezialisiert, ihre Leistungen feilbieten. Somit scheinen hier bereits ausreichende Marktprozesse möglich.

Zusammenfassend läßt sich festhalten, daß die Regulierung der Rechnungslegung in Deutschland durch die Einführung eines de facto Monopols für das staatliche Rechnungslegungssystem mitverantwortlich für den fehlenden Wettbewerb auf dem Markt für Rechnungslegungsdatenerstellung ist. Dieses Monopol erscheint vor dem Hintergrund des staatlichen Gewaltmonopols und der festen Bindung von Verfügungsrechten an die Ergebnisse des Rechnungslegungsprozesses zwar verständlich, eben jene Kopplung der Verfügungsrechte ist vor dem Hintergrund der hier skizzierten Wettbewerbswirkungen jedoch kritisch zu hinterfragen. Im Gegensatz zu der eigentlichen Erstellung der Daten,

17 Die Offenlegung des Jahresabschlusses über Datenbanken wurde in der Literatur bereits vor einigen Jahren diskutiert, vgl. z.B. Müller-Bader (1989); Mohren (1992) und Küting/Mohren (1992). Vgl. zu der Nutzung des Intemets als Kommunikationskanal durch deutsche Unternehemen Deller/Stubenrath/Weber (1997; 1999) und Wagenhofer/Pirchegger (1999).

18 Die Informationstransmission auf Kapitalmärkten wird im Rahmen der Mirkrostrukturanalyse modelltheoretisch und empirisch analysiert. Vgl. als Einführung O'Hara (1995) und zur Rolle der Finanzanalysten Löffler (1998), einführend insb. S. 9-28.

19 Vgl. allgemein zur Prognosehaftung in Deutschland Kersting/Sohbi (1998); zu der Situation in den USA vgl. allgemein zur Publiztitätshaftung Skousen (1991), S. 124133; speziell zu den Besonderheiten der durch die Safe Harbor Rule eingeschränkte Prognosehaftung Skousen (1991), S. 133; Markus (1996) und Lenenbach (1999).

20 Diese Regelungen umfassen z.B. Haftungsregeln und die allgemeinen Regeln zur Kaufmannstätigkeit. Lediglich die Erstellung von Jahresabschlußanalyse zu bestimmten Zwecken, wie z.B. zur Ermittlung der Deckungsstockfähigkeit im Versicherungsumfeld ist auf Verordnungsebene reguliert; vgl. hierzu Linnhoff/Pellens (1994). Im Gegensatz dazu ist der Bereich der Finanzdienstleistung in jüngster Zeit stärker reguliert worden, vgl. dazu Zimmer (1998). 
die von den berichtenden Unternehmen vertikal integriert wird und mehrere konkurrierende Rechenwerke in der Regel nicht entstehen läßt, herrscht auf dem Markt für Distribution der resultierenden Geschäftsberichte und auf dem Markt für die sich mit diesen befassende Analyse eine gewisse Vielfalt, die auf einen grundsätzlich funktionierenden Wettbewerb schließen läßt.

\section{Einfluß des US-amerikanischen Rechnungslegungspublizitätssystems auf die identifizierten Teilmärkte}

\subsection{Markt für Rechnungslegungssysteme}

Im Gegensatz zu der Entwicklung der deutschen Rechnungslegung gibt es in den USA keine gesetzlich fundierte Entwicklung hin zu einem übergreifenden einheitlichen Rechnungslegungspublizitätssystem für alle Unternehmensformen. $\mathrm{Da}$ die gesellschaftlichen Regelungen, wie in Kapitel III aufgezeigt, kein explizites Rechnungslegungssystem vorschreiben, gibt es de jure lediglich für börsennotierte Unternehmen ein zu befolgendes Normengebäude. Die Aufgabe, dieses System anzubieten, hat die SEC im Rahmen der SA Gesetzgebung mit Section 19(a) erhalten. ${ }^{21}$ Über die Vorschriften zur Veröffentlichung von Proxyberichten wirkt diese Kompetenz auch auf die Jahresabschlüsse, die den Aktionären zuzuleiten sind. Die SEC jedoch, eventuell vom Handlungsdruck und fehlender Sachkompetenz in ihrer Entstehungsphase gedrängt, delegierte die Normensetzungskompetenz auf Widerruf zurück an den privaten Sektor. Dabei hält sich die SEC jedoch das Recht vor, jederzeit eigene Standards mit bindendem Charakter für berichtspflichtige Unternehmen zu erlassen. Diese stecken somit quasi den Bereich der erlaubten Rechnungslegungssysteme ab. ${ }^{22}$ Innerhalb dieser Normen besitzen das FASB und seine Vorgängerinstitutionen allerdings den authoritative support nach ASR No. 4 und No. 150. $\mathrm{Da}$ der authoritative support bislang nur jeweils einem Normenanbieter gleichzeitig gewährt wurde, und da das Beseitigen der Normenvielfalt eines der erklärten Ziele der Implementierung der SEC seitens des Kongresses war, ${ }^{23}$ ist davon auszugehen, daß die SEC auch in Zukunft nur einen Normenanbieter am Markt für SEC-konforme Rechnungsle-

21 Bis zur Etablierung der SEC durch den SEA 1934 war diese Aufgabe an die Federal Trade Commission (FTC) übertragen. Durch den SEA, Section 13(b) wurde die Kompetenz auf die regelmäßig bei der SEC einzureichende Publizität erweitert. Eine noch weitere Ausweitung der Regelungsbefugnisse der SEC auf das interne Ablaufsystem der Rechnungslegungsdatenerstellung wurde vom Kongreß durch section 20(a) des Public Utility Holding Company Act von 1935 verabschiedet, vgl. Skousen (1991), S. 116.

22 Zu nennen sind hier die Regulation SX, Regulation S-K, die Financial Reporting Releases (FRR), welche sich mit speziellen Rechnungslegungs- und Offenlegungsfragen befassen und die Accounting and Auditing Enforcement Releases (AAER), die sich mit der Prüfung und Durchsetzung der Normen des Rechnungslegungssystem befassen. Vgl. zu den Veröffentlichungen der SEC zu Rechnungslegungsfragen Carmichael/Lilien/Mellman (1999), chapter 3; überblicksartig Brotte (1997), S. 129-137; Pellens (1999), S. 80-87.

23 Vgl. so Skousen (1991), S. 116. 
gung anerkennen wird und somit in diesem Marktsegement eine Art künstliches Monopol herrscht. ${ }^{24}$ Die mit einem protektionierten Monopol normalerweise verbundenen Effizienzverluste könnten jedoch durch das Verhalten der SEC reduziert werden. Da sich die SEC im Zeitablauf bereits zweimal für einen neuen Anbieter entschieden hat, so bei der Ablösung des Committee on Accounting Procedure (CAP) durch das Accounting Principles Board (APB) und dessen Ablösung durch das FASB, kann davon ausgegangen werden, daß die SEC durch die Entscheidung, wem sie den authorative support gewährt, quasi eine „Als-ob“25 Wettbewerbssituation generiert. Somit sehen sich die von der SEC ermächtigten Institutionen immer der Gefahr ausgesetzt, daß die SEC ihnen ihre Normensetzungskompetenz entziehen könnte. Dieser Anreiz fördert ein Engagement für Rechnungslegungsnormen, die von der SEC befürwortet werden. Die SEC fungiert hier als Nachfrageintermediär und bietet ihrerseits das Angebotspaket „Börsenzulassung“ den Unternehmensleitungen an. Bei dem hier beobachteten Marktsegement handelt es sich also tendenziell um ein bilaterales Monopol, wobei jedoch zumindest die theoretische Möglichkeit für die SEC besteht, auf andere Anbieter auszuweichen. ${ }^{26}$

Außerhalb des Marktsegmentes für das Rechnungslegungssystem börsennotierter Unternehmen bestehen im US-amerikanischen Bereich nur wenig marktbeschränkende Regulierungen. Grundsätzlich sind die Publizitätsersteller in der Wahl des zu verwendenden Rechnungslegungssystems freigestellt. De facto aber ist das von dem FASB und seinen Vorgängerinstitutionen geprägte System der US-GAAP für den Großteil der rechnungslegenden Unternehmen maßgeblich. Dies liegt einerseits an privatrechtlichen Verträgen, vor allem mit institutionellen Kapitalanbietern, welche die Verwendung von speziellen Rechnungslegungssystemen vorschreiben und andererseits in der Funktion der Wirtschaftsprüfer als verifizierende Instanz. Wirtschaftsprüfer bürgen im Rahmen der Principle-AgentBeziehung zwischen rechnungslegendem Management und informationsempfangenden Adressaten mit ihrer Reputation für die US-GAAP-Konformität und damit für die Glaubwürdigkeit der übermittelten Daten. ${ }^{27}$ In dieser Funktion übernehmen sie einen

24 Fraglich ist, inwieweit das IASC als alternativer Normenanbieter zugelassen wird. Vgl. zur aktuellen Diskussion aus regulierungstheorischer Sicht Cox (1999). Es steht jedoch zu erwarten, daß die SEC das IASC nur anerkennen wird, wenn das Rechnungslegungssystem des IASC große Ähnlichkeit zu dem FASB-System aufweisen wird.

25 Vgl. zu dem Begriff „Als-Ob-Wettbewerb“ Lenel (1968); geprägt wurder er von Eucken 1952 in den "Grundsätzen der Wirtschaftspolitik", vgl. die aktuelle Auflage Eucken (1990), S. 295.

26 Horngren (1972) behauptet, daß die Einflußnahme der SEC im Fall des APB dazu geführt hat, daß von einem privaten Standardsetting nicht mehr ausgegangen werden könne. Er bezeichnet das APB als lower management des SEC, daß letztlich nur Vorarbeit leistet. Diese Sichtweise kann jedoch wohl kaum auf das FASB übertragen werden, dagegen spricht allein schon das wesentlich höhere Lohnniveau der FASBMitarbeiter im Vergleich zu den Angestellten der SEC, vgl. zur Lohndifferenz Miller/Redding/Bahnson (1998), S. 158

27 Vgl. zur Rolle der Wirtschaftsprüfung im Rahmen der Rechnungslegungspubliztät Ewert (1990); zur ökonomischen Analyse von Haftungsregeln Dye (1993); jüngst Ewert (1999) und Hillegeist (1999). 
Großteil des ökonomischen Risikos einer Rechnungslegungssystemwahl. Somit wird es für Wirtschaftsprüfer rational, diese Wahl des Managements zu beeinflussen. Dies geschieht in der Regel dadurch, daß der Dachverband der Wirtschaftsprüfer das Befolgen bestimmter Rechnungslegungsregeln für die Testatserteilung voraussetzt. ${ }^{28}$ Damit wird das testatnachfragende Unternehmen de facto zur Verwendung eines bestimmten Rechnungslegungssystems gezwungen. Es verringert sich somit der Prüfungsaufwand aus Sicht des Wirtschaftsprüfers, da er nur noch spezifische Investitionen zum Erlemen eines Rechnungslegungssystems leisten muß. Dadurch reduziert sich auch die Gefahr der Fehlprüfung, und die Vergleichbarkeit unterschiedlicher testierter Jahresabschlüsse wird erhöht. Diese Tendenz zur Marktmonopolisierung erscheint ökonomisch nachvollziehbar und erweckt per se nicht den Anschein, daß marktfremde Kräfte am Werk sind.

Es ist also nur ein Teilbereich der Normenerstellung zur Rechnungslegung gesetzlich reguliert. Aufgrund der Entscheidung der Bundesbehörde SEC, ihre Normensetzungskompetenz auf Widerruf an den privaten Sektor rückzudeligieren, herrschen auch in diesem regulierten Marktsegment zumindest in Teilen marktwirtschaftliche Koordinationsprozesse. Zwar erzwingt die SEC künstlich ein Monopol, aber da sich das von diesem Monopol angebotene Rechnungslegungssystem de facto auch auf den nicht regulierten Teilmärkten durchsetzt, muß hier nicht unbedingt von einer groben Verletzung der marktwirtschaftlichen Koordination ausgegangen werden.

\subsection{Publizitätsprozeß}

Analog zu den Ausführungen zum deutschen Publizitätsprozeß soll hier untersucht werden, wie die Produktion der Rechnungslegungsdaten, deren Distribution im Rahmen der Unternehmenspublizität und die Analyse aus Adressatensicht im US-amerikanischen Wirtschaftsraum wirtschaftlich koordiniert werden. $\mathrm{Da}$ der eigentliche technische Prozeß der Buchführung und Jahresabschlußerstellung im wesentlichen vom nationalen Umfeld unabhängig ist, brauchen die möglichen Schnittstellen innerhalb dieses Prozesses nicht erneut analysiert $\mathrm{zu}$ werden.

Im Gegensatz zu dem deutschen Rechtssystem sind an die Rechnungslegungspublizität in den USA, von der Börsenzulassung abgesehen, keine wesentlichen direkten Rechtsfolgen gebunden, so sind weder die Ermittlung der Steuerbemessungsgrundlage noch die Dividendenzahlungen direkt von den Daten des Rechnungslegungspublizitätsprozesses ab-

28 Vgl. SARB Opinion No. 6, Appendix A, der die Befolgung der Verlautbarungen des APB für alle testierenden Wirtschaftsprüfer verbindlich macht, für die Vorschriften des FASB gilt analor Rule 203 aus den Rules of Conduct of the Code of Professional Ethics of the AICPA. 
hängig. ${ }^{29}$ Dadurch wird die direkte Verteilungswirkung einer Rechnungslegungsänderung wesentlich geringer und das Management hat tendenziell weniger Anreize, die Rechnungslegungsdatenerstellung in Eigenregie vorzunehmen. Andererseits ist in den USA die erfolgsabhängige Entlohnung des Managements in der Ausgestaltung der direkten oder indirekten Kopplung der Vergütung an Maßgrößen der Rechnungslegung wesentlich weiter verbreitet. ${ }^{30}$ Dies könnte für ein stärkeres Interesse des Managements an einer Manipulation der Rechnungslegung sprechen. ${ }^{31}$ Jedoch ist in den USA auch die direkt an den Aktienkurs gekoppelte Entlohnung von großer Bedeutung. ${ }^{32}$

Das Rechnungslegungssystem US-GAAP enthält tendenziell weniger Wahlrechte als die deutsche Umsetzung der vierten und siebenten EG-Richtlinie, somit ist der Einflußvektor im Rahmen der Rechnungslegungsdatenerstellung geringer, wobei Sachverhaltsgestaltungen natürlich weiterhin möglich erscheinen. Dies spricht an sich für einen eher geringen Wettbewerb im Bereich der US-GAAP konformen Rechnungslegungsdatenproduktion, da die möglichen alternativen Rechnungslegungsdaten aufgrund des kleinen Einflußvektors relativ homogen sein dürften. Wie im vorhergehenden Abschnitt schon erwähnt wurde, ist jedoch nur ein kleiner Teil der US-amerikanischen Unternehmen zur Erstellung eines US-GAAP konformen Jahresabschlusses verpflichtet, der Großteil der Unternehmen wählt das Rechnungslegungssystem lediglich vor dem Hintergrund der oben skizzierten Marktprozesse. Somit ist auch ein Abweichen von US-GAAP Vorschriften für nicht börsennotierte Unternehmen denkbar, wenn dies von den Publizitätsadressaten gefordert wird. Diese Möglichkeit wird vor allem von institutionellen Fremdkapitalgebern genutzt, die durch einzelne Regelungen im Kreditvertrag mit dem Schuldnerunternehmen eine bestimmte Ausprägung der Rechnungslegung vereinbaren. An die Daten, die aus diesem Rechnungslegungsprozeß entstehen, werden dann teilweise Ausschüttungsgrenzen oder ähnliches vertraglich gebunden. ${ }^{33}$ Dieses explizite Nachfragen von bestimmten Bilanzierungsverfahren wird nur möglich, wenn der Einflußvektor des Bilanzierenden hinreichend groß ist, oder wenn das (teilweise) Verlassen des verwendeten Rechnungslegungssystems nicht von regulatorischer Seite untersagt ist. Das Zustandekommen dieser privat-

29 Vgl. für den Zusammenhang von informationsorientierter und steuerrechtlicher Rechnungslegung in den USA beispielhaft Kahle (1999); zu anderen Auszahlungsregeln Leuz/Deller/Stubenrath (1998), insb. S. $113 \mathrm{f}$.

30 Vgl. Pellens/Crasselt/Rockholtz (1998), S. 7-11

31 Vgl. zu dieser Fragestellung auch die empirische Untersuchungen von Healy (1985); Holthausen/Larcker/Sloan (1995) und Guidry/Leone/Rock (1999), die zu dem Ergebnis kommen, daß Manager, die abhängig vom Jahresüberschuß entlohnt werden, den Jahresüberschuß tendenziell manipulierend nach unten korrigieren, wenn er über ihrer oberen Bonusbemessungsgrenze liegt.

32 Vgl. zu unterschiedlichen Arten der an Rechnungslegungsdaten gekoppelten Managemententlohnung auch Heintges (1997), S. 27-30; Ittner/Larcker/Rajan (1997).

33 Vgl. zu diesem Vorgehen Leftwich (1983); Alberth (1998); Schildbach (1998); Leuz/Deller/Stubenrath (1998). 
rechtlichen Vorschriften zur Anwendung einzelner Rechnungslegungsnormen ist ein deutlicher Beweis dafür, daß eine gewisse Vielfalt im Ablauf des Rechnungslegungsprozesses möglich und marktwirtschaftlich sinnvoll ist.

Der sich an die Erstellung der Rechnungslegungsdaten anschließende Prozeßschritt der Datenaufarbeitung zu einem Jahresabschluß und zur medialen Präsentation desselben im Rahmen der Unternehmenspublizität ist durch die SEC für die börsennotierten Unternehmen recht detailliert geregelt. So wird der Umfang der jährlichen Publizität börsennotierter Unternehmen im Rahmen des Basisinformationspakets durch Formular 10-K nach Regulation S-K und S-X geregelt. ${ }^{34}$ Dieses Formblatt beschreibt den gesamten Umfang der bei der SEC von berichtspflichtigen Unternehmen jährlich einzureichenden Informationen. Der den Aktionären zuzuleitende annual report und die bei Beantragung von Stimmrechtsübertragung ebenfalls zu veröffentlichen proxy statements sind im wesentlichen eine Teilmenge von Formular 10-K Durch die Vorgabe einer formalisierten Struktur erzwingt die SEC zwar nicht das genaue Einhalten einer bestimmten Datenpräsentation, trägt aber doch erheblich zu einer Standardisierung bei. Diese Standardisierung wird durch die Verwendung eines einheitlichen Distributionskanals noch weiter verstärkt. Das EDGAR-System, welches u.a. auch die elektronische Zuleitung von ausgefullten 10-K Formblättern ermöglicht, führt zu einer weitgehenden Vereinheitlichung der regelmäßigen SEC-Publizität. Davon losgelöst bleibt der eigentliche annual report, der nicht die schlichte Form der elektronisch eingereichten Formularblätter besitzt, sondern mehr aus visuellen Gesichtspunkten für den lesenden Adressaten gestaltet und mit zusätzlicher vorrangig qualitativer Information angereichert wird. ${ }^{35}$

Die detaillierten Vorschriften der SEC zu Publizitätsinhalten und zum Publizitätskanal haben zu einer starken Standardisierung der formularbasierten Publizität und somit zu einem nahezu kompletten Erliegen des Wettbewerbs um eine gelungene Publizitätsdistrubition geführt. Dies gilt jedoch nur für die bei der SEC einzureichenden Berichte. Für die den Aktionären zugesendeten annual reports gilt analog zur deutschen Situation, daß US amerikanische Unternehmen versuchen, durch eine individuelle visuelle und inhaltliche Gestaltung gegenüber ihren Konkurrenten Vorteile zu gewinnen. Ebenso werden neben dem traditionellen Distributionskanal der Schriftform weitere Wege wie die der elektronischen Datendistribution genützt. Da die US-amerikanischen Unternehmen ihren deutschen Konkurrenten in diesem Bereich zwar voraus sind ${ }^{36}$, andererseits jedoch nicht so-

34 Vgl. zu der regelmäßigen jährlichen und unterjährigen SEC-Publizität Brotte (1997), S. 151-159; Carmichael/Lilien/Mellman (1999), chapter 3.

35 Vgl. zur Struktur des US-amerikanischen annual reports, sowie zur Abgrenzung zum Financial Statement SFAC No. 5, Abbildung (ohne Nummer, sortiert um Par. 10).

36 Vgl. Deller/Stubenrath/Weber (1999). Speziell für den US-amerikanischen Markt Ashbaugh/Johnstone/Warfield (1999). 
weit wie bei anderen Bereichen der Nutzung von elektronischen Medien ${ }^{37}$, läßt sich allerdings vermuten, $\mathrm{da} ß$ die relativ frühe Einführung eines elektronischen Publizitätssystems durch die SEC und die damit verbundene öffentliche Verfügbarkeit elektronisch aufbereiteter Rechnungslegungsdaten dazu geführt hat, daß der Wettbewerb um eine effiziente Nutzung elektronischer Kommunikationskanäle für die Unternehmenspublizität in den USA nicht in dem möglichen Maße stattgefunden hat.

Die sich an die Publizität anschließende Analyse der Rechnungslegungsdaten findet in den USA auf einem sehr aktiven Markt statt, der abgesehen von sehr rigiden Verbraucherschutzregeln weitgehend frei von Wettbewerbsregulierungen ist. Die Tatsache, daß dieser Markt in der internationalen Geschichte der Finanzanalyse einer der Vorreiter war und heute eine nahezu marktbeherrschende Position einnimmt, ${ }^{38}$ läßt darauf schließen, daß dieser Markt offensichtlich auch keiner weitergehenden Regulierung bedarf.

Die US-amerikanischen Regulierungen zum Prozeß der Unternehmenspublizität scheinen wesentlich mehr als ihr deutsches Pendant von der Devise geprägt zu sein, nur dort einzugreifen, wo ein Eingriff auch wirklich nötig erscheint. Da sie für einen Großteil der Unternehmen weite Bereiche der Rechnungslegungspublizität unreguliert lassen, können zahlreiche Marktprozesse im Rahmen des Publizitätsprozesses ausgemacht werden. Andererseits herrscht im Bereich für börsennotierte Unternehmen eine sehr hohe Regulierungsdichte. Diese zahlreichen Vorschriften behindern die Evolution der Rechnungslegungspublizität. Eine Weiterentwicklung ist im wesentlichen nur noch durch die SEC und die jeweils von ihr beauftragte private Standard Setting Organisation möglich. Der kleine Einflußvektor der Rechnungslegungsdatenersteller ist nicht in der Lage, innerhalb des Rechnungslegungssystems US-GAAP einen wirklichen Wettbewerb um die am Markt erfolgreichste Rechnungslegungspublizität entstehen zu lassen.

37 So ergeben Zählungen der im Internet registrierten Rechner, daß zum Juli 1999 ca. 40 Millionen Rechner der US-Domänen (ca. 21 Millionen ohne die zunehmend international genutzte com-Domäne) ca. 1,5 Millionen Rechnern aus der deutschen de-Domäne gegenüberstehen, Vgl. zu diesen Zählungen die URL: http://www.isc.org, Stand: 12.11.99. Die nur über Befragung zu ermittelnden Nutzerzahlen des Internets werden gegen Ende 1999 auf rund 50\% der Amerikaner und ca. 20\% der Europäer geschätzt, vgl. die statistischen Informationen unter URL: http://www.nua.ie/surveys/, Stand 12.11.99.

38 Die Größenordnung des US-amerikanischen Marktes für Finanzanalyse läßt sich über einen Vergleich der Mitgliederstärke des US-amerikanischen Verbandes Association for Investment Management and Research (AIMR) mit der Mitgliederanzahl der korrespondierenden deutschen Organisation Deutsche Vereinigung für Finanzanalyse und Anlageberatung e.V. (DVFA) erahnen. Hier stehen etwa 36.000 AIMR-Mitglieder (davon etwa 28.000 aus den USA, Stand: 30.6.1999) etwa 1100 DVFA-Mitgliedern (Stand 1998) gegenüber. Vgl. zu den Daten URL: http://www.aimr.org/professionalism/about/profile.html, Stand 13.11.1999 und URL: http://www.dvfa.de/vorstell.htm, Stand 13.11.1999. 
Wie in diesem Kapitel deutlich wurde, behindert sowohl das deutsche als auch das USamerikanische Rechnungslegungssystem das Entstehen von marktwirtschaftlichen Prozessen im Bereich der Rechnungslegungspublizität. Speziell im eigentlichen Erstellungspro$z e ß$ der Jahresabschlußdaten, in den Fragen der Geschäftsvorfalldefinition, des Ansatzes, der Bewertung, der Konsolidierung und des Ausweises findet quasi kein Wettbewerb statt, alle Entscheidungen werden von dem berichtenden Untemehmen vertikal integriert. Lediglich bei den nicht der SEC-Berichtspflicht unterworfenen Unternehmen in den USA läßt sich die Wirkung von Adressatennachfrage nach bestimmten Bewertungsverfahren auf den Prozeß der Jahresabschlußerstellung feststellen.

Für alle Unternehmen jedoch, die entweder der Berichtspflicht der EG-Richtlinien oder der der US-amerikanischen Börsenaufsichtsbehörde unterliegen, ist der Einflußvektor auf die Jahresabschlußerstellung zu gering, um den Adressaten der Rechnungslegungspublizität signifikanten Einfluß auf die Generierung der Rechnungslegungsdaten zu ermöglichen. Das staatlich vergebene Monopol zur Erstellung eines Rechnungslegungssystems führt sowohl in Deutschland als auch in den USA dazu, daß nur ein Anbieter Verfahren zu Ansatz-, Bewertung- und Ausweisverfahren erstellt. Ohne alternative Rechnungslegungssysteme ist eine wirkliche Ausweitung der wettbewerblichen Rechnungslegungspublizität nicht möglich.

Neben diesem Grund spricht auch die Bedeutung der Wirtschaftsprüfer gegen die Entwicklung von Wettbewerbsprozessen im Rahmen der Jahresabschlußerstellung. Da diese für die Testierung eines Jahresabschlusses unabhängig davon entlohnt werden, welche speziellen Rechnungslegungsvorschriften bei seiner Erstellung angewendet wurden, andererseits aber für die Korrektheit dieses Abschlusses mit ihrer Reputation haften, tragen sie nur die Kosten, nicht per se aber auch den Nutzen eines Wettbewerbs von unterschiedlichen Jahresabschlußdaten. Deswegen tun sie sich sowohl in Deutschland als auch in den USA als Befürworter von klaren Standards hervor und verlangen deren Befolgung als Bedingung zur Testatserteilung.

Schließlich hat auch die Position des Managements Auswirkungen auf die Wettbewerbsinstensität auf dem Markt für Jahresabschlußerstellung. Je stärker der private Nutzen ist, den das Management aus der Abschlußproduktion ziehen kann, desto höher werden die Markteintrittsbarrieren für andere Anbieter, vorausgesetzt, daß das Management das Verfügungsrecht über die Entscheidung hält, welcher Anbieter auszuwählen ist. In diesem Zusammenhang sind vor allem die an den Jahresabschluß mittel- und unmittelbar gebunden Verteilungswirkungen von Bedeutung. So wirkt die deutsche Maßgeblichkeit der Handels- für die Steuerbilanz de facto wie eine Limitierung der möglichen Produktalternativen, da eine starke Präferenz aller direkten Unternehmensbeteiligten außer des Staates 
für ein Rechnungslegungssystem mit möglichst später Gewinnrealisierung entsteht. ${ }^{39}$ Auch wenn in den USA der publizierte Jahresabschluß weniger Verteilungswirkungen induziert, so besitzt doch auch dort das Management einen recht starken Anreiz, die Jahresabschlußerstellung zu kontrollieren, da sie durch die über den Jahresabschluß transformierten Informationen ihr Bild bei ihren Prinzipalen beeinflussen kann.

In diesem vierten Kapitel wurde deutlich, daß das US-amerikanische System tendenziell mehr Marktprozesse im Bereich der Rechnungslegungspublizität zuläßt als das deutsche System. Dies könnte durch den in der US-amerikanischen Öfentlichkeit weiter verbreiteten Glauben an die Selbststeuerungskräfte des Marktes zu erklären sein. Doch letztlich ist der Kernbereich der Erstellung von Jahresabschlußdaten sowohl in den USA als auch in Deutschland weit von einer marktwirtschaftlichen Koordination entfernt. Dies ist an sich nicht unbedingt problematisch, wenn die vertikale Integration der Erstellung und der mehr oder weniger monopolistische Markt für Rechnungslegungssysteme selber durch marktwirtschaftliche Prozesse zustande gekommen ist. Wie jedoch hier gezeigt wurde, läßt sich vermuten, daß letztendlich Regulierungen sowohl in den USA als auch in Deutschland für den Ausschluß des Wettbewerbs verantwortlich waren. Die Festschreibung eines bestimmten Rechnungslegungssystems behindert den theroetisch nachgelagerten Wettbewerb auf den Markt für Rechungslegungsdatenerstellung und -distribution wesentlich.

Bevor der Fokus der Arbeit im nächsten Kapitel auf die Entwicklung eines altemativen Publizitätssystems umschwenkt, läßt sich als Zwischenergebnis festhalten: Es gibt kein intersubjektiv vertretbares, ökonomisch fundiertes, normatives Leitbild für die Ausgestaltung der Rechnungslegungspublizität. Deswegen ist aus ökonomischer Sicht eine weitgehende Einbindung von marktnahen Koordinationsmechanismen in den Rechnungslegungspublizitätsprozeß zu fordern. Weder die deutsche noch die US-amerikanische Rechnungslegungspublizitätsregulierung ist erkennbar vor diesem Hintergrund gestaltet worden. Vielmehr lassen sich beide vorwiegend durch einen Rückgriff auf die positive Regulierungstheorie als dirigistische Eigriffe zur Bewältigung vermeintlicher Krisen erklären. Ungeachtet der festgestellten Objektive der Regulierungen weisen beide Systeme deutliche Defizite hinsichtlich der formulierten Zielvorstellung auf. So behindern künstliche Monopole im Bereich der Rechnungslegungssysteme, ein recht starr geregeltes Publizitätssystem im US-amerikanischen und zahlreiche Rechtsfolgen von Rechnungslegungsdaten im deutschen Bereich die freie Evolution der Rechnungslegungspublizität insbesondere im Bereich der Rechnungslegungsdatenerstellung.

39 Vgl. zur Steuerbelastungswirkung von unterschiedlichen Rechnungslegungssystemen beispielhaft Oestreicher/Spengel (1999). 
Die durch die Regulierung vor allem in den USA erzeugte Quasimonopolstellung des FASB, die umfangreichen Publizitätsregulierungen der SEC und die Verteilungswirkungen des deutschen Rechtssystems sind Ergebnisse eines politischen Prozesses und sollen im Rahmen dieser Arbeit als Fakten akzeptiert werden. Nicht akzeptiert werden soll jedoch das bestehende System der monodimensionalen, vorwiegend papierformorientierten Rechnungslegungsdatenerstellung und -distribution, die das gleichzeitige Anbieten von alternativen Rechnungslegungsdaten, die wiederum auf unterschiedlichen Rechnungslegungssystemen beruhen können, mit nahezu prohibitiv hohen Transaktionskosten belegt. Deswegen soll im nunmehr folgenden, praktischen Teil eine Publizitätskonzeption vorgestellt werden, die das gleichzeitige Angebot von unterschiedlichen Rechnungslegungsdatensätzen unterstützt und somit ungeachtet der Rahmenbedingungen der Rechnungslegungspublizität in der Lage sein sollte, einen Beitrag zur Evolutionsfähigkeit der Rechnungslegung zu leisten. 


\section{Kapitel V}

\section{Entwurf eines datenbankbasierten Publizitätssystems}

Im folgenden Kapitel soll basierend auf den festgestellten Schwachpunkten der deutschen und US-amerikanischen Rechnungslegungspublizität ein neues Konzept vorgestellt werden, daß Wettbewerbsprozesse fur unterschiedliche Ansatz-, Bewertungs- und Ausweismethoden unterstützt, ohne dabei jedoch den Rechnungslegungsadressaten der Gefahr der Informationsüberflutung auszusetzen. Diese Aufgabe wird erfüllt, indem die bisher eindimensional strukturierten Rechenwerke $\mathrm{GuV}$ und Bilanz zu einem multidimensionalen Datenmodell uminterpretiert werden, welches dann nicht mehr auf bestimmte eindeutige Bewertungs- und Gliederungskonzeptionen angewiesen ist, sondem direkt auf die Kontenebene der Buchführung aufsetzt. Die Realisation dieses Datenmodells wird durch die Implementierung eines Data-Warehouse-ähnlichen Ansatzes geleistet, der die Multidimensionalität von Rechnungslegungsinformationen berücksichtigt und aus dem operationalen Datenbestand des Unternehmens nur diejenigen Daten dem Nutzer präsentiert, die dieser für seine Abfragen benötigt.

\section{Theoretische Vorläufer}

\subsection{Normative Arbeiten}

Im Rahmen der in den USA geführten normativen Debatte zur Theorie der Rechnungslegung wurde schon in den 60er Jahren von Sorter ein Ansatz entwickelt, der eine disaggregierte Rechnungslegung über die Wertentwicklung vorschlug. ${ }^{1}$ Er differenziert zwischen dem Wertansatz (value theory) und dem Ereignisansatz (event theory) und kritisiert an dem Wertansatz vor allem die fehlende Möglichkeit, bei multiplen Adressaten das beste Bewertungsverfahren zu ermitteln und die mit der Bewertung verbundene Aggregation, welche unweigerlich zum Informationsverlust führt. ${ }^{2}$ Diese Kritikpunkte sieht er durch den Ereignisansatz zumindest teilweise als geheilt an, da die Annahmen über die Entscheidungsfunktion der Rechnungslegungsadressaten stark reduziert werden: Nunmehr muß nicht mehr eine Hypothese über die präferierten Wertansätze gebildet werden, son-

1 Vgl. Sorter (1969); seinerseits betont dieser die Bedeutung der Überlegungen von Vatter. Vgl. hierzu z.B. Vatter (1963).

2 Vgl. zum Problem der Aggregation auch schon Ijiri (1967), S. 120; Lev (1968) und aus Sicht der Informationsökonomie Feltham (1977); zu einer analogen Betrachtung des informationstheoretischen Prozesses der Rechnungslegung vgl. Lee/Bedford (1969). 
dern vielmehr nur noch über die relevanten Ereignisse, die dann einer Bewertung des Adressaten zugrunde liegen. ${ }^{3}$ Somit ist der Ereignisansatz seiner Ansicht nach eher in der Lage, die Adressaten der Rechnungslegung mit Informationen zu versorgen.

Der eher abstrakte Ansatz Sorters wird von Johnson weiterentwickelt. ${ }^{4}$ Er legt dabei vor allem Wert auf die Spezifizierung von einigen Begriffen, welche bei Sorter eher unreflektiert benutzt werden. So ordnet Johnson den Begriff „Ereignis“ wissenschaftstheoretisch als beobachtbare Größe ein, während er den Begriff „Wert“ als Ableitung auf der Basis von Beobachtung faßt. Seine Definition der Aggregationstätigkeit umfaßt unter der Bezeichnung Summierung die Subelemente Aggregation im engen Sinne, Kombination und Zusammenstellung. Eine Aggregation liegt vor, wenn wiederholtes Auftreten identischer Ereignisse summiert wird. Unter Kombination wird die Zusammenfassung von unterschiedlichen Ereignissen, die gleiche Ausprägungscharakteristika besitzen, verstanden. Eine Komposition hingegen bedeutet, daß unterschiedliche Ereignisse mit unterschiedlichen Charakteristika zu einer Größe zusammengefaßt werden. Jobnson geht davon aus, daß Sorter die Anwendung von Komposition im Rahmen der Jahresabschlußerstellung ablehnt, ist sich jedoch unschlüssig für den Bereich der Aggregation und Kombination. ${ }^{5}$ Ferner betont Johnson, daß Rechnungslegungsdaten nur durch ihre Prognosetauglichkeit den Adressaten Nutzen stiften können und unterscheidet die Prognose in Extrapolation und Vorhersage. Unter einer Extrapolation wird hierbei die rein statistisch fundierte Prognose einer Merkmalsausprägung aus den anderen im Zeitablauf gemessenen Ausprägungen des gleichen Merkmals verstanden, während die Vorhersage unter Verwendung eines Zusammenhangmodells aus den Ausprägungen unterschiedlicher Merkmale eine andere Merkmalsausprägung prognostiziert.

Die beiden Arbeiten von Sorter und Jobnson bilden zusammen den Grundstock der Event Theory. Während die Ausführungen von Sorter eher visionär und recht wenig spezifiziert waren, versuchte Johnson, die Begriffe der Event Theory so zu definieren, daß eine weitere wissenschaftliche Erschließung möglich wurde. Im Laufe der 70er Jahre versuchten sich einige Autoren an der Entwicklung von datenbankgestützten Rechnungslegungssystemen

3 Vgl. Sorter (1969), S. 14.

4 Vgl. Johnson (1970); vgl. auch Sorter/Ingberman (1987), die den Eventbegriff vor dem Hintergrund von Vertragsbeziehungen weiter analysieren.

5 Diese differenzierte Betrachtung wird fortgesetzt von Havelka/Khazanchi (1994), die Aggregation temporal und strukturell differenzieren. Femer versuchen sie, Verbindungen zwischen unterschiedlichen Aggregationsleveln und der Struktur von Entscheidungsproblemen herzustellen. Sie kommen zu dem Ergebnis, daß gut strukturierte Probleme tendenziell niedrig aggregierte Informationen benötigen, während schlecht strukturierte Fragestellungen eine hohe Aggregation erfordern. 
vorwiegend für das interne Rechnungswesen, die auch auf den Ansätzen von Sorter und Johnson fußten. ${ }^{6}$

Colantoni/Manes/Whinston nutzten die Fortschritte der Informationstechnologie, um ein neues Rechnungslegungssystem zu skizzieren. ${ }^{7}$ Dabei wendeten sie sich bewußt vom klassischen System der doppelten Buchführung ab, da dies bei Nutzung von EDV-Methoden unnötige Einschränkungen für das Design von Rechnungslegungssystemen mit sich bringe. Auf der Basis des Ansatzes von Sorter und dem Konzept der multiplen Wertansätze von Ijiri $^{8}$ entwerfen sie ein System, welches die Erfassung beliebig vieler Merkmalsausprägungen eines Ereignisses erlaubt und somit implizit auch unterschiedliche Bewertungskonzepte zuläßt. Ferner verzichten sie ebenfalls auf die Limitierung der zur Erfassung qualifizierten Ereignisse. Einzige Erfassungsbedingung ist, daß die Erignisse den ökonomischen Entscheidungsprozeß innerhalb des Unternehmens beeinflussen können. Diese weite Spezifikation des Datenbestandes impliziert einige Probleme bei der Gliederung und Aufbereitung der erfaßten Ereignisse. Colantoni/Manes/Whinston gehen hierbei davon aus, daß die Ereignisse anhand den ihnen jeweils zugeordneten Merkmalsausprägungen gegliedert werden können. Bemerkenswert an dem vorgestellten Konzept ist weiterhin, daß sie bereits die Notwendigkeit für die Ereignisse verbindende Relationen, wie z.B. den Zusammenhang von Zahlungseingängen und korrespondierenden Forderungen erkannt haben.'

Der Ansatz von Lieberman/Whinston ${ }^{10}$ skizziert ein Datenbanksystem, welches sich in drei Subsysteme aufteilen läßt. Die Mass Data Base enthält sämtliche Daten zu allen innerhalb des Unternehmens identifizierten Ereignissen, wobei eine abschließende Definition des Ereignisbegriffes hier nicht erfolgt. Die in der Mass Data Base implizite Struktur ist jedoch nicht für die Anwender bindend, da diese in dem zweiten Subsystem der UserDefined-Structure ihre eigene beschreibende Struktur für die von ihnen identifizierten Ereignisse verwenden können. Die in dieser Struktur erfaßten Ereignisdaten sind vom Datenbanksystem mit der Mass Data Base abzugleichen, so daß andere Anwender ebenfalls Zugriff auf die gespeicherten Daten haben. Die Aufbereitung der in der Datenbank

6 Vgl. u.a. Colantoni/Manes/Whinston (1971); Lieberman/Whinston (1975); Haseman/Whinston (1976) und Everest/Weber (1977).

7 Vgl. Colantoni/Manes/Whinston (1971).

8 Vgl. Ijiri (1967), S. 101-115 und Ijiri (1975), S. 92-105.

9 Weiterhin gehen sie noch auf Probleme der Speicherung der Daten, sowie auf eine mögliche Beschreibungssprache ein. $\mathrm{Da}_{\mathrm{a}}$ diese Ausführungen vor dem Hintergrund der Weiterentwicklungen der Informationstechnologie nur noch tangential relevant sind, sollen sie hier nicht wiedergegeben werden. Vgl. Colantoni/Manes/Whinston (1971), S. 97-101.

10 Vgl. Lieberman/Whinston (1975); auch in dieser Veröffentlichung finden sich zahlreiche hier nicht wiedergegebene Hinweise auf eine technische Realisierung des vorgestellten Konzeptes. 
gespeicherten Informationen geschieht mit Hilfe des dritten Subsystems, den UserDefined-Functions. Jeder Anwender hat die Möglichkeit, einmal formulierte Datenbankabfragen für die spätere Nutzung zu speichern, die bereits gespeicherten Abfragen beliebig zu kombinieren und auf vom System standardmäßig bereitgestellte Funktionen zurückzugreifen. Ein zentrales Charakteristikum des Ansatzes von Lieberman/Whinston ist somit die starke Flexibilität des Datenbankkonzeptes hinsichtlich der Bedürfnisse der Nutzer, wobei jedoch als Nutzer lediglich unternehmensinteme Subjekte, vor allem die mit der Datenerfassung betrauten, gesehen werden.

Everest/Weber" stellten in einer nachfolgenden Untersuchung die bislang vorgestellten Konzepte einer datenbankgestützten Rechnungslegung vor dem Hintergrund der Entwicklung der Datenbanktheorie in Frage. Sie kamen zu dem Ergebnis, daß nur ein relationaler Datenbankansatz in der Lage sei, eine konsistente unabhängige Datenbasis zu gewährleisten und skizzierten vor diesem Hintergrund ein relationales Datenbanksystem für das interne und exteme Rechnungswesen. Ein auf einem abstrakten EntitätenRelationen-Modell (ERM) ${ }^{12}$ aufbauendes System präsentierte McCartby. ${ }^{13}$ Aufbauend auf einer partiellen Erfassung der ökonomischen Realität eines Unternehmens bildet das von McCarthy präsentierte Rechnungslegungssystem nicht die Realität in Kontenform in Verbindung mit den Gesetzen der doppelten Buchführung ab. Vielmehr wird das berichtende Unternehmen als ein Gemenge von Entitäten und korrespondierenden Relationen betrachtet. Diesen werden im Rahmen der Rechnungslegung Eigenschaften zugesprochen. Durch diese Methode bleibt die eigentliche Grundlage der Rechnungslegung, nämlich die Ereignisse im Sinne von Sorter, als Entitäten und Relationen den Adressaten der Rechnungslegung erhalten. Laut McCartby eignet sich diese Vorgehensweise auch, um die traditionellen Berichtsgrößen der Rechnungslegung, wie Umsatzerlöse, Herstellungskosten etc. zu generieren. ${ }^{14}$

An seine eigenen Ausführungen anschließend entwickelt MrCarthy ein abstraktes und stark vereinfachtes ERM als Basis für den gesamten Datenbedarf des Unternehmens. ${ }^{15} \mathrm{Er}$ tituliert seinen Ansatz, mit Ressourcen-Event-Agent-Ansatz übersetzbar, als „REA Ac-

11 Vgl. Everest/Weber (1977).

12 Die Konzeption eines ERM geht grundlegend auf Chen zurück; vgl. Chen (1976); zum Überblick auch Date (1995), S. 347-371. Er charakterisiert Entitäten als unterscheidbare Objekte, die von Attributen (properties im Sinne von Eigenschaften) beschrieben werden und Relationen als Beziehungen zwischen diesen Objekten. Auch Relationen können wiederum durch Attribute charakterisiert werden. Das ERM ist mit seinen Weiterentwicklungen wohl das zentrale Instrument der semantischen Datenmodellierung, vgl. Date (1995), S. 347-351; Balzert (1996), S. 147-155; zur grundlegenden Technik der relationalen Datenbanksysteme vgl. Codd (1970).

13 Vgl. McCarthy (1979).

14 Vgl. McCarthy (1979), S. $681 \mathrm{f}$.

15 Vgl. McCarthy (1982). 
counting Model“". Aus dieser Bezeichnung werden die wichtigsten Komponenten des Datenmodells deutlich: Ökonomische Ressourcen sind knappe Güter, die einen Nutzen besitzen und sich unter Kontrolle des berichtenden Unternehmen befinden. Ein Event ist ein beobachtbares Ereignis, das zu Änderungen der Ressourcen des Unternehmens führt, z.B. durch Produktion, Verbrauch oder Verteilung. Agenten sind Akteure, die an den Events des Unternehmens teilnehmen können oder die für die Teilnahme der ihnen unterstellten Agenten verantwortlich sind. Eine Untergruppe dieser Agenten sind die betrieblichen Einheiten. Das sind die Agenten, die innerhalb des berichtenden Unternehmens arbeiten. ${ }^{16}$ Diese drei Entitätentypen dienen als Strukturierungsgrundlage des Datenmodells, dessen schematische Grundstruktur in Abb. 7 wiedergegeben wird. Die Zuordnungen von Entitäten zu Relationen werden durch die Linien wiedergegeben.

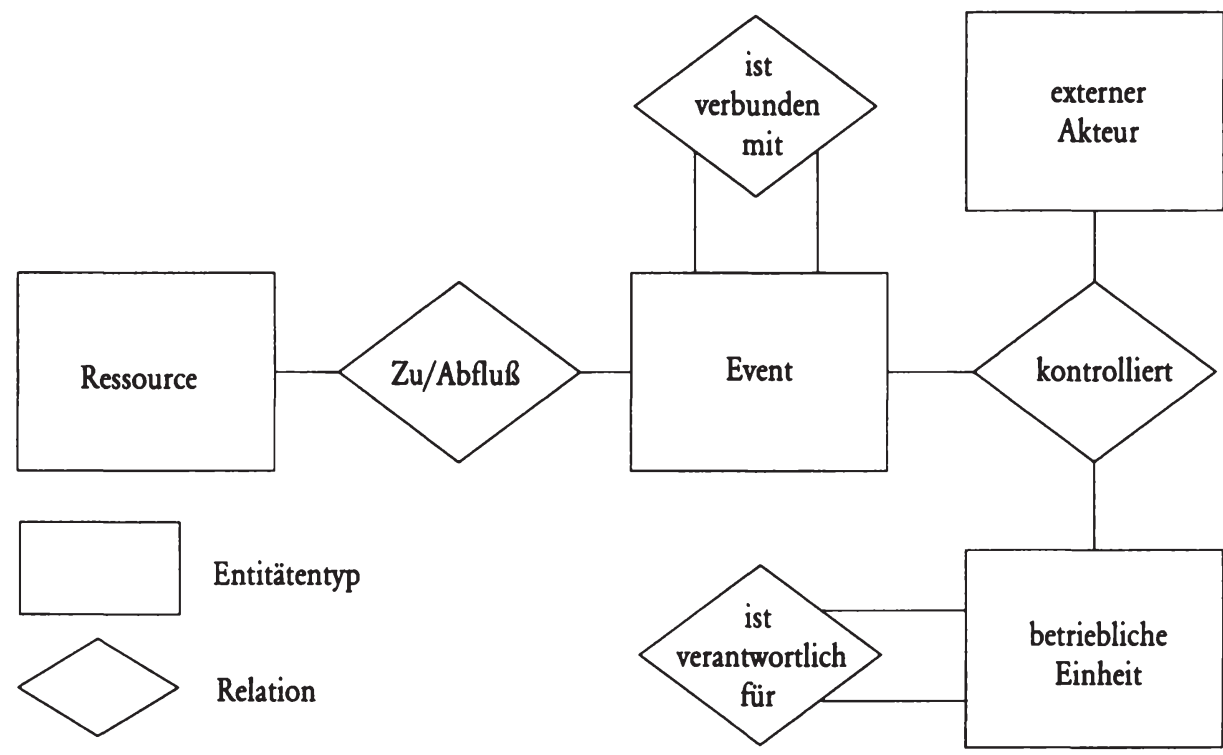

Abb. 7: REA Modell nach McCarthy

Quelle: Vgl. McCarthy (1982), S. 564.

Basierend auf diesem Grundkonzept wird eine Datenbasis skizziert, die auch die paarweise Verbindung von Events zu Dualitäten erlaubt. Diese Event-Dualitäten stellen laut MrCarthy einen Ersatz für die traditionelle Debit/Credit-Betrachtung dar, indem z.B. Kasseneingänge als Ressourcenzuwachs eine Relation mit korrespondierenden Forde-

16 Diese von McCarthy (1982), S. 563 übernommene Definition wirft einige Probleme hinsichtlich der Trennschärfe der verwendeten Unternehmensdefinition auf. Es ist davon auszugehen, daß McCarthy die Abgrenzung der rechnungslegenden Einheit als exogen geklärtes Problem unterstellt und somit die Abgrenzung zwischen ökonomischen Einheiten und sonstigen Agenten als unproblematisch ansieht. 
rungsreaktionen als Ressourcenreduktion eingehen. Die Informationsgewinnung und Datenaktualisierung soll durch sogenannte Prozeduren gewährleistet werden. Diese Prozeduren können mit und ohne Datenmodifikation und unmittelbar oder periodisch ausgelöst werden. Aus diesen Eigenschaften ergibt sich eine Vierfeldermatrix, denen Mc Cartby die vier Prozedurtypen Trigger, Datensicht, Anpassung und Ableitung zuordnet. ${ }^{17}$ Trigger sorgen für eine möglichst zeitgleiche Erfassung von realwirtschaftlichen Events in der Datenbasis, Anpassungen führen diese Änderungen periodisch und aggregiert durch, implizieren somit einen Verzicht auf die ständige Konsistenz der Datenbasis. Eine Datensicht ermöglicht die Präsentation ausgewählter Daten auf Basis des aktuellen Datenbestandes, während eine ableitende Prozedur die Daten periodisierend aufarbeitet. Ein Beispiel für eine ableitende Prozedur ist die Erstellung der jährlichen Gewinn- und Verlustrechnung.

Durch die Möglichkeit, innerhalb der Datenbasis Events in dualen Relationen zu erfassen, lassen sich auch Ungleichgewichte in diesen Relationen protokollieren. Diese Ungleichgewichte können z.B. den Charakter von Forderungen (gelieferte und noch nicht bezahlte Güter), Verbindlichkeiten (erhaltene und noch nicht bezahlte Güter) oder Rechnungsabgrenzungsposten (erhaltene oder geleistete Zahlungen für Güter, die noch nicht geliefert wurden) haben. Obwohl diese Posten nicht selber als Entitäten abgebildet werden, können ihnen im Rahmen von Datensichten entsprechende Attribute aus den unterliegenden Entitäten und Relationen zugeordnet werden. Des weiteren geht McCarthy noch auf einige ausgewählte Probleme des Datenmodells, wie auf die Möglichkeiten der Partionierung und Kombination der auf Basis der Eventdefinition strukturierten Daten, sowie auf das Verfahren der zeitlichen Aggregation ein. Hierbei betont er, daß sein Datenmodell nicht in der Lage sei, die Probleme der zeitlichen Zuordnung und Aggregation von kontinuierlichen ökonomischen Prozessen zu lösen. Er weist jedoch darauf hin, daß diese Probleme erst in der aggregierten Datensicht entstehen und die eigentliche Datenbasis davon unberührt bleibt und somit anderen Nutzern weiterhin unverzerrte Daten zur Verfügung stellen kann. Femer geht er kurz auf die Techniken der Zuordnung von unterschiedlichen Ressourcen zu Events auf der von ihm so titulierten Makroebene ein. So sieht er die Schlüsselung von Gemeinkosten zu expliziten Umsatzevents als eher virtuelle Relation an, die per se in einer von ihm skizzierten Datenbasis zwar möglich, aber nicht notwendig sei. Als ebenso ein Artefakt der doppelten Buchhaltung betrachtet er die Konzeption von außerordentlichen Ergebniskomponenten.

Der Ansatz Mcarthys ist vor allem gekennzeichnet durch eine gelungene Strukturierung der Datenbasis unter Verwendung der durch Sorter, Mattesich und Ijiri geprägten Defini-

17 Vgl. McCarthy (1982), S. 569. 
tionen. ${ }^{18}$ Die konsequente Anwendung des Entity-Relationship-Modelling ermöglicht die Bildung eines generalisierten ERM. ${ }^{19}$ Fraglich ist, ob die von MrCarthy verwendete Methode der Abstraktion und Generalisierung letztendlich die Datenmodellierung erleichtert, oder ob für die Umsetzung doch die generellen Entitäten-Relationen-Paare durch eine Vielzahl von spezifischen Entitätstypen ersetzt werden müssen. Wird eine solche weitgehende Zergliederung des grundsätzlichen ERM unterstellt, so verliert der Ansatz von Mc Carthy einiges an praktischer Handhabbarkeit, da zur Erstellung der Datensichten dann wiederum sehr spezielle Methoden notwendig werden. ${ }^{20}$

Als Reaktion auf die unterschiedlichen Ansätze einer EDV-orientierten disaggregierten Unternehmensberichterstattung wurden im folgenden einige experimentelle Untersuchungen vorgenommen, mit dem Ziel, die Auswirkung von unterschiedlichen Berichtssystemen zu untersuchen. Auf diese Arbeiten wird im folgenden Abschnitt eingegangen.

\subsection{Experimentelle Arbeiten}

Die in den vorgestellten Untersuchungen skizzierten Rechnungslegungskonzeptionen sind von einigen Autoren auf die Wirksamkeit im experimentellen Umfeld hin untersucht worden. So vergleichen Benbasat/Dexter ${ }^{21}$ das Bewertungs- und das Ereigniskonzept auf ihre Eignung als Informationsgrundlage für den Entscheidungsprozeß von unterschiedlichen Adressatentypen. Die tendenziell eher an internen Unternehmensbeteiligten ausgerichtete Studie kommt zum Ergebnis, daß stärker analytische Entscheidungstypen hochaggregierte Abschlüsse präferieren, während eher intuitive Entscheider den Datenbankansatz bevorzugen. Die Performance der Entscheidungen mit beiden Ansätzen war vergleichbar, der Entscheidungsprozeß mit Bezugnahme auf den aggregierten Report war kürzer. Diese Ergebnisse sind auch auf das Modelldesign zurückzuführen, da die Datenbank und der aggregierte Report auf der gleichen Datenbasis beruhen und der aggregierte Report speziell auf das recht spezifische Entscheidungsproblem des Managements im

18 Vgl. Sorter (1969); Mattessich (1964), insb. S. 98-139; Ijiri (1975), insb. S. 109-160.

19 Der letztendlich in der Dissertation von MrCarthy entwickelte Ansatz, vgl. McCarthy (1977) ist der Ausgangspunkt einer Forschungsrichtung, die sich generell mit der Anwendung von unterschiedlichen Datenbanksystemen auf Probleme des Rechnungswesen befaßt. Vgl. beispielhaft Armitage (1983); Adamson (1993) zur Verwendung von objektorientierten Datenbanksystemen und jüngst Debreceny (1998), der experimentell untersucht, ob relationale oder objektoreintierte Datenbanksysteme für Rechnungslegungsinformationen besser geeignet sind. Sein Ergebnis favorisiert objektorientierte Datenbanksysteme.

20 Nichtsdestotrotz ist der Ansatz von MrCartby von großer Bedeutung für den Bereich des Management Accounting, vgl. auch Gal/McCarthy (1986); Armitage/McCarthy (1987); Geerts/McCarthy (1991) und Bailey et al. (1992). Auch im deutschen Sprachraum wurde und wird die Verbindung vom intemen Rechnungswesen (speziell der Kostenrechung) und der Datenbanktheorie diskutiert. Vgl. grundlegend Sinzig (1990); Wedekind (1993); als Überblick Müller-Merbach (1997); ein neueres Implementierungsbeispiel liefert Lackes (1997).

21 Vgl. Benbasat/Dexter (1979); zu der Vorgehensweise vgl. Mason/Mitroff (1973). 
Experiment zugeschnitten ist. In einem auf dem gleichen Versuchsaufbau basierenden Experiment stellen Benbasat/Dexter fest, daß durch den Einsatz von Entscheidungshilfen, z.B. von Simulationstools, ex ante bestehende Unterschiede zwischen der Entscheidungsqualität von analytischen und weniger analytischen Wirtschaftssubjekten weitgehend beseitigt werden können. ${ }^{22}$

In einer experimentellen Untersuchung, die sich speziell mit den Fragen der idealen Aggregation von Managementberichten befaßt, kommen Otley/Dias zu dem Ergebnis, daß stark disagreggierte Berichte die Entscheidungsdauer und -qualität signifikant negativ beeinflussen. ${ }^{23}$ Sie gehen in der Hypothesenbildung von einer konkaven Relation zwischen Aggregationsniveau als erklärender und Entscheidungsqualität als erklärter Variable aus, die sie durch ihre experimentellen Ergebnisse auch bestätigt sehen. Dafür entwickelten sie vier unterschiedliche Berichte mit zunehmendem Aggregationsniveau. Die ersten drei Berichte reduzieren die Menge der Daten, jedoch nicht die Menge der Information. Der vierte Bericht mit der maximalen Aggregationsstufe reduziert auch die verfügbare Information. ${ }^{24}$ Otley/Dias sprechen hierbei von Typ I und Typ II Aggregation. Erwartungsgemäß nimmt die Entscheidungsqualität mit der informationsverlustfreien Typ I Aggregation zu und mit der Typ II Aggregation wieder ab.

Die experimentellen Ansätze beweisen vor allem eins: Unterschiedliche Präsentationsformen und unterschiedliche Aggregationsniveaus machen einen Unterschied. Dies gilt sowohl hinsichtlich der aus ihnen abgeleiteten Entscheidungen, als auch für unterschiedliche Typen von Wirtschaftssubjekten. Wenig Aufschluß hingegen läßt sich durch die experimentellen Einschränkungen darüber gewinnen, ob ein konsequent an Ereignissen orientiertes datenbankgestütztes externes Rechnungslegungssystem bei unterschiedlichen Wirtschaftssubjekten zu besseren Entscheidungen führt. Hierzu sind die simulierten Entscheidungssituationen zu simpel und die jeweils zur Verfügung gestellten Daten zu eindimensional angelegt.

Als Ansatz, diese impliziten Schwächen der experimentellen Rechnungslegungsforschung zu umgehen, sind die Ausführungen von Cushing zu sehen. ${ }^{25}$ Er versucht, unter Bezugnahme auf Ergebnisse der Rechnungslegungs- und Finanzierungstheorie deduktiv abzuleiten, welchen Nutzen, bzw. welche Nutzeneinbuße unterschiedliche, vom Prozeß der externen Rechnungslegung betroffene Gruppen aus der Einführung einer datenbankgestütz-

22 Vgl. Benbasat/Dexter (1982).

23 Vgl. Otley/Dias (1982).

24 Vgl. zu der in der Untersuchung verwendeten Definition von Informationsmenge (hier als Datenmenge bezeichnet) Lev (1968) und zum gemessenen Wert der Information (hier als Informationsgehalt bezeichnet) Feltham (1977).

25. Vgl. Cushing (1989). 
ten, ereignisorientierten Untemehmenspublizität ziehen bzw. erleiden werden. Als unterschiedliche Gruppen identifiziert er Investoren, Finanzanalysten, Kunden, Rechnungslegungsforscher, Mitarbeiter, Lieferanten, Wirtschaftsprüfer, Jahresabschlußersteller, staatliche Regulierer, Manager und die datenverarbeitende Industrie. Er kommt zum Ergebnis, daß staatliche Aufsichtsstellen für Unternehmenstätigkeiten, die Investoren ohne Zugang $\mathrm{zu}$ privaten Informationen über das Unternehmen, die Kunden sowie die datenverarbeitende Industrie mehr oder weniger stark ein datenbankgestütztes Publizitätssystem befürworten dürften, während er die Finanzanalysten, die Manager, die Rechnungsleger innerhalb der Unternehmen, die Lieferanten, die bisherigen Regulierer von Rechnungslegungsnormen, die Wirtschaftsprüfer und die Investoren mit Insiderwissen als Opponenten des Ansatzes identifiziert. Keine eindeutige Prognose gibt er für Arbeitnehmer. Darauf aufbauend prognostiziert Cushing als Reaktion auf eine datenbankgestützte Unternehmenspublizität einen Trend kleiner, öffentlich notierter Unternehmen weg vom organisierten Kapitalmarkt, sowie eine Vorreiterrolle der Unternehmen mit Bezug zum Markt für Informationstechnologie. So interessant die Ausführungen von Cushing sein mögen, so fehlt ihnen doch aufgrund des eher spekulativen und deduktiven Charakters die wissenschaftliche Fundierung. Ein eindeutiger Schluß für oder wider ein datenbankgestütztes ereignisorientiertes Rechnungslegungspublizitätssystem läßt sich daraus nicht ableiten.

\subsection{Engebnisse}

Die von Sorter und Johnson geprägte Konzeption der ereignisorientierten Rechnungslegungspublizität ermöglicht die Entwicklung von unterschiedlichen Aggregations- und Bewertungsmethoden auf der Grundlage einer einheitlichen Datenbasis. Somit können die unterschiedlichen Adressaten selber entscheiden, welche Informationen für ihren Entscheidungsprozeß notwendig sind. Dies verlagert die Schnittstelle, an der die Transformation der Information vom Rechnungslegungsdatenersteller zum Rechnungslegungsdatenempfänger erfolgt, in eine frühere Phase des Erstellungsprozesses. Die auf diesem Entwurf basierenden Konzepte einer datenbankgestützten Publizität bürden jedoch die gesamte Last der Datenaggregation und Bewertung a priori dem Adressaten auf. Aus diesem Blickwinkel ist auch zu verstehen, warum die experimentellen Ergebnisse nicht uneingeschränkt für eine Datenbankpublizität sprechen: Während bei der klassischen hochaggregierten Publizität die Erstellung vollkommen auf Seiten des berichtenden Unternehmens liegt, ist bei der Umsetzung des ereignisorientierten Ansatzes der Adressat der Rechnungslegungsdaten für die Auswertung verantwortlich. Auch bedingt durch die noch nicht so weit entwickelten technischen Möglichkeiten, sehen die Protagonisten der datenbankgestützten Rechnungslegungspublizität nicht die Möglichkeit, durch eine geeignete Standardisierung der Datenbankschnittstelle zur Außenwelt die Bewertung und Aggregation der angebotenen Daten Softwareprodukten von Dritten und damit Markt- 
prozessen zu überlassen. Diese wären sowohl in der Lage, aus der vorliegenden Datenbasis einen hochaggregierten Jahresabschluß mit einem einheitlichen Bewertungsverfahren zu erstellen als auch auf Benutzerwunsch hin unterschiedliche Wertansätze und Datensichten gegenüber zu stellen. Ohne diese weitergehende Aufbereitung der aggregierten Daten kann auch der in dieser Arbeit erwartete normative Vorteil eines solchen Modells, mehr Wettbewerb im Ablauf der Rechnungslegungspublizität zu ermöglichen, nicht realisiert werden.

Die datenbanktechnischen Ansätze, speziell die Konzeption eines ERM von McCarthy, können als gedankliche Grundlage für die nun folgende Konzeption eines Systems zur datenbankgestützten Rechnungslegungspublizität gesehen werden. ${ }^{26}$ Zur sprachlichen Vereinfachung soll dieses System im folgenden als Public Accounting Information System (PAIS) bezeichnet werden. Zentrale Anforderung an ein solches System soll die klare Standardisierung der extemen Datenbankschnittstelle sein, da nur diese einen Zugriff über unterschiedliche konkurrierende Retrievalsysteme erlaubt. Dem ereignisorientierten Ansatz soll insofern gefolgt werden, als daß die inhaltliche Grundlage der Datenbank die ökonomischen Transaktionen des Unternehmens darstellen und nicht die monetäre Erfassung derselben im Rahmen einer traditionellen Finanzbuchhaltung. Andererseits wird nicht dem von MCarthy vorgestelltem ERM gefolgt, da dies durch seine recht geringe Operationalisierung nicht zur Entwicklung von standardisierten Kommunikationsschnittstellen für die Rechnungslegungspublizität geeignet erscheint. Statt dessen wird auf Konzepte zur Verdeutlichung multidimensionaler Datenstrukturen zurückgegriffen. Hierbei wird, wo es sinnvoll erscheint, auf Parallelen zu im internen Rechnungswesen bereits verbreiteten Management Support Systems (MSS) verwiesen. ${ }^{27}$

26 Eine gewisse, eher technisch geprägte Verbindung besteht auch zu den unterschiedlichen Ansätzen, auf Matrizen als Beschreibungssprache für Rechnungslegungsdaten zurückzugreifen. Vgl. als Überblicksartikel für diese hier nicht weiter vertiefte Forschungsrichtung Leech/Mepham (1991). Es wurden weiterhin einige Arbeiten veröffentlicht, die sich mit einer multidimensional strukturierten disaggregierten Rechnungslegung befassen und die als konzeptionell beeinflussend für die folgenden Überlegungen gelten können. Vgl. z.B. die Ausführungen von Field (1969); Rappaport (1986), S. 45-49; Puterill/Debreceny/Kerkin (1994); Wallman (1995; 1996; 1997); Pratt/Storrar (1987), S. 217; Pellens/Fülbier/Gassen (1998; 1999) und Ordelheide (1999; 1999a). Des weiteren werden auch interne Berichtssysteme vorgestellt, die konzeptionelle Ähnlichkeiten zu dem hier diskutierten Entwurf aufweisen. Vgl. Fischer (1997); Boggs (1999).

27 Vgl. grundlegend zu MSS Gluchowski/Gabriel/Chamoni (1997); Gabriel/Gluchowski (1997). Vgl. auch Mauldin/Ruchala (1999), die die Bedeutung von MSS für die Entwicklung einer breiteren Theorie der Accounting Information Systems betonen. Als aktuelle Monographie vgl. Fritz (1999). 


\subsection{Datenhaltung im Unternehmensverbund}

Das in diesem Kapitel konzipierte Publizitätssystem greift auf die Daten des berichtenden Unternehmens zurück. Deswegen kann seine Konzeption auch in dem hier anvisierten Abstraktionsgrad nicht ohne explizite Berücksichtigung der unternehmensinternen Datenorganisation erfolgen. Empirische Untersuchungen belegen, daß Unternehmen u.a. abhängig von ihrer Geschichte, Größe, Marktsituation und Branchenzugehörigkeit sehr unterschiedliche Datenhaltungskonzeptionen und -techniken einsetzen. ${ }^{28} \mathrm{Je}$ nach dem Grad der Zentralisierung werden Daten in verteilten Systemen oder in einem zentralen System vorgehalten. Unter zentralen Systemen wird hier die Bündelung der Daten in einem Netzwerkpunkt verstanden, während sie bei einem verteilten System auf mehrere Netzwerkpunkte verteilt werden. ${ }^{29}$ Bei verteilten Systemen mit einer logischen Gesamtsicht ist die Möglichkeit der Verbindung der einzelnen Datenbestände gegeben, wohingegen sie in sogenannten Insellösungen nicht realisierbar ist. In der wirtschaftsinformatischen Literatur wird in der Regel von einer Strukturierung der Daten entsprechend ihrer Verwendung ausgegangen. ${ }^{30}$ Die Grundlage aller Unternehmensdaten bildet der Transaktionsdatenbestand, welcher die mengenmäßigen Güter- und Finanzströme innerhalb des Unternehmens erfaßt. Diese Transaktionsdaten werden durch Administrationssysteme kontrolliert. In der Unternehmenspraxis werden diese Datenbestände häufig nach den unternehmerischen Funktionsbereichen gegliedert erfaßt, wobei regelmäßig Redundanzen zu beobachten sind. ${ }^{31}$ Die Summe dieser Datenbestände stellt in der Regel die Gesamtheit der über das interne Unternehmensgeschehen vorrätigen Daten dar, auf die nachfolgende Datenverarbeitungssysteme zurückgreifen.

Unmittelbar mit der Transaktionsdatenbank verbunden sind in der Regel die unternehmensinternen Abrechnungssysteme. Ihre Aufgabe ist es, aus den mengenorientierten Transaktionsdaten monetäre Wertansätze zu gewinnen. Klassische Vertreter sind die traditionelle Finanzbuchhaltung und Kostenrechnungssysteme. Diese Systeme gewinnen aus dem Transaktionsdatenbestand in Verbindung mit zur Verfügung gestellten Wertansätzen, Bewertungsmethoden und Aggregationsverfahren eine neue Datenmenge, welche als Grundlage für Steuerungs- und Kontrollanalysen dient. ${ }^{32}$ Aufbauend auf diesen Daten

\footnotetext{
28 Vgl. z.B. die Untersuchung von Henneböle (1995), insb. S. 35-44 zu Informationsstrategien und Entscheidungsunterstützungssystemen.

29 Vgl. auch Scheer (1997), S. 7481.

30 Vgl. z.B. Gluchowski/Gabriel/Chamoni (1997), S. 4451.

31 Vgl. Scheer (1997), S. 410:

32 Vgl. Gluchowski/Gabriel/Chamoni (1997), S. 46.
} 
dienen Berichts- und Kontrollsysteme zur Erstellung von Reports und zur Bearbeitung gutstrukturierter Entscheidungsprobleme, wie das Debitorenmahnwesen, Lagerhaltung und einfache Kostenkontrollsysteme. ${ }^{33}$

Diese grundlegenden Datenverarbeitungssysteme werden zusammenfassend auch als operative Informationssysteme bezeichnet. ${ }^{34}$ Auf deren Daten bauen wiederum die Planungsund Kontrollsysteme auf, die auch als analyseorientierte Informationssysteme bezeichnet werden. ${ }^{35}$ Diese werden in Abhängigkeiten von der Benutzerinteraktion als passive oder aktive Systeme eingeteilt. Ein aktives System wird ohne explizite Aufforderung durch den Benutzer tätig. So wird ein reines Berichtssystem wie eine Kostenstellenrechnung in der Regel nach dem Überschreiten bestimmter Zeitpunkte selbständig aktiv und produziert den periodischen Bericht. Im Gegensatz zu diesen periodischen Systemen werden aperiodische Berichtssysteme von dem Vorliegen bestimmter Datenkonstellationen ausgelöst. Passive Planungs- und Kontrollsysteme werden von Benutzereingriffen gestartet. Wenn die Interaktion zwischen Bediener und System einmalig ist, wird das System als Abfragesystem bezeichnet. Bei der Möglichkeit der Weiterverwendung der Abfrageergebnisse in neuen Kommunikationsschritten handelt es sich um ein Dialogsystem. In die Planungsund Kontrollsysteme können gegebenenfalls ein oder mehrere alternative Entscheidungsmodelle eingebunden werden. Diese ermöglichen dann nicht nur das spezifische Retrieval der Datenbasis, sondern bieten gleich eine Entscheidungsempfehlung.

Die analyseorientierten Informationssysteme dienen der Versorgung von Entscheidern mit relevanten Informationen, während die operativen Informationssysteme vorwiegend der Datenorganisation und der Lösung von einfach strukturierten Entscheidungsproblemen dienen. ${ }^{36}$ Deswegen ist eine enge Verbindung und datenbanktechnische Kopplung der Systeme an die Transaktionsdaten von großer Bedeutung. Nur durch diesen Nexus hat das Management überhaupt die Chance, für seine Entscheidungsmodelle relevante Informationen nachzufragen. Die Probleme bei der Realisierung einer unternehmensweiten Datenbasis in Verbindung mit einem aufbauenden MSS liegen in der Heterogenität der datenverarbeitenden Infrastruktur, welche die Hardware, die Betriebssysteme, die Datenbanksysteme und die Datenmodelle umfaßt. ${ }^{37} \mathrm{Im}$ folgenden wird $\mathrm{zu}$ untersuchen

33 Vgl. Gluchowski/Gabriel/Chamoni (1997), S. 46; Behme/Mucksch (1998), S. 14; allgemein zum EDVgestützten betrieblichen Berichtswesen Gluchowski (1998a).

34 Vgl. Chamoni/Gluckowski (1999), S. 10 f.; Müller (1999), S. 11-14; speziell zu operativen Informationssystemen S. $12-86$.

35 Vgl. Chamoni/Gluckowski (1999), S. 10 f.; Müller (1999), S. 11-14; speziell zu analyseorientierten Informationssystemen S. 87-152; zur Grundsätzlichen Konzeption von unternehmensweiten Informationssystemen Kim/Everest (1994).

36 Vgl. Müller (1999), S. 87; Behme/Mucksch (1998), S. 13 f.

37 Vgl. speziell zur Schnittstelle zwischen operativen und analyseorientierten Informationsystemen umfassend Müller (1999), insb. S. 153-217. 
sein, an welcher Ebene der unternehmensinternen Datenbasis ein öffentlich verfügbares Informationssystem zur Rechnungslegungspublizität anzusetzen ist und wie der Unternehmensdatenbestand der operativen Informationssysteme integriert werden kann. Dazu soll zunächst auf die Unterschiede zwischen einem öffentlich erreichbaren und einem internen Informationssystem eingegangen werden.

\section{2 Öfentliches Informationssystem vs. internes Informationssystem}

Zentrales Problem der Informationsselektion, -aggregation und -präsentation ist die fehlende Möglichkeit, intersubjektiv nachvollziehbare Regeln für diese Prozesse zu entwikkeln. Dieses Problem erschwert bereits die Entwicklung von MSS, da keine Klarheit darüber herrscht, welche Daten für welchen Entscheider in welcher Präsentationsalternative optimal sind. ${ }^{38}$ Durch die Ausweitung des Nutzerkreises von internen auf externe Adressaten wird dieses Problem noch verschärft. In einem hierarchisch organisierten Unternehmensverbund ist es noch denkbar, daß die unterschiedlichen Informationsnachfrager von der Unternehmensleitung ggf. diktatorisch agierend auf eine einheitliche Linie gebracht werden und so die Diversität der unterschiedlichen Anforderungsprofile reduziert wird. ${ }^{39}$ Für ein öffentliches Informationssystem hingegen ist eine solche Vorgehensweise nicht möglich, da es, wie insbesondere im zweiten Kapitel gezeigt wurde, keine normative Hilfe für die Selektion des intersubjektiv optimalen Informationssystems gibt. Somit gilt für ein öffentlich verfügbares Informationssystem in gesteigertem Maße die Forderung nach Flexibilität. Dies gilt sowohl im Rahmen der Datenselektion als auch im Rahmen der Präsentation.

$\mathrm{Da}$ durch ein öffentlich verfügbares Informationssystem der Adressatenkreis über die Unternehmensgrenzen hinweg ausgeweitet wird, ist der Grad der Vertraulichkeit der präsentierten Daten von großer Bedeutung, wenn von der Möglichkeit ausgegangen wird, $\mathrm{da}$ die unternehmensinternen Daten von Wirtschaftssubjekten zum Nachteil für das Unternehmen genutzt werden könnten. $\mathrm{Da}$ bei einem öffentlichen Informationssystem die Daten grundsätzlich frei zugänglich sind, müssen die Nutzer der Daten in keiner Weise vertraglich an das berichtende Unternehmen gebunden sein, dem somit wichtige Möglichkeiten zur Sanktionierung von unerwünschtem Verhalten fehlen. Im Gegensatz dazu sind die Nutzer von intemen Informationssystemen Unternehmensangehörige, so

38 Vgl. Gluchowski/Gabriel/Chamoni (1997), S. 21-28; Henneböle (1995), S. 6472.

39 Hierbei ist allerdings zu beachten, daß auch bei Fokus auf Einzelpersonen deren generelle Akzeptanz für Informationssysteme nicht vorausgesetzt werden kann. Vgl. zu dem Problem der Anwenderakzeptanz den Übersichtsartikel von Dillon/Morris (1996). Choe (1998) zeigt, daß diese Akzeptanz durch Beteiligung der Nutzer am Entwurfs- und Implementierungsprozeß des Infomationssystems erhöht werden kann. Zur Anwendung der Agencytheory auf Führungsinformationssysteme Hess (1999). 
daß vertrauliche Daten eher angeboten werden können..$^{40}$ Deswegen können in MSS andere Daten bereitgestellt und eine andere Recherchetiefe ermöglicht werden, als bei öffentlicher Verfügbarkeit, wo nur die Informationen angeboten werden sollten, deren Auswertung und Verbreitung unter Berücksichtigung komplementärer Kosten keinen negativen Einfluß auf das Unternehmen hat. Diese Forderung schränkt den Bereich der theoretisch denkbaren Informationsmenge nicht unwesentlich ein. Zusammen mit den positiv mit der Informationsbreite verbundenen Erstellungskosten skizzieren diese beiden Ansprüche das grundsätzliche Dilemma der Unternehmenspublizität.

\subsection{Eingliederung des öffentlichen Informationssystems in den Verbund der Un- ternehmensdaten}

Aus dem Vorabschnitt wird einerseits deutlich, daß interne und externe Informationssysteme auf derselben breiten Datenbasis der operativen Informationssysteme fußen sollten. Andererseits wird auch das Problem der Veröffentlichung von internen Unternehmensdaten klar. Als Datenbasis für jedes Informationssystem dient die Transaktionsdatenbank, ggf. verbunden mit dem Abrechnungs- und Bewertungssystem des Unternehmens. Die simple Veröffentlichung einer so erweiterten Datenbasis erscheint aufgrund der in ihr enthaltenen detaillierten Information mit aus Managementsicht teilweise vertraulichem Charakter nicht möglich, also muß die Datenbasis durch die Verwendung von Aggregationsmethoden so verdichtet werden, daß ein ungewollter Rückgriff auf die vertraulichen Daten verhindert werden kann. Durch die Bewertung kann die ökonomische Bedeutung eines realen Sachverhalts kommuniziert werden, ohne daß die Angaben zu den einzelnen werttreibenden Details veröffentlicht werden müssen. So können die Probleme der Informationsveröffentlichung reduziert werden.

Bei vielen Untemehmen dürfte die interne Datenbasis eine bestimmte strukturelle Schwäche aufweisen. Die Transaktionsdatenbank ist in der Regel sehr operativ ausgelegt und nach den einzelnen funktionalen Einheiten des Unternehmens produktionsorientiert gegliedert. ${ }^{41}$ Die Tätigkeiten der eher führenden und strategischen Bereiche werden somit nicht in der operativen Datenbasis mengen- oder wertmäßig erfaßt, da sie nicht problemlos einzelnen Produktionsprozessen zuzuordnen sind. Somit ist eine Auswertung und Bewertung dieser untemehmerischen Tätigkeiten auch in den anschließenden analyseorientierten Informationssystemen nicht möglich. Dies ist bei MSS von untergeordneter Bedeutung, da die Informationsasymmetrie zwischen den strategischen und führenden

40 Dies gilt vor allem bei MSS, die aufgabengemäß nur mittleren und hohen Managementvertretern zur Verfügung stehen, welche auch aufgrund von dem Unternehmensziel konformer Entlohnungsschemata in der Regel nicht zu unternehmensschädigenden Verhalten greifen sollten.

41 Vgl. Müller (1999), S. 23; Scheer (1997), S. 410. 
Bereichen und dem entscheidenden Topmanagement naturgemäß eher gering ist, wohingegen zu den operativ tätigen Einheiten ein höherer Grad der Informationsasymmetrie herrscht, der durch analyseorientierte Informationssysteme reduziert werden soll. Unternehmensexteme Adressaten hingegen haben eine eher gleichverteilte Informationsasymmetrie über alle Teileinheiten des Unternehmensverbundes hinweg. Es ist deswegen zu erwarten, daß sie tendenziell aus allen Bereichen Informationen nachfragen werden. Somit ist die datenbanktechnische Erfassung der Tätigkeiten des Managements und der beigeordneten Stabsstellen unerläßlich, um den externen Adressaten die Möglichkeit zu geben, auch die Leistung der Unternehmensleitung im weiteren Sinne beurteilen zu können.

Die Erfassung dieser Daten in einer Transaktionsdatenbank erscheint jedoch nicht unproblematisch, da es sich in der Regel beim Output der strategischen Abteilungen nicht um materielle, eindeutig identifizierbare Güter handelt, sondern ein Großteil der Leistung in der Generierung neuer Ideen und Konzepte besteht, die erst nach der Implementierung im operationalen Unternehmensbereich, eventuell noch deutlich zeitlich verzögert, zu meßbaren Outputveränderungen führen. Basierend auch auf der schlechten Greifbarkeit des Outputs ist des weiteren in den aufbauenden bewertungsorientierten Informationssystemen die Bewertung der Leistung problematisch. Dies gilt auch deswegen, weil das Topmanagement als letztlich nach außen verantwortliche Teilinstitution gerade für diese Bewertungskonzepte verantwortlich ist. Der externe Adressat der Unternehmensinformation hat also im bildlichen Sinne den „Bock zum Gärtner gemacht“.

Die Verträge zwischen dem Management des Unternehmens und seinen Auftraggebern werden entsprechend dem Informationsstand der Prinzipale geschlossen. Da deren Informationsstand wesentlich von dem öffentlich zugänglichen Informationssystem abhängt, ist es naheliegend, daß Vertragskonditionen mehr oder weniger eng an die beobachtbaren Signale des öffentlichen Informationssystems gebunden werden. Somit ist die Auswertung der Daten nicht nur für externe Adressaten von Interesse, auch das Management hat ein Bedürfnis, die frei verfügbaren Informationen auszuwerten und ggf. ihr Verhalten an dieser beobachtbaren Auswirkung auszurichten. In diesem Sinne ist eine gewisse Konvergenz interner und externer Berichtssysteme und damit verbunden eine Rückwirkung des externen Berichtswesens auf die internen MSS zu erwarten. ${ }^{42}$ Es läßt sich also festhalten, daß ein öffentliches Informationssystem andere Spezifika aufweißt, als ein rein internes Informationssystem. Dies wirkt sich auch auf den Datenfluß aus, der im folgenden Unterkapitel analysiert wird.

42 Vgl. zu der schon länger diskutierten Konvergenz zwischen internem und extemem Rechnungswesen Ziegler (1994); Küpper (1995); Coenenberg (1995); Küting/Lorson (1998); Pfaff/Bärtel (1998); Erichsen (2000); zur empirischen Relevanz Horváth/Amaout (1997); kritisch zur Diskussion jüngst Dirrigl (1998). 


\subsection{Spezifikation des Datenflusses}

Zur Entwicklung einer Anforderungsdefinition soll nun die benötigte Datenstruktur mittels einer groben Analyse des Datenflusses genauer spezifiziert werden. Zunächst sind die gewünschten Inhalte begrifflich zu definieren. Dazu sind zunächst einige Begriffe zu klären. Der Adressat erhält von dem PAIS auf Anfrage einen Abschluß des berichtenden Unternehmens. Ein Abschluß im Sinne der PAIS-Konzeption besteht aus einem oder mehreren Rechenwerken, die zu einem bestimmten Zeitraum oder Zeitpunkt erstellt werden. Die Gesamtheit der zu einem Abschluß gehörenden Rechenwerke und ihre Erstellungsregeln werden durch einen Abschlußstil wiedergegeben. Ein Rechenwerk besteht aus beliebig, aber endlich vielen monetären Posten und einer äußeren Form, die dem Nutzer des Rechenwerks die Analyse und Auswertung der Daten erleichtert. Die Postenbeträge resultieren aus der PAIS-Datenbasis, sowie aus von anderen Postenbeträgen rein mathematisch funktional abhängigen Größen. Zusätzlich zu den Postenbeträgen wird als weiteres Merkmal der Posten eine sprachliche Bezeichnung angeboten. Wenn mehrere Rechenwerke einen Abschluß bilden, dann ist der Rückgriff auf Posten eines oder mehrerer anderer Rechenwerke zur Ermittlung eines Postenbetrags möglich. In diesem Fall ist die Reihenfolge der Erstellung der Rechenwerke von Bedeutung. Beispiele für Rechenwerke sind die Bilanz und die Gewinn- und Verlustrechnung. Beide bilden zusammen einen Abschluß. Sie greifen auf die PAIS-Daten zurück. Die GuV enthält derivative Posten, die jeweils die additive Verknüpfung der in der Staffelform über ihnen liegenden Posten sind. Der letzte additive Posten, der Jahresüberschuß/Jahresfehlbetrag wird als derivativer Posten in die Bilanz übernommen.

Das Ziel des zu entwickelnden PAIS-Systems ist also die Präsentation eines vom Anwender spezifizierten Abschlusses, der Abschlußinstanz, wobei die einzelnen Posten nach wählbaren Kriterien aufgegliedert werden können. Außerdem soll der Anwender spezifizieren können, welche Bilanzierungsregeln für die Bildung der Posten der jeweiligen Rechenwerke angewendet werden sollen. Somit ist der letztendliche Output von der äußeren Form abgesehen ein Zahlenvektor, dessen einzelne Elemente spezifischen Abschlußposten entsprechen. Als Inputfaktoren stehen dem Anwender demzufolge ein Vektor von Ansatz- und Bewertungsregeln, der Typ des gewünschten Abschlusses, ein oder zwei Zeitpunkte und eine Matrix aus beschreibenden Kriterien mit jeweils unterschiedlichen Merkmalsausprägungen zur Aufgliederung der einzelnen Posten zur Verfügung. Vereinfachend ist dieser Datenfluß in Abb. 8 wiedergegeben. 


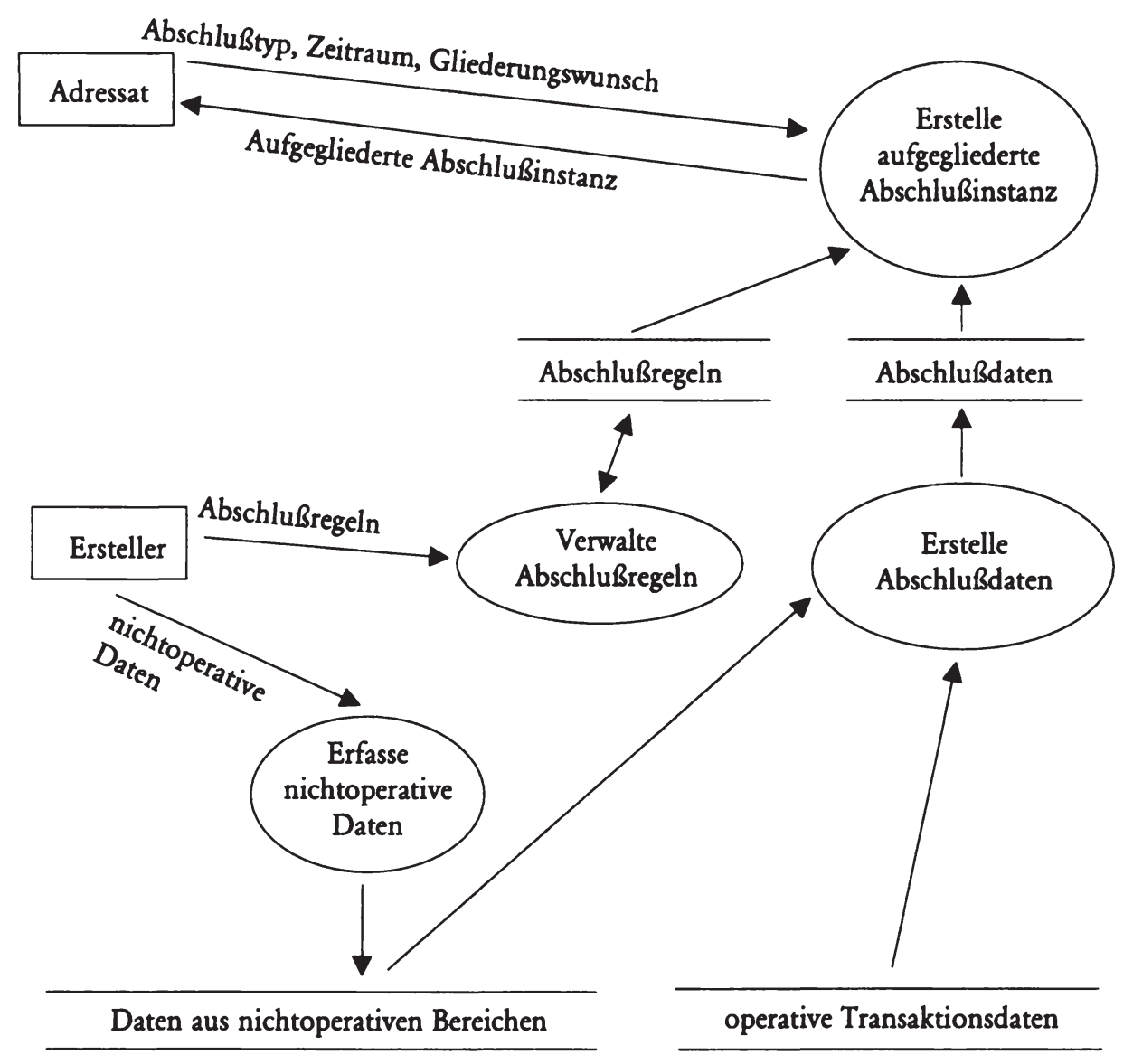

Abb. 8: Grobes Datenfluß-Diagramm des PAIS ${ }^{43}$

Auf Basis dieser Inputfaktoren ermittelt das zu entwickelnde System die angeforderte Abschlußinstanz und liefert die korrespondierenden Daten an den Anwender. Um die Inputfaktoren entsprechend setzen zu können, stellt das PAIS dem Adressat in einem separaten Interaktionsprozeß auf Anforderung die dafür notwendigen Daten zur Verfügung. Ein schreibender Zugriff des Anwenders auf den Datenbestand ist nicht erforderlich. Jede Anfrage wird direkt durch eine Antwort erwidert. Eine Protokollierung der Anfragen auf der Serverseite ist aus Sicht der Grundfunktionalität ebenfalls unnötig. ${ }^{44}$ Somit ist eine Implementierung dieses Datenaustausches in einem klassischen verbindungslosen Client-Server-System problemlos möglich. Um die Anfragen des Nutzers zu

43 Zu der Art der graphischen Darstellung vgl. Balzert (1996), S. 118-121.

44 Selbstverständlich ist zu Analysezwecken eine Protokollierung der Clientanfragen sinnvoll, da das berichtende Unternehmen so einen Überblick über die Informationsinteressen seiner Adressaten erhalten kann, vgl. zu entsprechenden Untersuchungen für interne Berichtssysteme Borgman (1998). 
erfuillen, muß das PAIS seinerseits aus dem operativen Datenbestand des berichtenden Unternehmens die Daten so aufbereiten, daß eine schnelle Beantwortung der Anwenderanfragen möglich wird, ohne die operative Datenhaltung zu beeinträchtigen.

\subsection{Zusammenfassung der Anforderungsdefinition}

Aus diesem Unterabschnitt läßt sich nun ein erstes Anforderungsprofil an das zu entwikkelnde Publizitätssystem skizzieren. Die wesentlichen Punkte sind in der Tab. 1 wiedergegeben.

\begin{tabular}{|l|l|}
\hline Komponente & Ausgestaltung \\
\hline Grundkonzeption & $\begin{array}{l}\text { Unterschiedliche Adressaten machen eine sehr flexible Archi- } \\
\text { tektur des Informationssystems notwendig. Dem Nutzer } \\
\text { müssen zahlreiche Wahlmöglichkeiten in der Datenselektion, } \\
\text {-aggregation und -präsentation eingeräumt werden. Die Mög- } \\
\text { lichkeit zur Generierung neuer Methoden und Sichten durch } \\
\text { den Nutzer sollte grundsätzlich gegeben sein. }\end{array}$ \\
\hline Datenbasis & $\begin{array}{l}\text { Ein Rückgriff auf die operativen Transaktions- und Bewer- } \\
\text { tungsdaten des berichtenden Unternehmens ist notwendig. } \\
\text { Die von dort extrahierten Daten sind um mengen- und wert- } \\
\text { mäßige Informationen zu den Tätigkeiten der nicht operati- } \\
\text { ven Einheiten zu erweitem. Das verwendete Datenmodell } \\
\text { wird zur Erhöhung der überbetrieblichen Vergleichbarkeit } \\
\text { generalisiert. }\end{array}$ \\
\hline Vertraulichkeit & $\begin{array}{l}\text { Die relevanten Daten sind bislang aus unternehmensinterner } \\
\text { Sicht private Information, deren Veröffentlichung unter } \\
\text { Umständen nicht wünschenswert erscheint. Deswegen ist die } \\
\text { Datenbasis so zu gestalten, daß vertrauliche Daten entweder } \\
\text { durch Aggregation oder durch Methodenkopplung privat } \\
\text { bleiben können. Es wird lediglich lesender Zugriff der Nutzer } \\
\text { benötigt. }\end{array}$ \\
\hline
\end{tabular}

Tab. 1: Anforderungen an die Konzeption des Publizitätssystems

Ein monolithischer Entwurf, der Datenhaltung, -selektion, -aggregation und -präsentation in einem Softwareprogramm vereint, kann nicht den in Tab. 1 skizzierten Ansprüchen genügen, da die Alternativen der Auswahl und Aufbereitung der Daten und zur Erstellung der gewünschten Sichten nicht ex ante komplett erfaßt werden können. Die Daten müssen allein aus Sicherheitsüberlegungen schon unbedingt innerhalb der datentechni- 
schen Infrastruktur des Unternehmens verbleiben. ${ }^{45}$ Die aufbereitenden Systeme hingegen sind auf die direkten Kontrollmöglichkeiten des Adressaten angewiesen. Da keine Möglichkeit besteht, die gewünschten Methoden der Adressaten abschließend zu ermitteln, ist die Datenbasis mit einer standardisierten und intensiv dokumentierten Schnittstelle zu versehen, auf die neu entwickelte Selektions-, Aggregations- und Präsentationsmodule (verkürzend zusammengefaßt: Retrievalmodule) zugreifen können.

Diese hier spezifizierten Anforderungen sollen nun bei dem folgenden Systementwurf explizit berücksichtigt werden. ${ }^{46}$ Zunächst wird die Datenbasis des PAIS konzeptionell und logisch im Rahmen des Datenbankentwurfs spezifiziert. Im Anschluß daran wird anhand einer exemplarischen Implementierung aufgezeigt, wie aus disaggregierten Transaktions- und Bewertungsdaten diese Datenbasis gefüllt werden kann, wie der Anwender auf diese Daten zugreifen kann und welche Analysemöglichkeiten er dadurch erhält. Abschließend wird auf mögliche Veränderungen und Erweiterungen der Implementierung eingegangen. ${ }^{47}$

\section{Datenbankentwurf}

\subsection{Konzeptionelles Schema}

\subsubsection{Starmodel als Konzeptionsgrundlage}

Wie im obigen Abschnitt beschrieben, handelt es sich bei den geplanten Zugriffen auf die Unternehmensdaten lediglich um lesende Anfragen. Auch wenn eine große Zeitnähe der angebotenen Informationen gewünscht ist, ist eine feste Kopplung der Datenbasis an den Transaktionsdatenbestand des Unternehmens trotzdem nicht sinnvoll, da der lesende und aggregierende Zugriff von außen auf diese Daten zu erheblichen Performanceeinbußen in den operativen Systemen der betrieblichen Datenverarbeitung führen würde. Daher sollten die für die Adressaten der datenbankgestützten Rechnungslegungspublizität relevanten Daten von dem allgemeinen Datenbestand des berichtenden Unternehmens grund-

45 Hiermit soll nicht der Eindruck erweckt werden, daß der folgende Entwurf Sicherheitsaspekte hinreichend berücksichtige. Die Sicherheit von Computersystemen und -daten ist ein Gebiet, dessen Komplexität im Rahmen der Implementierung wohl häufig unterschätzt wird. Vgl. allein für die grundlegende Systemsicherheit Loscocco et al. (1998) sowie die anderen Beiträge zur 21" National Information Systems Security Conference unter der URL http://csrc.nist.gov/nissc/1998/papers.html, Stand: 4.11.1999.

46 Im Rahmen dieses Systementwurfs werden nicht alle Phasen der Modellierungstheorie durchlaufen, da nicht für ein explizites Unternehmen sondern eher abstrakt argumentiert wird. Vgl. zu den Grundlagen der Informationsmodellierung im betriebswirtschaftlichen Umfeld überblicksartig Picot/Maier (1994).

47 Vgl. zur Struktur der Vorgehensweise Balzert (1996), insb. S. 699-734. 
sätzlich separat gehalten und nur in periodischen Abständen aktualisiert werden. ${ }^{48}$ Diese Konzeption schützt gleichzeitig den vertraulichen Charakter der Transaktionsdaten, da dadurch die operativen internen Unternehmensdaten auch konzeptionell von der öffentlich verfügbaren Datenbasis des PAIS getrennt sind.

Das PAIS-Datenmodell kann also grundsätzlich unabhängig von dem innerhalb des Unternehmens verwendeten Datenmodell konzipiert werden, solange die grundsätzliche Generierung des PAIS-Datenbestandes aus den operativen Unternehmensdaten möglich ist. $\mathrm{Da}$ bei einem öffentlichen Informationsangebot das Datenbankmanagementsystem $(D B M S)^{49}$ eine Vielzahl von teilweise zeitgleichen Anfragen bedienen muß, sollte die Datenhaltung möglichst rasche Zugriffe erlauben. Wie oben erläutert, werden die Daten des PAIS derivativ aus dem operationalen Datenbestand des Unternehmens generiert, so daß einige der klassischen Argumente für die Normalisierung der Daten nicht greifen. ${ }^{50}$ Die Daten werden redundant gehalten und sind jederzeit aus dem Datenbestand des Unternehmens reproduzierbar. $\mathrm{Da}$ sie, verglichen mit den unterliegenden Transaktionsdaten, bereits eine gewisse Aggregationsstufe besitzen, ist der von ihnen beanspruchte Speicherplatz verhältnismäßig gering. Dadurch sinkt auch der Umfang der durch eine konsequente Normalisierung der Daten zu sparende Speicherplatz. Im Gegensatz dazu weisen Abfragen auf normalisierte Datenbestände durch die notwendige Verbindung von Tabellen teilweise erheblich längere Antwortzeiten auf, was sich vor allem bei häufig formulierten Abfragen negativ bemerkbar macht. ${ }^{51}$

48 Diese Bedingungen entsprechen auch den Rahmenbedingungen für Data-Warehouse-Konzepte. Vgl. grundlegend Devlin/Murphy (1988); Gabriel/Chamoni/Gluchowski (2000) und Behme/Mucksch (1998). Deswegen weist die Konzeption des PAIS einige Parallelen zum Systementwurf eines Data Warehouse auf, vgl. dazu beispielhaft Golfarelli/Rizzi (1999).

49 Ein DBMS stellt als Teil eines Datenbanksystems (DBS) die Schnittstelle zwischen den Benutzerzugriffen einerseits und den eigentlichen Datenbanken und deren Aufbaubeschreibungen andererseits dar. Vgl. dazu Balzert (1996), S. 670-674; zur Aufbaustruktur von DBS generell Härder/Rahm (1999), insb. S. 3-37.

50 Durch Normalisierung werden relationale Datenstrukturen in eine redundanzfreie Form gebracht, indem intrarelationale Abhängigkeiten in einem mehrstufigen Prozeß eliminiert werden. Vgl. Codd (1990), S. $317-$ 319; Date (1995), S. 288-313; Balzert (1996), S. 721-727; Braun (1999).

51 Vgl. einführend zu Performancevorteilen von Starmodelqueries Nußdorfer (1999), S. 215-222; Holthuis (1998), S. 165. 


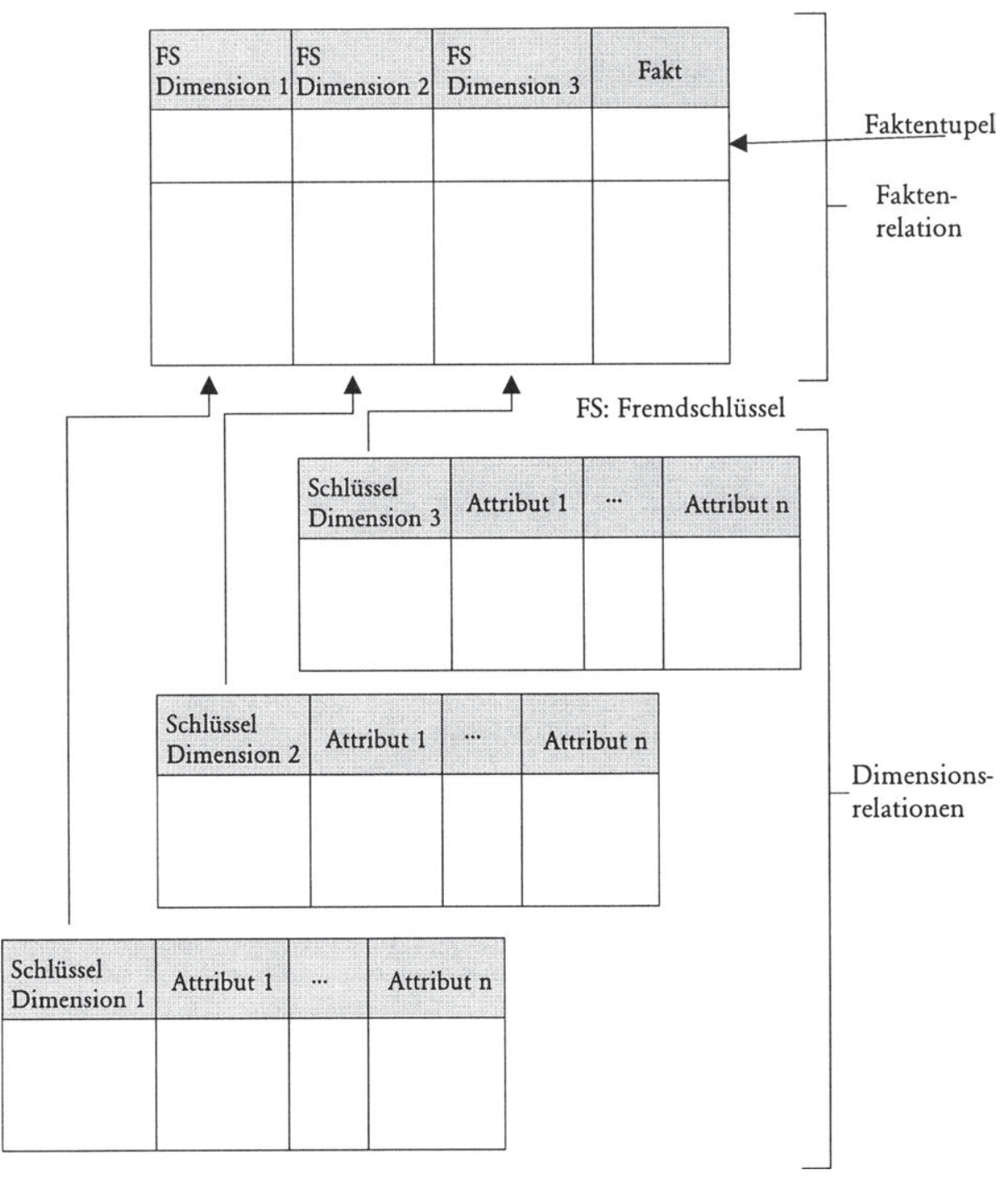

Abb. 9: Starmodel im Überblick

Im Gegensatz zu einem normalisierten ERM wird im Prozeß des dimensionalen Modellentwurfs darauf verzichtet, die Daten in die dritte Normalform zu transformieren. Eine Relation in der dritten Normalform besitzt kein Attribut, das von einem Nichtschlüsselelement der Relation abhängig ist. Durch Verzicht auf die dritte Normalform wird das verwendete Datenmodell für den Endanwender transparenter und die abfragenden $\mathrm{Zu}$ griffe können wesentlich schneller durchgeführt werden. ${ }^{52}$ Das für dieses Konzept ver-

52 Vgl. Kimball (1997). 
wendete Modell wird als „Starmodel“ bezeichnet und vom logischen Schema her in Abb. 9 wiedergegeben. ${ }^{53}$ Datenmodelle dieser Art bilden in der Regel die Grundlage von DataWarehouse-Konzepten ${ }^{54}$ und eignen sich im besonderen Maße für die Zwecke von Online Analytical Processing (OLAP) ${ }^{55}$. Die Aufgabe des PAIS liegt im Bereich des OLAP. Es soll dem Endanwender ermöglicht werden, die ihm zur Verfügung stehenden Rechnungslegungsdaten nach eigenem Ermessen multidimensional analysieren und aggregieren zu können. Insofern scheint die Verwendung eines Starmodel zur Entwicklung des konzeptionellen Schemas des PAIS sinnvoll.

Wenn das Starmodel zur Konzeption des Datenangebots verwendet werden soll, so müssen im folgenden die zu verwendenden Dimensionen des Modells und die „im Zentrum des Sterns" liegenden Fakten definiert werden. Während in der Faktenrelation die eigentliche Kerninformation des Starmodel wiedergegeben wird (z.B. Umsatzmengen), finden sich in den Dimensionsrelationen Merkmale, welche den jeweiligen Fakten beigeordnet werden können (z.B. Absatzort, Produkttyp, Zeiträume). Dieser Fakt und die korrespondierenden Dimensionen sollen in den unmittelbar folgenden zwei Abschnitten bestimmt werden.

\subsubsection{Definition der Fakten}

Die Fakten eines Starmodel stellen die Kerninformation des Datenbestandes dar und werden von den unterschiedlichen Dimensionen charakterisiert. An sie werden insbesondere folgende Anforderungen geknüpft: Sie sollten numerisch, additiv verknüpfbar und in der Lage sein, kontinuierlich unterschiedliche Werte anzunehmen. ${ }^{56}$ Durch diese Bedingungen ermöglichen sie die typische Vorgehensweise der Datenanalyse in DataWarehouse-Systemen. Eine elementstarke Menge von Fakten wird durch eine Abfrage ausgewählt, die für bestimmte Dimensionen das Vorhandensein spezifischer Merkmalsausprägungen fordert (z.B. der gesamte Umsatz für Produkt $\mathrm{X}$ in den deutschen Großmärkten im Jahr 1999). Die Ergebnisfaktenmenge wird jedoch in der Regel nicht als Menge präsentiert, sondern als durch Addition aggregierte Größe. Deswegen sollten die

53 Laut Kimball (1997) geht der Begriff Starmodel auf die Verknüpfungsmethode „star join“ und die wiederum auf die frühen Tage der relationalen Datenbankentwicklung zurück.

54 Vgl. Delvin/Murphy (1988); Cheng/Chang (1998), einführend Behme (1998); zur Konzeption und Implementierung Inmon (1996) und Kimball (1996), zur praktischen Bedeutung in unterschiedlichen Unternehmensbereichen Schumann (1998); Gluchowski (1998); Kaiser (1998).

s5 Vgl. zur Konzeption von OLAP grundlegend Codd/Codd/Salley (1993); zum Überblick auch Kusterer (1998); zur Anwendung innerhalb des Rechnungswesens beispielhaft Oehler (1998). Für aktuelle Entwicklungen und Veröffentlichungen zum Themenbereich OLAP/Data Warehouse sei femer auf die umfangreiche Datenbank von Chamoni, Gabrieh, Gluchowski, Müller, Schelp und Dittmar verwiesen, die unter URL: http://olap.winf.ruhr-uni-bochum.de, Stand 16.11.1999 recherchiert werden kann.

36

Vgl. Kimball (1996), S. 12. 
Faktenelemente in der Regel additiv verknüpfbar und numerisch sein. ${ }^{57}$ Die Additivität der identifizierten Fakten einer Datenbasis ist jedoch nicht immer gegeben. So können semi-additive Fakten vorliegen, die sich lediglich hinsichtlich einer Submenge der spezifizierten Dimensionen addieren lassen (z.B. ist eine Addition der Umsatzmengen über unterschiedliche Orte sinnvoll, über unterschiedliche Produkte jedoch nicht). Nichtadditive Fakten lassen sich über keine der gewählten Dimensionen additiv verknüpfen. Die einzigen Möglichkeiten zur Präsentation nicht-additiver Fakten liegen in der Angabe der Anzahl der abgefragten Faktenelemente und in der totalen Enumeration. Daß diese beiden Altemativen nicht den Informationsgehalt, bzw. die geringen Informationskosten der additiven Verknüpfung besitzen, dürfte offensichtlich sein. Nichtsdestotrotz sind Situationen denkbar, bei denen die Verwendung von nicht-additiven oder semi-additiven Faktendefinitionen angezeigt ist.

Die Forderung nach Kontinuität der Faktenmenge dient vorrangig der Unterscheidung zwischen Fakten und Dimensionen. ${ }^{58}$ Vermeintliche Fakten, die nur diskrete Wertunterschiede annehmen können, lassen sich in der Regel als endlich viele Attributsausprägungen einer Dimension modellieren.

Zur Ermittlung der geeigneten Fakten des PAIS ist besonders die Eigenschaft der Additivität hinsichtlich aller möglichen Dimensionen von Bedeutung. Grundsätzlich kommt als Fakteneigenschaft lediglich der ermittelte Stand eines bestimmten Kontos in Frage, wobei dieser von unterschiedlichen Dimensionen abhängen kann. Diese numerische Information steht im Zentrum der Rechnungslegungspublizität und damit auch im Zentrum der von ihr verwendeten Rechenwerke. Eine Unterscheidung zwischen Aktiv-/Passivund Aufwands-/Ertragskonten und somit zwischen Konten, die Zeitpunktgrößen und Konten, die Zeitraumgrößen widerspiegeln, ist hier nicht von Bedeutung, da sich diese Unterschiede durch eine geeignete Abfragerestriktion der Zeitdimension abbilden lassen. $\mathrm{Da}$ die Kontenstände monetär bewertet sind, können sie (im Rahmen der Restriktionen der bezogenen Währung) auch als kontinuierlich angesehen werden, eine Uminterpretation des Kontenstandes als Dimensionsmerkmal erscheint nicht sinnvoll.

Die grundsätzliche additive Verknüpfbarkeit der Kontenstände erscheint jedoch zweifelhaft. So argumentiert Kimball zutreffend, daß Kontenstände semi-additiv in Hinblick auf die Zeitdimension seien. ${ }^{59}$ Diese Nicht-Additivität der Zeitdimension erklärt sich aus der

57 Es gibt auch noch andere Möglichkeiten der Aggregation, teilweise wird zwischen Aggregation im engen Sinne als Addition und Aggregation im weiten Sinne als Gruppenzusammenfassung und Merkmalsbeschreibung unterschieden. Vgl. hierzu Henneböle (1995), S. 75 mw.N.

58 Vgl. Kimball (1996), S. 12.

59 Vgi. Kimball (1996), S. 49 f. 
Verwendung von Kontenständen als Faktengröße, die ihrerseits ja bereits Ergebnis einer additiven Verknüfung sind (z.B. ergibt sich der Schlußsaldo eines Bestandskontos aus der additiven Verknüpfung des Anfangsbestandes mit den $\mathrm{Zu}$ - und Abgängen der laufenden Periode). Deswegen läßt sich eine Additivität über die Zeit erreichen, indem nicht die Kontenstände als Fakten interpretiert werden, sondern die einzelnen Buchungsbeträge (also die einzelnen $\mathrm{Zu}$ - und Abgänge des Bestandskontos). Eine weitere Möglichkeit besteht in dem Verzicht auf die Zeitdimension. Diese bei der Verwendung eines Starmodel äußerst ungewöhnliche Entscheidung wird möglich, da in der Regel die Rechenwerke der Rechnungslegungspublizität zu bestimmten Perioden (z.B. Jahres- oder Quartalsberichte) angefordert werden, so daß eine recht grobe zeitliche Aggregation möglich wird. In diesem Fall wird zwar die zeitliche Nicht-Additivität der Faktendimension umgangen, andererseits sind die Fakten hinsichtlich der dann notwendigen „Abschlußdimension“, welche den jeweiligen Zeitrahmen als Merkmal enthält, ebenfalls nicht-additiv. Dies ist jedoch nicht besonders problematisch, da die Kombination unterschiedlicher Elemente der Abschlußdimension ökonomisch nicht besonders sinnvoll erscheint.

In diesem Sinne hängt die Spezifikation der Faktenelemente auch in großem Maße von den verwendeten Dimensionen ab. Deswegen sollen im nächsten Abschnitt zunächst die zu präsentierenden Dimensionen diskutiert werden, um im Anschluß daran als Ergebnis das zusammengefaßte Datenmodell zu präsentieren.

\subsubsection{Definition der Dimensionen}

Die Dimensionen des PAIS-Datenmodells sollen es dem Nutzer ermöglichen, einerseits die von ihm präferierten Ansatz- und Bewertungsregeln zu wählen und andererseits, die daraus resultierenden Konten bzw. Buchungsbeträge nach für ihn relevanten Informationen zu aggregieren. Die Entscheidung, welche Dimension in das Datenmodell zu integrieren ist und welche nicht, soll letztlich von den berichtenden Unternehmen getroffen werden können. In dem Entwurf des Starmodel sollten an dieser Stelle alle Dimensionen Berücksichtigung finden, denen ein Informationsgehalt nicht per se abgesprochen werden kann. Ob sie dann implementiert werden, hängt einerseits von den Kosten der Datengewinnung und -aufbereitung und andererseits von der Nachfrage der Endnutzer des PAIS ab.

Eine offensichtliche Dimension des PAIS-Datenmodels ist die Kontendimension. Sie ist unabhängig von der verwendeten Faktendefinition und dient der Zuordnung der Faktenbeträge auf das jeweilige Konto. Die Kontendimension der Beispielimplementierung orientiert sich im wesentlichen am Industriellen Kontenrahmen (IKR). Wichtig ist in diesem Zusammenhang, daß die Kontendimension nicht in besonderem Maße von den später angebotenen Rechenwerken abhängig ist, sondern vielmehr realwirtschaftlichen 
Überlegungen entsprechend konzipiert werden kann. Die jeweilige Zuordnung von Konten zu bestimmten Rechenwerksposten ist neben der sprachlichen Kontenbezeichnung eine wesentliche Attributgruppe der Kontendimension, da die Auswahl der in einer Abfrage gewünschten Konten in der Regel über die Spezifizierung des gewünschten Rechenwerks erfolgt. Die Kontendimension ist nur eingeschränkt additiv verknüpfbar, da Kontenaggregationen nur zum Teil ökonomisch sinnvoll sind. So ist die Zusammenfassung von Vorratsunterkonten zu einem Rechenwerksposten „Vorräte“ sinnvoll, eine additive Verknüpfung der Umsatzerlöse mit dem Bestandskonto des Fahrzeugparks jedoch weniger. In der Regel werden diese Aggregationen ebenfalls über das vom Anwender angeforderte Rechenwerk determiniert.

Eine der Hauptanforderungen an das PAIS ist die Fähigkeit zur Wiedergabe von unterschiedlichen Ansatz- und Bewertungsmethoden. Konzeptionelle Umsetzung dieser Forderung ist die Bewertungsdimension, welche sämtliche Bewertungsprinzipien und damit auch die Ansatzprinzipien umfaßt. ${ }^{60} \mathrm{Zu}$ untersuchen ist, welche Granularität die Bewertungsdimension aufweisen sollte. Jeder Buchungswertfakt für ein bestimmtes Konto zu einem bestimmten Zeitpunkt $\left(w_{k, t}\right)$ kann letztendlich als funktionales Ergebnis eines Vektors von realwirtschaftlichen $(\vec{r})$ und bewertungstheoretischen Merkmalsausprägungen $(\vec{b})$ gesehen werden. Dieser Zusammenhang wird in der formalen Darstellung durch Gleichung (1) wiedergegeben.

$$
\text { (1) } w_{k, t}=f(\vec{r}, \vec{b})
$$

Eine Granularität der Bewertungsdimension auf Ebene der einzelnen Vektorelemente erscheint nur unter der Bedingung eines additiven Funktionalzusammenhangs denkbar. Dies entspräche formal einem in der Gleichung (2) wiedergegebenen Zusammenhang:

$$
\text { (2) } w_{k, t}=f(\vec{r})+\sum_{i=1}^{n} g\left(b_{i}\right) \text {. }
$$

Dafür müßten alle Elemente des Bewertungsvektors in ihrer Auswirkung auf den Buchungsfaktenwert unabhängig voneinander sein. $\mathrm{Daß}$ dies nicht der Fall ist, läßt sich an einem Beispiel leicht aufzeigen: So sind die betragsmäßigen Auswirkungen von Abschreibungen auf Anschaffungs-/Herstellungskosten abhängig von dem Betrag der Erstbewertung, welcher wiederum von weiteren Ansatz- und Bewertungswahlrechten determiniert wird. Eine Bewertungsdimension, die jede Bewertungsentscheidung separat erfaßt, ist

60 Die Entscheidung, die Resultate eines bestimmten Geschäftsvorfalls gar nicht, als Vermögensgegenstand oder als Schuld anzusetzen, kann als eine Bewertungsentscheidung gleich, größer als, oder kleiner Null interpretiert werden. 
somit nicht möglich, vielmehr muß jedes Element der Bewertungsdimension für einen gesamten Bewertungsvektor stehen.

Diese Bedingung schränkt unter Umständen die Wahlmöglichkeiten des Endanwenders erheblich ein. Er kann den Bewertungsvektor nicht mehr selbst zusammenstellen. Während das berichtende Unternehmen diesen Nachteil noch durch das Angebot aller technisch möglichen und ökonomisch sinnvollen Bewertungsvektoren beheben kann, wiegt ein zweiter Nachteil schwerer. Durch die gegenseitigen Abhängigkeiten der unterschiedlichen Bewertungsvorschriften hinsichtlich ihrer monetären Auswirkung auf den Buchungswert ist es nicht möglich, den Buchungswert widerspruchsfrei auf die Bewertungsmethoden zurückzuführen und somit eine Aufgliederung des Werts über die relevanten Bewertungsmethoden hinweg zu generieren. So ist z.B. nicht eindeutig bestimmbar, welcher Anteil einer Abschreibung auf zu Vollkosten bewerteten selbsterstellten Anlagevermögen auf der Abschreibung selber und welcher Teil auf dem Vollkosten- bzw. Teilkostenansatz basiert. Für die Lösung dieses Problems sind mindestens zwei Lösungsalternativen gangbar. Einerseits kann auf die Verwendung einer Bewertungsdimension verzichtet werden. Andererseits kann die Zuordnung eines Buchungsbetragfaktes zu einer bestimmten Bewertungsdimension vereinfacht werden, indem der Betrag immer der Bewertungsregel zugeordnet wird, welche die entsprechende Buchung induziert hat. In dem obigen Beispiel hieße dies, daß der Abschreibungsbuchungsbetrag voll der für die $\mathrm{Ab}$ schreibung verantwortlichen Buchungsregel zugeordnet wird, unabhängig davon, daß der Betrag ebenso von anderen vorgelagerten Bewertungsregeln, wie z.B. der Erstbewertung zu Voll- oder Teilkosten, abhängig ist. Da einer Aufgliederung nach Bewertungsregeln auch unter Berücksichtigung dieser vereinfachenden Annahmen ein möglicher Informationscharakter nicht abgesprochen werden kann, soll für den Dimensionsentwurf dem zweiten Ansatz gefolgt werden.

Somit wird klar, daß das Datenmodell eine hinsichtlich der Buchungsbetragsfakten nichtadditive Dimension enthalten muß, die den verwendeten Bewertungsvektor repräsentiert. Die Nicht-Additivität folgt daraus, daß die Fakten für jeden unterschiedlichen Bewertungsvektor den gleichen ökonomischen Sachverhalt abbilden. Eine Addition dieser Sachverhalte führte zu einer Doppelerfassung und engibt deswegen keinen Sinn. Eine Entscheidung für einen bestimmten Bewertungsvektor ist immer eine Entscheidung gegen alle anderen möglichen Vektoren. Diese Dimension des Starmodel soll als Abschlußtypdimension bezeichnet werden und umfaßt neben dem Bewertungsvektor auch den Verweis auf einen bestimmten Abschlußstil. Die Koordinaten eines Bewertungsvektors sind jeweils binär, das heißt, jede Koordinate steht für eine mögliche Bewertungsmethode, die entweder zur Anwendung kommt oder nicht. In einer relationalen Datenbank läßt sich dies auf zwei Arten umsetzen. Bei begrenzter Anzahl der möglichen Bewertungsmethoden könnten unter Verzicht auf die Vorteile der dritten Normalform alle möglichen 
Methoden als Attribute in die Abschlußtypdimensionsrelation aufgenommen werden. Bei vielen Bewertungsmethoden kann es sinnvoller sein, eine separate Relation mit Bewertungsmethoden als Tupel $^{61} \mathrm{zu}$ bilden und diese dann mit der Abschlußtypdimension in eine n:m-Relation ${ }^{62}$ zu setzen. Da die Abschlußtypdimension wegen ihrer NichtAdditivität in der Regel bei Abfragen eindeutig spezifiziert wird, also eine Abfrage mit bestimmten Bewertungsmethoden als Parameter nicht erfolgen wird, ist der Geschwindigkeitsvorteil der Nicht-Normalisierung zu vernachlässigen. Im Datenmodell des PAIS soll also der zweite Ansatz angewendet werden.

Neben der Abschlußtypdimension wird, wie oben bereits erläutert, ebenso eine Bewertungsdimension implementiert, welche die (nicht widerspruchsfreie) Identifikation der monetären Auswirkungen der für den entsprechenden Abschlußtyp verwendeten Bewertungsmethoden auf die Buchungsfakten erlaubt. Als Attribute dieser Dimension kommen neben der semantischen Bezeichnung der Bewertungsmethode erläuternde Eigenschaften wie pagatorisch/historisch fundiert, auf Marktwerten basierend (eventuell mit weiterer Unterteilung für reale Marktwerte und durch marktwertnahe Bewertungsmaßnahmen ermittelte Wertansätze), oder auf Prognosen basierend in Frage. Für Prognosedaten ist ggf. noch die verwendete Methode, die erwartete Eintrittswahrscheinlichkeit sowie die Charakterisierung des Prognoseerstellers relevant.

Eine weitere wichtige Dimensionsebene von Rechnungslegungsdaten ist die Zeit. Grundsätzlich ist die Zeitdimension zentraler Bestandteil des Starmodel und auch einer DataWarehouse-Konzeption. ${ }^{63}$ Bei den Überlegungen zur Faktendefinition wurde bereits auf die Probleme hinsichtlich der Additivität von Kontenständen über die Zeit hingewiesen. Hier stellt sich die Frage, ob als Fakten die Buchungsbeträge, also die Veränderungen von Kontenständen, oder die Kontenstände zu bestimmten Zeitpunkten angesehen werden. Die letztere Vorgehensweise erscheint nur zweckmäßig, wenn gleichzeitig eine Beschränkung der Zeitdimension vorgenommen wird, welche die Zeitdimensionstupel auf die vom Unternehmen gewünschten Abschlußzeitpunkte reduziert. Andernfalls würde die Faktenrelation durch die Vielzahl der Zeitdimensionstupel ohne Informationsgewinn aufge-

${ }^{61}$ Ein Tupel ist ein Element einer Relation, also bildlich gesprochen eine Zeile in der tabellarischen Abbildung einer Relation.

62 Eine n:m-Relation verbindet zwei Relationen dergestalt, daß die Tupel einer Relation jeweils beliebig viele Verbindungen zu Tupeln der anderen Relation eingehen können. Für den hier betrachten Fall bedeutet dies, daß ein Abschlußtyptupel mit beliebig vielen einzelnen Bewertungsverfahren verbunden werden kann und $\mathrm{da} B$ andersherum ein Bewertungsverfahren mit beliebig vielen Abschlußtyptupeln assozierbar ist.

63 Vgl. Kimball (1996), S. 7 f. der in der expliziten Bewahrung von historisch strukturierten Daten, die von der operationalen Datenbasis abgekoppelt sind, ein konstituierendes Merkmal eines Data Warehouse sieht; vgl. allgemein zu temporalen Aspekten der multidimensionalen Datenmodellierung Chamoni/Stock (1999), sowie zu dem grundlegenden Tradeoff zwischen Zeitnähe und Verläßlichkeit der Daten Ballou/Pazer (1995). 
bläht, da durch die Definition von Kontenständen zu Fakten die ansonsten vorherrschende Lückenhaftigkeit der Faktentabelle verlorenginge. Einen Kontenstand weist jedes Konto zu jedem Zeitpunkt und nach jedem Abschlußtyp für jede Bewertungsdimension auf, eine Veränderung des Kontenstands erfolgt jedoch nur zu bestimmten diskreten Zeitpunkten. Deswegen werden beim Datenmodell des PAIS Buchungsbeträge als Fakten interpretiert. Somit bleibt eine Zeitdimension im klassischen Sinn erhalten.

Eine Aggregation der Buchungen erfolgt zunächst auf Tagesbasis. Eine untertägige Aggregation ist zwar technisch möglich, erscheint aber schwer operationalisierbar und nur beschränkt ökonomisch sinnvoll. ${ }^{64}$ Problematisch sind einerseits Buchungen, die in der traditionellen Jahresabschlußerstellung nur zum Abschlußtermin stattfinden, wie z.B. die planmäßigen Abschreibungen und andererseits Zahlungsaus- und Eingänge, die über eine gewisse Dauer als Aufwand oder Ertrag periodisiert werden, wie Steuer- und Tantiemenzahlungen. Zwar ist es denkbar, diese Buchungen täglich durchzuführen, andererseits führt dies zu einem erheblichen Mehraufwand im Rahmen der Datengenerierung. Deswegen wurde im Starmodel des PAIS das Konzept der Berichtsperiode geschaffen. Die Länge dieser Berichtsperiode kann vom Unternehmen frei gewählt werden. In der Beispielimplementierung wurde eine monatliche Berichtsperiode unterstellt. Somit sind alle Abschlußbuchungen nur einmal innerhalb der Berichtsperiode und nicht unbedingt täglich vorzunehmen. Diese periodischen Abschlußbuchungen führen auch dazu, daß nur noch die zeitlichen Aggregationen des Datenbestandes ökonomisch gesehen Sinn ergeben, die den Berichtsperioden entsprechen. In diesem Fall ist eine separate Beibehaltung der Zeitdimension nicht mehr notwendig, die zeitliche Aggregation kann direkt durch die Berichtsperioden erfolgen. Fraglich ist, ob auch bei der Verwendung von Berichtsperioden an Buchungsbeträgen als Fakten festgehalten werden soll. Diese würden dann ggf. über die Berichtsperioden additiv verknüpft, um Bestandskontensalden zu ermitteln.

Im Datenmodell des PAIS wird hierfür ein „zweigleisiger“ Ansatz verwendet. Einerseits gibt es ein Starmodel, in dem auf Berichtsperioden verzichtet wird und Buchungsbeträge als Fakten unter Berücksichtigung einer traditionellen Zeitdimension abgeleitet werden. Aus diesem Datenmodell wird ein weiteres Starmodel abgeleitet, welches die Daten bereits sehr präsentationsnah vorhält. In diesem wurde die Abschlußtypdimension mit den unterschiedlichen Berichtsperioden so verbunden, daß eine neue Dimension entsteht, die als Abschlußdimension bezeichnet werden soll. Ein Tupel der Abschlußdimension enthält also indirekt einen bestimmten Bewertungsvektor und Abschlußstil, sowie direkt die

o4 Für international tätige Unternehmen ist schon die Definition eines Zeitraums als "Geschäftstag" wegen der Zeitverschiebung nicht unproblematisch. Wird von einer täglichen Aktualisierung des PAISDatenbestandes ausgegangen, könnten die dafür notwendigen lesenden Zugriffe auf die operationale Datenbasis zu nicht unerheblichen Performanceeinbußen für das rund um die Uhr beschäftigte Datenbanksystem führen. Eine untertägige Aggregation würde diese Effekte nur noch verstärken. 
gewünschte Berichtsperiode. Dementsprechend sind die Fakten als Kontenstände definiert, die bereits die Art, Bestand oder Aufwand/Ertrag, des jeweiligen Kontos berücksichtigen. Logischerweise sind die Fakten über die Kontendimension nicht-additiv.

Als weitere Dimensionen beider Starmodel kommen noch die Buchungsvorgänge weiter charakterisierenden Eigenschaften in Frage. Für das PAIS Datenmodell wurden hier interne und externe Akteure identifiziert, die mit dem realwirtschaftlichen Vorgang, der die Buchung induziert hat, in direkte ursächliche Verbindung zu bringen sind. Diese Akteure sind nicht für alle Geschäftsvorfälle und erst recht nicht für alle Abschlußbuchungen zu identifizieren, so daß im PAIS Datenmodell eine Art „Nullakteur“ sowohl für die interne als auch die externe Akteurdimension angeboten wird. Problematisch ist ebenfalls die geeignete Abgrenzung und Aggregation von Akteurgruppen oder ggf. die Aufsplittung von Buchungen auf verschiedene Akteure. Trotzdem kann, zumindest für bestimmte Kontengattungen, wie z.B Forderungen und Verbindlichkeiten und für die Unternehmenssegmentierung von dem Informationscharakter dieser Dimensionen ausgegangen werden. Weitere Dimensionen könnten analog zu den Akteurdimensionen von berichtenden Unternehmen in der Implementierung ihres spezifischen Datenmodells berücksichtigt werden.

\subsection{Logisches Schema}

Die in den Vorkapiteln geschilderte Konzeption des Datenmodells führt zu einer zweistufigen Struktur, deren logisches Schema in Abb. 10 und Abb. 11 wiedergegeben ist. ${ }^{65}$ Die Abbildungen enthalten nur die für den Endanwender wichtigen Teile des Datenmodells. Für die anderen eher technischen Bereiche der Datenkonzeption sei auf den Auszug der Datenbank im Anhang verwiesen. Die erste Stufe verwendet als Faktendefinition die Buchungsbeträge in täglicher Aggregation. Der jeweils zur Bewertung zu verwendende Vektor wird über die Abschlußtypdimension erfaßt. Die Bewertungsdimension selber charakterisiert also nur noch die einzelnen Buchungsbeträge hinsichtlich des jeweils einschlägigen Elements des Bewertungsvektors. Das Modell ist additiv für die Zeit-, Akteurund Bewertungsdimension, nicht-additiv für die Konten- und Abschlußtypdimension. Die Abschlußtypdimension enthält nicht direkt den Bewertungsvektor, dieser wird über eine n:m-Relation mit einer separaten Bewertungsverfahrenrelation abgebildet, welche in der Abbildung nicht wiedergegeben wird.

65 Auf eine semantische Modellierung als Vorstufe wurde wegen des hohen Abstraktionsgrads und der damit verbundenen geringen Komplexität der resultierenden Datenstruktur verzichtet. Vgl. zu unterschiedlichen semantischen Modellierungsnotationen für multidimensionale Datenstrukturen Gabriel/Gluchowski (1998). 


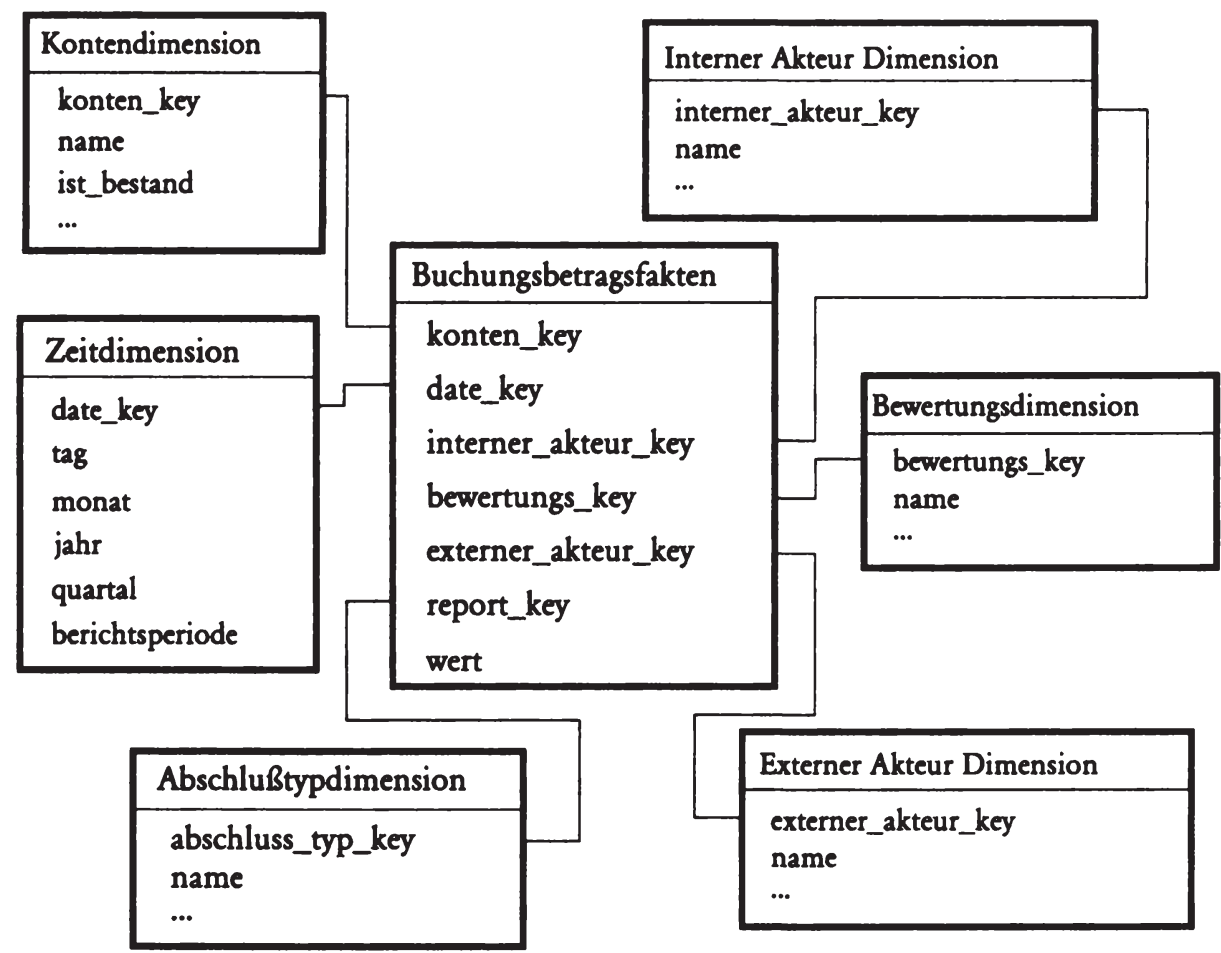

Abb. 10: PAIS-Datenmodell 1. Stufe

Die Kombination der Abschlußtyp- und Zeitdimension zur Abschlußdimension läßt aus dem PAIS-Datenmodell der 1. Stufe das Modell der 2. Stufe entstehen und erfolgt unter Berücksichtigung des Kontentyps. Für Bestandskonten wird der aktuelle Kontenstand am Ende der Berichtsperiode durch additive Verknüpfung aller vorher erfolgten Buchungen erreicht, während für Aufwands- und Ertragskonten nur die Buchungen der jeweiligen Periode aggregiert werden. Aus diesem Vorgehen resultiert die Definition der FaktengröBe, die sich für das Starmodel der zweiten Stufe auf Kontenstände bezieht. Das Starmodel der zweiten Stufe ist ebenfalls additiv für die Akteur- und Bewertungsdimension. Nichtadditiv ist es für die Abschluß und Kontendimension. Die neue Abschlußdimension enthält einerseits die Merkmale, welche den jeweiligen Berichtszeitraum bestimmen, also die Daten, die im Modell der 1. Stufe in der Zeitdimension wiedergegeben wurden. Andererseits umfaßt die Abschlußdimension ebenfalls den Verweis auf den angewendeten Bewertungsvektor, also die Information der Abschlußtypdimension der 1. Stufe. 


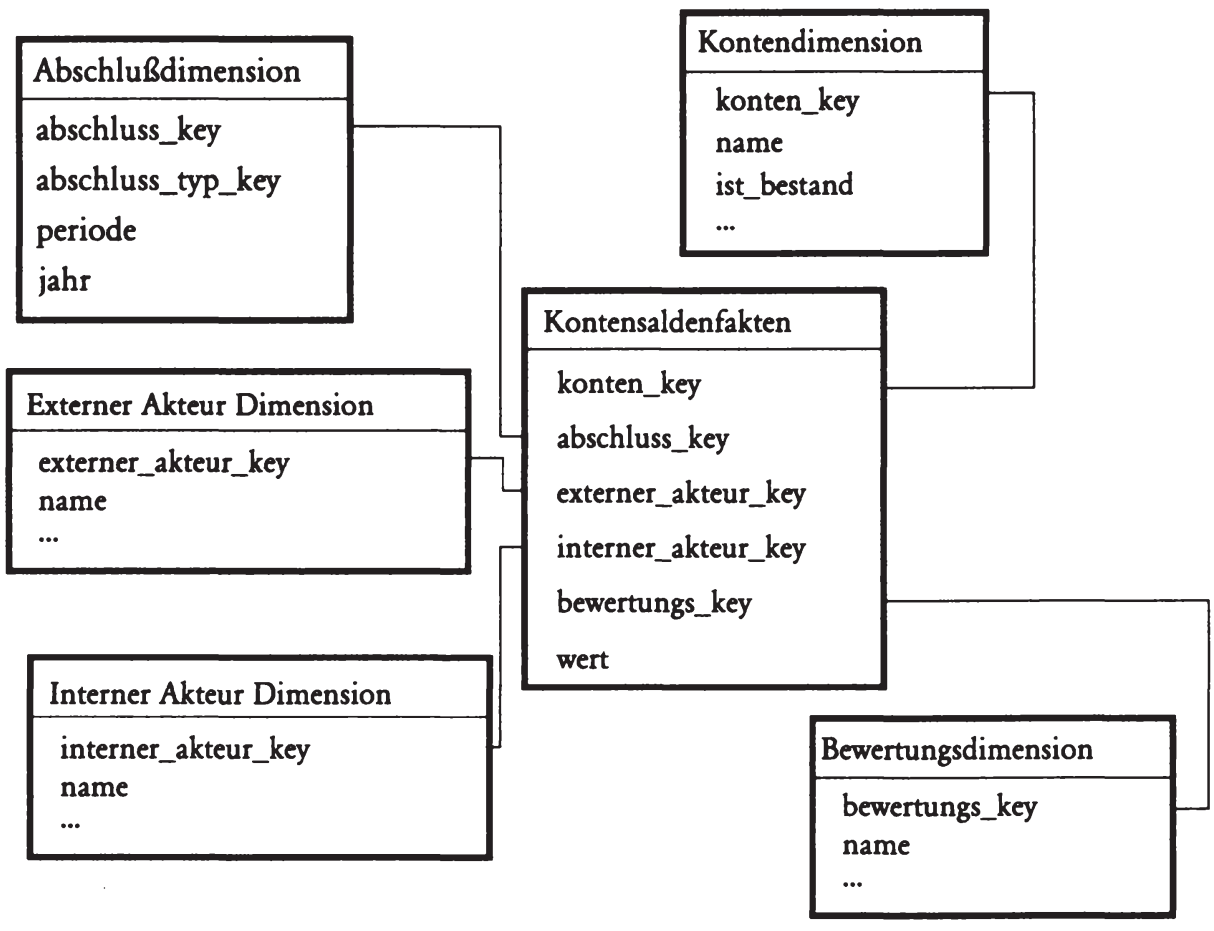

Abb. 11: PAIS-Datenmodell 2. Stufe

\section{Implementierung eines Public-Accounting-Information-System-Server}

\subsection{PAIS-Struktur und Verteilung}

Eines der Hauptziele dieser Arbeit ist die exemplarische Implementierung eines PAIS. Hierbei soll das Hauptaugenmerk auf der Verdeutlichung des Konzepts und nicht auf der Lösung von praktischen Anwendungsproblemen liegen. Diese sind hochgradig unternehmensspezifisch und somit nur in speziellen Fallstudien zu lösen. Somit ist das im Rahmen dieser Arbeit entwickelte PAIS-Konzept als ein Prototyp anzusehen, der den grundsätzlichen Ansatz verdeutlicht und somit als Vorlage für spätere Implementierungen dienen kann. Die nun folgenden Ausführungen beziehen sich auf diese prototypische Implementierung, wobei besonderer Wert auf die Spezifizierung der Schnittstellen und Kommunikationsmethoden gelegt wurde, da diese für die Eignung des PAIS als öffentliches Informationssystem von großer Bedeutung sind. 


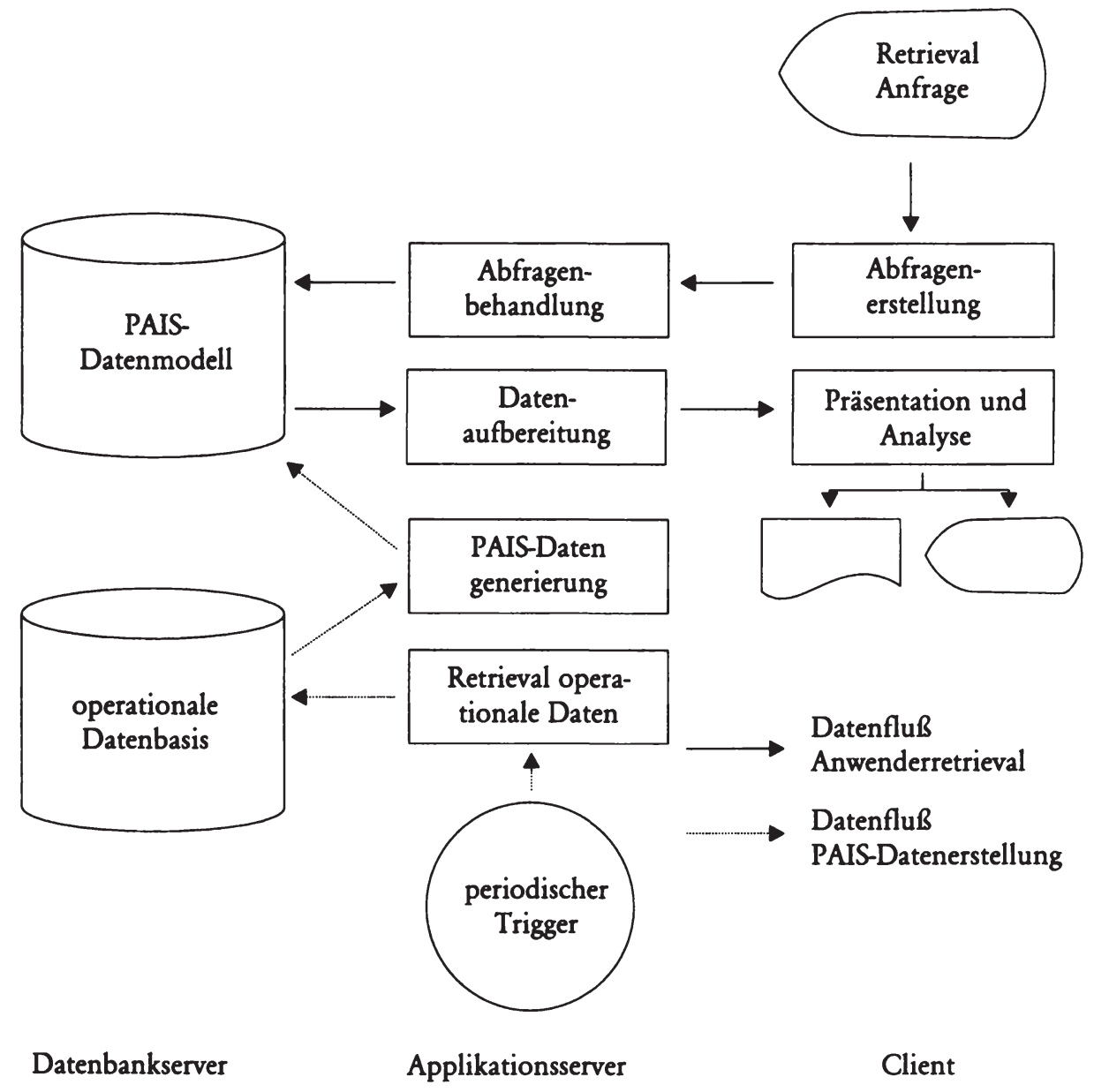

Abb. 12: Client/Server-Datenflußplan des PAIS ${ }^{66}$

Die Implementierung des PAIS basiert auf einer Client/Server Konzeption, deren korrespondierender Datenflußplan in Abb. 12 wiedergegeben wird. Ein Datenbankserver übernimmt das DBS. Die Funktionen zur Generierung der Daten des PAIS-Datenmodells, sowie zur Reaktion und Verarbeitung von Anwenderanfragen werden von einem separaten Rechner, dem Applikationsserver, bereitgestellt. Die Datenanfrage und die graphische Aufbereitung und Präsentation der Daten wird von dem Client des jeweiligen Anwenders erstellt. Für die Funktionsfähigkeit des PAIS-Konzepts ist es von zentraler Bedeutung, daß die Kommunikation zwischen Client und PAIS-Server über Wide Area Networks (WAN) problemlos möglich ist. Die Kommunikation zwischen Client und Applikationsserver

66 Zu den Sinnbildem vgl. die Spezifikation in DIN 66001. 
erfolgt deswegen, wie übrigens auch die Kommunikation zwischen Applikations- und Datenbankserver, über das Transmission Control Protocol/Intemet Protocol (TCP/IP), wobei unterschiedliche Protokolle des Process/Applications Layer Interfaces des TCP/IP Stacks zur Anwendung kommen können. ${ }^{67}$ Während durch die systemübergreifende Standardisierung von TCP/IP die Art des Clients praktisch frei gewählt werden kann und somit keine expliziten Spezifikationen formuliert werden müssen, sollen im folgenden die Spezifikationen für den Datenbank- und Applikationsserver kurz umrissen werden.

\subsection{Spezifikation des Datenbankservers}

Wegen des exemplarischen Charakters der PAIS-Implementierung sind viele in der Realität einschlägige Kriterien für die Identifikation des geeigneten Datenbankservers ${ }^{68}$ eher von untergeordneter Bedeutung, wohingegen andere Gesichtspunkte in den Vordergrund treten. Vor allem soll das System für wissenschaftliche Zwecke kostengünstig zu realisieren sein und weitgehende Eingriffe und Gestaltungsmöglichkeiten erlauben. Ferner ist auf gute Portierbarkeit der Datenbank und des Programmcodes zu achten. Andererseits ist die Verarbeitungsgeschwindigkeit des Systems von eher untergeordneter Bedeutung, da die Datenbestände wesentlich weniger umfangreich sind als in der betrieblichen Praxis. AuBerdem wird auf die PAIS-Daten, von der Erstellung abgesehen, nur lesend zugegriffen, so daß die Leseperformance höher als die Schreibperformance gewichtet werden sollte.

Durch die Konzeption des Datenmodells als Starmodel und die starke Retrievallastigkeit der Nutzung des PAIS wird für das DBS der relationale Ansatz dem objektorientierten vorgezogen, der eher in der komplexen Manipulierung und Modifikation von sehr heterogen typisierten Datenbeständen seine Vorteile hat. ${ }^{69}$ Als Abfragesprache für das DBS wird die Structured Query Language (SQL) verwendet, der De-facto-Standard für die Kommunikation mit relationalen DBS. ${ }^{70}$ Die Forderung nach einer hohen Manipulierbarkeit und Modifizierbarkeit des DBS führt zur Wahl eines Produktes, dessen Quellcode offengelegt wurde, damit eventuell fehlende Produkteigenschaften des DBS nachgerüstet

67 Das TCP/IP-Protokoll ist ein konstituierendes Merkmal des weltweiten Computernetzes Internet. Es ist weitgehend standardisiert und ermöglicht sowohl verbindungslose als auch verbindungsorientierte WANKommunikation zwischen unterschiedlichsten Computersystemen. Vgl. zur Technik von TCP/IP RFC 1122. Zur Bedeutung von TCP/IP für das Entstehen und die Verbreitung des Internets Borchers/Benning/Kuri (1999).

68 Vgl. zur Auswahl eines geeigneten Datenbankservers Nußdorfer (1999); zu den in der Praxis im Rahmen der Implementierung eines Data Warehouses notwendigen Arbeitsschritten Dittmar (1999), S. 40-50.

69 Vgl. zum Überblick für die Unterscheidung von relationalen und objektorientierten DBS Robie/Bartels (1994); Matthiessen/Unterstein (1997), S. 255-269; speziell für die Frage der Anwendungstauglichkeit in Data-Warehouse-Systemen Ohlendorf (1998). Vgl. ferner für die Anwendung von objektorientierten DBS im Bereich des Rechnungswesens Chu (1992); Inman (1998).

70 Vgl. einführend zur SQL-Syntax und -Abfrage Matthiesen/Unterstein (1997); zur Entstehung insb. S. 115 f; sowie die unterschiedlichen Versionen von ISO 9075. 
werden können. Die Wahl fiel hier auf das Produkt mySQL der Firma T.cX. DataKonsult $A B$, da dieses DBS einen sehr SQL95 nahen Standard pflegt, auf vielzähligen Unix-, Mainframe- und Windowsplattformen installierbar ist, komplett im Quellcode verfügbar ist und die Lizenz für die akademische Nutzung kostenlos ist. ${ }^{71}$

Als Betriebssystem fiel die Wahl auf das ebenfalls im Quellcode verfügbare Unix-Derivat Linux, das unter den progressiven Lizenzbedingungen der GNU General Public License $(G P L)^{72}$ frei vertrieben werden darf. Der aus dieser Lizenz resultierende Schutz des geistigen Eigentums der Entwickler bei der gleichzeitigen Garantie der ständigen öffentlichen Zugänglichkeit des produzierten Produkts haben erheblich dazu beigetragen, daß Linux, zusammen mit einer Vielzahl größtenteils ebenfalls unter der GPL vertriebenen Produkte, mittlerweile ein allgemein anerkanntes Serverbetriebssystem darstellt. ${ }^{73}$ In Verbindung mit mySQL ist somit die Serverplattform softwareseitig bestimmt. Als Hardwarebasis wurde primär aus Kosten- und Verfügbarkeitsgründen auf einen Standard-PC mit IntelMikroprozessor gesetzt.

\subsection{Spezifikation des Applikationsservers}

Die auf dem Applikationsserver bereitgestellten Funktionen bilden die Schnittstelle zwischen dem Datenbankserver und dem Anwender. Da ein Großteil der Systembelastung durch die Netznutzung entsteht, sollte die Vernetzung mit dem Datenbankserver und dem WAN zum Nutzer, im Regelfall wohl gleichbedeutend mit dem Internet, einen hohen Datendurchsatz ermöglichen. Das verwendete Betriebssystem benötigt eine standardkonforme und belastbare Implementierung des TCP/IP-Stacks. Wird für die Kommunikation mit den Clients auf Standardprotokolle des Process/Applications Layer Interfaces des TCP/IP-Stacks zurückgegriffen, sollte für das verwendete Betriebssystem dementsprechende Serversoftware verfügbar sein. $\mathrm{Da}$ die Funktionen nicht durch Standardkomponenten realisiert, sondern mit Hilfe der Programmiersprache $C$ entwickelt werden, benötigt der Applikationsserver zumindest in der Erstellungsphase eine dementsprechende Entwicklungsumgebung, welche die Produktion von möglichst portablem Quellcode erlaubt.

Auch diese Spezifikationen werden von einem linuxbasierten Rechner in Verbindung mit der GNU-C Entwicklungsumgebung mit dem gcc Compiler und einem apache HTTPServer erfüllt. Sämtliche Software ist kostenfrei im Quellcode verfügbar. Ein weiterer

71 Vgl. für Informationen URL: http://www.mysql.com.

72 Vgl. URL: http://www.gnu.org/copyleft/gpl.html.

73 Vgl. zu den Besonderheiten der Linuxentwicklung Raymond (1998); zu der zunehmenden Akzeptanz z.B. Riek (1998); zu ökonomischen und gesellschaftlichen Auswirkungen Nürnberger (1999). 
Vorteil in der Verwendung einer einheitlichen Betriebssystemplattform für Datenbankund Applikationsserver liegt in der Möglichkeit, beide Systeme ggf. auf einer Hardwareplattform zu realisieren.

\subsection{Generierung der PAIS-Datenbasis}

\subsubsection{Schnittstelle zu operationalen Unternehmensdaten}

$\mathrm{Da}$, wie bereits geschildert, die PAIS-Datenstruktur zumindest teilweise auf den operationalen Informationssystemen aufsetzt, ist in einem ersten Implementierungsschritt die Schnittstelle zu diesen Informationssystemen und die Funktionen zur Transformation des operativen Datenbestandes in das analyseorientierte PAIS-Datenmodell zu beschreiben. $^{74}$

Der Datenabgleich zum operationalen Bestand erfolgt nicht kontinuierlich, deswegen wird die Datentransformation für die PAIS-Datenbasis durch einen periodischen Trigger ausgelöst. Durch Rückgriff auf die operationalen Unternehmensdaten werden diese in ein bestimmtes Schema aggregiert. Die Hauptschnittstelle zwischen operationaler Datenhaltung und dem PAIS-Datenmodell liegt in der Relation buchungen, die vom Grundgerüst einem Hauptjournal mit zusätzlichen charakterisierenden Dimensionen ähnelt. In diese sind vor allem die Daten der Finanzbuchhaltung zu überführen. Der Bezeichner ist insofern irreführend, als daß nicht notwendigerweise als Quelle nur einzelne Buchungen in Frage kommen. Vielmehr ist eine Aggregation von Buchungen, die für alle Dimensionen gleiche Merkmalsausprägungen besitzen bereits bei dieser ersten Übertragung möglich.

Diese Schnittstelle zwischen den operationalen Daten und dem PAIS ist gleichzeitig die Datenbasis, in der die Buchungen für ein fiktives Beispielunternehmen erfaßt wurden.

74 Vgl. allgemein zur Transformation von operationalen Daten in analyseorienterte Informationssysteme umfassend Müller (1999); einführend Kemper/Finger (1999); Müller (1999a). Bei dieser Transformation ist auch die Qualität der Daten ein zu beachtendes Problem; vgl. mit dem Versuch einer Strukturierung der Einflußfaktoren auf die Datenqualität speziell für multidimensionale Informationssysteme mit mehreren Adressaten Ballou/Pazer (1985); sowie Ballou/Tayi (1999). 
Auf diese Daten wird im folgenden exemplarisch zurückgegriffen, um die Funktionsweise des PAIS zu verdeutlichen. ${ }^{75}$

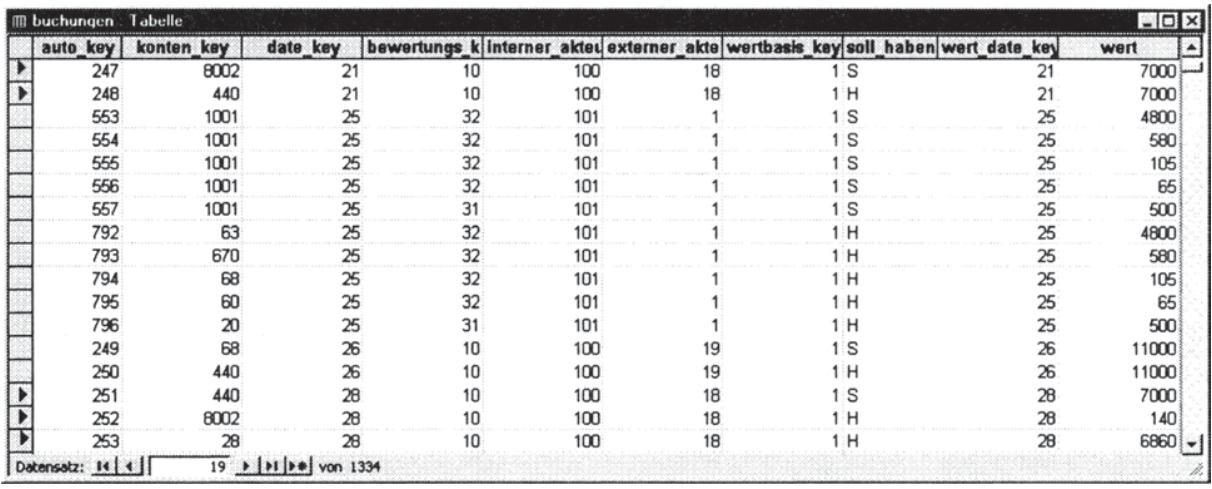

Abb. 13: Screenshot Relation Buchungen

Die Implementierung der Relation buchungen wird in der Abb. 13 wiedergegeben. Die fünf links durch Pfeile markierten Tupel korrespondieren zu den in Tab. 2 wiedergegebenen zwei Geschäftsvorfällen ${ }^{76}$.

\begin{tabular}{|l|l|}
\hline 21.01.98 & $\begin{array}{l}\text { Lieferung und Rechnungseingang TechConsult GmbH Re-Nr. 98002: } \\
\text { Entwicklungssystem: 7.000 DM }\end{array}$ \\
\hline 28.01 .98 & $\begin{array}{l}\text { Zahlungsausgang Firma TechConsult GmbH Re-Nr. 98002: 6.860 DM } \\
\text { (exkl. 2\% Skonto) }\end{array}$ \\
\hline
\end{tabular}

Tab. 2: Beispielgeschäftsvorfälle Anlagenbuchungen

$75 \mathrm{Zu}$ der Beispielsimplementierung sei angemerkt: Bei dem fiktiven Unternehmen handelt es sich um eine kleine Start-Up GmbH, deren Geschäftszweck in der Erstellung und Veräußerung von Sicherheitssoftware, sowie in der Beratung und Schulung in Sicherheitsfragen besteht. Gerade in der Anlaufphase besteht für dieses Unternehmen das Problem, daß ein Großteil der ökonomischen Wertschöpfung in der Produktion von klassisch nicht anzusetzenden immateriellen Vermögensgegenständen besteht. Anhand von unterschiedlichen Bewertungskonzeptionen läßt sich dieses Problem der bilanziellen Abbildung gut verdeutlichen. Bei der Verbuchung der im Anhang angegebenen Geschäftsvorfälle wurde eine gewisse „akademische Lässigkeit" an den Tag gelegt: So wurde u.a. auf die Berücksichtigung von Steuern aller Art verzichtet und recht stark vereinfachende Annahmen zur Gemeinkostenschlüsselung getroffen. Diese Vereinfachungen sind allerdings nur buchhalterischer Natur und beeinflussen nicht den Erklärungsgehalt des Beispiels.

76 Auf die Wiedergabe sämtlicher Geschäftsvorfälle und deren Verbuchung wurde im Rahmen dieser Arbeit verzichtet. Interessenten an der Beispielsimplementierung sind herzlich eingeladen, sich direkt an den Autor zu wenden. 
Traditionell formuliert lauten die Buchungen:

21. Januar 1998:

Betriebs- und Geschäftsausstattung (Konto-Nr. 8002)

an Verbindlichkeiten aus Lieferung und Leistung (Konto-Nr. 440) 7.000,00 DM

28. Januar 1998:

Verbindlichkeiten aus Lieferung und Leistung 7.000,00 DM

an Betriebs- und Geschäftsausstattung $140,00 \mathrm{DM}$

und an Liquide Mittel (Konto-Nr. 28) $\quad 6.860,00 \mathrm{DM}$

Diesen Buchungen werden in der Relation noch die entsprechenden internen und externen Akteure, das entsprechende Bewertungskonzept (10 steht für historische Kosten) und die Bezugsgröße (monetär) und Zeitpunkt (Zeitpunkt des Erwerbs) zugeordnet.

Um die Daten der Finanzbuchhaltung in das PAIS-Datenmodell übernehmen zu können, müssen die Merkmalsausprägungen der jeweiligen Dimensionen also für alle Geschäftsvorfälle wie in diesem Beispiel noch spezifiziert werden. Bei den meisten Dimensionen kann hierfür wiederum auf den operativen Datenbestand des berichtenden Unternehmens zurückgegriffen werden, so ist die Kontendimension evtl. wie hier aus dem verwendeten Kontenrahmen ableitbar und die Zeitdimension wird in der Finanzbuchhaltung bereits erfaßt. Für die externe Akteurdimension scheint ein Rückgriff auf die Kundenund Lieferantendaten der Kreditoren- und Debitorenbuchhaltung denkbar. Die interne Akteurdimension läßt sich ggf. durch Rückgriffe auf die inteme Organisation, auf die rechtliche Organisation oder auf die Verwendung von unterschiedlichen Buchungskreisen im Finanzbuchhaltungssystem spezifizieren. ${ }^{7}$

Im Regelfall neuartig wird die Bewertungsdimension sein, da gerade die Multidimensionalität hinsichtlich der Ansatz- und Bewertungsvorschriften eine Innovation des PAIS darstellt. Um die Buchungen hinsichtlich der Bewertungsdimension zu charakterisieren, sind zunächst die bestehenden Ansatz- und Bewertungsentscheidungen zu identifizieren und deren korrespondierende Buchungen zu erstellen. Dann können alternative Bewertungskonzepte mit ihren Buchungen in die Buchungsrelation eingefügt werden. Hierbei dürfte nur in Ausnahmefällen der Rückgriff auf bereits vorhandene Datenbestände möglich sein. Die Methoden zur Abbildung von Ansatz- und Bewertungswahlrechten werden im nächsten Abschnitt erläutert.

$\pi \quad$ Vgl. als weitere Beispiele für mögliche interne Akteure auch die von SAP R/3 eingeführten Organisationseinheiten, vgl. Wolf (1997), S. 68-72. 


\subsubsection{Erfassung von Ansatz- und Bewertungswahlrechten}

Durch die Einführung von multidimensionalen Buchungseigenschaften entstehen logisch bedingte Probleme. Sämtlichen Buchungen, deren Betrag nicht nur direkt von realökonomischen Sachverhalten, sondern auch von anderen Buchungswerten und Kontenständen abhängen, kann kein absoluter Buchungswert mehr zugeordnet werden, da die referenzierten Kontenstände ebenfalls von Bewertungsentscheidungen abhängen. Als einfaches Beispiel möge die Erfassung eines Umsatzprozesses dienen, wobei eine GuV nach Umsatzkostenverfahren erstellt werden soll. Im Rahmen der Produktfertigung werden die Herstellungskosten identifiziert. Hierbei hat das Unternehmen ein Bewertungswahlrecht zwischen Voll- und Teilkosten. Denkbar ist eine Umsetzung dieses Wahlrechts im Rahmen der Bewertungsdimension durch die Schaffung von zwei Bewertungsaltemativen: Sämtliche Buchungen, die zur Aktivierung der Teilkosten gehören, werden einem Bewertungsverfahren zugeordnet und alle Buchungen, die zur Aktivierung der anteiligen Gemeinkosten gehören, werden einem anderen Bewertungsverfahren zugeordnet. Je nachdem, ob beide Bewertungsverfahren oder nur die Teilkosten zur Anwendung kommen, werden im Vorratsvermögen die Voll- oder Teilkosten des fertigen Produkts ausgewiesen. Grundsätzlich wird der Wertansatz des Produktes in die Herstellungskosten eingestellt und aus den Vorratsvermögen dementsprechend ausgebucht. Der Buchungsbetrag steht jedoch erst mit der Spezifikation der anzuwendenden Bewertungsregeln (Teil- oder Vollkostenansatz) fest. Diese werden allerdings erst im Rahmen der PAIS-Datenerstellung festgelegt, somit kann in der Schnittstelle zur operationalen Datenbasis noch nicht darauf zurückgegriffen werden. Solche Buchungen werden deswegen als relative Buchungen erfaßt. Anstatt eines absoluten Buchungsbetrags wird das zu referenzierende Konto und der zu verwendende Prozentsatz in die Datenbasis eingetragen. Der eigentliche Buchungsbetrag wird durch die multiplikative Verknüpfung des Prozentsatzes mit dem aktuellen Kontensaldo ermittelt. Durch dieses Verfahren wird eine recht weitgehende Aufgliederung der einzelnen Konten notwendig. Im Beispiel müßte der Produktionsprozeß auf ein separates Vorratskonto gebucht werden, um die automatische Aggregation mit anderen Vorräten zu vermeiden.

Die Reihenfolge, in der die gewünschten Bewertungsregeln zur Anwendung kommen, ist wegen der Verwendung von relativen Buchungen von Bedeutung. Wird die Regel, welche für die Erfassung von Herstellungskosten verantwortlich ist, vor den Regeln zur Bewertung von selbsterstellten Vorräten angewendet, so wird bei der Ermittlung des Buchungsbetrags der relativen Buchung nicht der korrekte Wert für die Herstellungskosten ermittelt, sondern fälschlicherweise ein Betrag von Null, da dies dem aktuellen Saldo des Vorratskontos entspricht. Für jeden Bewertungsvektor ist also eine feste Reihenfolge vorzugeben, nach der die Bewertungsregeln durchzuführen sind, um die korrekte Ermittlung der Buchungsbeträge für die relativen Buchungen zu gewährleisten. Diese Reihenfolge wird in 
der Implementierung dieser Arbeit durch die Abfolge der numerischen Schlüssel ${ }^{78}$ der Wertbasisdimension definiert. Die Wertbasisdimension erlaubt die Differenzierung zwischen Buchungen, deren Betrag relativ zu einem anderen Kontenstand zu einem bestimmten Zeitpunkt angegeben wird und den nachfolgend erläuterten Buchungen, deren Betrag relativ zu einem Vergleichsvermögensgegenstand angegeben wird.

Diese Vorgehensweise soll noch anhand der Implementierung verdeutlicht werden. Die entsprechenden Buchungen sind in der Abb. 14 wieder durch kleine Pfeile markiert.

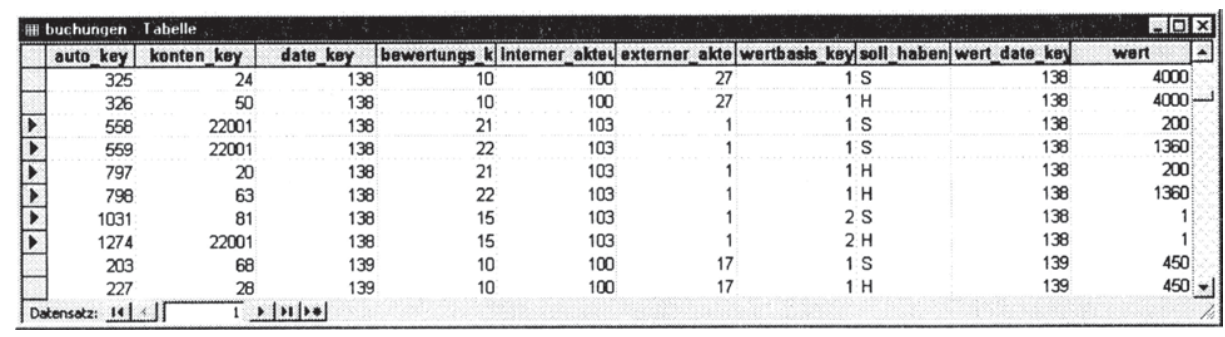

Abb. 14: Screenshot Herstellungskostenbuchungen

Der korrespondierende Geschäftsvorfall läßt sich aus Tab. 3 entnehmen.

\begin{tabular}{l|l} 
18.05.98 & $\begin{array}{l}\text { Rechnungsausgang für Beratungsleistung Schmidt Elektronik GmbH (2 } \\
\text { Ingenieurtage, 200 DM Materialeinzelkosten) } 4000 \mathrm{DM}, \text { Re-Nr. A98002 }\end{array}$
\end{tabular}

Tab. 3: Beispielgeschäftsvorfall Herstellungskosten

Um die relative Buchungskonzeption zu verdeutlichen, wird hier lediglich auf den Herstellungsprozeß eingegangen. Die Herstellungskostenbuchungen wären für den Teilkostenansatz:

Fertige Erzeugnisse (Konto-Nr. 22001) an Roh-, Hilfs- und Betriebsstoffe (Konto-Nr. 20) 200,00 DM

und

Herstellungskosten (Konto-Nr. 81)

an fertige Erzeugnisse (Konto-Nr. 22001) 200,00 DM

78 Unter einem Schlüssel wird ein spezielles Attribut oder eine Attributsgruppe einer Relation verstanden, welches jeden Tupel der Relation eindeutig definiert. 
sowie für Vollkosten:

Fertige Erzeugnisse (Konto-Nr. 22001)

$1.560,00 \mathrm{DM}$

an Roh-, Hilfs- und Betriebsstoffe (Konto-Nr. 20)

$200,00 \mathrm{DM}$

und an Gehälter (Konto-Nr. 63)

$1.360,00 \mathrm{DM}$

und

Herstellungskosten (Konto-Nr. 81)

an Fertige Erzeugnisse (Konto-Nr. 22001) 1560,00 DM

Beide Ansätze finden sich auch in der Relation buchungen. Die Teilkosten haben den Bewertungsdimensionsschlüssel 21 und die anteiligen Gemeinkosten den Bewertungsdimensionsschlüssel 22. Die sich an den „Produktionsprozeß“ anschließende Herstellungskostenbuchung ist, wie oben erwähnt, relativ. Sie hat den Bewertungsdimensionsschlüssel 15 und besteht in der vollständigen Ausbuchung des Kontos mit der Kontonummer 22001. Dieses Bezugskonto kann aus der Wertbasisdimension mit dem Schlüssel 2 entnommen werden. Das Konto mit der Nummer 22001 ist ein Unterkonto der fertigen Erzeugnisse und enthält nur das in der Tab. 3 wiedergegebene Beratungsprojekt. Somit werden durch die komplette Ausbuchung auf jeden Fall die korrekten Herstellungskosten ermittelt, unabhängig davon, ob der Bewertungsvektor einen Ansatz zu Voll- oder Teilkosten gefordert hat.

Neben der Möglichkeit, bestimmte Ansatz- und Bewertungswahlrechte durch dementsprechende Wahl des Bewertungsvektors auszuüben, wird als weitere bewertungsrelevante Schnittstelle zur operationalen Datenbasis die Möglichkeit eingeräumt, den Buchungsbetrag nicht relativ zu einem bezogenen Konto, sondern relativ zu einem Vergleichswertgegenstand anzugeben. Dieser Vergleichsgegenstand kann z.B. eine bestimmte Währung sein. Durch die Angabe des Wertes einer bestimmten Buchung relativ zu einer Währung wird die implizite Währungsumrechnung eines absoluten Buchungsbetrags vermieden. Neben dem Vergleichswertgegenstand wird ein Vergleichszeitpunkt der Buchung zugeordnet. Die Berechnung des Buchungsbetrags macht somit den Rückgriff auf eine Wertrelation notwendig, in der für die unterschiedlichen Vergleichsgegenstände Wertansätze für die unterschiedlichen Zeitpunkte vorgehalten werden. Zur Erstellung dieser Wertrelation kann z.B. auf untemehmensextem vorgehaltene Daten, wie z.B. auf Börsen- oder Devisenmarktdaten zurückgegriffen werden. Ein besonderer Fall dieser relativen Buchungen sind Buchungen, deren Betrag relativ zur Berichtswährung angegeben wird. Diese Buchungen sind im Vorfeld vereinfachend als absolute Buchungen bezeichnet worden.

Als letzte Bewertungsmöglichkeit können durch die Spezifikation eines dementsprechenden Bewertungsverfahrens für den Abschlußtyp ganze Kontenstände auf ein separat in 
der Relation marktwerte angegebenes Betragsniveau neubewertet werden. Die aus der Neubewertung entstehenden Aufwendungen und Erträge werden korrespondierenden Konten gutgeschrieben, die in dem jeweiligen Bewertungsdimensionstupel spezifiziert werden. Durch geeignete Kontenwahl läßt sich so sowohl eine erfolgsneutrale, als auch eine erfolgswirksame Erfassung abbilden. Dieses Bewertungsverfahren wird grundsätzlich immer als letztes Bewertungsverfahren angewendet. Als Bezugszeitpunkt wird der Zeitdimensionstupel des Berichtsperiodenendes gewählt.

In diesem Abschnitt sind die in der Beispielimplementierung verwendeten Verfahren beschrieben worden, mit denen die Ausgangsdaten für das PAIS-Datenmodell aus der operativen Datenbasis des Unternehmens erstellt werden. Selbstverständlich sind auch andere Methoden hierfür denkbar, z.B. eine direkte Erfassung der Daten im Starmodel. Basierend auf diesen Daten wird von den Funktionen des Applikationsservers die PAISDatenbasis für die Anwenderabfragen erstellt. Dieser zweistufige Prozeß wird in den beiden nächsten Abschnitten beschrieben.

\subsubsection{Erstellung des PAIS-Datenmodells 1. Stufe}

Aufbauend auf der originären Datenbasis, welche zumindest um multiple Ansatz- und Bewertungsverfahren bereichert wurde, wird in periodischen Abständen das PAISDatenmodell gefüllt. Die Häufigkeit der Datenproduktion hängt einerseits von der Aggregationsdichte der Zeitdimension ab und andererseits für die zweite Stufe des Datenmodells von den gewünschten Berichtsintervallen. Zum jeweiligen Erstellungszeitpunkt werden zunächst alle Buchungen, deren Betrag relativ zu einem Vergleichsvermögensgegenstand angeben wurde, jeweils über alle Dimensionen für alle Bewertungsvektoren aggregiert und mit den entstandenen Salden in die Faktentabelle eingetragen. Die relativen Buchungen, die sich auf einen anderen Kontenstand beziehen, werden anschließend erfaßt. Da, wie im Vorabschnitt beschrieben, für die korrekte Ermittlung der Buchungsbeträge die Reihenfolge der Berücksichtigung der unterschiedlichen Bewertungen maßgeblich ist, werden die einzelnen Buchungen in entsprechender Reihenfolge berücksichtigt.

Anschließend werden für alle Bewertungsvektoren, die Neubewertungsverfahren enthalten, die dementsprechenden Neubewertungen zum jeweiligen Abschlußzeitpunkt durchgeführt. Die dabei entstehenden Wertdifferenzen werden auf die entsprechenden Konten gebucht.

Die nach diesen Anpassungsmaßnahmen entstandene Relation buchungen_val ist noch nicht über alle Dimensionen aggregiert, weist also noch einen künstlichen Schlüssel auf. Außerdem sind die Buchungen für die unterschiedlichen Konten noch nicht saldiert, 
so daß die Art des Buchungsbetrags (Soll oder Haben) noch ein Element der Tabelle ist. Durch die Aggregation verschwindet der zusätzliche Key und ebenfalls die Unterteilung in Soll- und Habenbuchungen. Der aggregierte Buchungsbetrag ist positiv für Sollsalden und negativ für Habensalden. Mit der Relation buchungsbetrag_fakten ist die erste Stufe des PAIS-Datenmodells erreicht.

\subsubsection{Erstellung des PAIS-Datenmodells 2. Stufe}

Wie in den Ausführungen zum PAIS-Datenmodell geschildert, ist das Starmodel der ersten Stufe nicht uneingeschränkt logisch zu interpretieren. So wurden zum Beispiel die Neubewertungsbuchungen nicht zu jedem möglichen Zeitpunkt, sondern nur zum jeweiligen Periodenschluß vorgenommen. Das Starmodel der zweiten Stufe verzichtet komplett auf die Zeitdimension, indem für vordefinierte Berichtsperioden komplette Kontenstände ermittelt werden und als neue Fakten in das Starmodel eingehen. Für diese Aggregation müssen zunächst seitens des berichtenden Untemehmens die gewünschten Berichtsperioden spezifiziert werden. In der Implementierung dieser Arbeit gibt es monatliche, quartalsjährliche und jährliche Berichtsphasen. Für jeden gewünschten Abschluß werden dann durch Aggregation der Buchungen die Kontenstände nach den unterschiedlichen anderen Dimensionen aufgegliedert generiert. Bei der zeitlichen Aggregation der Buchungen wird mittels des entsprechenden Elements der Kontendimension zwischen Bestandskonten einerseits und Aufwands-/Ertragskonten andererseits unterschieden. Die Bestandskonten werden über sämtliche Zeitpunkte bis zum Berichtsperiodenende aggregiert, wohingegen die Aufwands/Ertragskonten nur von Berichtsperiodenstart bis Berichtsperiodenende aggregiert werden müssen. Als Ergebnis dieser Aggregation entsteht mit der Relation kontensalden_fakten das PAIS-Starmodel der zweiten Stufe, indem die Zeit- und Abschlußtypdimension zu einer neuen Abschlußdimension verschmolzen sind. Die Daten dieses Starmodel können somit direkt in die gewünschten Rechenwerke übernommen werden. Wie aus diesen Daten dann die gewünschten Rechenwerke generiert werden, wird im folgenden Abschnitt erläutert.

\subsection{Erstellung eines Abschlusses}

\subsubsection{Charakterisierende Daten für den Abschluß}

Die durch das PAIS angebotenen Abschlußstile werden in der Relation abschluss_stil als Tupel erfaßt und in ihren Merkmalen beschrieben, wobei die Implementierung dieser Arbeit lediglich ein textlich beschreibenes Merkmal vorsieht. Die Auswahl des gewünschten Abschlusses erfolgt also über die Selektion eines Tupels dieser Relation. Die Zuordnung von Rechenwerken zu den unterschiedlichen Abschlußstilen übernimmt die Relation rechenwerk_abschluss_stil_relation. Durch die 
Wahl eines Abschlußstils ist lediglich die Art des Abschlusses bestimmt. Die Wahl der gewünschten Periode und die Bestimmung des Bewertungsvektors stehen noch aus. Erst dann können die jeweiligen Postenbeträge ermittelt werden. Die Gesamtheit der Postenbeträge sind dann die Daten einer Abschlußinstanz.

Bereits bei der Erstellung der zweiten Stufe des PAIS-Starmodel war es nötig, Berichtsperioden zu spezifizieren. Diese sind gleichzeitig die Auswahlmöglichkeiten für die Einschränkung der Zeitdimension. Grundsätzlich erfolgt die Spezifizierung der angebotenen Berichtsperioden durch einen Rückgriff auf die Merkmalsausprägungen der Zeitdimension. $\mathrm{Da}$ das berichtende Unternehmen ein Interesse daran hat, daß die PAIS-Adressaten nur die Berichtsperioden nachfragen, für die es auch die entsprechenden Buchungen in den Faktendaten berücksichtigt hat, werden in der Implementierung dieser Arbeit die entsprechenden Datenbankabfragen funktional in den PAIS-Server integriert und die aus diesen Abfragen resultierenden Perioden dem Anwender zur Auswahl präsentiert.

In der Relation abschluss_typdimension sind die Abschlußtypen erfaßt, die aus einem bestimmten Abschlußstil einerseits und einem Bewertungsvektor andererseits bestehen. Dieser Vektor wird durch die Relation abschluss_typ_bewertungs_relation wiedergegeben. Durch Auswahl eines bestimmten Abschlußtyps, der den gewünschten Bewertungsvektor enthält, kann der Adressat die letzte fehlende Merkmalsausprägung seiner gewünschten Abschlußinstanz auswählen. Die Gesamtheit aller aus der beliebigen Kombination der drei Merkmale Abschlußstil, Abschlußtyp und Berichtsperiode resultierenden Abschlußinstanzen bildet die Relation abschlussdimension. Diese dient nicht der direkten Selektion durch den Adressaten, sondern über ihren automatisch erstellten Schlüssel der Zuordnung der Faktendaten zu jeweiligen Abschlußinstanzen.

Nachdem somit alle zur Ermittlung der Postenbeträge notwendigen Merkmale spezifiziert wurden, soll im folgenden auf die Ermittlung der einzelnen Postenbeträge der jeweiligen Abschlußinstanz eingegangen werden.

\subsubsection{Ermittlung der Postenbeträge}

Wie oben erwähnt, hat das PAIS des berichtenden Unternehmens vier konstituierende Bestandteile eines Abschlusses anzubieten. Erstens ist die Information vorzuhalten, welche Rechenwerke zum Abschluß gehören und in welcher Reihenfolge sie zu erstellen sind. Zweitens sind Datenbankabfragen zu generieren, welche es erlauben, die für die Rechenwerke erforderlichen PAIS-Daten aus dem Datenbestand zu extrahieren. Drittens benötigen die Adressaten mathematische Algorithmen zur Gewinnung derivativer Größen aus diesem Datenbestand und, viertens, ein Konstrukt, das die für die entsprechende Präsen- 
tation der einzelnen Rechenwerke notwendigen Daten und Methoden enthält. Im folgenden wird dargestellt, wie in der PAIS-Implementierung dieser Arbeit diese vier Bestandteile abgebildet werden.

Die Datenbankabfragen bedingen grundsätzlich eine additive Aggregation der Fakten der zweiten Stufe des Starmodel über unterschiedliche Tupelmengen der Kontendimension, wobei die gewünschte Abschlußinstanz als selektierende Dimension dient. So können z.B. sämtliche Konten mit der IKR-Kontengruppe 15 für das Rechenwerk Bilanz zu dem Posten „Wertpapiere des Anlagevermögens“ zusammengefaßt werden. Dies geschieht im Rahmen des konventionellen Jahresabschlusses durch Abschlußbuchungen. Dieses Prinzip der Abschlußbuchungen findet sich grundsätzlich auch in der Implementierung des PAIS-Datenmodells wieder. Technisch bedeutet dies eine additive Aggregation einer bestimmten Menge von Kontenständen. Die resultierende Summe wird, ggf. in Abhängigkeit vom Vorzeichen, einem bestimmten Rechenwerkposten als Betrag zugeordnet. Die Zuordnung der Konten zu den jeweiligen Posten des Rechenwerks geschieht durch die Daten der rechenwerk_regeln Relation. Anhand dieser Informationen werden die Abschlußbuchungen in der Tabelle rechenwerk_positionen_fakten festgehalten. Diese Tabelle enthält somit für alle Posten des zu erstellenden Rechenwerks die entsprechenden Summen als Saldo der Abschlußbuchungen jeweils entsprechend der übrigen Dimensionen aufgegliedert .

Aus den Posten der Relation rechenwerk_positionen_fakten müssen nun die mathematisch abhängigen Beträge für die derivativen Posten ermittelt werden. Dies kann grundsätzlich auf zwei Arten geschehen: Entweder erhält der Client die originären Daten und die funktionalen Regeln zur Ermittlung der davon abhängigen Summen oder die Ermittlung der abhängigen Summen erfolgt durch den Applikationsserver und der Client erhält sämtliche Daten zum angeforderten Rechenwerk auf einen Block, braucht also keine eigenen Berechnungen mehr durchzuführen. Aus zwei Gründen wurde für die Implementierung des PAIS der zweite Ansatz gewählt. Erstens sind die abhängigen Größen vieler Rechenwerke von zentraler Bedeutung, z.B. der Jahresüberschuß der Gewinnund Verlustrechnung, so daß berichtende Unternehmen ein Interesse besitzen, die Kontrolle über deren Ermittlung zu behalten. Zweitens sind ein Protokoll und eine Kommunikationssprache, welche in der Lage wären, beliebige mathematisch funktionale Zusammenhänge über Systemgrenzen hinweg eindeutig zu spezifizieren, alles andere als trivial, so daß eine Durchführung der Berechnungen auf dem Applikationsserver praktikabler erscheint. Also wurde die Berechnung der derivativen Größen der unterschiedlichen Rechenwerke direkt in dem Quellcode des PAIS-Servers realisiert.

Die aus der Berechnung resultierenden Postenbeträge werden in die Tabelle rechenwerk_positionen_fakten eingetragen. Eine Differenzierung dieser deri- 
vativen Posten hinsichtlich der Akteurs- und Bewertungsdimensionen ist grundsätzlich der jeweiligen Implementierung überlassen, erscheint aber nur unter bestimmten funktionalen Abhängigkeiten mathematisch logisch und ist ökonomisch auch nur beschränkt sinnvoll. Ggf. ist innerhalb der Berechnungsregeln auf die jeweilige Behandlung der Dimensionsmerkmale einzugehen. In der Implementierung dieser Arbeit wurde auf eine Zuordnung der derivativen Rechenwerkposten zu unterschiedlichen Dimensionen wegen der eingeschränkten ökonomischen Interpretierbarkeit des Ergebnisses verzichtet.

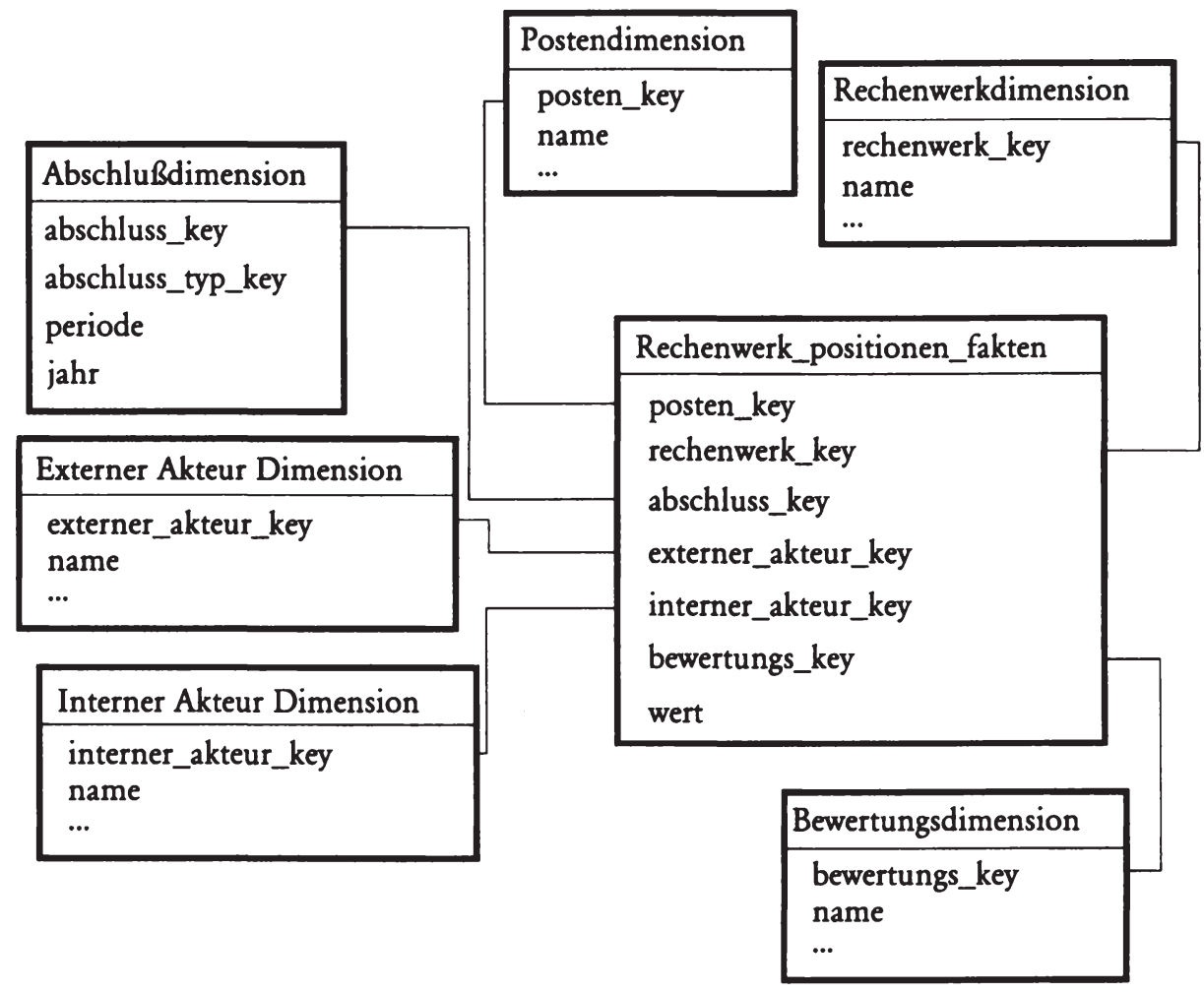

Abb. 15: PAISStarmodel 3. Stufe

Das Ergebnis sämtlicher Postenbeträge findet sich nach der Berechnung der derivativen Posten in der Relation rechenwerk_positionen_fakten. Diese stellt gleichzeitig die dritte Stufe des PAIS-Starmodel dar, die in Abb. 15 wiedergegeben wird und sich vom Modell der 2. Stufe im wesentlichen dadurch unterscheidet, daß die Kontensaldenfakten durch den regelkonformen Abschluß zu den unterschiedlichen Rechenwerkposten zusammengefaßt wurden. Aus der 3. Stufe des Starmodel lassen sich durch additive Verknüpfung der Fakten aller Tupel mit der gewünschten Abschlußinstanz und dem jeweiligen Posten als Dimensionsausprägungen die Postenbeträge der gewünschten Abschlußinstanz direkt ermitteln. Werden vom Adressaten weitere Informationen über die Zusam- 
mensetzung der einzelnen Posten über die unterschiedlichen Dimensionen gewünscht, so ist dies durch entsprechende Aufgliederung der Rechenwerkposten zu erreichen. Auf die hierfür notwendigen Schritte wird im nächsten Abschnitt eingegangen.

\subsubsection{Auswahl der Gliederungsebenen}

Unabhängig vom nachgefragten Rechenwerk können die einzelnen Posten anhand der Dimensionen der Relation rechenwerk_positionen_fakten aufgegliedert werden. Hierfür kommen die Dimensionen der internen und externen Akteure, sowie die Bewertungsdimension in Frage. Theoretisch ist es denkbar, daß die Aufgliederung der Rechenwerkposten für jeden Tupel einer bestimmten Dimensionsrelation erfolgt, also z.B. daß der Forderungsbestand aufgegliedert nach den einzelnen Kunden erfolgt. Im Regelfall wird die Aufgliederung jedoch nicht anhand der einzelnen Tupel, sondern vielmehr an bestimmten Tupelmerkmalen erfolgen. Zum Beispiel sind mögliche Merkmale der externen Akteure die Zuordnung zu bestimmten Stakeholdergruppen, ${ }^{79}$ die regionale Zugehörigkeit, sowie weitere, stakeholderspezifische Merkmale. Diese könnten für Kunden z.B. eine Charakterisierung in das $\mathrm{ABC}-\mathrm{Schema}^{80}$ und die Fakturierungswährung sein. Die Möglichkeiten der Kombination dieser Merkmale zu speziellen Untergliederungen sind so vielfältig, daß eine direkte Vorhaltung der Daten unsinnig wäre, da sie nicht die Vorteile des Starmodel nutzt. Vielmehr greift der Client auf die Relation rechenwerkposten_fakten zu, indem er die gewünschten Merkmalsausprägungen der Dimensionen setzt. Der PAIS-Server ermittelt die diesen Einschränkungen entsprechenden Postenanteile des spezifizierten Rechenwerks. Diese resultierenden Posten sind zwar für sich gesehen nur eingeschränkt informativ, doch in ihrer Summe entsprechen sie dem kompletten Posten, wie er durch die oben beschriebene Abfrage ermittelt wird.

Somit ist geklärt, wie die Abschlußinstanzen generiert und welche Einflußmöglichkeiten dem PAIS-Adressaten ermöglicht werden. Im folgenden Abschnitt wird auf die Kommunikation zwischen Client und PAIS-Server eingegangen, die es dem Client ermöglicht, die gewünschten Daten zu spezifizieren und die Anfrageergebnisse entgegen zu nehmen.

79 Vgl. zu den unterschiedlichen Stakeholdergruppen eines Untemehmens Pellens (1999), S. 12-20; Clarke (1998); zur Stakeholder versus Shareholderdiskussion in Deutschland beispielhaft Speckbacher (1997).

80 Das ABC-Schema geht konzeptionell auf die Lorenzkurve zurück. Vgl. allgemein zum ABC-Schema Welling (1999), S. 5456. 


\subsection{Abschlußanforderung durch den Client}

\subsubsection{Interaktionsproze $B$}

Aus dem vorhergehenden Abschnitt ist deutlich geworden, welche Variablen der Client spezifizieren muß, um seine gewünschte Abschlußinstanz zu selektieren. Er muß den Abschluß selektieren, die Berichtsperiode bestimmen und den Bewertungsvektor auswählen. Dafür müssen dem Client die altemativen Ausprägungen der Variablen bekannt sein. Eine Datenvorhaltung dieser unterschiedlichen Merkmalsausprägungen auf Clientseite ist nicht mit dem dynamischen Prinzip des PAIS vereinbar. Die Entscheidung über Umfang und Art der Wahlmöglichkeiten liegt beim berichtenden Unternehmen, deswegen sind dort auch die dementsprechenden Daten vorzuhalten. Eine lokale Spiegelung auf der Clientseite ist unnötig, da die Daten sich theoretisch jederzeit ändern könnten.

Der Interaktionsprozeß zwischen Client und PAIS-Server zur Auswahl der gewünschten Abschlußinstanz durchläuft mehrere Stufen. Zunächst erfragt der Client für die drei Variablen die zulässigen Ausprägungen. Diese werden ihm vom Server geliefert. Im Anschluß daran werden diese Alternativen dem Anwender zur Selektion präsentiert. Der Anwender wählt seine gewünschten Ausprägungen. Diese Selektion wird dem PAIS-Server übermittelt, der daraufhin die korrespondierende Abschlußinstanz bereitstellt. Dieser mehrstufige Kommunikationsprozeß macht die Verwendung eines Kommunikationsprotokolls notwendig. Da diese Kommunikation zwischen dem PAIS-Server und einem ex ante unbekannten Clientsystem von zentraler Bedeutung für die gesamte PAISKonzeption ist, soll auf die unterschiedlichen Protokollmöglichkeiten im nächsten Abschnitt recht detailliert eingegangen werden. Dies geschieht deswegen auf einem recht technischen Niveau, da in der Implementierung des PAIS-Konzepts nur eine Alternative realisiert wurde, die technische Möglichkeit der anderen Konzepte hier jedoch auch dargestellt werden soll.

\subsubsection{Protokollalternativen}

Kommunikation zwischen unabhängigen Programmsystemen benötigt ein Protokoll. ${ }^{81}$ Hierfür kann der Entwickler einerseits auf ein Standardprotokoll zurückgreifen, oder ein spezifisches Protokoll für den Kommunikationszweck entwickeln. Die Vorteile eines Standardprotokolls liegen in seiner weiten Verbreitung und in der Möglichkeit, Standardsoftware zur Implementierung einzusetzen. Die Vorteile eines Spezialprotokolls liegen u.a. in einer effizienteren Kommunikation ohne redundante Elemente.

${ }^{81}$ Vgl. zum Thema Interprozesskommunikation speziell in Unix-Systemen Herold (1996), S. 583-713. 
Im Rahmen dieser Arbeit soll auf den Clientsystemen Standardsoftware zum Einsatz kommen, die über möglichst viele Plattformen hinweg einsetzbar ist. Die Wahl fiel somit auf die Verwendung eines HTTP-Clients. Diese sogenannten Browser werden von unterschiedlichen Unternehmen entwickelt und vertrieben, sind kostengünstig bzw. kostenlos für alle wichtigen Plattformen verfügbar und nutzen mit HTTP ein standardisiertes Kommunikationsprotokoll zur Übertragung von Dokumenten. ${ }^{82}$ Der Großteil der Dokumente, die über HTTP erreichbar sind, sind in der Hypertext Markup Language (HTML) verfaßt. ${ }^{83}$ Der Gesamtbereich der über HTTP zu erreichenden Dokumentenmenge wird auch als World Wide Web (WWW) bezeichnet.

Ein großer Vorteil von HTML ist die Unterstützung von Forms, also von Schaltelementen auf der Clientseite, über die der Anwerder dem HTTP-Server bestimmte Daten übermitteln kann, die dieser wiederum auswertet. Dieses Verfahren wird auch in der Implementierung genutzt. Zur Spezifizierung seiner gewünschten Abschlußinstanz ruft der Anwender eine bestimmte HTML-Seite des PAIS-Servers auf. Diese HTML-Seite wird auf dem Server entweder statisch vorgehalten, oder auf Anfrage dynamisch durch ein Common Gateway Interface (CGI) Programm erstellt. ${ }^{84}$ Falls die Seite statisch vorgehalten wird, muß über geeignete Prozeduren dafür Sorge getragen werden, daß die Seite und der PAIS-Datenbestand für die Merkmale der Selektionsvariablen immer deckungsgleich sind. Ändert das Unternehmen häufig die Auswahlmöglichkeiten, ist entweder bei jeder Änderung des Datenbestandes die Auswahlseite automatisiert neuzuerstellen oder eine CGILösung sinnvoll. Um dieses Verfahren zu demonstrieren, wurde letzteres in dieser Arbeit implementiert.

Auf der Auswahlseite sind für die drei abschlußspezifizierenden Variablen dementsprechende Auswahlelemente anzubieten. In der Regel wird dies über sogenannte Drop-down lists realisiert, die über das HTML-Tag <select> innerhalb eines HTML-Formulars eingefügt werden. Der Anwender benutzt diese drei Auswahllisten und leitet seine Wahl über die „Submit"-Schaltfläche an den PAIS-Server weiter. Somit sind sämtliche Stufen der Auswahlkommunikation über HTTP und HTML, also mit Standardprodukten, reali-

82 HTTP ist ein Protokoll des Applikationslayers des TCP/IP Schichtenmodells. Vgl. zu den Implementierungsdetails von HTTP/1.0 RFC 1945, von HTTP/1.1 RFC 2068; dieser Request for Comment (RFC) wurde von der Internet Engineering Task Force (IETF) weiterentwickelt zu einem Internet-draft mit der Kennung draft-ietf-http-v11-spec-rev-06.

83 HTML kann mittlerweile durch viele proprietäre und browserspezifische Erweiterungen nur noch beschränkt als standardisiert gelten. Ein anerkannter HTML-Standard HTML 2.0 ist RFC 1866. Weitere wichtige Standardisierungen wurden vom World Wide Web Consortium (W3C) herausgegeben. Innerhalb des WWWW hat momentan HTML 3.2 weite Verbreitung, vgl. zur Standardisierung REC-html32, die neue Standardisierungsebene ist HTML 4.0, vgl. REC-html40. Zu den zahlreichen aktuellen Entwicklungen im Bereich des dynamischen HTML vgl. umfassend Goodman (1998).

84 Vgl. zur Spezfikation von CGI CGI/1.1; eine Weiterentwicklung dieses Standards zu einem RFC ist geplant. 
siert, ohne daß die Entwicklung eines eigenen Kommunikationsprotokolls notwendig wurde. Die resultierende Benutzerschnittstelle findet sich in Abb. 16.

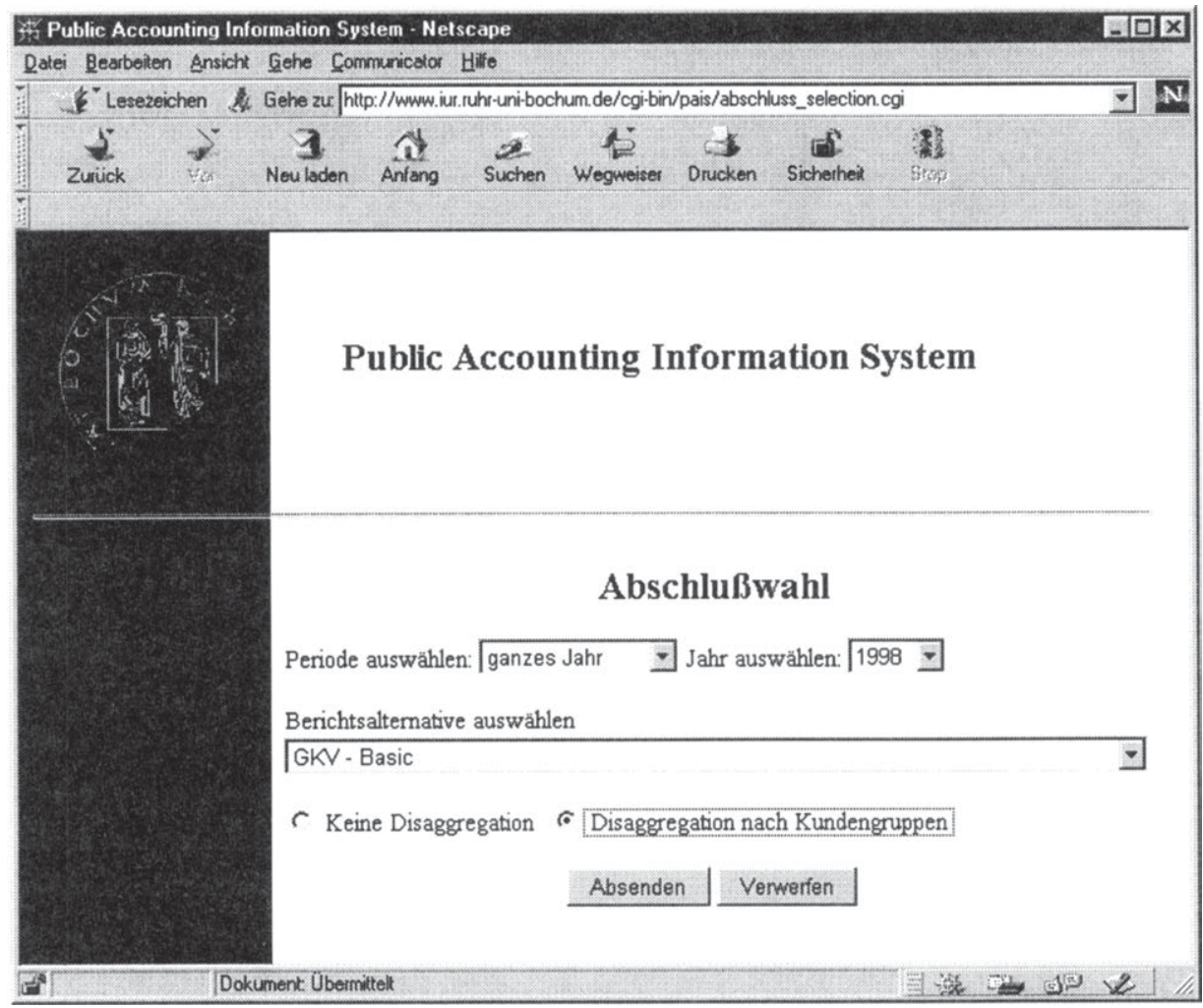

Abb. 16: HTML basierte PAIS-Benutzerschnittstelle

Eine sinnvolle Alternative zu dem hier vorgestellten HTTP-basierten Verfahren stellt die direkte Freigabe der wesentlichen Elemente des PAIS-Datenmodells für den Datenbankzugriff über WAN dar. In Frage kämen hier die Relationen rechenwerkposten_fakten, bewertungsvektoren, berichtsperioden, abschluesse und abschlussinstanzen. Wenn der Adressat direkten Zugriff auf diese Relationen hat, kann er die gewünschten Daten seiner Abschlußinstanz durch eine SQL-Abfrage ermitteln:

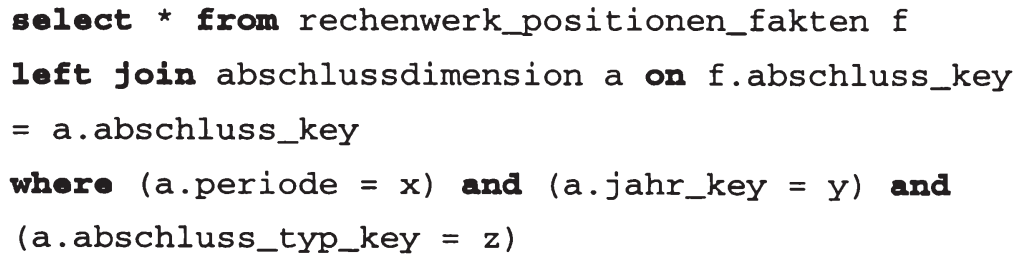


Hierbei ist es nicht notwendig, daß der Adressat seine Abfragen direkt über ein SQL Monitor dem PAIS-System zuführt. Im Gegenteil: In der Regel wird wohl geeignete Clientsoftware diese Aufgabe übernehmen und so dem Adressaten die Mühe abnehmen, seine Recherchefragen in SQL formulieren zu müssen.

Der WAN-Zugriff auf den PAIS-Datenbestand kann entweder über spezifische Protokolle des jeweiligen DBS erfolgen oder über den De-facto-Standard des systemübergreifenden Datenbankzugriffs Open Database Connectivity (ODBC). ODBC dient als Application Programming Interface (API) für DBS und benutzt SQL zur Kommunikation. Da für zahlreiche DBS entsprechende ODBC-Treiber verfügbar sind und vor allem unter dem marktbeherrschenden Betriebssystemen der Firma Microsoft zahlreiche Anwendungsprogramme auf die in das Betriebssystem integrierte ODBC-Schnittstelle zugreifen können, bietet sich die Verwendung von ODBC zur Kommunikation zwischen PAIS-Server und Client an. Der direkte Datenbankzugriff erleichtert vor allem die Aufbereitung und Präsentation der von dem PAISServer bereitgestellten Abschlußinstanz. Auf diese Fragen wird im nächsten Abschnitt eingegangen. In der Implementierung dieser Arbeit ist die Möglichkeit des direkten ODBC Zugriffes vorgesehen, indem bei der Auswahl des DBS die Verfügbarkeit von ODBC-Treibern für unterschiedliche Systemplattformen sichergestellt wurde. Auf die Entwicklung von speziellen Clientsystemen, die über bloße Standardsoftware, wie z.B. die Microsoft Office-Produktlinie, hinausgehen, wurde jedoch verzichtet.

\subsection{Ubertragung der Abschlußdaten}

\subsubsection{Mögliche Untergliederungen der Posten}

Die vom PAIS zur Verfügung gestellten Daten eines Postens einer bestimmten Abschlußinstanz entsprechen einem Datenwürfel, dessen Dimensionen die internen und externen Akteure und die angewendete Bewertungsregel sind. Schnitte durch den Würfel ermöglichen die Aufgliederung der Postendaten. Wie viele Elemente jede Würfelkante hat, hängt von der Gruppenaufgliederung der Dimensionen ab, deren Möglichkeiten bereits in Kapitel V-4.5.3 diskutiert wurden. Grundsätzlich kann eine Dimension anhand jedes ihrer Elemente alternativ gruppiert werden. Die Gruppierungen derjenigen Dimensionen, nach denen eine Aufgliederung gewünscht wird, sind vom Adressaten auszuwählen. Anhand dieser Gruppierungen werden dann die einzelnen Würfelelemente klassifiziert. Für jeden Posten der Rechenwerke gibt es einen Datenwürfel. Somit ist es theoretisch möglich, für jeden Posten unterschiedliche Dimensionsgruppierungen zu spezifizieren.

Hier soll zunächst die Visualisierung über eine tabellarische Präsentation der nach Dimensionen aufgegliederten Postenbeträge als einziges Präsentationsverfahren der Clients 
implementiert werden. ${ }^{85}$ Für diese Untergliederung ist die Reihenfolge der „Würfelschnitte“ maßgeblich. Zwar liefert jede Schnittreihenfolge letztlich jedes einzelne Würfelelement, insofern enthalten alle Reihenfolgen die gleiche Information, doch dürften für den Anwender weniger die einzelnen Würfelelemente, als vielmehr bestimmte Teilsummen von Interesse sein. Die Bildung der Teilsummen jedoch wird durch die Schnittreihenfolge determiniert.

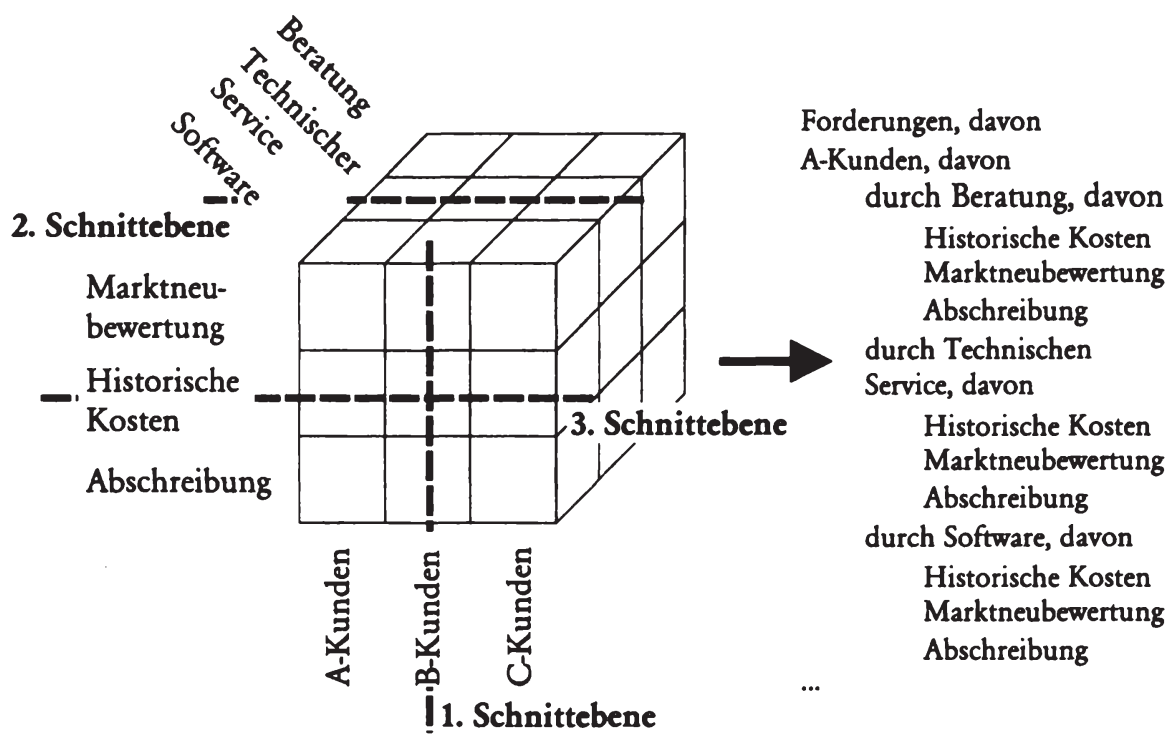

Würfelschnitt des Forderungspostens korrespondierende Gliederung

Abb. 17: Exemplarischer Datenwürfel des Forderungspostens

Wie sich diese Würfelschnitte auf die resultierende Untergliederung der unterschiedlichen Rechenwerkposten auswirken, wird in Abb. 17 am Beispiel des Forderungsbestandes verdeutlicht. Natürlich ist es auch möglich, den Postenwürfel nicht in alle drei möglichen Schnittebenen zu teilen. Das Clientsystem muß also vom Benutzer zur Untergliederung der Posten die gewünschten Schnittebenen sowie die Reihenfolge deren Anwendung erfragen. Daraufhin kann es ihm dann die gewünschte Gliederung präsentieren. Somit sind in Anschluß an die Selektion der gewünschten Abschlußinstanz und deren Bereitstellung durch den PAIS-Server folgende Ablaufschritte zu absolvieren: Der Anwender muß Schnittebenen und Schnittreihenfolgen bestimmen, ggf. kann dies für alle Posten einzeln oder einmal für alle Posten geschehen. Für jede zu schneidende Dimension muß das gruppierende Element angegeben werden, auch dies entweder für alle Posten einmal oder

85 Für graphische Präsentationtsaltemativen von multidimensionalen Datenstrukturen vgl. Cleveland (1993), Jacoby (1998); einige Ansätze für PAIS-Daten finden sich in Kapitel V-4.8.2 
für jeden Posten einzeln. Der Client muß ggf. die Daten vom PAIS-Server abrufen, die Gliederung muß erstellt, mit den Daten gefüllt und dem Anwender präsentiert werden. Wie schon bei der Selektion der gewünschten Abschlußinstanz gibt es auch für die Implementierung dieses Ablaufes unterschiedliche Konzepte, die kurz geschildert werden sollen.

\subsection{2 Übertragungsalternativen}

\subsubsection{HTML}

Nachdem die gewünschte Abschlußinstanz vom Anwender selektiert wurde, müssen die entsprechenden PAIS-Daten übertragen und entsprechend der Schnitt- und Gruppierungswünsche des Adressaten dimensional gegliedert und präsentiert werden. $\mathrm{Da}$ die Retrievalkommunikation bereits über HTTP/HTML abgewickelt wurde, liegt es nahe, auch die Datenübertragung und Präsentation auf dem gleichen Weg zu organisieren, zumal dann für die Clientsysteme komplett auf Standardsoftware zurückgegriffen werden kann und die Entwicklung von Spezialclientsoftware nicht nötig ist. ${ }^{86}$

Der HTML-Client ruft die Daten des PAIS-Server nicht im Rohzustand ab. Die einzigen Daten, die er vom PAIS-Server erhält, sind die fertig präsentierten und gegliederten Daten der Abschlußinstanz als dynamisch erstellte HTML-Seite. Im ersten Schritt jedoch muß der Anwender seine gewünschten Schnitte spezifizieren und die gruppierenden Elemente der zu schneidenden Dimensionen bestimmen. Dies geschieht über ein vom PAIS-Server erstelltes HTML-Formular. Da die Einstellmöglichkeiten des Anwenders nicht von den PAIS-Daten abhängen, sondern von dem unterliegenden Datenmodell und den vorhandenen Dimensionselementen, ist eine dynamische Erstellung dieses Formulars wohl in der Regel nicht notwendig. Je nachdem, ob die Möglichkeit gegeben sein soll, für unterschiedliche Rechenwerkposten unterschiedliche Schnitte und/oder Dimensionsgruppierungen zu fordern, kann das zur Eingabe notwendige HTML-Formular sehr groß und komplex werden, was zu einer wenig ergonomischen Oberflächengestaltung führt. Deswegen wurden in der HTML-Implementierung dieser Arbeit dem Anwender nur sehr restriktive Gliederungsgestaltungsmöglichkeiten eingeräumt, die in der in Abb. 16 wiedergeben Benutzerschnittstelle zu finden sind. Er hat lediglich die Wahl, eine gewünschte Schnittebene anzugeben, die bei allen Posten dann einheitlich zur Anwendung kommt. Die Möglichkeit zur Gruppierung auf Basis der unterschiedlichen Dimensionen wird ihm nicht gewährt. Diese Limitierungen sind nur mit dem exemplarischen Charakter der Implementierung zu rechtfertigen.

\footnotetext{
86 Vgl. analog Behme/Kruppa (1999); Mucksch/Behme (1998), S. 7484.
} 
Hat der Anwender diese Angaben gemacht, so produziert der PAIS-Server darauf basierend die entsprechend aufgegliederte Abschlußinstanz. Diese wird in einer HTML-Seite aufgebaut, wie in Abb. 18 exemplarisch wiedergegeben. Zur Strukturierung und Gestaltung der Ausgabe wird auf ein in der PAIS-Datenbasis vorgehaltenes HTML-Template zurückgegriffen. Stehen unterschiedliche HTML-Templates für die zu der Abschlußinstanz gehörenden Rechenwerke zur Verfügung, so wird dem Leser mittels eines zwischengeschalteten HTML-Formulars die Möglichkeit gegeben, eines auszuwählen.

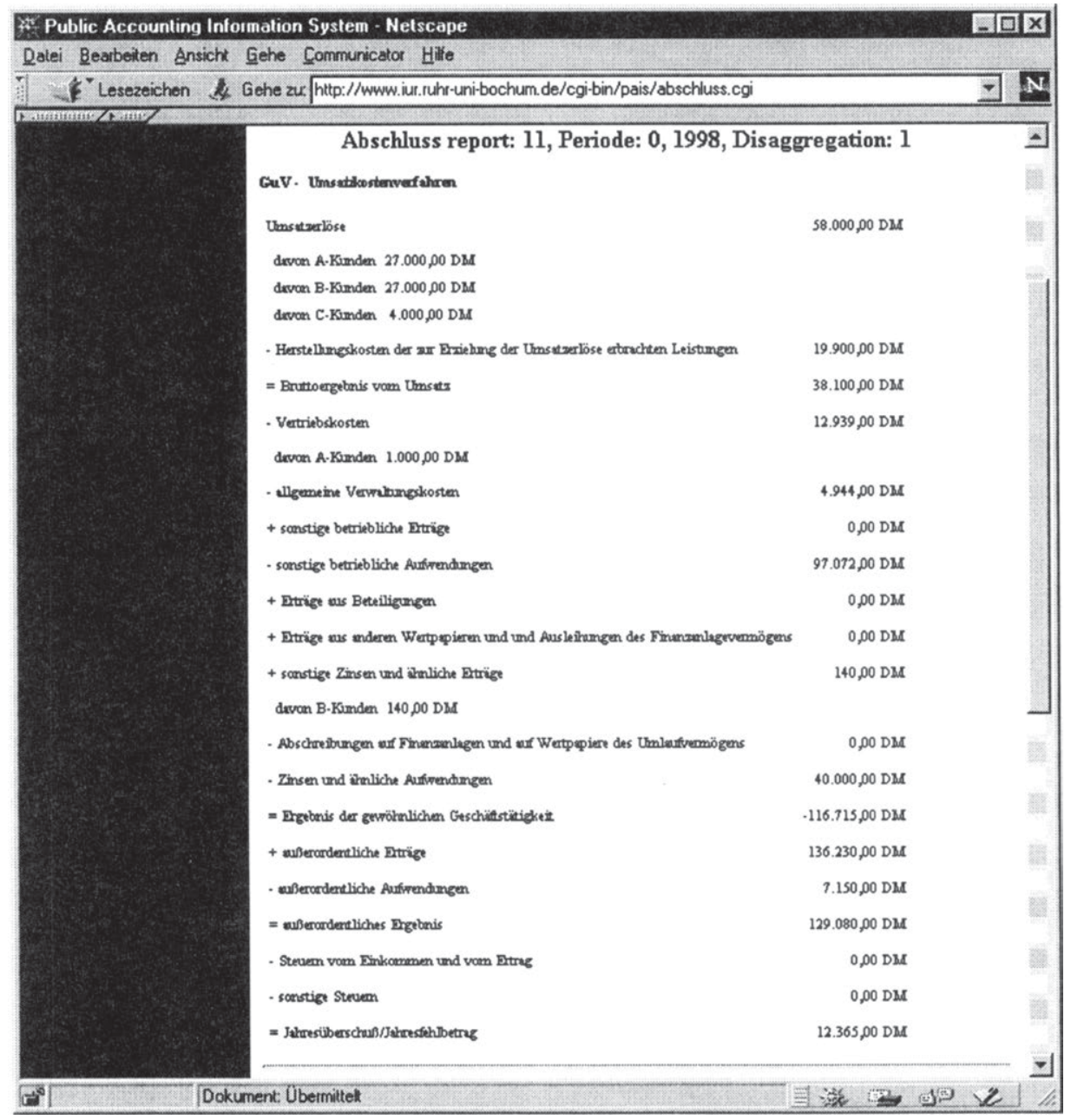

Abb. 18: HTML Ausgabe einer GuV

Der Rückgriff auf HTTP/HTML zur Präsentation der Abschlußinstanz beim Adressaten hat neben den bereits erwähnten Vorteilen auch eine Reihe teilweise schwerwiegender Nachteile. So stößt das Konzept der Eingabesteuerung über HTML-Formulare durch die 
Vielzahl der möglichen Einflußmöglichkeiten des Adressaten an seine Grenzen, da die Möglichkeiten zur Benutzerführung über HTML letztendlich beschränkt sind. Eine Reduktion dieser Einflußmöglichkeiten, wie in der Beispielimplementierung geschehen, konterkariert jedoch den Sinn von multidimensionalen Datenmodellen, der in der Vielzahl der Analysemöglichkeiten liegt. Des weiteren erhält der Client lediglich die HTML Seite mit der präsentierten Abschlußinstanz als Ergebnis der Anfrage. Dies mag für einen Endnutzer der Daten akzeptabel sein, da diese Sichtweise der traditionellen Form der Jahresabschlußpräsentation entspricht. Wenn jedoch auf der Clientseite eine EDVtechnische Weiterverarbeitung der Daten geplant ist, z.B. mit Tabellenkalkulationssoftware oder statistischen Analysepaketen, oder auch mit speziellen PAIS-Clients, so ist die Präsentationsform als HTML-Seite für die weitere Verarbeitung sehr hinderlich. ${ }^{87}$ Deswegen werden im folgenden zwei hinsichtlich dieser Schwachpunkte bessere Alternativen vorgestellt.

\subsubsection{Extensible Markup Language (XML)}

Die beschränkten Möglichkeiten von HTML, den Text einer HTML-Datei inhaltlich zu strukturieren und zu systematisieren, waren eine der Hauptgründe für die Entwicklung von XML durch das W3C. ${ }^{88}$ Gerade eine klare inhaltliche Struktur der Hypertextdaten ist für die automatisierte Auswertung und Weiterverarbeitung unerläßlich.

Wie schon HTML beruht XML auf der Standard Generalized Markup Language (SGML). SGML ist eine Beschreibungssprache für den Inhalt und die Struktur unterschiedlicher Arten elektronischer Dokumente. ${ }^{89}$ HTML ist eine spezielle Anwendung von SGML für Dokumente, die in Bereichen der täglichen hauptsächlich textbasierten Kommunikation auftreten. Deswegen enthält HTML strukturierende Tags für Absätze, Aufzählungen, Bilder usw. und keine für speziellere Daten wie Musiknoten, mathematische Formeln, Molekularstrukturen etc. Für diese spezielleren Kommunikationsaufgaben können wiederum andere SGML-Anwendungen entwickelt werden. XML hingegen ist keine SGML Anwendung, sondem eine vereinfachte Untermenge von SGML. Es fungiert also wie SGML als eine Art Metahypertextsprache, welche es dem Anwender ermöglicht, eigene

87 In Verbindung mit der üblichen Präsentation von Jahresabschlußdaten im Internet haben auf diesen Sachverhalt bereits Westarp et al. (1999) hingewiesen. Sie skizzieren ebenfalls einen XML-Entwurf für elektronische Jahresabchlußpräsentationen, der konzeptionell in die gleiche Richtung wie der XML-Ansatz des folgenden Abschnittes zielt. Weitere Hinweise zur Anwendung von XML im Rahmen der Rechnungslegungspublizität finden sich in Hoffman/Kurt/Koreto (1999); sowie jüngst vom AICPA, vgl. Harding (1999), im Rahmen der Entwicklung eines XML-Dialekts für finanzielle Berichterstattung, der XML-based Financial Reporting Markup Language (XFRML), vgl. dazu http://www.xfrml.org, Stand: 30.03.2000.

88 Vgl. Behme (1999).

89 Vgl. zur Definition und Spezifiaktion von SGML ISO 8879, zur Anwendung im praktischen Umfeld Rieger (1995). 
Hypertextbeschreibungssprachen zu entwickeln. Im Gegensatz zu SGML zeichnet sich XMI durch ein recht einfaches Konzept aus. Sie wurde speziell entwickelt, um die Verbreitung von SGML im Internet zu erhöhen und somit den engen Rahmen von HTML als De-facto-Standard zu verlassen.

Ein XML-Dokument ${ }^{90}$ muß speziellen lexikalischen Ansprüchen genügen, um als korrekt strukturiert (well-formed) zu gelten. Innerhalb eines korrekt strukturierten XMDokuments können beliebige Hypertext-Tags verwendet werden, um den Dokumentinhalt zu strukturieren. Wenn der Ersteller der XML-Datei eine Standardisierung der zu verwendenen Tags wünscht, benötigt er eine formale Strukturbeschreibung. Eine solche Strukturbeschreibung wird als Document Type Definition (DTD) bezeichnet. ${ }^{91}$ Ein korrekt strukturiertes XML-Dokument, welches einer innerhalb des Dokumentes spezifizierten DTD entspricht, wird als gültig (valid) bezeichnet. Durch die DTD wird also ein Rahmen vorgegeben, innerhalb dessen die Dokumentinhalte strukturiert werden können. Durch die formalisierte Darstellung der Dokumentstruktur wird die EDV-technische Weiterverarbeitung der innerhalb der Dokumente präsentierten Daten wesentlich erleichtert.

Ziel der Präsentation der Abschlußinstanz ist es, dem Adressaten möglichst weitgehende Analyse- und Auswertungsmöglichkeiten zu gewähren. Um die Weiterverarbeitung zu erleichtern, erscheint also die Spezifikation einer DTD nötig. Die DTD muß alle nötigen Elemente der Abschlußinstanz erhalten. Hierfür scheinen zwei Wege vorstellbar. Entweder wird die DTD ohne genaue Kenntnis des jeweiligen PAIS-Datenmodells entworfen, oder speziell auf eine bestimmte Implementierung des Modells hin angepaßt. Im ersten Fall muß die DTD neben den einzelnen Daten auch Strukturen beinhalten, mit deren Hilfe die in der jeweiligen Implementierung verwendeten Dimensionen und deren Elemente beschrieben werden können. Im zweiten Fall ist dies nicht nötig. Hier können die Dimensionen und Elemente direkt als Strukturelemente verwendet werden.

Eine DTD für die Darstellung einer Abschlußinstanz ohne Referenz auf ein bestimmtes PAIS-Datenmodell könnte wie folgt aussehen ${ }^{92}$ :

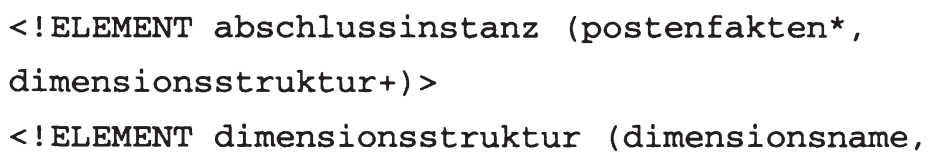

0 Vgl. zu den folgenden Ausführungen zu XML die Spezifikation von W3C REC-xml-19980210; sowie zu einer ersten Einführung Schinzer/Thome (1999); Behme (1999).

91 Vgl. Behme (1999a).

92 Vgl. zur Syntax W3C REC-xml-19980210, insb. Gliederungspunkt 3.2. 


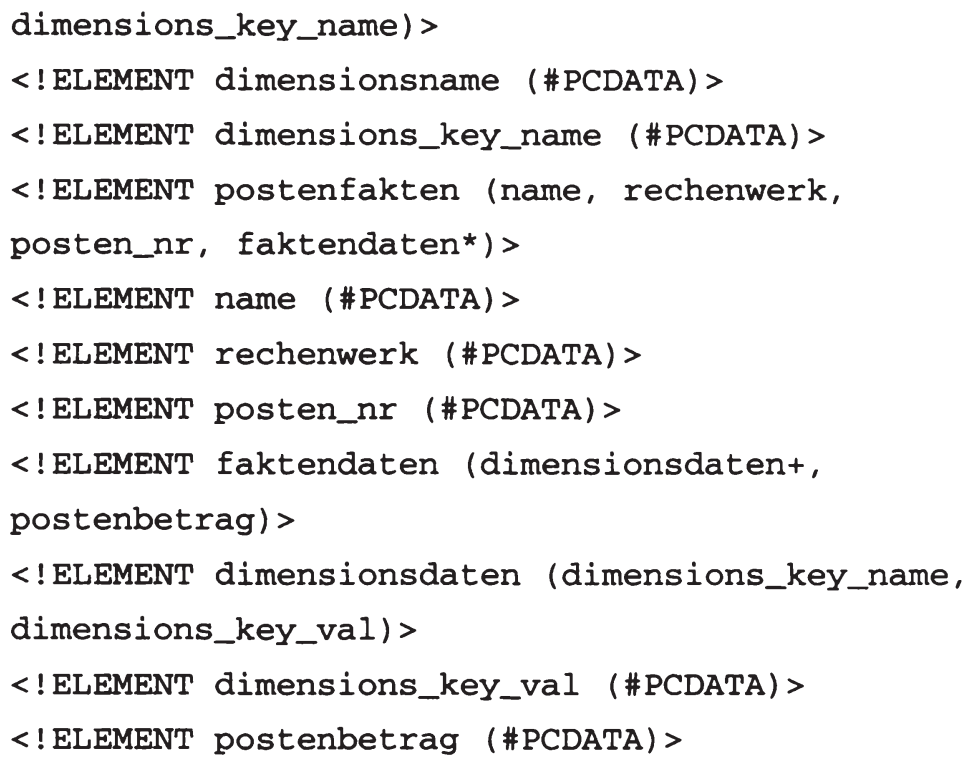

Die DTD spezifiziert zunächst das übergeordnete Strukturelement abschlussinstanz, das sich aus mehreren Rechenwerkposten zusammensetzt. AuBerdem wird jede Dimension des implementierten PAIS-Datenmodells in dem Strukturelement dimensionsstruktur beschrieben. Da die Elemente einer Dimensionsrelation selber in einer separaten DTD spezifiziert werden, reicht für diese DTD der Name des Dimensionsschlüsselelements und der Name der Dimensionsrelation aus. Die Rechenwerkposten werden durch das Strukturelement postenfakten beschrieben. postenfakten besteht einerseits aus Angaben zu dem jeweiligen Posten, also zu dessen Name, der ihm zugeordneten Nummer und zum Namen des Rechenwerks, zu dem er gehört. Andererseits sind die zu diesem Posten gehörenden Daten der zentrale Bestandteil der Struktur. Sie werden in dem Strukturelement faktendaten beschrieben. Dort enthält dimensionsdaten die Fremdschlüssel und Namen der Dimensionen und postenbetrag den korrespondierenden Betrag. Es wird hier bereits deutlich, daß die DTD nicht verhindert, daß in fehlerhaften XML-Dateien in dimensionsdaten auf Dimensionenschlüssel verwiesen wird, die in dimensionsstruktur nicht genannt wurden, ohne daß dies der XML-Parser ${ }^{93}$ erkennen würde. Die XML-Datei würde trotzdem als gut strukturiert und gültig hinsichtlich der o.a. DTD gelten.

93 Ein Parser ist ein Software-Modul, daß einen vom Entwickler verfaßten Quellcodetext oder sonstige Dokumente syntaktisch analysiert und in einzelne von nachfolgenden Programmen interpretierbare Subeinheiten (Token) aufteilt. 
Wie bereits erwähnt, reicht ein XML-Dokument, welches der obigen DTD entspricht, nicht aus, da auch noch die verwendeten Dimensionen beschrieben werden müssen. Auch hierfür wird ein DTD erstellt:

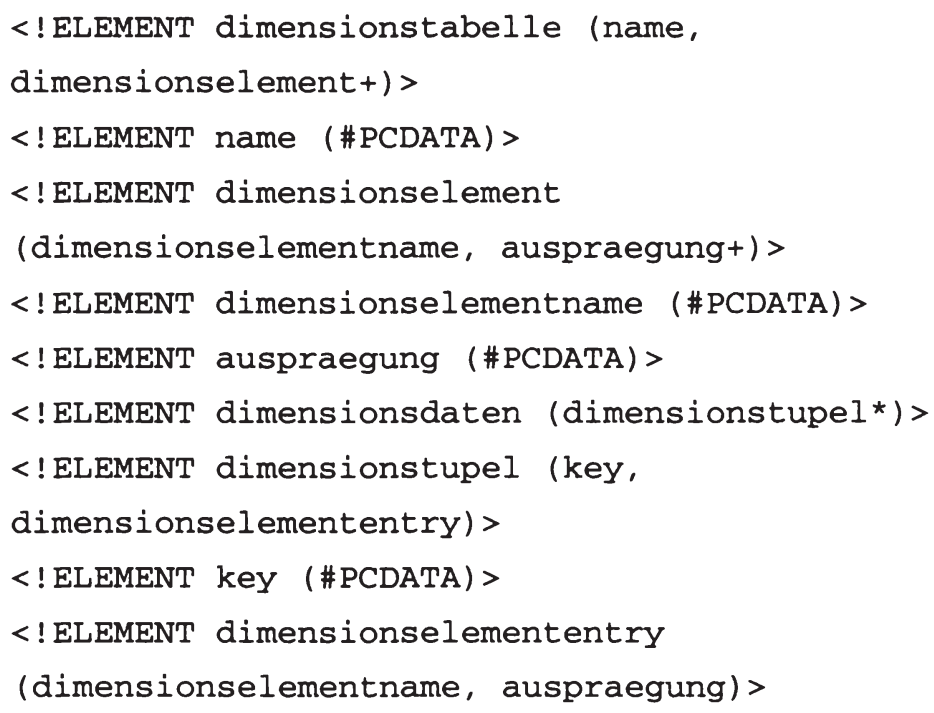

Die in dem Strukturelement dimensionstabelle beschriebene Dimension wird durch ihren Namen charakterisiert. Außerdem werden ihr die der PAIS-Datenbasis entsprechenden Elemente zugeordnet. Das für deren Beschreibung zuständige Strukturelement dimensionselement bietet Platz für einen Elementsbezeichner und eine bis unendlich viele Elementsausprägungen. Die Daten der beschriebenen Dimension werden durch das Strukturelement dimensionsdaten erfaßt, welches beliebig viele dimensionstupel aufnehmen kann. Ein dimensionstupel wiederum umfaßt ein bis unendlich Strukturen des Typs dimensionselemententry und den jeweiligen Dimensionsschlüssel des Tupels.

Diese beiden DTD enthalten die notwendigen Strukturen, um die Daten für alle Posten der gewünschten Abschlußinstanz multidimensional in einer XML-Datei zu präsentieren. Eine weitere XML-Datei, die auf dem DTD zu den Dimensionstabellen basiert, versorgt den Adressaten mit den notwendigen Informationen, um gewünschte Gruppierungen und Dimensionsschnitte zu spezifizieren. Als Ergebnis aus diesen Daten lassen sich bislang jedoch nur Postenlisten für unterschiedliche Rechenwerke ableiten. Auf die jeweiligen Namen und auf Präsentationsrichtlinien hat der Adressat noch keinen Zugriff. Ein DTD für die Gliederung eines Rechenwerks ändert dies: 


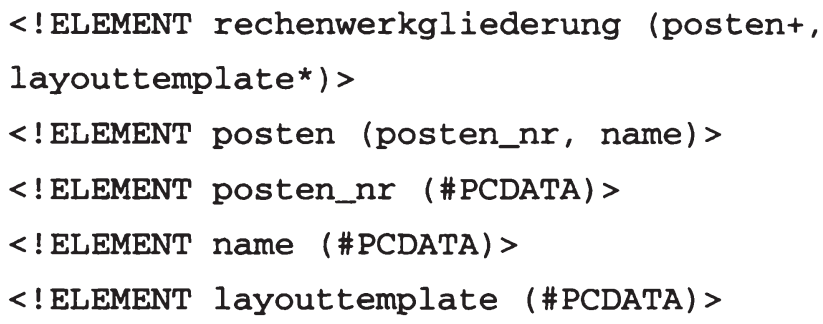

Das Strukturelement rechenwerkgliederung umfaßt einerseits die Posten des Rechenwerks, andererseits ein layouttemplate, in dem die Gestaltung des Rechenwerks in einer textuell-graphischen Beschreibungssprache geregelt ist. Das Strukturelement posten enthält den Namen und die Nummer des Posten. Mit einer XML-Datei des PAIS-Servers, die gültig hinsichtlich dieses DTD ist, kann der Adressat aus seiner Datensammlung entsprechende aufbereitete Rechenwerke erstellen.

Durch die Verwendung dieser abstrakten DTD, die unabhängig sind von der jeweiligen Implementierung des PAIS-Datenmodells, ist der PAIS-Server in der Lage, über Standardprotokolle wie HTTP und mit einer Standardkommunikationssyntax wie XML so spezifische Daten wie die Ergebnisse des PAIS-Datenmodells anzubieten, ohne daß die genaue Implementierung dem Clientsystem vorher bekannt sein muß. Der Client erfährt durch die gültigen XML-Dateien des PAIS-Servers gut strukturiert alle Informationen, die er benötigt, um die multidimensionalen Daten einer Abschlußinstanz auswerten zu können. Die mögliche Existenz von inkonsistenten aber gut strukturierten und gültigen XMLDateien ist unproblematisch. Diese werden vom PAIS-Server erstellt, so daß das berichtende Unternehmen die Kontrolle über die Dateien behält und Inkonsistenzen vermeiden kann. Ein momentan noch recht gewichtiger Nachteil ist vielmehr die geringe Verbreitung von XML außerhalb des Experimentierstadiums. Es fehlt noch an voll funktionsfähigen Standardclients, die in der Lage sind, XML-Dateien aufzubereiten und die darin enthaltenen Daten zu präsentieren. Doch selbst wenn diese Clients verfügbar sind, bleibt das Problem des relativ speziellen Charakters der präsentierten Daten. Zwar ist die Entwicklung von XMLClients zur Darstellung von multidimensionalen Datenzusammenhängen denkbar, doch um das Informationspotential der über XML bereitgestellten PAISDaten für den Adressaten komplett verfügbar zu machen, erscheint die Entwicklung spezieller Clientsysteme unausweichlich.

Im folgenden wird auf eine weitere Übertragungsalternative eingegangen, die bereits bei dem Abschlußselektionsprozeß zum Zuge kam. Im Gegensatz zu dem HTTP/XML Kommunikationskanal gibt es für diese zumindest im Bereich der Windowsbetriebssysteme eine recht breite Palette von auf Datenanalyse und Datenpräsentation spezialisierter Standardsoftware. 


\subsubsection{Datenbankzugriff über $\mathrm{ODBC} / \mathrm{SQL}$}

Wie bereits erwähnt, kann durch Verwendung des API ODBC und der Datenbankabfragesprache SQL auf lokale und entfernte DBMS zugegriffen werden. Die weite Verbreitung von $O D B C$ und die ansatzweise Standardisierung von SQL ermöglichen den Einsatz von Standardsoftware und erleichtern die Entwicklung von spezieller Software durch den Rückgriff auf Libraries zur ODBC/SQL-Kommunikation. Deswegen erscheint es sinnvoll, die Möglichkeit des Client-Zugriffs über ODBC zu diskutieren.

Der direkte Datenbankzugriff macht es für das berichtende Unternehmen notwendig, diejenigen Elemente der PAIS-Datenbank zu identifizieren, die dem Adressaten direkt zugänglich gemacht werden sollen. Ferner ist die Art des Zugriffs festzusetzen. Wegen des offenen PAIS-Adressatenkreises ist lediglich ein lesender Zugriff denkbar, schreibende oder strukturändernde Zugriffe scheiden naturgemäß aus. Zentraler Bestandteil der publizierten Daten ist die Faktenrelation rechenwerkposten_fakten mit den Dimensionen Abschlußinstanz, Posten, Bewertungsmethode, interner und externer Akteur und mit dem Fakt anteiliger Postenbetrag. Für die Dimensionsgruppierung und Aufgliederung werden zusätzlich sämtliche Dimensionsrelationen benötigt. Um die Posten bestimmten Rechenwerken zuzuordnen und die korrespondierenden Postenbezeichnungen zu ermitteln, müssen die Adressaten auf die Relation rechenwerkposten zurückgreifen, die diese Informationen vorhält. Ist die Verwendung von zentralen Rechenwerklayouts geplant, dann ist die korrespondierende Relation rechenwerkdesign ebenfalls den PAIS-Adressaten zugänglich zu machen. Durch den Zugriff auf diese Datenstruktur wird es dem Adressaten durch Verwendung von SQL-Abfragen ermöglicht, das PAIS gemäß seiner eigenen Zielvorstellungen zu analysieren. Die Entwicklung beliebig spezieller Clientsysteme ist technisch problemlos.

Im Vergleich zum Verfahren über HTTP/XML hat die Kommunikationsvariante $\mathrm{ODBC} / \mathrm{SQL}$ einige deutliche Vorteile. So ist Sprache und Protokoll direkt an die Zwecke der Datenanalyse angepaßt. Durch die weitere Verbreitung gibt es Standardsoftware, mit der sich bereits zielführende Analysen durchführen lassen. Da durch ODBC/SQL die Abschlußauswahl, der Abruf der Abschlußinstanz und die Datenanalyse über einen Kommunikationskanal erfolgt, ist der softwaretechnische Aufwand für die Kommunikation von Client und PAIS-Server denkbar gering. Letztendlich stellt für den Client der PAIS-Server nicht mehr als ein DBMS mit einem speziellen, öffentlich verfügbaren Datenbestand dar.

Der Hauptnachteil der reinen Nutzung eines ODBC/SQL-Kommunikationssystems liegt in der mangelnden Spezifikation der jeweiligen Implementierung des PAIS-Datenmodells. Der Client erhält über ODBC die notwendigen Informationen zu den vom PAIS-Server angebotenen Tabellen und zu deren Struktur. Eine Art Metastruktur, die z.B. von den 
DTD für die XML-Dateien geboten wird, fehlt jedoch. Somit ist der unternehmensübergreifende Vergleich von PAIS-Daten erschwert, da die Methoden zur Generalisierung des Datenbestandes fehlen. Auch DTD-Dateien garantieren jedoch keinen problemlosen Unternehmensvergleich, eine generelle Ablehnung von ODBC-Kommunikationsverfahren läßt sich also aus diesem vermeintlichen Nachteil nicht ableiten.

Aus dem Gesagten wird deutlich, daß eine eindeutige Empfehlung für einen Kommunikationskanal nicht ausgesprochen werden kann. Während die Kommunikation über HTTP/HTML den Vorteil hat, auf der Clientseite außer Standardsoftware keine weiteren Voraussetzungen zu benötigen, bieten HTTP/XML und ODBC/SQL wesentlich effizientere und weitgehendere Kommunikationsmöglichkeiten. Beide benötigen allerdings auf der Clientseite spezielle Software. Für ODBC kommt erschwerend hinzu, daß es sich um einen Firmenstandard handelt, der, obgleich weit verbreitet, nicht öffentlich verfügbar ist. XML hingegen ist ein öffentlicher Standard und stellt eine sinnvolle Plattform für die Spezifikation von Datenpräsentationsregeln dar. Für die Kommunikationskoordination selber muß jedoch dann weiterhin auf HTTP zurückgegriffen werden.

Glücklicherweise besteht keine Notwendigkeit, die Implementierung eines PAIS-Servers auf ein Kommunikationssystem zu beschränken. Sämtliche hier vorgestellten Kommunikationskanäle können vom Implementierer parallel angeboten werden. Des weiteren sind selbstverständlich noch weitere Verfahren denkbar. Somit muß die Entscheidung für ein bestimmtes Kommunikationssystem zwischen Client und PAIS-Server hier nicht gefällt werden, sondern kann der direkten Interaktion von PAIS-Adressaten und PAIS-Anbietern überlassen bleiben.

Im Anschluß an diese Überlegungen zur Kommunikation zwischen Client und PAISServer im Abschlußerstellungsprozeß soll noch kurz auf die Frage der Abschlußpräsentation eingegangen werden. Untersuchungen über die "Qualität" von Geschäftsberichten scheinen zu belegen, daß das ästhetische Auge des Adressaten bei der Jahresabschlußauswertung mitentscheidet. ${ }^{94}$ Außerdem kann eine klare und sinnvolle Gliederung der Rechenwerke die Informationsaufnahme erleichtern. ${ }^{95}$ Insofern besteht ein Rechenwerk, wie bereits in der Definition angemerkt, nicht nur aus einer Liste von Posten mit Titeln und Beträgen, sondern auch aus einer medialen Gestaltung. ${ }^{96}$ Das gleiche läßt sich grundsätz-

94 Vgl. Kirchhoff (1997), S. $61 \mathrm{f}$.

95 Vgl. z.B. Lusk (1973), der in einer experimentellen Studie belegt, daß die Gestaltung des Jahresabschlusses die Investitionsentscheidung beeinflußt; weiterführend speziell zu EDV-gestützten Informationssystemen Dillon/Morris (1996) und Singh (1998).

96 Der Gedanke der „multimedialen“ Geschäftsberichte findet sich aufgrund des ständigen Bedeutungszuwachses des WWWW immer wieder in der Literatur. Vgl. z.B. Flynn/Gowthorpe (1997); Labhart/Volkart (1997); Kirchhoff/Klein (1997); Helm (1998); Louwers/Pasewark/Typpo (1998) und Bury (1999). 
lich auch für Abschlüsse sagen, die ja gewissermaßen eine Klammer um eine Gruppe von Rechenwerken bilden. Deswegen sollen im folgenden zwei alternative Ansätze vorgestellt werden, mit der eine Präsentation der Abschlußinstanzdaten gewährleistet werden kann.

\subsection{Präsentation der Engebnisse}

\subsubsection{Rückgriff auf zentrale Präsentationsschemata}

Die erwähnte Bedeutung der medialen Abschlußpräsentation spricht dafür, daß berichtende Unternehmen ein Interesse haben dürften, Einfluß auf die Präsentation ihrer PAIS Abschlußinstanzen zu nehmen. Dieses Interesse läßt sich auch schon daraus ableiten, daß eine große Anzahl von Unternehmen ihren gedruckten Geschäftsbericht über Portable Document Format (PDF) Dateien auch im Internet verfügbar halten, anstatt die vorhandenen Daten über simple Tabellen bereitzustellen. ${ }^{97}$

Um diesem Bedürfnis Rechnung zu tragen, wurde in dem PAIS-Datenmodell die Möglichkeit integriert, Rechenwerken sogenannte Layout-Templates zuzuordnen. In der Implementierung dieser Arbeit wurden diese Templates als Binary Large Object (BLOB) definiert. Diese Datenstruktur erlaubt die nahezu beliebige inhaltliche Gestaltung der Templates. Um seine Aufgabe als Präsentationshilfe für Rechenwerkdaten zu erfüllen, muß ein Template auf eine bestimmte, dem Clientsystem bekannte Struktur zurückgreifen. Diese Struktur muß einerseits das Zusammenführen von Template, Abschlußinstanzdaten und Rechenwerkpostendaten unterstützen, damit aus dieser Gesamtheit die Präsentation des Rechenwerks entstehen kann. Andererseits muß das Template in der Lage sein, sämtliche gewrinschten medialen Inhalte zweifelsfrei und systemübergreifend zu beschreiben.

Für die Lösung der zweiten Aufgabe bietet sich der Rückgriff auf bereits bestehende Beschreibungssprachen an. Mit Postscript existiert zum Beispiel eine druckorientierte Seitenbeschreibungssprache, die in der Lage ist, Druckseiten systemübergreifend wiederzugeben. Nachteile von Postscript liegen in der monomedialen Ausrichtung und der mangelnden Berücksichtigung von Besonderheiten der Bildschirmdarstellung. In dieser Hinsicht ist das PDF der Firma Adobe besser geeignet. ${ }^{98}$ Es wurde speziell für plattformübergreifende bildschirm- und druckgetreue Verbreitung von Dokumenten entwickelt und

97 Vgl. einschlägige Untersuchungen zum Publizitätsverhalten im Internet, z.B. Lymer (1997; 1999); Lymer/Tallberg (1997); Flynn/Gowthrope (1997); Marston/Leow (1998); Deller/Stubenrath/Weber (1997; 1999); Wagenhofer/Pirchegger (1999); Ashbaugh/Johnstone/Warfield (1999); Craven/Marston (1999); Gowthrope/Amat (1999); Hedlin (1999); Pirchegger/Wagenhofer (1999); speziell zur Zeitnähe von Ergebnisberichten Petravick/Gillett (1998).

98 Vgl. als Referenz Adobe Systems Inc. (1999). 
gestattet in den neuen Versionen (ab PDF-1.2) auch die Verwendung von Links auf andere Medienquellen, wie Audio- und Videodaten. Damit lassen sich mit PDF-Dateien multimediale Daten hinreichend beschreiben. Zur eigentlichen Speicherung von Audio- und Videodaten kann dann auf jeweilige spezielle Formate zurückgegriffen werden. Audiodateien können auch in PDF-Dateien direkt gespeichert werden. Als weitere Möglichkeit kommt HTML in Frage, eventuell mit spezifischen mittels XML charakterisierten Erweiterungen. Auch mittels HTML lassen sich multimediale Dokumente erstellen, die sich plattformübergreifend am Bildschirm darstellen lassen. Sowohl die Bildschirmdarstellung als auch im noch größerem Maße die Druckausgabe sind jedoch von der jeweiligen Clientumgebung abhängig. Als weiterer Nachteil können die nur recht eingeschränkt vorhandenen graphischen Gestaltungsmöglichkeiten angeführt werden, die eine dem normalen Drucklayout entsprechende gestalterische Freiheit nicht zulassen. Als großer Vorteil von HTML muß die weite Verbreitung und die einfache Struktur der Sprache gelten.

Wenn die Wahl der Beschreibungssprache getroffen und das Layout-Template grundsätzlich erstellt wurde, muß dafür Sorge getragen werden, daß PAIS-Daten und das LayoutTemplate zu einem Gesamtdokument integriert werden. Im einfachsten Fall einer textbasierten gegliederten Wiedergabe der Posten müssen die entsprechenden Daten in den Text des Templates integriert werden. Dies kann z.B. geschehen, indem Platzhalter für die Posten des Rechenwerks eingefügt werden. Je komplexer jedoch die Visualisierung der PAIS-Daten geplant ist, desto komplexer wird die Implementierung des Templateparsings. So ist es im Extremfall vorstellbar, daß ein Template letztendlich nur noch das Datenumfeld für eine bestimmte Programmfunktion speichert, die dann die Präsentation des Rechenwerks unter Rückgriff auf den Datenbestand dynamisch erstellt. Diese Programmfunktion kann entweder auf dem PAIS-Server oder auf dem Client ablaufen. Im letzten Fall ist die Grenze zur zweiten Präsentationsalternative, welche die Präsentation der Abschlußinstanz im wesentlichen dem Clientsystem überläßt, fließend.

In dieser Implementierung wurde ein bewußt einfaches Prinzip der Rechenwerkpräsentation gewählt. Der PAIS-Server hält HTML-Templates für jedes Rechenwerk vor. In diese sind Platzhalter jeweils für den Postennamen und den Postenbetrag eingefügt. Ein einfacher Parser im PAIS-Server ersetzt diese mit den jeweiligen Postendaten. Die so entstandene HTML-Datei wird dem Client vom HTTP-Server gesendet.

Egal welche Beschreibungssprache verwendet wird und wie weit die zu präsentierenden Daten aufbereitet werden, bei dem präsentierten Rechenwerk handelt es sich um eine Präsentation nach Geschmack des berichtenden Unternehmens. Da die Vorstellung des PAIS-Adressaten von guter Präsentation von denen des berichtenden Unternehmens durchaus abweichen können, soll im folgenden dargestellt werden, welche Möglichkeiten sich durch eine Präsentation mittels des Clientsystems ergeben. 


\subsubsection{Lokale Aufbereitungsmöglichkeiten der Daten}

Ungeachtet der vom berichtenden Unternehmen angebotenen Präsentationsalternativen kann der Adressat mittels der ihm zur Verfügung stehenden Technik die übermittelten PAIS-Abschlußinstanzdaten weiterverarbeiten und gemäß seiner Interessen und Bedürfnisse präsentieren. Speziell durch den multidimensionalen Charakter der Daten ergibt sich ein Präsentationsproblem, für das es keine eindeutige Lösung gibt. Da die Daten aus unterschiedlichen Blickwinkeln betrachtet werden können, die herkömmlichen Präsentationsmedien jedoch zweidimensional sind, kann a priori keine allumfassende Präsentatonsmöglichkeit bestimmt werden. Mit den auch aus der Multidimensionalität von Daten entstehenden Visualisierungsproblemen von Daten befaßt sich eine ganze Forschungsrichtung, ${ }^{\text {9 }}$ so daß eine umfassende Behandlung der Möglichkeiten den Rahmen dieser Arbeit sprengen würde. Interessante Ergebnisse sind vor allem zu erwarten, wenn mehrere Abschlußinstanzen, die sich nur hinsichtlich des verwendeten Bewertungsvektors unterscheiden, zusammen visualisiert werden. Die daraus resultierenden unterschiedlichen Beträge für bestimmte Rechenwerkposten verdeutlichen anschaulich eines der Hauptprobleme der traditionellen Rechnungslegungspublizität: die Fiktion vom eindeutigen Wertansatz. Werden den Bewertungsvektoren, entweder vom Adressaten oder vom bilanzierenden Unternehmen als Dimensionsmerkmal, Eintrittswahrscheinlichkeiten der Prognosekorrektheit zugeordnet, so wird aus den unterschiedlichen Postenbeträgen eine Verteilung von Prognosedaten. ${ }^{100}$ Dem PAIS-Adressaten bietet sich durch die lokale Präsentation und Aufbereitung der Daten also die Möglichkeit, die Ergebnisse des PAIS für seine Zwecke optimal zu präsentieren. ${ }^{101}$

\section{Zusammenfassung und mögliche Erweiterungen}

Ziel dieses Kapitels war die Entwicklung eines Konzeptes für eine datenbankgestützte Rechnungslegungspublizität. Nach der Analyse der theoretischen Vorläufer wurden zunächst die der Literatur zu entnehmenden Klassifizierungen der normalen Unterneh-

99 Vgl. als Einstieg Degen (1999); als Überblick den Proceedings-Band zum 5. Internationalen Symposium für Informationswissenschaft Krause/Herfurth/Marx (Hrsg.) (1996); Cleveland (1993), Jacoby (1998); als Implementierungsbeispiel in anderen Anwendungsfeldern Davidson et al. (1998).

100 Vgl. allgemein zur Technik und Bedeutung der prognoseorientierten Berichterstattung Käfer (1962); Busse von Colbe (1968); Mennenöh (1984); Möhlmann/Bartels (1998); zur Kapitalmarktrelevanz von Prognoseberichten allgemein Pechtl (2000) und von Gewinnprognosen Löffler (1999). Auch für andere Rechenwerke mit prognostiven Charakter erscheint diese Vorgehensweise denkbar, wie z.B. für Technologiebilanzen, vgl. Hartmann (1997; 1998).

101 Im Rahmen der technischen Weiterverarbeitung ist auch vorstellbar, die Daten einem Expertensystem oder anderen Instrumenten der künstlichen Intelligenz zuzuführen. Vgl. einführend Poddig/Huber (1999); Ollmert/Schinzer (2000) und als Überblick zur Anwendung von künstlicher Intelligenz im Rahmen des Rechnungswesens Connell (1991) und Baldwin/Williams (1999) sowie zur Verbindung von künstlicher Intelligenz und Data-Warehouse-Konzepten Zabezhailo/Finn (1996) und Füllgraf (1999). 
mensdatenhaltung vorgestellt, um Ansatzpunkte für ein öffentliches Informationssystem zu ermitteln. Im Anschluß daran wurde die Konzeption des PAIS präsentiert. Ergänzt wurden die eher theoretischen Überlegungen zu den zentralen Datenstrukturen durch eine beispielhafte Implementierung, die im Anhang wiedergegeben ist. Die sich an die Beschreibung der konzeptionellen und logischen Datenstruktur anschließenden Ausführungen versuchten, den Datenfluß und funktionalen Programmablauf des PAIS-Servers nachzuzeichnen, der zur Erstellung einer Abschlußinstanz und zur Präsentation dieser Instanz bei dem PAIS-Adressaten führt.

Ziel der beispielhaften PAIS-Implementierung ist nicht, einen funktionsfähigen Prototyp für multidimensionale datenbankgestütze Rechnungslegungspublizität zu liefern. Wie aus dem entsprechenden Abschnitt dieses Kapitels in Verbindung mit der ihm zugrundeliegenden Literatur deutlich werden dürfte, ist die Datenrealität von Unternehmen viel zu komplex, als das solche Systeme allein am „grünen Tisch der Forschung“ entwickelt werden könnten. Die Daten und Algorithmen, aus denen die hier präsentierte Implementierung die Daten für das PAISStarmodel generiert, sind stark simplifiziert und nicht den Anforderungen der betrieblichen Realität gewachsen. Dies schmälert jedoch nicht den Kern des PAIS-Datenmodells: das dreistufige Starmodel. In dieser einfachen Datenstruktur lassen sich die für eine multidimensionale Rechnungslegungspublizität notwendigen Informationen erfassen. Es bildet somit die gesuchte Schnittstelle zwischen berichtendem Unternehmen und Adressat.

Diese Schnittstelle ermöglicht das gleichzeitige Angebot von unterschiedlichen Rechnungslegungskonzeptionen und Abschlußarten, ohne daß der Adressat selber die Vielzahl der Daten analysieren und auswerten muß. Dies ist jedoch bestenfalls die halbe Strecke zu einer Marktprozesse ermöglichenden Unternehmenspublizität. Ohne Clientsysteme, welche die Daten dieser Schnittstelle aufbereiten und Adressaten zur Verfügung stellen, ist mit den PAIS-Daten wenig anzufangen. In der Beispielimplementierung wurde für den Client auf eine Achse aus lokalem Browser, HTTP/HTML und vom Applikationsserver dynamisch erstellten HTML-Dateien gesetzt. Diese Lösung hat als Vorteil die geringen Anforderungen an die Clientausstattung. Um die Vorteile einer multidimensionalen datenbankgestützten Unternehmenspublizität wirklich zu nutzen, bedarf es noch mehr: Ein kompletter Zugriff auf die öffentlichen PAIS-Daten und weitreichende Analyse- und Präsentationsmöglichkeiten sind erforderlich. Hier greift die Hoffnung auf Marktprozesse: Wenn die PAIS-Daten dem Adressaten einen Informationsnutzen versprechen, dann besteht für den Adressaten, aber auch für andere Marktteilnehmer ein Anreiz, diesen Informationsnutzen freizulegen. Es sollten sich also Anbieter von Clientsoftware bilden, die versuchen, die PAIS-Daten durch gezielte Abfrage und Präsentation so zu filtern, daß 
der Nutzen aus der effizienteren und kompakteren Präsentation die Kosten der aggregationsbedingten Informationseinbuße möglichst stark überkompensiert. ${ }^{102}$ Durch diese Verwendung von standardisierten Informationsquellen und Informationsanalysetools kann somit das alte Dilemma der Informationsüberflutung zumindest teilweise gelöst werden.

Aber nicht nur der Markt für Auswertungssoftware ist für den hypothetischen Erfolg eines PAIS von großer Bedeutung. Weitere beigeordnete Märkte, wie z.B. der Markt für Qualitätssicherung der in einem PAIS enthaltenen Daten, der Markt für Implementierung von PAIS, der Markt für die Weiterentwicklung des PAIS-Datenmodells oder der Markt für die Weiterverarbeitung von PAIS-Daten zu realwirtschaftlichen Empfehlungen sind für die Entwicklung des PAIS-Konzeptes von großer Bedeutung. Auf der Suche nach Anhaltspunkten für die Art der zu erwartenden Marktprozesse rund um eine datenbankgestützte Rechnungslegungspublizität befindet sich das nächste und letzte Hauptkapitel dieser Arbeit.

102 Hierbei ist u.a. auch an Agentensoftware und ähnliche Informationssysteme zu denken, die in ähnlichen Bereichen bereits seit längerem eingesetzt werden. Vgl. beispielhaft Schumann et al. (1997); zu deren ökonomischen Analyse Avery/Resnick/Zeckhauser (1999). 
Joachim Gassen - 978-3-631-75496-2

Downloaded from PubFactory at 01/11/2019 04:12:56AM

via free access 


\section{Kapitel VI}

\section{Marktwirtschaftliche Auswirkungen einer}

\section{datenbankgestützten Unternehmenspublizität}

Das im Vorkapitel entwickelte PAIS würde die Rechnungslegungspublizität der berichtenden Unternehmen wesentlich verändern. Nachdem im vierten Kapitel festgestellt wurde, daß die im dritten Kapitel analysierten Systeme zur Rechnungslegungspublizität die Evolutionsfähigkeit der betroffenen Märkte gemäß des im zweiten Kapitel entwickelten Leitsatzes behindern können, soll in dem nun folgenden beleuchtet werden, welche marktwirtschaftlichen Prozesse durch die Verwendung des PAIS-Konzeptes induziert werden könnten. In diesem Zusammenhang kann aufgrund der nicht zu ermittelnden Einflußfaktoren und Wirkungsinterdependenzen keine gesamtwirtschaftliche Analyse der zu erwartenden Auswirkungen erfolgen, vielmehr soll die Betrachtung auf einige, direkt mit einer datenbankgestützen Rechnungspublizität verbundene Marktbereiche beschränkt werden. Dies ist einerseits der Markt für PAIS-Implementierungen und andererseits vorund nachgelagerte Märkte, wie z.B. der Markt für die Erstellung von Rechnungslegungsregeln, der Markt für Wertpapierhandelsdienstleistungen, der Markt für Prüfung von Rechnungslegungsdaten und für die Abschlußerstellung sowie der Markt für die Analyse von Unternehmen und speziell für die Analyse von Rechnungslegungsdaten. Des weiteren werden noch Anhaltspunkte für eine Rückwirkung des PAIS-Konzeptes auf das regulatorische Umfeld der Rechnungslegungspublizität entwickelt.

\section{Marktwirkungsanalysen}

\subsection{Zur verwendeten Methodik}

Um die sich in diesem Kapitel stellenden Fragen zu beantworten, muß die Reaktion und das Verhalten von Organisationen im Marktprozeß vorhergesagt werden. Dazu bieten sich unterschiedliche Methoden an. Einerseits versucht die traditionelle neoklassisch geprägte Industrieökonomik, das Ergebnis von Marktprozessen vor dem Hintergrund des Paradigmas „Marktstruktur-Marktverhalten-Marktergebnis“ zu erklären. ${ }^{1}$ Aufbauend auf die aus der Marktstruktur ableitbare Marktform verwendet die neoklassisch geprägte

1 Vgl. stellvertretend für viele zur neoklassischen Industrieökonomik Kaufer (1980) und Scherer/Ross (1990). 
Industrieökonomik gleichgewichtstheoretische und partialanalytische Ansätze, die das Marktverhalten der Akteure und damit auch das Marktergebnis unter Ausklammerung der institutionellen und organisatorischen Rahmenbedingungen prognostizieren. ${ }^{2} \mathrm{Da}$ für die Prognose der Marktwirkungen eines PAIS speziell das Verhalten von beteiligten Organisationen und der sich fernab der Neoklassik bewegende juristisch/politische Meinungsbildungsprozeß von Bedeutung sind, soll diese Methodik im Rahmen dieses Kapitels nicht zur Anwendung kommen. Anderseits sind im Bereich der Industrieökonomik in letzer Zeit einzelne Fragestellungen mittels der Spieltheorie unter Berücksichtigung der einzelnen Akteure detailliert analysiert worden. ${ }^{3} \mathrm{Da}$ diese Methodik eher erlaubt, besondere informationstechnische und institutionelle Rahmenbedingungen $\mathrm{zu}$ erfassen, soll auf ihre Ergebnisse für den zentralen Markt der PAIS-Anwendung zurückgegriffen werden.

Doch auch in einer spieltheoretischen Untersuchung läßt sich das Entstehen und die Modifikation der Regeln, also der Institutionen, nicht untersuchen. Die neue Institutionenökonomik geht hingegen auf die Organisationsstruktur der Unternehmen und auf den institutionellen Charakter der dazugehörenden Märkte ein. Als Gegenentwurf zu den primär auf dem Modell des homo oeconomicus mit statischen Präferenzen basierenden Ansätzen versteht sich weiterhin das Konzept der evolutorischen Ökonomik, welche als spezielles Untersuchungsobjekt die Analyse von Ungleichgewichtsprozessen hat. Diese beiden theoretischen Ansätze sind bereits im zweiten Kapitel vorgestellt worden und sollen nunmehr auch auf die Fragestellungen des sechsten Kapitels angewendet werden.

\subsection{Zur Auswahl der betrachteten Märkte}

Ein PAIS dient der Information von Unternehmensbeteiligten über die Tätigkeit des Unternehmens und somit dem Abbau der asymmetrischen Informationsverteilung zwischen dem Management und den anderen Unternehmensbeteiligten. Als Beschreibungssprache werden quantifizierte in Zahlungsgrößen normierte und auf realwirtschaftlichen Phänomenen beruhende Daten verwendet. ${ }^{4}$ Daher beeinflußt ein PAIS die Informationskosten aller Unternehmensbeteiligten und somit quasi den gesamten volkswirtschaftlichen Bereich.

2 Vgl. zu Unterscheidung der neoklassischen und neo-instutionalen Theorie einführend Cezanne/Meyer (1998).

3 Vgl. als ersten Überblick zur Spieltheorie z.B. Tirole (1988); Kreps (1990), S. 353-573; Holler/Illing (1996).

4 Rechnungslegungsdaten werden häufig auch als Ergebnis eines Prozesses erfaßt. Vgl. Schneider (1997), S. 67159, der von auf Hypothesen beruhenden Meßergebnissen spricht, und Pellens (1999), S. 1. 
Diese Überlegung ist für die Selektion der zu analysierenden Märkte nicht sonderlich zielführend. Die theoretische Möglichkeit, die Einführung der Produktinnovation PAIS in einem neoklassisch geprägten Totalmodell zu analysieren, soll wegen der methodischen Grundlagen eines solchen Modells und der damit verbundenen geringen empirischen Relevanz nicht gewählt werden. Somit ist eine partialanalytische Vorgehensweise unumgänglich. Um die Anzahl der betrachteten Unternehmensbeteiligten und mit ihnen die Anzahl der zu analysierenden Märkte zu reduzieren, sollen nur die Anbieter von Risikokapital als Unternehmensbeteiligte berücksichtigt werden. ${ }^{5}$

Die Risikokapitalgeber bieten die Überlassung von Kapital an, wobei sie im Austausch die Verfügungsrechte an dem Unternehmen erhalten. Im hier zu betrachtenden Regelfall werden die Nutzungsrechte des Unternehmens auf Widerruf von den Eigentümern an sogenannte Manager übertragen. Aus Sicht der Risikokapitalgeber entsteht erst durch diese Trennung von Eigentum und Verfügungsrecht das Problem der Informationsasymmetrie. Wegen der fehlenden Information erkennen sie die Änderungen des Wertes ihrer Verfügungsrechte durch den Marktprozeß nicht mit gleicher Genauigkeit wie die Manager. Rechnungslegung ist eine Institution, die in der Lage sein sollte, auf diese Informationsasymmetrie Einfluß zu nehmen.

Grundsätzlich können nur die Manager des berichtenden Unternehmens Rechnungslegungsdaten produzieren, da deren spezifische Investition der Einarbeitung in das betreffende Unternehmen sie zu de facto Monopolisten macht. Somit sind sie auch die relevanten Entscheider für die Einführung eines PAIS. Damit soll nicht gesagt sein, daß eine Eigentümergruppe nicht den Managem die Verwendung eines PAIS vorschreiben kann. Der aus dieser Nachfrage resultierende Markt für PAIS-Implementierungen soll als erster betrachtet werden.

Wegen der oben erwähnten Monopolstellung der Manager hinsichtlich der Bereitstellung der operationalen Daten, haben diese die Möglichkeit zu opportunistischem Verhalten. Insbesondere können sie versuchen, durch gezielte Manipulation der Datenbasis, ihren Nutzen auf Kosten der Eigentümer zu erhöhen, sich also die Quasirente der Transaktion anzueignen. Ein Lösungsansatz aus Sicht der Vertragstheorie für solche Probleme ist die Einführung einer bilateralen Kontrolle. ${ }^{6}$ Derzeit ist eben diese Kontrolle durch die Wirtschaftsprüfung für traditionelle Rechnungslegungsdaten empirisch international evident. Deswegen soll der daraus resultierende Markt für Wirtschaftsprüfungsdienstleistungen anschließend diskutiert werden.

5 Diese Beschränkung entspricht der Stoßrichtung der international führenden Rechnungslegungssysteme. Vgl. SFAC No. 1, Par. 2431, insb. Par. 30; IASC Framework, Par. 2-11, insb. Par. 10.

6 Vgl. in anderem Zusammenhang auch Hoffmann/Postel-Vinay/Rosenthal (1998). 
In der oben skizzierten Beziehung zwischen Eigentümem und Managem sind keine Annahmen über die Marktform des Marktes für Unternehmenseigentumstitel gemacht worden. Als empirischer Fakt läßt sich festhalten, daß ein Großteil der Wirtschaftsleistung von Unternehmen bereitgestellt wird, deren Eigentumsrechte über eine Vielzahl von Wirtschaftssubjekten verteilt sind. ${ }^{7}$ Dadurch stehen viele Eigentümer wenigen Managern gegenüber. Die Koalitionsbildung in großen Gruppen fällt schwerer, ${ }^{8}$ somit reduzieren sich c.p. die notwendigen Anstrengungen des Managements für die Kaschierung von opportunistischen Verhalten, da die große Gruppe der Eigentümer segmentiert zu Vertragsverhandlungen auftritt. Andererseits besitzen kleine Eigentumsanteile an Unternehmen eine wesentlich geringere Transaktionsspezifität, so daß eine marktnahe Transaktion von Unternehmensanteilen möglich wird. ${ }^{9}$ Für die Besitzer von wertmäßig geringen Eigentumsanteilen ist das direkte Angebot und das Eruieren von potentiellen Nachfragen mit sehr hohen Anbahnungskosten verbunden, so daß eine Veräußerung von Eigentumsanteilen wenig vorteilhaft erscheint. $\mathrm{Da}$ der wirtschaftliche Planungshorizont von Haushalten im allgemeinen wesentlich kürzer ist als der von Unternehmen, ist die VeräuBerung von Eigentumsanteilen zur Nutzenrealisation allerdings für Haushalte unerläßlich. Deswegen hat auch das Management des betroffenen Unternehmens ein Interesse daran, die Transaktionskosten des Eigentumsübergangs durch die Etablierung geeigneter Institutionen $\mathrm{zu}$ reduzieren. ${ }^{10}$ Somit ist $\mathrm{zu}$ erwarten, daß untemehmerisch veranlagte Wirtschaftssubjekte entsprechende Dienstleistungen anbieten. Diese Dienstleistungen werden von Börsen angeboten, die als Gut eine Feststellung von Preisen durch die gesteuerte Zusammenführung und ggf. auch Generierung von Angebot und Nachfrage produzieren. Je höher die Anzahl der Marktteilnehmer, desto höher ist die Qualität der von der Börse angebotenen Dienstleistungen. Da auch die Anbieter und Nachfrager von Eigentumsanteilen gegenüber dem Management nur asymmetrisch über ihr Unternehmen informiert sind, erhoffen sich Börsen, durch bessere Information der Marktteilnehmer über die gehandelten Titel den Umsatz der Titel und damit ihre Konkurrenzsituation gegenüber anderen Börsen zu verbessern. ${ }^{11}$ Deswegen verlangen Sie von Unternehmen,

7 Vgl. zur Bedeutung und der Motivation von Börsengängen Pagano/Panetta/Zingales (1998).

8 Vgl. grundlegend Buchanan/Tullock (1962), insb. S. 11-15; Truman (1971); als Übersichtsartikel Tollison (1982; 1988). Diese Argumentation findet sich ebenso in der capture theory. Vgl. grundlegend Stigler (1971; 1975); Posner (1974) und Peltzman (1976). Vgl. einführend zur Willensbildung in Organisationen Oelsnitz (1999).

9 Vgl. allgemein zu dem vermeintlichen Spannungsverhältnis von Untemehmenskontrolle und Anteilsliquidität Maug (1998).

10 So läßt sich auch zeigen, daß Unternehmen, die organisierte Kapitalmärkte zur Finanzierung in Anspruch nehmen, zu mehr freiwilliger und besser gewerteter Publizität neigen. Vgl. zu dementsprechenden Untersuchungen Frankel/McNichols/Wilson (1995); Sengupta (1998).

11 Des weiteren läßt sich spieltheoretisch zeigen, daß strenge Börsenvorschriften auch vom Management der gelisteten Unternehmen als glaubhafte Versicherung gegenüber den Marktteilnehmern genutzt werden kann. Vgl. Fürst (1998). 
welche ein Listing nachfragen, die Erfüllung spezieller Informationspflichten. ${ }^{12}$ Damit werden die Börsen auch $\mathrm{zu}$ möglichen indirekten Nachfragern von PAISImplementierungen, was die Analyse des Marktes für Eigenkapitalhandelsdienstleistungen rechtfertigt.

Eins der konstituierenden Prinzipien des PAIS-Ansatzes ist die Nichtfestschreibung bestimmter Rechnungslegungs- und Bewertungsregeln. Nur so ist ein Wettbewerb von konkurrierenden Ansätzen möglich. Insofern wird durch das PAIS die Nachfrage nach Rechnungslegungsregeln eher positiv als negativ beeinflußt. $\mathrm{Ob}$ und wie die gewünschten Regeln erzeugt werden, soll hier analysiert werden.

Die Informationen aus dem PAIS lösen per se noch nicht die Qualitätsunsicherheit der Eigentümer. Dafür müssen diese die PAIS-Daten analysieren, um mit anderen Informationen letztlich zu Entscheidungen zu kommen. Dieser Prozeß erfordert teilweise recht spezifische Kenntnisse, so daß ein gewisser Grad der Arbeitsteilung vorstellbar ist. ${ }^{13}$ Bislang gibt es zahlreiche Anbieter, die Unternehmensanalysen aus unterschiedlichen Blickwinkeln vornehmen und deren Ergebnisse anbieten. Es soll daher auch beleuchtet werden, wie das PAIS-Konzept auf diesem Markt wirken könnte. Somit sind alle zu analysierenden Märkte identifiziert. Zunächst soll auf den zentralen Markt für PAISImplementierungen eingegangen werden.

\subsection{Markt für PAIS-Implementierungen}

\subsubsection{Verbale Analyse}

Das im Rahmen dieser Arbeit vorgestellte PAIS-Modell kann als erster Prototyp einer Produktinnovation gesehen werden. Um zu untersuchen, ob und ggf. wie ein darauf basierendes Gut angeboten und nachgefragt würde, soll der Charakter des Gutes genauer beleuchtet werden. Es ist also zunächst zu fragen, ob zwischen zwei potentiellen Nutzern des Gutes eine Rivalität im Konsum herrscht, das heißt, ob und in welchem Grad der Konsum des Gutes durch ein Wirtschaftssubjekt andere Wirtschaftssubjekte von dem Konsum ausschließt. Güter wie Gesellschaftsrecht oder Verbraucherschutz weisen keine

12 Vgl. z.B. die privatrechtlichen Publizitätspflichten, welche von der Deutschen Börse AG Unternehmen des Neuen Markts oder des Small Cap Exchange (SMAX) auferlegt werden. Vgl. im Regelwerk Neuer Markt, Abschnitt 2, Gliederungspunkt 4 zum Emissionsprospekt, Gliederungspunkt 7.1 zum Quartalsbericht, 7.2.2 zum Jahresabschluß; 7.2.4 zum Unternehmenskalender und 7.2.11 zur Analystenveranstaltung, zusammengefaßt werden diese Vorschriften u.a. bei Gerke (1999), S. 205. Zum SMAX vgl. die Vorschriften in den SMAX Teilnahmebedingungen, Gliederungspunkt 3.1 zum Quartalsbericht, 3.2 zum Jahresabschluß und Lagebericht und 3.4 zur Analystenveranstaltung, zusammenfassend Strieder/Ammedick (1999).

13 Vgl. auch Barker (1998). 
derartigen Rivalitätseigenschaften auf. Der Konsum eines bestimmten Lebensmittels hingegen ist nur einem Wirtschaftssubjekt vorbehalten, es herrscht vollkommene Rivalität im Konsum.

Darüber hinaus ist unter Berücksichtigung der Kontrollkosten zu überprüfen, ob Nichtzahler von der Inanspruchnahme der mit dem Gut verbundenen Verfügungsrechte ausgeschlossen werden können. Auch dies ist bei vielen Gütern, wie Natur, guter Luft etc., kaum möglich, wohingegen bei vielen materiellen Gütern die Zuordnung und Durchsetzung von Verfügungsrechten unproblematisch erscheint. Aus dieser Unterteilung können vier Güterklassen abgeleitet werden: ${ }^{14}$

- Rein private Güter. Es besteht Rivalität im Konsum und ebenso ist die Ausschließbarkeit von Nichtzahlern jederzeit gewährleistet.

- Quasikollektivgüter. Es besteht zwar Rivalität im Konsum, jedoch besteht keine Exkludierbarkeit.

- Klubleollektivgüter. Hier besteht keine Rivalität im Konsum, free-rider können jedoch von der Nutzung des Gutes ausgeschlossen werden.

- Reine bzw. prototypische Kollektivgüter. Es gibt keine Rivalität im Konsum und keine Ausschließbarkeit von Nichtzahlern.

Die jeweilig zu erwartende Bereitstellung hängt somit vom jeweiligen Gutscharakter ab. Um ein Kollektivgut aufgrund der Nachfrage-/Produktionskostenelastizität von einem privaten Gut zu unterscheiden, muß einerseits die Elastizität $(\gamma)$ der Ausbringungsmenge $(m)$ hinsichtlich der Nachfrage $(x)$ und andererseits die Elastizität $(\delta)$ der Produktionskosten $(K)$ hinsichtlich der Ausbringungsmenge betrachtet werden. Formal bedeutet dies: $:^{15}$

$$
\text { (3) } m=x^{\gamma}
$$

für die Ausbringungsmenge und

$$
\text { (4) } K=f m^{\delta}
$$

\footnotetext{
14 Vgl. Musgrave/Musgrave/Kullmer (1994), S. 71-72; sowie Grossekettler (1995), S. 499.

15 Vgl. Grossekettler (1995), S. $501 \mathrm{f}$.
} 
für die Kostenfunktion, die gemäß eines Funktionalzusammenhangs $(\delta)$ abhängig von der Ausbringungsmenge ist. Daraus folgt für die Elastizität der Produktionskosten $(\rho)$ hinsichtlich der Nachfrage:

$$
\text { (5) } \rho=\frac{d K}{d x} \frac{x}{K}=\gamma \delta f x^{(\gamma \delta-1)} \frac{x}{K}=\gamma \delta \frac{f x^{\gamma \delta}}{K}=\gamma \delta
$$

Somit ist die Gesamtelastizität das Produkt der Elastizitäten der Ausbringungsmenge und der Produktionskosten. Bei einer Gesamtelastizität von 0 liegt ein prototypisches Kollektivgut vor, da zusätzliche Nachfrage aus Sicht des Unternehmens kostenlos befriedigt werden kann. Bei einer Gesamtelastizität von 1 verursacht jeder zusätzliche Nutzer genau anteilig zusätzliche Kosten.

Die Elastizität der Ausbringungsmenge von PAIS in Abhängigkeit der Nachfrage ist von der physischen Distribution her gesehen nahe 1. Jeder neue Nutzer braucht eine Nutzungslizenz, Implementierungsservice, Codeanpassungen, Schulungen und Wartung. Die Elastizität der Produktionskosten ist jedoch deutlich kleiner als 1, da ein Großteil der Entwicklungskosten fix hinsichtlich der Ausbringungsmenge sind. Somit ist die Gesamtelastizität zwischen 0 und 1 und es handelt sich bei dem PAIS weder um ein prototypisches Kollektivgut noch um ein rein privates Gut. Verantwortlich dafür ist die deutliche Kostendegression der Produktion.

Neben der Betrachtung der Produktionskosten ist des weiteren zu überprüfen, ob institutionelle Arrangements vorstellbar sind, mit deren Hilfe Anbieter Nichtzahler von der Nutzung des Gutes ausschließen können. Die Nutzer von PAIS sollten normalerweise die resultierenden PAIS-Daten veröffentlichen, somit ist die Identifikation der Nutzer problemlos. Da diese PAIS freiwillig einsetzen, erscheint eine privatrechtliche Sanktionierung der Trittbrettfahrer unproblematisch. Es liegt also eine grundsätzliche Exkludierbarkeit vor, die das Bilden von privaten Bereitstellungsverbänden ermöglicht.

Bislang wurde zur Charakterisierung von Gütern lediglich auf die Exkludierbarkeit und auf die Rivalität im Konsum abgestellt. Aus Sicht der Anwender beeinflußt jedoch eine weitere Guteigenschaft die Konsumentscheidung nicht unwesentlich. Es gibt Güter, für deren Konsumtion ein sog. Anwendernetzwerk nutzenbeeinflussend ist. Diese Beziehung wird als Netzwerkexternalität bezeichnet. ${ }^{16}$ Ein klassisches Beispiel für ein Gut mit Netzwerkexternalität ist die Telekommunikation. Der Nutzen, den ein einzelner Konsument aus der Konsumtion der Telekommunikation ziehen kann, ist positiv korreliert mit der

16 Vgl. grundlegend Katz/Shapiro (1985; 1986); einführend Woeckener (1994) und Hess (2000). 
Anzahl der anderen Telekommunikationsnutzer; es entstehen also durch jeden neuen Nutzer für die alten Nutzer positive Netzwerkexternalitäten. ${ }^{17}$

Eine Mindeststandardisierung der PAIS-Implementierungen vorausgesetzt, bedeutet jeder zusätzliche Nachfrager eines PAIS, der damit zu einem Anbieter von PAIS-Daten wird, eine Verbreiterung der Datenbasis, in der dann die Nutzer von PAIS-Daten recherchieren können. Je breiter die Datenbasis wird, desto interessanter ist es auch für potentielle Analysedienstleister, Tools zur Analyse der PAIS-Daten zu entwickeln. Insofern gehen von hinreichend standardisierten PAIS-Implementierungen nicht unerhebliche Netzwerkexternalitäten aus. Als weitere indirekte Externalitäten kommen statische und dynamische Skaleneffekte der PAIS-Implementierung in Frage, die jedoch grundsätzlich unabhängig von der Netzwerkeigenschaft des Gutes sind. Während der erste Effekt direkt auf die Nachfragefunktion der Konsumenten wirkt, üben die Skaleneffekte über die Produktionsfunktion des Anbieters einen Einfluß auf seine Preisangebotsfunktion aus. ${ }^{18}$

Eine weiteres Problemfeld der Güter mit Netzwerkexternalitäten ist die Sicherung derjenigen Gutscharakterisika, welche die Netzwerkeigenschaften definieren, also die Sicherung der Verfügungsrechte über die Standardisierung der PAIS-Implementierung. Ein Standard gewährleistet die Interoperabilität des Netzwerks. Wenn das produzierende Unternehmen durch patentrechtliche Regelungen die alleinigen Nutzungsrechte an dem Standard hält, ist eine andere Entwicklung zu erwarten als bei offenen Standards. Probleme dieser Art lassen sich mit Modellen, die u.a. auf nicht-kooperativer Spieltheorie beruhen, analysieren. Je nachdem, wie heterogen das betrachtete Gut ist, kann es zu Gleichgewichtssituationen mit einem oder mehreren konkurrierenden Standards kommen. Im Extremfall ist auch vorstellbar, daß es wegen mangelnder Durchsetzbarkeit der Verfügungsrechte an den selbstproduzierten Standards überhaupt nicht zu einer Produktion von PAIS-Standards kommt. Von Bedeutung ist hier auch die Reihenfolge des Marktzutritts der unterschiedlichen Konkurrenten. ${ }^{19}$

Fraglich ist, ob diese Externalitäten und die Kostendegression die Bereitstellung über marktwirtschaftliche Prozesse so behindern, daß sie unterbleibt. Zur Beantwortung dieser Frage soll ein vergleichbarer Produktmarkt herangezogen werden. Im Bereich von betriebswirtschaftlicher Standardsoftware gibt es aufgrund der vielen an die jeweilige Software gekoppelten Folgeprodukte und Märkte erhebliche Netzwerkexternalitäten. Trotz-

17 Die Standards zur Nutzung dieser Netzwerkextemalitäten können auch als relationale Verträge interpretiert und analysiert werden. Vgl. hierzu Hansen (1999), insb. S. 43-64.

18 Vgl. Woeckener (1994), S. $236 \mathrm{f}$.

19 Vgl. als Ansätze zu marktwirtschaftlichen Auswirkungen von Netzwerk-Externalitäten grundlegend Kindleberger (1983); Braunstein/White (1985); Berg (1988; 1989); Katz/Shapiro (1985; 1986); Farrell/Saloner (1985; 1986); sowie jüngst Choi/Thum (1998). 
dem erfolgt die Bereitstellung problemlos ohne staatlichen Interventionismus. Allerdings läßt sich, ähnlich wie in anderen Bereichen der Softwareindustrie eine recht starke Tendenz zur Marktkonzentration feststellen. Ob diese Marktkonzentration positiv oder negativ zu bewerten ist, hängt vom gerade politisch opportun erscheinenden wirtschaftspolitischen Leitbild ab. Weitgehend unproblematisch erscheint sie jedenfalls, wenn sie nicht mit einer massiven Beschränkung des Markteintritts einhergeht. Der Markteintritt geschieht in der Softwareindustrie in der Regel über die Kenntnisse der Schnittstellen der marktbeherrschenden Programme. Gelingt es einem PAIS-Anbieter, einen de facto Standard für PAIS zu entwickeln und die genaue Spezifikation dieses Standards geheim zu halten, so kann er sich unter Umständen eine marktbeherrschende Stellung sichern. Diese Position ist aus marktwirtschaftlicher Sicht jedoch nicht nur negativ zu sehen. Schließlich hat das Unternehmen, welches den Standard entwickelt hat, offensichtlich den Markt verändert und somit zur Systemevolution beigetragen. Da eine mögliche Grundkonzeption einer PAIS-Schnittstelle bereits hier veröffentlicht wurde und da für den wirtschaftlichen Erfolg des PAIS-Konzepts die Netzwerkexternalitäten von tendenziell hoher Bedeutung sind, kann wohl davon ausgegangen werden, daß eine marktbeherrschende Stellung von properitären Standards relativ unwahrscheinlich sein dürfte.

Für die Entwicklung des Angebots ist die Entwicklung der Nachfrage logischerweise von großer Bedeutung. Da der Nachfrager nicht aus eigenem, direktem Konsumtionsbedürfniss das Gut nachfragt, sondern ggf. auf Weisung seiner Prinzipale oder aus anderen Motiven handelt, ist die Nachfrage nach PAIS-Implementierungen für einen Entrepreneur relativ schwer abzuschätzen. Dies wiegt aufgrund der Kostendegression um so schwerer. Speziell in der Experimentierphase des Marktes ist es nahezu unmöglich, fundiert zu prognostizieren, ob sich Anbieter und Nachfrager für PAIS-Implementierungen finden werden. Nichtsdestotrotz ist diese Frage für die praktische Relevanz dieser Arbeit von großer Bedeutung. Deswegen soll im folgenden versucht werden, weitere mögliche Einflußfaktoren für die Marktentwicklung zu identifizieren. Dies geschieht unter Bezugnahme auf spieltheoretische Modelle ${ }^{20}$, welche generell die Frage der freiwilligen Publizität behandeln.

20 Erste spieltheoretische Modelle im Zusammenhang mit Untemehmenspublizität finden sich bereits bei Moxter (1962), S. 29-40. Vgl. weiter u.a. Akerlof (1970); Grossman (1981); Verrecchia (1983); Dye (1985; 1985a); Wagenhofer (1990; 1990a); Gigler (1994). Speziell zur Frage der Wirkung von freiwilligen Veröffentlichungen auf Lohnverhandlungen Frantz/Walker (1997); vgl. zum folgenden Überblick auch Kuhner (1998), S. 181-212. Ferner wird auch die Informationsasymmetrie innerhalb von Unternehmen spieltheoretisch untersucht, vgl. z.B. Alles/Newman/Noel (1998). 


\subsubsection{Modelltheoretische Analyse}

Die Entscheidung eines Managers, bestimmte ihm privat verfugbare Informationen zu veröffentlichen, ist dann nicht trivial, wenn er einerseits einen Nutzen aus diesen privaten Informationen zieht und andererseits Nachfrage von außen besteht. ${ }^{21}$ Diese Nachfrage entsteht, sobald die Information ex post das Nutzenniveau der Unternehmensbeteiligten tangiert und diese ex ante die Möglichkeit besitzen, auf die Information durch Handlungen zu reagieren und dadurch wiederum ihren Nutzen zu modifizeren. Mittels der Spieltheorie läßt sich diese Situation als ein unvollkommenes, aber vollständiges nichtkooperatives Spiel zwischen der Natur, dem Management und den extemen Untemehmensbeteiligten analysieren. In der ersten Spielphase trifft die Natur die Entscheidung über die Ausprägung der Information und präsentiert diese Information dem Manager. In der zweiten Phase entscheidet sich der Manager, diese Information zu veröffentlichen oder nicht. In der dritten Phase reagiert der externe Unternehmensbeteiligte auf die Veränderung seines Informationsstandes und determiniert damit seine Auszahlung und die des Managements. ${ }^{22}$ Dieser grundsätzliche Aufbau des Veröffentlichungsspiels ist in Abb. 19 in der extensiven Form wiedergegeben. Zur besseren Visualisierbarkeit wurde davon ausgegangen, daß die Natur zwischen zwei unterschiedlichen Zuständen unterscheidet und $\mathrm{da} ß$ auch die Adressaten nur zwei unterschiedliche Möglichkeiten wahrnehmen können.

Es läßt sich zeigen, daß dieses Spiel nur einen Gleichgewichtszustand besitzt, indem der Manager die Information offenlegt, wenn folgende Bedingungen kumulativ erfüllt sind:

- Das Management möchte den informationsabhängigen Marktwert des Unternehmens maximieren.

- Die Informationen stehen dem Management kostenfrei zur Verfügung, das Vorliegen der Information und die möglichen Ausprägungen sind den Adressaten bekannt. Die

- Publizität führt zu keinen Kosten, also zu keinen Kosten, die beim Manager direkt im Rahmen der Veröffentlichung oder durch nachgelagerte Wettbewerbswirkungen anfallen.

- Die publizierte Information ist absolut glaubwürdig und korrekt.

21 Die Komplexität der Ausweisentscheidung wird auch deutlich, wenn die unterschiedlichen Kommunikationswege, die Manager für freiwillige Prognosepublizität benutzen, analysiert werden. Vgl. Bamber/Cheon (1998).

22 Vgl. Ross (1979); der Ansatz geht grundsätzlich auch auf Akerlof (1970) zurück. 
- Die unterschiedlichen Ausprägungen der veröffentlichten Information können vom Adressaten in eine ordinal skalierte Reihenfolge hinsichtlich ihrer Vorteilhaftigkeit gebracht werden.

Natur

Manager

Adressat

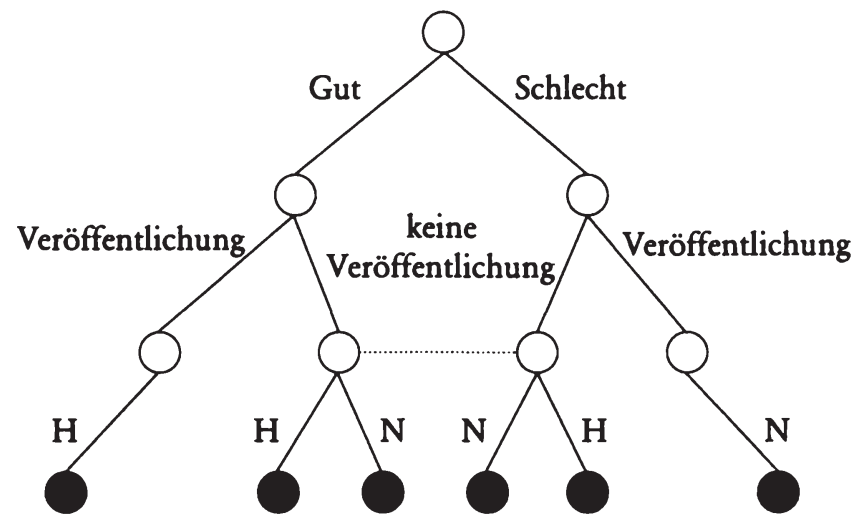

H: Hohe Zahlungsbereitschaft

N: Niedrige Zahlungsbereitschaft

Abb. 19: Extensive Darstellung des Veröffentlichungsspiels

Auf die formale Herleitung dieser Aussage soll hier verzichtet werden. ${ }^{23}$ Verbal läßt sich der Gleichgewichtsprozeß folgendermaßen nachvollziehen: Das Management besitzt Transparenz darüber, wie seine private Information von den Adressaten bewertet wird. Die Manager, die die attraktivste private Information besitzen, werden diese offenlegen, um sich von den anderen Managern abzugrenzen. Dieses Verhalten antizipierend werden auch die Manager mit den „zweitbesten“ Informationen diese offenlegen, um sich wiederum von den restlichen Managern abzugrenzen. Dieser Prozeß setzt sich iterativ fort, bis nur noch die Manager mit der schlechtesten aller denkbaren Informationen verbleiben. Diese sind indifferent zwischen dem Ausweis dieser Information und dem Nichtausweis, da die rational spielenden Adressaten diesen in der Literatur als unraveling bezeichneten Prozeß ihrerseits antizipiert haben und bei Nichtausweis davon ausgehen, daß der Manager die schlechteste aller verfügbaren Informationen erhalten hat.

Ein zentraler Pfeiler dieses Spielergebnisses ist die Annahme, daß der Adressat über die Gesamtheit der möglichen Informationen und über die Tatsache, daß der Manager eine bestimmte Informationsausprägung erhalten hat, informiert ist. Realiter ist diese Annahme wohl in der Regel nicht erfüllt. Die Adressaten können einerseits nicht den gesamten Bereich der möglichen Information überblicken, andererseits wissen Sie nicht mit

23 Vgl. Ross (1979); Dye (1985); Wagenhofer (1990), S. 18-35. 
Sicherheit, ob das Management überhaupt eine Information aus der Grundgesamtheit der Informationen erhalten hat. Ein erster Schritt zur Abbildung dieses Umstandes im Rahmen eines spieltheoretischen Modells besteht darin, daß die Natur nur mit einer bestimmten Wahrscheinlichkeit die Manager mit einer Information versorgt, wobei diese Wahrscheinlichkeit für die Adressaten zu beobachten ist. ${ }^{24}$ Dieses Spieldesign führt zu dem Ergebnis, daß sich ein vollständiges Offenlegungsgleichgewicht nicht mehr einstellt. Vielmehr werden Manager mit einer Information, die bei Offenlegung zu einem Unternehmenswert unter dem momentanen Marktwert führen würde, diese nicht offenlegen. Dies wird wiederum von den Adressaten antizpiert, die ihre Erwartungen entsprechend anpassen. Ein kompletter unraveling Prozeß findet allerdings nicht statt, da die Wahrscheinlichkeit besteht, daß das Management keine Information von der Natur erhalten hat und somit nicht in der Lage ist, Offenlegung zu betreiben. Je geringer die Wahrscheinlichkeit ist, daß das Management keine Information erhalten hat, desto geringer ist die Schwelle, ab der das Management seine Information offenlegt, desto ungünstigere Informationen werden also vom Management veröffentlicht. Wenn unterstellt wird, daß die Verfügbarkeitswahrscheinlichkeit von Informationen im Zeitablauf zunimmt, decken sich die Modellergebnisse mit dem empirisch beobachtbaren Phänomen, daß gute Informationen eher veröffentlicht werden als schlechte Informationen. ${ }^{25}$

Ein anderer Ansatz geht davon aus, daß die Informationen, die dem Management vorliegen, nicht eindeutig sind, sondern nur als Wahrscheinlichkeitsverteilung vorliegen und dem Management über ein unscharfes Informationssystem übertragen werden. ${ }^{26} \mathrm{Als} \mathrm{Er-}$ gebnis läßt sich verfeinernd festhalten, daß die Adressaten, wenn sie Informationen über das Informationssystem der Manager besitzen, auf unschärfere Informationssysteme mit niedrigeren Zahlungsbereitschaften reagieren.

Wenn davon ausgegangen wird, daß das Vorliegen von Informationen für den einzelnen Manager auch davon abhängig ist, ob die anderen Manager diese Information erhalten haben, können Erklärungen dafür geliefert werden, daß Manager ihre Veröffentlichungsentscheidung von dem Verhalten anderer Manager abhängig machen. Dieses Verhalten wird spieltheoretisch modelliert, indem zwei mögliche Veröffentlichungszeitpunkte abgebildet werden. Diejenigen Manager, die zu dem ersten Zeitpunkt ihre Information nicht offengelegt haben, ziehen unter Umständen im zweiten Zeitpunkt nach, wenn zum ersten

\footnotetext{
24 Vgl. Jung/Kwon (1988); erweitert von Ewert (1995); Penno (1997).

25 Vgl. Jung/Kwon (1988); Kuhner (1998), S. 187.

26 Vgl. Shin (1994).
} 
Zeitpunkt mehr als eine bestimmte Anzahl von Managern ihre Information offengelegt haben. $^{27}$

Durch die unterschiedlichen Modellansätze, welche die Art der Information und den Grad der Informationsasymmetrie zwischen Management und Adressat beleuchten, wird deutlich, daß das simple unraveling Gleichgewicht nur aufrecht erhalten werden kann, wenn die Adressaten über die Art der Information des Managements genau informiert sind. Ansonsten gibt es einen Gleichgewichtsbereich, in dem vor allem Manager, die aufgrund der Information eher schlechter stehen, nicht veröffentlichen. Des weiteren wird deutlich, daß bei Unterschieden in den Informationssystemen von Managern die Adressaten auf ein schlechteres Informationssystem mit einer niedrigeren Zahlungsbereitschaft reagieren. ${ }^{28}$

Neben der Art der Informationsverteilung ist als weiteres die Annahme der kostenfreien Informationsübermittlung kritisch zu reflektieren. Unter Kosten sind hier nicht nur die direkt mit der Informationsproduktion verbundenen betriebswirtschaftlichen Kosten zu verstehen, sondern auch die von der Informationsoffenlegung eventuell induzierten Wettbewerbswirkungen, die das Nutzenniveau der Manager beeinflussen können. ${ }^{29}$ Um diese Kostenwirkung auf die Veröffentlichungsentscheidung spieltheoretisch zu modellieren, sind grundsätzlich zwei Möglichkeiten denkbar: Entweder werden die Kosten modellexogen erfaßt und somit als Fixkostenblock oder abhängig vom Charakter der Veröffentlichungsentscheidung abgebildet und in den Auszahlungsfunktionen der Spieler berücksichtigt, oder sie werden modellendogen abgebildet, indem neben den Adressaten und dem Manager weitere Spieler modelliert werden, die ihrerseits auf die Veröffentlichung der Manager reagieren und so das Nutzenniveau der anderen Spieler tangieren. Der letzte Ansatz wird unter anderem gewählt, um die Auswirkungen von Konkurrentenverhalten auf die Veröffentlichungsentscheidung des Managements zu modellieren. ${ }^{30}$

Das einfachste Modell zur exogenen Erfassung von Ausweiskosten bildet die Kosten des Ausweises als Fixkostenblock ab. ${ }^{31}$ Ausgehend von dem unraveling Gleichgewicht ohne Veröffentlichungskosten läßt sich zeigen, daß die Fixkosten dazu führen, daß alle Manager, deren Vorteil der Differenzierung von den „schlechteren“ Konkurrenten von den Informationskosten überkompensiert wird, auf eine Veröffentlichung ihrer privaten In-

27 Vgl. Dye/Sridhar (1995).

28 Vgl. Shin (1994); Kuhner (1998), S. 191; vgl. erweiternd Watson (1996), der die Möglichkeit berücksichtigt, $\mathrm{da}$ das Management unsicher über seine private Information ist.

29 Verrecchia wählt hier den verdeutlichenden Begriff „proprietary cost“, vgl. Verrecchia (1983), S. 181-183; Wagenhofer (1990), S. 61 spricht von direkten und indirekten Ausweiskosten.

30 Vgl. hier grundlegend Wagenhofer (1990), insb. S. 85-114; sowie Wagenhofer (1990a).

31 Vgl. Verrecchia (1983). 
formation verzichten. Interessant ist dieses Ergebnis vor allem vor dem Hintergrund, daß z.B. durch einen besonders intensiven Wettbewerb hohe Veröffentlichungskosten dazu führen, daß der Schwellenwert zwischen Offenlegung und Nicht-Offenlegung sich in Richtung Nicht-Offenlegung verschiebt und die Adressaten auf diese Nicht-Offenlegung mit relativ moderaten Preisabschlägen reagieren.

Speziell vor dem Hintergrund des Konkurrenzverhaltens ist es naheliegend, Ausweiskosten nicht als Fixblock, sondem als Funktion des offengelegten Signals zu modellieren. Über den funktionalen Zusammenhang zwischen Information und Offenlegungskosten können unterschiedlichste Annahmen getroffen werden. So geht Verrecchia in einer Erweiterung seines Fixkostenansatzes von einer Relation aus, die sowohl bei „überraschend guten“ als auch bei „überraschend schlechten“ Signalen von höheren Ausweiskosten ausgeht. ${ }^{32} \mathrm{Zu}$ erklären ist dieser Zusammenhang durch sich überlagernde Wechselwirkungen, wie z.B. das Eintreten von Konkurrenten in den Markt bei guter Ausgangslage und die Erhöhung der Kapitalkosten durch eine schlechte Unternehmensentwicklung. Andererseits sind auch monoton mit den Eigenschaften der Information steigende Ausweiskosten vorstellbar. Je nach Modellierung der Ausweiskosten kann es somit zu komplett unterschiedlichen Gleichgewichtskombinationen wie einem Vollausweis, einem Teilausweis mit einem Schwellenwert, einem Teilausweis mit zwei Schwellenwerten oder einem kompletten Nichtausweis kommen. ${ }^{33}$

Da zumindest teilweise die Ausweiskosten vom Verhalten anderer wirtschaftlicher Akteure abhängen, ist es naheliegend, daß diese Aktionen spieltheoretisch endogenisiert werden, um eine detailliertere Analyse zu erhalten. Dies gilt insbesondere, da exogene Modelle in der Regel negieren, daß Ausweiskosten durch Wettbewerbsreaktionen auch entstehen können, wenn der betreffende Manager nicht ausgewiesen hat. ${ }^{34}$ Dadurch, daß z.B. der Konkurrent auch aus dem Nichtausweis Rückschlüsse auf den Informationsstand des Managers treffen kann, verändern sich die möglichen Gleichgewichtslösungen tendenziell in Richtung Vollausweis der Manager, wobei wesentlich detailliertere Analysen der strategischen Wechselwirkungen ermöglicht werden. ${ }^{35}$ Generell läßt sich ungeachtet von der jeweiligen Modellierung festhalten, daß Kosten der Informationsgenerierung und transmission das Vollausweisgleichgewicht der Ausgangssituation relativieren. Allerdings bleibt auch weiterhin ein Anreiz zum freiwilligen Ausweis bestehen. ${ }^{36}$

32 Vgl. Verrecchia (1983), S. $190 \mathrm{f}$.

33 Vgl. Wagenhofer (1990), S. 62-66.

34 Vgl. Wagenhofer (1990), S. 71-77.

35 Vgl. Wagenhofer (1990), S. 105-107; Dye (1985a).

36 Vgl. dazu auch Newman/Sansing (1993), die neben endogenen Ausweiskosten auch noch die Möglichkeit berücksichtigen, daß das Management unscharfe Informationen berücksichtigt. 
In den gesamten bisherigen Betrachtungen war die Verifikationsmöglichkeit der von den Managern ausgewiesenen Information gegeben. Wie wichtig diese Grundannahme ist, läßt sich leicht nachvollziehen, wenn unterstellt wird, daß die Informationen nicht verifizierbar sind. $\mathrm{Da}$ der Adressat dann nicht davon ausgehen kann, daß das Informationssignal die wahrhaftige Information des Managers wiedergibt, gibt es für den Adressaten grundsätzlich keine Veranlassung, das Signal des Managers in seiner Entscheidung zu berücksichtigen. Anderes gilt nur, wenn die Ziele des Managers und des Adressaten miteinander konform gehen. ${ }^{37}$ Insofern kann nichtverifizierte Information als wertlos angesehen werden. Solange Verifikation von Informationen kostenfrei möglich ist, wird der veröffentlichungswillige Manager seine Information immer verifizieren lassen.

Wenn Verifikation Kosten verursacht, gelten im wesentlichen die Ergebnisse für exogene Veröffentlichungskosten. Da im Regelfall die Verifikationskosten von der Ausprägung des Informationssignals unabhängig sind, wird sich wie bei exogenen Fixkosten ein Nichtausweisbereich von Managern mit schlechten Informationen einstellen. ${ }^{38}$

Fraglich ist, wie der Ausweis von nicht-verifizierbaren Information vom Adressaten aufgenommen wird. Wenn keinerlei Möglichkeit besteht, eine Verifizierung direkt vorzunehmen, sind zwei Möglichkeiten denkbar: Entweder kann die nicht-verifizierbare Information im Zeitablauf durch neue Informationen bestätigt oder widerlegt werden, oder der Manager kann durch Entsenden eines anderen Signals deutlich machen, daß seine Information glaubwürdig ist. Dafür muß die Entsendung des Signals mit Kosten verbunden sein, die im Fall einer Vortäuschung falscher Information prohibitiv hoch sind. ${ }^{39}$ Letztendlich kann diese Situation auch interpretiert werden, indem das entsendete Signal als eigentliche Information mit korrespondierenden exogenen Ausweiskosten interpretiert wird. ${ }^{40}$ Informationen, die erst im Zeitablauf verifiziert werden, erfordern eine neue zeitliche Komponente der Analyse. Fraglich ist, ob ein Manager hinreichende Anreize besitzt, richtig zu berichten, wenn für die Adressaten erst im Zeitablauf Klarheit über den Wahrheitsgehalt seiner Aussage besteht. Ein solches Verhalten könnte entweder über Haftungsregeln sichergestellt werden oder über die Reputation des Managers als Geisel informell induziert werden. ${ }^{41}$ Letztere Methode bietet sich vor allem im Bereich der Prognosepubli-

37 Vgl. Crawford/Sobel (1982); Wagenhofer (1990), S. 116 f; im Extremfall der identischen Entlohnungsfunktion wird diese Aussage allerdings trivial.

38 Vgl. Wagenhofer (1990), S. $119 \mathrm{f}$.

39 Vgl. Gigler (1994); weiterführend auch Rajan/Sarath (1996). Dieser Modellansatz entspricht im wesentlichen der signaling theory, die diese Möglichkeit der Informationsübertragung eingehend und unter unterschiedlichen Rahmenbedingungen analysiert. Vgl. grundlegend Spence (1974); Riley (1979); zum Überblick Kreps (1990), S. 625-660; vgl. beispielhaft zu Anwendungen auf Fragen der Untemehmenspublizität Leland/Pyle (1977); Milgrom (1981); Sarath/Natarajan (1996) und Frantz (1999).

40 Vgl. Wagenhofer (1990), S. 125.

41 Vgl. zur Absicherung von Verträgen durch Geiseln Williamson (1983). 
zität an, da dieses sehr variable und unscharfe Gebiet nur schwer durch Haftungsregeln koordiniert werden kann. Im Gegensatz dazu scheint es eher denkbar, auf unsorgfältig gestellte Prognosen mit einer Abstrafung des Managers durch Reputationsreduktion zu reagieren. ${ }^{42}$

Anders als die hier geschilderten Modellerweiterungen bedroht die Lockerung eines Annahmenkomplexes die spieltheoretische Analyse in ihren Grundfesten. Bislang wurde immer davon ausgegangen, daß die Adressaten die von den Managem übermittelten Informationen in Nutzenauswirkungen transformieren und somit in eine ordinal skalierte Reihenfolge hinsichtlich ihrer Erwünschtheit bringen können. Des weiteren wird davon ausgegangen, daß diese Transformation für alle Adressaten zum gleichen Ergebnis führt. Es besteht also annahmegemäß Einigkeit darüber, wie sich ein bestimmtes Signal auf den Nutzen des einzelnen Adressaten auswirkt. Diese in der Informationsökonomie als monotone likelihood ratio property bekannte Annahme ${ }^{43}$ ermöglicht erst das unraveling Gleichgewicht des Ausgangsmodells, da anhand der veröffentlichten Informationen die Unternehmen in eine objektive Ordinalskala gebracht werden können. Wenn es ein solches verifizierbares Informationssignal gäbe, wäre die Suche nach einem normativen Instrument zur Bewertung von unterschiedlichen Rechnungslegungssystemen im zweiten Kapitel wesentlich leichter gefallen. Da Rechnungslegungspublizität, freiwillig oder nicht, immer für einen ex ante unbekannten Adressatenkreis erstellt wird, erscheint die Annahme eines ordinal skalierten Informationssystems in der Realität kaum haltbar. Unterschiedliche Adressaten werden aufgrund eines unterschiedlichen Informationsstandes und unterschiedlicher Präferenzen neue Informationen anders interpretieren und bewerten. So kann z.B. die Mitteilung, Investitionsaufwendungen in eine bestimmte neue Technologie getätigt zu haben, den unkundigen Adressaten indifferent lassen, den einen vermeintlich kundigen Adressaten zu einer Erhöhung seiner Zahlungsbereitschaft für die betreffenden Unternehmensanteile bewegen, wohingegen ein anderer vermeintlich informierter Adressat die neue Technologie womöglich für einen Irrweg hält und deswegen seine Zahlungsbereitschaft reduzieren wird. Wenn solche Interpretationsunterschiede zugelassen werden, dann besteht seitens des Managements Unsicherheit darüber, wie die Adressaten auf die zu veröffentlichende Information reagieren werden. In diesem Fall sind Aussagen über das zu erwartende Verhalten der Akteure von der Spieltheorie nicht zu erwarten. ${ }^{44}$

Wie lassen sich die Modellergebnisse zur freiwilligen Publizität auf die hier zu untersuchende Fragestellung übertragen? Grundsätzlich scheint für rational handelnde Manager

42 Vgl. zu Möglichkeiten, Reputation modelltheoretisch abzubilden, Sobel (1985) und Kim (1996).

43 Vgl. Kreps (1990), S. 595 f.

44 Vgl. jedoch Watson (1996), der einige Anhaltspunkte dafür liefert, daß ein unsicheres Management ggf. auch zur Veröffentlichung neigen könnte. 
ein gewisser Anreiz zur freiwilligen Publizität zu bestehen, der unter anderem maßgeblich von der vorhandenen Informationsasymmetrie abhängt. ${ }^{45}$ Eine PAIS-Publizität unterscheidet sich von den traditionellen Konzepten vor allem durch die multidimensionale Datenstruktur und die damit verbundene Intensivierung der System-AdressatenInteraktion sowie durch die größere zeitliche Flexibilität. Die zeitliche Flexibilität kann u.a. dazu genutzt werden, erst im Zeitablauf verifizierbare Informationen zeitnah zu legitimieren. Die multidimensionale Struktur der PAIS-Daten dürfte das Problem der unterschiedlichen Interpretation der Daten tangieren. Hier ist einerseits vorstellbar, daß Adressaten aufgrund der Datenmenge noch unterschiedlicher auf die Informationen reagieren und daß somit die Ex-ante-Beurteilung der Vor- und Nachteile einer freiwilligen PAIS-Publizität den Managern noch schwerer fällt. Andererseits kann vor allem die Möglichkeit der spezifischeren Informationsgestaltung für unterschiedliche Adressatengruppen verbunden mit der zeitnahen Verifizierung von Daten mit prognostiven Charakter dazu führen, daß eine Abschätzung der Informationswirkung wegen der größeren Zweckeignung der Daten für den einzelnen Adressaten überhaupt erst möglich wird.

Die Analyse von unterschiedlich scharfen Informationssystemen scheint dafür zu sprechen, daß Adressaten schärfere Signale bevorzugen. ${ }^{46}$ Wenn unterstellt wird, daß das Management durch die Verwendung eines PAIS in der Lage ist, schärfere Signale als mit der traditionellen Rechnungslegungspublizität zu liefern, dann könnte sich in Abhängigkeit der Informationskosten eine "Zweiklassengesellschaft" der Publizität aufbauen: Unternehmen mit guten Umweltlagen und hohem Interesse an scharfer Informationsvermittlung verwenden ein PAIS, während die anderen Unternehmen aufgrund der Kosten und des eigenen inferioren Informationsstands bei der traditionellen Publizität verbleiben und deswegen eine Reduktion der Zahlungsbereitschaft der Adressaten in Kauf nehmen. Generell läßt sich aus spieltheoretischer Sicht keine klare Antwort geben, ob eine Pflichtpublizität freiwillige Publizität fördert, behindert oder unbeeinflußt läßt. ${ }^{47}$ Somit kann zumindest nicht per se behauptet werden, daß freiwillige Publizitätssysteme neben einer Publizitätspflicht keinen Bestand haben können.

45 Vgl. mit empirischem Beleg Bartov/Bodnar (1996) und Coller/Yohn (1997).

46 Hierbei ist allerdings von Bedeutung, daß die Adressaten die Schärfe des Signals auch beurteilen können. Ist dies nicht der Fall, neigt das Management zur Verwendung von unschärferen Informationssystemen. Vgl. zum Zusammenhang von freiwilliger Publizität und Bilanzierungsspielräumen Kasznik (1999). Dieses Dilemma kann unter Umständen durch die Einbindung von externen Akteuren gelöst werden, welche die Qualität des Publizitätssystems festsetzen. Vgl. Sarath (1996).

47 Vgl. Dye (1985a); Wagenhofer (1990), S. 186 f. Einen anderen analytischen Ansatz wählen Gigler/Hemmer (1998), die zu dem Ergebnis kommen, daß zeitnahe Pflichtberichterstattung unter Umständen die Vermittlung von privaten Signalen behindert. 
Tendenziell scheint sich also eine Nachfrage nach PAIS-Implementierungen spieltheoretisch eher zu bestätigen. Fraglich ist, ob die hohe Unsicherheit hinsichtlich der Adressatenreaktion in Verbindung mit den Fixkosten einer Implementierung erste Anwender von einer Nachfrage abhalten könnte. Dazu ist auch von Bedeutung, inwiefern die Adressaten die gleichen Erwartungen über die Implementierungskosten haben wie die Manager und wie sich die variablen Kosten der Informationsproduktion im Verhältnis zu den einmaligen Implementierungskosten im Zeitablauf entwickeln.

\subsubsection{Zusammenfassung}

Aus den Analysen dieses Abschnittes wird deutlich, daß für Unternehmen ein Anreiz besteht, ein PAIS als Veröffentlichungsinstrument zu benutzen. Ob dieser Anreiz auch zur Implementierung führt, hängt von den Kosten der Einführung und des fortwährenden Betriebs des PAIS ab, davon ob die PAIS-Daten hinsichtlich ihrer Wirkung von Adressaten und Manager gleich und klar beurteilt werden können und von dem generellen Vorteil, den der Adressat aus den veröffentlichten PAIS-Daten ziehen kann. Ferner ist von Bedeutung, wie sich die Kostendegression in der PAIS-Erstellung auf die Marktstruktur und damit auf die Ausnutzung der Netzwerkexternalitäten auswirkt. Zu viele Anbieter mit proprietären Schnittstellen können das Entstehen dieser Externalitäten verhindern und somit die Kosten der PAIS-Auswertung erhöhen. Auf die Wirkung von Auswertungskosten wird im Abschnitt 1.7 dieses Kapitels noch einmal eingegangen, da Kosten der PAIS-Auswertung eventuell Intermediäre auf den Plan rufen können, die auf Basis der ihnen zur Verfügung stehenden Daten Unternehmensanalysen anbieten. Zuletzt läßt sich festhalten, daß durch die privatrechtliche Exkludierbarkeit die Möglichkeit gegeben ist, auch bei starker Kostendegression eine Bereitstellung von PAIS-Implementierungen über Bereitsstellungsverbände zu koordinieren.

Die Gefahr der Manipulation von PAIS-Daten kann eventuell durch die Etablierung einer bilateralen Kontrollinstitution gelöst werden..$^{48}$ Auf die Besonderheiten des resultierenden Marktes für Daten- und Methodenprüfungen wird im folgenden eingegangen.

\subsection{Markt für Daten- und Methodenprüfungen}

Wie bereits erwähnt, wurde im Vorabschnitt zum Teil davon ausgegangen, daß das Management wahrheitsgemäß berichtet und somit das PAIS nur korrekte Angaben enthält. In den methodischen Grundlagen wurde jedoch dargestellt, daß eine solche Verhaltensweise wenig realistisch erscheint. In der Realität hat das Management die Möglichkeit, Informa-

\footnotetext{
48 Vgl. allgemein zur Vertrauenssicherung in elektronischen Märkten Kuhlen (2000).
} 
tionen zurückzuhalten und falsche Informationen in das PAIS einzuspeisen. Wird dem Management realitätsnah opportunistisches Verhalten unterstellt, so wird es die PAISDaten fälschen, wenn es sich dadurch einen persönlichen Vorteil erhofft. Da dieses Verhalten wiederum von den PAIS-Adressaten antizipiert wird, verschlechtert sich die Signalwirkung des PAIS. Aus evolutorischer Sicht ist deswegen zu erwarten, daß diejenigen Manager, welche die Signalwirkung des PAIS nachfragen, nach institutionalen Altemativen suchen werden, um die von Ihnen veröffentlichten Daten für die Adressaten glaubhaft $\mathrm{zu}$ machen. Um zu skizzieren, auf welche Institutionen zurückgegriffen werden könnte, soll im folgenden mit dem Verfahren der Wirtschaftsprüfung die zentrale qualitätssichernde Institution der traditionellen Rechnungslegung untersucht werden. ${ }^{49}$

Die Dienstleistung der Wirtschaftsprüfung wird in Deutschland gesetzlich reguliert vom Berufsstand der Wirtschaftsprüfer angeboten. Seit der Aktienrechtsnovelle 1931 besteht für den Jahresabschluß eine Pflicht zur Prüfung gem. \262a HGB 1931. Erste Vorschläge dazu finden sich bereits $1873 .^{50}$ In dem ADHGB 1861 war in Art. 209, Nr. 6 lediglich geregelt, daß der Gesellschaftervertrag die Verfahren der Prüfung festzulegen habe. Diese Prüfung erfolgte in der Regel durch den Aufsichtsrat (Art. 225 ADHGB 1861). Die Berufung extemer Revisoren wird erstmals in der Aktienrechtsnovelle von 1884 ermöglicht. ${ }^{51}$

Eine zentrale Aufgabe der Wirtschaftsprüfer besteht darin, die Einhaltung der gesetzlichen Vorschriften im Rahmen der Erstellung des Jahresabschlusses und Lageberichts zu überprüfen und auf eventuelle Verstöße hinzuweisen. Aus theoretischer Sicht bedeutet ein uneingeschränktes Testat die glaubhafte Versicherung einer dritten Stelle gegenüber dem Adressaten, daß die Informationen des Jahresabschlusses und Lageberichts ein tatsächliches Bild der Vermögens-, Finanz- und Ertragslage sowie des Geschäftsverlaufs und somit der Lage der Gesellschaft vermitteln. ${ }^{52}$

49 Vgl. zur Funktionsweise der Wirtschaftsprüfung und deren unterschiedlichen internationalen Ausgestaltung Sell (1999); als Überblick zu den Prüfungsregeln auch Baetge/Sell (1999); zum Prüfungsmarkt für deutsche börsennotierte Gesellschaften Lenz/Ostrowski (1999) und Hüllweck/Ostrowski (2000); als kritische Reflektion zum Prüfungsmechanismus Power (1997).

50 Vgl. den Gesetzesvorschlag im Rahmen der ersten Tagung des Vereins für Socialpolitik Wiener (1873) Seite 20-23, vgl. ferner erläutemd und diskutierend Barth (1953), S. $325 \mathrm{f}$.

51 Vgl. Art. 239a ADHGB 1884, der die Berufung eines Revisors durch die Hauptversammlung fordert. Auch die Bestellung eines Revisors durch eine 10 \%ige Minderheit der Aktionäre war unter bestimmten Umständen möglich, vgl. Art. 222a ADHGB 1884.

$52 \mathrm{Zu}$ beachten ist hierbei, daß Wirtschaftsprüfer letztendlich lediglich die Einhaltung der gesetzlichen Normen bestätigen können, wobei diese jedoch qualitative Anforderungen wie in $\$ 297$ (2) HGB enthalten. Die Wirkung des uneingeschränkten Testats läßt sich unter der Annahme, daß ein uneingeschränkt testierter Jahresabschluß und Lagebericht einen höheren Erklärungsgehalt für den Aktienkurs besitzt, als ein mit Einschränkungen testierter, empirisch messen. Vgl. mit positivem Ergebnis Teoh/Wong (1993). 
Doch auch Wirtschaftsprüfer sollten realistischer Weise als opportunistisch handelnde Individuen aufgefaßt werden. Sie sind keine mechanistisch handelnde Qualitätssicherungssysteme und können somit nicht als ein simpler Kostenfaktor der Unternehmenspublizität behandelt werden. Wird die Wirtschaftsprüfung als eigenständige Institution in die Analyse mit einbezogen, so bildet sich eine zweistufige Prinzipal-Agenten-Relation vom Management über den Prüfer zum Adressaten. ${ }^{53}$ Zwei Faktoren könnten neben dem Verfügungsrecht über die Vertragsentscheidungen das Verhalten der Wirtschaftsprüfer in die von den Adressaten präferierte Richtung beeinflussen: Reputation und vertraglich geregelte Haftung für falsche Information. ${ }^{54}$

$\mathrm{Da}$ die Implementierung eines PAIS eine nicht gesetzlich regulierte Entscheidung von Wirtschaftssubjekten darstellt, ist davon auszugehen, daß auch die Prüfung von PAIS grundsätzlich nicht der gesetzlichen Kontrolle unterliegen wird. Somit ist zunächst zu hinterfragen, ob und ggf. warum eine Nachfrage nach prüfungstechnischer Validierung von PAIS-Informationen entstehen könnte. Im Anschluß daran ist zu untersuchen, ob und ggf. mit welchen Einschränkungen ein solches Produkt angeboten werden kann.

Wie bereits im Vorabschnitt diskutiert, haben opportunistisch handelnde Individuen einen Anreiz, Informationen zurückzuhalten und/oder zu verfälschen, wenn sie sich dadurch einen Vorteil verschaffen können. Auch ohne dies formaltheoretisch abzuleiten, ist es evident, daß ohne Prüfungsmöglichkeit die Signalqualität eines PAIS im einmaligen Spiel durch das opportunistische Verhalten des Managements stark beeinträchtigt wird. Ist die Manipulation der PAIS-Daten kostenfrei, dann ist davon auszugehen, daß die veröffentlichten PAIS-Daten dem Adressaten keinerlei Informationsverbesserung hinsichtlich der Qualität des berichtenden Untemehmens mehr bietet. Diese negative Prognose über den Informationsgehalt ungeprüfter PAIS-Daten relativiert sich allerdings, wenn die Analysedauer über den erstmaligen Zeitpunkt hinweg ausgebaut wird. Adressaten, die ex post feststellen mußten, daß sie von ihrem Management hintergangen wurden, werden ihre Erwartungen hinsichtlich des Verhaltens des Managements verändern. Wird des weiteren noch eine sich im Zeitablauf ändernde Umweltlage unterstellt, besitzen auch Manager von schlechten Untemehmen einen gewissen Anreiz, sich eine Reputation aufzubauen. Der Aufbau von Reputation erfolgt in der Regel durch das Realisieren von spezifischen Investitionen, deren Wert in alternativen Verwendungsmöglichkeiten stark

53 Vgl. für eine modelltheoretische Analyse der Prüferbeziehung für Deutschland grundlegend Ewert (1990) und einführend Schildbach (1996); jüngst in einem allgemeinen Modell Faure-Grimaud/Laffont/Martimort (1999). Realiter umfaßt die Prinzipal-Agenten-Relation in Deutschland sogar noch den Aufsichtsrat, der nach $\int 111$ (2) Satz 3 AktG dem Prüfer den Prüfungsauftrag erteilt und die Hauptversammlung die gem. $\int 119$ (1) Nr. 4 AktG den Prüfer bestellt. Vgl. zur Funktion des Aufsichtsrates und der Prüfung nach dem KonTraG Mattheus (1999).

54 Vgl. Dye (1993); sowie jüngst Ewert (1999); Datar/Alles (1999) und Hillegeist (1999). 
sinken würde. Eine solche Investition kann auch eine PAIS-Implementierung sein. Schließlich ist die erstmalige Einführung der Infrastruktur mit wesentlich höheren Kosten belegt als der spätere Betrieb. Insofern würde ein Manager, der ein PAIS einführt, um damit falsche Informationen zu verbreiten, seine soeben etablierte Reputation sofort wieder zerstören.

Dieser Reputationseffekt mag Managern dienen, die sich bereits eine Zeit im Markt aufhalten, was aber ist mit Entrepeneuren, die ihr Unternehmen veräußern wollen oder mit Managern, die in den Ruhestand gehen? Wenn Sie ein Interesse haben, die hohe Qualität ihres Unternehmens zu signalisieren, dann müssen sie Reputation zukaufen, falls die spezifische Investition in ein PAIS als reputationsbildende Maßnahme vom Markt als nicht ausreichend angesehen wird. Reputation können Prüfer anbieten, die schon länger am Markt tätig sind, bzw. für Gesellschaften arbeiten, die ihrerseits eine Firmenreputation besitzen.

Zur Analyse der Manager-Prüfer-Beziehung ist es von Bedeutung, ob der Manager die Möglichkeit hat, den Prüfer zu hintergehen. Kann er dies, so wird er versucht sein, dem Prüfer Daten vorzulegen, die seinem Unternehmen realiter nicht vorhandene positive Qualitätseigenschaften attestieren. Da der wirkliche Prüfungsaufwand des Prüfers für den Adressaten in der Regel nicht erkennbar ist und dieser statt dessen nur den Wahrheitsgehalt des Informationssystems ex post beurteilen kann, bedroht solch opportunistisches Verhalten des Managements auch die Reputation des Prüfers. $\mathrm{Da}$ somit der Manager unter Umständen in der Lage ist, die Reputation des Prüfers zu nutzen, um einen eigenen Vorteil zu erzielen, wird die zweite klassische Sicherungsinstitution der Prüfungstätigkeit notwendig: die Haftungsregeln.

Haftungsregeln greifen auch bei einmaligen Prüfungsvorgängen. Sie regeln die Ansprüche, welche von falschen Informationen betroffene Wirtschaftssubjekte an das berichtende Management und den Prüfer geltend machen können, wenn diesen ein Verstoß gegen bestehende Prüfungsregeln nachgewiesen werden kann. ${ }^{55}$ Müssen diese ex post mit Haftungszahlungen rechnen, so reduziert sich für das Management der Anreiz zu opportunistischem Verhalten, und die Prüfer erhalten einen weiteren Anreiz, ihre nur privat zu beobachtende Prüfungsanstrengung zu erhöhen. Ob das Management, die Prüfer oder beide Parteien in die Haftung genommen werden, hängt von der einzelnen rechtlichen

55 Die Frage, ob gegen Prüfungsregeln verstoßen wurde, oder nicht, kann letztlich nur von der Rechtsprechung entschieden werden. In diesem Zusammenhang ist von großer Bedeutung, wie scharf die Prüfungsregeln sind. Für den Bereich des PAIS lassen sich wegen des großen Entscheidungsspielraums des Managements wohl keine scharfen Prüfungsregeln entwickeln. Ewert (1999) zeigt jedoch mittels eines spieltheoretischen Ansatzes, daß scharfe Regeln die Prüfer beim Vorliegen von Haftungsregeln nicht notwendigerweise zu größerer Prüfungsanstrengung veranlassen. 
Ausgestaltung ab, ist aber von eher untergeordneter Bedeutung, wenn der haftenden Partei die Möglichkeit zur Nachverhandlung mit der anderen Partei eingeräumt wird. Fraglich ist, inwieweit Haftungsregeln für die Prüfung eines PAIS implementiert werden können, ohne daß die Bereitstellung der PAIS-Daten gesetzlich reguliert ist. Grundsätzlich greifen beim Fehlen von speziellen Vorschriften die normalen privatrechtlichen Normen. Somit ist eine bewußte Täuschung von Vertragspartnern unzulässig. Dies geht einerseits aus $₫ 400$ (1) Nr. 1 AktG hervor, darauf basierend können Vertragspartner Schadensersatzansprüche gem. $₫ 823$ (2) BGB gegen den Manager und ggf. i.V.m. \31 BGB gegen seine Gesellschaft geltend machen. ${ }^{56}$ Somit scheint grundsätzlich eine privatrechtliche Grundlage für ausreichende Haftungsregeln zu bestehen.

Da einerseits die Möglichkeit des Reputationsaufbaus durch Manager und Prüfer und andererseits die Grundlage für Haftungsregeln besteht, ist davon auszugehen, daß sich eine Prüfung von PAISSystemen grundsätzlich als wirkungsvolle Institution ausgestalten läßt, ohne daß es der expliziten Regulierung bedarf. Ferner wurde argumentiert, daß es auch Unternehmen geben könnte, die ohne staatliche Regulierung einen Anreiz besitzen, ihre PAIS-Implementierung prüfen zu lassen, um sich von den "schwarzen Schafen“ abzugrenzen. Fraglich bleibt jedoch: Läßt sich eine PAIS-Implementierung überhaupt im klassischen Sinn prüfen?

Die Prüfung der handelsrechtlichen Untemehmenspublizität ist im wesentlichen an dem Rechenwerk ausgerichtet. Somit erfolgt die Prüfung periodisch. Sie kontrolliert den zur Veröffentlichung anstehenden Jahresabschluß und Geschäftsbericht. ${ }^{57}$ Durch die Implementierung eines PAIS wird die Berichterstattung tendenziell kontinuierlicher, somit erscheint eine periodische Prüfung der Daten nicht mehr hinreichend. Andererseits dürfte ein kontinuierlicher Prüfungsprozeß der unter Umständen täglich modifizierten $\mathrm{Da}$ tenbasis zu hohen Kosten führen, die den Nutzen eines PAIS eventuell überkompensieren könnten. Um diese Prüfungskosten zu reduzieren, ist eine gewisse Fokussierung auf die Methodenprüfung denkbar. ${ }^{58}$ Während die Kosten der Datenprüfung stark vom Datenaufkommen abhängig sind, lassen sich Methoden zur Datengewinnung unabhängig von dem Zeitpunkt der Aktualisierung und der Verarbeitung der Daten überprüfen. Im Regelfall sollte der Großteil der Daten ohne operativen Eingriff generiert werden, so daß eine Überprüfung der Methoden und die wirksame Verhinderung von späteren menschlichen Eingriffen in den Datenproduktionsprozeß hinreichend sein könnte. Nichtsdestotrotz

56 Vg. allgemein zu Haftungsregeln innerhalb der Untemehmenspublizität Kersting/Sohbi (1998), hier insb. S. $305 \mathrm{f}$.

57 Vg. zum Prüfungsablauf und -2ufbau umfassend Leffson (1988), insb. S. 138-289.

so Diese Fokussierung findet sich bereits als Systemprüfung im traditionellem Prüfungsprozeß. Vgl. Leffson (1988), S. 226-250. 
kann eine Methodenprüfung nicht eine Prüfung der letztendlich veröffentlichten Daten komplett ersetzen, solange der gesamte Datengenerierungsprozeß nicht vollkommen vom menschlichen Eingriff abgeschirmt ist.

Abgesehen vom Datenvolumen und dem kontinuierlichen Charakter der Datenerhebung und -veröffentlichung bestehen weitere Probleme in der Verifizierung von PAIS-Daten. Dem offenen Konzept folgend, erlaubt eine PAIS-Implementierung unter Umständen hochsubjektive Bewertungsmethoden, die sich einer Objektivierung in einem Prüfungsprozeß weitgehend verschließen. Aus Sicht der klassischen Rechnungslegungstheorie wird diese fehlende Objektivität oder intersubjektive Nachprüfbarkeit als ein Ausschlußkriterium für mögliche Wertansätze gesehen. ${ }^{59} \mathrm{Da}$ diese Wertansätze trotzdem Informationscharakter besitzen können, schließlich determinieren sie letztlich den Marktpreis, sollten solche Wertansätze nicht a priori ausgeschlossen werden. Ähnliches gilt auch für den gesamten Bereich der Prognosepublizität innerhalb des PAIS. ${ }^{60}$ Insofern erscheint es vorstellbar, daß gewisse Bereiche eines PAIS anhand der Methoden geprüft werden, andere anhand der Daten und wieder andere gar nicht. Es ist dann eine zentrale Aufgabe der jeweiligen PAIS-Implementierung, dem Adressaten zu verdeutlichen, welchen Grad der Zertifizierung die gerade präsentierten Daten haben. In der Regel kann dies durch ein bestimmtes Merkmal in einer der Dimensionen geschehen. Im Ausnahmefall kann auch die Anfügung einer weiteren Dimension notwendig sein.

Es läßt sich festhalten, daß sich eine gewisse Nachfrage nach Prüfung der PAIS-Daten entwickeln dürfte. Auch auf den speziellen Charakter einer PAIS-Prüfung kann durch die Wahl eines geeigneten Prüfungskonzepts eingegangen werden. Somit ist zu erwarten, daß sich auch Anbieter einer solchen Prüfungsleistung finden werden. Ob diese Lücke ganz oder teilweise von den gleichen Anbietem, die bereits im traditionellen Markt für Wirtschaftsprüfung tätig sind, geschlossen wird, oder ob sich andere Wirtschaftssubjekte auf diese Dienstleistung spezialisieren, bleibt abzuwarten. ${ }^{61}$ Eine weitere Gruppe von Marktteilnehmem, die eventuell auch eine gewisse Zertifizierungsaufgabe übernehmen oder

59 Vgl. AAA (1977), S. 13; ein klarer Wertansatz als Ansatzkriterium wird bereits von Simon gesehen, vgl Simon (1899), S. 169; vgl. für den korrespondierenden Begriff der „Objektivierbarkeit“ Baetge (1996), S. 79 f.

60 Vgl. zu den aus der Prognosepublizität resultierenden Prüfungsproblemen überblicksweise Kersting/Sohbi (1998).

61 Dies ist u.a. auch davon abhängig ob die traditionellen Anbieter die notwendige technische Kompetenz vorweisen, bzw. sich erarbeiten können. $D_{a}$ schon seit geraumer Zeit die traditionelle Wirtschaftsprüfung regen Gebrauch von EDV-Infrastruktur macht, vgl. Meisel (1997); die Befragung Hertzsch/Schmitz (1997) und für den amerikanischen Bereich Lanza (1998); Helms/Mancino (1998), und auch die interne Revision sich mit dem Themengebiet schon seit einiger Zeit befaßt, vgl. beispielhaft Luippold/Wagner (1996) und Kohler/Beuchat (1996), ist wohl von ausreichender Kompetenz auszugehen, zumal zumindest im intemationalen Bereich die Notwendigkeit zur kontinuierlichen Weiterentwicklung der Profession seitens der Wirtschaftsprüfer erkannt wird, vgl. z.B. Carlozzi (1998) und Debreceny/Gray (1999). 
auch als Nachfrager von Prüfungsdienstleistungen auftreten könnte, sind die Wertpapierbörsen. Ihre Aufgabe soll in dem nächsten Abschnitt beleuchtet werden.

\subsection{Markt für Eigenkapitalhandelsdienstleistungen}

In diesem Abschnitt sollen die Auswirkungen eines PAIS auf den Markt für Wertpapierhandel analysiert werden. Wegen des besonderen Informationsbedürfnisses der Eigenkapitalgeber beschränken sich die Ausführungen auf den Markt für Eigenkapitaltitel. ${ }^{62}$

Wertpapierbörsen können sowohl als Märkte als auch als Unternehmen analysiert werden. ${ }^{63}$ Hier wird der letzte Ansatz gewählt, da so die über die reine Preisfindung hinausgehenden Dienstleistungen der Wertpapierbörsen greifbar werden. Zu diesen Dienstleistungen gehören u.a. das Präsentieren und Verbreiten von Unternehmensinformationen, das Organisieren von Analystenkonferenzen sowie die Etablierung von Betreuern im Preisfindungsprozeß. All dies dient dem Hauptziel der Wertpapierbörsen, für die Eigenkapitaltitel einen liquiden Markt bereitzustellen, da ihre Erträge im wesentlichen aus der Umsatzbeteiligung resultieren. ${ }^{64}$

In den letzten Jahren ist speziell bei der Deutschen Börse AG ein Trend zu beobachten, den Gesamtmarkt der Aktien zu unterteilen und bestimmte Segmente mit Unternehmen zu bilden, die hinsichtlich bestimmter kapitalmarktrelevanter Eigenschaften ähnlich sind. Ein erstes Beispiel hierfür ist der Neue Markt. ${ }^{65}$ Hier will die Deutsche Börse AG hauptsächlich produktinnovative Unternehmen mit Expansionstendenzen versammeln, da sie davon ausgeht, daß diese Unternehmen im traditionellen Aktienhandel zu wenig Aufmerksamkeit von der Nachfrageseite erhalten. ${ }^{66} \mathrm{Um}$ diese Aufmerksamkeit zu sichern, werden die Unternehmen nicht nur in einem eigenen Marktsegment plaziert, die Deutsche Börse AG verlangt auch durch privatrechtliche Vertragsbindungen eine über die traditionellen gesellschaftsrechtlichen und kapitalmarktrechtlichen Vorschriften hinausgehende Unternehmenspublizität. ${ }^{67}$ Durch diese Verpflichtung dokumentieren die parti-

62 Vgl. allgemein zur instutionellen Struktur von Wertpapierbörsen Köndgen (1998); Segna (1999); generell zur institutionellen Struktur des deutschen Kapitalmarkts und zu dem (ehemals) recht großen Gewicht der Banken Cable (1985).

63 Vgl. Mulherin/Netter/Overdahl (1991), insb. S. 643 f.; Mahoney (1997).

64 Vgl. allgemein zur Bedeutung von Wertpapierbörsen für die Volkswirtschaft Levine/Zervos (1998); relativierend Stiglitz (1981); zum positiven Zusammenhang von Unternehmenspublizität und Marktliquidität Verrecchia (1996); sowie relativierend für das Vorliegen von heuristischen Händlem, die auf Informationen überreagieren, Fischer/Verrecchia (1999); modelltheoretisch grundlegend für den Zusammenhang zwischen Liquidität und Kapitalkosten Merton (1987); einen empirischen Beleg für den japanischen Aktienmarkt liefern Amihud/Mendelson/Uno (1999).

65 Vgl. Förschle/Helmschrott (1998; 1999); Gerke (1999); Gerke/Bank/Seitz (1999); Maute (1999).

66 Vgl. Infoordner Neuer Markt, S. 1-5.

67 Vgl. Infoordner Neuer Markt, S. 6-8. 
zipierenden Unternehmen ihre Bereitschaft, eine progressive Unternehmenspublizität vorzunehmen. Da bei Nichtbefolgung der privatrechtlichen Publizitätspflichten ein Delisting im entsprechenden Segment droht, wird diese Verpflichtung auch für externe Adressaten glaubwürdig. Aus Sicht der Börse bietet sich so die Möglichkeit, die Informationsasymmetrie zwischen Unternehmen und Kapitalgebern abzubauen und somit die Preisfindung zu erleichtern. Deswegen findet diese Strategie vor allem bei Gruppen von Kapitalnachfragern Anklang, welche die Informationsasymmetrie zwischen Angebot und Nachfrage als besonders gravierend einschätzen. Neben den erweiterten Publizitätspflichten erwartet die Deutsche Börse AG von innovativen Unternehmen anscheinend eine größere Kompetenz im Umgang mit digitalen Medien. Ein Großteil der Kapitalmarktkommunikation des Neuen Markts wird über das Internet abgewickelt. Die Geschäftsberichte werden für die Adressaten einfach erreichbar als PDF-Datei auf dem zentralen Kommunikationsserver des Neuen Marktes abgelegt.

Speziell in dem Bereich der stark wachsenden Unternehmen herrscht eine recht große Nachfrage nach Kapitalgebern. ${ }^{68}$ Diese Firmen besitzen wegen ihres großen Wachstums einen hohen Kapitalbedarf, den sie nur schwer über Fremd- und Eigenkapital decken können. Da das recht hohe Risiko von Entrepreneuren bei Fremdkapitalgebern wegen der vertraglich determinierten Rückzahlung tendenziell asymmetrisch wirkt, stellt die Finanzierung mit zusätzlichem Eigenkapital ab einer gewissen Unternehmensgröße eine absolute Notwendigkeit dar. ${ }^{69}$ Insofern ist es vorstellbar, daß Wertpapierbörsen besonders für dieses Marktsegment spezialisierte Informationssysteme zur Kapitalanbieterinformation nachfragen. Zwar ist bereits die Nutzung des Internets zur Distribution der Unternehmenspublizität vor allem für die potentiellen Aktionäre ein wesentlicher Fortschritt, doch behindern die noch vorhandenen Medienbrüche die EDV-gestützte Analyse und Weiterverarbeitung der Daten. Diese Probleme könnten durch die Implementierung eines PAIS gelöst werden. Wenn die PAIS-Implementierung auf Veranlassung der jeweiligen Börse hin geschieht, kann diese auch die genaue Datenstruktur spezifizieren, um neben dem auf das einzelne Unternehmen spezialisierten Informationsangebot auch eine bessere Vergleichbarkeit zwischen den Unternehmen gewährleisten zu können. Somit wäre sie dann in der Lage, den Kapitalmarktteilnehmern ein aus Sicht der Börse geeignet gestaltetes Informationssystem zu bieten, mit dem der Unternehmensvergleich erleichtert wird. Ferner besteht durch diese Dienstleistung die Möglichkeit zur Schaffung eines komparativen Konkurrenzvorteils gegenüber anderen Wertpapierbörsen. Dies ist insofern von

68 Vg. Infoordner Neuer Markt. S. 1. Dies gilt vor allem, da für Venture-Capital-Finanziers ein geeignetes Börsensegement von großer Bedeutung ist. Vgl. Perlitz/Seger/Ackermann (1999).

69 Vgl. zu Finanzierungsproblemen von Entrepreneuren Trester (1998); hierbei ist wiederum die Prüfungsdienstleistung von großer Bedeutung, vgl. Willenborg (1999). 
Bedeutung, als daß sich die Konkurrenzsituation der Wertpapierbörsen in den letzten Jahren durch die Zunahme des internationalen Wertpapierhandels noch verstärkt hat. ${ }^{70}$

Es ist also vorstellbar, daß Wertpapierbörsen in ihrer Rolle als Marktdienstleister die Verbreitung von PAIS-Implementierungen fördern. Entscheidend hierfür kann unter anderem das börsentechnische Abschneiden der Wertpapiere von PAIS-Pionieren sein. Wenn von der Börsenleitung in zeitlicher Koexistenz mit der Verbreitung der PAIS-Daten ein gestiegener Umsatz der Aktie des berichtenden Unternehmens festgestellt wird, dann liegt der Rückschluß auf eine marktbelebende Wirkung einer PAIS-Publizität nahe. In diesem Fall könnte für die Börse der Versuch naheliegen, andere umsatzschwache Unternehmen zu einer PAIS-Implementierung zu bewegen, wenn als ein Grund für die geringen Umsätze eine mangelnde Unternehmenspublizität gesehen wird.

\subsection{Markt für Rechnungslegungsregeln}

Ungeachtet von der Art der PAIS-Implementierung und der maßgeblichen Motive für die Implementierung braucht der Ersteller von PAIS-Daten weiterhin Rechnungslegungsregeln, die ihm die Abbildung der realökonomischen Situation durch quantitative, multidimensional strukturierte Rechnungslegungsdaten ermöglichen. Diese Regeln sollen im folgenden zur deutlichen Unterscheidung PAIS-Rechnungslegungsregeln, oder verkürzt PAIS-Regeln genannt werden, um sie von den normalen monodimensional konzipierten Rechnungslegungsregeln abzugrenzen. Ungeachtet dieses Unterschieds könnte der PAISDatenersteller die PAIS-Regeln von einem Anbieter monodimensionaler Rechnungslegungsregeln beziehen, zumal diese durch den politischen und teilweise auch wirtschaftlichen Prozeß der Normensetzung aus evolutorischer Sicht auch eine gewisse Legitimation besitzen. $\mathrm{Da}$ in einem PAIS auch unterschiedliche Regeln parallel zur Anwendung kommen können, dürfte sich jedoch auch eine Nachfrage nach weiteren alternativen PAISRegelsätzen herausbilden. Durch die Möglichkeiten der Multidimensionalität entfällt eine der zentralen Anforderungen an traditionelle Rechnungslegungsregeln: Die Entwicklung eines einzigen Wertansatzes mit möglichst guter Informationsübermittlung. Deswegen ist davon auszugehen, daß die monodimensionalen Rechnungslegungsregeln nur dann eine superiore Eignung für PAIS-Datengenerierung besitzen könnten, wenn nur ein einziges Regelsystem zur Anwendung kommen soll. Sobald ein berichtendes Unternehmen mehrere alternative Regelsysteme in die PAIS-Datenbank einstellen möchte, spricht die eindi-

70 Vgl. für EASDAQ und den Neuen Markt Gerke/Bank/Seitz (1999). Es ist auch ein deutlicher Trend zu internationalen Allianzen zu beobachten, vgl. Gajo (1999); des weiteren verschärfen elektronische Handelssysteme in Verbindung mit längeren Handelszeiten den Wettbewerb, vgl. Unser/Oehler (1998). Daß die Börsen dennoch segmentiert sind, beweisen Unterschiede in den Aktienrenditen, vgl. Jorion/Goetzmann (1999). 
mensionale Ausrichtung der bestehenden Systeme tendenziell gegen eine Anwendung. Die in der Realität zu beobachtende Mischform von monodimensionalen Regeln, die z.B. teilweise fortgefuihrte Anschaffungskosten und zum Teil Marktwerte als Bewertungsgrundlage favorisiert, ist wohl nur vor dem Hintergrund der Notwendigkeit eines eindeutigen Wertansatzes zu rechtfertigen. Unter Verwendung des PAIS scheint es vielmehr angeraten, beide Ansätze alternativ für alle abzubildende Sachverhalte anzubieten.

Die für die Implementierung eines PAIS anzuwendenden Regeln divergieren stark in Abhängigkeit der jeweiligen daten- und realökonomischen Struktur des berichtenden Unternehmens. So mag ein Rohstofflieferant, der mit komplizierten Terminkontrakten handelt, PAIS-Regeln nachfragen, mit denen er diese Verträge anhand seiner operationalen Datenbasis und externer Marktdaten marktnah bewerten kann, während ein Dienstleister Verfahren benötigt, um das in seinem Unternehmen gebundene Know-How zu bewerten. In beiden Fällen sind die PAIS-Regeln nach Möglichkeit so zu formulieren, daß eine weitgehend automatisierte Anwendung zur Generierung der korrespondierenden Wertansätze möglich ist. Insofern handelt es sich bei PAIS-Rechnungslegungsregeln um ein auch technisch geprägtes Produkt. Zu deren Generierung dürfte teilweise recht detailliertes Know-How über betriebliche Datenverarbeitung notwendig sein. Ob die traditionellen Produzenten von Rechnungslegungsregeln diese notwendigen spezifischen Investitionen vornehmen, um diese Regeln anzubieten, bleibt abzuwarten, erscheint jedoch nur beschränkt wahrscheinlich. Insofern ist es zu erwarten, daß sich einerseits strategische Allianzen $^{71}$ zwischen bislang monodimensional ausgerichteten Standardsettern und Unternehmen mit dem spezifischen Fachwissen bilden und daß andererseits neue Anbieter in den Markt eintreten werden.

Die theoretische Vorarbeit zur Generierung möglicher Wertansätze wurde in der Vergangenheit und wird auch in der Gegenwart von der rechnungslegungstheoretischen Forschung und Praxis geleistet, so daß ein potentieller Anbieter sich zur Generierung und Legitimierung unterschiedlicher Ansätze im Regelfall auf die Literatur berufen kann. Größeren Aufwand für den Anbieter stellt die technische Realisierung der Anwendungsmethoden und die Kommunikation der Zweckdienlichkeit des verwendeten Wertansatzes dar. Die technische Realisierung erfordert detaillierte Kenntnisse der implementierten PAIS und der operationalen Datenbasen der berichtenden Unternehmen. Die Zweckdienlichkeit läßt sich unter Umständen argumentativ theoretisch oder empirisch begründen. Die theoretische Ableitung einer Rechnungslegungsregel erscheint wegen des komplexen Informationsbedürfnisses der unterschiedlichen Adressaten nur sehr begrenzt möglich.

71 Selbstverständlich sind auch andere Formen der Koordination denkbar. Da es sich bei der unterliegenden Transaktion um eine recht spezifische und wiederkehrende handelt, erscheint die Bildung von strategischen Allianzen allerdings recht wahrscheinlich. 
Die empirische Begründung eines Wertansatzes hingegen kann durch den Nachweis einer Korrelation von regelkonformen PAIS-Daten mit Zielgrößen, wie z.B. künftigen Börsenkursen oder Renditen, erfolgen. Gelänge ein solcher empirischer Nachweis, so wäre dies wohl ein für viele Adressaten nachvollziehbares Qualitätsmerkmal. Da die Ergebnisse der empirischen Kapitalmarktforschung für traditionelle Rechnungslegungsdaten zwar einen signifikanten Zusammenhang zwischen Rechnungslegungsdaten und Börsenkursen belegen, aufgrund der nur niedrigen Erklärungsquote bislang jedoch eine Prognoseeignung noch nicht gegeben ist, ${ }^{72}$ ist wohl zumindest kurzfristig nicht zu erwarten, daß PAISDaten nach anderen Regeln bahnbrechende Verbesserungen erbringen. ${ }^{73}$ Nichtsdestotrotz stellt die Prognosetauglichkeit der PAIS-Daten für Zukunftsereignisse eine geeignete Meßlatte für die PAIS-Rechnungslegungsregeln dar. Welches Zukunftsereignis prognostiziert werden soll, hängt von den unterschiedlichen Adressateninteressen ab. Wenn die Prognosetauglichkeit als Qualitätsmerkmal unterschiedlicher Rechnungslegungsregeln akzeptiert wird, dürfte dies die Produktion von Regeln mit hoher Qualität erschweren, da die Datenbasis eines berichtenden Unternehmens hauptsächlich vergangenheitsorientiert organisiert ist. Zur Generierung von prognoserelevanten Informationen aus dieser Datenbasis ist eine erhebliche Fachkenntnis über das berichtende Unternehmen notwendig, die in der Regel am ehesten das Management des Unternehmens haben dürfte. ${ }^{74}$ Der daraus resultierende Prinzipal-Agenten-Konflikt kann, wie oben bereits diskutiert, unter Umständen durch die Einbindung externer Prüfungsinstanzen, welche auch die Regeleinhaltung durch Methodenprüfung zertifizieren, gemindert werden.

$\mathrm{Daß}$ die Qualität der PAIS-Rechnungslegungsregeln intersubjektiv beobachtbar ist, spricht für einen möglichen Wettbewerb von alternativen PAIS-Regeln. Problematisch könnte sein, daß die Qualität der Regeln höchstwahrscheinlich stark mit den spezifischen Investitionen des Produzenten korreliert. Dies läßt tendenziell eine vertikale Integration der Regelproduktion einerseits in die Produktion von PAIS-Implementierungen und andererseits in die Produktion der PAIS-Daten erwarten. Letzteres ist um so wahrscheinlicher, je mehr die private Information der Datenersteller zur Formulierung und Anwendung einer qualitativ hochstehenden PAIS-Rechnungslegungsregel vonnöten ist. $\mathrm{Da}$ in der Regel für die Datenerstellung das Management des berichtenden Untemehmens verantwortlich sein wird, ist in diesem Fall die Möglichkeit der Adressaten, auf das Managementverhalten Einfluß zu nehmen, von großer Bedeutung.

72 Vgl. dazu die Ausführungen in Kapitel II-2.2.3.

73 Wobei mittel und langfristig durch einen starken evolutionären Wettbewerb für PAIS Rechnungslegungsregeln sehr wohl eine Verbesserung des Prognosecharakters denkbar erscheint.

74 Inwieweit die untemehmensberatende und -analysierende Branche in diesem Zusammenhang von Bedeutung ist, wird im nächsten Abschnitt beleuchtet werden. 
Auch ohne empirisch meßbare Qualität können unterschiedliche PAIS Rechnungslegungsregeln hinsichtlich ihres Markterfolges bewertet werden. Wenn die PAIS-Implementierungen protokollieren, welche Daten von den Adressaten häufig abgerufen werden, läßt sich darüber die Nachfrage nach bestimmten Regeln ermitteln. $\mathrm{Da}$ realiter davon ausgegangen werden kann, daß das Abrufen und die Analyse von PAISDaten für den Adressaten nicht kostenfrei ist, wird dieser nur die Daten nachfragen, von denen er einen Nettonutzen erwartet.

Der Markt für PAIS-Rechnungslegungsregeln unterscheidet sich also recht deutlich vom Markt für monodimensionale Rechnungslegungsregeln, da diese mit Blick auf unterschiedliche Informationsbedürfnisse als jeweils ausschließliche Norm gestaltet werden müssen, während die PAIS-Regeln auf ein spezielles Informationsbedürfnis hin gestaltet werden können und eng mit der technischen Implementierung und mit der jeweiligen Datenbasis verbunden sind. Trotzdem können PAIS-Rechnungslegungsregeln einen Einfluß auf monodimensionale Rechnungslegungsregeln ausüben. Ist das jeweilige traditionelle Rechnungslegungssystem zentral auf eine weitgehend homogene Adressatengruppe zugeschnitten, dann können vom Markt „auserwählte“ PAIS-Rechnungslegungsregeln, die für dieselbe Gruppe zugeschnitten sind, Anhaltspunkte für die Ermittlung einer monodimensionalen Rechnungslegungsregel geben. Dies gilt vor allem, wenn entweder die qualitativen Eigenschaften der beobachteten PAIS-Regel sehr hoch sind, oder sich diese Norm im Wettbewerb der unterschiedlichen Normen als erfolgreich herausgestellt hat.

Als Ergebnis dieses Abschnittes ist festzuhalten, daß die Wirkung der PAIS-Konzeption auf den Markt für Rechnungslegungsregeln evolutionsfördernd ist. Durch ein PAIS entsteht die Möglichkeit, spezifische Regeln, deren Qualität für den einzelnen Nachfrager meßbar ist, anzubieten und zu nutzen. Zwei zentrale Fragen bleiben: Erstens, gibt es überhaupt Regeln, die in der Lage sind, die Informationsbedürfnisse der Adressaten zu bedienen? Vor allem, da ein Großteil der Informationsbedürfnisse auf das Ergebnis von Marktprozessen hinzielt, dürfen hier wohl keine Wunder erwartet werden. Die zweite darauf aufbauende Frage lautet: Wenn zur Bildung von Regeln und damit zur Generierung der Daten private Informationen notwendig sind, werden die involvierten Wirtschaftssubjekte bereit sein, sie offenzulegen? Bei beiden Fragen ist die Antwort ungewiß. Da jedoch ein PAIS den potentiellen Wettbewerb zwischen den unterschiedlichen Abbildungssystemen fördert und seinerseits wettbewerblichen Grundsätzen gehorcht, ist wohl davon auszugehen, daß es einer Findung von Antworten eher zu- als abträglich ist.

\subsection{Markt für externe Unternehmensanalyse}

Wie im Vorabschnitt deutlich geworden, muß der Entwickler von PAIS Rechnungslegungsregeln das berichtende Unternehmen oder zumindest die korrespondie- 
rende Branche gut kennen. Dies gilt wohl für alle prognoserelevanten Informationen. Somit ist der mögliche Anbieterkreis stark eingeschränkt. Neben dem Management der berichtenden Unternehmen kommen wohl nur noch die beratenden und analysierenden Unternehmensdienstleister in Frage. Während die Unternehmensberatungen eng mit der Führung zusammenarbeiten und im Regelfall eine eher geringe Informationsasymmetrie zwischen ihnen und dem Management herrscht, sind Unternehmensanalysten ${ }^{75}$ im Sinne dieser Arbeit extem angesiedelt. Im Rahmen von Analystenkonferenzen erfahren sie zwar unter Umständen bislang als privat eingestufte Informationen, aber nur, um sie im Anschluß als öffentliche Information verfügbar zu machen. Insofern ist ihr Informationsvorteil gegenüber der Öffentlichkeit nur temporär. Als Ersteller von Regeln zur Generierung von PAIS-Daten mit Prognosecharakter kommen also neben dem Management letztlich wohl nur Unternehmensberater in Frage, wenn das Management seine Informationsasymmetrie zu den Untemehmensanalysten aufrecht erhalten will.

Somit stehen die Analysten tendenziell auf der Adressatenseite. Sie erhalten die PAISDaten zusammen mit den anderen Adressaten. Um ihre traditionelle Aufgabe als Informationsintermediär wahmehmen zu können ${ }^{76}$, ist es von großer Bedeutung, ob diese Leistung noch nachgefragt wird. Grundsätzlich ist das PAIS in der Lage, Informationen so anzubieten, daß aus ihnen EDV-gestützt Entscheidungen abgeleitet werden können, oder zumindest Entscheidungsvorlagen erstellt werden können. Dies deckt sich im wesentlichen mit der klassischen Analystenaufgabe. Insofern ist die Wirkung eines PAIS auf die Analyse zweigeteilt. Einerseits wird durch die Erweiterung der Unternehmenspublizität die Datenbasis der Analyse verbreitert, was ggf. auch zu einer Verbesserung der Analysequalität führen kann. Andererseits wird durch das PAIS ein gewisser Teil der Analystentätigkeit vorweggenommen, so daß die Wertschöpfung der Analyse und damit auch der Nettonutzen der Analysenachfrager aus der Analyse reduziert wird. Das kann im Extremfall dazu führen, daß die Adressaten den Nutzenzuwachs durch eine Analyse eines Unternehmens, welches seinerseits ein PAIS-System implementiert hat, so gering einschätzen, daß sie die Analyseleistung nicht mehr nachfragen. Dies gilt vor allem für den Fall, daß

75 Der Begriff Unternehmensanalyst ist hier als Überbegriff für alle Formen der externen Analyse zu verstehen. Im folgenden wird für diese Berufsgruppe auch verkürzend der Begriff Analyst verwendet. Vgl. für eine ähnliche Eingrenzung Löffler (1998), S. 17.

76 Vgl. zur grundsätzlichen Vorgehensweise von Unternehmensanalysten bei der Analyse von Rechnungslegungsdaten Kang/O'Brian/Sivaramakrishnan (1994); Block (1999); Hunton/McEwan (1997); McEwan/Hunton (1999); Barker (1998; 1998a); zu Determinanten ihres Erfolges Clement (1999) und Mikhail/Walther/Willis (1999). Zur Rolle der Finanzanalyse auf dem deutschen Kapitalmarkt Hüfner/Möller (1997); Löffler (1998; 1999); ferner zur Wirkung von US-amerikanischen Analysten auf die Preisbildung Stickel (1992); Branson/Guffey/Pagach (1998); Alexander/Ang (1998); zur Auswirkung auf die Handelsvolumen Alforrd/Berger (1999); generell zur modelltheoretischen Analyse ihrer Tätigkeit beispielhaft Benston/Smith (1976) und Chemamanur/Fulghieri (1994). Einen guten Literaturüberblick über die Untersuchungen zu Vorhersagen von Finanzanalysten bieten Keane/Runkle (1998). 
es den PAIS-Erstellern gelingt, Regeln zu entwickeln, die für die entsprechende Adressatengruppe eine hohe Qualität erreichen.

Ungeachtet der Qualität der PAIS-Daten wird eine Nachfrage nach Weiterverarbeitung der Daten bestehen. Im Idealfall kann diese Weiterverarbeitung auf Basis von standardisierten EDV-Produkten automatisch geschehen. In diesem Fall beschränkt sich die Aufgabe der klassischen Unternehmensanalyse auf die Entwicklung und Vermarktung von solchen Softwarelösungen für unterschiedliche Adressatengruppen. Da der gesamte Softwaremarkt sich durch starke Veränderungen in relativ kurzen Zeiträumen auszeichnet, ist aus evolutorischer Sicht nicht davon auszugehen, daß auf diesem Markt für PAISDatenverarbeitung kein hinreichender Wettbewerb zu erwarten wäre.

Des weiteren besteht ein Bedarf an unternehmensübergreifendem Vergleich von PAISDaten. Während es grundsätzlich vorstellbar ist, daß die publizierenden Unternehmen durch Kollusion ein EDV-gestütztes Vergleichssystem generieren, ist es wegen der damit verbundenen Wettbewerbswirkungen wahrscheinlicher, daß sie diese Aufgabe Dritten überlassen. In der Entwicklung solcher vergleichenden Analysetools könnte durch das PAIS ein neues Tätigkeitsfeld für die Unternehmensanalyse entstehen.

Folgende Überlegungen zum Markt für Unternehmensanalyse lassen sich festhalten: Einerseits verbreitert ein PAIS die der Analyse zur Verfügung stehende Datenbasis und erleichtert aufgrund der EDV-technischen Standardisierung auch die Analysetätigkeiten. Andererseits nimmt mit steigender Qualität der PAIS-Daten und der komplementären Datenverarbeitung das Wertschöpfungspotential der Analyse ab, im Extremfall, also bei 100\%iger Zweckeignung der PAIS-Daten aus Adressatensicht, wird eine Analyse sogar unnötig. Die Analysetätigkeit wird also noch mehr als bisher in der Entwicklung von standardisierten, auf den jeweiligen Adressaten zugeschnittenen Softwareprodukten und in der Entwicklung von unternehmensvergleichenden Analysetools bestehen. Durch den Vergleich mit anderen Softwaremärkten läßt sich dort ein recht intensiver Wettbewerb erwarten.

Bei dieser Betrachtung soll nicht verschwiegen werden, daß eine 100\%ige Zweckeignung der PAIS-Daten illusorisch ist. Deswegen wird wohl immer Bedarf an Analyseleistungen verbleiben. Aus diesem Grund wird auch ein PAIS die Nachfrage nach nicht rechnungslegungsorientierter Publizität, also an verbalen Informationen des berichtenden Unternehmens und nach Unternehmensinformationen aus dritter Hand, nicht zum Erlöschen bringen. 


\section{Auswirkungen auf die gesetzlichen Rahmenbedingungen}

In den Vorabschnitten wurden die Auswirkungen eines PAIS auf die unterschiedlichen von Rechnungslegungspublizität tangierten Märkte analysiert. Nicht eingegangen wurde bislang auf die Frage, wie sich das PAIS auf die bestehende, in Deutschland weitgehend gesetzlich regulierte Rechnungslegungspublizität auswirken könnte. Dies soll in diesem Abschnitt kurz geschehen."

Vorweg zur Abgrenzung. Die steuerbilanzielle Rechnungslegung wird hier nicht beleuchtet, da die Auswirkungen eines PAIS auf diese zumindest direkt gering sein dürften. ${ }^{78} \mathrm{Da}$ die Steuerbilanz einen eindeutigen Adressaten mit einer gesetzlich kodifizierten Zweckdefinition besitzt, besteht keine Notwendigkeit für eine multidimensionale Rechnungslegung. Im Gegenteil, die Ermittlung von eindeutigen Werten ist konstituierendes Merkmal der Steuerbilanz. ${ }^{79}$ Die in der Literatur in ähnlichem Zusammenhang diskutierte Möglichkeit, daß alternative vor allem prognostiv ausgerichtete Wertansätze Begehrlichkeiten bei der Finanzbürokratie wecken könnten, ${ }^{80}$ soll hier nicht kategorisch bestritten werden. Dies spricht jedoch nicht gegen die Verwendung eines PAIS, sondern zunächst für eine kritische Prüfung der Finanzbürokratie und des politischen Prozesses der Steuergesetzgebung.

Die traditionelle deutsche Rechnungslegungspublizität wiederum ist eingebettet in ein Geflecht von gesellschafts- und kapitalmerktrechtlichen Vorschriften. Vor allem die Gesetzesnormen zur Einkommensverwendung ( $\$ 58$ (2) Akt $G$ und $\ 29 \mathrm{GmbHG}$ ) induzieren direkte realwirtschaftliche Wirkungen des Ergebnisses des handelsrechtlichen Einzelabschlusses. Der daraus resultierende Zielkonflikt wird noch verschärft durch die in \ 5 EStG manifestierte Maßgeblichkeit des handelsbilanziellen Einzelabschlusses für die steuerbilanzielle Gewinnermittlung. Für diese Zwecke ist eine Rechnungslegung, die auf einem PAIS aufbaut, nur bedingt geeignet. Einerseits ist die Möglichkeit multipler Wertansätze zwar aus Informationssicht positiv, zur Ermittlung eines verteilungsfähigen Gewinns jedoch eher negativ zu beurteilen. Andererseits ist auch die bewrßte Inkaufnahme von intersubjektiv nur begrenzt nachvollziehbaren Wertansätzen für eine Festset-

$\pi$ Unabhängig von der Entwicklung neuer Publizitätssysteme werden in der juristischen Literatur die Auswirkungen der neuen Kommunikationstechnologie auf das Kapitalmarkt- und Gesellschaftsrecht bereits intensiv diskutiert. Vgl als umfassenden Einstieg Noack (1998).

78 Dies setzt voraus, daß keinerlei Maßgeblichkeit zwischen PAIS und Steuerbilanz besteht. Vgl. zur Diskussion der Maßgeblichkeit im sich wandelnden Umfeld der Rechnungslegung z.B. Ballwieser (1990); WeberGrellet (1999), sowie jüngst und umfassend Pannen (1999); zur Situation in den USA als Überblick Kahle (1999) und vor dem Hintergrud der internationalen Rechnungslegung Herzig (1999).

79 Vgl. Schneider (1997), S. 199 f.; Weber-Grellet (1997), S. 389; zusammenfassend Robisch/Treisch (1997) und in anderem Zusammenhang Wagner (1998) sowie Fülbier/Gassen (1999).

* Vgl. beispielhaft Schneider (1978), S. 1578; Crezelius (1994), S. 690 f.; Robisch/Treisch (1997), S. 168. 
zung der Dividenden als problematisch anzusehen. Die gesamte Konzeption eines PAIS basiert auf dem Ziel, ein möglichst flexibles Informationssystem zu schaffen. Dieses Ziel deckt sich nicht mit der Ausrichtung des deutschen Gesellschaftsrechts, Verteilungsprobleme durch Regulierung zu lösen.

Insofern ist nicht zu erwarten, daß ein PAIS einen Gegenentwurf zur traditionellen deutschen Rechnungslegung darstellen kann. Vielmehr handelt es sich um eine freiwillige Erweiterung der rechnungslegungsorientierten Unternehmenspublizität, die eher als die handelsrechtliche Rechnungslegungspublizität in der Lage ist, die Informationsfunktion der Rechnungslegung zu erfüllen.

Somit ist zunächst nicht zu erwarten, daß die Einführung von PAIS Bewegung in den Bereich der deutschen Rechnungslegung bringen könnte. Anders sieht dies jedoch im Rahmen der internationalen Rechnungslegung aus. Diese ist, im Gegensatz zu ihrem deutschen Pendant, ausschließlich der Informationsfunktion verpflichtet. Insofern erscheint es zumindest denkbar, daß Teile der PAIS-Konzeption Eingang in die Überlegungen zur Regelung der Rechnungslegungspublizität finden. So ist speziell in Fragen des elektronischen Ausweises von Rechnungslegungsdaten bereits Bewegung bei den einschlägigen Standardsettem zu registrieren. ${ }^{81}$ In diesem Zusammenhang ist es möglich, daß PAIS, die unter anderem standardkonforme Wertansätze anbieten, von diesen Standardsettern als zulässiges Publizitätssystem anerkannt werden. Des weiteren erscheint vorstellbar, daß Börsenaufsichtsbehörden wie die SEC, die zur Übermittlung von Publizitätsinformation bereits die Hilfe der elektronischen Datenübertragung in Anspruch nehmen, Teile der PAIS-Konzeption für diese Zwecke verwenden. So sind bislang die mangelnden Möglichkeiten der elektronischen Weiterverarbeitung der EDGAR-Daten ein großer Nachteil des US-Systems ${ }^{82}$, der durch Verwendung von Datenbankkonzepten wie dem PAIS behoben werden könnte. Ob die im PAIS angewendeten Rechnungslegungsregeln Eingang in das jeweilige Standardsetting finden, ist unklar. Einerseits kann sich eine Organisation wie das FASB einem Wertansatz, dessen Informationsrelevanz empirisch belegt wurde, nur schwer vollkommen verschließen, anderseits widersprechen die PAISRegeln unter Umständen dem Conceptual Framework des FASB, z.B. dem Anspruch auf Verläßlichkeit. Des weiteren ist auch das FASB eine Organisation von Individuen, die ein gewisses Beharrungsvermögen aufweist und sich deswegen nicht ohne weiteres von einem Marktprozeß substituieren lassen wird.

81 Vgl. zum Electronic Financial Reporting Project des LASC das jüngst erschienene Discusson Paper Lymer et al. (1999); zusammenfassend o.V. (2000) und die Überlegung des FASB zur Veröffentlichung von Financial Statements über das WWW, vgl. zu dem Projekt FauxCOM URL: http://www.rutgers.edu/Accounting/ raw/fasb/fauxcom/Default.htm, Stand 18.11.1999 und Upton (1998).

82

Vgl. Lymer et al. (1999), S. 57 f. 
Wenn der Trend zur elektronischen Publizität international anhält und auch von den Börsen und deren Aufsichtsbehörden gefördert wird, ist denkbar, daß auch deutsche Unternehmen vermehrt elektronisch publizieren werden. ${ }^{83}$ In diesem Fall besteht für diese ein starker Anreiz, der PAIS-Konzeption ähnelnde Publizitätsdatenbanksysteme zu implementieren. Dadurch unterwerfen sich die Unternehmen freiwillig einer Doppelbelastung: Einerseits publizieren sie einen traditionellen Jahresabschluß und Lagebericht, andererseits publizieren sie zusätzlich weitere Rechnungslegungsdaten auf elektronischem Wege über das PAIS. Wenn die PAIS-Daten von den Adressaten als primäre Informationsquelle angenommen werden, besteht für den Informationszweck der traditionellen Publizität ein gewisser Legitimierungszwang. Da die doppelte Publizität einen Mehraufwand für die Unternehmen bedeutet, ist mit einem lobbyistischen Druck auf den Gesetzgeber zu rechnen, die handelsrechtliche Publizität einzuschränken oder abzuschaffen. Dies zöge u.a. eine Modifizierung des $₫ 58$ (2) AktG und $₫ 29 \mathrm{GmbHG}$ mit sich sowie eine weitere Auflösung der Maßgeblichkeit. Ob der Gesetzgeber einer solchen Beschneidung seiner Kompetenz vor allem im Verbund der EU kampflos zustimmen wird, darf zumindest bezweifelt werden. Eher vorstellbar erscheint in diesem Zusammenhang eine befreiende Wirkung eines Internet-basierten Jahresabschlusses und Lageberichts für die Druckform. Unter Umständen wird dann ein PAIS, welches die gesetzlich konformen und geprüften Rechnungslegungsdaten entsprechend betont, als befreiend für die korrespondierenden Rechenwerke des Jahresabschlusses angesehen.

Es ist also nicht anzunehmen, daß der deutsche Gesetzgeber und auch die internationalen Standardsetter sofort auf das Aufkommen von PAIS reagieren werden. Wenn das PAISKonzept sich allerdings am Markt bewähren sollte und somit sein Informationscharakter für die Adressaten belegbar ist, dann kann zumindest von den weltweit führenden Standardsettem LASC und FASB/SEC erwartet werden, daß sie eine Übernahme zumindest von Teilen des PAIS-Konzeptes in das jeweilige Rechnungslegungssystem öffentlich zur Diskussion stellen. $\mathrm{Da}$ der deutsche Gesetzgeber bereits bei der Anwendung von Rechnungslegungsnormen letztlich dem Druck der internationalen Bewegung gefolgt ist, kann dann auch eine Änderung des deutschen Rechtsrahmens nicht mehr ausgeschlossen werden. Inwiefern das DRSC im Rahmen seiner Tätigkeit sich auch solchen, primär als Ausweisfragen zu interpretierenden Themen zuwendet und somit eventuell als Promoter eines PAIS in Frage käme, bleibt abzuwarten.

83 Vgl. zu Untersuchungen zum elektronischen Publizitätsverhalten im intemationalen Vergleich jüngst Deller/Stubenrath/Weber (1999) sowie den Überblick in Lymer et al. (1999), S. 4459. 


\section{Kapitelfazit}

In diesem Kapitel wurde untersucht, wie sich das PAIS-Konzept in die bestehenden Strukturen der Rechnungslegungspublizität einfinden könnte. Selbstverständlich sind solche prognostischen Überlegungen sehr unsicher. Nichtsdestotrotz gehört es im Rahmen einer Neuentwicklung zu den maßgeblichen Aufgaben, die Auswirkungen der Neuerung auf bestehende Strukturen zu analysieren. Als Ergebnis bleibt festzuhalten: Der Erfolg des PAIS-Konzeptes hängt maßgeblich von den aus den Implementierungen resultierenden Daten ab. Der zentrale Vorteil gegenüber der traditionellen Unternehmenspublizität besteht neben der Multidimensionalität und der Zeitnähe darin, daß die Qualität der PAIS-Daten zumindest theoretisch aufgrund ihrer jeweiligen monokausalen Zielsetzung gemessen werden kann. Somit wird ein Wettbewerb zwischen unterschiedlichen Wertansätzen und Publizitätskonzeptionen zu vertretbaren Transaktionskosten möglich. Je größer die beobachtete Qualität der PAIS-Daten ist, desto größer ist die Wahrscheinlichkeit, $\mathrm{da} ß$ sich PAIS-Implementierungen am Markt etablieren und dann auch wiederum andere Märkte und die Gesetzgebung beeinflussen werden. Insofem kann dieses Kapitel nur einige Wenn-Dann-Aussagen bieten. $\mathrm{Da}$ das PAIS-Konzept letztlich nur die evolutorische Potenz der Märkte in der Entwicklung neuer Bewertungs- und Abbildungsregeln freisetzen soll, konnten klare Prognosen auch nicht erwartet werden. 
Joachim Gassen - 978-3-631-75496-2

Downloaded from PubFactory at 01/11/2019 04:12:56AM

via free access 


\section{Kapitel VII}

\section{Zusammenfassung}

Ziel dieser Untersuchung war die Entwicklung eines Rechnungslegungspublizitätssystems, das die Evolutionsfähigkeit der Rechnungslegungspublizität steigem kann. Im folgenden sollen die Ergebnisse der Arbeit thesenmäßig zusammengefaßt werden.

Ein Rechnungslegungspublizitätssystem ist ein quantitatives Abbildungsverfahren der unternehmerischen Realität, dessen Produkt, die Rechnungslegungspublizität, das Metaziel besitzt, die Informationsasymmetrie zwischen den unterschiedlichen Vertragspartnern im Vertragsgeflecht Unternehmen zu reduzieren. Das Ergebnis der Rechnungslegungspublizität sind die öffentlich verfügbaren Rechnungslegungsdaten.

Diese Rechnungslegungsdaten werden von Wirtschaftssubjekten als Grundlage für Entscheidungsprozesse genutzt. Sobald mehr als ein Wirtschaftssubjekt die Daten verwendet, können keine eindeutigen Erstellungsregeln mehr ermittelt werden. Somit läßt sich eine normative Theorie der Rechnungslegungspublizität nicht generieren.

In Ermanglung dieser normativen Theorie wird im Rahmen der Arbeit auf die Grundlagen der evolutionären Ökonomik zurückgegriffen. Der daraus zu gewinnende Leitsatz fordert die konsequente Einbeziehung von marktwirtschaftlichen Prozessen in die Erstellung von Rechnungslegungspublizitätssystemen einerseits und in die Produktion der Rechnungslegungsdaten andererseits. Diese abstrakte Forderung wird durch den Rückgriff auf die Vertragstheorie weiter operationalisiert. Innerhalb des Prozesses der Rechnungslegungsdatenerstellung werden zahlreiche Schnittstellen identifiziert, an denen theoretisch marktnahe Koordinationsformen greifen könnten. Ein operationalisierter Leitsatz zur Rechnungslegungspublizität fordert dementsprechend die Etablierung von marktnahen Koordinationsformen an diesen Schnittstellen, wobei jedoch die jeweiligen Organisationskosten zu beachten sind.

Sowohl das deutsche als auch das US-amerikanische System der Rechnungslegungspublizität sind historisch gewachsene Konstrukte. Speziell für das deutsche Rechnungslegungspublizitätssystem ist die enge Einbettung in das Gesellschaftsrecht ein konstituierendes Merkmal, während das US-amerikanische System neben dem Wettbewerb der Einzelstaaten im wesentlichen vom Kapitalmarktrecht auf Bundesebene geprägt ist. 
Aus Sicht der ökonomischen Analyse des Rechts lassen sich sowohl das US-amerikanische als auch das deutsche Rechnungslegungspublizitätssystem vor allem durch Rückgriff auf die Public Choice Theory erklären. Zentrale Motivation zum regulatorischen Eingriff war vor allem die Reaktion auf vermeintliche Krisen im wirtschaftlichen Bereich. Die Identifikation dieser Krisen hängt auch von dem jeweilig opportun erscheinenden wirtschaftspolitischen Leitbild ab. Speziell für den deutschen (und europäischen) Gesetzgeber ist weiterhin $\mathrm{zu}$ beobachten, daß im Sinne einer „Follow the Leader" Strategie als positiv angesehene Regulierungen von führenden Volkswirtschaften (also hauptsächlich von den USA) in nationale Regulierungssysteme übernommen werden, ohne daß eine genaue und reflektierte Einbettung in den bestehenden Rechtsrahmen erfolgt.

Ungeachtet der jeweiligen Intention beeinflussen die Regulierungen unterschiedliche marktwirtschaftliche Prozesse. Sowohl die deutsche als auch die US-amerikanische Rechnungslegungspublizität behindern die Evolutionsfähigkeit der Rechnungslegungspublizität, wenn auch in teilweise unterschiedlichen Bereichen. Die weitgreifende Pflichtpublizität sowie die Verteilungsfolgen der Rechnungslegungsdaten schränken den Entscheidungsspielraum deutscher Untemehmen beträchtlich ein. So wird die Entscheidung des Ansatzes und der Bewertung bei deutschen Unternehmen vertikal in das berichtende Unternehmen integriert, während bei US-amerikanischen Unternehmen, die nicht der Berichtspflicht nach US-GAAP unterstehen, Nachfrage nach bestimmten Bewertungsverfahren von Vertragspartnern der Unternehmen festgestellt werden kann. Andererseits unterliegen börsennotierte Unternehmen in den USA einem sehr rigiden Rechnungslegungspublizitätssystem, das ihnen nur einen sehr kleinen Einflußvektor überläßt. Auch das von der SEC entwickelte EDGAR-System behindert durch sein implizites Standardisierungsangebot die evolutionäre Weiterentwicklung der elektronischen Rechnungslegungspublizität. Somit entsprechen beide Systeme nicht dem Leitsatz einer evolutionsfördernden Rechnungslegungspublizität.

Die zentralen Probleme der analysierten Rechnungslegungspublizitätssysteme bestehen in dem mangelnden Einflußvektor der Adressaten. Aufgrund der monodimensionalen Bewertungskonzeption und der über Jahrhunderte tradierten Form des schriftlichen Ausweises sind alternative Konzeptionen der Rechnungslegungspublizität für den Adressaten wegen prohibitiv hohen Transaktionskosten nicht zu erhalten. Dies verhindert weitgehend jede Evolution im Bereich der Rechnungslegung. Durch die vertikale Integration der Rechnungslegungspublizität in das berichtende Unternehmen wird der Wettbewerb der unterschiedlichen Konzepte der quantitativen Unternehmensberichterstattung behindert. Die im Vergleich zu anderen Märkten, wie dem Markt für allgemeine Informationsübertragung, im Zeitablauf recht konstante Ausprägung der Rechnungslegungspublizitätssysteme spricht weiterhin für einen aus evolutionärer Sicht mangelhaften Wettbewerb. 
Traditionell ließ sich die vertikale Integration der Rechnungslegungspublizität noch über die mangelnde technische Separierbarkeit der Schnittstellen rechtfertigen. Mit der Entwicklung der Netzwerk- und Datenbanktechnik stehen allerdings Verfahren bereit, multidimensionale, disagreggierte Daten über geographische Grenzen hinweg zur Weiterverarbeitung zur Verfügung zu stellen. Andererseits gibt es in der Rechnungswesentheorie immer wieder die Forderung, auf die starke Aggregation der Daten zu verzichten.

Unter Rückgriff auf das multidimensional geprägte Starmodel wurde ein abstraktes Datenmodell für Rechnungslegungsdaten entwickelt, welches in der Lage ist,

- unterschiedliche Ansatz-, Bewertungs- und Ausweiskonzeptionen,

- unterschiedliche Rechenwerke,

- unterschiedliche Berichtszeitpunkte und

- unterschiedliche Merkmale der unterliegenden Transaktionen zu erfassen.

Auf Basis dieses Modells wurde eine beispielhafte Implementierung eines Public Accounting Information System (PAIS) realisiert, das als Serversystem in der Lage ist, die Daten des PAIS-Datenmodells anfragenden Clients zur Verfügung zu stellen, und so Rechenwerke on-the-fly generiert.

Für die Kommunikation zwischen Server und Client und für den Client selber wurden unterschiedliche Konzepte vorgestellt. Sie unterscheiden sich hinsichtlich der Anforderungen, die sie an die Clientumgebung stellen und ihrer Zweckeignung zu einer friktionsfreien Weiterverarbeitung der vom Server erhaltenden Daten. Da alle vorgestellten Konzepte grundsätzlich nebeneinander existieren können, muß im Rahmen der Arbeit kein Konzept eindeutig präferiert werden.

Diejenigen Clientsysteme, die eine EDV-technische Weiterverarbeitung der PAIS-Daten unterstützen, ermöglichen dem Adressaten neben der klassischen Rechenwerkspräsentation zahlreiche weitere Möglichkeiten der Datenanalyse. Speziell die Verbindung unterschiedlicher Wertansätze zu Bandbreiten oder Wahrscheinlichkeitsverteilungen sowie die graphische Interpretation von disagreggierten Datenbeständen sind hier als attraktive Möglichkeiten anzuführen.

Für die erwünschte Eigenschaft der Evolutionsförderung ist es von großer Bedeutung, welche marktwirtschaftlichen Reaktionen auf die Entwicklung des PAIS zu erwarten sind. Zentrale Frage ist hier, inwieweit Unternehmen überhaupt ein PAIS-System nachfragen werden, um ihre Unternehmenspublizität zu erweitern. Durch den Rückgriff auf spieltheoretische Modellansätze läßt sich zeigen, daß die Entscheidung eines Unternehmens, 
ein PAIS zu implementieren u.a. von der Unsicherheit des Anlegers über die zu erwartende Entwicklung des Unternehmenswertes, von der Qualität des PAIS und von der Höhe der Kosten der Einführung abhängig ist. Die Kosten sind hierbei nur insofern von Belang, als daß sie den Bruttonutzen der Implementierung nicht überkompensieren dürfen.

Wesentlich für den Nutzen des PAIS ist, daß die Qualität der Daten sichergestellt ist. Während einerseits der marktwirtschaftliche Selektionsprozeß Manager, die dolose Handlungen vornehmen, mittelfristig sanktionieren sollte, ist andererseits die Prüfung der PAIS-Methoden durch Dritte vorstellbar, während die Prüfung der PAIS-Daten aufgrund der Zeitnähe und Datenmenge wohl mit zu hohen Kosten verbunden sein dürfte.

Neben diesen Märkten tangiert das PAIS-Konzept auch wesentlich den Markt für Wertpapierhandel und Unternehmensanalyse. Es erscheint vorstellbar, daß Wertpapierbörsen von Unternehmen die Verwendung von PAIS-Implementierungen verlangen, um die Informationsasymmetrie am Markt abzubauen und dadurch den Umsatz und letztlich die Konkurrenzsituation der Börse zu verbessern. Aus Sicht der externen Unternehmensanalyse bedeutet die Anwendung des PAIS-Konzepts zunächst eine Verbreiterung der quantitativen Analysebasis. Aufgrund der gesunkenen Transaktionskosten der Weiterverarbeitung erscheint die Analyse der PAIS-Daten weitgehend durch den Rückgriff auf Softwareprodukte möglich, so daß die mögliche Wertschöpfung durch die Unternehmensanalyse tendenziell sinkt. Deswegen ist zu erwarten, daß sich das Tätigkeitsprofil der Unternehmensanalyse von der eigentlichen Analyse zur Entwicklung von Softwareprodukten zur Datenweiterverarbeitung und Entscheidungsvorbereitung wandelt.

Außer den marktwirtschaftlichen Reaktionen ist es theoretisch möglich, daß auch das Regulierungsumfeld auf das PAIS-Konzept reagiert. Hierbei ist jedoch zu beachten, daß ein Großteil des deutschen Rechtsrahmens zur Koordination von Verfügungsrechten einen explizit eindeutigen Wertansatz erfordert. Eine Verwendung von PAIS-Daten zur Koordination von Verteilungsproblemen erscheint indes unsinnig. Anders sieht es bei der gesetzlich geforderten Unternehmenspublizität für reine Informationszwecke aus. Hier erscheint vorstellbar, daß langfristig das PAIS-Konzept einen Gegenentwurf zur traditionellen monodimensionalen Unternehemenspublizität darstellen kann. Mittelfristig ist das Diffundieren von PAIS-Regeln in die Vorschriften zur monodimensionalen Rechnungslegungspublizität möglich. 


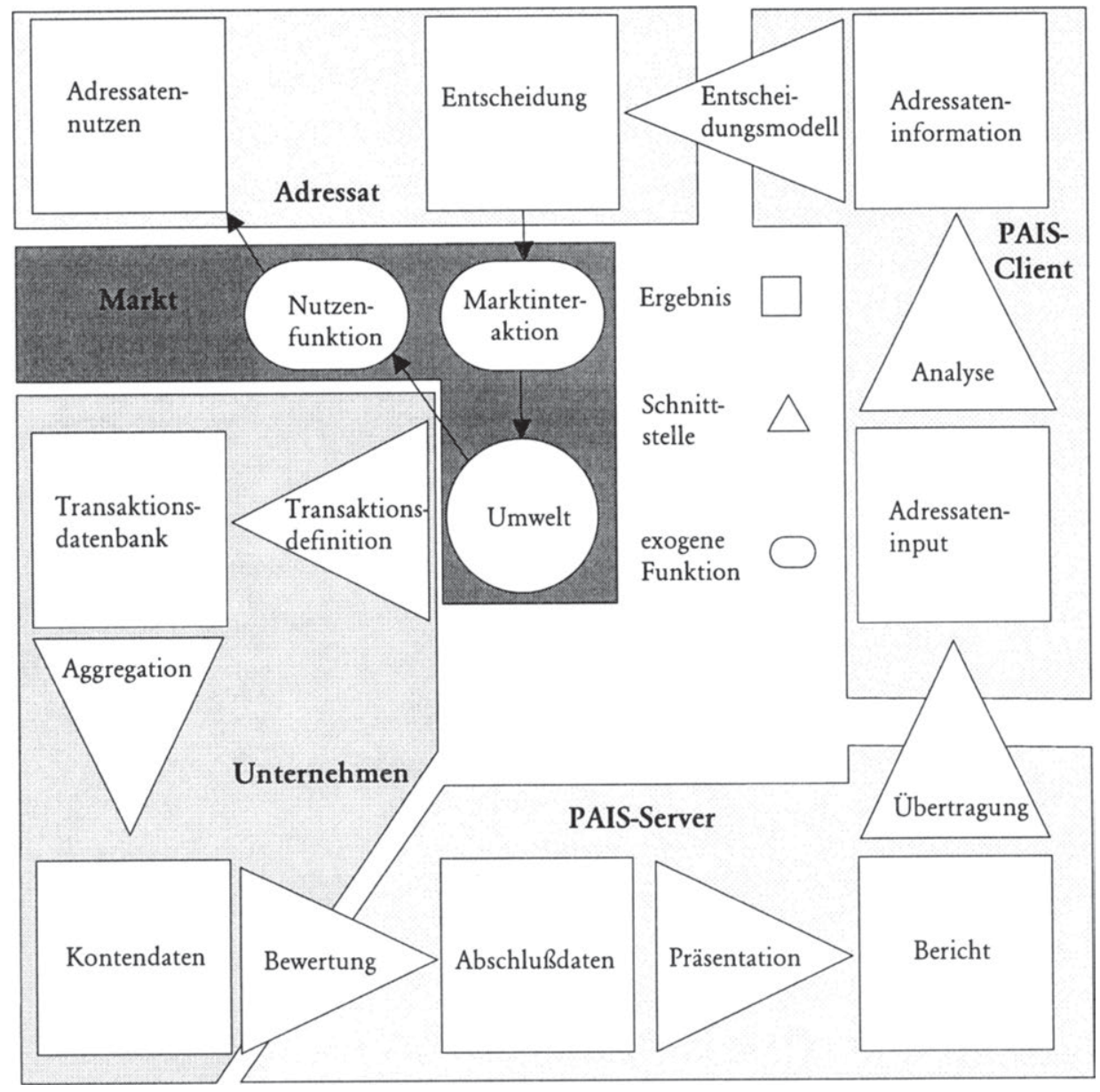

Abb. 20: Rechnungslegungspublizitätsprozeß mit PAIS

Die Arbeit bietet also einen Gegenentwurf zur traditionellen Rechungslegungspublizität. Ob sie auch ihr Ziel erreicht, einen Beitrag zur Stärkung der Evolutionsfähigkeit der Rechnungslegung zu leisten, muß abgewartet werden. Die traditionelle Rechnungslegungspublizität befindet sich aufgrund der größtenteils regulierten Rahmenbedingungen in einer evolutionären Sackgasse, da sie nicht die aktuellen technischen Möglichkeiten zur Datenanalyse gebraucht, und somit die kreativen Kräfte des Marktes nicht im möglichen Umfang für sich arbeiten läßt. Das PAIS verändert die Grenze zwischen Markt und Unternehmen, wie aus dem Vergleich von Abb. 2 und Abb. 20 deutlich wird. Dabei verläuft die Grenze zwischen PAIS-Server und PAIS-Client fließend. Für die Implementierungen des PAIS-Client ist ein direkter innovativer Wettbewerb wahrscheinlich. Die von den PAIS-Servern angebotenen Daten unterliegen zwar grundsätzlich noch dem Entscheidungsbereich der berichtenden Unternehmen, aufgrund der Möglichkeit des multidimen- 
sionalen Datenangebots ist aber auch dort mit einer Belebung des Wettbewerbs durch Druck der Datennachfrager zu rechnen.

Somit werden mehr technisch separierbare Schnittstellen für marktwirtschaftliche Prozesse erreichbar, da sie nunmehr außerhalb des Bereichs des vertikal integrierenden Unternehmens realisiert werden. Der Markt als Entdeckungsverfahren sollte die Evolutionsfähigkeit der Rechnungslegung im Vergleich zu einer vertikalen Integration im berichtenden Unternehmen stärken können. 


\section{Anhang}

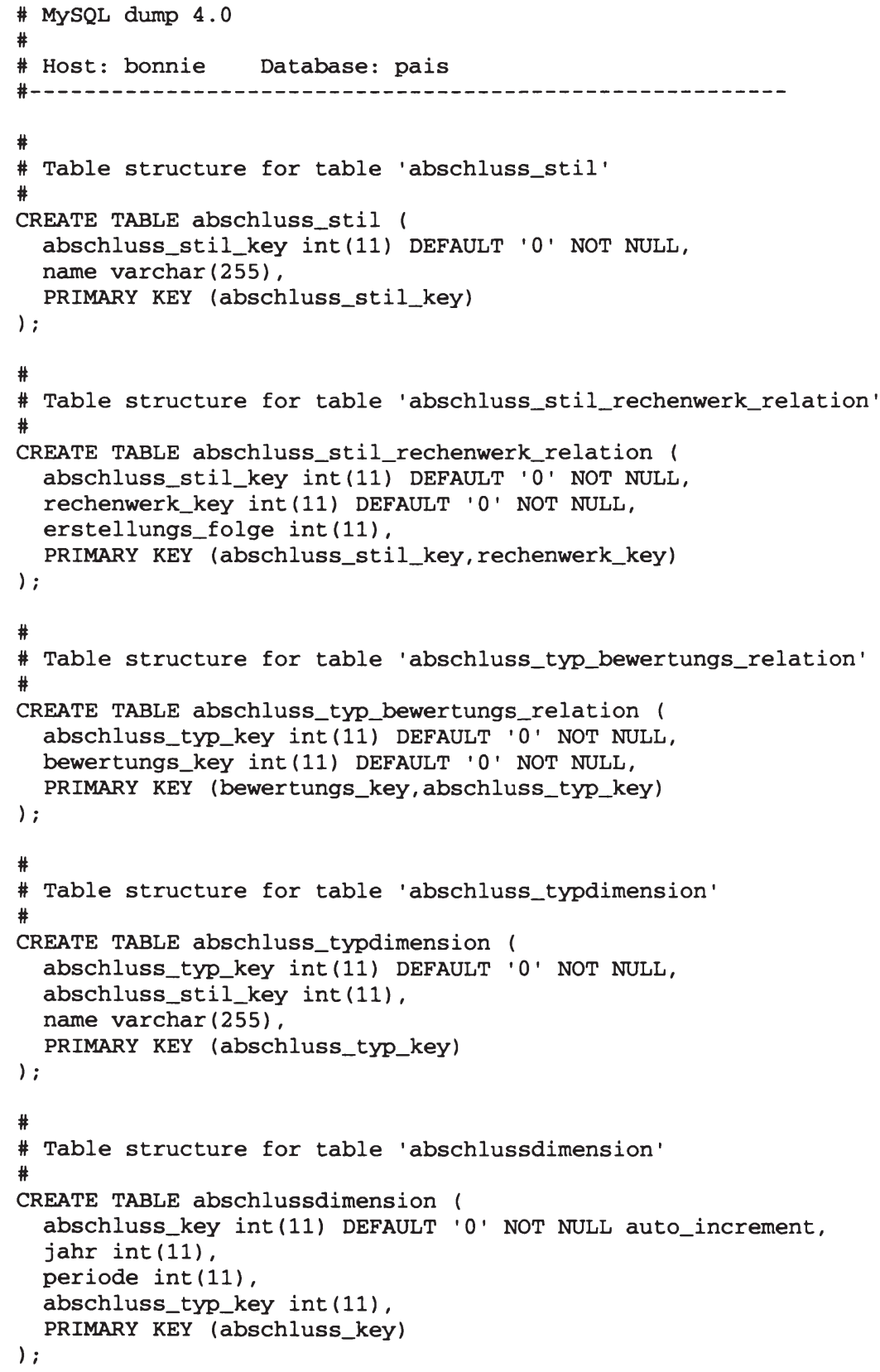




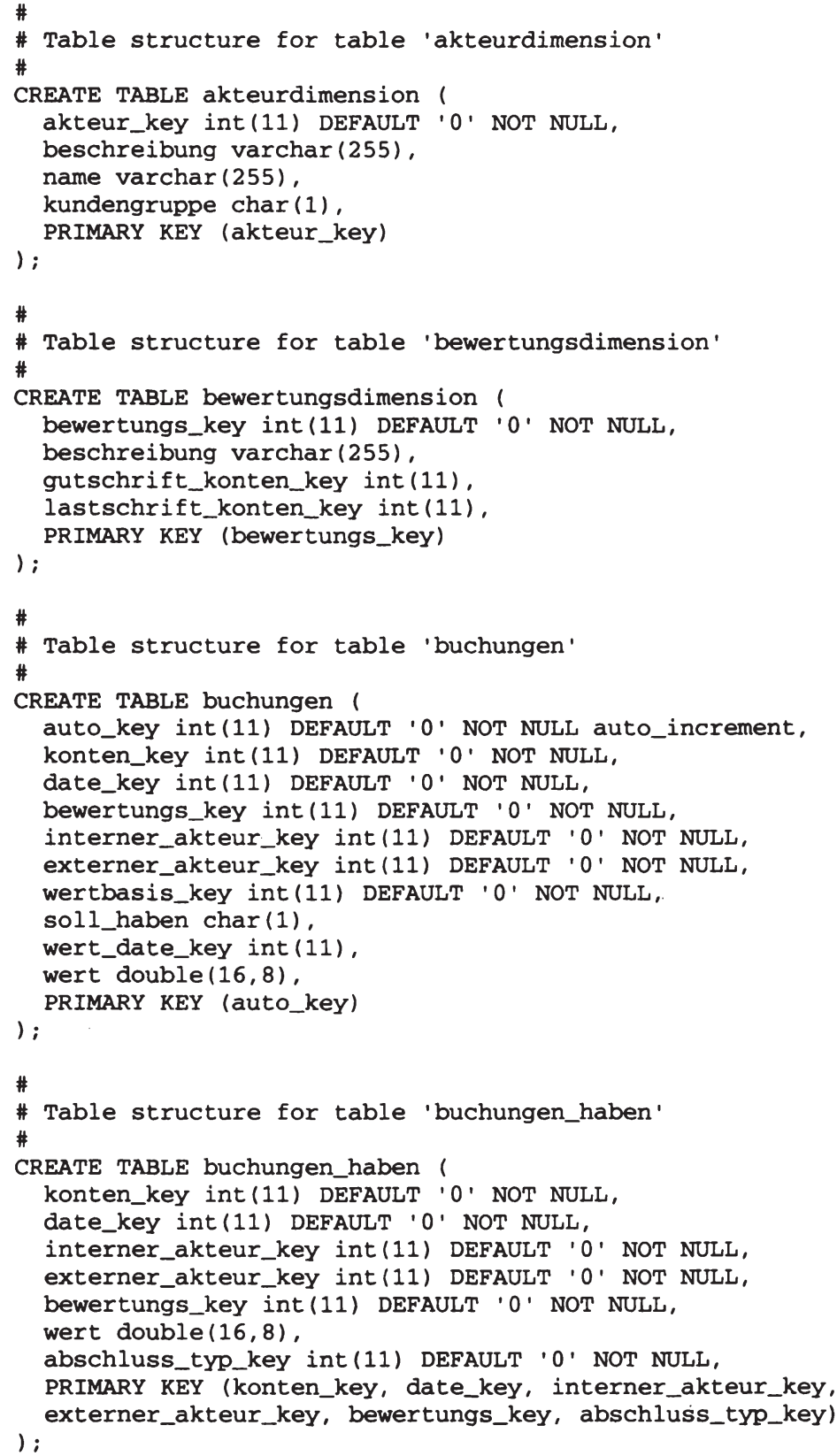




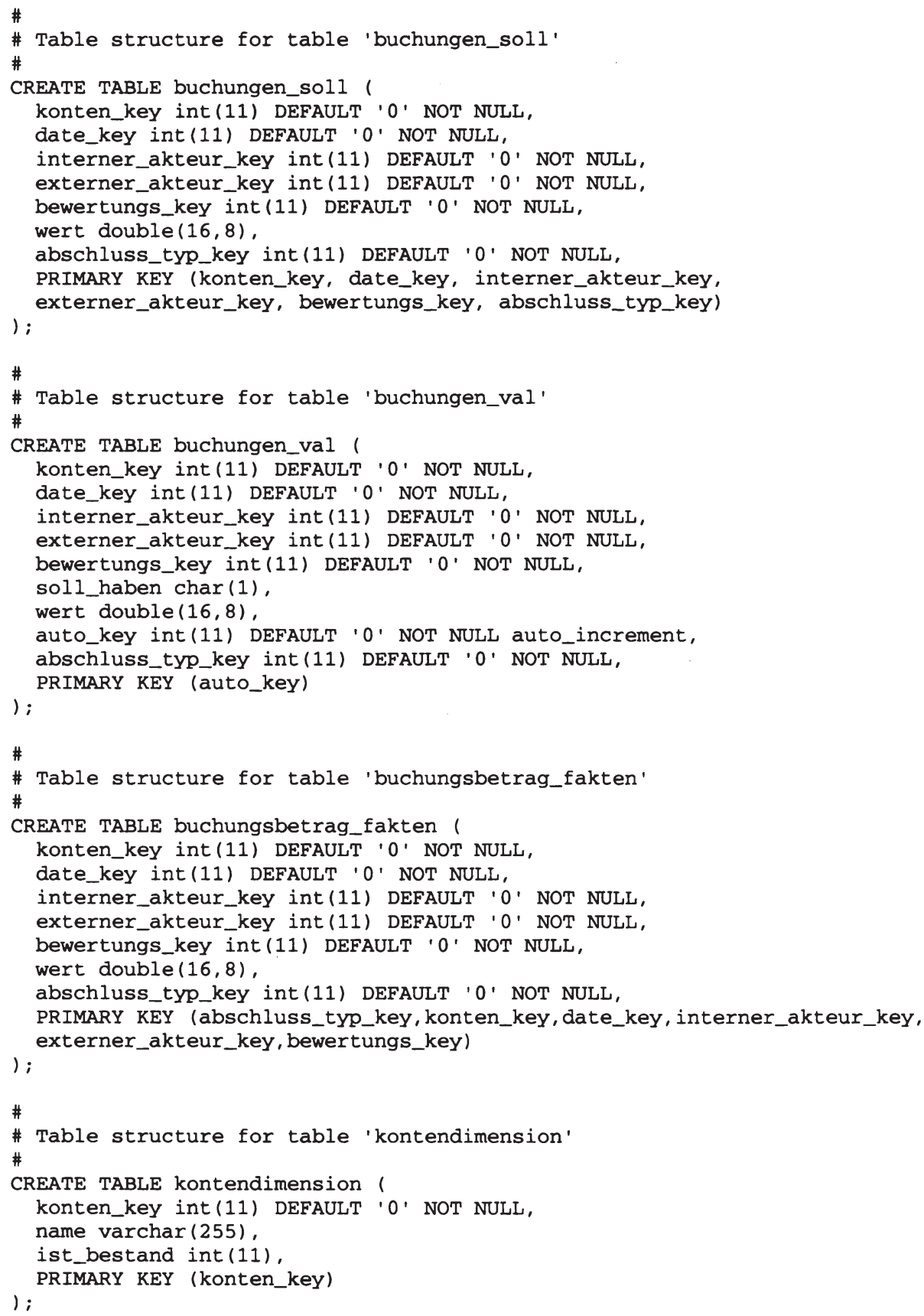




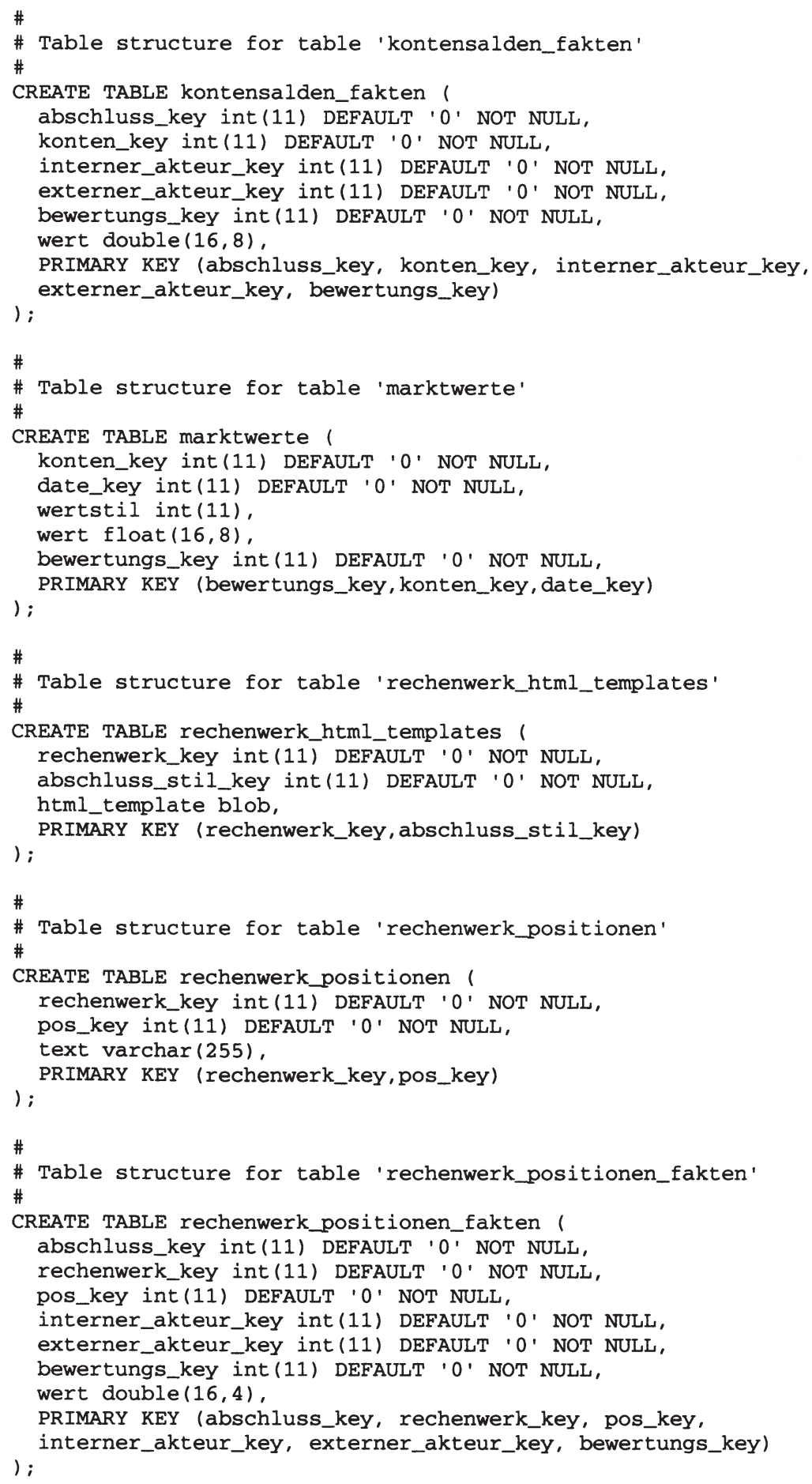




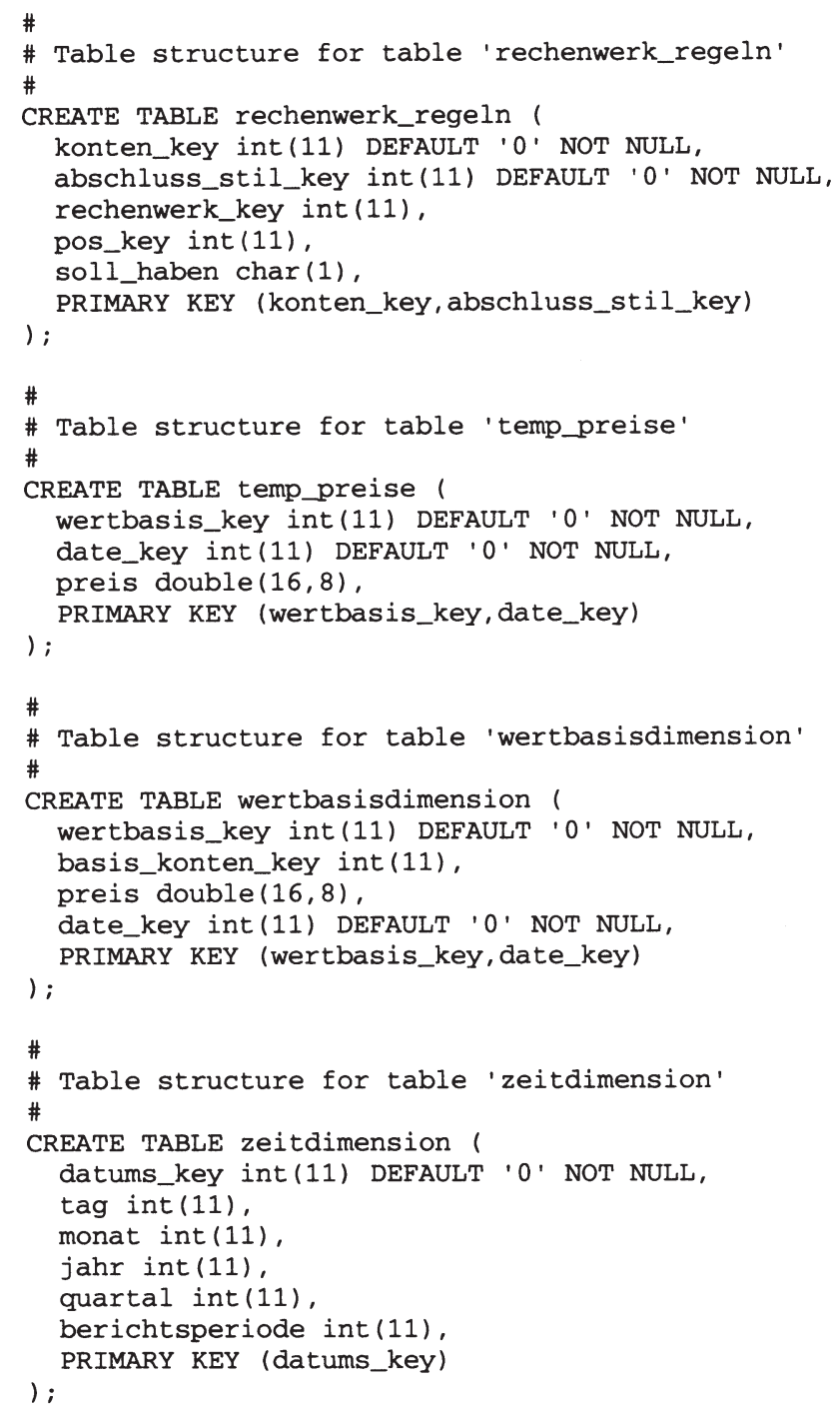


Joachim Gassen - 978-3-631-75496-2

Downloaded from PubFactory at 01/11/2019 04:12:56AM

via free access 


\section{Literaturverzeichnis}

AAA (1966): A Statement of Basic Accounting Theory. Chicago.

AAA (1977): A Statement on Accounting Theory and Theory Acceptance. Sarasota.

AAA's Financial Accounting Standards Committee (1998): Response to FASB Exposure Draft, "Proposed Statement of Financial Accounting Concepts: Using Cash Flow Information in Accounting Measurements". In: Accounting Horizons, Vol. 12, S. 304-311.

Abdel-Khalik, A. Rashad (1973): The Effect of Aggregating Accounting Reports on the Quality of Lending Decision: An Empirical Investigation. In: JAR Supplement, Vol. 12, S. 104-138.

Aboody, David/Lev, Baruch (1998): The Value Relevance of Intangibles: The Case of Software Capitalization. In: JAR Supplement, Vol. 36, S. 161-191.

Adam, Dietrich (1996): Planung und Entscheidung. 4. Auflage, Wiesbaden.

Adamson, Ian L. (1993): An Object-Oriented Financial Accounting Information System. Dissertation University of Waterloo.

Adobe Systems Inc. (1999): Portable Document Format: Reference Manual; Version 1.3, o. O.

Admati, Anat R./Pfleiderer, Paul (1988): Selling and Trading on Information in Financial Markets. In: AER, Papers and Proceedings, Vol. 78, S. 96-103.

Akerlof, George A. (1970): The Market of "Lemons": Quality Uncertainty and the Market Mechanism, in: Quarterly Journal of Economics, Vol. 84, S. 488-500.

Alberth, Markus R. (1998): US-amerikanische Gläubigerbilanzen durch Covenants in Verträgen der Versuch einer weltweiten Kategorisierung der Rechnungslegung und Folgen für die Internationalisierung der Rechnungslegung. In : ZfB, 68. Jg., S. 803-824.

Alchian, Armen A./Demsetz, Harold (1972): Production, Information Costs, and Economic Organization. In: AER, Vol. 62, S. 777-795.

Alchian, Armen A./Woodward, Susan (1987): Reflections on the Theory of the Firm. In: JITE, Vol. 143, S. 110-136.

Alexander, John C. Jr./Ang, James S. (1998): Do Equity Markets Respond to Earnings Paths? In: Financial Analysts Journal, Vol. 34 (4), S. 81-94. 
Alford, Andrew W./Benger, Phillip G. (1999): A Simultaneous Equations Analysis of Forecast Accuracy, Analyst Following, and Trading Volume. In: Journal of Accounting, Auditing and Finance, Vol. 14, S. 219-246.

Alles, Michael/Newman, Paul/Noel, James (1998): The value of information in internal management communication. In: Journal of Economic Behavior and Organization, Vol. 36, S. 295-317.

Alsmöller, Horst (1982): Wettbewerbspolitische Ziele und kooperationstheoretische Hypothesen im Wandel der Zeit: Eine dogmengeschichtliche Untersuchung von Einstellungen zu Verbundsystemen und von Gründen für diese Einstellungen. Tübingen.

Altenburger, Otto A. (1996): Rechnungslegung und Unsicherheit. Berlin.

Altman, Edward J. (1968): Financial Ratios, Discriminant Analysis and the Prediction of Corporate Bankruptcy. In: JoF, Vol. 23, S. 589-609.

Amihud, Yakov/Mendelson, Haim/Uno, Jun (1999): Number of Shareholders and Stock Prices: Evidence from Japan. In: JoF, Vol. 54, S. 1169-1184.

Amir, Eli/Lev, Baruch (1996): Value-relevance of nonfinancial information: The wireless communication industry. In: Journal of Accounting \& Economics, Vol. 22, S. 330.

Anders, Ulrich/Szczesny, Andrea (1998): Prognose von Insolvenzwahrscheinlichkeiten mit Hilfe logistischer neuronaler Netze. In: ZfbF, 50. Jg., S. 892-915.

Aranson, Peter H. (1998): The New Institutional Analysis of Politics. In: JITE, Vol. 154, S. 744753.

d'Arcy, Anne/Leuz, Christian (2000): Rechungslegung am Neuen Markt - Eine Bestandsaufnahme. In: DB, 53. Jg. S. 385-391.

Armitage, Howard M. (1983): Toward an Improved Distribution Accounting Information System Through an Entity Relationship Data Modelling Approach. Dissertation Michigan State University.

Armitage, Howard M./McCarthy, William E. (1987): Decision Support Using EntityRelationship Modeling. In: Financial \& Accounting Systems, Vol. 3, No. 3, S. 12 19.

Arrow, Kenneth J. (1962): Economic Welfare and the Allocation of Ressource for Invention. In: The Rate and Direction of Incentive Activity: Economic and Social Factors: A Conference of the Universities-National Bureau Committee for Eco- 
nomic Research and The Committee on Economic Growth of the Social Science Research Council, Princeton, S. 609-625.

Ashbaugh, Hollis/Johnstone, Karla M./Warfield, Terry D. (1999): Corporate Reporting on the Internet. In: Accounting Horizons, Vol. 13, S. 241-257.

Assmann, Heinz-Dieter (1993): Die Regelung der Primärmärkte für Kapitalanlagen mittels Publizität im Recht der Europäischen Gemeinschaft. In: AG, 38. Jg., S. 549-563.

Atiase, Rowland K./Tse, Senyo (1986): Stock valuation models and accounting information: A Review and Synthesis. In: Journal of Accounting Literature, Vol. 5, S. 133.

Avery, Christopher/Resnick, Paul/Zeckhauser, Richard (1999): The Market for Evaluations. In: AER, Vol. 89, S. 564-584.

Baetge, Jörg (1996): Bilanzen. 4. Auflage, Düsseldorf.

Baetge, Jörg/Fischer, Thomas R. (1987): Zur Aussagefähigkeit der Gewinn- und Verlustrechnung nach deutschem Recht. In: ZfB-Ergänzungsheft 1/1987. S. 175-201.

Baetge, Jörg/Fischer, Thomas R. (1988): Exteme Erfolgsanalyse auf der Grundlage des Umsatzkostenverfahrens. In: BFuP, 40. Jg., S. 1-21.

Baetge, Jörg/Schmedt, Ulrich/Hüls, Dagmar/Krause, Clemens/Uthoff, Carsten (1994): Bonitätsbeurteilung von Jahresabschlüssen nach neuem Recht (HGB 1985) mit Künstlichen Neuronalen Netzen auf der Basis von Clusteranalysen. In: DB, 1994, 47. Jg., S. 337-343.

Baetge, Jörg/Sell, Kirsten (1999): Die US-amerikanischen, internationalen und deutschen Prüfungsgrundsätze im Vergleich. In: StuB, 1. Jg., S. 517-525.

Bailey, Andrew D./Han, Keyong S./Stansifer, Ryan D./Whinston, Andrew B. (1992): A Formal Algorithmic Model Compatible with the Conceptual Modeling of Accounting Information Systems. In: Accounting, Management and Information Technology, Vol. 2, S. 57-76.

Bain, Joe S. (1971): Barriers to new competition: their character and consequences in manufacturing industries. 5. Auflage, Cambridge.

Baker, H. Kent/Haslem, John A. (1973): Information Needs of Individual Investors. In: JoA, Vol. 136, November, S. 64-69.

Baldwin, Amelia A./Williams, S. L. M. (1999): The future of intelligent Internet agents in European financial reporting. In: EAR, Vol. 8, S. 303-319. 
Ball, Ray/Brown, Philip (1968): An Empirical Evaluation of Accounting Income Numbers. In: JAR, Vol. 6, S. 159-178.

Ballou, Donald P./Pazer, Harold L. (1985): Modeling Data and Process Quality in Multi-Input Multi-Output Information Systems. In: Management Science, Vol. 31, S. $150-162$.

Ballou, Donald P./Pazer, Harold L. (1995): Designing Information Systems to Optimize the Accuracy-timeliness Tradeoff. In: Information Systems Research, Vol. 6, S. 51-72.

Ballou, Donald P./Tayi, Giri K. (1999): Enhancing Data Quality in Data Warehouse Environments. In: Communications of the ACM, Vol. 42, No. 1, S. 73-78.

Ballwieser, Wolfgang (1982): Zur Begründbarkeit informationsorientierter Jahresabschlußverbesserungen. In: ZfbF, 34. Jg., S. 772-793.

Ballwieser, Wolfgang (1985): Informationsökonomie, Rechnungslegungstheorie und Bilanzrichtlinie-Gesetz In: ZfbF, 37. Jg., S. 47-66.

Ballwieser, Wolfgang (1990): Ist das Maßgeblichkeitsprinzip überholt? In: BFuP, 42. Jg., S. $477-498$.

Ballwieser, Wolfgang (1993): Die Entwicklung der Theorie der Rechnungslegung in den USA. In: ZfbF, Sonderheft 32/1993, S. 107-138.

Ballwieser, Wolfgang (1995): Aktuelle Aspekte in der Unternehmensbewertung. In: WPg, 48. Jg., S. 119-129.

Ballwieser, Wolfgang (1996): Ein Überblick über Ansätze zur ökonomischen Analyse des Bilanzrechts. In: BFuP, 48. Jg., S. 503-527.

Ballwieser, Wolfgang (1997): Grenzen des Vergleichs von Rechnungslegungssystemen: Dargestellt anhand von HGB, US-GAAP und IAS. In: Aktien- und Bilanzrecht: Festschrift für Bruno Kropff, hrsg. von Forster,Karl-Heinz/Grunewald, Barbara/Lutter, Marcus/Semler, Johannes, Düsseldorf, S. 371-391.

Balzert, Helmut (1996): Lehrbuch der Software-Technik: Software-Entwicklung. Heidelberg et al.

Bamber, Linda S./Cheon, Youngsoon S. (1998): Discretionary Management Earnings Forecast Disclosures: Antecedents and Outcomes Associated with Forecast Venue and Forecast Specificity Choices. In: JAR, Vol. 36, S. 167-190.

Bamberg, Günter/Coenenberg, Adolf G. (1996): Betriebswirtschaftliche Entscheidungslehre. 9. Auflage, München. 
Barker, Richard G. (1998): The market for information: Evidence from finance directors, analysts and fund managers. In: Accounting and Business Research, Vol. 29, S. 3-20.

Barker, Richard G. (1998a): How Analysts use Accounts. In: Accountancy, Vol. 122, November, S. 152.

Barth, Mary E./Beaver, William H./Landsman, Wayne R. (1998): Relative valuation roles of equity book value and net income as a function of financial health. In: Journal of Accounting \& Economics, Vol. 25, S. 1-34.

Barth, Mary E./Clinch, Greg (1998): Revalued Financial, Tangible, and Intangible Assets: Association with Share Prices and Non-Market-Based Value Estimates. In: JAR Supplement, Vol. 36, S. 199-233.

Barth, Kuno (1953): Die Entwicklung des deutschen Bilanzrechts und der auf ihm beruhenden Bilanzauffassungen handelsrechtlich und steuerrechtlich. Band 1, Stuttgart.

Bartov, Eli/Bodnar, Gordon M. (1996): Alternative Accounting Methods, Information Asymmetry and Liquidity: Theory and Evidence. In: AccRev, Vol. 71, S. 397-418.

Barzel, Yoram (1982): Measurement Cost and the Organization of Markets. In: Journal of Law and Economics, Vol. 25, 27-48.

Bator, Francis M. (1958): The Anatomy of Market Failure. In: Quarterly Journal of Economics, Vol. 72, S. 351-379.

Baumol, William J./Panzar, John C./Willig, Robert D. (1988): Contestable markets And the Theory of Industry Structure. Revised Edition, San Diego et al.

Bayes, Thomas (1763): An Essay Towards Solving a Problem in the Doctrine of Chances. In: Philosophical Transactions, Vol. 53, S. 370-418. Reprint New York et al. 1963.

Beaver, William H. (1966): Financial Ratios as Predictors of Failure. In: JAR Supplement, Vol. 4, S. 71-111.

Beaver, William H. (1989): Financial Reporting: An Accounting Revolution. 2. Auflage, Englewood Cliffs.

Beaver, William H./Demski, Joel S. (1974): The Nature of Financial Accounting Objectives: A Summary and Synthesis. In: JAR Supplement, Vol. 12, S. 170-187.

Beaver, William H./Dukes, Roland E. (1972): Interperiod Tax Allocation, Eamings Expectations, and the Behavior of Security Prices. In: AccRev., Vol. 47, S. 320332. 
Beaver, William H./Dukes, Roland E. (1973): Interperiod Tax Allocation and $\delta$ Depreciation Methods: Some Empirical Results. In: AccRev., Vol. 48, S. 549-559.

Beaver, William H./Kennely, John W./Voss, Wiliam M. (1968): Predictive Ability as a Criterion for the Evaluation of Accounting Data. In: AccRev., Vol. 43, S. 675683.

Beermann, Klaus (1976): Prognosemöglichkeiten von Kapitalverlusten mit Hilfe von Jahresabschlüssen. Düsseldorf.

Behme, Henning (1999): Kunst der Stunde: Wozu die Extensible Markup Language gut ist. In: iX, o. Jg., Februar, S. 36-41.

Behme, Henning (1999a): Ein Titel ist ein Titel: Namensräume in der Extensible Markup Language. In: iX, o. Jg., Mai, S. 142-145.

Behme, Wolfgang (1998): Das Datawarehouse-Konzept. In: WISU, 27. Jg., S. 148-150.

Behme, Wolfgang/Mucksch, Harry (1998): Die Notwendigkeit einer entscheidungsorientierten Informationsversorgung. In: Das Data Warehouse-Konzept, hrsg. von Mucksch, Harry/Behme, Wolfgang, 3. Auflage, Wiesbaden, S. 3-31.

Behme, Wolfgang/Kruppa Stephan (1999): Web Warehousing: Nutzen von Synergieeffekten zweier bewährter Systeme. In: Analytische Informationssysteme, hrsg. von Chamoni, Peter/Gluchowski, Peter, 2. Auflage Berlin et al., S. 191-211.

Behn, Bruce K. /Riley, Richard A. Jr. (1999): Using Nonfinancial Information to Predict Financial Performance: The Case of the U.S. Airline Industry. In: Journal of Accounting, Auditing and Finance, Vol. 14, S. 29-56.

Beisse, Heinrich (1999): Normqualität und Normstruktur von Bilanzvorschriften und Standards: Adolf Moxter zum 70. Geburtstag. In: BB, 54. Jg., S. 2180-2186.

Belkaoui, Ahmed R. (1992): Accounting Theory. 3. Auflage, London et al.

Benbasat, Izak/Dexter, Albert S. (1979): Value and Events Approaches to Accounting: An Experimental Evaluation. In: AccRev., Vol. 54, S. 735-749.

Benbasat, Izak/Dexter, Albert S. (1982): Individual Differences in the Use of Decision Support Acts. In: JAR., Vol. 20, S. 1-11.

Bens, Nadja/Kiwitz, Thorsten (1999): Der Neue Markt der Frankfurter Wertpapierbörse - Zulassung und Folgepflichten. In: DStR, 37. Jg., S. 1162-1168

Benston, George J. (1969): The Value of the SEC's Accounting Disclosure Requirements. In: AccRev., Vol. 44, S. 515-532. 
Benston, George J. (1973): Required Disclosure and the Stock Market: An Evaluation of the Securities Act of 1934. In: AER, Vol. 63, S. 132-155.

Benston, George J./Smith, Clifford J. (1976): A Transactions Cost Approach to the theory of Financial Intermediation. In: JoF, Vol. 31, S. 215-231.

Berg, Sanford V. (1988): Duopoly Compatibility Standards with Partial Cooperation and Standards Leadership. In: Information Economics and Policy, Vol. 3, S. 35-53.

Beng, Sanford V. (1989): The Production of Compatibility: Technical Standards as collective Goods. In: Kyklos, Vol. 42, S. 361-383.

Berndsen, Hans-Peter (1978): Unternehmenspublizität in Deutschland: Stand der empirischen Forschung. In: DBW, 38. Jg., S. 121-134.

Berndsen, Hans-Peter (1979): Untemehmenspublizität: Eine empirische Untersuchung zur Messung des Publizitätsverhaltens großer börsennotierter Aktiengesellschaften und der Auswirkung auf die Anlageentscheidungen am Aktienmarkt. Dissertation, Augsburg.

Bhattacharya, Utpal/Daouk, Hazem/Jongenson, Brian/Kehr, Carl-Heinrich (2000): When an event is not an event: the curious case of an emerging market. In: JFE, Vol. 55, S. 69-101.

Biener, Herbert (1989): Die Publizität der Rechnungslegung im Wandel. In: BFuP, 41. Jg., S. 213-230.

Biener, Herbert (1996): Fachnormen statt Rechtsnormen: Ein Beitrag zur Deregulierung der Rechnungslegung. In: Rechnungslegung: Warum und Wie? Festschrift für Hermann Clemm zu 70. Geburtstag, hrsg. von Ballwieser, Wolfgang/Moxter, Adolf/Nonnenmacher, Rolf, München, S. 59-79.

Biener, Herbert/Berneke, Wilhelm (1986): Bilanzrichtlinien-Gesetz. Düsseldorf.

Bihr, Dietrich (1999): Der Entwurf des Kapitalgesellschaften- und Co.-Richtlinie-Gesetes (KapCoRiLiG) vom 13.8.1999. In: BB, 54. Jg., S. 1862-1864.

Billings, Bruce K. (1999): Revisiting the Relation between the Default Risk of Debt and the Earnings Response Coefficient. In: AccRev, Vol. 74, S. 509-522.

Bird, Ronald G./Locke, Stuart M. (1981): Financial Accounting Reports: A Market Model of Disclosure. In: Journal of Business Finance and Accounting, Vol. 8, S. 27-44.

Blochwitz, Stefan/Eigermann, Judith (2000): Unternehmensbeurteilung durch Diskriminanzanalyse mit qualitativen Merkmalen. In: ZfbF, 52. Jg., S. 58-73. 
Block, Stanley B. (1999): A Study of Financial Analysts: Practice and Theory. In: Financial Analysts Journal, Vol. 55, S. 86-95.

Böckem, Hanne/d'Arcy, Anne (1999): Evolution of (International) Accounting Systems. In: ZfbF, 51. Jg., S. 61-76.

Böcking, Hans-Joachim/Nowak, Karsten (2000): Die Bedeutung des Börsnkurses bei Unternehmensbewertungen. In: FB, 2. Jg.,S. 17-24.

Bode, Jürgen (1997): Der Informationsbegriff in der Betriebswirtschaftslehre. In: ZfbF, 49. Jg., S. 449-468.

Boggs, Scott M (1999): Accounting the Digital Way: How Microsoft adds it up. In: JoA, Vol. 187, May, S. 99-108.

Borchers, Detlef/Benning, Maria/Kuri, Jürgen (1999): „Hätt ich Dich heut erwartet...“: Das Internet hat Geburtstag - Oder nicht? In: Magazin für Computertechnik (c't), o. Jg., Heft 21, S. 128-133.

Borgman, Hans P. (1998): Managers' information search behavior using Management Support Systems. In: Wirtschaftsinformatik, 40. Jg., S. 527-535.

Brandi, Ernst (1977): Informationswirkungen der Jahresabschlußveröffentlichung auf Entscheidungen am Aktienmarkt. Dissertation, Augsburg

Branson, Bruce C./Guffey, Daryl, M./Pagach, Donald P. (1998): Information Conveyed in Announcements of Analyst Coverage. In: CAR, Vol. 15, S. 119-143.

Braun, Brigitte (1999): Entwurf eines relationalen Datenmodells mit Hilfe des Normalisierungsverfahrens. In: WISU, 28. Jg., S. 975-982.

Braunstein, Yale M./White, Lawrence J. (1985): Setting technical compatibility standards: an economic analysis. In: The Antitrust Bulletin, Vol. 30, S. 337-355.

Breidenbach, Karin (1997): Normensetzung für Rechnungslegung: Bisherige Ausgestaltung und mögliche Fortentwicklung in Deutschland. Wiesbaden.

Breidenbach, Karin (1999): Aufgaben und Organisation des Deutschen Rechnungslegungs Standards Committee (DRSC). In: StuB, 1. Jg., S. 641-647.

Breuer, Michael (1998): Die Theorie der Clubs. In: WISU, 27. Jg., S. 139-141.

Brooks, LeRoy D./Buckmaster, Dale A./Dascher, Paul E. (1975): Alternative Income Measurement Models and Common Stock Prices. In: Review of Business and Economic Research, Vol. 12, S. 21-34.

Brotte, Jörg (1997): US-amerikanische und deutsche Geschäftsberichte: Notwendigkeit, Regulierung und Praxis jahresabschlußergänzender Informationen. Wiesbaden. 
Buchanan, James M. (1965): An Economic Theory of Clubs. In: Economica, Vol. 32, S. $1-14$.

Buchanan, James M./Tullock, Gordon (1962): The Calcus of Consent: Logical Foundations of Constitutional Democracy. 4. Druck 1971, Ann Arbor.

Budde, Wolfgang D./Steuber, Elgin (1998): Normsetzungsbefugnis eines deutschen Standard Setting Body. In: DStR, 36. Jg., S. 1181-1187.

Bury, Liz (1999): On line and on time. In: Accountancy, Vol. 124, August, S. 38-39.

Busse von Colbe, Walther (1966): Aufbau und Informationsgehalt von Kapitalflußrechnungen. In: ZfB-Ergänzungsheft, 36. Jg. S. 82-114.

Busse von Colbe, Walther (1968): Prognosepublizität von Aktiengesellschaften. In: Beiträge zur Lehre der Unternehmung: Festschrift für Karl Käfer, hrsg. von Angehrn, Otto/Künzi, Hans P., Stuttgart, S. 91-118.

Busse von Colbe, Walther (1987): Die neuen Rechnungslegungsvorschriften aus betriebswirtschaftlicher Sicht. In: WPg, 40. Jg, S. 117-126.

Busse von Colbe, Walther (1996): Accounting and the business economics tradition in Germany. In: EAR, Vol. 5, 1996, S. 413-434.

Busse von Colbe, Walther/Becker, Winfried/Berndt, Helmut/Geiger, Klaus/Haase, Heidrun/Schellmoser, Friedrich/Schmitt, Günter/Seeberg, Thomas/Wysocki, Klaus von (Hrsg.) (2000): Ergebnis je Aktie nach DVFA/SG: Gemeinsame Empfehlung. 3. Auflage, Stuttgart.

Busse von Colbe, Walther/Ordelheide, Dieter (1993): Konzernabschlüsse: Rechnungslegung für Konzerne nach betriebswirtschaftlichen Grundsätzen und gesetzlichen Vorschriften. 6. Auflage, Wiesbaden.

Butterworth, John E. (1972): The Accounting System as Information Function. In: JAR, Vol. 10, S. 1-27.

Cable, John (1985): Capital Market Information and Industrial Performance: The Role of West German Banks. In: The Economic Journal, Vol. 95, S. 118-132.

Cahn, Andreas (1998): Grenzen des Markt- und Anlegerschutzes durch das WpHG. In: ZHR, 162. Jg., S. $1-50$.

Canning, John B. (1929): The Economics of Accountancy: A Critical Analysis of Accounting Theory. New York.

Caplan, Edwin H. (1972): Accounting Research as an Information Source for Theory Construction. In: Report of the Committee on Research Methodology in Ac- 
counting, hrsg. von Committee on Research Methodology in Accounting, AccRev. Supplement, Vol. 47, S. 399-520, hier S. 437-444.

Carlozzi, Catherine L. (1998): Visionary: The CPA's New Role. In: JoA, Vol. 185, January, S. $44-46$.

Carmichael, D.R./Lilien, Steven B./Mellman, Martin (1999): Accountants' Handbook: Volume One: Financial Accounting and General Topics. 9. Auflage, New York et al.

Carter, Mary E./Soo, Billy S. (1999): The Relevance of Form 8-K Reports. In: JAR, Vol. 37, S. 119-131.

Cezanne, Wolfgang/Mayer, Anette (1998): Neue Institutionenökonomik: Ein Überblick. In: WISU, 27. Jg., S. 1345-1353.

Chambers, Raymond J. (1955): Blueprint for a Theory of Accounting. In: Accounting Research, Vol. 6, S. 17-25.

Chambers, Raymond J. (1966): Accounting, Evaluation and Economic Behavior. Englewood Cliffs.

Chamoni, Peter/Gluchowski, Peter (1999): Analytische Informationssyteme: Einordnung und Überblick. In: Analytische Informationssyteme: Data Warehouse, OnLine Analytical Processing, Data Mining, hrsg. von Chamoni, Peter/Gluchowski, Peter, 2. Auflage Berlin et al., S. 3-25.

Chamoni, Peter/Stock, Steffen (1999): Temporale Aspekte in Data Warehouse. In: WISU, 28. Jg., S. 720-726.

Charitou, Andreas (1997): The Role of Cash Flows and Accruals in Explaining Security Returns: Evidence for the UK. In: EAR, Vol. 6, S. 629-652.

Charitou, Andreas/Panagiotides, George (1999): Financial analysis, future earnings and cash flows, and the prediction of stock returns: evidence from the UK. In: Accounting and Business Research, Vol. 29, S. 281-298.

Chemamanur, Thomas J./Fulghieri, Paolo (1994): Investment Bank Reputation, Information Production, and Financial Intermediation. In: JoF, Vol. 49, S. 57-79.

Chen, Peter P. (1976): The Entity Relationship Model: Toward a Unified View of Data. In: ACM Transactions on Database Systems, Vol. 1, S. 9-35.

Cheng, C. S. Agnes/Liu, Chao-Sin/Schaefer, Thomas F. (1996): Eamings Permanence and the Incremental Information Content of Cash Flow from Operations. In: JAR, Vol. 34, S. 173-181. 
Cheng, Paul S./Chang, Pintsang (1998): Transforming corporate information into value through data warehousing and data mining. In: Aslib Proceedings, Vol. 50, S. 109-113.

Chmielewicz, Klaus (1993): Periodisierung. In: HWR, hrsg. von Chmielewicz, Klaus/Schweizer, Marcell, 3. Auflage, Stuttgart, Sp. 1514-1525.

Choe, Jong-Min (1998): The effects of user participation on the design of accounting information systems. In: Information \& Management, Vol. 34, S. 185-198.

Choi, Jay P./Thum, Marcel (1998): Market Structure and the timing of technology adoption with network externalities. In: European Economic Review, Vol. 42, S. 225-244.

Chu, Pai-Cheng (1992): An Object-Oriented Approach to Modeling Financial Accounting Systems. In: Accounting, Management \& Information Technology, Vol. 2, S. 39-56.

Clarke, Thomas (1998): The stakeholder corporation: A business philosophy for the information age. In: Long Range Planning, Vol. 31, S. 182-194.

Clement, Michel B. (1999): Analyst forecast accuracy: Do ability, resources and portfolio complexity matter? In: Journal of Accounting and Economics, Vol. 27, S. 285303.

Cleveland, William S. (1993): Visualizing Data. Murray Hill.

Coase, Ronald H. (1937): The nature of the firm. In: Econometrica N. S., Vol. 4, S. 386405.

Coase, Ronald H. (1984): The New Institutional Economics. In: JITE, Vol. 140, S. 229231.

Codd, Edgar F. (1970): A Relational Model of Data for Large Shared Data Banks. In: Communications of the ACM, Vol. 13, No. 6, S. 377-387.

Codd, Edgar F. (1990): The Relational Model for Database Management: Version 2. Reading et al.

Codd, Edgar F./Codd, S. F./Salley, C. T. (1993): Providing OLAP to User-Analysts: An IT Mandate. White Paper E.F. Codd Associates. URL: http://www.hyperion.com/olapcoddwp.pdf, Stand 09.04.1999.

Coenenberg, Adolf G. (1968): Gewinnbegriff und Bilanzierung. In: ZfbF, 20. Jg. S. 442469. 
Coenenberg, Adolf G. (1995): Einheitlichkeit und Differenzierung von internem und externem Rechnungswesen: Die Anforderung der internen Steuerung. In: DB, 48. Jg., S. 2077-2083.

Coenenberg, Adolf G. (1997): Jahresabschluß und Jahresabschlußanalyse: Grundfragen der Bilanzierung nach betriebswirtschaftlichen, handelsrechtlichen, steuerrechtlichen und internationalen Grundsätzen. 16. Auflage, Landsberg am Lech.

Coenenberg, Adolf G. (1998): Bilanztheorien. In: Lexikon des Rechnungswesens, hrsg. von Busse von Colbe, W./Pellens, B., 4. Auflage, München et al., S. 125-131.

Coenenberg, Adolf G./Möller, Peter (1979): Entscheidungswirkungen von Jahresabschlußinformationen vor und nach der Aktienrechtsreform von 1965. In: BFuP, 31. Jg., S. 438-454.

Coenenberg, A. G./Berndsen, H. P./Möller, P./Schmidt, F./Schönbrodt, B. (1978): Empirische Bilanzforschung in Deutschland: Eine Bestandsaufnahme. In: DBW, S. 495-507.

Coenenberg, Adolf G./Federspieler, Christian (1999): Zwischenberichtspublizität in Europa: Der Informationsgehalt der Zwischenberichterstattung deutscher, britischer und französischer Untemehmen. In: ZfbF Sonderheft 41/99, Rechnungswesen und Kapitalmarkt: Beiträge anläßlich eines Symposiums zum 70. Geburtstag von Prof. Dr. h.c. mult. Walther Busse von Colbe, hrsg. von Gebhardt, Günther/Pellens, Bernhard. Düsseldorf, S. 167-198.

Coing, Helmut (1968): Die ursprüngliche Einheit der europäischen Rechtswissenschaft. Wiesbaden.

Colantoni, Claude S./Manes, Rene P./Whinston, Andrew (1971): A Unified Approach to the Theory of Accounting and Information Systems. In: AccRev., Vol. 46, S. 90-102.

Coller, Maribeth/Yohn, Teri L. (1997): Management Forecasts and Information Asymmetry: An Examination of Bid-Ask Spreads. In: JAR, Vol. 35, S. 181-191.

Collins, Daniel W./Pincus, Morton/Xie, Hong (1999): Equity Valuation and Negative Earnings: The Role of Book Value of Equity. In: AccRev, Vol. 74, S. $29-61$.

Commons, John R. (1934): Institutional Economics: Its Place in Political Economy. Reprint in zwei Bänden, New Brunswick 1990.

Connell, Nigel A. (1991): Artificial Intelligence and Accounting. In: IT and Accounting: The impact of information technology, hrsg. von Williams, Bernard C./Spaul, Barry. London et al., S. 401-417. 
Copeland, Ronald M./Francia, Arthur J./Strawser, Robert H. (1973): Students as Subjects in Behavioral Business Research. In: AccRev., Vol. 48, S. 365-374.

Cox, J. D. (1999): Regulatory duopoly in US securities markets In: Columbia Law Review, Vol. 99, S. 1200-1252.

Crandall, Robert H. (1969): Information Economics and Its Implications for the Further Development of Accounting Theory. In: AccRev., Vol. 44, S. 457-466.

Craven, Barrie M./Marston, C. L. (1999): Financial reporting on the Internet by leading UK companies. In: EAR, Vol. 8, S. 321-333.

Crawford, Vincent P./Sobel, Joel (1982): Strategic Information Transmission. In: Econometrica, Vol. 50, S. 1431-1451.

Crezelius, Georg (1994): Maßgeblichkeitsgrundsatz in Liquidation? In: DB, 47. Jg., S. 689-691

Cushing, Barry E. (1977): On the Possibility of Optimal Accounting Principles. In: AccRev., Vol. 52, S. 308-321.

Cushing, Barry E. (1989): On the Feasibility and the Consequences of a Database Approach to Corporate Financial Reporting. In: Journal of Information Systems, o. Jg., S. 29-52.

Cyert, Richard M./Ijiri, Yuji (1974): Problems of Implementing the Trueblood Objectives Report. In: JAR Supplement, Vol. 12, S. 29-42.

Datar, Srikant/Alles, Michael (1999): The Formation and Role of Reputation and Litigation in the Auditor-Manager Relationship. In: Journal of Accounting, Auditing and Finance, Vol. 14, S. 401-428.

Date, C. J. (1995): An Introduction to Database Systems. 6. Auflage, Reading et al.

Däubler-Gmelin, Herta (1999): Neue Ansätze für Entwicklungen im Wirtschaftsrecht. In: WM, 53. Jg., S. 169-170.

David, René/Grasmann, Günther (1988): Einführung in die großen Rechtssysteme der Gegenwart. 2. Auflage, München.

Davidson, George S./Hendrickson, Bruce/Johnson, David K./ Meyers, Charles E./Wylie, Brian N. (1998): Knowledge Mining With VxInsight: Discovery Through Interaction. In: Journal of Intelligent Information Systems, Vol. 11, S. 259-279. 
Davis-Friday, Paquita Y./Folami, L. Buky/Liu, Chao-Shin/Mittelstaedt, H. Fred (1999): The Value Relevance of Financial Statement Recognition vs. Disclosure: Evidence from SFAS No. 106. In: AccRev, Vol. 74, S. 403-423.

Debreceny, Roger (1998): Object and Relational Database Accounting Information Systems: An Experimental Evaluation. Dissertation Southern Cross University.

Debreceny, Roger/Grey, Glen L. (1999): Financial Reporting on the Internet and the external audit. In: EAR, Vol. 8, S. 335-350.

Dechow, Patricia M. (1994): Accounting Earnings and Cash Flows as Measures of Firm Performance: The Role of Accounting Accruals. In: Journal of Accounting and Economics, Vol. 18, S. 3-42.

Dechow, Patricia M./Kothari S. P./Watts Ross L. (1998): The relation between earnings and cash flows. In: Journal of Accounting and Economics, Vol. 25, S. 133-168.

Degen, Horst (1999): Statistische Methoden zur visuellen Exploration mehrdimensioneller Daten. In: Analytische Informationssysteme, hrsg. von Chamoni, Peter/Gluchowski, Peter, 2. Auflage Berlin et al., S. 393-414.

Deller, Dominik/Stubenrath, Michael/Weber, Christoph (1997): Die Intemetpräsenz als Instrument der Investor Relations: Rechnungslegungsdaten deutscher Unternehmen im Internet. In: DB, 50. Jg., S. 1577-1583.

Deller, Dominik/Stubenrath, Michael/Weber, Christoph (1999): A survey on the use of the Internet for investor relations in the USA, the UK and Germany. In: EAR, Vol. 8, S. 351-364.

Delvin, Barry A./Murphy, Paul T. (1988): An architecture for a business and information system. In: IBM Systems Journal, Vol. 27, S. 60-80.

Demski, Joel S. (1973): The General Impossibility of Normative Accounting Standards. In: AccRev., Vol. 48, S. 718-723.

Demski, Joel S. (1974): Choice Among Financial Accounting Altematives. In: AccRev., Vol. 49, S. 221-232.

Demski, Joel S./Kreps, David M. (1982): Models in Managerial Accounting. In: JAR Supplement, Vol. 20, S. 117-148.

Demsetz, Harold (1968): Why Regulate Utilities? In: Journal of Law and Economics, Vol. 11, S. 55-65.

Deng, Zhen/Lev, Baruch/Narin, Francis (1999): Science and Technology as Predictors of Stock Performance. In: Financial Analysts Journal, Vol. 55, May/June, S. 20 32. 
Devine, Carl T. (1960): Research Methodology and Accounting Theory Formation. In: AccRev., Vol. 35, S. 387-399.

Devlin, Barry A./Murphy, Paul T. (1988): An architecture for a business and information system. In: IBM Systems Journal, Vol. 27, S. 60-80.

Dhaliwal, Dan/Subramanym, K. R./Trezevant, Robert (1999): Is comprehensive income superior to net income as a measure of firm performance? In: Journal of Accounting \& Economics, Vol. 26, S. 43-67.

Dillon, Andrew/Morris, Michael G. (1996): User acceptance of information technology: Theories and models. In: Annual Review of Information Science and Technology, hrsg. von Williams, Martha E., Vol. 31, S. 3-32.

Dirrigl, Hans (1998): Wertorientierung und Konvergenz in der Unternehmensrechnung. In: BFuP, 50. Jg., S. 541-579.

Dittmar, Carsten (1999): Erfolgsfaktoren für Data Warehouse-Projekte: Eine empirische Studie aus Sicht der Anwendungsunternehmen. Institut für Unternehmungsführung und Unternehmensforschung der Ruhr-Universität Bochum, Arbeitsbericht Nr. 78.

Dittmar, Thomas/Hilbert, Andreas (1998): Bonitätsprüfung mit Hilfe Künstlicher Neuronaler Netze. In: ZBB, 10. Jg, S. 343-352.

Dodd, Peter /Leftwich, Richard (1980): The Market for Corporate Charters: "Unhealthy Competition" Versus Federal Regulation. In: Journal of Business, Vol. 53, S. 259283.

Drescher, Sebastian (1998): Gründung eines privaten Rechnungslegungsgremiums in Deutschland: „public versus private standard setting?“. In: StuW, 75. (28.) Jg., S. 240-253.

Dyckman, Thomas R./Gibbins, Michael/Swieringa, Robert J. (1978): Experimental and Survey Research in Financial Accounting: A Review and Evaluation. In: The Impact of Accounting Research on Practice and Disclosure, hrsg. von Abdelkhalik, A. Rashad/Keller, Thomas F., Durham, S. 48-105.

Dyckman, Thomas R./Zeff, Stephen A. (1984): Two Decades of the Journal of Accounting Research. In: JAR, Vol. 22, S. 225-297.

Dye, Ronald A. (1983): Communication and Post-Decision Information. In: JAR, Vol. 21, S. 514533.

Dye, Ronald A. (1985): Disclosure of Nonproprietary Information. In: JAR, Vol. 23, S. 123-145. 
Dye, Ronald A. (1985a): Strategic Accounting Choice and the Effects of Alternative Financial Reporting Requirements. In: JAR, Vol. 23, S. 544-574.

Dye, Ronald A. (1993): Auditor Standards, Legal Liability, and Auditor Wealth. In: Journal of Political Economy, Vol. 101, S. 887-914.

Dye, Ronald A./Sridhar Sri S. (1995): Industry-Wide Disclosure Dynamics. In: JAR, Vol. 33, S. 157-174.

Dye, Ronald A./Verrecchia, Robert E. (1995): Discretion vs. Uniformity: Choices Among GAAP. In: AccRev., Vol. 70, S. 389-415.

Edwards, Edward O./Bell, Phillip W. (1961): The Theory and Measurement of Business Income. Berkley et al., 7. Nachdruck 1973.

Eggertsson, Thrainn (1990): Economic Behavior and Institutions. Cambridge.

Elliot, Robert K./Jacobson, Peter D. (1994): Costs and Benefits of Business Information Disclosure. In: Accounting Horizons, Vol. 8, S. 80-96.

Ely, Kirsten/Waymire, Gregory (1999): Accounting Standard-Setting Organizations and Earnings Relevance: Longitudinal Evidence from NYSE Common Stocks, 192793. In: JAR, Vol. 37, S. 293-317.

Erichsen, Jörgen (2000): Verbesserung der Effizienz des Controllings. In: Bilanz \& Buchhaltung, 46. Jg., Heft 2, S. 55-59.

Erlei, Mathias (1998): Institutionen, Märkte und Marktphasen: Allgemeine Transaktionskostentheorie unter spezieller Berücksichtigung der Entwicklungsphasen von Märkten. Tübingen.

Erlei, Mathias/Leschke, Martin/Sauerland, Dirk (1999): Neue Institutionenökonomik. Stuttgart.

Emst, Christoph (1999): Die Grundzüge des Referentenentwurfs zum Kapitalgesellschaften- \& Co.-Richtlinie-Gesetz. In: DStR, 37. Jg., 903-907.

Eucken, Walter (1965): Die Grundlagen der Nationalökonomie. 8. Auflage, Berlin.

Eucken, Walter (1990): Grundsätze der Wirtschaftspolitik. 6. Auflage, Tübingen.

Everest, Gordon C./Weber, Ron (1977): A Relational Approach to Accounting Models. In: AccRev., Vol. 52, S. 340-359.

Ewert, Ralf (1989): Bilanzielle Publizität im Lichte der Theorie öffentlich verfügbarer Information. In: BFuP, 41. Jg., S. 245-263.

Ewert, Ralf (1990): Wirtschaftsprüfung und asymmetrische Information. Berlin et al. 
Ewert, Ralf (1995): Untemehmenspublizität und Insiderhandel. In: Unternehmenstheorie und Besteuerung. Dieter Schneider zum 60. Geburtstag, hrsg. von Elschen, Rainer/Siegel, Theodor/Wagner, Franz W. Wiesbaden, S. 229-265.

Ewert, Ralf (1999): Auditor Liability and the Precision of Auditing Standards. In: JITE, Vol. 155, S. 181-206.

Falk, Haim/Ophir, Tsvi (1973): The Effect of Risk on the Use of Financial Statements by Investment Decision-Makers: A Case Study. In: AccRev., Vol. 48, S. 323-338.

Fama, Eugene F. (1970): Efficient Capital Markets: A Review of Theory and Empirical Work. In: JoF, Vol. 25, S. 383-417.

Fama, Eugene F. (1991): Efficient Capital Markets: II. In: JoF, Vol. 46, S. 1575-1617.

Fama, Eugene F./Fisher, Lawrence/Jensen, Michael C./Roll, Richard (1969): The Adjustment of Stock Prices to New Information. In: International Economic Review, Vol. 10, S. 1-21.

Fama, Eugene F./Laffer, Arthur B. (1971): Information and Capital Markets. In: Journal of Business, Vol. 44, S. 289-298.

Farrell, Joseph/Saloner, Garth (1985): Standardization, compatibility, and information. In: Rand Journal of Economics, Vol. 16, S. 70-83.

Farrell, Joseph/Saloner, Garth (1986): Installed Base and Compatibility: Innovation, Product Preannouncements and Predation. In: AER, Vol. 76, S. 940-955.

Faure-Grimaud, Antoine/Laffont, Jean-Jacques/Martimort, David (1999): The endogenous transaction costs of delegated auditing. In: European Economic Review, Vol. 43, S. 1039-1048.

Feldhoff, Michael (1992): Die Regulierung der Rechnungslegung. Frankfurt am Main et al.

Feldhoff, Michael (1992a): Versagt der Markt für Informationen? Eine spieltheoretische Analyse. In: ZfbF, 44. Jg., S. 1012-1022.

Feldhoff, Michael (1994): Staat und Rechnungslegung: Eine regierungspolitische Analyse. In: WPg, 47. Jg., S. 529-536.

Feltham, Gerald A. (1968): The Value of Information. In: AccRev., Vol. 43, S. 684-696.

Feltham, Gerald A. (1977): Cost Aggregation: An Information Economics Analysis. In: JAR, Vol. 15, S. 42-70.

Feltham, Gerald A./Ohlson, James A. (1995): Valuation and Clean Surplus Accounting for Operating and Financial Activities. In: CAR, Vol. 11, S. 689-731. 
Feltham, Gerald A./Ohlson, James A. (1999): Residual Eamings Valuation With Risk and Stochastic Interest Rates. In: AccRev, Vol. 74, S. 165-183.

Field, John E. (1969): Toward A Multi-Level, Multi-Goal Information System. In: AccRev., Vol. 44, S. 593-599.

Fischer, Joachim (1997): Einsatzmöglichkeiten zeitorientierter Vertragsdatenbanken im Controlling. In: Wirtschaftsinformatik, 39. Jg., S. 55-63.

Fischer, Paul E./Verrecchia, Robert E. (1999): Public Information and heuristic trade. In: Journal of Accounting and Economics, Vol. 27, S. 89-124.

Fischer, Rudolf (1909): Über die Grundlagen der Bilanzwerte. Leipzig.

Fitzgerald, Julie/Carnall, Wayne (1999): What an accountant should know about the SEC. In: Der Schweizer Treuhänder, 73. Jg., S. 975-982.

Flynn, Gerard/Gowthrope, Catherine (1997): Volunteering financial data on the World Wide Web: A study of financial reporting from a stakeholder perspective. Working Paper präsentiert auf der 1. Financial Reporting and Business Communication Conference, Cardiff, Juli 1997. URL: http://www.summa.org.uk/corp/ papers/gowthrope/gowthrope.htm, Stand 16.11.1999.

Fogarty, Timothy J. (1992): Financial Accounting Standard Setting as an Institutionalized Action Field: Constraints, Opportunities and Dilemmas. In: Journal of Accounting and Public Policy, Vol. 11, S. 331-355.

Förschle, Gerhart/Glaum, Martin/Mandler, Udo (1995): US-GAAP, LAS und HGB: Ergebnisse einer Umfrage unter deutschen Rechnungslegungsexperten. In: BFuP, 47. Jg., S. 392-413.

Förschle, Gerhart/Glaum, Martin/Mandler, Udo (1998): Internationale Rechnungslegung und Kapitalaufnahmeerleichterungsgesetz: Meinungswandel bei den Führungskräften deutscher Unternehmen? In: DB, 51. Jg., S. 2281-2288.

Förschle, Gerhart/Helmschrott, Harald (1998): Neuer Markt an der Frankfurter Wertpapierbörse: Eine Erste Bilanz. 2. Auflage, Frankfurt am Main.

Förschle, Gerhart/Helmschrott, Harald (1999): Neuer Markt an der Frankfurter Wertpapierbörse: Update 1999 - Bedeutung, wesentliche Änderungen, Ausblick. In: WPK-Mitteilungen, 38. Jg., S. 210-220.

Frankel, Richard/McNichols, Maureen/Wilson, Peter G. (1995): Discretionary Disclosure and External Financing. In: AccRev., Vol. 70, S. 135-150. 
Frantz, Pascal (1999): Discretionary write-downs, write-offs, and other restructuring provisions: a signalling approach. In: Accounting and Business Research, Vol. 29, S. 109-121.

Frantz, Pascal/Walker, Martin (1997): Information Disclosure To Employees and Rational Expectations: A Game-Theoretical Perspective. In: Journal of Business, Finance \& Accounting, Vol. 24, S. 1421-1431.

Fraser, Ian A. M. (1993): Triple-Entry Bookkeeping: A Critique. In: Accounting and Business Research, Vol. 23, S. 151-158.

Fritsch, Michael/Wein, Thomas/Ewers, Hans-Jürgen (1999): Marktversagen und Wirtschaftspolitik: Mikroökonomische Grundlagen staatlichen Handelns. 3. Auflage, München.

Fritz, Burkhard (1999): DV-gestützte Führungsinformationssysteme: Konzeptionelle Anforderungen und Gestaltungsmöglichkeiten. Frankfurt am Main et al.

Fülbier, Rolf Uwe (1998): Die Regulierung der Ad-hoc-Publizität: Ein Beitrag zur ökonomischen Analyse des Rechts. Wiesbaden.

Fülbier, Rolf Uwe (1999): Regulierung: Ökonomische Betrachtung eines allgegenwärtigen Problems. In: WiSt, 28. Jg., S. 468-473.

Fülbier, Rolf Uwe (1999a): 5 Jahre Ad-hoc-Publizität: Ein Zwischenergebnis. In: StuB, 1. Jg., S. 1260-1267.

Fülbier, Rolf Uwe/Gassen, Joachim (1999): Wider die Maßgeblichkeit der International Accounting Standards für die steuerliche Gewinnermittlung: Erwiderung zu Oestreicher/Spengel, DB 1999, S. 593-600. In: DB, 52. Jg., S. 1511-1513.

Füllgrav, Nicola (1999): Trends und Zukunftspersepktiven für Data Warehouses. In: Information Management \& Consulting, 14. Jg., S. 29-34.

Fürst, Oren (1998): A Theoretical Analysis of the Investor Protection Regulations Argument for Global Listing of Stocks. Working Paper, International Center for Finance at Yale.

Gabriel, Roland/Chamoni, Peter/Gluchowski, Peter (2000): Data Warehouse und OLAP - Analyseorientierte Informationssysteme für das Management. In: ZfbF, 52. Jg., S. 74-93.

Gabriel, Roland/Gluchowski, Peter (1997): Management Support Systeme (Teil I-III). In: WiSt, 26. Jg., S. 308-313, S. 422-427 und S. 535-540. 
Gabriel, Roland/Gluchowski, Peter (1998): Grafische Notationen für die semantische Modellierung multidimensionaler Datenstrukturen in Management Support Systemen. In: Wirtschaftsinformatik, 40. Jg., S. 493-502.

Gaertner, Wulf (1998): The Theory of Social Choice since 1951. In: JbfNatStat, Vol. 217, S. $657-680$.

Gajo, Marianne (1999): Neue Börsenallianzen. In: AG, 44. Jg., S. R 164.

Gal, Graham/McCarthy, William E. (1986): Operation of a Relational Accounting System. In: Advances in Accounting, Vol. 3, S. 83-112.

Gebhardt, Günther (1980): Insolvenzprognosen aus handelsrechtlichen Jahresabschlüssen: Eine Beurteilung der Reform der Rechnungslegung durch das Aktiengesetz 1965 aus der Sicht unternehmensexterner Adressaten, Wiesbaden.

Geerts, Guido/McCarthy, William E. (1991): Database Accounting Systems. In: IT and Accounting: The impact of information technology, hrsg. von Williams, Bernard C./Spaul, Barry. London et al., S. 159-183.

Gerke, Wolfgang (1999): Der Neue Markt. In: WiSt, 28. Jg., S. 204206.

Gerke, Wolfgang/Bank, Matthias/Seitz, Steffen (1999): EASDAQ versus Neuer Markt: Entwicklung, Erfolgsfaktoren und Perspektiven. In: FB, 1. Jg., S. 199-205.

Gessler, Ernst (1965): Der Bedeutungswandel der Rechnungslegung im Aktienrecht. In: 75 Jahre Deutsche Treuhand-Gesellschaft 1890-1965, hrsg. von Volkmar Muthesius, Frankfurt am Main, S. 129-166.

Gibbins, Michael/Richardson, Alan/Waterhouse, John (1990): The Management of Corporate Disclosure: Opportunism, Ritualism, Policies, and Processes. In: JAR, Vol. 28, S. 121-143.

Gigler, Frank (1994): Self-Enforcing Voluntary Disclosures. In: JAR Supplement, Vol. 32, S. 224240 .

Gigler, Frank/Hemmer, Thomas (1998): On the Frequency, Quality, and Informational Role of Mandatory Financial Reports. In: JAR Supplement, Vol. 36, S. 117-147.

Gluchowski, Peter (1998): Data Warehouse: Konzepte, Produkte und Einsatzmöglichkeiten. In: Data Warehouse: Einsatzmöglichkeiten in der Praxis. Arbeitsberich Nr. 70 des IUU Ruhr-Universität Bochum, hrsg. von Gabriel, Roland, Bochum, S. 119.

Gluchowski, Peter (1998a): Werkzeuge zur Implementierung des betrieblichen Berichtswesens. In: WISU, 27. Jg., S. 1174-1188. 
Gluchowski, Peter/Gabriel, Roland/Chamoni, Peter (1997): Management Support Systeme: Computergestützte Informationssysteme für Führungskräfte und Entscheidungsträger. Berlin et al.

Goerdeler, Reinhard (1992): Publizität der Rechnungslegung in Rückblick und Ausblick. In: Rechnungslegung: Entwicklung bei der Bilanzierung und Prüfung von Kapitalgesellschaften, Festschrift zum 65. Geburtstag von Prof. Dr. Dr. h.c. K.-H. Förster, hrsg. von Moxter, Adolf/Müller, Hans-Peter/Windmöller, Rolf/Wysocki, Klaus von, Düsseldorf, S. 235-252.

Goldberg, Victor P. (1976): Regulation and Administered Contracts. In: Bell Journal of Economics, Vol. 7, S. 426-448.

Golfarelli, Matteo/Rizzi, Stefano (1999): Designing the Data Warehouse: Key Steps and Crucial Issues. In: Journal of Computer Science and Information Management, Vol. 2, Heft 3.

Gonedes, Nicholas J. (1972): Efficient Capital Markets and External Accounting. In: AccRev., Vol. 47, S. 11-21.

Gonedes, Nicholas J. (1973): Capital Market Equilibrium and Annual Accounting Numbers: Empirical Evidence. In: JAR, Vol. 12, S. 26-62.

Gonedes, Nicholas J. (1975): Information Production and Capital Market Equilibrium. In: JoF, Vol. 30, S. 841-864.

Gonedes, Nicholas J./Dopuch Nicholas (1974): Capital Market Equilibrium, Information Production, and Selecting Accounting Techniques: Theoretical Framework and Review of Empirical Work. In: JAR Supplement, Vol. 12, S. 48-129.

Gonedes, Nicholas J./Dopuch Nicholas/Penman, Stephan H. (1976): Disclosure Rules, Information Production and Capital Market Equilibrium: The Case of Forecast Disclosure Rules. In: JAR, Vol. 14, S. 89-137.

Goodman, Danny (1998): Dynamic HTML: The Definitive Reference. Cambridge et al.

Gowthorpe, Catherine/Flynn, Gerard (1997): Reporting on the Web: State of the Art. In: Accountancy, Vol. 121, August, S. 58-59.

Gowthorpe, Catherine/Oriol, Amat (1999): External reporting of accounting and financial information via the Internet in Spain. In: EAR, Vol. 8, S. 365-371.

Gray, Sidney J. (1988): Towards a Theory of Cultural Influence on the Development of Accounting Systems Internationally. In: ABACUS, Vol. 24, S. 1-15. 
Grossekettler, Heinz (1995): Öffentliche Finanzen. In: Vahlens Kompendium der Wirtschaftstheorie und Wirtschaftspolitik: Band 1, hrsg. von Bender, Dieter et al., 6. Auflage, München, S. 483-627.

Groß, Wolfgang (1999): Die börsengesetzliche Prospekthaftung. In: AG, 44. Jg., S. 199209.

Großfeld, Bernhard (1997): Internationales Bilanzrecht/Intemationale Rechnungslegung. In: AG, 42. Jg., S. 433-448.

Großfeld, Bernhard (2000): Börsenkurs und Unternehmenswert. In: BB, 55. Jg., S. 261266.

Grossman, Stanford J. (1981): The Informational Role of Warranties and Private Disclosure About Product Quality. In: Journal of Law and Economics, Vol. 24, S. 461483.

Grossman, Stanford J./Hart, Oliver D. (1986): The Costs and Benefits of Ownership: A Theory of Vertical and Lateral Integration. In: Journal of Public Economy, Vol. 94, S. $691-719$.

Grossman, Stanford J./Stiglitz, Joseph E. (1980): On the Impossibility of Informationally Efficient Markets. In: American Economic Review, Vol. 70, S. 393-408.

Guidry, Flora/Leone, Andrew J./Rock, Steve (1999): Earnings-based bonus plans and earnings management by business-unit managers. In: Journal of Accounting and Economics. Vol. 26, S. 113-142.

Gümbel, Rudolf (1996): Betriebswirtschaftslehre und ökonomische Theorie. Stuttgart.

Guntz, Peter (1995): Das Ende des Anlegerschutzes in den USA? In: ZBB, 7. Jg., S. 206210.

Härder, Theo/Rahm, Erhard (1999): Datenbanksysteme: Konzepte und Techniken der Implementierung. Berlin et al.

Hakansson, Nils H. (1969): Normative Accounting Theory and the Theory of Decision. In: International Journal of Accounting Education and Research, Vol. 4, S. 3347.

Hakansson, Nils H. (1977): Interim Disclosure and Public Forecasts: An Economic Analysis and a Framework for Choice. In: AccRev., Vol. 52, S. 396-416.

Hakansson, Nils H. (1981): On the Politics of Accounting Disclosure and Measurement: An Analysis of Economic Incentives. In: AccRev., Vol. 52, S. 396-416. 
Hakansson, Nils H./Kunkel, J. Greogory/Ohlson, James A. (1982): Sufficient and Necessary Conditions for Information to have Social Value in Pure Exchange. In: JoF, Vol. 37, S. 1169-1181.

Haller, Axel (1988): Das Verhältnis von steuerrechtlicher und "handelrechtlicher" Rechnungslegung in den USA. In: DBW, 48. Jg. S. 723-733.

Haller, Axel (1994): Die Grundlagen der externen Rechnungslegung in den USA: Unter besonderer Berücksichtigung der rechtlichen, institutionellen und theoretischen Rahmenbedingungen. 4. Auflage, Stuttgart.

Hansen, Frank-Peter (1999): Standardisierung als relationaler Vertrag: Die Entwicklung von technischen Vorschriften in der europäischen Telekommunikation. Frankfurt am Main et al.

Harding, Wayne E. (1999): AICPA establishes a Language for Electronic-Based Financial Reporting. In: JoA, Vol. 188, September, S. 15.

Harrer, Herbert/Heidemann, Dieter (1999): Going Public: Einführung in die Thematik. In: DStR, 37. Jg., S. 254260.

Harris, Trevor S./Lang, Mark H./ Möller Hans P. (1994): The Value Relevance of German Accounting Measures: An Empirical Analysis. In: JAR, Vol. 32, S. 187-209.

Harris, Mary S./Muller Karl A. III (1999): The Market Valuation of IAS versus USGAAP accounting measures using Form 20-F reconciliations. In: Journal of Accounting and Economics, Vol. 26, S. 285-312.

Hart, Oliver/Moore, John (1990): Property Rights and the Nature of the Firm. In: Journal of Political Economy, Vol. 98, S. 1119-1158.

Hartmann, Matthias (1997): Technologie-Bilanzierung. Instrument einer zukunftsorientierten Unternehmensbeurteilung. Göttingen.

Hartmann, Matthias (1998): Die Technologiebilanz als Instrument einer zukunftsorientierten Unternehmensbeurteilung. In: WiSt, 27. Jg., S. 134136.

Hartmann-Wendels, Thomas (1994): Agency-Theorie und Publizitätspflicht nichtbörsennotierter Kapitalgesellschaften. In: BFuP, 44. Jg., S. 412-425.

Haseman, William D./Whinston, Andrew B. (1976): Design of a Multidimensional Accounting System. In: AccRev., Vol. 51, S. 65-79.

Hatzis-Schoch, Brent (1992): Die Bedeutung von Delaware für das US-amerikanische Gesellschaftsrecht. In: RIW, 38. Jg., S. 539-543. 
Hauser, Heinz (1993): Harmonisierung oder Wettbewerb nationaler Regulierungssysteme in einem integrierten Wirtschaftsraum. In: Aussenwirtschaft, 48. Jg., S. 459-476,

Havelka, Douglas/Khazanchi, Deepak (1994): An "Events“ Model for Information Aggregation. In: Journal of Computer Information Systems, Vol. 35, Winter 1994-95, S. 72-81.

Hax, Herbert (1988): Rechnungslegungsvorschriften: Notwendige Rahmenbedingungen für den Kapitalmarkt? In: Unternehmenserfolg: Planung - Ermittlung - Kontrolle, Walther Busse von Colbe zum 60. Geburtstag, hrsg. von Domsch, Michel/Eisenführ, Franz/Ordelheide, Dieter/Perlitz, Manfred, Wiesbaden, S. 187201.

Hayek, Friedrich A. von (1945): The Use of Knowledge in Society. In: AER, Vol. 35, S. 519-530.

Hayek, Friedrich A. von (1960): The Constitution of Liberty. Chicago.

Hayek, Friedrich A. von (1973): Law, Legislation and Liberty: Vol. 1: Rules and Order. London.

Hayek, Friedrich A. von (1989): The Pretence of Knowledge: Nobel Memorial Lecture, December, 11, 1974. In: AER (Special Issue), Vol. 79, S. 3-7.

Hayek, Friedrich A. von (1994): Freiburger Studien: Gesammelte Aufsätze. 2. Auflage, Tübingen.

Healy, Paul M. (1985): The effect of bonus schemes on accounting decisions. In: Journal of Accounting and Economics, Vol. 7, S. 85-107.

Heap, Shaun H./Varoufakis, Y. (1991): Economic theories of information: a critique. In: IT and Accounting: The impact of information technology, hrsg. von Williams, Bernard C./Spaul, Barry. London et al., S. 60-76.

Hedlin, Pontus (1999): The Internet as a vehicle for investor relations: the Swedish case. In: EAR, Vol. 8, S. 373-381.

Hegarty, John (1997): Accounting for the Global Economy: Is National Regulation Doomed to Disappear? In: Accounting Horizons, Vol. 11, S. 75-90.

Helm, Leonhard (1998): Electronic Publishing - Neue Möglichkeiten der Aktionärsinformation am Beispiel Internet. In: Shareholder Value Reporting: Veränderte Anforderungen an die Berichterstattung börsennotierter Unternehmen, hrsg. von Müller, Michael/Leven, Franz-Josef, Wien, S. 103-119.

Helms, Glenn L./Mancino, Jane (1998): The Electronic Auditor: Wave good-bye to the old paper trail. In: JoA, Vol. 185, April, S. 45-48. 
Heintges, Sebastian (1997): Bilanzkultur und Bilanzpolitik in den USA und in Deutschland: Einflüsse auf die Bilanzpolitik börsennotierter Unternehmen. 2. Aufl., Stemenfels et al.

Heintzen, Markus (1999): Zur Verfassungsmäßigkeit von $\int 292$ a Abs.2 Nr. 2 a) HGB. In: BB, 54. Jg., S. 1050-1054.

Heni, Bernhard (1999): Transformation der GmbH \& Co.-Richtlinie: Neuer Schub für die Konzernrechnungslegung. In: DStR, 37. Jg., S. 912-916.

Henneböle, Jörg (1995): Executive Information Systems für Unternehmensführung und Controlling: Strategie - Konzeption - Realisierung. Wiesbaden.

Herbig/Geßler/Hefermehl (1937): Das Neue Aktiengesetz. In: Deutsche Juristenzeitung, 99. Jg., S. 184-197.

Herold, Helmut (1996): UNIX-Systemprogrammierung. Bonn et al.

Hertzsch, Andrea/Schmitz, Eckhard (1997): Die EDV als ein Werkzeug der Datenprüfung im Rahmen der Jahresabschlußprüfung: Eine empirische Untersuchung. In: WPg, 50. Jg., S. 525-532.

Herzig, Norbert (2000): Internationalisierung der Rechnungslegung und steuerliche Gewinnermittlung. In: WPg, 53. Jg., S. 104119.

Hess, Thomas (1999): Die Agency-Theorie als Gestaltungshilfe für Führungsinformationssysteme. In: WISU, 28. Jg., S. 1503-1509.

Hess, Thomas (2000): Netzeffekte: Verändern neue Informations- undKommunikationstechnologien das klassische Marktmodell? In: WiSt, 29. Jg., S. 96-98.

Hillegeist, Stephen A. (1999): Financial reporting and auditing under alternative damage apportionment rules. In: AccRev., Vol. 74, S. 347-369.

Hirshleifer, Jack (1971): The Private and Social Value of Information and the Reward to Incentive Activity. In: AER, Vol. 61, S. 561-573.

Hoffman, Charles/Kurt, Christopher/Koreto, Richard J. (1999): The XML Files: In the 21 st century economy, XML is the way you will move information. In: JoA, Vol. 187, May, S. 71-77.

Hoffmann, Philip T./Postel-Vinay, Gilles/Rosenthal, Jean-Laurent (1998): What do Notaries do? Overcoming Asymmetric Information in Financial Markets: The Case of Paris, 1751. In: JITE, Vol. 154, S. 499-530.

Holland, John B. (1998): Private Disclosure and financial reporting. In: Accounting and Business Research, Vol. 28, S. 255-269. 
Holler, Manfred J./Illing, Gerhard (1996): Einführung in die Spieltheorie. 3. Auflage, Berlin et al.

Holmstrom, Bengt R./Tirole, Jean (1989): The Theory of the Firm. In: Handbook of Industrial Organization, Vol. I., hrsg. von Schmalensee, R./Willig, R. D., Amsterdam, S. 61-133.

Holthausen, Robert W./Larcker, David F. (1992): The prediction of stock returns using financial statement information. In: Journal of Accounting and Economics, Vol. 15, S. $373-411$.

Holthausen, Robert W./Larcker, David F./Sloan, Richard G. (1995): Annual bonus schemes and the manipulation of earnings. In: JAE, Vol. 19, S. 29-74.

Holthuis, Jan (1998): Multidimensionale Datenstrukturen: Modellierung, Strukturkomponenten, Implementierungsaspekte. In: Das Data Warehouse-Konzept, hrsg. von Mucksch, Harry/Behme, Wolfgang, 3. Auflage, Wiesbaden, S. 143-193.

Hommelhoff, Peter (1985): Eigenkontrolle statt Staatskontrolle: Rechtsdogmatischer Überblick zur Aktienrechtsreform 1884. In: Hundert Jahre modernes Aktienrecht: Eine Sammlung von Texten und Quellen zur Aktienrechtsreform 1884 mit zwei Einführungen, hrsg. von Schubert, Werner/Hommelhoff, Peter, Berlin et al., S. 53-105.

Hommelhoff, Peter/Mattheus, Daniela (1998): Corporate Govemance nach dem KonTraG. In: AG, 43. Jg., S. 249-259.

Hommelhoff, Peter/Schilling, Wolfgang (Hrsg.) (1987): Die Aktienrechtsreform: Die Protokolle der Verhandlungen im Aktienrechtsausschuß des Vorläufigen Reichswirtschaftsrats unter dem Vorsitz von Max Hachenburg. Berlin et al.

Hommelhoff, Peter/Schwab, Martin (1998): Gesellschaftliche Selbststeuerung im Bilanzrecht-Standard Setting Bodies und staatliche Regulierungsverantwortung nach deutschem Recht. In: BFuP, 50. Jg., S. 38-56.

Hopf, Michael (1983): Informationen für Märkte und Märkte für Informationen. Frankfurt am Main.

Hoppmann, Erich (1966): Das Konzept der optimalen Wettbewerbsintensität: Rivalität oder Freiheit des Wettbewerbs: Zum Problem eines wettbewerbspolitisch adäquaten Ansatzes der Wettbewerbstheorie. In: JbeNatStat, Band 179, S. 286-323.

Hopt, Klaus J. (1980): Die Publizität von Kapitalgesellschaften: Grundsätzliche Überlegungen zum Stand nach der 4. EG-Richtlinie und zur Reformdiskussion in den USA. In: ZGR, 9. Jg., S. 225-253. 
Hopt, Klaus J. (1995): Grundsatz- und Praxisprobleme nach dem Wertpapierhandelsgesetz: Insbesondere Insidergeschäfte und Ad-hoc-Publizität. In: ZHR, 159. Jg., S. 135-163.

Horngren, Charles T. (1955): Security Analysts and the Price Level. In: AccRev., Vol. 30, S. 575-581.

Horngren, Charles T. (1972): Accounting Principles: Public or Private Sector? In: JoA, Vol. 133, May, S. 37-41.

Horngren, Charles T. (1985): Institutional Alternatives for Regulating Financial Reporting. In: Journal of Comparative Business and Capital Market Law, Vol. 7, S. 267289.

Horton, James L. (1993): Electronic filing portends market volatility. In: Public Relations Journal, Vol. 49, July/August, S. 27-28.

Horváth, Péter/Arnaout, Ali (1997): Internationale Rechnungslegung und Einheit des Rechnungswesens: State-of-the-Art und Implementierung der in der deutschen Praxis. In: Controlling, 9. Jg., S. 254268.

Hüfner, Bernd/Möller, Hans-Peter (1997): Erfolge börsennotierter Unternehmen aus Sicht von Finanzanalysten: Zur Verläßlichkeit von DVFA-Ergebnissen und deren Prognosen. In: ZBB, 9. Jg., S. 1-14.

Hulle, Karel van (1998): Die Zukunft der europäischen Rechnungslegung im Rahmen einer sich ändernden internationalen Rechnungslegung. In: WPg, 51. Jg., S. 138153.

Hüllweck, Jörg-Karsten/Ostrowski, Markus (2000): Die Abschlußprüfer börsennotierter Gesellschaften: Veränderungen im Markt für Abschlußprüfungen zwischen 1996 und 1997. In: WPK-Mitteilungen, 39. Jg., S. 2-9.

Hunton, James E./McEwan, Ruth A. (1997): An Assessment of the Relation Between Analysts' Earnings Forecast Accuracy, Motivational Incentives, and Cognitive Information Search Strategy. In: AccRev., Vol. 72, S. 497-515.

Ijiri, Yuji (1967): The Foundations of Accounting Measurement: A Mathematical, Economic, and Behavioral Inquiry. Englewood Cliffs.

Ijiri, Yuji (1975): Theory of Accounting Measurement. Sarasota.

Ijiri, Yuji (1978): Cash Flow Accounting and Its Structure. In: Journal of Accounting, Auditing \& Finance, Vol. 1, S. 331-348.

Ijiri, Yuji (1986): A Framework for Triple-entry Bookkeeping. In: AccRev., Vol. 61, S. 745-759. 
Ijiri, Yuji (1989): Momentum Accounting and Triple-Entry Bookkeeping: Exploring the Dynamic Structure of Accounting Measurements. Sarasota.

Ingrim, Robert W./Chewing, Eugene G. (1983): The Effect of Financial Disclosure Regulation on Security Market Behavior. In: AccRev., Vol. 58, S. 562-580.

Inman, Eric E. (1998): Enterprise modelling advantages of San Francisco for general ledger systems. In: IBM Systems Journal, Vol. 37, S. 170-180.

Inmon, William H. (1996): Building the Data Warehouse. 2. Auflage, New York et al.

Ittner, Christopher D./Larcker, David (1998): Are Nonfinancial measures Leading Indicators of Financial Performance? An Analysis of Customer Satisfaction. In: JAR Supplement, Vol. 36, S. 1-35.

Ittner, Christopher D./Larcker, David/Rajan, Madhav V. (1997): The Choice of Performance Measures in Annual Bonus Contracts. In: AccRev., Vol. 72, S. 231-255.

Jacoby, William G. (1998): Statistical Graphics for Visualizing Multivariate Data. Thousand Oaks.

Johnson, Orace (1970): Toward an "Events" Theory of Accounting. In: AccRev., Vol. 45, S. 641-653.

Johnson, Orace (1981): Some Implications of the United States Constitutions for Accounting Institution Alternatives. In: JAR Supplement, Vol. 19, S. 89-119.

Jonas, Greogory J./Young, Stephen J. (1998): Bridging the Gap: Who Can Bring a User Focus to Business Reporting? In: Accounting Horizons, Vol. 12, S. 154-159.

Jorion, Phillipe/Goetzmann, William N. (1999): Global Stock Markets in the Twentieth Century. In: JoF, Vol. 54, S. 953-980.

Joskow, Paul L. (1974): Inflation and Environmental Concem: Structural Change in the Process of Public Utility Price Regulation. In: Journal of Law and Economics, Vol. 17, S. 291-327.

Jensen, Michael C./Meckling, William H. (1976): Theory of the Firm: Managerial Behavior, agency costs, and capital structure. Journal of Financial Economics, Vol. 3, S. 305-360.

Jung, Wooh-Oh/Kwon, Young K. (1988): Disclosure When the Market is Unsure of Information Endowment of Managers. In: JAR, Vol. 26, S. 146-153.

Kahle, Holger (1999): Zur Bedeutung der US-GAAP für die steuerliche Maßgeblichkeit in den USA. In: StuB, 1. Jg., S. 1145-1151. 
Kaiser, Bernd-Ulrich (1998): Erfahrungen beim Aufbau eines Data Warehouse und Betrieb eines unternehmensweiten Informationssystems bei der Bayer AG. In: Data Warehouse: Einsatzmöglichkeiten in der Praxis. Arbeitsbericht Nr. 70 des IUU Ruhr-Universität Bochum, hrsg. von Gabriel, Roland, Bochum, S. 51-93.

Kaiser, Joseph H. (1978): Die Repräsentation organisierter Interessen. 2. Auflage, Berlin.

Käfer, Karl (1962): Die Bilanz als Zukunftsrechnung: Eine Vorlesung über den Inhalt der Unternehmungsbilanz. Zürich.

Kamar, Ehud (1998): A Regulatory Competition Theory of Indeterminacy in Corporate Law. In: Columbia Law Review, Vol. 98, S. 1908-1959.

Kang, Sok-Hyon/O'Brian, John/Sivaramakrishnan, K. (1994): Analysts' Interim Eamings Forecasts: Evidence on the Forecasting Process. In: JAR, Vol. 32, S. 103-112.

Kantzenbach, Erhard (1967): Die Funktionsfähigkeit des Wettbewerbs. 2. Auflage, Göttingen.

Kaplan, David/Fender, Elizabeth A. (1998): The Development of Comment Letters of FASB Proposals by the AICPA Accounting Standards Executive Committee. In: Accounting Horizons, Vol. 12, S. 184187.

Kaplan, Robert S. (1978): The Information Content of Financial Accounting Numbers: A Survey of Empirical Evidence. In: The Impact of Accounting Research on Practice and Disclosure, hrsg. von Abdel-Khalik, A. Rashad/Keller, Thomas F., Durham, S. 134-173.

Karpe, Jan (1999): Ökonomische Verhaltenskonzepte. In: WiSt, 28. Jg., S. 605-607.

Kasznik, Ron (1999): On the Association between Voluntary Disclosure and Earnings Management. In: JAR, Vol. 37, S. 57-81.

Katz, Michael L./Shapiro, Carl (1985): Network externalities, competition, and compatibility. In: AER, Vol. 75, S. 424440.

Katz, Michael L./Shapiro, Carl (1986): Technology Adoption in the presence of network externalities. In: Journal of Political Economy, Vol. 94, S. 822-841.

Kaufer, Erich (1980): Industrieökonomik: Eine Einführung in die Wettbewerbstheorie. München.

Keane, Michael P./Runkle, David E. (1998): Are Financial Analysts' Forecasts of Corporate Profits rational? In: Journal of Political Economy, Vol. 106, S. 768-805.

Kemper, Hans-Georg/Finger, Ralf (1999): Datentransformation im Data-Warehouse: Konzeptionelle Überlegungen zur Filterung, Harmonisierung, Verdichtung und 
Anreicherung operativer Datenbestände. In: Analytische Informationssysteme, hrsg. von Chamoni, Peter/Gluchowski, Peter, 2. Auflage, Berlin et al., S. 77-94.

Kersting, Mark O./Sohbi, Hassan (1998): Haftungsrechtliche Aspekte einer vorausschauenden Berichterstattung. In: Shareholder Value Reporting: Veränderte Anforderungen an die Berichterstattung börsennotierter Unternehmen, hrsg. von Müller, Michael/Leven, Franz-Josef, Wien, S. 293-312.

Kim, Jeong-Yoo (1996): Cheap talk and reputation in repeated pretrial negotiation. In: Rand Journal Of Economics, Vol. 27, S. 787-802.

Kim, Young-Gil/Everest, Gordon C. (1994): Building an IS architecture: Collective Wisdom from the field. In: Information \& Management, Vol. 26, S. 1-11.

Kimball, Ralph (1996): The Data Warehouse Toolkit: Practical Techniques for Building Dimensional Data Warehouses. New York et al.

Kimball, Ralph (1997): A Dimensional Modeling Manifesto. In: DBMS Online, Vol. 10, No. 9 1997. URL: http://www.dbmsmag.com/9708d15.html, Stand 09.04.99.

Kindlebenger, Charles P. (1983): Standards as Public, Collective and Private Goods. In: Kyklos, Vol. 36, S. 377-396.

King, Ronald/Waymire, Gregory (1993): Accounting Standard-Setting Institutions and the Govemance of Incomplete Contracts. In: Journal of Accounting, Auditing \& Finance, Vol. 8, S. 579-605.

Kirchgässner, Gebhard (1991): Homo oeconomicus. Tübingen.

Kirchhoff, Klaus R. (1997): Die Optik des Geschäftsberichts: Die visuelle Bilanz. In: Der Geschäftsbericht: Bedeutung, Inhalt, Sprache, Design, Servicefunktion, Praxisbeispiele, hrsg. von Baetge, Jörg/Kirchhoff, Klaus R., Wien et al., S. 61-91.

Kirchhoff, Klaus R./Klein, Sonja (1997): Multimediale Präsentationen. In: Der Geschäftsbericht: Bedeutung, Inhalt, Sprache, Design, Servicefunktion, Praxisbeispiele, hrsg. von Baetge, Jörg/Kirchhoff, Klaus R., Wien et al., S. 61-91.

Kleine-Doepke, Reiner (1981): Informationsökonomische Analyse der externen Rechnungslegung. Frankfurt am Main et al.

Knorr, Liesel (1998): In FASB's Footsteps? In: Accountancy, Vol. 122, July, S. 62.

Knutson Peter H./Napolitano, Gabrielle U. (1998): Criteria Employed by the AIMR Financial Accounting Policy Committee in Evaluating Financial Accounting Standards. In: Accounting Horizons, Vol. 12, S. 170-176. 
Kohler, Thomas/Beuchat, Alain (1996): Sicherheit in vernetzten Systemen: Veränderte betriebliche Aspekte und Sicherheitsanforderungen. In: Der Schweizer Treuhänder, 70. Jg., S. 673-683.

Koller, Heinrich (1999): Die Selbstregulierung im neuen Rechnungslegungs- und Revisionsrecht. In: Der Schweizer Treuhänder, 73. Jg., S. 427-434.

Köndgen, Johannes (1998): Ownership and Corporate Governance of Stock Exchanges. In: JITE, Vol. 154, S. 224-256.

Kothari, S. P./Zimmerman Jerold L. (1995): Price and Retum Models. In: Journal of Accounting and Economics, Vol. 20, S. 155-192.

Krämling, Markus (1998): Der Goodwill aus der Kapitalkonsolidierung: Bestandsaufnahme der Bilanzierungspraxis und deren Relevanz für die Aktienbewertung. Frankfurt am Main et al.

Krause, Clemens (1993): Kreditwürdigkeitsprüfung mit Neuronalen Netzen. Düsseldorf

Krause, Jürgen/Herfurth, Matthias/Marx, Jutta (Hrsg.) (1996): Herausforderungen an die Informationswirtschaft: Informationsverdichtung, Informationsbewertung und Datenvisualisierung; Proceedings des 5. Internationalen Symposiums für Informationswissenschaft (ISI '96). Konstanz.

Kreps, David M. (1990): A Course in Microeconomic Theory. New York et al.

Krisement, Vera M. (1994): Ansätze zur Messung des Harmonisierungs- und Standardisierungsgrades der externen Rechnungslegung. Frankfurt am Main et al.

Kronstein, Heinrich/Claussen, Carsten P. (1960): Publizität und Gewinnverteilung im neuen Aktienrecht. Frankfurt am Main.

Kropff, Bruno (1993): Publizität. In: HWR, hrsg. von Chmielewicz, K/Schweizer, M, 3. Auflage, Stuttgart, Sp. 1669-1678.

Küffner, Peter/Hock, Burkhard (1998): Internationalisierung der Rechnungslegung aus der Sicht mittelständischer Unternehmen. In: BFuP, 50. Jg., S. 57-76.

Kuhlen, Rainer (2000): Vertrauenssicherung auf elektronischen Märkten. In: WISU, 29. Jg., S. 220-226.

Kuhner, Christoph (1998): Verfügungsrechte an Unternehmensinformationen: Die Verrechtlichung des Informationsflusses zwischen Unternehmen und Kapitalmarkt im Blickfeld ökonomischer Analysen. Habilitation München.

Kunz, Harald (1985): Marktsystem und Information: „Konventionelle Unwissenheit“ als Quelle von „Ordnung“. Tübingen. 
Küpper, Hans-Ulrich (1995): Unternehmensplanung und -steuerung mit pagatorischen oder kalkulatorischen Erfolgsrechnungen. In: ZfbF Sonderheft 34/95, S. 19-50.

Kusserow, Berthold/Hofmann, Mark J. (1998): Drittes Finanzmarktförderungsgesetz. In: BBK, o.Jg., Fach 2, S. 1139-1150.

Kusterer, Frank (1998): Online Analytical Processing. In: WiSt, 27. Jg., S. 207-209.

Küting, Karlheinz/Lorson, Peter (1998): Konvergenz von intemen und externen Rechnungswesen: Anmerkungen zu Strategien und Konfliktfeldern. In: WPg, 51. Jg., S. 483-493.

Küting, Karlheinz/Mohren, Stephan (1992): Jahresabschlußpublizität und Datenbanken: Die Offenlegung und Analyse von Jahresabschlüssen mit Hilfe von Datenbanken. In: BB, 47. Jg., Beilage 2 zu Heft 3/1992, S. 1-14.

Labhart, Peter/Volkart, Rudolf (1997): Das Internet als Medium für Investor Relations: $\mathrm{Zu}$ den Charakteristiken und zum Potential dieses neuen Mediums. In: Der Schweizer Treuhänder, 71. Jg., S. 515-524.

Lackes, Richard (1997): DV-orientierte Umsetzung von Kostenrechnungssystemen auf der Basis von Datenbankmodellen. In: Kostenmanagement: Neuere Konzepte und Anwendungen, hrsg. von Freidank, Carl-Christian/Götze, Uwe/Huch, Burkhard/Weber, Jürgen, Berlin et al., S. 357-378.

Laitinen, Teija/Kankaanpää, Maria (1999): Comparative analysis of failure prediction models: the Finnish case. In: EAR, Vol. 8, S. 67-92.

Lamont, Owen (1998): Earnings and Expected Returns. In: JoF, Vol. 53, S. 1563-1587.

Lanza, Richard B. (1998): Take My Manual Audit, please. In: JoA, Vol. 185, June, S. 3336.

La Porta, Rafael/Lopez-de-Silanes, Florencio/Shleifer, Andrei/Vishny, Robert W. (1998): Law and Finance. In: Journal of Political Economy, Vol. 106, S. 11131155.

Laupenmühlen, Michael/Münz, Sandra M. (1998): Die neue SEC-Berichtsvorschrift zum Marktrisiko. In: DB, 51. Jg., S. 2025-2032.

Lawrence, David B. (1999): The Economic Value of Information. New York et al.

Lee, Lucy C./Bedford, Norton M. (1969): An Information Theory Analysis of the Accounting Process. In: AccRev., Vol. 43, S. 256-275. 
Leech, Stewart/Mepham, Michael J. (1991): The development of matrix-based accounting. In: IT and accounting: The impact of information technology, hrsg. von Williams, Bernard C./Spaul, Barry. London et al., S. 127-148.

Leffson, Ulrich (1988): Wirtschaftsprüfung. 4. Auflage, Wiesbaden.

Leftwich, Richard (1980): Market failure fallacies and accounting information. In: Journal of Accounting and Economics, Vol. 2, S. 193-211.

Leftwich, Richard (1983): Accounting Information in Private Markets: Evidence from Private Lending Agreements. In: AccRev., Vol. 58, S. 23-42.

Leland, Hayne E./Pyle, David H. (1977): Informational Asymmetries, Financial Structure, and Financial Intermediation. In: JoF, Vol. 32, S. 371-387.

Lenel, Hans O. (1968): Vollständiger und freier Wettbewerb als Leitbilder für die Wettbewerbspolitik gegenüber mächtigen Unternehmen. In: Wirtschaftsordnung und Staatsverfassung: Festschrift für Franz Böhm zum 80. Geburtstag, S. 317-340.

Lenenbach, Markus (1999): Reformen des U.S.-amerikanischen Kapitalanlagerechts: Abwehr mißbräuchlicher Schadensersatzklagen und ein "safe harbor" für Unternehmensprognosen. In: WM, 53. Jg., S. 1393-1402.

Lenk, Thomas/Teichmann, Volkmar (1999): Arrows Unmöglichkeitstheorem. In: WISU, 28. Jg., S. 866-870.

Lenz, Hansrudi/Ostrowski, Markus (1999): Der Markt für Abschlußprüfungen bei börsennotierten Aktiengesellschaften. In: DBW, 59. Jg., S. 397-411.

Lev, Baruch (1968): The Aggregation Problem in Financial Statements: An Informational Approach. In: JAR, Vol. 6, S. 247-261.

Lev, Baruch (1988): Toward a Theory of Equitable and Efficient Accounting Policy. In: AccRev., Vol. 63, S. 1-22.

Lev, Baruch/Ohlson, James A. (1982): Market-Based Empirical Research in Accounting: A Review, Interpretation, and Extension. In: JAR Supplement, Vol. 20, S. 249322.

Lev, Baruch/Thiagarajan, Ramu (1993): Fundamental Information Analysis. In: JAR, Vol. 31, S. 190-215.

Lev, Baruch/Zarowin, Paul (1999): The Boundaries of Financial Reporting and How to Extend Them. In: JAR, Vol. 37, S. 353-385.

Leuz, Christian/Deller, Dominic/Stubenrath, Michael (1998): An International Comparison of Accounting-Based Payout Restrictions in the United States, United 
Kingdom and Germany. In: Accounting and Business Research, Vol. 28, S. 111129.

Levine, Ross/Zervos, Sara (1998): Stock Markets, Banks, and Economic Growth. In: AER, Vol. 88, S. 537-558.

Levitt, Arthur (1998): The Importance of High Quality Accounting Standards. In: Accounting Horizons, Vol. 12, S. 79-82.

Lieberman, Arthur Z./Whinston, Andrew B. (1975): A Structuring of an EventsAccounting Information System. In: AccRev., Vol. 50, S. 246-258.

Liener, Gerhard (1992): Intemationale Unternehmen brauchen eine globalisierte Rechnungslegung. In: ZfB, 62. Jg., S. 269-292.

Lindenberg, Siegwart (1998): The Cognitive Turn in Institutional Analysis: Beyond NIE and NIS? In: JITE, Vol. 154, S. 716-727.

Linnhoff, Ulrich/Pellens, Bernhard (1994): Kreditwürdigkeitsprüfung mit den neuen Jahresabschlußkennzahlen des Bundesaufsichtsamtes für das Versicherungswesen (BAV). In: DB, 47. Jg., S. 589-594.

Löffler, Gunter (1998): Der Beitrag von Finanzanalysten zur Informationsverarbeitung: Eine empirische Untersuchung zum deutschen Aktienmarkt. Wiesbaden.

Löffler, Gunter (1999): Die Verarbeitung von Gewinnprognosen am deutschen Aktienmarkt. In: ZfbF, 51. Jg., S. 128-147.

Loscocco, Peter A./Smalley, Stephen D./Muckelbauer, Patrick A./Taylor, Ruth C./Turner, S. Jeff/Farell, John F. (1998): The Inevitability of Failure: The Flawed Assumption of Security in Modem Computer Environments. URL: http://csrc.nist.gov/nissc/1998/proceedings/paperF1.pdf, Stand 4.11.99.

Louwers, Timothy J./Pasewark, William R./Typpo, Eric W. (1998): Silicon Valley meets Norwalk. In: JoA, Vol. 186, August, S. 20-26.

Ludewig, Rainer/Ludewig-Husheer, Bettina (1999): Der „wahre“, „wirkliche“ Wert im Gesellschaftsrecht. In: Festschrift für Bernhard Großfeld zum 65. Geburtstag, hrsg. von Hübner, Ulrich/Ebke, Werner F., Heidelberg, S. 713-726.

Luippold, Hans-Peter/Wagner, Stefan (1996): Internet und Revision://Revision im Internet. In: Interne Revision, 31. Jg., S. 192-200.

Lundholm, Russell J. (1991): Public Signals and the Equilibrium Allocation of Private Information. In: JAR, Vol. 29, S. 322-349. 
Lusk, Edward J, (1973): Cognitive Aspects of Annual Reports: Field Independence/Dependence. In: JAR Supplement, Vol. 11, S. 191-202.

Lutter, Marcus (2000): ECLR: Das Europäische Unternehmensrecht im 21. Jahrhundert. In: ZGR, 29. Jg., S. 1-18.

Lymer, Andrew (1997): The use of the Internet for Corporate Reporting: A discussion of the issues and survey of current usage in the UK. In: Journal of Financial Information Systems, Accepted Paper 1997, URL: http://www.shu.ac.uk/schools/ fsl/fisjnl/vol1996/pprs1997/lymer97.htm, Stand 15.11.1999.

Lymer, Andrew (1999): The Internet and the future of corporate reporting in Europe. In: EAR, Vol. 8, S. 289-301.

Lymer, Andrew/Debreceny, Roger/Gray, Glen L./Raman, Asheq (1999): Business Reporting on the Internet: A Report Prepared for the International Accounting Standards Committee. London.

Lymer, Andrew/Tallberg, Anders (1997): Corporate Reporting and the Internet - a survey and commentary on the use of the WWW in corporate reporting in the UK and Finland. Working Paper präsentiert auf dem 20. EAA Kongreß in Graz April 1997. URL: http://www.summa.org.uk/corp/papers/lymer/lymereaa.htm, Stand 15.11.1999.

Macneil, Ian R. (1974): The Many Futures of Contracts. Southern California Law Review, Vol. 47, S. 691-816.

Macneil, Ian R. (1978): Contracts: Adjustment of long-term economic relations under classical, neoclassical and relational contract law. In: Northwestem University Law Review, Vol. 72, S. 854906.

Mahoney, Paul G. (1997): The Exchange as Regulator. In: Virginia Law Review, Vol. 83, S. $1453-1500$.

Markus, Hugh B. (1996): Haftungsbeschränkungen gegenüber dem Abschlußprüfer in den USA. In: WPK-Mitteilungen, 35. Jg., S. 93-94.

Marshall, John M. (1974): Private Incentives and Public Information. In: AER, Vol. 64, S. 373-390.

Marshall, Ronald M. (1972): Determining an Optimal Accounting Information System for an Unidentified User. In: JAR, Vol. 10, S. 286-307.

Marston, Claire and Leow, C.Y. (1998): Financial Reporting on the Internet by leading UK companies. Working Paper präsentiert auf dem 21. EAA Kongreß in Ant- 
werpen April 1998. URL: http://www.summa.org.uk/corp/papers/marston/ marston.html, Stand 15.11.1999.

Marx, Franz-Jüngen/Scharenberg, Sigrun (1999): Neue Sanktionen bei Verstößen gegen die Jahresabschlußpublizität. In: StuB, 1. Jg., S. 1138-1145.

Mason Edward S. (1939): Price and Production Policies of Large-Scale Enterprise. In: AER Supplement, Vol. 29, S. 61-74.

Mason Edward S. (1949): The Current State of the Monopoly Problem in the United States. In: Harvard Law Review, Vol. 62, S. 1265-1285.

Mason, Richard O./Mitroff, Ian I. (1973): A Program for Research on Management Information Systems. In: Management Science, Vol. 19, S. 475-487.

Mattessich, Richard (1964): Accounting and Analytical Models: Measurement and Projection of Income and Wealth in the Micro- and Macro-Economy. Homewood.

Mattheus, Daniela (1999): Die gewandelte Rolle des Wirtschaftsprüfers als Partner des Aufsichtsrats nach dem Kon'TraG. In: ZGR, 28. Jg., S. 682-714.

Matthiessen, Günter/Unterstein, Michael (1997): Relationale Datenbanken und SQL: Konzepte der Entwicklung und Anwendung. Bonn et al.

Maug, Ernst (1998): Large Shareholders as Monitors: Is There a Trade-off between Liquidity and Control? In: JoF, Vol. 53, S. 65-98.

Maute, Hanspeter (1999): Börsenfähigkeit mittelständischer Unternehmen: Empirische Studie am Neuen Markt Frankfurt und Prädikatsmarkt München. In: DStR, 37. Jg., S. 687-692.

Mauldin, Elaine G./Ruchala, Linda V. (1999): Towards a meta-theory of accounting information systems. In: Accounting, Organizations and Society, Vol. 24, S. 317331.

May, Robert G./Sundem Gary L. (1976): Research for Accounting Policy: An Overview. In: AccRev., Vol. 51, S. 747-763.

McCarthy, William E. (1977): A Relational Model for Events-Based Accounting Systems. Dissertation University of Massachusetts.

McCarthy, William E. (1979): An Entity-Relationship View of Accounting Models. In: AccRev, Vol. 54, S. 667-686.

McCarthy, William E. (1982): The REA Accounting Model: A Generalized Framework for Accounting Systems in a Shared Data Environment. In: AccRev., Vol. 57, S. 554578 . 
McEwan, Ruth A./Hunton, James E. (1999): Is Analyst Forecast Accuracy Associated with Accounting Information Use? In: Accounting Horizons, Vol. 13, S. 1-16.

McLeay, Stuart/Ordelheide, Dieter/Young, Steven (1999): Constitutent Lobbying and its Impact on the Development of Financial Reporting Regulation: Evidence from Germany. Erscheint demnächst in Accounting, Organizations and Society, Vol. 24.

Meier, Johann C. (1992): Die Entstehung des Börsengesetzes vom 22. Juni 1896, St. Katharinen.

Meisel, Bernd S. (1997): Moderne Informations- und Kommunikationsmittel in der Praxis der Wirtschaftsprüfer und vereidigten Buchprüfer. In: WPK-Mitteilungen 2/1997, 36. Jg., S. 113-127.

Mennenöh, Hartwig (1984): Prognosepublizität: Vorschläge zur ungewißheitsorientierten Gestaltung einer prospektiven Berichterstattung von Unternehmen an externe Adressaten. Bochum.

Merkt, Hanno (1991): US-amerikanisches Gesellschaftsrecht. Heidelberg.

Merton, Robert C. (1987): A simple model of capital market equilibrium with incomplete information. In: JoF, Vol. 42, S. 483-510.

Mikhail, Michael B./Walther, Beverly R./Willis, Richard H. (1999): Does Forecast Accuracy Matter to Security Analysts? In: AccRev, Vol. 74, S. 185-200.

Milgrom, Paul R. (1981): Good News and Bad News: Representation Theorems and Applications. In: Bell Joumal of Economics, Vol. 12, S. 380-391.

Miller, Paul B. W./Redding, Rodney J./Bahnson, Paul R. (1998): The FASB: The People, the Process, and the Politics. 4. Auflage, Boston et al.

Miller, Herbert E. (1974): Discussion of Opportunities and Implications of the Report on Objectives of Financial Statements. In: Studies of Financial Accounting Objectives, JAR Supplement, Vol. 12, S. 18-20.

Möhlmann, Thomas/Bartels, Dörte (1998): Zur Aussagefähigkeit von Planbilanzen und anderen Instrumenten prognoseorientierter Berichterstattung. In: BBK, o. Jg., Fach 29, S. 903-914.

Mohren, Stephan W. (1992): Der Jahresabschluß - Was leisten Datenbanken?: Offenlegung und Analyse von Jahresabschlüssen mit Hilfe von Datenbanken. Stuttgart.

Möller, Hans P. (1985): Die Informationseffizienz des deutschen Aktienmarktes: Eine Zusammenfassung und Analyse empirischer Untersuchungen. In: ZfbF, 37. Jg., S. 500-518. 
Möller, Hans P. (1986): Bilanzkennzahlen und Ertragsrisiken des Kapitalmarkts: Eine empirische Untersuchung des Ertragsrisiko-Informationsgehaltes von Bilanzkennzahlen deutscher Aktiengesellschaften, Stuttgart.

Möller, Hans P./Keller, Erich (1999): Financial Reporting and the Stock Market in Germany. In: Empirical Research on the German Capital Market, hrsg. von Bühler, Wolfgang/Hax, Herbert/Schmidt, Reinhard, Heidelberg et al., S. 135148.

Möllers, Thomas M. J. (1999): Kapitalmarkttauglichkeit des deutschen Gesellschaftsrechts: Kritik am sachlichen und persönlichen Anwendungsbereich der Novellierung von AktG und $\mathrm{HGB}$ im Bereich von Rechnungslegung, Corporate Governance und Transparenz. In: AG, 44. Jg., S. 433-442.

Morel, Mindy (1999): Multi-Lagged Specification of the Ohlson Model. In: Journal of Accounting, Auditing and Finance, Vol. 14, S. 147-161.

Moxter, Adolf (1962): Der Einfluß von Publizitätsvorschriften auf das unternehmerische Verhalten. Köln et al.

Moxter, Adolf (1966): Die Grundsätze ordnungsmäßiger Bilanzierung und der Stand der Bilanztheorie. In: ZfbF, 18. Jg., S. 28-59.

Moxter, Adolf (1984): Bilanzlehre: Band I: Einführung in die Bilanztheorie. 3. Auflage, Wiesbaden.

Moxter, Adolf (1998): Deutsches Rechnungslegungs Standards Committee: Aufgaben und Bedeutung. In: DB, 51. Jg., S. 1425-1428.

Mucksch, Harry/Behme, Wolfgang (1998): Das Data Warehouse-Konzept als Basis einer unternehmensweiten Informationslogistik. In: Das Data Warehouse-Konzept, hrsg. von Mucksch, Harry/Behme, Wolfgang, 3. Auflage, Wiesbaden, S. 33-100.

Mues, Jochen (1997): Hundert Jahre deutsches Börsengesetz: Zum Inkrafttreten des Börsengesetzes am 1. Januar 1897. In: ZBB, 9. Jg., S. 15-24.

Mulherin, J. Harold/Netter, Jeffry M./Overdahl, James A. (1991): Prices are Property: The Organization of Financial Exchanges from a Transaction Cost Perspective. In: Journal of Law \& Economics. Vol. 34, S. 591-644.

Müller, Jochen (1999): Transformation operativer Daten zur Nutzung im Data Warehouse. Dissertation Ruhr-Universität Bochum, Veröffentlichung in Vorbereitung. 
Müller, Jochen (1999a): Datenbeschaffung im Data Warehouse. In: Analytische Informationssysteme, hrsg. von Chamoni, Peter/Gluchowski, Peter, 2. Auflage, Berlin et al., S. 95-117.

Müller-Bader, Peter (1989): Publizität und neue Medien: Wird der Jahresabschluß zu einem neuen Informationsinstrument? In: BFuP, 41. Jg., S. 231-244.

Müller-Merbach, Heiner (1997): Kostenrechnung und Datenbanken. In: Das Rechnungswesen im Spannungsfeld zwischen strategischem und operativen Management: Festschrift für Marcell Schweitzer zum 65. Geburtstag, hrsg. von Küpper, Hans-Ulrich/Troßmann, Emst, Berlin, S. 495-515.

Münstermann, Hans (1966): Dynamische Bilanz: Grundlagen, Weiterentwicklung und Bedeutung in der neuesten Bilanzdiskussion. In: ZfbF, 18. Jg., S. 512-531.

Musgrave, Richard A./Musgrave, Peggy B./Kullmer, Lore (1994): Die öffentlichen Finanzen in Theorie und Praxis: 1. Band. 6. Auflage, Tübingen.

Nelson, Phillip (1970): Information and Consumer Behavior, Journal of Political Economy, Vol. 78, S. 311-329.

Newman, Paul/Sansing, Richard (1993): Disclosure Policies with Multiple Users. In: JAR, Vol. 31, S. 92-112.

Neus, Werner (1998): Einführung in die Betriebswirtschaftslehre aus institutionenökonomischer Sicht. Tübingen.

Noack, Ulrich (1998): Moderne Kommunikationsformen vor den Toren des Unternehmensrechts. In: ZGR, 27. Jg., S. 592-616.

North, Douglass C. (1990): Institutions, Institutional Change and Economic Performance. Cambridge.

Nowak, Klaus (1999): Ökonomik, Erkenntnisfortschritt und theoretische Integration: Eine methodische Analyse der Entwicklungsperspektiven der evolutorischen Ökonomik. Lohmar et al.

Nürnberger, Christian (1999): Freie Software für alle. In: Süddeutsche Zeitung, Nr. 185 vom 13.09.1999, S. 13.

Nußdorfer, Richard (1999): Moderne Technologie für leistungsfähige DW-Datenbanken: Datenbank-Technologie und Performance. In: Analytische Informationssysteme, hrsg. von Chamoni, Peter/Gluchowski, Peter, 2. Auflage Berlin et al., S. 213-230.

O.V. (1997): SEC Adopts New Disclosure Rules for Derivatives. In: JoA, Vol. 183, April, S. 9-10. 
O.V. (1999): Delay on Derivatives Standard. In: JoA, Vol. 188, August, S. 10-11.

O.V. (2000): Online Financial Reports Show Problems and Promise. In: JoA, Vol. 189, February, S. 16-21.

Oberender, Peter/Cachej, Monty (1999): Wettbewerb der Systeme: Chancen und Risiken für die europäische Integration. In: WiSt, 28. Jg., S. 577-581.

Oberender, Peter/Daumann, Frank (1995): Industriepolitik. 3. Auflage, München.

Oehler, Karsten (1998): OLAP-Konzeption einer geschlossenen Kostenrechnung. In: krpSonderheft, 42. Jg., Heft 2, S. 85-95.

Oelsnitz, Dietrich von der (1999): Mikropolitik in Organisationen. In: WISU, 28. Jg., S. $710-716$.

Oestreicher, Andreas/Spengel, Christoph (1999): International Accounting Standards, Maßgeblichkeitsprinzip und Besteuerung. In: DB, 52. Jg., S. 593-600.

O'Hara, Maureen (1995): Market Microstructure Theory. Cambridge et al.

Ohlendorf, Thomas (1998): Objektorientierte Datenbanksysteme für den Einsatz im Data Warehouse-Konzept. In: Das Data Warehouse-Konzept, hrsg. von Mucksch, Harry/Behme, Wolfgang, 3. Auflage, Wiesbaden, S. 219-241.

Ohlson, James A. (1995): Eamings, Book Values, and Dividends in Equity Valuation. In: CAR, Vol. 11, S. 661-687.

Ohlson, James A./Buckman, A. Gregory (1980): Toward a Theory of Financial Accounting. In: JoF, Vol. 35, S. 537-547.

Ohlson, James A./Buckman, A. Gregory (1981): Toward a Theory of Financial Accounting: Welfare and Public Information. In: JAR, Vol. 19, S. 399-433.

Ohlson, James A./Penman, Stephen H. (1992): Disaggregated Accounting Data as Explanatory Variables for Returns. In: Journal of Accounting, Auditing \& Finance, Vol. 7, S. 553-573.

Ohlson, James A./Zhang, Xiao-Jun (1998): Accrual Accounting and Equity Valuation. In: JAR Supplement, Vol. 36, S. 85-111.

Ollmert, Clemens/Schinzer, Heiko (2000): Software-Agenten im Internet. In: WISU, 29. Jg., S. 213-220.

Ordelheide, Dieter (1995): Brauchen wir für die Unternehmensüberwachung mehr Publizität? In: Corporate Governance: Unternehmensüberwachung auf dem Prüfstand, hrsg. von Picot, Amold. Stuttgart. 
Ordelheide, Dieter (1997): Regulierung der Rechnungslegung: Ökonomische Zwänge und kulturelle Unterschiede. In: Interkulturelles Management: Theoretische Fundierung und funktionsbereichspezifische Konzepte, hrsg. von Engelhard, Johann. Wiesbaden, S. 235-259.

Ordelheide, Dieter (1998): Rechnungslegung und internationale Aktienanalyse In: Rechnungswesen als Instrument für Führungsentscheidungen: Festschrift für Prof. Dr. Dr. h. c. Adolf G. Coenenberg zum 60. Geburtstag, hrsg. von Möller, H. P./Schmidt, F., Stuttgart, S. 505-524.

Ordelheide, Dieter (1998a): Zur politischen Ökonomie der Rechnungslegung. In: ZfbF Sonderheft 40/98, Rechnungslegung und Steuern International, hrsg. von Ballwieser, Wolfgang/Schildbach, Thomas, Düsseldorf, S. 1-16.

Ordelheide, Dieter (1999): Rechnungslegung im digitalen Zeitalter. In: ZfbF Sonderheft 41/99; Rechnungswesen und Kapitalmarkt: Beiträge anläßlich eines Symposiums zum 70. Geburtstag von Prof. Dr. h.c. mult. Walther Busse von Colbe, hrsg. von Gebhardt, Günther/Pellens, Bernhard. Düsseldorf, S. 229-253.

Ordelheide, Dieter (1999a): Rechnungslegung im Zeichen globaler Finanzmärkte. In: Globale Finanzmärkte und Europäische Währungsunion: Konsequenzen für die Geschäftsbereiche und ihre Steuerung, hrsg. von Krahnen, Jan P./Rudolph, Bernd, Frankfurt am Main, S. 81-99.

Osbahr, Wilhelm (1923): Die Bilanz vom Standpunkt der Unternehmung: Die bisherige und zukünftige Gestaltung des Bilanzproblems. 3. Auflage, bearbeitet von Nicklisch, H., Berlin et al..

Ostrom, Elinor (1985): An Agenda for the study of Institutions. In: Public Choice, Vol. 48 , S. 3-25.

Ostrom, Elinor (1990): Governing the Commons: The Evolution of Institutions of Collective Action. Cambridge.

Ostrowski, Markus (1999): Die Offenlegung der Jahresabschlüsse von im Freiverkehr an der Börse gehandelten Aktiengesellschaften. In: ZBB, 11. Jg., S. 19-26.

Otley, David T./Diaz, Francisco J. B. (1982): Accounting Aggregation and Decision Making Performance: An Experimental Investigation. In: JAR, Vol. 20, S. 171188.

Ou, Jane A. (1990): The information content of non-eamings accounting numbers as earnings predictors. In: JAR, Vol. 28, S. 144-163. 
Ou, Jane A./Penman, Stephen A. (1989): Financial Statement Analysis and the Prediction of Stock Returns. In: Journal of Accounting and Economics, Vol. 11, S. 295-329.

Pagell, Ruth A. (1995): EDGAR: Electronic Data Gathering and Receiving. In: Bus. Info. Rev., Vol. 11, S. 56-68.

Pagano, Marco/Panetta, Fabio/Zingalis, Luigi (1998): Why Do Companies Go Public? An Empirical Analysis. In: JoF, Vol. 53, S. 27-64.

Pannen, Michael (1999): Meßtheoretische Grundprobleme des Maßgeblichkeitsprinzips. Dissertation Ruhr-Universität Bochum.

Pape, Jochen (1997): A Milestone in German Accounting. In: Accountancy, Vol. 119, May, S. 58-59.

Paton, W. A. /Littleton, A. C. (1940): An Introduction to Corporate Accounting Standards. Columbus 1940, 12. Nachdruck, Ann Arbor 1965.

Pechtl, Hans (2000): Die Prognosekraft des Prognoseberichts: Eine empirische Untersuchung am Beispiel deutscher Aktiengesellschaften. In: ZfbF, 52. Jg., S. 141-159.

Peemöller, Volker H./Finsterer, Hans/Neubert, Martin (1999): Bilanzierung von Unternehmen des Neuen Markts nach IAS und US-GAAP: Analyse der Ergebnisse einer Umfrage. In: BB, 54. Jg., S. 1103-1107.

Pellens, Bernhard (1989): Der Informationswert von Konzemabschlüssen: Eine empirische Untersuchung deutscher Börsengesellschaften. Wiesbaden.

Pellens, Bernhard (1991): Ad-hoc-Publizitätspflicht des Managements börsennotierter Mutterunternehmen nach $₫ 44$ a BörsG. In: AG, 36. Jg., S. 62-69.

Pellens, Bernhard (1994): Aktionärsschutz im Konzern: Empirische und theoretische Analyse der Reformvorschläge der Konzernverfassung. Wiesbaden.

Pellens, Bernhard (1995): Publizität. In: HWF, hrsg. von Gerke, W./Steiner, M., 2. Auflage, Stuttgart, Sp. 1589-1600.

Pellens, Bernhard (1999): Internationale Rechnungslegung. 3. Auflage, Stuttgart.

Pellens, Bernhard/Bonse, Andreas (1999): Annäherung des deutschen Aktionärsschutzes an US-amerikanische Vorstellungen. In: Festschrift für Bernhard Großfeld zum 65. Geburtstag, hrsg. von Hübner, Ulrich/Ebke, Werner F., Heidelberg, S. 851874.

Pellens, Bernhard/Bonse, Andreas/Gassen, Joachim (1998): Perspektiven der deutschen Konzernrechnungslegung - Auswirkungen des Kapitalaufnahmeerleichterungsge- 
setzes und des Gesetzes zur Kontrolle und Transparenz im Unternehmensbereich. In: DB, 51. Jg., S. 785-792.

Pellens, Bernhard/Crasselt, Nils/Rockholtz, Carsten (1998): Wertorientierte Entlohnungssysteme für Führungskräfte: Anforderungen und empirische Evidenz. In: Unternehmenswertorientierte Entlohnungssysteme, hrsg. von Pellens, Bernhard, Stuttgart, S. 1-28.

Pellens, Bernhard/Fülbier, Rolf Uwe (1994): Publizitätspflichten nach dem Wertpapierhandelsgesetz: Ausgestaltung unter Berücksichtigung anglo-amerikanischer Regulierungen. In: DB, 47. Jg., S. 1381-1388.

Pellens, Bernhard/Fülbier, Rolf Uwe/Gassen, Joachim (1997): Unternehmenspublizität unter veränderten Marktbedingungen. In: Controlling und Rechnungswesen im internationalen Wettbewerb, hrsg. von Coenenberg, Adolf G./Börsig, Clemens. Stuttgart, S. 55-69.

Pellens, Bernhard/Fülbier, Rolf Uwe/Gassen, Joachim (1999): Herausforderungen an die Rechnungslegungs- und Publizitätsregulierung. In: Intemationale Rechnungslegung: Festschrift für Professor Dr. Claus-Peter Weber zum 60. Geburtstag, hrsg. von Küting, Karlheinz/Langenbucher, Günther. Stuttgart, S. 543-558.

Pellens, Bernhard/Gassen, Joachim (1998): Die Bereitstellung von Rechnungslegungssystemen: Eine Aufgabe des Staates oder des Marktes? In: Rechnungswesen als Instrument für Führungsentscheidungen: Festschrift für Prof. Dr. Dr. h. c. Adolf G. Coenenberg zum 60. Geburtstag, hrsg. von Möller, H. P./Schmidt, F., Stuttgart, S. 633-650.

Pellens, Bernhard/Tomaszewski, Claude (1999): Kapitalmarktreaktionen auf den Rechnungslegungswechsel zu IAS bzw. US-GAAP. In: ZfbF Sonderheft 41/99; Rechnungswesen und Kapitalmarkt: Beiträge anläßlich eines Symposiums zum 70. Geburtstag von Prof. Dr. h.c. mult. Walther Busse von Colbe, hrsg. von Gebhardt, Günther/Pellens, Bernhard. Düsseldorf, S. 199-228.

Peltzman, Sam (1976): Towards a more General Theory of Regulation. In: Journal of Law and Economics, Vol. 19, S. 211-240.

Penndorf, Balduin (1913): Geschichte der Buchhaltung in Deutschland. Leipzig.

Penndorf, Balduin (1930): Inventar, Bilanz und Bewertung in der italienischen Buchhaltung des 14. Jahrhunderts. In: ZfhF, 24. Jg., S. 489-495.

Penno, Mark C. (1997): Information quality and voluntary disclosure. In: AccRev., Vol. 72, S. $275-284$. 
Perlitz, Manfred (1973): Die Prognose des Unternehmenswachstums aus Jahresabschlüssen deutscher Aktiengesellschaften. Wiesbaden.

Perlitz, Manfred/Seger, Frank/Ackermann, Niels (1999): Eignung des Neuen Markts für die Desinvestition von Venture Capital-Beteiligungen im Vergleich zu alternativen Börsensegmenten. In: ZfB-Ergänzungsheft 3/99, 69. Jg., S. 107-130.

Petravick, Simon/Gillett, John W. (1998): Distributing Eamings Reports on the Internet. In: Management Accounting, 90. Jg., October, S. 54-56.

Pfaff, Dieter (1989): Zur allokativen Begründung von Ausschüttungsregeln: Ein Beitrag zur Diskussion um $₫ 58$ AktG. In: ZfbF, 41. Jg., S. 1013-1028.

Pfaff, Dieter/Bärtel, Oliver (1998): Externe Rechnungslegung, internes Rechnungswesen und Kapitalmarkt. In: ZfbF, 50. Jg., S. 757-777.

Pfeiffer, Ray J. Jr./Elgers, Pieter T./Lo, May H./Rees, Lynn L. (1998): Additional Evidence on the Incremental Information Content of Cash Flows and Accruals: The Impact of Errors in Measuring Market Expectations. In: AccRev., Vol. 73, S. 373-385.

Picot, Arnold/Dietl, Helmut (1994): Informations(de)regulierung am Kapitalmarkt aus institutionenökonomischer Sicht. In: Jahrbuch für Neue Politische Ökonomie, Band 13: Neue politische Ökonomie der Regulierung, Deregulierung und Privatisierung. Tübingen.

Picot, Arnold/Meier, Matthias (1994): Ansätze der Informationsmodellierung und ihre betriebswirtschaftliche Bedeutung. In: ZfbF, 46. Jg., S. 107-126.

Picot, Gerald/Land, Volker (1999): Going Public: Typische Rechtsfragen des Ganges an die Börse. In: DB, 52. Jg., S. 570-575.

Pirchegger, Barbara/Wagenhofer, Alfred (1999): Financial information on the Internet: a survey of the homepages of Austrian companies. In: EAR, Vol. 8, S. 383-395.

Platon (399-347 v. Chr.): Euthymedos. In: Gesammelte Werke, Band 1: Frühdialoge, Übertragen von Rudolf Rufener, Zürich et al., S. 267-329.

Poddig, Thorsten/Huber, Claus (1999): Data Mining und Knowledge Discovery in Databases. In: WiSt, 28. Jg., S. 663-666.

Pohlmann, Peter (1981): Die Wahl von Bewertungskonzeptionen im externen Jahresabschluss unter besonderer Berücksichtigung von Preisänderungen. Bochum.

Popper, Karl (1989): Logik der Forschung. 9. Auflage, Tübingen. 
Porter, Michael E. (1985): Competitive Advantage: Creating and Sustaining Superior Performance. New York et al.

Posner, Richard A. (1974): Theories of economic regulation. In: Bell Journal of Economics and Management Science, Vol. 5, S. 335-358.

Posner, Richard A. (1992): The Economic Analysis of Law. 4. Auflage, Boston et al.

Power, Michael (1997): The Audit Society: Rituals of Verification. Oxford.

Pownall, Grace/Schipper, Katherine (1999): Implications of Accounting Research for the SEC's Consideration of International Accounting Standards for U.S. Securities Offerings. In: Accounting Horizons, Vol. 13, S. 259-280.

Pratt, Ken C./Storrar, A. Colin (1997): UK Shareholders' Lost Access to Management Information. In: Accounting and Business Research. Vol. 27, S. 205-218.

Priest, George L. (1993): The Origins of Utility Regulation and the "Theories of Regulation" Debate. In: Journal of Law and Economics, Vol. 36, S. 289-323.

Puterill, Martin/Debreceny, Roger/Kerkin, Darien (1994): The Search for a Better Accounting System: The Overlooked Concern. In: Accounting Education for the 21st Century: The Global Challenges, hrsg. von Burns, Jane O./Needles, Belverd E. Jr., Oxford et al., S. 465-472.

Quassowski (1937): Die Grundzüge des neuen Aktienrechts. In: Deutsche Juristenzeitung, 99. Jg., S. 182-184.

Rajan, Madvah V./Sarath, Bharat (1996): Limits to Voluntary Disclosures in Efficient Markets. In: Journal of Accounting, Auditing and Finance, Vol. 11, S. 361-387.

Rappaport, Alfred (1986): Creating Shareholder Value. New York et al.

Rawls, John (1990): A Theory of Justice. Oxford et al.

Raymond, Eric S. (1998): The Cathedral and the Bazaar. URL: http://www.ssc.com/linux/Eric/cathedral-paper.ps, Stand 13.04.99.

Reither, Cheri L. (1998): What are the Best and the Worst Accounting Standards? In: Accounting Horizons, Vol. 12, S. 283-292.

Remmer, Werner/Theile, Carsten (1998): Die Auswirkungen von „KonTraG“ und „KapAEG“ auf die GmbH. In: GmbH Rundschau, 89. Jg., S. 909-915.

Revsine, Lawrence (1973): Replacement Cost Accounting. Englewood Cliffs.

Rhode, John G. (1972): Behavioral Science Methodologies with Application for Accounting Research: References and Source Materials. In: Report of the Committee on 
Research Methodology in Accounting, hrsg. von Committee on Research Methodology in Accounting, AccRev. Supplement, Vol. 47, S. 399-520, hier S. 494 504.

Ricardo, David (1817): Principles of Political Economy and Taxation. Great Mind Series, Amherst 1996.

Richter, Rudolf/Furubotn, Eirik (1996): Neue Institutionenökonomik: Eine Einführung und kritische Würdigung. Tübingen.

Rieger, Wilhelm (1928): Einführung in die Privatwirtschaftslehre. Nürnberg.

Rieger, Wolfgang (1995): SGML für die Praxis: Ansatz und Einsatz von ISO 8879; mit einer Einführung in HTML; enthält DOS-Diskette mit SGML-Parser. Berlin et al.

Riek, Werner (1998): Linux findet immer mehr Anhänger. In: HB, Nr. 248 vom 23.12.98, S. 56.

Rieter, Heinz (1994): Historische Schulen. In: Geschichte der Nationalökonomie, hrsg. von Issing, Otmar, 3. Auflage, München, S. 127-162.

Riley, John G. (1979): Informational Equilibrium. In: Econometrica, Vol. 47, S. 331-359.

Robie, Jonathan/Bartels, Dirk (1994): A Comparison between Relational and Object Oriented Databases for Object Oriented Application Development. White Paper. URL: http://www.poet.com/products/object_development_solutions/ white_papers/relational_vs_object/relational_vs_object.html, Stand 13.04.99.

Robisch, Martin/Treisch, Corinna (1997): Neuere Entwicklungen des Verhältnisses von Handelsbilanz und Steuerbilanz: Anhaltspunkte für eine Trendwende? In: WPg, 50. Jg., S. 156-169.

Rockholtz, Carsten (1999): Marktwertorientiertes Akquisitionsmanagement: Due Dilligence-Konzeption zur Identifikation, Beurteilung und Realisation akquisitionsbedingter Synergiepotentiale. Frankfurt am Main et al.

Röder, Klaus (1999): Auswirkungen von Meldungen deutscher Aktiengesellschaften. Lohmar et al.

Rogero, L. Hal (1998): Characteristics of High Quality Accounting Standards. In: Accounting Horizons, Vol. 12, S. 177-183.

Ross, Stephen A. (1979): Disclosure Regulations in Financial Markets: Implications of Modern Finance Theory. In: Issues in Financial Regulation, hrsg. von Edwards, Franklin D., New York, S. 177-200. 
Rothschild, Michael/Stiglitz, Joseph E. (1976): Equilibrium in Competitive Insurance Markets: An Essay on the Economics of Imperfect Information. In: Quarterly Journal of Economics, Vol. 90, S. 629-649.

Rückle, Dieter (1993): Bilanztheorie. In: HWR, hrsg. von Chmielewicz, K/Schweizer, M, 3. Auflage, Stuttgart, Sp. 249-261.

Samuelson, Paul A. (1954): The Pure Theory of Public Expenditure. In: Review of Economics and Statistics, Vol. 36, S. 387-289.

Sarath, Bharat (1996): Public Information Quality with Monopolistic Sellers. In: Games and EconomicBehavior, Vol. 16, S. 261-279.

Sarath, Bharat/Natarajan, Ramachandran (1996): Unobservable Risk Preferences and Value of Information in Financial Markets with Adverse Selection. In: Journal of Accounting, Auditing and Finance, Vol. 11, S. 197-222.

Sauerland, Dirk (2000): Wettbewerb zwischen Jurisdiktionen: Ein Modellvergleich. In: WiSt, 29. Jg., S. 90-95.

Savary, Jaques (1675): Le parfait négociant ou instruction général pur ce qui regarde le commerce de toute sorte de Marchandises, tant de France, que de pays etrangers. Nachdruck Düsseldorf 1993.

Scheer, August-Wilhelm (1997): Wirtschaftsinformatik: Referenzmodelle für industrielle Geschäftsprozesse. 7. Auflage, Berlin et al.

Scheffler, Eberhard (1999): Der Deutsche Standardisierungsrat: Struktur, Aufgaben und Kompetenzen. In: BFuP, 51. Jg., S. 407-417.

Scheffler, Eberhard (1999a): Internationale Rechnungslegung und deutsches Bilanzrecht. In: DStR, 37. Jg., S. 1285-1292.

Schefold, Bertram/Carstensen, Kristian (1994): Die klassische Politische Ökonomie. In: Geschichte der Nationalökonomie, hrsg. von Issing, Otmar, 3. Auflage, München, S. 63-87.

Scherer, Frederic M./Ross, David (1990): Industrial Market Structure and Economic Performance. 3. Auflage, Boston et al.

Schildbach, Thomas (1986): Jahresabschluß und Markt. Berlin et al.

Schildbach, Thomas (1996): Die Glaubwürdigkeitskrise der Wirtschaftsprüfer: Zu Intensität und Charakter der Jahresabschlußprüfung aus wirtschaftlicher Sicht. In: BFuP, 48. Jg., S. 1-22. 
Schildbach, Thomas (1998): Harmonisierung der Rechnungslegung: Ein Phantom. In: BFuP, 50. Jg., S. 1-22.

Schildbach, Thomas (1999): Das private Rechnungslegungsgremium gemäß § 342 HGB und die Zukunft der Rechnungslegung in Deutschland. In: DB, 52. Jg., S. 645652.

Schildbach, Thomas (1999a): Öffnung der Jahresabschlüsse für IAS und US-GAAP: Ein Fortschritt? In: StuB, 1. Jg., S. 421-428.

Schildbach, Thomas/Feldhoff, Michael (1993): Adressaten. In: HWR, hrsg. von Chmielewicz, Klaus/Schweizer, Marcell, 3. Auflage, Stuttgart, Sp. 30-36.

Schinzer, Heiko/Thome, Rainer (1999): Extensible Markup Language. In: WISU, 28. Jg., S. 208-215.

Schmalenbach, Eugen (1919): Grundlagen dynamischer Bilanzlehre. In: ZfhF, 13. Jg., S. 1-101.

Schmalenbach, Eugen (1962): Dynamische Bilanz. Bearbeitet von Bauer, Richard, 13. Auflage, Köln et al.

Schmidt, Karl-Heinz (1994): Merkantilismus, Kameralistik, Phyiokratie. In: Geschichte der Nationalökonomie, hrsg. von Issing, Otmar, 3. Auflage, München, S. 37-62.

Schmidt, Karsten (1997): Woher, Wohin? ADHGB, HGB und die Besinnung auf den Kodifikationsgedanken. In: ZGR, 161. Jg., S. 2-12.

Schmidt, Reinhard H. (1976): Aktienkursprognose. Wiesbaden.

Schmidt, Reinhard H. (1982): Rechnungslegung als Informationsproduktion auf nahezu effizienten Kapitalmärkten. In: ZfbF, 34. Jg., S. 728-748.

Schmidtchen, Dieter (1983): Property Rights, Freiheit und Wettbewerbspolitik. Tübingen.

Schmidtchen, Dieter (1991): Jenseits von Maximierung, Gleichgewicht und Effizienz: Neuland für die ökonomische Analyse des Rechts. In: Ökonomische Probleme des Zivilrechts: Beiträge zum 2. Travemünder Symposium zur ökonomischen Analyse des Rechts, 21.-24. März 1990, hrsg. von Ott, Claus/Schäfer, HansBernd, Berlin et al., S. 316-341.

Schmidt-Jortzig, Edzard (1997): Aktuelle Gesetzgebungsvorhaben im Wirtschaftsrecht: Eine Zwischenbilanz. In: WM, 51. Jg., S. 1043-1044.

Schmoller, Gustav von (1900): Grundriß der Allgemeinen Volkswirtschaftslehre: Erster, größerer Teil. Leipzig. 
Schneider, Dieter (1978): Maßgeblichkeit der Handelsbilanz für die Steuerbilanz und Besteuerung nach der Leistungsfähigkeit. In: BB, 33. Jg., S. 1577-1581.

Schneider, Dieter (1986): Ausweichhandlungen vor Regulierungen auf Finanzmärkten als Prüfstein wettbewerbspolitischer Konzepte. In: Ordo, Band 37, S. 155-181.

Schneider, Dieter (1993): Wider Insiderhandelsverbot und die Informationseffizienz des Kapitalmarkts. In: DB, 46. Jg., S. 1429-1435.

Schneider, Dieter (1993a): Geschichte der Buchhaltung und Bilanzierung. In: HWR, hrsg. von Chmielewicz, Klaus/Schweizer, Marcell, 3. Auflage, Stuttgart, Sp. 712721.

Schneider, Dieter (1995): Betriebswirtschaftslehre: Band 1: Grundlagen. 2. Auflage, München et al.

Schneider, Dieter (1996): Theorie der Unternehmung und Theorie der Rechnungslegung. In: BFuP, 48. Jg., S. 471-502.

Schneider, Dieter (1997): Betriebswirtschaftslehre: Band 2: Rechnungswesen. 2. Auflage, München et al.

Schneider, Dieter (1997a): Betriebswirtschaftslehre: Band 3: Theorie der Unternehmung. 2. Auflage, München et al.

Schneider, Dieter (1998): Marktwertorientierte Unternehmensführung: Pegasus mit Klumpfuß. In: DB, 51. Jg., S. 1473-1478.

Schön, Wolfgang (1997): Entwicklung und Perspektiven des Handelsbilanzrechts: vom ADHGB zum IASC. In: ZHR, 161. Jg., S. 133-159.

Schörner, Peter (1991): Gesetzliches Insiderhandelsverbot: Eine ordnungspolitische Analyse. Wiesbaden.

Schredelseker, Klaus/Kopetsch, Gerd/Maybüchen, Bernd (1986): Publizität und Unternehmensverfassung: Das Beispiel der französischen Sozialbilanz. Frankfurt et al.

Schulz, Wolfgang (1994): Das deutsche Börsengesetz: Die Entwicklungsgeschichte und wirtschaftlichen Auswirkungen des Börsengesetzes von 1896. Frankfurt am Main et al.

Schumann, Jochen (1992): Grundzüge der mikroökonomischen Theorie. 6. Auflage, Berlin et al.

Schumann, Matthias (1998): Aktuelle Entwicklungen im DV-gestützten Controlling. In: krp-Sonderheft, 42. Jg., Heft 2, S. 5-13. 
Schumann, Matthias/Itter, Ralf/Müller, Jörg/Stegemann und Stein, Ernst von (1997): Informationsagenten zur online-basierten Entscheidungsunterstützung am Beispiel einer Kreditversicherung. In: Information Management, 12. Jg., Heft 4, S. 30-37.

Schwab, Manfred (1999): Der Standardisierungsvertrag für das DRSC: Eine kritische Würdigung (Teil I und II). In: BB, 54. Jg., S. $731-738$ (Teil I) und S. 783-788 (Teil II).

Schwark, Eberhard (1978): Probleme der Unternehmenspublizität im Licht der 4. und 7. gesellschaftsrechtlichen EG-Richtlinie. In: AG, 23. Jg., S. 269-279.

Schwark, Eberhard (1987): Das neue Kapitalmarktrecht. In: NJW, 34. Jg., S. 2041-2048.

Schubert, Werner (1985): Die Entstehung des Aktiengesetzes vom 18. Juli 1884. In: Hundert Jahre modemes Aktienrecht: Eine Sammlung von Texten und Quellen zur Aktienrechtsreform 1884 mit zwei Einführungen, hrsg. von Schubert, Werner/Hommelhoff, Peter, Berlin et al., S. 1-52.

Segna, Ulrich (1999): Die Rechtsform deutscher Wertpapierbörsen: Anmerkungen zur Reformdiskussion. In: ZBB, 11. Jg., S. 144152.

Sell, Kirsten (1999): Die Aufdeckung von Bilanzdelikten bei der Abschlußprüfung: Berücksichtigung von Fraud \& Error nach deutschen und internationalen Vorschriften. Düsseldorf.

Sengupta, Partha (1998): Corporate Disclosure Quality and the Cost of Debt. In: AccRev., Vol. 73, S. 459-474.

Shachar, Ron/Nalebuff, Barry (1999): Follow the Leader: Theory and Evidence on Political Participation. In: AER, Vol. 89, S. 525-547.

Shevlin, Terry (1996): The value-relevance of nonfinancial information: A discussion. In: Journal of Accounting \& Economics, Vol. 22, S. 31-42.

Shin, H. S. (1994): News Management and the value of firms. In: Rand Journal of Economics, Vol. 25, S. 58-71.

Sieben, Günter/Schildbach, Thomas (1994): Betriebswirtschaftliche Entscheidungstheorie. 4. Auflage, Düsseldorf.

Siebert, Horst/Koop, Michael J. (1990): Institutional Competition. A Concept for Europe? In: Aussenwirtschaft, 45. Jg., S. 439-462.

Simko, Paul J. (1999): Financial Instrument Fair Values and Nonfinancial Firms. In: Journal of Accounting, Auditing and Finance, Vol. 14, S. 247-278. 
Simon, Herbert A. (1957): Models of Man: Social and Rational. New York et al.

Simon, Herbert A. (1976): Administrative Behavior: A Study of the Decision-Making Processes in Administrative Organization. 3. Auflage, New York et al.

Simon, Hermann V. (1899): Die Bilanzen der Aktiengesellschaften und der Kommanditgesellschaften auf Aktien. 3. Auflage, Berlin.

Singh, Danièle T. (1998): Incorporating cognitive aids into decision support systems: the case of the strategy execution process. In: Decision Support Systems, Vol. 24, S. 145-163.

Sinn, Hans-Werner (1997): The Selection Principle and Market Failure in Systems Competition. In: Journal of Public Economics, Vol. 66, S. 247-274.

Sinzig, Werner (1990): Datenbankorientiertes Rechnungswesen: Grundzüge einer EDVgestützten Einzelkosten- und Deckungsbeitragsrechnung. 3. Auflage, Berlin et al.

Skousen, K. Fred (1991): An Introduction to the SEC. 5. Auflage, Cincinnati.

Sloan, Richard G. (1996): Do Stock Prices Fully Reflect Information in Accruals and Cash Flows about Future Earnings? In: AccRev., Vol. 71, S. 289-315.

Smith, Adam (1776): An Inquiry into the Nature and Causes of The Wealth of Nations. Edited, with an introduction, notes, marginal summary, and enlarged index by Edwin Cannan, New York 1994.

Smith, John T. (1998): Responding to FASB Standard-Setting Proposals. In: Accounting Horizons, Vol. 12, S. 163-169.

Sobel, Joel (1985): A Theory of Credibility. In: Review of Economic Studies, Vol. 52, S. 557-573.

Sorter, George H. (1969): An "Events" Approach to Basic Accounting Theory. In: AccRev., Vol. 44, S. 12-19.

Sorter, George H./Gans, Martin S. (1974): Opportunities and Implications of the Report on Objectives of Financial Statements. In: JAR Supplement, Vol. 12, S. 1-20.

Sorter, George H./Ingberman, Monroe (1987): The Implicit Criteria for the Recognition, Quantification, and Reporting of Accounting Events. In: Journal of Accounting, Auditing and Finance, Vol. 2, S. 99-114

Speckbacher, Gerhard (1997): Shareholder Value und Stakeholder Ansatz. In: DBW, 57. Jg., S. 630-639.

Spence, A. Michael (1974): Market Signalling: Information Transfer in Hiring and Related Screening Processes. Cambridge et al. 
Spindler, Gerald (1998): Deregulierung des Aktienrechts? In: AG, 43. Jg., S. 53-74.

Stickel, Scott E. (1992): Reputation and Performance Among Security Analysts. In: JoF, Vol. 47, S. 1811-1836.

Stigler, George J. (1961): The Economics of Information. In: Journal of Political Economy, Vol. 69, S. 213-225.

Stigler, George J. (1964): Public Regulation of the Securities Markets. In: Journal of Business, Vol. 37, S. 117-142.

Stigler, George J. (1971): The Theory of economic regulation. In: Bell Journal of Economics and Management Science, Vol. 2, S. 3-21.

Stigler, George J. (1975): The citizen and the State: Essays on Regulation. Chicago et al.

Stiglitz, Joseph E. (1981): The Allocation Role of the Stock Market: Pareto Optimality and Competition. In: JoF, Vol. 36, S. 235-251.

Streim, Hannes (1985): Freie oder regulierte Rechnungslegung? Working Paper anläßlich des Vortrages vor dem Ausschuß „Unternehmensrechnung“ des Vereins für Socialpolitik, Trier.

Streit, Manfred E./Mussler, Werner (1994): The Economic Constitution of the European Community From Rome to Maastricht. In: Constitutional Political Economy, Vol. 5, S. 319-353.

Strieder, Thomas/Ammedick, Oliver (1999): Die Informationspflichten der Teilnehmer am SMAX In: FB, 1. Jg., S. 143-149.

Sürken, Silke (1999): Abgrenzung der wirtschaftlichen Einheit nach US-GAAP: Neuere Entwicklungen und Vergleich mit den deutschen Vorschriften. Frankfurt am Main et al.

Swieringa, Robert J./Weick, Karl F. (1982): An Assessment of Laboratory Experiments in Accounting. In: JAR Supplement, Vol. 20, S. 56-101.

Tas, Leo G. van der (1992): Evidence of EC financial reporting practice harmonization: The Case of deferred taxation. In: EAR, Vol. 1, S. 69-104.

Templin, Hans-Ulrich (1998): Unternehmensrisiko und Bilanzkennzahlen. Wiesbaden.

Teoh, Siew H. (1997): Information disclosure and voluntary contributions to public goods. In: Rand Journal of Economics, Vol. 28, S. 385-406.

Teoh, Siew H./Wong, T. J. (1993): Perceived Auditor Quality and the Earnings Response Coefficient. In: AccRev., Vol. 68, S. 346-366. 
Thomas, Arthur L. (1974): The Allocation Problem: Part Two. Sarasota.

Tirole, Jean (1988): The Theory of Industrial Organization. Cambridge et al.

Tollison, Robert D. (1982): Rent Seeking: A Survey. In: Kyklos, Vol. 35, S. 575-602.

Tollison, Robert D. (1988): Public Choice and Legislation. In: Virginia Law Review, Vol. 74, S. 339-371.

Tomaszewski, Claude (1999): Realoptionen in der strategischen Unternehmensbewertung. Dissertation Ruhr-Universität Bochum.

Trester, Jeffrey J. (1998): Venture Capital contracting under asymmetric information. In: Journal of Banking and Finance, Vol. 22, S. 675-699.

Trigueiros, Duarte/Taffler, Richard (1996): Neural Networks and Empirical Research in Accounting. In: Accounting and Business Research, Vol. 26, S. 347-355.

Trueman, Brett (1983): Optimality of the Disclosure of Private Information in a Production-Exchange Economy. In: JoF, Vol. 38, S. 913-924.

Truman, David B. (1971): The Governmental Process: Political Interests and Public Opinion. 2. Auflage, New York.

Uecker, Wilfred C. (1978): A Behavioral Study of Information System Choice. In: JAR, Vol. 16, S. 169-189.

Unser, Matthias/Oehler, Andreas (1998): Das Börsenhandelssystem XETRA. In: WiSt, 27. Jg., S. 463-468.

Upton, Wayne S. Jr. (1998): The Internet and Financial Information - Is there a Future here? In: FASB Status Report No. 299, Financial Accounting Series No. 183 vom 27. März 1998, S. 46.

Vatter, William J. (1963): Operating Confusion in Accounting: Two Reports or One? In: Journal of Business, Vol. 36, S. 290-301.

Vehn, A. ter (1929): Die Entwicklung der Bilanzauffassungen bis zum AHGB. In: Zeitung für Betriebswirtschaft, 5. Sonderheft.

Verrecchia, Robert E. (1982): The Use of mathematical Models in Financial Accounting. In: JAR Supplement, Vol. 20, S. 1-42.

Verrecchia, Robert E. (1983): Discretionary Disclosure. In: Journal of Accounting and Economics. Vol. 5, S. 179-194.

Verrecchia, Robert E. (1996): Disclosure and the cost of capital: A discussion. In: Journal of Accounting and Economics. Vol. 26, S. 271-283. 
Wagenhofer, Alfred (1990): Informationspolitik im Jahresabschluß: Freiwillige Informationen und strategische Bilanzanalyse. Heidelberg.

Wagenhofer, Alfred (1990a): Voluntary Disclosure With a Strategic Opponent. In: Journal of Accounting and Economics. Vol. 12, S. 341-363.

Wagenhofer, Alfred/Ewert, Ralf (1997): Unternehemensrechnung und ökonomische Theorie. In: Homo Oeconomicus, Band XIV, S. 31-62.

Wagenhofer, Alfred/Pirchegger, Barbara (1999): Nutzung des Internet für die Kommunikation von Finanzinformationen. In: DB, 52. Jg., S. 1409-1416.

Wagner, Franz W. (1998): Aufgabe der Maßgeblichkeit bei einer Internationalisierung der Rechnungslegung? In: DB, 51. Jg., S. 2073-2077.

Walker, Martin (1988): The Information Economics Approach to Financial Reporting. In: ABR, Vol. 18, S. 170-182.

Wallman, Steven M. (1995): The Future of Accounting and Disclosure in an evolving world: The need for a dramatic change. In: Accounting Horizons, Vol. 9, S. 8191.

Wallman, Steven M. (1996): The Future of Accounting and Financial Reporting Part II: The Colorized Approach. In: Accounting Horizons, Vol. 10, S. 138-148.

Wallman, Steven M. (1997): The Future of Accounting and Financial Reporting Part IV: Access Accounting. In: Accounting Horizons, Vol. 11, S. 103-116.

Walz, W. Rainer (1993): Ökonomische Regulierungstheorien vor den Toren des Bilanzrechts. In: Ökonomische Analyse des Bilanzrechts: Entwicklungslinien und Perspektiven, ZfbF-Sonderheft 32/93, Düsseldorf et al., S. 85-106.

Warfield, Terry D./Wild, John J./Wild, Kenneth L. (1995): Managerial Ownership, accounting choices, and informativeness of earnings. In: Journal of Accounting and Economics, Vol. 20, S. 61-91.

Watson, Joel (1996): Information Transmission When the Informed Party is Confused. In: Games and Economic Behavior, Vol. 12, S. 143-161.

Watts, Ross L./Zimmerman, Jerold L. (1979): The Demand for and the Supply of Accounting Theories: The Market for Excuses. In: AccRev., Vol. 54, S. 273-305.

Watts, Ross Lo/Zimmerman, Jerold L. (1986): Positive Accounting Theory. Englewood Cliffs.

Watts, Ross L./Zimmerman, Jerold L. (1990): Positive Accounting Theory: A Ten Years Perspective. In: AccRev., Vol. 65, S. 131-156. 
Weber, Max (1897): Börsengesetz. In: Handwörterbuch der Staatswissenschaften, zweiter Supplementband, hrsg, von Conrad, J./Elster, L./Lexis, W./Loening, Ed., Jena, S. 222-246.

Weber-Grellet, Heinrich (1997): Maßgeblichkeitsgrundsatz in Gefahr? In: DB, 50. Jg., S. 385-391.

Weber-Grellet, Heinrich (1999): Der Maßgeblichkeitsgrundsatz im Lichte aktueller Entwicklungen. In: BB, 54. Jg., S. 2959-2666.

Wedekind, Hartmut (1993): Kaufmännische Datenbanken. Mannheim et al.

Wegner, Gehrard (1996): Wirtschaftspolitik zwischen Selbst- und Fremdsteuerung: Ein neuer Ansatz. Baden-Baden.

Weingast, Barry R. (1984): The congressional-bureaucratic system: a principle agent perspective (with applications to the SEC). In: Public Choice, Vol. 44, S. 147-191.

Welling, Michael (1999): Möglichkeiten und Grenzen einer geschäftsbeziehungsorientierten Deckungsbeitragsrechnung. Institut für Unternehmungsführung und Unternehmensforschung der Ruhr-Universität Bochum, Arbeitsbericht Nr. 77.

Wengert, Georg/Wengert, Katharina (1997): Deutsches Gesetz zur Kontrolle und Transparenz im Unternehmensbereich im Referentenentwurf: Neue Perspektiven für den deutschen Wirtschaftsprüfer. In: Der Schweizer Treuhänder, 71. Jg., S. 171175.

Werkheim, Michael (1998): Die Diskriminanzanalyse als Instrument der Bonitätsprüfung. In: BBK, o. Jg.,Fach 19, S. 449-456

Wessling, Ewald (1991): Individuum und Information: Die Erfassung von Information und Wissen in ökonomischen Handlungstheorien. Tübingen.

Westarp, Falk von/Ordelheide, Dieter/Stubenrath, Michael/Buxmann, Peter/König, Wolfgang (1999): Internet-Based Corporate Reporting: Filing the Standardization Gap. In: Proceedings of the 32nd Hawaii International Conference on System Sciences, Maui, S. 1-10

Wheeler, John T. (1970): Accounting Theory and Research in Perspective. In: AccRev., Vol. 45, S. 1-10.

White, Gerald I./Sondhi, Ashwinpaul C./Fried, Don (1997): The Analysis and Use of Financial Statements. 2. Auflage, New York et al.

Wied-Nebbeling, Susanne (1997): Markt- und Preistheorie. 3. Auflage, Berlin et al. 
Wiener, Heinrich (1873): Zur Reform des Actiengesellschaftswesens: 3 Gutachten auf Veranlassung der Eisenacher Versammlung zur Besprechung der socialen Frage. Leipzig.

Wiese, Harald (1998): Externe Effekte. In: WiSt, 27. Jg, S. 404-408.

Wilde, Christian (1998): Informationsrechte und Informationspflichten im Gefüge der Gesellschaftsorgane. In: ZGR, 27. Jg., S. 423-465.

Willenborg, Michael (1999): Empirical Analysis of the Economic Demand for Auditing in the Initial Public Offerings Market. In: JAR, Vol. 37, S. 225-238.

Williamson, Oliver E. (1973): Markets and Hierarchies: Some Elementary Considerations. In: AER, Vol. 63, S. 316-325.

Williamson, Oliver E. (1976): Franchise bidding for natural monopolies: in general and with respect to CATV. In: Bell Journal of Economics, Vol. 7, S. 73-104.

Williamson, Oliver E. (1979): Transaction-Cost Economics: The Govemance of Contractual Relations. In: Journal of Law and Economics, Vol. 22, S. 233-261.

Williamson, Oliver E. (1981): The Modern Corporation: Origin, Evolution, Attributes. In: Journal of Economic Literature, Vol. 19, S. 1537-1568.

Williamson, Oliver E. (1983): Credible Commitments: Using Hostages to Support Exchange. In: AER, Vol. 73, S. 519-540.

Williamson, Oliver E. (1985): Economic Institutions of Capitalism. New York et al.

Williamson, Oliver E. (1991): Comparative Economic Organization: The Analysis of Discrete Structural Alternatives. In: Administrative Science Quarterly, Vol. 36, S. 269-296.

Wilson, Charles A. (1977): A Model of Insurance Markets with Incomplete Information. In: Journal of Economic Theory, Vol. 16, S. 167-207.

Windbichler, Christine (1988): Handelsrechtliche Publizität durch private Datenverbreiter. In: CR, 4. Jg., S. 447-452.

Wittmann, Waldemar (1959): Unternehmung und unvollkommene Information: Unternehmerische Voraussicht - Ungewißheit und Planung. Köln et al.

Woeckener, Bernd (1994): Dynamische Marktprozesse bei Netzwerk-Externalitäten und begrenzter Rationalität. In: Probleme der unvollkommenen Konkurrenz, hrsg. von Ott, Alfred E., Tübingen et al., S. 235-254.

Wöhe, Günter (1999): Die Entwicklung der Rechnungslegungsvorschriften der Kapitalgesellschaften im 20. Jahrhundert. In: Internationale Rechnungslegung: Fest- 
schrift für Professor Dr. Claus-Peter Weber zum 60. Geburtstag, hrsg. von Küting, Karlheinz/Langenbucher, Günther. Stuttgart, S. 65-90.

Wolf, Peter (1997): Finanzbuchhaltung aus Sicht der R/3-Referenzmodelles. In: SAP R/3 Anwendungen in der Praxis: Anwendung und Steuerung betriebswirtschaftlichintegrierter Geschäftsprozesse mit ausgewählten R/3-Modulen, hrsg. von Wenzel, Paul, Braunschweig et al., S. 67-110.

Wolk, Harry I./Francis, Jere R./Tearney, Michael G. (1991): Accounting Theory: A Conceptual and Institutional Approach. 3. Auflage, Cincinatti.

Wysocki, Klaus von (1976): Ergebnisse empirischer Untersuchungen über das Publizitätsverhalten deutscher Unternehmen. In: ZfbF, 28. Jg., S. 744755.

Zabeshailo, M. I./Finn, V. K. (1996): Intelligent Information Systems. In: International Forum on Information and Documentation, Vol. 21, No. 2, S. 21-31.

Zeff, Stephen A. (1972): Chronology of Significant Developments in the Establishment of Accounting Policies in the United States 1926-1972. In: JAR, Vol. 10, S. 217232.

Zeff, Stephen A. (1978): The Rise of Economic Consequences. In: JoA, Vol. 146, December, S. 56-63.

Zeff, Stephen A. (1984): Some Junctures in the Evolution of the Process of Establishing Accounting Principles in the U.S.A. In: AccRev., Vol. 59, S. 447-468.

Ziegler, Hasso (1994): Neuorientierung des internen Rechnungswesens für das Unternehmens-Controlling im Hause Siemens. In: ZfbF, 46. Jg., S. 175-188.

Zimmer, Daniel (1998): Neue Vorschriften für den nicht organisierten Kapitalmarkt: Eine Bewertung der den "grauen Kapitalmarkt" betreffenden Bestimmungen der 6. KWG-Novelle. In: DB, 51. Jg, S. 969-973.

Zimmer, Daniel (1998a): Das Gesetz zur Kontrolle und Transparenz im Untemehmensbereich. In: NJW, 51. Jg., S. 3521-3554. 
Joachim Gassen - 978-3-631-75496-2

Downloaded from PubFactory at 01/11/2019 04:12:56AM

via free access 


\title{
Verzeichnis der Rechtsquellen,
}

\section{Rechnungslegungspublizitätsnormen, Urteile}

\author{
und technischen Normen
}

\section{A. Rechtsquellen}

\section{Deutschland}

ADHGB: Einführungsgesetz zum Allgemeinen Deutschen Handelsgesetzbuch vom 24. Juni 1861, GS. 1861, S. 449.

AktG 1937: Gesetz über die Aktiengesellschaften und Kommanditgesellschaften auf Aktien vom 30. Januar 1937, RGBl. I 1937, S. 107.

AktG: Aktiengesetz vom 6. September 1965, BGBl. I 1965, S. 1089.

Aktienrechtsnovelle 1870: Gesetz betreffend die Kommanditgesellschaften auf Aktien und die Aktiengesellschaften vom 11. Juni 1870, BGBl. des Norddeutschen Bundes 1870, S. 375 .

Aktienrechtsnovelle 1884: Gesetz, betreffend die Kommanditgesellschaften auf Aktien und die Aktiengesellschaften vom 18. Juli 1884, RGBl. 1884, S. 123.

Aktienrechtsnovelle 1931: Verordnung des Reichspräsidenten über Aktienrecht, Bankenaufsicht und über eine Steueramnestie, RGBl. I 1931, S. 493.

Aktienregulativ 1856: Zirkularverfügung wegen der bei der Bestätigung der Statuten von Aktiengesellschaften festzuhaltenden allgemeinen Grundsätze vom 29.3.1856.

ALR: Allgemeines Landrecht für die Preußischen Staaten von 1794, Textausgabe, Frankfurt am Main et al. 1970.

BiRiLiG: Gesetz zur Durchführung der Vierten, Siebenten und Achten Richtlinie des Rates der Europäischen Gemeinschaften zur Koordinierung des Gesellschaftsrechts (Bilanzrichtlinien-Gesetz) vom 19. Dezember 1985, BGBl. I 1985, S. 2355.

Börsengesetzreform 1908: Gesetz, betreffend Änderung des Börsengesetzes vom 8. Mai 1908, RGBl. 1908, S. 183. 
Börsengesetzreform 1934: Gesetz zur Änderung des Börsengesetzes vom 5. März 1934, RGBl. I 1934, S. 169.

BörsG 1896: Börsengesetz vom 22. Juni 1986, RGBl. 1896, S. 183.

EStG: Einkommensteuergesetz 1997 in der Fassung der Bekanntmachung vom 16. April 1997, BGBl. I 1997, S. 821.

Gesetz über die Aktiengesellschaften: Preußisches Gesetz über die Aktiengesellschaften vom 9.11.1843, GS. 1843 , S. 341.

HGB: Handelsgesetzbuch vom 10. Mai 1897, RGB1. 1897, S. 219.

KapAEG: Gesetz zur Verbesserung der Wettbewerbsfähigkeit deutscher Konzerne an Kapitalmärkten und zur Erleichterung der Aufnahme von Gesellschafterdarlehen (Kapitalaufnahmeerleichterungsgesetz) vom 20. April 1998, BGBl. I 1998, S. 707.

KonTraG: Gesetz zur Kontrolle und Transparenz im Unternehmensbereich vom 27. April 1998, BGBI. I 1998, S. 786.

PublG: Gesetz über die Rechnungslegung von bestimmten Unternehmen und Konzernen (Publizitätsgesetz) vom 15. August 1969, BGBl. I 1969, S. 1189

StückAG: Gesetz über die Zulassung von Stückaktien (Stückaktiengesetz) vom 25. März 1998, BGBl. I 1998, S. 590.

VerkProspG: Wertpapier-Verkaufsprospektgesetz (VerkaufsprospektG) vom 17. Juli 1996, BGBl. I 1996, S. 1047.

VerkProspVO: Verordnung über Wertpapier-Verkaufsprospekte (VerkaufsprospektVerordnung) vom 17. Dezember 1990, BGBl. I 1990, S. 2869.

WpHG: Gesetz über den Wertpapierhandel (Wertpapierhandelsgesetz) vom 26. Juli 1994, BGBl. I 1994, S. 1749.

\section{Europäische Union}

Achte EG-Richtlinie: Achte Richtlinie des Rates vom 10. April 1984 aufgrund von Artikel 54 Absatz $3 g$ des Vertrags über die Zulassung der mit der Pflichtprüfung der Rechnungslegungsunterlagen beauftragten Personen, 84/253/EWG, ABl. EG Nr. L 126/20 vom 12. Mai 1984.

Emissionsprospektrichtlinie: Richtlinie des Rates vom 17. April 1989 zur Koordinierung der Bedingungen für die Erstellung, Kontrolle und Verbreitung des Prospekts, der im Falle öffentlicher Angebote von Wertpapieren zu veröffentlichen ist, 89/298/EWG, ABl. EG Nr. L 124/8 vom 5.5.1989.

Erste EG-Richtlinie: Erste Richtlinie des Rates vom 9. März 1968 zur Koordinierung der Schutzbestimmungen, die in den Mitgliedstaaten den Gesellschaften im Sinne des Artikel 
58 Absatz 2 des Vertrages im Interesse der Gesellschafter sowie Dritter vorgeschrieben sind, um diese Bestimmungen gleichwertig zu gestalten, 68/151/EWG, ABl. EG Nr. L 65/8 vom 14.3.1968.

GmbH \& Co-Richtlinie: Richtlinie des Rates vom 8. November 1990 zur Änderung der Richtlinien 78/660/EWG und 83/349/EWG über den Jahresabschluß bzw. den konsolidierten Abschluß hinsichtlich ihres Anwendungsgebiets, 90/605/EWG, ABl. EG Nr. $\mathrm{L} / 317 / 60$ vom 16.11 .1990 .

Insiderrichtlinie: Richtlinie des Rates vom 13. November 1989 zur Koordinierung der Vorschriften betreffend Insider-Geschäfte, 89/592/EWG, ABl. EG Nr. L 334/30 vom 18.11.1989.

Prospektrichtlinie: Richtlinie des Rates vom 17. März 1980 zur Koordinierung der Bedingungen für die Erstellung, die Kontrolle und die Verbreitung des Prospekts, der für die Zulassung von Wertpapieren zur amtlichen Notierung an einer Wertpapierbörse zu veröffentlichen ist, 80/390/EWG, ABl. EG Nr. L 100/1 vom 17. April 1980.

Siebente EG-Richtlinie: Siebente Richtlinie des Rates vom 13. Juni 1983 aufgrund von Artikel 54 Abs. $3 g$ des Vertrages über den konsolidierten Abschluß, 83/349/EWG, ABl. EG Nr. L 193/1 vom 18. Juli 1983.

Transparenzrichtlinie: Richtlinie des Rates vom 12. Dezember 1988 über die bei Erwerb und Veräußerung einer bedeutenden Beteiligung an einer börsennotierten Gesellschaft zu veröffentlichenden Informationen, 88/627/EWG, ABl. EG Nr. L 348/62 vom 17.12.1988.

Vierte EG-Richtlinie: Vierte Richtlinie des Rates vom 25. Juli 1978 auf Grund von Artikel 54 Abs. $3 \mathrm{~g}$ des Vertrages über den Jahresabschluß von Gesellschaften bestimmter Rechtsformen, 78/660/EWG, Abl. EG Nr. L 222/11 vom 14. August 1978.

Wertpapierdienstleistungsrichtlinie: Richtlinie des Rates vom 10. Mai 1993 über Wertpapierdienstleistungen, 93/22/EWG, Abl. EG Nr. L 141/27 vom 11.6.1993.

Zulassungsrichtlinie: Richtlinie des Rates vom 5. März 1979 zur Koordinierung der Bedingungen für die Zulassung von Wertpapieren zur amtlichen Notierung an einer Wertpapierbörse, 79/279/EWG, Abl. EG Nr. L 66/21 vom 16. März 1979.

Zwischenberichtsrichtlinie: Richtlinie des Rates vom 15. Februar 1982 über regelmäßige Informationen, die von Gesellschaften zu veröffentlichen sind, deren Aktien zur amtlichen Notierung an einer Wertpapierbörse zugelassen sind, 82/121/EWG, Abl. EG Nr. L 48/26 vom 22. Februar 1987. 


\section{Ausland}

Cal.Gen.Corp.L.: California Corporations Code, devision 1 (section 100-2319) as amended by act of the California Legislature, 1975 -76 Regular Session, effective January 1, 1977.

Code de Commerce: Code de Commerce von 1807. Relevante Teile für die Rechnungslegungspublizität finden sich in Barth (1953).

Const.: The Constitution of the United States, September 28, 1787.

Del.Gen.Corp.L.: Delaware General Corporation Law, Delaware Code, Title 8, \\ 101 619. Abgedruckt in Merkt (1991).

N.Y.Bus.Corp.L.: New York State Business Corporation Law, New York State Consolidated Laws, Chapter 4, Secs. 101-2001.

Ordonnance de Commerce: Ordonnance des Louis XIV pour le Commerce vom 23.3.1673. Relevante Teile für die Rechnungslegungspublizität finden sich in Barth (1953).

Public Utility Holding Company Act: Public Utility Holding Company Act of 1935 (Aug 26, 1935), 49 Stat. 838, 15 U.S. Code, Secs. 79 to $79 z-6$.

R.M.B.C.A.: Revised Model Business Corporation Act. In: Model Business Corporation Act Annotated by the Committee on Corporate Laws, ABA Section of Business Law, 3. Auflage, Chicago 1994, Supplement 1997.

SA: Securities Act of 1933 (May 27, 1933), 48 Stat. 74, 15 U.S. Code, Secs. 77a-77aa.

SEA: Securities Exchange Act of 1934 (June 6, 1934), 48 Stat. 881, 15 U.S. Code, Secs. 78a-78ji.

Securities Litigation Reform Act: Securities Litigation Reform Act of 1995 (Dec 22, 1995), 109 Stat. 737, 15 U.S. Code, Sec. 78a note.

Unif.S.A. National Conference of Commissioners on Uniform State Laws: Uniform Securities Act (1985) with 1988 Amendments, URL: http://www.law.upenn.edu/ bll/ulc/fnact99/usa88.htm, Stand 25.11.1999.

\section{B. Rechnungslegungspublizitätsnormen}

\section{Deutschland}

HFA 1/1978: Stellungnahme des HFA 1/1978: Die Kapitalflußrechnung als Ergänzung des Jahresabschlusses, WPg, 21. Jg., S. 207-208. 
HFA 1/1995: Stellungnahme HFA 1/1995: Die Kapitalflußrechnung als Ergänzung des Jahres- und Konzernabschlusses, Fachnachrichten des IDW, o. Jg., S. 72-76.

Infoordner Neuer Markt: Gruppe Deutsche Börse, Neuer Markt, Infoordner. URL: http://www.neuer-markt.de/nm30/start.html, Stand 01.05.1999, Option Download Infoordner Neuer Markt.

Regelwerk Neuer Markt: Gruppe Deutsche Börse, Neuer Markt, Regelwerk. URL: http://www.neuer-markt.de/nm30/start.html, Stand 01.05.1999, Option Download Infoordner Neuer Markt.

Teilnahmebedingungen SMAX: Deutsche Börse, SMAX Teilnahmebedingungen, Stand: 27. Januar 1999. URL: http://www.smax.de/INTERNET/SMAX/smax.nsf, Stand 17.11.1999, Option Download, Teilnahmebedingungen SMAX.

\section{International/USA}

APB Statement No. 4: APB Accounting Principles, Original Pronouncements as of Febuary 1, 1971, Vol. 2, New York 1971.

ASR Nr. 4 und ASR Nr. 150: Wiedergegeben und aufgegangen in FRR Nr. 1, Sec. 101: Statement of Policy on the Establishment and Improvement of Accounting Principles and Standards, 1997 SEC Rules and Regulations.

IAS: LASC, International Accounting Standards, London 1999.

Securities Act Release Nr. 33-7386: 17 CFR Parts 210, 228, 229, 239, 240, and 249 (Release Nos. 33-7386; 34-38223; IC-22487; FR-48; International Series No. 1047; File No. S735-95). Disclosure of Accounting Policies for Derivative Financial Instruments and Derivative Commodity Instruments and Disclosure of Quantitative and Qualitative Information About Market Risk Inherent in Derivative Financial Instruments, Other Financial Instruments, and Derivative Commodity Instruments, January 31, 1997. URL: http://www.sec.gov/rules/final/33-7386.txt, Stand 25.11.1999.

Securities Act Release Nr. 33-7558: 17 CFR Parts 231, 241, 271, 276 (Release Nos. 337558; 34-40277; IA-1738; IC-23366; International Series Release No. 1149). Statement of the Commission regarding Disclosure of Year 2000 Issues and Consequences by Public Companies, Investment Advisers, Investment Companies and Municipal Securities Issuers, July 29, 1998. URL: http://www.sec.gov/rules/concept/33-7558.htm, Stand 10.11.1999.

SFAC: FASB, Original Pronouncements 1999/2000 Edition: Accounting Standards as of June $1^{\text {st }}$, Vol. 1 und 2, Norwalk 1999. 
SFAS 137: Statement of Financial Accounting Standards No. 137; Accounting for Derivative Instruments and Hedging Activities: Deferral of the Effective Date of FASB Statement No. 133. An amendment of FASB Statement No. 133, No. 196-E, June 1999.

SFAS: FASB, Original Pronouncements 1999/2000 Edition: Accounting Standards as of June $1^{\text {st }}$, Vol. 1 und 2, Norwalk 1999.

\section{Urteile}

ROHG (1873): Entscheidungen des ROHG. Band XII.

\section{Technische Normen}

CGI/1.1: The CGI Specification CGI version 1.1. URL: http://hoohoo.ncsa.uiuc.edu/ cgi/interface.html.

DIN 66001: Informationsverarbeitung: Sinnbilder und ihre Anwendung. In: SoftwareEntwurf Programmierung Dokumentation Schaltzeichen: Normen (Informationsverarbeitung 4), 2. Auflage, Stand der Normen 28. Februar 1985, hrsg. vom DIN Deutsches Institut für Normung e.V., Berlin et al., S. 154-174.

draft-ietf-http-v11-spec-rev-06: HTTP-Working Group of the IETF, Internet-draft "Hypertext Transfer Protocol - HTTP/1.1", Fielding, R./Gettys, J./Mogul, J. C./Frystyk, H./Masinter, L./Leach, P./Bemers-Lee, T., November 18, 1998, Eypiration date: May 18, 1999. URL: http://www.w3c.org/Protocols/HTTP/1.1/draft-ietf-http-v11-spec-rev-06.txt, Stand 26.11.1999.

ISO: ISO standards handbook, Genf.

REChtml32: HTML 3.2 Reference Specification, W3C Recommendation 14Jan-1997, Ragget, Dave. URL: http://www.w3c.org/TR/REC-html32.html, Stand 26.11.1999.

REChtm140: HTML 4.0 Specification, W3C Recommendation revised on 24Apr-1998, Ragget, Dave/Le Hors, Amaud/Jacobs, Ian, URL: http://www.w3c.org/TR/REC-html40/, Stand 26.11.1999.

REC-xml-19980210: Extensible Markup Language (XM) 1.0, W3C Recommendation 10 Feb-98, Bray, Tim/Paoli, Jean/Sperberg-McQueen, C. M., URL: http://www.w3c.org/TR/ REC-xml, Stand 26.11.1999.

RFC 1122: Requirements for Internet Hosts - Communication Layers, hrsg. von Braden, R., October 1989. 
RFC 1866: Proposed Standard "Hypertext Markup Language - 2.0", Berners-Lee, T./Connoly, D., November 1995.

RFC 1945: Informational "Hypertext Transfer Protocol - HTTP/1.0", Berners-Lee, T./Fielding, R./Frystyk, R., May 1996.

RFC 2068: Proposed Standard "Hypertext Transfer Protocol - HTTP/1.1", Fielding, R./Gettys, J./Mogul, J./Frystyk, H./Berners-Lee, T., January 1997. 


\section{Bochumer Beiträge zur Unternehmungsführung und Unternehmensforschung \\ Herausgegeben vom Direktorium des Instituts für Unternehmungsführung und Unternehmensforschung der Ruhr-Universităt Bochum}

Band 1 Busse von Colbe, Walther/Mattessich, Richard (Hrsg.): Der Computer im Dienste der Unternehmungsführung (1968)

Band 2 Busse von Colbe, Walther/Meyer-Dohm, Peter (Hrsg.): Unternehmerische Planung und Entscheidung (1969)

Band 3 Anthony, Robert N.: Harvard-Fälle aus der Praxis des betrieblichen Rechnungswesens. Herausgegeben von Richard V. Mattessich unter Mitarbeit von Klaus Hermberger und Wolf Lange (1969)

Band 4 Mattessich, Richard: Die wissenschaftlichen Grundlagen des Rechnungswesens (1970)

Band 5 Schweim, Joachim: Integrierte Unternehmungsplanung (1969)

Band 6 Busse von Colbe, Walther (Hrsg.): Das Rechnungswesen als Instrument der Unternehmungsführung (1969)

Band 7 Domsch, Michel: Simultane Personal- und Investitionsplanung im Produktionsbereich (1970)

Band 8 Leunig, Manfred: Die Bilanzienung von Beteiligungen. Eine bilanztheoretische Untersuchung (1970)

Band 9 Franke, Reimund: Betriebsmodelle. Rechensystem für Zwecke der kurzfristigen Planung, Kontrolle und Kalkulation (1972)

Band 10 Wittenbrink, Hartwig: Kurzfristige Erfolgsplanung und Erfolgskontrolle mit Betriebsmodellen (1975)

Band 11 Lutter, Marcus (Hrsg.): Recht und Steuer der intemationalen Unternehmensverbindungen (1972)

Band 12 Niebling, Helmut: Kurzfristige Finanzrechnung auf der Grundlage von Kosten- und Erlösmodellen (1973)

Band 13 Perlitz, Manfred: Die Prognose des Untemehmenswachstums aus Jahresabschlüssen deutscher Aktiengesellschaften (1973)

Band 14 Niggemann, Walter: Optimale Informationsprozesse in betriebswirtschaftlichen Entscheidungssituationen (1973)

Band 15 Reichardt, Harald: Der aktienrechtliche Abhăngigkeitsbericht unter ökonomischen Aspekten (1974)

Band 16 Backhaus, Klaus: Direktvertrieb in der Investitionsgüterindustrie - Eine Marketing-Entscheiung (1974)

Band 17 Plinke, Wulff: Kapitalsteuerung in Filialbanken (1975)

Band 18 Steffen, Rainer: Produktionsplanung bei Fließbandfertigung (1977)

Band 19 Kolb, Jürgen: Industrielle Erlösrechnung - Grundlagen und Anwendungen (1978)

Band 20 Busse von Colbe, Walther/Lutter, Marcus (Hrsg.): Wirtschaftsprüfung heute: Entwicklung oder Reform? (1977)

Band 21 Uphues, Peter: Untemehmerische Anpassung in der Rezession (1979) 
Band 22 Gebhardt, Günther: Insolvenzprognosen aus aktienrechtlichen Jahresabschlüssen (1980)

Band 23 Domsch, Michel: Systemgestützte Personalarbeit (1980)

Band 24 Schmied, Volker: Altemativen der Arbeitsgestaltung und ihre Bewertung (1982)

Band 25 Wäscher, Gerhard: Innerbetriebliche Standortplanung bei einfacher und mehrfacher Zielsetzung (1982)

Band 26 Weber, Martin: Entscheidungen bei Mehrfachzielen - Verfahren zur Unterstūtzung von Individual- und Gruppenentscheidungen (1983)

Band 27 Kroesen, Alfred: Instandhaltungsplanung und Betriebsplankostenrechnung (1983)

Band 28 Plinke, Wulf: Erlösplanung im industriellen Anlagengeschäft (1985)

Band 29 Chamoni, Peter: Simulation störanfälliger Systeme (1986)

Band 30 Aming, Andreas: Die wirtschaftliche Bewertung der Zentrenfertigung - Dargestellt am Beispiel einer Fertigungsinsel (1987)

Band 31 Gebhardt, Günther: Finanzielle Planung und Kontrolle bei internationaler Unternehmenstātigkeit

Band 32 Markiewicz, Michael: Ersatzteildisposition im Maschinenbau - Betriebswirtschaftliche Methoden der Planung und Überwachung (1988)

Band 33 Pellens, Bernd: Der Informationswert von Konzernabschlüssen - Eine empirische Untersuchung deutscher Börsengesellschaften (1989)

Band 34 Mrotzek, Rüdiger: Bewertung direkter Auslandsinvestitionen mit Hilfe betrieblicher Investitionskalküle (1989)

Band 35 Deppe, Joachim: Quality Circle und Lernstatt - Ein integrativer Ansatz (1989, 3. Auflage 1993)

Band 36 Rademacher, Michael: Arbeitszeitverkürzung und -flexibilisierung - Formen und betriebliche Auswirkungen (1990)

Band 37 Kaiser, Klaus: Kosten- und Leistungsrechung bei automatisierter Produktion (1991, 2. Auflage 1993)

Band 38 Müller, Hermann: Industrielle Abfallbewältigung - Entscheidungsprobleme aus betriebswirtschaftlicher Sicht (1991)

Band 39 Schömer, Peter: Gesetzliches Insiderhandelsverbot - Eine ordnungspolitische Analyse (1991)

Band 40 Bentler, Martin: Grundsätze ordnungsmäßiger Bilanzierung für die Equitymethode (1991)

Band 41 Brüggerhoff, Jürgen: Management von Desinvestitionen (1992)

Band 42 Bröker, Erich W.: Erfolgsrechnung im industriellen Anlagengeschäft - Ein dynamischer Ansatz auf Zahlungsbasis - (1993)

Band 43 Frankenberg, Peter: Transnationale Analyse US-amerikanischer und deutscher Jahresabschlüsse - Eine theoretische und empirische Untersuchung (1993)

Band 44 Kleinaltenkamp, Michael: Standardisierung und Marktprozeß - Entwicklungen und Auswirkungen im CIM-Bereich (1993)

Band 45 Pellens, Bernhard: Aktionärsschutz im Konzern - Empirische und theoretische Analyse der Reformvorschläge der Konzernverfassung (1994)

Band 46 Reckenfelderbäumer, Martin: Marketing-Accounting im Dienstleistungsbereich - Konzeption eines prozeßkostengestützten Instrumentariums (1995) 
Band 47 Knittel, Friedrich: Technikgestützte Kommunikation und Kooperation im Büro. Entwicklungshindemisse - Einsatzstrategien - Gestaltungskonzepte (1995)

Band 48 Riezler, Stephan: Lebenszyklusrechnung - Instrument des Controlling strategischer Projekte (1996)

Band 49 Schulte, Jöm: Rechnungslegung und Aktienkursentwicklung - Erklärung und Prognose von Aktienrenditen durch Einzel- und Konzernabschlußdaten (1996)

Band 50 Muhr, Martin: Zeitsparmodelle in der Industrie - Grundlagen und betriebswirtschaftliche Bedeutung mehrjähriger Arbeitszeitkonten (1996)

Band 51 Brotte, Jörg: US-amerikanische und deutsche Geschäftsberichte. Notwendigkeit, Regulierung und Praxis jahresabschlußergänzender Informationen (1997)

Band 52 Gersch, Martin: Vernetzte Geschäftsbeziehungen. Die Nutzung von EDI als Instrument des Geschäftsbeziehungsmanagement (1998)

Band 53 Währisch, Michael: Kostenrechnungspraxis in der deutschen Industrie. Eine empirische Studie (1998)

Band 54 Völkner, Pér: Modellbasierte Planung von Geschäftsprozeßabläufen (1998)

Band 55 Fülbier, Rolf Uwe: Regulierung der Ad-hoc-Publizität. Ein Beitrag zur ökonomischen Analyse des Rechts (1998)

\section{Band 1 - 55 erschienen beim Gabler Verlag Wiesbaden}

Band 56 Ane-Kristin Reif-Mosel: Computergestützte Kooperation im Büro. Gestaltung unter Berücksichtigung der Elemente Aufgabe, Struktur, Technik und Personal (2000)

Band 57 Claude Tomaszewski: Bewertung strategischer Flexibilität beim Unternehmenserwerb. Der Wertbeitrag von Realoptionen (2000)

Band 58 Thomas Erler: Business Objects als Gestaltungskonzept strategischer Informationssystemplanung (2000)

Band 59 Joachim Gassen: Datenbankgestützte Rechnungslegungspublizität. Ein Beitrag zur Evolution der Rechnungslegung (2000)

Band 60 Frauke Streubel: Organisatorische Gestaltung und Informationsmanagement in der lernenden Untemehmung. Bausteine eines Managementkonzeptes organisationalen Lernens (2000) 


\section{Informationsmanagement im Wandel}

\section{Praxisorientierte Lösungsansätze und Managementmodelle zur Bewältigung von Veränderungen im Informationsma- nagement}

Frankfurt/M., Berlin, Bern, New York, Paris, Wien, 1999.

LXVIII, 410 S., zahlr. Tab. u. Graf.

Europäische Hochschulschriften: Reihe 5,

Volks- und Betriebswirtschaft. Bd. 2422

ISBN 3-631-34212-8 • br. DM 128. -*

Die seit Jahren zu beobachtende Beschleunigung der Veränderungen in unseren Arbeits- und Wirtschaftsprozessen verlangt vom Informationsmanagement ein Umdenken. Das in dieser Arbeit geforderte veränderungsorientierte, strategisch-dynamische Informationsmanagement versucht, die vielfachen Schwächen des Informationsmanagements in der Unternehmenspraxis zu heilen und die erkannten Schwächen in Stärken umzukehren. Auf Basis von Situationen, Schwachstellen und Veränderungen im Informationsmanagement werden praxisorientierte Lösungsansätze und Managementmodelle erarbeitet. Diese sind eine Orientierungsleitlinie für Geschäftsfeldund IT-Manager und erleichtern den Übergang von einem status-quoorientierten zu einem veränderungsorientierten Informationsmanagement.

Aus dem Inhalt: Informationsmanagement im Wandel · Grundlagen für das Informationsmanagement - Situation und Schwachstellen des Informationsmanagements in der Praxis · Veränderungen im Informationsmanagement . Lösungsansätze zur Bewältigung von Veränderungen im Informationsmanagement · Managementmodelle zur Bewältigung von Veränderungen im Informationsmanagement

Frankfurt/M - Berlin · Bern · New York · Paris · Wien

Auslieferung: Verlag Peter Lang AG

Jupiterstr. 15, CH-3000 Bern 15

Telefax (004131) 9402131

*inklusive Mehrwertsteuer

Preisänderungen vorbehalten 
Joachim Gassen - 978-3-631-75496-2

Downloaded from PubFactory at 01/11/2019 04:12:56AM

via free access 